\title{
Internet et politique en Chine
}

Séverine Arsène

Manuscrit publié par Karthala, 2011

Version auteur avant travail éditorial 
Séverine Arsène - Internet et politique en Chine - version auteur

\section{Remerciements}

Cet ouvrage est issu d'une thèse de doctorat soutenue à l'IEP de Paris en décembre 2009. Je dois les plus chaleureux remerciement à Jean-Philippe Béja, mon directeur de thèse, qui a grandement contribué à l'aboutissement de cette recherche par son impressionnante connaissance de la Chine, ses conseils méthodologiques, son soutien et sa disponibilité exemplaires. Il a su me laisser suivre des intuitions théoriques qu'il ne partageait pas toujours, non sans me porter une contradiction stimulante, à laquelle j'espère avoir répondu de manière convaincante.

Mes remerciements vont également aux membres de mon jury de thèse, Françoise Mengin (présidente), Loïc Blondiaux, Luigi Tomba et Chantal de Gournay, qui, par leurs commentaires, leurs recommandations et leurs conseils, ont joué un rôle non négligeable dans la transformation de ce manuscrit en livre. Sur ce point, ma gratitude va naturellement à JeanFrançois Bayart qui a bien voulu accueillir cet ouvrage dans la collection Recherches Internationales qu'il dirige.

Cette enquête n'aurait pas été possible sans le soutien financier, mais aussi l'émulation scientifique que j'ai trouvés auprès d'Orange Labs, à Issy-les-Moulineaux et à Pékin. Je remercie Dominique Cardon, Damien Schaepelynck, Jean-François Doulet, Shang Dan, Tong Hui, et Zhao Lianfei pour avoir défendu ce projet, et pour les conversations passionnantes que nous avons échangées au quotidien.

Cinquante-sept personnes ont accepté de me consacrer deux heures de leur temps pour parler de leurs usages d'Internet. Ils n'imaginent pas toute la valeur des mots qu'ils m'ont livrés. Je leur suis infiniment reconnaissante pour la confiance qu'il m'ont accordée.

De nombreuses rencontres avec d'autres chercheurs m'ont appris que la recherche n'est pas du tout une aventure solitaire. Je tiens à remercier Stéphanie Wojcik, Thierry Vedel et les membres du réseau de recherche DEL pour leurs conseils, pistes de recherche et relectures critiques, qui m'ont été très utiles. Surtout, je remercie les membres de notre groupe de travail à Sciences-po, et plus particulièrement Yasmine Berriane, Marie-Laure Geoffray, Soline Laplanche et Amélie Le Renard, qui détiennent la palme des échanges comparatifs 
Séverine Arsène - Internet et politique en Chine - version auteur

improbables, ainsi que tous les jeunes chercheurs qui peuplent la Bibliothèque Nationale et celle de l'école doctorale de l'IEP. 
Séverine Arsène - Internet et politique en Chine - version auteur

\section{Avant-propos}

A la Fête de l'Humanité de septembre 2009, le stand du Quotidien du Peuple présente une image très officielle de la Chine actuelle. De grands panneaux thématiques décrivent une Chine florissante, en plein développement économique, et ouverte sur l'international. L'un de ces panneaux détaille la catégorie "Démocratie". En son milieu, figure une photographie de Hu Jintao, souriant, participant à une discussion en ligne avec des internautes chinois ${ }^{1}$. Elle est accompagnée du texte explicatif suivant: "La participation et la délibération du peuple dans les affaires publiques via Internet a connu des progrès. Cela est encouragé par le président $\mathrm{Hu}$ Jintao, le premier ministre Wen Jiabao ainsi que les dirigeants de différents ministères et provinces. Le respect à l'égard des avis des internautes traduit le développement de la démocratie de la société chinoise."

Alors que la Chine s'apprête à célébrer soixante ans de direction du pays par le Parti Communiste Chinois, cette affirmation attire l'attention sur la manière dont le PCC "réinvente sa légitimité" ${ }^{2}$, après avoir lancé en 1978 une série de réformes qui ont profondément transformé la société chinoise.

Sous l'égide de Deng Xiaoping, le PCC a en effet abandonné les grands principes de l'économie planifiée, pour adopter progressivement l'économie de marché. Il a ainsi remplacé l'idéologie maoïste par un "pragmatisme" économique censé garantir le développement et l'enrichissement progressif de toute la nation chinoise. Cet important tournant s'est traduit notamment par l'encouragement de l'entreprise privée et par la réduction progressive du rôle des institutions qui assuraient tout à la fois la prise en charge et le contrôle des individus, comme l'unité de travail. L'éducation, la santé, mais aussi le logement et l'emploi sont

${ }^{1}$ Cette photographie date de juin 2008. "Hu Jintao talks to netizens via People's Daily Online", 20/06/2008, People's Daily Online, consulté le 16/10/2010 sur http://english.peopledaily.com.cn/90001/90776/90785/6433952.html

${ }^{2}$ LALIBERTE André et LANTEIGNE Marc (dir.). The Chinese Party-state in the 21st century. Adaptation and the reinvention of legitimacy, Londres et New York: Routledge, 2008. 
Séverine Arsène - Internet et politique en Chine - version auteur

désormais largement du ressort des individus, qui acquièrent ainsi une autonomie sans précédent.

Contrairement à ce qui a souvent été pronostiqué ou réclamé ${ }^{3}$, ce retrait de l'Etat de pans entiers du monde économique et social ne s'est pas traduit par la démocratisation du régime. Tout au contraire, le PCC a réaffirmé régulièrement et fermement son rôle de leader politique incontestable, réprimant sévèrement toute contestation, au nom de l'unité de la nation et de la stabilité du pays, qui sont présentées comme les conditions sine qua non du développement économique.

Si la prise en compte des attentes des individus ne passe pas par le développement d'institutions démocratiques, elle n'est cependant pas totalement inexistante. Le PCC a mis en place de nombreux mécanismes destinés à construire un consensus autour de ses décisions et à montrer qu'il est à l'écoute de l'"opinion publique". Il s'appuie sur des analyses d'experts, met en place diverses formes de consultation de la population, des sondages, et procède même à des élections locales en zones rurales ${ }^{4}$. Trente ans après le début des réformes, ces méthodes dites de "gouvernance" ${ }^{5}$ ne semblent plus relever de la transition ${ }^{6}$, ni même de la "résilience" ${ }^{7}$. La cooptation des élites économiques ${ }^{8}$, le développement encadré d'associations ou d'ONG et

${ }^{3}$ Sur le Mur de la Démocratie en 1979, le dissident Wei Jingsheng défendait par exemple l'idée que les "Quatre modernisations" engagées par le PCC devaient logiquement se prolonger par une "cinquième modernisation": la Démocratie. WEI Jingsheng. La cinquième modernisation et autres écrits du Printemps de Pékin, Paris: C. Bourgois, 1997; voir aussi BEJA Jean-Philippe. A la recherche d'une ombre chinoise, Paris: Seuil, 2004.

${ }^{4}$ Ce que Richard Baum nomme "léninisme consultatif". BAUM Richard. "The limits of 'authoritarian resilience' in China", conférence au CERI, Paris, 2007, consulté le 15/10/2010 sur http://www.cerisciences-po.org/archive/jan07/art_rb.pdf.

${ }^{5}$ GOLDMAN Merle et PERRY Elizabeth (dir.). Grassroots political reform in contemporary China, Cambridge: Harvard University Press, 2007; HOWELL Jude (dir.). Governance in China, Oxford: Rowman \& Littlefield, 2004; SAICH Tony. Governance and politics of China, New York: Palgrave, 2001.

${ }^{6}$ Voir par exemple le dossier "China in transition", Social Research, vol. 73, $n^{\circ} 1,2006$.

${ }^{7}$ NATHAN Andrew. "Authoritarian resilience", Journal of democracy, vol.14, n¹, p. 6-17, 2003. Ce terme désigne une capacité d'adaptation et de transformation du régime, qui permet sa survie après un choc.

8 CABESTAN Jean-Pierre. "La Chine évoluerait-elle vers un autoritarisme 'éclairé' mais ploutocratique?", Perspectives chinoises, n84, 2004, p.21-29; DICKSON Bruce. "Cooptation and corporatism in China: the logic of Party adaptation", Political science quarterly, vol.115, $\mathrm{n}^{\circ} 4$, hiver 2000-2001, p. 517-540. 
Séverine Arsène - Internet et politique en Chine - version auteur

le recours insistant à une rhétorique "scientifique" sembleraient plutôt être l'apanage d'un régime technocratique ${ }^{9}$. La Chine aurait-elle sauté l'étape de la démocratie pour évoluer directement vers une forme $\mathrm{d}^{\prime}$ "autoritarisme post-politique" ${ }^{10}$ ? Indéniablement, définir la nature du régime chinois demeure un exercice très délicat.

Comme le montre le discours sur le "développement de la démocratie" diffusé jusqu'en France par le Quotidien du Peuple, l'"énigme chinoise" ${ }^{11}$ que constitue la nature des relations entre le Parti et la population a trouvé une illustration particulièrement emblématique dans le développement d'Internet. La parole des internautes a en effet commencé à prendre une importance considérable dès le début des années 2000, avec le développement des premières mobilisations en ligne, d'abord à teneur nationaliste, et dès 2003, dans le sens de la défense des droits des citoyens, sans donner lieu pour autant à un véritable bouleversement du régime.

Sur ce point, le discours présenté par le Quotidien du Peuple est diamétralement opposé à celui des militants pour la démocratisation du régime, qui voient souvent dans Internet un cheval de Troie de la démocratie contre le Parti Communiste Chinois. En permettant à tout individu de s'exprimer publiquement, Internet créerait une brèche considérable dans le système de censure, et surtout dans le monopole étatique de la parole politique, ce qui permettrait aux citoyens de réclamer des droits politiques plus importants. Dans cette vision, les progrès de la "participation et la délibération du peuple dans les affaires publiques via Internet" conduiraient inéluctablement à la transformation du régime chinois et seraient donc un danger direct pour le Parti $^{12}$.

${ }^{9}$ ZANG Xiaowei. "The consolidation of political technocracy in china: The fourteenth and fifteenth central committees of the CCP", Journal of Communist Studies and Transition Politics, vol. 15, n³, p.101, 1999.

${ }^{10}$ BEJA Jean-Philippe. "The changing aspects of civil society in China", Social research, vol. $73, \mathrm{n}^{\circ} 1$, 2006, p. 53-74.

11 "L'énigme chinoise", dossier consacré à la nature du régime chinois dans la revue Le Débat. Sur cette question, voir aussi l'ensemble du dossier "China's changing of the guard", dans la revue Journal of Democracy en 2003.

${ }^{12}$ HAUTER François. "Tandis que la Chine s'éveille, le Parti communiste resserre ses griffes sur la société civile", Le Figaro, 26/05/2006; MEVEL Jean-Jacques. "En Chine, la société civile sort de l'ombre", Le Figaro, 15/10/2007; "Chine, le modèle se fissure", dossier Courrier International, n902, 14 février 2008. 
Séverine Arsène - Internet et politique en Chine - version auteur

Les internautes chinois ont en effet montré leur capacité à se saisir d'Internet pour parler politique en ligne, même s'ils l'ont fait sous des formes parfois déroutantes. En effet la contestation du régime proprement dite reste extrêmement minoritaire, tandis que les internautes ont massivement pris la parole sur diverses questions de société. Les blogs ou forums hébergent des discussions très animées, parfois même violentes, par exemple sur le Tibet ou Taiwan. Sur les sujets les plus sensibles, comme la corruption au sein du Parti, on trouve des prises de parole critiques ou ironiques, des caricatures et des vidéos satiriques, mais aussi de nombreuses formes de double langage ou de détournement du sens des caractères chinois. Cette effervescence conduit parfois à de véritables mobilisations, collectives mais pas forcément organisées, autour de problèmes aussi disparates que l'interdiction des gros chiens dans le centre-ville de Pékin, l'installation d'une usine chimique à proximité de zones d'habitations, ou encore l'esclavage d'enfants dans des briqueteries. Elles ont parfois eu un impact politique important, forçant les autorités à intervenir sur le terrain et même, dans certains cas, à changer la loi.

Or le discours officiel ne présente pas la prise de parole des internautes chinois comme un danger pour le régime, mais au contraire comme un élément central de son fonctionnement, qui se trouve au fondement de la légitimité du PCC. Elle est décrite comme l'un des canaux au travers desquels le Parti prend connaissance des "avis" des internautes, envisagés comme une incarnation de l'opinion publique. Cette place centrale donnée à la parole des internautes n'est cependant pas sans ambiguïtés. En effet, le développement d'Internet n'a absolument pas aboli la censure et il s'accompagne d'efforts importants des autorités locales et centrales pour "guider l'opinion publique". Le Parti n'est visiblement pas prêt à prendre en considération n'importe quelle forme d'expression publique.

En quoi consistent alors ces mobilisations, que reconnaissent les dirigeants et auxquelles ils sont obligés de réagir ostensiblement? Qu'est-ce qui peut expliquer que certaines causes, plutôt que d'autres, franchissent ainsi les barrières de la censure, et soient soutenues par de nombreux internautes? Sous quelles conditions ces causes très disparates 
Séverine Arsène - Internet et politique en Chine - version auteur

peuvent-elles s'imposer, dans la société chinoise et par le biais d'Internet, comme de véritables "problèmes publics"13?

Beaucoup de travaux décrivent en détail les profils des militants et les stratégies qu'ils mettent en place pour contourner la censure et rallier des publics à leurs causes. Le succès de leurs démarches dépend aussi des prises de position d'experts qui y voient l'occasion de pousser un agenda politique, ainsi que du relais des médias traditionnels qui leur assurent une certaine publicité et entérinent la légitimité de leurs démarches. De nombreuses études de cas mettent ainsi en évidence combien les mobilisations en ligne sont faites de contingences, de stratégies, de jeux d'influences et de cadrages thématiques, ce qui les rend très complexes.

D'autre part des travaux de plus en plus nombreux suggèrent que l'horizontalité des échanges en ligne favorise la constitution de publics sensibles à certaines questions d'intérêt général. La manière dont ces deux éléments se répondent reste cependant peu étudiée. Il faut en effet expliquer les raisons qui poussent de nombreux internautes ordinaires à s'indigner devant certaines causes plutôt que d'autres, détailler les modes d'expression qu'ils choisissent et, le cas échéant, éclairer les situations dans lesquelles ils préfèrent se taire. Cela implique de prêter une attention particulière aux justifications et argumentaires qui sont susceptibles de toucher des internautes "ordinaires", ainsi qu'aux modalités d'expression qui leur semblent légitimes sur la place publique.

C'est pourquoi je me propose d'adopter une approche goffmanienne ${ }^{14}$ de ce phénomène, sur le modèle de ce que propose Cefai ${ }^{15}$. La notion de "cadrage" des problèmes publics permet de saisir les contraintes, formelles mais aussi normatives, qui s'imposent à la

${ }^{13}$ CEFAI Daniel. "La construction des problèmes publics. Définitions de situations dans des arènes publiques", Réseaux, n 75 , 1996, p. 43-65; GUSFIELD Joseph. La culture des problèmes publics: l'alcool au volant: la production d'un ordre symbolique, Paris: Economica, 2009.

${ }^{14}$ Voir en particulier GOFFMAN Erving. Façons de parler, Paris: Minuit, 1987. Pour un aperçu plus large des théories de Goffman, voir La mise en scène de la vie quotidienne, Paris: Minuit, 1979, 2 vol ou Les cadres de l'expérience, Paris: Minuit, 1991.

${ }^{15}$ CEFAI Daniel. Pourquoi se mobilise-t-on?, Paris: La Découverte, 2007; ainsi que "La construction des problèmes publics. Définitions de situations dans des arènes publiques", Réseaux, n75, 1996, p. 43-65. 
Séverine Arsène - Internet et politique en Chine - version auteur

formulation des problèmes en public, tout en tenant compte des divergences et des transgressions dont elles font l'objet, et de leurs transformations.

Il me semble en effet que l'on peut comprendre en grande partie l'irruption de la parole politique des internautes, tout autant que sa limitation, au travers d'un certain discours dominant en Chine. Largement impulsé par la propagande étatique, ce discours porte essentiellement sur les conditions nécessaires pour garantir la cohésion de la société et le développement économique, c'est à dire qu'il articule un projet de société autour duquel repose le contrat social chinois. Il met en scène un style de vie nouveau et confortable, emblématique d'une certaine conception de la modernité à la chinoise, et dans lequel les technologies de l'information tiennent une place privilégiée. Il trouve des échos dans tous les pans de la société, où il est sans cesse reformulé, réinterprété, critiqué. L'objectif de ce travail est de repérer les traces de ce discours dominant dans les modalités de formation des mobilisations en ligne, sans occulter pour autant les malentendus dont il fait l'objet, ni les transgressions, ou encore les stratégies mises en oeuvre par les internautes pour le détourner au profit des causes qu'ils soutiennent.

Cela suppose tout d'abord de prendre en considération la participation des individus eux-mêmes à cette dynamique complexe, depuis la définition des normes et leur imposition dans l'ordre social, jusqu'à leur remise en question. Si les autorités sont en mesure d'imposer des interdits, un nombre bien plus grand de règles informelles sont mises en actes par les internautes au fil de leurs interactions. Or ces règles sont variables en fonction du type de scène concerné, et leur définition est tributaire de l'interprétation personnelle de chaque individu, laquelle peut changer avec le temps. Il s'agit dès lors d'expliquer en quoi le projet de société moderne qui se diffuse au sein de la société chinoise sert de repère aux internautes dans les moments clés où ces règles sont transgressées, remises en question, redéfinies.

Cela permettra de montrer que les possibilités de mobilisation qui s'offrent aux citoyens chinois sur Internet sont à la fois très larges et très restreintes. Si le discours sur la modernisation du pays fournit parfois des arguments pour défendre des droits individuels contre les autorités, il exclut aussi toute remise en cause radicale du contrat social sur lequel il est fondé. Or celui-ci fait constamment l'objet de mises en récit contradictoires par une diversité d'acteurs, porteurs de causes, médias, experts, autorités, qui se disputent l'attention 
Séverine Arsène - Internet et politique en Chine - version auteur

des publics afin d'inscrire des problèmes sur l'agenda politique. Il s'agit donc, en dernier ressort, d'étudier la manière dont la définition de ce contrat social, incarné dans un idéal de modernité à la chinoise, devient l'enjeu des recompositions du pouvoir politique en Chine.

Donner autant d'importance aux normes sociales supposait de procéder par entretiens approfondis auprès d'une population concernée par la question de la prise de parole en ligne. Que signifie publier des opinions en ligne? Quels sont les sujets sur lesquels les internautes ont envie de s'exprimer? Quelles sont les limites qui s'imposent à leur parole? Sont-ils tentés de transgresser ces limites? Le font-ils parfois involontairement? Ce sont ces questions, et bien d'autres encore, que j'ai posées à cinquante internautes pékinois "ordinaires" en 2006 et 2007. Recrutés au sein de la génération née après 1978, menant une vie urbaine, ils font partie de la portion de la population chinoise la mieux représentée sur le Web chinois. Au travers de leurs réponses, je souhaite restituer la place du contexte idéologique, l'appropriation des règles et leur transgression, mais aussi les contingences, les émotions, et la diversité des positions individuelles qui façonnent les multiples modes de prise de parole de ces internautes.

Après une exploration du contexte et des enjeux liés à la recrudescence de ces mobilisations en ligne, et quelques précisions méthodologiques (introduction), le plan de ce travail s'articulera donc en trois parties. Dans un premier temps, je détaillerai de quelle manière l'idéologie de la "modernisation" se décline dans une représentation d'Internet en général (chapitre 1) et des outils de prise de parole en particulier (chapitre 2). Cette représentation se prolonge dans un discours relativement dépréciateur sur la politique, ce qui peut sembler paradoxal étant donné l'intérêt relatif que de nombreux internautes accordent à d'importantes questions de société (chapitre 3).

Je montrerai ensuite que cette idéologie fonde un "régime de civilité" qui encadre la prise de parole publique plus sûrement que ne le fait la seule censure (chapitre 4). Fondée sur le principe d'une représentation unanime de la nation chinoise, cette civilité exclut tout comportement politique en public (chapitre 5). Elle n'empêche pas cependant de nombreuses réserves, critiques, et autres allusions, qui ont un statut privé, mais auxquelles Internet donne une visibilité toute particulière (chapitre 6). 
Séverine Arsène - Internet et politique en Chine - version auteur

La dernière partie sera l'occasion de montrer que la norme dominante fournit des argumentaires qui permettent de rendre des problèmes publics. Or elle est mise à l'épreuve et réinterprétée au quotidien, ce qui peut aussi se retourner contre les autorités (chapitre 7). La mobilisation des publics se joue donc beaucoup dans la mise en récit des problèmes. Elle est l'objet d'une négociation permanente entre de nombreux acteurs, "porteurs de causes", médias, autorités locales et centrales (chapitre 8). Cette compétition autour de la formation de l'agenda politique ne remet cependant pas en cause la nature du régime politique. Elle est au contraire un élément central de son fonctionnement (chapitre 9). 


\section{Introduction}

\section{Internet et prise de parole politique en Chine}

S'il est un sujet qui nécessite des précautions théoriques et méthodologiques, c'est bien celui des enjeux politiques d'Internet en contexte autoritaire. Chacun de ces termes est chargé d'imaginaires et de programmes idéologiques puissants. Dès l'arrivée d'Internet auprès du grand public, les commentateurs y ont vu tour à tour un sauvetage ou un coup de grâce pour la participation politique des citoyens. Pourtant, à l'épreuve des faits, il s'avère que les usages politiques d'Internet accompagnent les recompositions politiques de manière plus subtile, que ce soit en contexte démocratique ou autoritaire. Si Internet permet à des formes de prise de parole relativement hétérodoxes de se développer, il n'échappe pas aux luttes pour la visibilité, aux inégalités de ressources et de compétences, ou encore aux stratégies argumentatives inhérentes à tout espace public.

Ces dimensions complexes de la prise de parole politique sur Internet peuvent également nourrir une réflexion sur certaines ambivalences des situations autoritaires, notamment en éclairant les contours de la prise en compte de la parole des citoyens par les autorités. Le cas de la Chine en est un exemple emblématique. Alors qu'aucune démocratisation ne s'amorce, le Parti Communiste Chinois ne peut pas manifester une indifférence complète vis-à-vis de l'opinion publique, mais il s'efforce de la canaliser, parfois non sans succès.

Cette réflexion doit également prendre en compte la diversité des mobilisations qui prennent corps dans les échanges en ligne. Comment peut-on appréhender ensemble des formes de protestation aussi hétérogènes que la critique du régime par les dissidents, la défense des droits d'un groupe de propriétaires lésés et les lamentations de milliers d'internautes à propos des mésaventures rencontrées dans la vie quotidienne? Ces différents modes de participation contribuent pourtant tous à la formation des mobilisations en ligne. Il faut saisir la diversité des formes d'engagement, des positions (et donc des contraintes) qui caractérisent tant les "porteurs de causes" que les nombreux internautes anonymes, lorsqu'ils décident d'apporter leur voix à certaines protestations plutôt qu'à d'autres, ou lorsqu'ils choisissent de garder le silence. 
Pour éclairer ces questions, je souhaite montrer que l'idéologie dominante, qui fait reposer la légitimité du régime sur une promesse de prospérité et de modernisation du pays, constitue une sorte de "cadrage" de la prise de parole des internautes ordinaires. Cette idéologie, qui n'est certes pas adoptée de la même manière ni avec la même sincérité par l'ensemble de la population, constitue une référence commune autour de laquelle se redéfinissent au quotidien les règles du vivre ensemble. Souvent transgressée, moquée ou contournée, elle n'en détermine pas moins les conditions de la formation des mobilisations sur le Web chinois.

\section{1 - La formation d'un espace public complexe et conflictuel}

\section{Internet et participation politique}

Partout dans le monde, le développement d'Internet s'est accompagné d'un renouvellement des réflexions sur les formes de participation politique des citoyens ${ }^{1}$. Dans les démocraties occidentales, son utilisation s'est répandue au moment même où l'on constatait une certaine désaffection des citoyens pour les formes de participation traditionnelles, telles que le vote ou l'adhésion aux partis politiques ${ }^{2}$. Le militantisme en général semble en perte de vitesse $^{3}$, remplacé par des formes d'action plus diversifiées et moins coûteuses pour les individus. Les jeunes recherchent des formes d'engagement plus souples et modulables ${ }^{4}$, moins coûteuses en temps et en ressources. Signer une pétition, ou participer ponctuellement à une manifestation, apparaissent par exemple comme des formes d'action plus aisées que s'inscrire dans un syndicat ou militer dans un parti politique.

Ce désinvestissement des formes institutionnalisées de participation politique, et en particulier le fort taux d'abstention aux élections, nuit à la légitimité des décisions prises par

\footnotetext{
${ }^{1}$ Pour de bonnes synthèses de ces débats, voir par exemple FLICHY Patrice. "Internet, un outil de la démocratie?", laviedesidees.fr, 2008; GREFFET Fabienne et WOJCIK Stéphanie. "Parler politique en ligne. Une revue des travaux français et anlo-saxons", Réseaux, vol. 26, n¹50, p. 19-50.

${ }^{2}$ Pour une synthèse, voir MAYER Nonna et PERRINEAU Pascal. Les comportements politiques, Paris: Armand Colin, 1992.

3 ION Jacques. La fin des militants?, Paris: L'Atelier, 1997. ION Jacques (dir.). L'engagement politique, Saint-Etienne: Publications de l'Université, 2001.

${ }^{4}$ MUXEL Anne. Les jeunes et la politique, Paris: Hachette, 1996.
} 
les pouvoirs publics. Pour pallier ce phénomène, les collectivités réfléchissent à d'autres modalités de consultation des citoyens, ce qui a conduit à la prolifération des consultations, panels, jurys citoyens et sondages délibératifs ${ }^{5}$. Un véritable "impératif délibératif" ${ }^{6} \mathrm{~s}^{\prime}$ est ainsi développé. Internet, qui offre la possibilité d'organiser ce type de consultations en ligne, donne à beaucoup de ces collectivités l'espoir de revitaliser le débat démocratique ${ }^{7}$. Les partis politiques misent aussi sur leurs sites Internet pour apporter une dimension délibérative à la construction de leurs programmes, et pour réduire les contraintes de l'adhésion des militants.

Ces initiatives soulèvent cependant un certain nombre de questions, depuis leur mise en place jusqu'au moment de la prise de décision par les autorités. On peut d'une part s'interroger sur l'influence des dispositifs ${ }^{8}$ mis en place, ainsi que des modérateurs ${ }^{9}$, sur l'issue des délibérations. D'autre part, la prise en compte effective des idées issues de ces délibérations et leur application dans les politiques publiques pose problème, car elles n'ont généralement qu'une force consultative. Du côté des partis, la délibération peut également se réduire à une forme de consultation sans véritable impact sur la formulation des programmes et qui n'a d'autre but que d'être utilisée à des fins de communication ${ }^{10}$. Enfin, il n'est pas évident que la consultation touche l'ensemble des individus concernés. La question de la fracture numérique $^{11}$ ne viendrait que remplacer, dans ce cas, d'autres formes de freins à la participation politique des citoyens.

${ }^{5}$ Dont le principal théoricien est certainement FISHKIN James. The voice of the people. Public opinion and democracy, New Haven et Londres: Yale University Press, 1995.

${ }^{6}$ BLONDIAUX Loïc et SINTOMER Yves. "L'impératif délibératif", Politix, vol.15, n57, 2002, p 1735 .

${ }^{7}$ Les exemples sont pléthoriques. Voir par exemple WOJCIK Stéphanie. Délibération électronique et démocratie locale: le cas des forums municipaux des régions Aquitaine, Languedoc-Roussillon et Midi-Pyrénées, Thèse pour le doctorat de science politique, Université Toulouse 1, 2005.

${ }^{8}$ WRIGHT Scott et STREET John. "Democracy, deliberation and design: the case of online discussion forums", New media \& society, vol.9, n5, p. 849-869.

${ }^{9}$ WOJCIK Stéphanie. "Les modérateurs des forums de discussion municipaux, des intermédiaires démocratiques?", Questions de communication, n²12, p. 335-354.

${ }^{10}$ Sur les partis, voir notamment BLANCHARD Gersende. La communication politique partisane sur Internet: des pratiques et des stratégies nouvelles?, Thèse pour le doctorat en Sciences de l'information et de la communication, Université Stendhal Grenoble 3, 3 vol, 2007.

${ }^{11}$ Voir par exemple le dossier "Fracture numérique et justice sociale", Cahiers du numérique, vol.5, $\mathrm{n}^{\circ} 1,2009, \mathrm{p} .12-247$. 
Au-delà des dispositifs organisés par des collectivités ou des partis, la capacité d'Internet à favoriser l'émergence de la délibération citoyenne pose problème. Les discussions en ligne ont très tôt été comparées aux salons de l'espace public idéal habermassien ${ }^{12}$. L'apparente facilité de prendre la parole en ligne et le fait que l'on puisse rester anonyme pourraient faire tomber un certain nombre de barrières liées au statut social, en mettant les individus à égalité devant leur écran. Dans cette optique, Internet aurait donc le potentiel de favoriser des formes plus démocratiques de participation politique ${ }^{13}$.

Pourtant de nombreuses recherches empiriques montrent que les débats en ligne sont rarement conformes à l'idéal délibératif. Les débats sont fréquemment monopolisés par quelques utilisateurs ${ }^{14}$, lorsqu'ils ne tournent pas tout simplement au pugilat. D'autre part certains travaux suggèrent le fait qu'Internet donne lieu à une polarisation des opinions, plus qu'il ne permet la recherche du consensus ${ }^{15}$. Les citoyens seront-ils prêts à se confronter à des idées contradictoires, ne serait-ce que par hasard ${ }^{16}$ ? L'infinie possibilité de choix qui est offerte aux internautes ne conduirait-elle pas à une fragmentation de l'espace public, dangereuse pour les principes républicains du vivre ensemble ${ }^{17}$ ?

En contrepoint de ces inquiétudes, le modèle idéal de délibération qui fonde ces travaux est lui-même remis en question. En effet les théories critiques de l'espace public montrent que

12 RHEINGOLD Howard. The virtual community : homesteading on the electronic frontier, Cambridge: MIT Press, 2000.

${ }^{13}$ Surtout dans la recherche anglo-saxonne. GIMMLER Antje. "Deliberative democracy, the public sphere and the internet", Philosophy \& Social Criticism, vol. 27, n ${ }^{\circ}$, 2001, p. 21-39; SAVIGNY Heather. "Public opinion, political communication and the Internet", Politics, vol. 22, n 1, 2002, p. 18; WITSCHGE Tamara. "Online deliberation: possibilities of the Internet for deliberative democracy", in SHANE Peter (dir.) Democracy online. Prospects for political renewal through the Internet, London: Routledge, 2004, p. 109-122.

${ }^{14}$ SCHNEIDER Steven. "Creating a democratic sphere through political discussion", Social science computer review, vol.14, $\mathrm{n}^{\circ} 4$, p. 373-393.

15 POSTER Mark. "Cyberdemocracy: the Internet and the public sphere", in HOLMES D.(dir.), Virtual politics, identity and community and cyberspace, Londres: Sage, p. 212-228 DUMOULIN Michaël. "Les forums électroniques: délibératifs ou électroniques?", in MONIERE D. (dir.), Internet et la démocratie: les usages politiques d'Internet en France, au Canada et aux Etats-Unis, Québec: Monière et Wollank, p. 141-157.

${ }^{16}$ LEV-ON Azi et MANIN Bernard. "Internet: la main invisible de la délibération", Esprit, n ${ }^{\circ}$, 2006, p.195-212.

${ }^{17}$ SUNSTEIN Cass. Republic.com, Princetown: Princetown University Press, 2001. 
la valorisation d'une certaine forme de délibération et de rationalité s'est accompagnée de l'exclusion de catégories entières de la société, notamment les illettrés et les femmes ${ }^{18}$. Dans cette perspective, l'ouverture d'Internet à des formes de discours hétérodoxes dans leurs formes comme dans leur fond peut se lire comme une salutaire contestation de la culture dominante $^{19}$. Au demeurant, le caractère policé des échanges délibératifs n'est pas nécessairement un gage de démocratie ${ }^{20}$, car celle-ci suppose l'engagement des citoyens dans la confrontation de points de vue contradictoires, ce qui n'est jamais confortable ${ }^{21}$. Ces réflexions conduisent à considérer plus positivement le conflit, mais aussi des modes d'expression qui étaient auparavant dépréciés (témoignage, narration, récit personnel, indignation $^{22}$ etc), et à mieux prendre en compte les "formes profanes du rapport au politique" 23 .

La plasticité des formes d'expression sur Internet donne également naissance à de nouvelles formes d'activisme en ligne ${ }^{24}$. Il ne s'agit pas seulement de tenir un site Internet, ou d'envoyer une lettre d'information à des abonnés, mais de développer des modes de participation beaucoup moins conventionnels, comme le détournement d'image de marque, la création de réseaux d'éducation alternatifs, l'organisation de projets culturels voire le piratage

${ }^{18}$ CALHOUN Craig (dir). Habermas and the public sphere, Cambridge, Massachusetts.: MIT Press, 1992 dont FRASER Nancy. "Repenser la sphère publique: une contribution à la critique de la démocratie telle qu'elle existe réellement", traduit dans Hermès, n³1, 2001, p. 125-158.

19 DAHLBERG Lincoln. "Rethinking the fragmentation of the cyberpublic: from consensus to contestation", New Media \& Society, vol. 9, n5, 2007, p. 827-847.

${ }^{20}$ PAPACHARISSI Zizi. "Democracy online: civility, politeness, and the democratic potential online political discussion groups", New media and society, vol.6, n², 2004, p. 259-283.

${ }^{21}$ SCHUDSON Michael. "Why conversation is not the soul of democracy", Critical studies in mass communication, $\mathrm{n}^{\circ} 14,1997$, p. 297-309.

${ }^{22}$ Entre autres, voir CARDON Dominique et HEURTIN Jean-Philippe. "La critique en régime d'impuissance, une lecture des indignations des auditeurs de France Inter", in FRANCOIS Bastien et NEVEU Erik (dir.). Espaces publics mosä̈ques, acteurs, arènes et rhétoriques des débats publics contemporains, Rennes: Presses universitaires de Rennes, 1999, p 85-119; CARDON Dominique, HEURTIN Jean-Philippe et LEMIEUX Cyril. "Parler en public", Politix, vol. 8, n³1, p. 5-19; LIVINGSTONE Sonia et LUNT Peter. "Se faire entendre dans l'espace public. Les femmes, la télévision et le citoyen-spectateur", Réseaux, vol.12, nº3, 1994, p. 59-74.

23 JOIGNANT Alfredo. "Compétence politique et bricolage. Les formes profanes du rapport au politique", Revue française de science politique, vol. 57, n 6, p. 799-817.

${ }^{24}$ MCCAUGHEY Martha et AYERS Michael D. Cyberactivism, New York et Londres: Routledge, 2003. 
de sites Internet. L'efficacité politique de ces procédés repose moins sur la capacité à peser sur les mécanismes de la prise de décision politique au sein des instances de représentation élues, que sur la faculté de rendre publiques leurs causes et d'y rallier une opinion publique locale, nationale, voire internationale ${ }^{25}$. Des individus ou des groupes qui ne disposent habituellement pas d'accès aux arènes traditionnelles de la représentation politique peuvent ainsi espérer faire entendre leur voix et se donner une certaine visibilité. L'enjeu de la participation politique des citoyens bascule dès lors de la difficulté de prendre la parole à celle de se faire entendre. C'est pourquoi Wojcik invite entre autres à un repérage plus précis des ressources argumentatives employées par les citoyens ordinaires pour imposer leurs causes dans l'agenda politique, ainsi qu'à explorer le rôle de la culture politique nationale dans ce processus $^{26}$.

Internet accompagne ainsi (certainement plus qu'il ne cause) les transformations de la participation politique des individus et devient un "révélateur d'une recomposition politique" ${ }^{27}$ : ouverts à des grammaires de prise de parole plus diversifiées, les différents espaces investis par les citoyens donnent une visibilité sans précédent à des opinions hétérodoxes ainsi qu'à des revendications micro-politiques. Ainsi que le soulignent les questionnements normatifs précédemment évoqués, cette diversification des modes de participation politique est considérée avec une certaine circonspection dans les pays occidentaux, car elle bouscule les formes les plus traditionnelles de la participation politique, telles qu'elles sont mises en place dans les institutions démocratiques. Il est intéressant de constater que cette caractéristique est tout au contraire louée par les défenseurs de la démocratie dans le cas des pays autoritaires, car elle permet l'émergence d'une parole "non autorisée" sur la place publique, ce qui a souvent été envisagé comme une chance de démocratisation. Là encore, la littérature qui s'est développée sur le sujet invite à adopter une approche plus nuancée de ce phénomène.

${ }^{25}$ GRANJON Fabien. L'Internet militant, Rennes: Apogée, 2001.; et GRANJON Fabien. "Les répertoires d'action télématiques du néo-militantisme", Le Mouvement social, № 200, 2002, p. 11-32; TRAUTMANN Flore. "Internet au service de la démocratie? Le cas d'ATTAC", Les Cahiers du CEVIPOF, $\mathrm{n}^{\circ} 30$.

${ }^{26}$ GREFFET Fabienne et WOJCIK Stéphanie. "Parler politique en ligne. Une revue des travaux français et anlo-saxons", Réseaux, vol. 26, n¹50, p. 19-50.

${ }^{27}$ MAIGRET Eric. Sociologie de la communication et des médias, Paris: Armand Colin, 2003. 
Dans un premier temps, beaucoup d'études de cas ont été consacrées à des exemples de "résistance" 28 aux pouvoirs autoritaires dans différents pays. Elles documentent l'utilisation d'Internet par des groupes ou organisations dissidents ${ }^{29}$, qui cherchent à faire connaître leurs idées, à se coordonner, et à toucher l'opinion, à l'échelle nationale et internationale. Par exemple, les stratégies de mobilisation des Zapatistes ${ }^{30}$ au Mexique, ou encore les sites Internet dédiés à l'Ingouchie ${ }^{31}$ montrent que le web peut permettre de donner une visibilité particulière à des situations, des messages ou à des événements qui sans cela seraient couverts par les autorités. Pour les auteurs les plus optimistes, ces actions, en bravant les limites de la censure et en forçant ponctuellement les autorités concernées à modérer leurs politiques répressives, grignoteraient peu à peu la rigidité des régimes autoritaires, ce qui constituerait les prémisses d'un processus progressif mais inexorable de transformation du régime politique $^{32}$. Une partie de ce tropisme peut s'expliquer par le fait qu'un grand nombre de ces recherches sont financées par des organismes qui s'intéressent de près ou de loin à la promotion de la démocratie, comme la Rand Corporation, la Croix Rouge, ou le Carnegie Endowment for International Peace ${ }^{33}$.

En dépit de l'optimisme affiché par ces organismes, les diverses monographies disponibles montrent que l'efficacité des mobilisations en ligne menées par les dissidents politiques est très relative. Si les militants pour la démocratie réussissent généralement à faire connaître leurs idées auprès d'une certaine opinion internationale, ils obtiennent rarement gain

${ }^{28}$ Voir par exemple ce cas singapourien. TAN Suqin. Resistance on the Internet: a study of the Singapore case, Mémoire pour l'obtention du Master de Sciences sociales, Singapour: National University of Singapore, 2006.

${ }^{29}$ CHASE Michael et MULVENON James. You've got dissent! Chinese dissident use of the Internet and Beijing's counter-strategies, Santa Monica: RAND, 2002.

${ }^{30}$ RONFELDT David. The zapatista "social netwar" in Mexico, New York: Rand Corporation, 1998.

${ }^{31}$ LYSENKO Volodymyr et DESOUZA Kevin. Cyberprotest in contemporary Russia: The cases of Ingushetiya.ru and Bakhmina.ru, Technological Forecasting \& Social Change, 2010.

32 DAVIS Creighton Powell. "The Internet as a Source of Political Change in Egypt and Saudi Arabia", Al Noor, 1(1), 2008, p. 32-41.

${ }^{33}$ Il est intéressant de constater que les mêmes organismes produisent des études alarmistes sur le cyber-terrorisme. Le rôle des think-tanks américains, et en particulier de la Rand, est central dans la formation de la notion de "société de l'information", et dans la diffusion de l'idée de "guerre informationnelle", selon laquelle la maîtrise de l'information est centrale dans les conflits. Voir MATTELART Armand. Histoire de la société de l'information, Paris: La Découverte, 2009. 
de cause auprès des autorités concernées. C'est par exemple le cas de la révolution $\operatorname{Safran}^{34}$ en Birmanie, qui a donné lieu à des actions importantes en ligne, sans faire ployer les autorités locales. Il en est de même pour la secte Falun Gong ${ }^{35}$ ou les séparatistes tibétains ${ }^{36}$ en Chine. De plus le caractère très informel de ces mobilisations ne permet pas forcément de structurer des collectifs pérennes. Lonkila note par exemple que le mouvement antimilitariste en Russie $^{37}$ reste très fragmenté et peu organisé.

Ces échecs relatifs sont souvent mis sur le compte de l'efficacité de la censure ${ }^{38}$, qui empêche les dissidents de faire passer leurs messages et les conduit fréquemment en prison. Cette censure peut prendre des formes très variées, qu'elles soient techniques (filtrage par URL ou mots-clés), juridiques (responsabilisation des fournisseurs de services) ou qu'elles s'appuient sur le contrôle social et l'autocensure. C'est pourquoi Taubman ${ }^{39}$ et Hughes en ont conclu, par exemple, «qu'Internet ne démocratisera pas la Chine ${ }^{40}$. Pourtant il est relativement facile de contourner cette censure grâce à l'ironie ou au double langage ${ }^{41}$, et les exemples de messages critiques qui sont publiés au moyen de ces procédés ne manquent pas. Cette explication, si elle est importante, ne peut pas justifier à elle seule la faiblesse de la contestation en ligne des régimes politiques.

\footnotetext{
${ }^{34}$ CHOWDHURY Mridul. The role of the Internet in Burma's Saffron Revolution, Berkman Center for Internet and Society, 2008, consulté le 17/07/2010 sur http://cyber.law.harvard.edu/publications/2008/Role_of_the_Internet_in_Burmas_Saffron_Revolution. ${ }^{35}$ O'LEARY Stephen. "Falun Gong and the Internet", Online Journalism Review, 2000, consulté le 13/07/2010 sur http://www.ojr.org/ojr/ethics/1017964337.php.

${ }^{36}$ BRAY John. "Tibet, democracy and the Internet bazaar", Democratization, vol.7, n¹, 2000, p.157173.

${ }^{37}$ LONKILA Markku. "The Internet and anti-military activism in Russia", Europe-Asia Studies, vol. $60, n^{\circ} 7,2008$, p.1125-1149.

${ }^{38}$ Sur ce sujet la synthèse la plus complète est celle de DEIBERT Ronald et al. Access controlled, Cambridge (Mass): MIT Press, 2010.

39 TAUBMAN Geoffry. "A not-so World Wide Web: The Internet, China and the challenges to nondemocratic rule", Political Communication, vol. 15, n²2, 1998, p.255-273.

${ }^{40}$ HUGHES Christopher. "Pourquoi Internet ne démocratisera pas la Chine", Critique internationale, $\mathrm{n}^{\circ} 15$, avril 2002, p.85-104.

${ }^{41}$ ESAREY Ashley et XIAO Qiang. "Political expression in the Chinese blogosphere. Below the radar", Asian Survey, vol. 48, n 5, 09/10 2008, p. 752-772.
} 
Il est remarquable que les combats pour la démocratie ne soulèvent pas forcément l'enthousiasme des internautes dans les différents pays en question. Tout d'abord les outils de coordination et de publication en ligne peuvent servir à défendre des causes de toutes natures, parfois très éloignées de la promotion de la démocratie. On s'alarme par exemple que le net soit aussi un vecteur de communication très important pour des réseaux terroristes ${ }^{42}$, jihadistes $^{43}$ ou encore néo-nazis ou talibans ${ }^{44}$ qui défendent des agendas très différents. Cette catégorie est toutefois très minoritaire parmi les multiples usages d'Internet. En fait, quelle que soit leur teneur, les messages contestataires ne semblent trouver que très peu d'écho auprès des internautes, souvent jeunes, qui semblent plus préoccupés par le divertissement en ligne que par le militantisme politique ${ }^{45}$ (ce en quoi ils ne sont pas différents des jeunes internautes occidentaux).

Ces derniers travaux semblent expliquer cette apparente apathie politique par une forme d'égoïsme postmoderne qui serait l'apanage de cette génération d'internautes. Cet argument ne tient cependant pas tout à fait. En effet beaucoup d'entre eux expriment à l'occasion leur indignation devant des faits divers, ou se joignent à des mobilisations, qui ne sont généralement pas directement tournées contre leurs régimes respectifs, mais qui néanmoins mettent les autorités en difficulté et les forcent à réagir en faveur des citoyens. De nombreux exemples en sont recueillis partout dans le monde ${ }^{46}$. Ainsi des internautes chinois postent-ils des messages indignés face à l'arbitraire policier ou à des jugements iniques ${ }^{47}$. En Iran, on discute des conséquences du 11 septembre et on télécharge des livres et des films occidentaux

${ }^{42}$ WEIMANN Gabriel. Terror on the Internet: the new arena, the new challenges, US Institute of Peace Press, 2006.

${ }^{43}$ ADHAMI Wael. "The strategic importance of the Internet for armed insurgent groups in modern warfare", International Review of the Red Cross, vol. 89, n868, 2007, p. 857-878.

${ }^{44}$ CHROUST Peter. "Neo-Nazis and Taliban on-line: anti-modern political movements and modern media", Democratization, vol. 7, n 1, 2000, p. 102-118.

45 AMIR-EBRAHIMI Masserat. "La jeunesse iranienne dans le miroir du blog", Les cahiers de l'Orient, 79, 2005, p.43-56; SIMA Yangzi et PUGSLEY Peter. "The rise of a 'me culture' in postsocialist China", The International Communication Gazette, vol. 72, n³, 2010, p. 287-306; RUTTEN Ellen. "More than a poet? Why Russian writers didn't blog on the 2008 elections", Russian Cyberspace, vol. 1, $\mathrm{n}^{\circ}$ 1, 2009, p. 25-30.

${ }^{46}$ Voir BOAS Taylor et KALATHIL Shanthi. Open networks, closed regimes: the impact of the Internet on authoritarian rule, Washington DC: Carnegie Endowment for International Peace, 2003.

${ }^{47}$ Sur le cas chinois, voir HASKI Pierre. Internet et la Chine, Paris: Seuil, 2008. 
interdits par le régime ${ }^{48}$. En Arabie Saoudite ou en Égypte, les bavures policières, ou encore les comportements condescendants des princes et des puissants créent de véritables scandales en ligne ${ }^{49}$. A minima, il semble exister une « opinion publique » en ligne qui peut à l'occasion s'affirmer face aux autorités ${ }^{50}$.

Comme dans les pays démocratiques, cette place centrale de l'opinion publique montre que la relation entre gouvernants et gouvernés ne se résume pas au fonctionnement des institutions, aussi importantes soient-elles, mais qu'elle se joue aussi dans les mécanismes complexes de construction de l'agenda politique, d'accès à la visibilité et a fortiori de construction des problèmes publics ${ }^{51}$. En donnant une place privilégiée à des formes de prise de parole profanes, parfois désordonnées ou imprévisibles, Internet se trouve désormais au cœur de ces enjeux.

Ayant tout à la fois la population d'internautes la plus nombreuse du monde, le système de censure le plus sophistiqué, et un nombre de mobilisations en ligne en constante augmentation, la Chine semble constituer un exemple privilégié pour explorer toute la subtilité de ces recompositions du jeu politique en contexte autoritaire. En particulier, la recrudescence des mobilisations qui ont lieu en dépit d'une censure en constante adaptation souligne le poids nouveau de la parole politique des citoyens.

${ }^{48}$ Sur le cas iranien, voir ALAVI Nasrin. We are Iran, Londres: Portobello Books, 2006; RAHIMI Babak. "The politics of the Internet in Iran", dans SEMATI Mehdi (dir.). Media, culture and society in Iran: living with globalization and the Islamic state, Londres: Routledge, 2008, p. 37-55.

${ }^{49}$ ELTING Bruce et al. Mapping the Arabic blogosphere: politics, culture, and dissent, Internet and democracy case studies, Harvard University: Berkman Center for Internet and Society, 2009.

${ }^{50}$ LAGERKVIST Johan. "The rise of online public opinion in the People's Republic of China", China: An international journal, vol.3, $\mathrm{n}^{\circ} 1$, mars 2005, p. 119-130; XIAO Qiang. "The rise of online public opinion and its political impact", communication à la Chinese Internet Research Conference, Université de Hong Kong, 2008, consulté le 09/07/2009 sur http://jmsc.hku.hk/blogs/circ/files/2008/06/xiao_qiang.pdf.

${ }^{51}$ CEFAI Daniel. "La construction des problèmes publics. Définitions de situations dans des arènes publiques", Réseaux, nº75, 1996, p. 43-65. 


\section{Le poids nouveau de la parole politique des citoyens en Chine}

La mobilisation populaire de très grande ampleur qui a émergé lors de l'affaire Sun Zhigang en 2003 représente en Chine un événement fondateur ${ }^{52}$. Sun, un jeune travailleur nouvellement arrivé à Canton, a été arrêté et conduit dans un centre de rétention pour migrants car il ne pouvait pas produire de papiers en règle. Il est décédé dans des circonstances obscures trois jours plus tard. En quelques jours, ses proches ont réussi à faire connaître son cas sur Internet, son histoire a été reprise de manière extensive dans la presse, et de nombreux internautes ont exprimé leur indignation en ligne. Cette grande publicité a permis d'obtenir une enquête officielle, des condamnations et même un changement de la loi. Pour la première fois, des milliers de personnes prenaient fait et cause publiquement pour que justice soit faite, et cette action a eu un véritable impact politique. Il ne fait aucun doute que la publicité permise par Internet a joué un rôle crucial dans le déroulement de cette affaire.

Depuis, les cas les plus divers se multiplient. On a enquêté publiquement sur d'autres morts suspectes ${ }^{53}$. Des citadins se mobilisent pour arrêter l'installation d'usines chimiques à proximité des zones habitées ${ }^{54}$, ou pour obtenir de meilleures compensations lors de leur expropriation $^{55}$. A au moins deux reprises, les internautes chinois ont pris la défense d'assassins au moment de leur procès, faisant d'eux des icônes de la lutte des citoyens contre l'arbitraire policier ou les abus des fonctionnaires ${ }^{56}$. Après le tremblement de terre qui a

52 THIREAU Isabelle et HUA Linshan. "De l'épreuve publique à la reconnaissance d'un public: le scandale Sun Zhigang", Politix, vol.18, n²71, 2005, p 137-164. HAND Keith J. "Using law for the righteous purpose: the Sunzhigang incident and evolving forms of citizen action in the people's republic of China", Columbia Journal of transnational law, $\mathrm{n}^{\circ}$ 114, 2006.

${ }^{53}$ PEDROLETTI Brice. "Pékin invite les internautes à enquêter sur la mort d'un gardé à vue", Le Monde.fr, 20/02/2009.

${ }^{54}$ ZHU Hongjun. "Xiamen calls an abrupt halt to the PX project to deal with the public crisis", Southern Weekend, 28/05/2007, traduit par SOONG Roland, East South West North, consulté le 16/10/2010 sur http://www.zonaeuropa.com/20070601_1.htm.

${ }^{55}$ YANG Zhizhu. "Dingzihushijian he jie yangai zhidu quexian" Sina.com.cn, 03/04/2007, consulté le 14/10/2010 à l'adresse http://news.sina.com.cn/c/2007-04-03/090612682608.shtml.

${ }^{56}$ Le premier, Yang Jia, a assassiné six policiers pour se venger d'un contrôle d'identité où il avait été violenté. La deuxième, Deng Yujiao, est une jeune pédicure qui a assassiné un officiel alors qu'il tentait de la forcer à avoir un rapport sexuel. HASKI Pierre. "La peine de mort pour le meurtrier héros du Web chinois", Rue89, 02/09/2008, consulté le 14/10/2010 sur http://www.rue89.com/chinatown/lapeine-de-mort-pour-le-meurtrier-heros-du-web-chinois, et "La meurtrière devenue héroine du web 
secoué le Sichuan au printemps 2008, une polémique a éclaté autour des détournements de fonds publics et de leurs conséquences funestes. En effet, les écoles, moins solides que tous les autres bâtiments, se sont effondrées les premières ${ }^{57}$. Les exemples se multiplient ainsi à $l^{\prime} e n v i^{58}$.

Ces mouvements ne sont généralement pas présentés comme des actes politiques, dans le sens où les intéressés ne réclament pas une plus grande participation au pouvoir politique. Les revendications, lorsqu'il y en a, ne portent presque que sur des cas particuliers et locaux. Elles naissent comme des "protestations de poche" ${ }^{59}$. Elles portent sur des problèmes relativement concrets, liés par exemple à la propriété privée, à l'éducation, à des cas d'injustice manifestes, c'est à dire qu'elles ont trait à la défense des droits des citoyens, mais jamais à la nature du régime ou à la démocratisation. Lorsque des injustices sont dénoncées, elles sont formulées comme des appels en direction des autorités pour qu'elles interviennent au nom de l'ordre public, et non pour qu'elles se retirent. Il ne s'agit même pas toujours de mobilisations à proprement parler. Il n'y a parfois ni organisateurs identifiés, ni revendications précises, mais seulement des injustices qui sont rendues publiques et qui suscitent de nombreuses réactions parmi les internautes.

Pourtant, ces mouvements peuvent être considérés comme politiques dans un sens plus large. Les problèmes qui sont relayés en ligne peuvent illustrer les inégalités sociales, la corruption, l'arbitraire policier, autant de notions qui soulèvent une réflexion sur l'ordre social et remettent en question les conditions du vivre ensemble. Ces mobilisations, qui font appel à l'autorité publique pour régler des questions liées à la chose commune, donnent naissance à ce que l'on peut appeler des problèmes publics ${ }^{60}$.

libérée par la justice chinoise", Rue89, 17/06/2009, consulté le 14/10/2010 sur http://www.rue89.com/chinatown/2009/06/17/la-meurtriere-devenue-heroine-du-web-liberee-par-lajustice-chinoise.

57 LI Datong. "China: after the quake, the debate", 20/06/2008, consulté le 14/10/2010 sur http://www.opendemocracy.net/article/china-after-the-quake-the-debate.

${ }^{58}$ HASKI Pierre. Internet et la Chine, Paris: Seuil, 2008.

${ }^{59}$ LYALL Jason. "Pocket protests. Rhetorical coertion and the micropolitics of collective action in semiauthoritarian regimes", World politics, vol. 58, n³, 2006, p. 378-412.

${ }^{60}$ GUSFIELD Joseph. La culture des problèmes publics: l'alcool au volant: la production d'un ordre symbolique, Paris: Economica, 2009. 
La recrudescence de ces événements suggère le fait que le PCC n'est pas en mesure d'empêcher l'expression de certaines revendications ou aspirations sur Internet. Cependant la censure n'a pas disparu. Bien au contraire, la stratégie de contrôle et de propagande des autorités s'est renforcée et transformée pour mieux s'adapter aux caractéristiques spécifiques d'Internet.

Les techniques de contrôle du Net mises en place en Chine sont certainement parmi les plus sophistiquées au monde ${ }^{61}$. La mise en place du réseau a été strictement encadrée par les autorités, et les infrastructures sont faites de sorte à pouvoir tracer toujours plus précisément les dits et les faits des internautes ${ }^{62}$. Le système surnommé "Bouclier d'Or" ou "Grand Parefeu" repose sur plusieurs niveaux de filtrage de l'information, qui permettent à la fois d'empêcher l'accès à certains sites, mais aussi d'effectuer une surveillance des internautes et de signaler les sujets sensibles dans les blogs ou sur les forums. De plus les fournisseurs d'espaces tels que les plates-formes de blogs ou de mails sont responsabilisés pénalement pour les contenus publiés par leurs utilisateurs, de sorte qu'ils sont juridiquement forcés de mettre en place des systèmes de filtrage des contenus a priori ou a posteriori, et ils sous-traitent de fait une bonne partie de la censure ${ }^{63}$. Un billet contenant des critiques du régime ou des allusions aux événements du 4 juin 1989 sur la place Tiananmen n'a ainsi presque aucune chance de rester publié plus de quelques minutes avant d'être effacé. Il arrive que les auteurs de tels messages soient signalés et fassent l'objet d'une surveillance plus spécifique.

${ }^{61}$ Pour une analyse comparative des différents systèmes de censure d'Internet dans le monde, voir DEIBERT Ronald, PALFREY John, ROHOZINSKI Rafal et ZITRAIN Jonathan (dir.). Access denied, Cambridge, Massachussets: MIT Press, 2008.

${ }^{62}$ Sur le filtrage en Chine en particulier, voir entre autres: Internet Filtering in China, Opennet Initiative, consulté le 15/10/2010 sur http://opennet.net/sites/opennet.net/files/ONI_China_2009.pdf. TSUI Lokman. Internet in china: big mama is watching you, Mémoire de Master, Chinese Languages \& Cultures, University of Leiden, July 2001, consulté le 15/10/2010 sur http://lokman.nu/thesis/. "CHINE. Voyage au cour de la censure d'Internet", Reporters sans frontières, octobre 2007, consulté le 15/10/2010 sur http://www.rsf.org/IMG/pdf/Voyage_au_coeur_de_la_censure_FR.pdf.

${ }^{63}$ GOLDKORN Jeremy. "Who's doing the censoring, exactly?", Danwei.org, 04/09/2006, consulté le 15/10/2010 sur http://www.danwei.org/film/whos_doing_the_censoring_exact.php 
Internet est donc à la fois perçu comme le porte-voix qui permet à des millions de personnes de s'exprimer, et comme le panoptique qui les dénonce en un clin d'œil ${ }^{64}$. De ce point de vue, tout Internet semblerait consister en une course-poursuite entre un gendarme et des voleurs, l'ensemble des observateurs étant suspendus à des "coups" joués par les deux parties: diffusion d'un tract par e-mail, enregistrement de l'identité des blogueurs, développement de nouveaux proxys, arrestation d'un internaute... C'est un jeu du chat et de la souris $^{65}$, qui rend bien peu vraisemblable un changement politique important à court terme ${ }^{66}$.

Or il est possible de contourner cette censure de diverses manières. Les internautes les plus doués en informatique utilisent des proxys ${ }^{67}$ pour accéder aux sites qui sont bloqués en Chine. D'une manière générale, on trouve beaucoup de propos critiques "en-dessous du radar" $^{68}$, qui échappent à la censure parce qu'ils sont formulés dans des termes ambigus, ou sous forme d'allusions. Comme le montrent Esarey et Xiao, les internautes ont souvent recours à un langage codé sur Internet pour passer au travers des filtres automatiques, par exemple en jouant sur des homonymes ou du vocabulaire argotique. Ils utilisent abondamment l'euphémisme ou la métaphore, ainsi que la satire ou le second degré. Ils font parfois précéder leurs textes critiques d'avertissements pour s'en dédouaner ou pour les

${ }^{64}$ GIESE Karsten. "Speakers corner or virtual panopticon: discursive construction of Chinese identities online", in MENGIN Françoise (dir). Cyber China, Reshaping national identities in the age of information, New York: Palgrave Macmillan, 2004, p. 19-36.

${ }^{65}$ NEEDHAM Kirsty. "Mice starting to win in the Beijing blogosphere", The Age, 25/11/2008, consulté le 15/10/2010 sur http://www.theage.com.au/news/technology/web/mice-starting-to-win-inthe-beijing-blogosphere/2008/11/24/1227491517178.html?page $=1$.

66 "The Internet and other technologies have potential to serve as a democratizing force - providing more access to information, building a virtual space for community gathering and grassroots development - but these sophisticated technologies (such as biometrics) can also be manipulated into tools for repression, propaganda and enforcing authoritarian control." HOM Sharon, TAI Amy et NICHOLS Gabriel. "The rise of the Internet and advancing human rights", Human Rights in China, China Rights Forum n ${ }^{\circ}$ 3, 2004, consulté le 16/10/2010 sur http://www.hrichina.org/fs/view/downloadables/pdf/downloadable-

resources/The_Rise_of_the_Internet_and_Advancing_Human_Rights.pdf. Voir aussi HUGHES Christopher. "Pourquoi Internet ne démocratisera pas la Chine", Critique internationale, $\mathrm{n}^{\circ} 15$, avril 2002, p.85-104; SAUTEDE Eric. "Les leurres de la modernité, Internet, information et crise du "SRAS" en Chine", Perspectives chinoises, mars-avril 2003, nº 76, p. 21-27.

${ }^{67}$ Comme Tor par exemple. Ce sont des programmes qui empêchent l'identification de l'origine des paquets d'informations, neutralisant les systèmes de blocage des adresses IP.

${ }^{68}$ ESAREY Ashley et XIAO Qiang. "Political expression in the Chinese blogosphere. Below the radar", Asian Survey, vol. 48, n 5, 09/10 2008, p. 752-772. 
attribuer à d'autres. Ils manient ainsi toute une palette de techniques pour parler sincèrement de la situation de leur pays en dépit des contrôles, et ils sont très bien compris de leurs lecteurs. Toutes ces "transcriptions cachées" ${ }^{69}$ permettent la révélation (shai, ou mise en lumière) et la diffusion massive $(h u o)^{70}$ d'informations qui ne seraient jamais devenues publiques avant le développement d'Internet.

Au-delà de ces stratégies de contournement, il est surtout très difficile de censurer les mouvements d'opinion qui se développent au quotidien à la suite de faits divers ou d'anecdotes dans l'actualité. Ceux-ci ne sont pas officiellement contestataires. Ils portent chaque jour sur des thématiques différentes et leur recensement est difficile à actualiser. Faisant le portrait de trois blogueurs relativement politisés, Zhou montre bien qu'il est possible de tenir une variété de discours critiques sur le Net à condition de ne pas transgresser l'interdit fondamental que représente la remise en cause du régime ${ }^{71}$. Ces blogueurs se jouent des définitions mouvantes de ce qui est politiquement correct pour rendre publics leurs points de vue, qui sont au demeurant très différents les uns des autres. Les mobilisations qui se sont développées ces dernières années ont été rendues possibles par l'existence de ces interstices sur Internet, qui échappent à la censure mais dans lesquels peuvent se tenir des échanges entre les internautes sur l'état de la société.

La censure pure et simple ne peut donc pas empêcher le développement de ces échanges, et elle s'avère même parfois contre-productive, car elle met en évidence de manière concrète le manque de transparence des autorités, sans même parler du désagrément pour les internautes qui s'intéressent à ces sujets et qui représentent une précieuse audience. Sur ce plan, la situation des portails Internet ressemble beaucoup à la situation nouvelle des médias de presse ${ }^{72}$. Concurrents, et faisant l'objet d'une politique de développement intensif, ils sont

${ }^{69}$ SCOTT James. Domination and the arts of resistance: hidden transcripts, Yale University Press, 1990.

${ }^{70}$ XIAO Qiang. "The rise of online public opinion and its political impact", communication à la Chinese Internet Research Conference, Université de Hong Kong, 2008, consulté le 16/10/2010 sur http://jmsc.hku.hk/blogs/circ/files/2008/06/xiao_qiang.pdf.

${ }^{71}$ ZHOU Yongming. "Living on the cyber border. Minjian political writers in Chinese cyberspace", Current anthropology, vol.46 n5, Dec.2005, p 779-803.

${ }^{72}$ Sur cette question, voir notamment LEE Chin-Chuan (dir.). Power, money, and media. Communication patterns and bureaucratic control in cultural China, Evanston: Northwestern University Press, 2000 LYNCH Daniel. After the propaganda State. Media, politics, and "thought 
contraints de rechercher de l'audience en ouvrant leur politique éditoriale, et en donnant la plus grande marge de manœuvre possible à leurs utilisateurs, tout en se pliant aux contraintes très strictes de la surveillance des informations sensibles ${ }^{73}$.

C'est pourquoi on assiste à une lente transformation du contrôle effectué par les autorités (et par les fournisseurs de services) sur les internautes. Depuis quelques années, les instruments de filtrage de l'information se sont faits plus discrets. On ne bloque plus l'accès à des plates-formes de blogs entières, mais à certains blogs en particulier. Les technologies sont affinées en permanence, pour atteindre un niveau de contrôle plus précis, et réduire les désagréments pour les utilisateurs qui ne font qu'un usage anodin des instruments de publication en ligne. Surtout, les outils de filtrage ne servent plus seulement à bloquer l'accès à des contenus, mais aussi à signaler les problèmes le plus rapidement possible et à les faire remonter vers les autorités compétentes en vue d'une réaction rapide et adéquate. Il ne s'agit plus de cacher les événements ${ }^{74}$, mais de détecter les problèmes en amont, et d'en imposer l'interprétation la plus favorable possible aux autorités.

Les autorités s'appuient aussi sur une armée d'internautes rémunérés (le "Parti à 50 centimes", ou wumaodang) pour poster sur les forums des avis favorables au régime ${ }^{75}$. Enfin, de gros efforts sont faits pour mettre en place des services de "e-gouvernement", dans le but affiché de faciliter les démarches des citoyens auprès des différentes administrations, mais qui

work" in reformed China, Stanford: Stanford University Press, 1999; ZHAO Yuezhi. Media, market, and democracy in China. Between the party line and the bottom line. Urbana et Chicago: University of Illinois Press, 1998.

${ }^{73}$ LAGERKVIST Johan. "In the crossfire of demands. Chinese news portals between propaganda and the public", in DAMM Jens et THOMAS Simona, Chinese cyberspaces. Technological changes and political effects, Londres et New York: Routledge, 2006, p. 42-63.

${ }^{74}$ Sauf dans les cas les plus graves. Par exemple, les émeutes opposant des Chinois Han et Ouighours au Xinjiang en juillet 2009 ont conduit à une fermeture totale d'Internet, mais les autorités ont pris malgré tout la peine d'ouvrir d'autres canaux d'investigations aux journalistes pour simuler une certaine transparence. BEJA Jean-Philippe. "Après les émeutes au Xinjiang: répression et communication", CERI/Alternatives Internationales, 2009, consulté le 16/10/2010 sur http://www.cerisciencespo.com/archive/2009/juillet/chr_jb.pdf. Je développerai dans la dernière partie les raisons pour lesquelles les mobilisations de ce type n'entrent pas dans le champ très spécifique au sein duquel les autorités sont obligées de laisser les internautes s'exprimer.

75 BANDURSKI David. "China's Guerrilla War for the Web", Far Eastern Economic Review, 04/07/2008. 
servent aussi d'outils de communication politique ${ }^{76}$. Ces instruments sont donc de plus en plus souvent associés à une propagande gouvernementale mieux ciblée et mieux anticipée, destinée à influencer l'opinion publique, et non à l'étouffer ${ }^{77}$. Au-delà des techniques, c'est donc l'objet même de la propagande qui se transforme. Elle doit se faire plus informative et ludique. Il faut influencer sans cesser de plaire, car le public peut se soustraire d'un clic au discours officiel, et forger son opinion dans le clair-obscur des conversations informelles qui se tiennent en ligne. Lagerkvist nomme "ideotainment" ${ }^{78}$ cette transformation des techniques de propagande.

Cette ambivalence du contrôle d'Internet par les autorités est révélatrice du rapport complexe que celles-ci entretiennent avec la parole et l'opinion des citoyens. Il est très significatif que le Président Hu Jintao et son Premier Ministre Wen Jiabao aient tour à tour reconnu, par voie de presse, l'importance de ce qu'ils nomment "opinion publique en ligne", se prêtant même au jeu des questions-réponses avec des internautes ${ }^{79}$. En attribuant aux internautes un rôle de "supervision" de la politique du Parti au plus proche du terrain, ce qui était jusque là l'apanage des journalistes de la presse officielle ${ }^{80}$, ils leur ont accordé une importance politique de premier plan, qui n'est pas sans ambivalence.

Rappelons que le Parti Communiste Chinois se présente depuis sa fondation comme au "service du peuple". Loin d'être seulement l'objet de la propagande, la prise en compte des aspirations de la population se trouve au cour de la question de la légitimité du pouvoir

76 ZHANG Junhua. "Will the government 'serve the people'? The development of Chinese egovernment", New media and society, vol. 4, n 2, p. 163-184.

${ }^{77}$ Pour une présentation plus détaillée de ces transformations, voir Internet Filtering in China, Opennet Initiative, consulté le $16 / 10 / 2010$ sur http://opennet.net/sites/opennet.net/files/ONI_China_2009.pdf.

78 LAGERKVIST Johan. "Internet Ideotainment in the PRC: national responses to cultural globalization", Journal of Contemporary China, vol. 17, n54, 2008, p. 121-140.

79 "Hu Jintao talks to netizens via People's Daily Online", 20/06/2008, People's Daily Online, consulté le 16/10/2010 sur http://english.people.com.cn/90001/90776/90785/6433952.html.

${ }^{80}$ Les journalistes pouvaient faire remonter des cas problématiques auprès de la hiérarchie du Parti, alors même qu'il leur était interdit de les publier. ZHAO Yuezhi et SUN Wusan. "Public opinion supervision: possibilities and limits of the media in constraining local officials". 
politique ${ }^{81}$. Or l'Etat chinois ne peut compter sur sa seule bureaucratie pour faire remonter les informations. C'est pourquoi les réformes des trente dernières années en Chine ont donné lieu à d'importantes transformations des modalités de représentation des préoccupations des citoyens. L'Etat central a donné de plus en plus d'importance aux sondages d'opinion, a permis une certaine diversification des médias, et a même mis en place des procédures (limitées) de participation politique locale, avec notamment des élections locales en milieu rural ${ }^{82}$. Dans le discours officiel, la mise en place de ces dispositifs est explicitement liée à l'optimisation de la prise en compte de l'opinion publique par le Parti et à la modernisation de la gouvernance du pays. Cette tendance se prolonge par une politique active de mise en place de services gouvernementaux sur le Web. Le "e-gouvernement" peut ainsi être analysé comme un effort de légitimation par le PCC, sur le mode technocratique.

Cependant l'opinion publique qui donne lieu à des mobilisations nombreuses et diverses n'est bien évidemment pas celle recueillie par les sites de e-gouvernement chinois. Elle est beaucoup plus diverse, spontanée et imprévisible, beaucoup moins contrôlable. C'est pourquoi cette reconnaissance de la parole des citoyens s'accompagne dans le discours officiel de la notion de "direction de l'opinion publique" ${ }^{83}$. Celle-ci n'est pas nouvelle, mais son adaptation à Internet fait l'objet d'une intense préoccupation politique et académique ${ }^{84}$. Dans les travaux académiques chinois recensés par Lagerkvist, l'opinion publique est très souvent envisagée

${ }^{81}$ CHEN Jie. Popular political support in urban China, Washington: Woodrow Wilson Center Press et Stanford (California): Stanford University Press, 2004; LALIBERTE André et LANTEIGNE Marc (dir.). The Chinese Party-state in the 21st century. Adaptation and the reinvention of legitimacy, londres et New York: Routledge, 2008; RIGBY Thomas H. et FEHER Ferenc (dir.). Political legitimacy in communist states, New York: St. Martin's Press, 1982.

82 "It is difficult to imagine that any regime, democratically elected or not, can sustain itself for very long without taking public opinion into consideration. The Chinese rulers, long before the invention of modern elections, compared public opinion to the river and the state to the boat - a boat that, if misguided, could easily be overturned (shui neng zai zou, yi neng fu zou)." TANG Wenfang. Public opinion and political change in China, Stanford: Stanford University Press, 2005, p. 198.

${ }^{83}$ BANDURSKI David. "Propaganda leaders scurry off to carry out the 'spirit' of Hu Jintao's 'important' media speech", China Media Project, 25/06/2008, consulté le 14/10/10 sur http://cmp.hku.hk/2008/06/25/1079/.

${ }^{84}$ Pour une excellente présentation du vocabulaire sur la "supervision par l'opinion publique" et la "direction de l'opinion publique", voir QIAN Gang. "Guidance • Supervision • Reform • Freedom: Plotting the direction of Chinese media through an analysis of the all-important buzzword" [traduit par David Bandurski], China media project, 13/07/2005, consulté le 16/10/2010 sur http://cmp.hku.hk/2005/07/13/33/. 
comme une masse aisément manipulable, y compris par des "forces étrangères". Les mobilisations sont souvent présentées comme des menaces à l'ordre public, des situations de "crise" ${ }^{85}$. La reconnaissance officielle de l'opinion publique est donc indissociable d'un discours qui ramène les mobilisations incontrôlées au rang de mouvements irrationnels, dangereux, et qui justifie ainsi la nécessité du "guidage de l'opinion" par les autorités. Elle peut donc être considérée comme une manière de circonscrire les mobilisations au sein d'un dispositif rationalisé et maîtrisé par les pouvoirs publics ${ }^{86}$.

Il serait cependant réducteur de considérer cette canalisation des mobilisations en ligne comme une action purement unilatérale de la part des autorités ${ }^{87}$. Certes, tout acteur qui souhaiterait mobiliser un public autour d'un problème est contraint par l'action des pouvoirs publics. Cependant les efforts rhétoriques consentis par les autorités montrent qu'elles sont tenues de justifier leur traitement de la parole des internautes au regard d'une certaine conception de l'ordre public. L'espace étroit dans lequel se glisse la parole des internautes est chaque jour remodelé par cette contrainte réciproque.

Le processus au cours duquel se configurent les mobilisations en ligne est donc le reflet d'un compromis, provisoire et en constante renégociation, entre des conceptions concurrentes de la relation entre gouvernants et gouvernés. Autrement dit, ce qui se joue ici relève de la définition même de la nature du régime chinois. En décrire les ressorts constitue l'objectif de ce travail.

${ }^{85}$ BANDURSKI David. "China and the 'crisis' of public opinion", China Media Project, 17/08/2009, consulté le 16/10/2010 sur http://cmp.hku.hk/2009/08/17/1706/.

${ }^{86}$ Sur le modèle de ce qui s'est fait en France par exemple. REYNIE Dominique. Le triomphe de l'opinion publique, Paris: Odile Jacob, 1998. De manière proche, une analyse foucaldienne conduirait à considérer la notion d'opinion publique comme un dispositif d'imposition d'un certain ordre social, masculin et bourgeois. FRASER Nancy. "Repenser la sphère publique: une contribution à la critique de la démocratie telle qu'elle existe réellement", Hermès, n³1, 2001, p. 125-158.

${ }^{87}$ Par exemple, dans le cas des sondages, la reconnaissance à un moment donné d'une forme spécifique de représentation de la volonté populaire est le fruit d'une lutte entre divers acteurs, journalistes, publicistes, partis politiques, élus qui tous revendiquent un rôle de porte-parole de l'opinion publique, en s'appuyant sur des conceptions différentes de la représentation idéale et légitime de l'opinion publique. BLONDIAUX Loïc. La fabrique de l'opinion : une histoire sociale des sondages, Paris: Seuil, 1998. 


\section{De la dissidence à la contestation}

Dans le contexte chinois, la dimension réticulaire des échanges sur Internet permet de contourner l'impossibilité pour les citoyens de s'associer de manière formelle autour de projets politiques. La mise en réseau informelle remplace ainsi l'association qui n'est pas envisageable, et la publication à titre personnel permet une publicité qui ne serait pas possible autrement, compte tenu des restrictions qui touchent l'édition et la presse traditionnelle. Les formes d'action de ces individus ou groupes ne se limitent pas à l'utilisation des forums ou des blogs. Elles sont très diverses, et peuvent comprendre aussi bien la publication de dessins ${ }^{88}$ ou de vidéos satiriques, que le piratage de sites Internet. Plus les propos tenus sont contestataires, plus cela nécessite de contourner la censure en utilisant l'humour, le sous-entendu ou des jeux de mots, voire en postant des images sans commentaires ${ }^{89}$. Ces possibilités sont saisies par un grand nombre d'individus et de groupes, pour faire circuler des opinions politiques contestataires ou des revendications diverses. La diversité des mots choisis pour désigner ces mouvements sociaux dans les travaux existants souligne cependant la difficulté qu'il y a à les qualifier compte tenu de leur extrême hétérogénéité et des ambivalences inhérentes au contexte politique autoritaire. Si certaines initiatives relèvent complètement de la dissidence au sens où elles sont tournées vers une critique du régime et une remise en cause du rôle du Parti Communiste, d'autres relèvent plutôt de la protestation sur des événements particuliers à l'échelle locale, ou de la "contestation" pour reprendre la distinction proposée par Geoffray ${ }^{90}$.

En s'inspirant des travaux de James Scott sur les transcriptions cachées ${ }^{91}$, l'utilisation stratégique d'Internet pour contourner la censure pourrait s'analyser comme une forme de "résistance" des "dominés" face aux contraintes qui limitent l'expression politique en Chine. Il est vrai que certaines de ces actions sont clairement destinées à diffuser des idées

${ }^{88}$ CODY Edward. "Cartoonist's social spoofs attract young Chinese", Washington Post, 26/01/2006.

${ }^{89}$ ESAREY Ashley et XIAO Qiang. "Political expression in the Chinese blogosphere. Below the radar", Asian Survey, vol. 48, n 5, 09/10 2008, p. 752-772.

90 "La notion de protestation est associée à l'idée d'opposition et de refus et sa connotation est plus proche de l'hostilité, tandis qu'il y a contestation quand il y a controverse, donc quand il existe un espace commun d'interaction, de débat et de discussion, même si cet espace peut aussi être un lieu d'affrontement". GEOFFRAY Marie-Laure. Culture, politique et contestation à Cuba (1989-2009), Thèse pour le doctorat de science politique, Institut d'Etudes Politiques de Paris, 2010, p. 23.

${ }^{91}$ SCOTT James. Domination and the arts of resistance: hidden transcripts, Yale University Press, 1990. 
contestataires au sein de la société chinoise. Par exemple de nombreux dissidents politiques diffusent des articles plaidant pour une démocratisation du régime sur leurs blogs ou par courrier électronique, que ce soit depuis l'étranger ${ }^{92}$ ou depuis le territoire chinois. De telles initiatives tournées directement vers la critique du régime et du Parti Communiste sont réprimées sévèrement et la liste des dissidents emprisonnés ${ }^{93}$ par les autorités pour leurs écrits en ligne s'allonge progressivement. Ainsi le blogueur Hu Jia a-t-il été arrêté en juillet 2008. Son épouse, en liberté surveillée, parvient parfois à donner quelques nouvelles sur son blog ${ }^{94}$. C'est également le cas des auteurs de la Charte 08, qui ont fait circuler par Internet ce manifeste pour une démocratisation du pays, inspirée de la Charte 77 tchécoslovaque. L'un de ses principaux signataires, Liu Xiaobo, arrêté en décembre 2008 et inculpé pour "incitation à la subversion du pouvoir de l'Etat" en juin 2009, est toujours emprisonné. Son implication dans la rédaction et la diffusion de cette charte lui a valu le prix Nobel de la Paix en $2010^{95}$.

Les nombreux cas d'arrestations liées à des prises de parole politiques sur Internet rendent la question de la diffusion des idées dissidentes particulièrement saillante, soulignant par là même le fait qu'elles sont prises très au sérieux par les autorités. Cependant la revendication d'un changement de régime ne représente qu'une petite partie des nombreuses mobilisations en ligne qui marquent l'actualité chinoise. Les motivations des internautes qui se saisissent d'Internet comme outil de mobilisation sont particulièrement variées, et elles sont loin d'être toujours contestataires vis-à-vis du régime. C'est pourquoi le développement de ces

${ }^{92}$ Par exemple, Cai Chongguo me déclarait en 2005 avoir recours à des listes de diffusion par e-mail pour faire connaître ses articles politiques et ses commentaires de l'actualité en Chine métropolitaine. Il tient également un blog en chinois dont l'accès est bloqué depuis la Chine.

${ }^{93}$ Il y aurait soixante-quatorze personnes incarcérées en raison de leur activité en ligne en 2009. Source Reporters Sans Frontières, le 16/10/2010 sur http://fr.rsf.org/barometre-de-la-liberte-de-lapresse-net-citoyens-emprisonnes.html?annee $=2010$.

94 Voir son portrait sur le blog de France 2 à Pékin, consulté le 16/10/2010 sur http://blog.france2.fr/pascal-golomer/index.php/2007/06/03/53907-18-ans-apres-tian-an-men-portraitdun-dissident, et sa biographie sur Wikipedia http://fr.wikipedia.org/wiki/Hu_Jia .

95 "L'opposant chinois Liu Xiaobo reçoit le prix Nobel de la paix 2010", LeMonde.fr, 08/10/2010, consulté le 31/10/2010 sur http://www.lemonde.fr/asie-pacifique/article/2010/10/08/1-opposantchinois-liu-xiaobo-recoit-le-prix-nobel-de-la-paix-2010_1422166_3216.html. 
mouvements est souvent évoqué en termes d'empowerment ${ }^{96}$ de la "société civile" ${ }^{97}$ face à l'Etat $^{98}$.

Les forums de discussion ont été la première cible des investigations allant dans ce sens. Par exemple, Yang observe sur les forums une utilisation efficace d'Internet par une "société civile" incarnée notamment par des associations, et la formation de communautés en ligne autour de discussions sur des sujets de société, ce qui selon lui ouvre un troisième domaine (third realm) entre l'Etat et les individus ${ }^{99}$.

Sur les blogs, un petit nombre de personnalités influentes publient des opinions ou des analyses et peuvent dans une certaine mesure être considérés comme des leaders d'opinion ${ }^{100}$. C'est par exemple le cas des trois blogueurs "minjian" étudiés par Zhou ${ }^{101}$. C'est le cas aussi de quelques experts, avocats ${ }^{102}$, juristes ${ }^{103}$, écrivains ou universitaires célèbres, qui publient sur leurs blogs des commentaires et analyses sur la société chinoise et qui ont une influence sur le déroulement de certaines mobilisations. Il y a d'autre part des individus qui utilisent Internet pour dénoncer des injustices dont ils sont victimes personnellement ${ }^{104}$ ou dont ils ont

${ }^{96}$ Cefaï traduit empowerment par "capacitation", ce qui reflète bien le fait qu'il s'agit d'une capacité à défendre des droits ou des intérêts, et non d'une participation effective au pouvoir politique ou d'une contestation du pouvoir en place. CEFAI CEFAI Daniel. Pourquoi se mobilise-t-on?, Paris: La Découverte, 2007, p. 722.

${ }^{97}$ Celle-ci comprend généralement, dans ce cas, les individus, associations, ou ONG qui défendent des causes de manière militante.

${ }^{98}$ TAI Zixue. The Internet in China. Cyberspace and civil society, New York et Londres: Routledge, 2006.

${ }^{99}$ YANG. Guobin. "The co-evolution of the Internet and civil society in China", Asian Survey, vol. XLIII, n³, mai-juin 2003, p.405-422.

${ }^{100}$ KATZ Elihu et LAZARSFELD Paul. Personal influence, Glencoe: The Free Press, 1955.

${ }^{101}$ Minjian signifie dans ce cas que l'action de ces citoyens se fait en-dehors de toute structure institutionnalisée, en-dehors de l'Etat. Ce terme est parfois proposé comme traduction pour "société civile". ZHOU Yongming. "Living on the cyber border. Minjian political writers in Chinese cyberspace", Current anthropology, vol.46 n5, Dec.2005, p 779-803.

${ }^{102}$ ZYW Anna. "Les avocats chinois, promoteurs d'un réseau juridique virtuel", Hermès, n 55,2009 , p. 65-70.

${ }^{103}$ Des juristes ont par exemple pris position pour une réforme de la gestion des migrants au moment de l'affaire Sun Zhigang. THIREAU Isabelle et HUA Linshan. "De l'épreuve publique à la reconnaissance d'un public: le scandale Sun Zhigang", Politix, vol.18, n71, 2005, p 137-164.

${ }^{104}$ Par exemple, des pères d'enfants disparus qui lancent un appel public sur Internet pour qu'on les aide à briser le silence. ZHAO Kate. "Who can save our children? - Fathers of 400 enslaved children", 
été témoins, et que j'appellerai des "porteurs de causes". On a vu par exemple se développer le phénomène des "journalistes-citoyens" ${ }^{105}$, blogueurs qui sillonnent la Chine et publient des billets en forme de reportages très critiques.

Comme je l'ai mentionné, même les mouvements qui prennent la forme d'une défense des droits des citoyens peuvent s'appuyer sur certaines autorités politiques, au lieu de les critiquer. Ils utilisent aussi très souvent la rhétorique du régime dans leurs arguments. Par exemple, Wu Ping, qui luttait pour obtenir des indemnités d'expropriation plus élevées, a accroché un drapeau chinois sur sa maison, qui trônait au milieu d'un chantier excavé sur plusieurs mètres de profondeurs, et dont l'image insolite a fait le tour du pays sur Internet ${ }^{106}$. Il arrive même que des internautes se mobilisent pour défendre le régime lui-même contre ce qu'ils perçoivent comme des agressions. Très tôt, des internautes chinois ont par exemple utilisé Internet pour réagir à des événements internationaux impliquant la Chine, en affirmant leur patriotisme en ligne, en appelant à des manifestations contre des pays étrangers, ou en piratant des sites étrangers par exemple ${ }^{107}$. Ce type de mouvements patriotiques est récurrent et ils sont parfois d'une telle ampleur qu'ils échappent même au contrôle des autorités. Les internautes qui publient des avis favorables au gouvernement, contre espèces sonnantes et trébuchantes, constituent en revanche un cas très particulier ${ }^{108}$.

Si Internet est utilisé par certains individus comme une forme de participation politique, la remise en cause du régime ne représente qu'une petite partie des causes défendues en ligne.

China Digital Times, 14/06/2007, consulté le 16/10/2010 sur http://chinadigitaltimes.net/2007/06/whocan-save-our-children-fathers-of-400-enslaved-children/.

${ }^{105}$ Voir le cas de "Zola", un jeune blogueur qui couvre des problèmes sociaux relativement sensibles. KENNEDY John. "China: Nation's first citizen reporter?", Global Voices Online, 30/03/2007, consulté le 16/10/2010 sur http://globalvoicesonline.org/2007/03/30/china-nations-first-citizen-reporter/.

${ }^{106}$ BANDURSKI David. "Chinese media and Web users discuss the winners and losers following demolition of China's 'toughest nail house'", China Media Project, 04/04/2007.

${ }^{107}$ Le bombardement de l'ambassade chinoise à Belgrade en 1999 a donné lieu à l'une des premières mobilisations vraiment importantes du Web chinois, inaugurant sans doute, sur un mode politiquement irréprochable, un mode de participation politique qui allait être utilisé plus tard pour des motifs très différents. WU Xu. Chinese Cyber Nationalism: Evolution, Characteristics and Implications, Plymouth: Lexington Books, 2007.

${ }^{108}$ Ils sont connus sous le nom de "50 cents party" à cause de la rémunération qu'ils perçoivent pour chaque commentaire posté. BANDURSKI David. "China's Guerrilla War for the Web", Far Eastern Economic Review, 04/07/2008. 
Certains y voient l'effet d'une censure efficace, qui ne laisse passer que les protestations qui attaquent le régime de biais. Colomb y voit de son côté l'expression d'une auto-limitation des internautes propre à la dimension "paradoxale" de la culture chinoise ${ }^{109}$. Zheng nous invite cependant à dépasser cet apparent paradoxe en décortiquant la complexité des relations de pouvoir au sein de l'Etat d'une part, et au sein de la société d'autre part ${ }^{110}$. Les associations ou organisations "non gouvernementales" ne peuvent se développer en Chine qu'avec la bienveillance de l'Etat, et dans les domaines autorisés par lui ${ }^{111}$. D'autre part Zheng montre bien qu'il existe des divergences d'intérêts entre les différents organes de l'Etat, notamment entre les gouvernements locaux et le pouvoir central. Certains internautes sont capables d'adopter de véritables stratégies de mise en récit pour présenter leurs causes sous le meilleur jour possible et les adresser aux autorités les plus susceptibles de leur être favorables.

Compte tenu de cette diversité d'acteurs, d'organisations, de stratégies et de buts poursuivis, on peut se poser la question de la représentativité des opinions exprimées ainsi. Or l'efficacité de ces acteurs repose essentiellement sur leur capacité à s'attirer le soutien de publics très nombreux qui leur donnent une grande publicité, et tous ne réussissent pas dans cette entreprise. Comment expliquer que certaines thématiques, à des moments particuliers, donnent lieu à des mobilisations véritablement massives et qui donnent l'impression d'un unanimisme très fort? A ce stade, on comprend encore mal comment une mobilisation "prend"112, et pourquoi elle retient à un moment donné le regard d'un nombre important d'internautes, qui le manifestent en ligne d'une manière ou d'une autre.

C'est pourquoi d'autres auteurs proposent de se focaliser sur la diffusion d'une sorte de culture citoyenne au travers d'Internet. Dépassant la question de la contestation du régime, de nombreux travaux attirent l'attention sur une diffusion horizontale de la culture populaire, des centres d'intérêts, des liens interpersonnels, qui entraîne à long terme la formation de visions

109 COLOMB Dominique. Médias et communication en Chine. Au-delà des paradoxes, Paris: L'Harmattan, 2008.

${ }^{110}$ ZHENG Yongnian. Technological empowerment. The Internet, State, and society in China, Stanford University Press, 2008.

${ }^{111}$ BEJA Jean-Philippe. "The changing aspects of civil society in China", Social research, vol. $73, \mathrm{n}^{\circ}$ 1, 2006, p. 53-74.

${ }^{112}$ DOBRY Michel. "Les causalités de l'improbable et du probable: Notes à propos des manifestations de 1989 en Europe centrale et orientale", Culture et Conflits, 1995, p. 111-136. 
du monde de plus en plus autonomes (et critiques) vis-à-vis du discours officiel. Le concept habermassien d'espace public fournit la plupart du temps le cadre d'analyse de ces études qui décrivent l'apparition d'espaces de sociabilité autonomes par rapport à l'Etat et favorables à la prise en compte collective du bien commun, ce qui, dans une vision quelque peu déterministe, serait un préalable nécessaire à une plus grande implication politique du public.

Pour $\mathrm{Hu}$, les nombreuses mobilisations qui ont eu lieu en ligne sont le signe d'un inéluctable développement d'une "société citoyenne", remplaçant des "sujets" obéissants par des "auditoires" désormais prêts à se battre pour défendre leurs droits ${ }^{113}$. Hung, lui, parle d'une "sphère publique virtuelle", qui représenterait un préalable obligatoire à la politisation des individus et à la formation d'une opinion publique ${ }^{114}$. Les blogueurs, quant à eux, sont pour Zhou des précurseurs d'une forme d'expression "populaire" (minjian). Ils ne se contentent pas de reprendre le discours et l'agenda officiels, mais proposent leurs propres visions de l'intérêt du pays ${ }^{115}$. De même, selon McKinnon, les blogs sont surtout importants parce que leur existence même élargit l'espace disponible pour les collaborations, les conversations, et pour la naissance d'un "discours civique", ce qui est porteur de conséquences politiques de long terme ${ }^{116}$. Ce phénomène est souvent désigné comme la formation d'une véritable opinion publique en ligne, qui a désormais une influence considérable sur l'agenda politique $^{117}$. Ici: dernier ouvrage de Yang?

Dans un ouvrage où il compare les débuts de la télégraphie et les débuts d'Internet en Chine à cent ans de distance, Zhou met cependant en garde contre une application trop déterministe de la notion d'espace public à l'Internet chinois. Il rappelle que la technologie en elle-même ne conditionne pas complètement la nature des usages qui en sont faits et que

${ }^{113}$ HU Yong. "Why remain independent and how to be alternative?", in HU Yong, FENG Chien San et MCKINNON Rebecca. Info-Rhizome: Report on independent media in the Chinese-speaking world, Hong Kong: Hong Kong In-Media, 2009, p. 13-16, p.16.

${ }^{114}$ HUNG Ching-Fu. "Public discourse and 'virtual' political participation in the PRC: the impact of the Internet", Issues \& Studies, vol. 39, n4, 2003

${ }^{115}$ ZHOU Yongming. "Living on the cyber border. Minjian political writers in Chinese cyberspace", Current anthropology, vol.46 n5, Dec.2005, p 779-803.

${ }^{116}$ MCKINNON Rebecca. "Flatter world and thicker walls ? Blogs, censorship and civic discourse in China", Public Choice, n 134, 2008, p. 31-46.

${ }^{117}$ LAGERKVIST Johan. "The rise of online public opinion in the People's Republic of China", China: An international journal, vol.3, ${ }^{\circ} 1$, mars 2005, p. 119-130. 
l'existence d'espaces d'expression nouveaux ne conduit pas nécessairement à une plus grande politisation des individus ${ }^{118}$. Force est de constater en effet que le degré de politisation de la plupart des internautes reste très faible. Les usages les plus répandus du Web sont consacrés au divertissement, c'est à dire au partage de photos entre amis, à l'écoute ou au téléchargement de musique par exemple ${ }^{119}$. Comme partout ailleurs dans le monde, les sites qui traitent de potins mondains ont beaucoup plus d'audience que les pages d'actualité politique. De plus, l'intérêt politique des différentes mobilisations qui ont eu lieu en ligne est tout à fait inégal ${ }^{120}$.

Certains travaux s'intéressent à la formation de l'opinion publique sous un angle plus procédural, le plus souvent en recherchant des formes de délibération en ligne, notamment sur les forums. Or ceux-ci semblent souvent trop désordonnés pour favoriser le développement d'échanges raisonnés et constructifs entre les citoyens chinois. Lorsqu'elles existent, les discussions qui contiennent une dimension politique ne réunissent que rarement les critères idéaux d'une discussion d'intérêt général (qui, au demeurant, sont difficiles à définir). S'il y a des formes de délibération en ligne, elles sont encore embryonnaires ou confinées sur de petites plates-formes $^{121}$. Ailleurs, la violence des échanges est particulièrement marquante sur des questions qui mettent en cause l'unité de la nation ${ }^{122}$. Des faits divers ont même conduit à

118 ZHOU Yongming. Telegraphy, the Internet, and Political Participation in China, Stanford University Press, 2006.

119 Pour un aperçu global des usages les plus courants d'Internet en Chine, voir les statistiques du CNNIC, ou pour une approche plus fine dans les zones urbaines, GUO Liang. Surveying Internet usage and its impact in seven Chinese cities, Markle Foundation, 2007.

${ }^{120}$ Peut-on par exemple mettre sur le même plan un appel patriotique au boycott de Carrefour, une indignation générale devant un cas d'esclavage d'enfants, et une chasse à l'homme contre un mari coupable d'adultère?

${ }^{121}$ MIN Jiang. "Authoritarian deliberation: public deliberation in China", article présenté au Congrès annuel de l'International Communication Association - ICA, Montréal, mai 2008; ZHOU Xiang. "Is an online public sphere emerging in China? A comparative study of deliberativeness of Chinese online discussion forums", Article présenté à la Convention de l'Association internationale de communication, Montréal, Mai 2008.

122 TAYLOR Sophie. "Chinese seethe on Web over rare riots in Tibet", Reuters, 15/03/2008; MACKINNON Rebecca. "Tibet... Is discussion possible?", Rconversation, 16/03/2008, consulté le 16/10/2010 sur http://rconversation.blogs.com/rconversation/2008/03/tibet-is-discus.html. 
plusieurs reprises à des campagnes de harcèlement contre des individus, comparables à de véritables chasses à l'homme en ligne ${ }^{123}$.

Le Web chinois ne propose donc pas seulement des échanges pacifiés et rationnels entre des individus éclairés, mais il s'apparente bien plus souvent à une multiplicité d'espaces désarticulés, animés par des individus aux motivations hétérogènes, où règne en général un très grand désordre. En somme, l'espace public proposé par le Web est tout aussi mosaïque ${ }^{124}$ et conflictuel en Chine que n'importe où dans le monde. Cela n'empêche pourtant pas l'apparition fréquente de mobilisations qui semblent presque unanimes.

\section{2 - Le cadrage de la prise de parole}

Il me semble qu'une approche interactionniste (ou "praxéologique"125) permettrait de rendre compte de cette complexité. La "construction des problèmes publics" ${ }^{126}$ peut s'analyser comme le résultat d'interactions entre divers acteurs qui confrontent des points de vue sur des "arènes publiques", au moyen de récits, de témoignages, de discours d'experts et d'autres formes de représentations. Au cours de ces interactions, les acteurs procèdent à des opérations de "cadrage"127 qui leur permettent d'attirer 1"attention publique" sur ces problèmes en justifiant de leur "pertinence". Ce cadrage comprend notamment le respect des modalités légitimes de la prise de parole ${ }^{128}$ et des types de justifications ${ }^{129}$ ou d'argumentaires recevables dans les espaces publics concernés, et notamment sur Internet. C'est une convention, un compromis normatif en constante renégociation au fil des interactions et des conflits d'interprétation entre les acteurs. En permettant à certains problèmes plutôt qu'à

${ }^{123}$ FRENCH Howard. "Mob rule on China's Internet: The keyboard as weapon", The New York Times, 01/06/06.

124 FRANCOIS, Bastien, et NEVEU, Erik (dir.), Espaces publics mosaïques, acteurs, arènes et rhétoriques des débats publics contemporains, Presses universitaires de Rennes, 1999.

${ }^{125}$ QUERE Louis. "Opinion: l'économie du vraisemblable. Introduction à une approche praxéologique de l'opinion publique", Réseaux, vol. 8 n43, 1990, p. 33-58.

${ }^{126}$ QUERE Louis. "Opinion: l'économie du vraisemblable. Introduction à une approche praxéologique de l'opinion publique", Réseaux, vol. 8 n43, 1990, p. 33-58.

${ }^{127}$ GOFFMAN Erving. Les cadres de l'expérience, Paris: Minuit, 1991.

${ }^{128}$ GOFFMAN Erving. Façons de parler, Paris: Minuit, 1987.

${ }^{129}$ BOLTANSKI Luc et THEVENOT Laurent. De la justification. Les économies de la grandeur, Paris: PUF, 1987. 
d'autres de recueillir l'attention de publics, et a fortiori de porter des conséquences politiques, ce processus fait partie intégrante du fonctionnement politique du pays.

En me fondant sur cette approche, je souhaite analyser la formation de l'opinion publique chinoise en ligne comme le produit d'une idéologie dominante dans la Chine urbaine, qui place l'expression personnelle des individus au cœur d'un important projet de "modernisation" du pays. Cette idéologie fonctionne comme une norme sociale qui définit, ou "encadre" ${ }^{130}$ au sens de Goffman, les modalités légitimes de la prise de parole individuelle et collective sur Internet. Bien plus que la censure proprement dite, cette norme donne aux internautes des indications sur ce qui se dit et ce qui ne se dit pas, ce qui se dit à demi-mot ou en privé. Je souhaite montrer que si cette norme fonde largement l'autocensure de nombreux internautes, elle fournit aussi des argumentaires sur la base desquels de nombreuses causes individuelles ont trouvé un écho auprès d'un large public. C'est à partir de cette idéologie dominante que je souhaite décrire la formation complexe des mobilisations en ligne. Il est donc nécessaire d'en dresser une description détaillée.

\section{La modernité à la chinoise: une promesse}

Les transformations économiques et politiques des trente dernières années se sont accompagnées d'une révision complète des fondements idéologiques du contrat qui lie le Parti Communiste Chinois à la population ${ }^{131}$. Depuis 1978 , le Parti Communiste Chinois fonde sa légitimité $^{132}$ dans sa capacité à assurer les conditions d'un développement économique

${ }^{130}$ GOFFMAN Erving. Les cadres de l'expérience, Paris: Minuit, 1991 GOFFMAN Erving. Façons de parler, Paris: Minuit, 1987. Pour une analyse détaillée de l'apport des théories de Goffman à l'analyse des mobilisations, voir CEFAI Daniel. Pourquoi se mobilise-t-on?.

131 Pour une bonne synthèse des réformes et de leurs enjeux, voir par exemple SAICH Tony. Governance and politics of China, New York: Palgrave, 2001 ou ZHAO Yuezhi et SUN Wusan. "Public opinion supervision: possibilities and limits of the media in constraining local officials", in GOLDMAN Merle et PERRY Elizabeth (dir.). Grassroots political reform in contemporary China, p. 300-324.

132 J'entends cet effort de légitimation comme "l'entretien par les gouvernants et les groupes dominants de l'image d'un pouvoir politique accordé à des valeurs qui sont, dans le même temps, présentées comme constitutives de la cohésion morale de la société". LAGROYE Jacques, FRANCOIS Bastien et SAWICKI Frédéric. Sociologie politique, Paris: Presses de Sciences-po et Dalloz, 2006, p. 448. Souligné par l'auteur. 
soutenu ${ }^{133}$. En échange de l'introduction de l'économie de marché et d'un assouplissement du contrôle des individus, le PCC réaffirme régulièrement et fermement son rôle de leader politique incontestable, garant de l'unité de la nation et de la stabilité du pays, qui sont présentées comme les conditions sine qua non du développement économique.

Par conséquent la montée des inégalités sociales engendrée par trente ans de croissance économique $^{134}$ est peu à peu devenue un enjeu crucial pour le gouvernement chinois ${ }^{135}$. Les différences territoriales entre les zones urbaines plus développées et les zones rurales se déclinent en inégalités d'accès à l'éducation, à la santé ou même à l'emploi. Si le pays s'est considérablement enrichi, tous les Chinois n'en bénéficient pas de la même manière, alors que dans le même temps les attentes de chacun sont de plus en plus élevées. La préoccupation visible dont font preuve les sociologues chinois ${ }^{136}$ sur ces questions montre à quel point les clivages retiennent l'attention en plus haut lieu. Comment comprendre autrement le slogan du président $\mathrm{Hu}$ Jintao mettant au premier plan le concept de "société harmonieuse"?

Dans ce contexte, l'accession d'une certaine frange de la population à un niveau de prospérité confortable soulève de nombreuses spéculations ${ }^{137}$. Cette portion de la population étant moins riche que les milliardaires "parvenus", et n'étant plus vraiment pauvre, elle a rapidement été nommée la "classe moyenne". Ce terme recouvre une signification relativement vague et qui repose d'abord sur une observation intuitive. Tous les observateurs de la Chine contemporaine (journalistes, sociologues, mais aussi résidents) peuvent décrire dans leur entourage l'émergence d'un mode de vie nouveau, marqué par un niveau de

${ }^{133}$ HOWELL Jude (dir.). Governance in China, Oxford: Rowman \& Littlefield, 2004; LALIBERTE André et LANTEIGNE Marc (dir.). The Chinese Party-state in the 21st century. Adaptation and the reinvention of legitimacy, londres et New York: Routledge, 2008.

${ }^{134}$ A l'occasion de l'anniversaire des réformes à l'hiver 2008/2009, les articles de synthèse ne manquent pas dans la presse. ANG Audra. "30 years transform China, but not its politics", Associated Press, 17/12/2008; BRANIGAN Tania. "30-year journey from Mao to the market", The Guardian, $18 / 12 / 2008$.

135 GIROIR Guillaume. "Les fractures socioterritoriales en Chine, ou l'impossible 'société harmonieuse'?", Perspectives chinoises, n²007/3, p. 88-97.

${ }^{136}$ LI Peilin. "The building of a harmonious society and the 'China experience'", Social sciences in China, vol.28, automne 2007, p. 98-107.

${ }^{137}$ ZHOU Xiaohong. "La classe moyenne chinoise: réalité ou illusion?", traduit par Mylène Hardy et Jean-Louis Rocca, Actes du colloque Quels outils pour analyser les sociétés contemporaines?, Pékin: Université Tsinghua, 19 et 20 mai 2007. 
consommation plus élevé, des ambitions professionnelles débordantes, un intérêt plus marqué pour le cinéma, la musique ou la décoration intérieure... Ces observations convergentes restent cependant diffuses, floues. Cette "classe moyenne" reste très difficile à saisir, à définir et à quantifier.

Le premier réflexe pour tenter de saisir les contours de la classe moyenne est de procéder par une classification des revenus. Or les plus grandes difficultés se manifestent déjà. Quels critères adopter? En 2004, le Bureau national des statistiques ${ }^{138}$ retenait une fourchette de revenus annuels de 60000 à 500000 RMB par an et par ménage. Cela a-t-il un sens d'agréger dans un même groupe des personnes dont les salaires s'échelonnent de un à dix? De même, peut-on mesurer ensemble des niveaux de revenus dans des villes différentes, ou même dans les campagnes, où le coût de la vie est tout à fait incomparable?

Selon les critères retenus, les chiffres peuvent varier considérablement. Ainsi un rapport $^{139}$ de 2001 sur la question donnait-il lieu à une grande controverse en chiffrant à 80 millions de personnes la classe moyenne chinoise. Le Quotidien du Peuple annonce, lui, dès 2001, 200 millions de personnes pour 2006, en citant une étude prospective du Centre national des informations ${ }^{140}$. Le China Daily ${ }^{141}$ cite quant à lui, en 2004, trois sources contradictoires. L'Académie des sciences sociales de Chine aurait évalué la classe moyenne à 19\% de la population, soit 247 millions de personnes. Le même article cite un chiffre de BNP Paribas de 2002 qui fixerait le niveau de revenus à 75000 RMB annuels, ce qui aurait alors concerné 50 millions de personnes. Il cite enfin les "chiffres officiels" du Bureau national des statistiques qui prévoyait que 75 millions de personnes appartiendraient à la classe moyenne en 2005 . Une autre étude, en $2006^{142}$, où les chercheurs ont retenu le critère de revenu 2000

${ }^{138}$ Cité par ZHOU Xiaohong. "La classe moyenne chinoise: réalité ou illusion?", op. cit.

${ }^{139}$ LU Xueyi. Dangdai Zhongguo shehui jieceng yanjiu baogao [Rapport d'étude sur les couches sociales de la Chine actuelle], Beijing: Shehui kexue wenxian chubanshe, 2001

140 "La classe moyenne chinoise comptera d'ici cinq années 200 millions de personnes", Le Quotidien $d u$ peuple, 20/07/2001, consulté le $16 / 10 / 2010 \quad$ sur http://french.peopledaily.com.cn/french/200107/20/fra20010720_48311.html.

${ }^{141}$ XIN Zhigang. "Dissecting China's 'middle class'", China Daily, 27/10/2004, consulté le 16/10/2010 sur http://www.chinadaily.com.cn/english/doc/2004-10/27/content_386060.htm .

${ }^{142}$ CAMBIER Claire. "Une étude de la classe moyenne chinoise controversée", Aujourd'hui la Chine, 13/11/2006, consulté le 16/10/2010 sur http://www.aujourdhuilachine.com/actualites-chine-une-etudede-la-classe-moyenne-chinoise-controversee-1034.asp?1=1. 
RMB mensuels dans les villes et 1500 RMB mensuels dans les campagnes, a soulevé l'indignation générale par l'invraisemblance de ses résultats. Une majorité des répondants annonçaient en effet des revenus très élevés, mettant en doute les méthodes d'enquête.

Le critère du revenu permet toutefois de préciser le fait que cette classe n'est pas véritablement conçue comme "moyenne". Elle se distingue certes de la catégorie des "parvenus" qui sont soupçonnés de tenir leurs privilèges de leurs relations et de la corruption, et elle est porteuse de valeurs différentes fondées sur le mérite, l'éducation, l'esprit d'entreprise. Cependant la catégorie généralement désignée par le terme "classe moyenne" a un mode de vie très au-dessus de la moyenne des citoyens chinois. Compte tenu de l'écart très important entre les différentes catégories de revenus, il est hasardeux de situer ce groupe en formation au "milieu" de l'échelle des revenus ou du niveau de vie, alors que ce groupe se situe plus vraisemblablement dans la catégorie des hauts revenus. Dès lors cette classe ne représenterait qu'une petite portion de la population, d'un nombre comparable par exemple aux 150 millions de migrants qui peupleraient les villes sans être l'objet d'un discours aussi enthousiaste $^{143}$. Néanmoins la classe moyenne chinoise reste toujours aussi insaisissable.

D'autres articles mentionnent des critères de niveaux de vie, comme la propriété d'un appartement $^{144}$ ou d'une voiture. L'appartement surtout garantit une certaine stabilité à leurs propriétaires dans la mesure où il existe très peu de mécanismes de protection sociale. Acheter son logement est une priorité pour les jeunes actifs. C'est bien souvent la condition sine qua non pour pouvoir s'installer, et se marier. Les annonces matrimoniales ont d'ailleurs consacré l'expression "you fang, you che" [appartement, voiture] comme un critère de sélection rédhibitoire. Il semblerait donc que la possession d'un appartement soit un indicateur intéressant de l'amélioration du niveau de vie de certaines catégories de la population. Cependant ce critère est biaisé par une grande diversité dans les modalités d'accès à ce type de ressources. Ceux qui possèdent un appartement ne sont-ils pas, plutôt que des gens relativement aisés, ceux qui disposent de bonnes connexions dans les administrations, ou bien tout simplement d'anciens employés des entreprises d'Etat qui ont pu acheter leur logement à

\footnotetext{
143 "China now has 150 million migrant workers", Reuters via China Daily, 29/10/2006, consulté le 16/10/2010 sur http://www.chinadaily.com.cn/china/2006-10/29/content_719289.htm.

${ }^{144}$ LI Jian et NIU Xiaohan. "Accès à la propriété et formation d'une classe moyenne à Pékin", Perspectives chinoises , $\mathrm{n}^{\circ} 74,11-12 / 2008$, p. 4-20.
} 
des tarifs préférentiels auprès de leur unité de travail ${ }^{145}$ Ainsi le critère du logement peut-il rassembler des entrepreneurs très riches qui collectionnent les appartements à des fins de spéculation (ce qui est désormais limité par la loi) et des retraités aux revenus faibles, et qui n'ont pour seul patrimoine que l'appartement qu'ils ont obtenu de leur unité de travail pour une bouchée de pain.

Un autre critère envisagé pour désigner la classe moyenne est celui de l'activité professionnelle, et en particulier de la catégorie des "entrepreneurs", ceux-là même qui sont au cœur de la "Théorie des trois représentations"146 de Jiang Zemin. Cette dernière explicite les trois catégories que le PCC se doit de représenter depuis que les ouvriers ne sont plus au cœur de la stratégie de développement du gouvernement. Désormais le PCC représente les "forces productives progressistes", la "culture chinoise moderne" et les "intérêts fondamentaux de la majorité de la population" chinoise. Cette théorie est généralement comprise comme une façon de coopter les entrepreneurs dans les sphères du pouvoir ${ }^{147}$. Ces nouveaux admis, capitalistes ${ }^{148}$, sont désormais invités à entrer au Parti. Bien souvent cependant, les cadres du Parti ont pu bénéficier de leur capital politique pour faciliter leur conversion à l'entreprise capitaliste ${ }^{149}$. Cette cooptation ne fait ainsi qu'entériner des liens déjà très complexes et ambigus. Les entrepreneurs chinois "sont moins une classe moyenne qu'une future part centrale de la classe dirigeante" ${ }^{150}$. Ainsi Gentelle voit-il naître une bourgeoisie

145 TOMBA Luigi. "Creating an urban middle class: social engineering in Beijing", The China Journal, n51, 2004, p. 1-26.

146 TRAN Emilie. "Trois représentations", in SANJUAN Thierry (dir.). Dictionnaire de la Chine contemporaine, Paris: Armand Colin, 2007.

147 ZHANG Houyi. "The position of the private entrepreneur stratum in China's social structure", Social Sciences in China, vol.16, nº 4, 1995, p.29-36.

${ }^{148}$ BERGERE Marie-Claire. Capitalismes et capitalistes en Chine: XIXe-XXIe siècle, Paris: Perrin, 2007; GUIHEUX Gilles. "Le renouveau du capitalisme familial: défense et illustration par un entrepreneur du Zhejiang", Perspectives chinoises, n 87 , janvier/février 2005, p.22-32.

${ }^{149}$ NEE Victor. "The emergence of a market society", American sociological review, ${ }^{\circ} 56$, vol.3, p. 267-282.

${ }^{150}$ GOODMAN David. "Why China has no new middle class", in GOODMAN David (dir.). The new rich in China. Future rulers, present lives, London: Routledge, 2008, p. 23-37, p. 24. 
industrielle dans les sphères du pouvoir ${ }^{151}$, et Chang voit la résurgence d'une sorte de bourgeoisie ${ }^{152}$.

Cependant, il semble que la classe moyenne puisse recouvrir une population plus large que ce groupe particulier d'entrepreneurs. Ceux-ci représentent certes une attitude nouvelle par rapport à l'entreprise privée et à l'enrichissement, mais la notion de classe moyenne ne se limite pas forcément aux seules caractéristiques de ce groupe. Pour beaucoup de sociologues, elle regroupe en fait l'ensemble des travailleurs non manuels, et en particulier la catégorie des "cols blancs", qui avait retenu l'attention de Mills dans un ouvrage classique sur les classes moyennes américaines ${ }^{153}$. Il est vrai que les réformes économiques des trente dernières années ont donné lieu à d'importantes transformations des formes d'emploi et notamment à une augmentation du nombre de salariés des entreprises privées, qui forment un contingent de plus en plus important d'employés de bureaux, de commerciaux ou de cadres, autrement dit des "cols blancs".

L'amélioration rapide du niveau de vie est en effet une réalité pour un grand nombre de cadres salariés de grandes entreprises privées ou publiques, et en premier lieu les employés des entreprises étrangères. Selon Luigi Tomba, cette catégorie se caractérise par la propriété du logement, un haut niveau d'éducation, des responsabilités managériales, et une identité de consommateurs bien définie, ce qui les place au cœur du développement économique ${ }^{154}$. Le marché intérieur dont la Chine aurait tant besoin serait assuré par la classe moyenne qui aurait du temps et de l'argent à dépenser. Les salariés, ou plus généralement les employés de bureaux ou "cols blancs", formeraient ainsi une sorte de bourgeoisie sur laquelle reposent de nombreux espoirs de développement.

${ }^{151}$ GENTELLE Pierre. "Une société en mouvement", Questions internationales, nº, mars-avril 2004, p.26-38.

${ }^{152}$ CHANG Chen-pang. "The resurgence of the bourgeoisie", Issues and Studies, vol. 30, n5, mai 1994, p. 31-44.

${ }^{153}$ MILLS Charles Wright. Les cols blancs : essai sur les classes moyennes américaines, Paris: Seuil, 1970.

154 TOMBA Luigi. "Creating an urban middle class: social engineering in Beijing", The China Journal, $\mathrm{n}^{\circ} 51,2004$, p. 3-4. 
Ces dernières remarques montrent que l'idée de classe moyenne recouvre une grande diversité de situations, selon le mode de résidence, le niveau de revenus ou le statut professionnel entre autres critères. Un seul critère ne peut donc suffire à caractériser la classe moyenne chinoise (peut-être pas plus que toute autre classe moyenne). C'est sur ce constat que des enquêtes tentent le pari de délimiter une classe moyenne à l'aide de critères croisés. Par exemple, Zhou utilise le revenu, la catégorie professionnelle ainsi que le niveau d'études dans un sondage, pour arriver au chiffre de $11.9 \%$ des habitants des villes, soit 105 millions de personnes en Chine en $2005^{155}$. Zhou suggère une liste des types d'activités qui forment le groupe de la classe moyenne: y figurent les chefs d'entreprises privées, les petits commerçants et indépendants, ainsi que les cadres du gouvernement, les cols blancs des entreprises étrangères, les managers d'entreprises et enfin "le groupe à hauts revenus apparu avec l'utilisation des hautes et nouvelles technologies et les nouveaux secteurs".

Il en ressort une impression très disparate et l'idée que ce sont des individus concernés par une certaine ascension sociale, qu'elle soit due à un bon niveau d'éducation, une bonne situation professionnelle ou à un capital social ou patrimonial. Luigi Tomba décrit par exemple une "classe moyenne urbaine, éduquée et riche en ressources", définie de manière très floue: un groupe social large, qui partage le potentiel ou l'expérience d'un accès accru à des ressources (éducation, information et richesse) et d'une ascension sociale rapide, et qui devient l'objet, l'inspiration et l'exemple sur lequel s'appuient les discours gouvernementaux actuels sur le développement personnel. ${ }^{156}$

Cette idée d'ascension sociale semble fédérer de plus en plus de chercheurs. Wang Xin constate par exemple que les individus d'un (petit) échantillon ont des "identités divergentes, et des intérêts convergents" 157 . Plutôt qu'une classe "moyenne", Goodman propose de parler des "nouveaux riches" que sont les "bénéficiaires de la croissance économique". Loin d'ignorer la disparité des situations au sein même de cette catégorie, en termes de revenus,

${ }^{155}$ ZHOU Xiaohong. "La classe moyenne chinoise: réalité ou illusion?", traduit par Mylène Hardy et Jean-Louis Rocca, Actes du colloque Quels outils pour analyser les sociétés contemporaines?, Pékin: Université Tsinghua, 19 et 20 mai 2007.

${ }^{156}$ TOMBA Luigi. "Of quality, harmony and community: civilization and the middle class in urban China", Positions: East Asia cultures critique, 2009.

${ }^{157}$ WANG Xin. "Divergent identities, convergent interests: the rising middle-income stratum in China and its civic awareness", Journal of contemporary China, vol. 17, n ${ }^{\circ}$ 4, 2008, p. 53-69. 
d'activités professionnelles ou de statuts, il propose d'en faire un objet d'analyse. "Comprendre les interactions entre ces hiérarchies de richesse, de pouvoir et de statuts aide à expliquer les dynamiques de la société chinoise et l'exercice du pouvoir politique."158

L'ascension, de fait, est en grande partie l'apanage de la jeune génération qui a évidemment plus de cartes en mains pour se positionner sur le nouvel échiquier social que leurs aînés, pour qui les dés sont jetés. Les jeunes nés après 1980 en particulier sont affublés d'un surnom, "post 80" (balinghou), qui souligne leur spécificité dans la société chinoise. N'ayant connu que la Chine de l'ouverture, enfants uniques pour beaucoup (surtout dans les villes), ces jeunes ont été élevés dans un esprit très différent de celui qui a vu grandir leurs parents. Dans leur génération prime surtout l'esprit de compétition et l'individualisme, qui se traduit bien souvent par des ambitions assez élevées. C'est ce qui fait que Rosen opte par exemple pour l'expression "jeunesse en cours d'ascension sociale"159 et qu'il prend des étudiants comme objet d'étude.

Les ambitions de ces jeunes ne sont pas toujours réalisées, et beaucoup sont même cruellement déçues, mais elles se construisent dans un cadre commun, à l'école, devant la télévision, avec leurs parents, entre amis, et désormais, sur Internet. En ce sens, la "classe moyenne" serait peut-être mieux définie au travers des aspirations des individus qu'au travers de leur statut matériel ou de leur niveau de vie effectif. Cette notion d'ambitions suggère une approche de la classe par les représentations.

Ces atermoiements sur la définition de la classe moyenne suggèrent qu'il existe en fait très peu de fondements empiriques pour parler de l'existence d'une classe moyenne à proprement parler en Chine. Pour autant, la circulation de l'idée de classe moyenne, et son appropriation par un nombre grandissant de personnes ne doivent pas être ignorées. Il faut analyser l'effort même de délimitation et d'identification de la classe moyenne, et, selon les termes de Boltanski, le "travail de regroupement, d'inclusion et d'exclusion, dont il est le produit, et en analysant le travail social de définition et de délimitation, qui a accompagné la

${ }^{158}$ GOODMAN David (dir.). The new rich in China. Future rulers, present lives, London: Routledge, 2008 , p. 3.

159 ROSEN Stanley. "The victory of materialism: aspirations to join China's urban moneyed classes and the commercialisation of education", The China Journal, n51, 2004, p. 29. 
formation du groupe et qui a contribué, en l'objectivant, à le faire être sur le mode du cela-vade-soi". ${ }^{160}$

Reprenant cette profession de foi, Rocca plaide pour une approche de la classe par les représentations et les discours, tenant compte du contexte historique, qui est riche d'enseignements sur le projet de société promu par les autorités et son écho dans diverses sphères. ${ }^{161}$ Dans les efforts que les sociologues chinois déploient pour définir la classe moyenne il décèle une grande normativité, et la volonté de cerner les contours d'une société idéale qu'il s'agit de faire émerger au plus vite dans l'intérêt de la stabilité et du développement du pays, et a fortiori dans l'intérêt du PCC. C'est à eux en effet qu'est confiée la tâche d'identifier la classe moyenne, de la délimiter et de formuler des recommandations pour favoriser son expansion.

Ce processus est particulièrement problématique en Chine, dans le contexte du remplacement de la classe ouvrière par les entrepreneurs au cœur du Parti. Comme le souligne Touraine, "en parlant de position moyenne, on introduit l'idée d'échelle: les strates moyennes sont aussi celles qui occupent des positions non extrêmes sur des échelles de stratification classant les individus selon leur qualification, leur revenu, leur niveau d'éducation, leur prestige, leur statut, etc. D'autre part, en parlant de classes, on se réfère au thème des rapports de classes" ${ }^{162}$. Les sociologues chinois hésitent entre de nombreuses formulations - classes, couches, groupes etc - pour désigner cette catégorie de la population. Utiliser le terme marxiste de "classe" revient à introduire l'idée que de nouveaux conflits de classes peuvent apparaître. Le terme de "couches" sociales au contraire permet d'entériner l'idée d'une stratification de la société sans nécessairement souligner les intérêts divergents entre les différentes couches. Une tendance générale est de promouvoir l'idée qu'une société "en forme

${ }^{160}$ BOLTANSKI Luc. Les cadres: la formation d'un groupe social, Paris: Minuit, 1982, p.51-52. Souligné par l'auteur.

${ }^{161}$ ROCCA Jean-Louis. "Political crossroad, social representations and academic intervention: the formation of a middle class in China", communication au International Forum on Comparative Studies on Middle Class, Changsha (Hunan), Juillet 2007, p. 2 et p. 9.

162 TOURAINE Alain. "Classes moyennes", Encyclopaedia Universalis [CD ROM], 2008. 
d'olive", c'est à dire avec une proportion importante de gens de statut intermédiaire, est souhaitable pour la stabilité du pays ${ }^{163}$.

La classe moyenne peut donc être envisagée comme un modèle donné à l'ensemble de la population chinoise, une direction vers laquelle les autorités invitent la population à tendre. Ils le font par la voix des chercheurs et par de nombreux autres canaux, que ce soit de manière directe ou indirecte. Par exemple, les promoteurs immobiliers sont des relais particulièrement efficaces de l'image de la classe moyenne ${ }^{164}$. Ils entourent les chantiers urbains de grandes palissades recouvertes d'images de lieux de vie idéalisés, grandes villas, salons luxueux, et autres terrains de golfs. La publicité, l'éducation sont des vecteurs de diffusion de ce modèle idéalisé, et c'est en cela qu'il est possible de parler d'ingénierie sociale (social engineering) ${ }^{165}$.

L'image véhiculée par cette "classe moyenne" illustre en effet un mode de vie nouveau. Avec elle est définitivement entériné le mot d'ordre de l'enrichissement (modéré) qui devient ouvertement une priorité. La classe moyenne incarne la fameuse prospérité dont la majorité de la population ne profite pas encore. Cette prospérité est due, en principe, à leur capacité d'innovation, leur niveau d'éducation et leur énergie. Ils sont en effet décrits comme des forces vives de la nation, et les moteurs du développement économique. Les plus riches étalent volontiers leurs berlines aux vitres fumées et leurs téléphones portables. L'appartement, la voiture, les bijoux, mais aussi des loisirs dispendieux comme le golf ou l'équitation, font partie du niveau de vie théorique de cette classe. Ils rassemblent en somme toutes les caractéristiques extérieures d'un mode de vie nouveau et exemplaire. Dans cette représentation, le bonheur passe essentiellement par la consommation ${ }^{166}$. Ainsi l'individualisation et la diversification des pratiques de consommation marquent un changement radical des styles de vie et des mentalités, une véritable "révolution

163 GUO Yingjie. "Class, stratum and group: the politics of description and prescription", in GOODMAN David (dir.). The new rich in China. Future rulers, present lives, London: Routledge, 2008, p.38-52.

${ }^{164}$ HAWES Colin. "Corporate CEOs as cultural promoters", in GOODMAN David (dir.). The new rich in China. Future rulers, present lives, London: Routledge, 2008, p. 85-98.

165 TOMBA Luigi. "Creating an urban middle class: social engineering in Beijing", The China Journal, n51, 2004, p. 1-26.

${ }^{166}$ MEYER Eric. Sois riche et tais-toi ! Portrait de la Chine d'aujourd'hui, Paris: Robert Laffont, 2002. 
consumériste" $^{167}$. L'individu, ses désirs ${ }^{168}$, ses intérêts, ses spécificités, ses goûts sont désormais mis en valeur, une tendance encore accentuée par la politique de l'enfant unique.

Cette image n'est pas sans attirer quelques critiques. Les personnes très fortunées font même l'objet d'une forme de "haine de la richesse", basée sur le constat que les richesses d'un tout petit nombre se sont amassées aux dépens de la plus grande partie de la population ${ }^{169}$. Leur richesse a ceci d'ambigu qu'elle est à la fois le symbole de la réussite professionnelle tant encouragée pour le développement du pays, et celui d'une certaine perversion des valeurs morales ${ }^{170}$. On critique aussi dans la jeunesse un consumérisme ${ }^{171}$ excessif et la perte des valeurs communistes qui nourriraient la corruption du régime ${ }^{172}$.

C'est pourquoi on encourage en même temps la poursuite d'autres idéaux. Si le niveau de vie des entrepreneurs fait rêver, c'est officiellement la dimension de l'entreprise et du travail qui est valorisée.

L'explication populaire pour le succès des entrepreneurs chinois a toujours été leurs attributs et états d'esprits personnels, basés sur le travail acharné, la résilience, et l'autonomie. Certains chercheurs ont prétendu que la période post-1978 a produit une génération d'entrepreneurs qui sont rompus au travail acharné, qui sont prêts à endurer des difficultés, et qui veulent saisir l'opportunité. [...] Les entrepreneurs

${ }^{167}$ DAVIS Deborah (dir.). The consumer revolution in urban China, Berkeley: California University Press, 2000.

${ }^{168}$ ROFEL Lisa. Desiring China. Experiments in neoliberalism, sexuality, and public culture, Durham et Londres: Duke University Press, 2007.

169 ZANG Xiaowei. "Market transition, wealth and status claim", in GOODMAN David (dir.). The new rich in China. Future rulers, present lives, London: Routledge, 2008.

170 JEFFREYS Elaine. "Advanced producers or moral polluters?" China's bureaucrat-entrepreneurs and sexual corruption", in GOODMAN David (dir.). The new rich in China. Future rulers, present lives, London: Routledge, 2008, p. 229-244.

${ }^{171}$ HEWITT Duncan. China: getting rich first : a modern social history, New York: Pegasus Books, 2008; MEYER Eric. Sois riche et tais-toi! Portrait de la Chine d'aujourd'hui, Paris: Robert Laffont, 2002; ROSEN Stanley. "The victory of materialism: aspirations to join China's urban moneyed classes and the commercialisation of education", The China Journal, n51, 2004, p. 27-52.

${ }^{172}$ KWONG Julia. "Ideological crisis among China's youths: values and official ideology", The British journal of sociology, vol. 45, n² ${ }^{\circ}$ juin 1994, p. 247-264. 
chinois qui ont réussi semblent passionnés, dévoués, respectueux, dynamiques, talentueux et acharnés. ${ }^{173}$

Les grands entrepreneurs manifestent une certaine volonté de se donner une image vertueuse. Par exemple, des patrons de mines dans le Shanxi se lancent dans des investissements d'intérêt général comme des écoles ou des cliniques ${ }^{174}$. De nombreux patrons se présentent également comme des amateurs d'art ou de philosophie ${ }^{175}$.

Porter un regard sur les "nouveaux riches" est donc surtout l'occasion d'articuler un discours autour de valeurs nouvelles qu'il s'agit de promouvoir ou de rejeter. Cette démarche est généralement subsumée dans la notion de "qualité" (suzhi) ${ }^{176}$, qui souligne clairement un jugement de valeur. La "qualité" est omniprésente dans les discours à tous les niveaux. Il s'agit de qualité de la population, des individus. Elle pourrait se définir par un certain niveau d'éducation, mais aussi une forme de culture, d'attitude ou de savoir vivre. En ce sens, elle met le capital humain au centre des nouvelles hiérarchies sociales ${ }^{177}$. Elle implique l'adoption de certains comportements, d'un certain type de consommation (c'est ce que suggère la publicité), ou l'acquisition de compétences particulières comme la maîtrise de langues étrangères. L'éducation viserait ainsi à améliorer la "qualité" de la population, dans une perspective très développementaliste, où l'idée de progrès est centrale. Comme le fait remarquer Kipnis, cette idée d'amélioration de la population, qui est associée à une sélection des meilleurs par la compétition, frôle parfois l'eugénisme.

${ }^{173}$ GOODMAN David (dir.). The new rich in China. Future rulers, present lives, London: Routledge, 2008, p.11.

${ }^{174}$ CARILLO Beatriz. "From coal black to hospital white: new welfare entrepreneurs and the pursuit of a cleaner status", in GOODMAN David (dir.). The new rich in China. Future rulers, present lives, London: Routledge, 2008, p.99-112.

${ }^{175}$ HAWES Colin. "Corporate CEOs as cultural promoters", in GOODMAN David (dir.). The new rich in China. Future rulers, present lives, London: Routledge, 2008, p. 85-98.

${ }^{176}$ KIPNIS Andrew. "Suzhi: a keyword approach", The China Quarterly, n ${ }^{\circ} 186$, juin 2006, p. 294313.

${ }^{177}$ HSU Carolyn. Creating market socialism. How ordinary people are shaping class and status in China, Durham et Londres: Duke University Press, 2007. 
La classe moyenne est ainsi présentée comme l'archétype d'une population de "qualité", "civilisée", "moderne" ou encore de "culture avancée" ${ }^{178}$. Les valeurs qui lui sont attribuées ont une force prescriptive. On les retrouve dans les cours de morale et de politique ${ }^{179}$ dispensés aux étudiants tout au long de leur scolarité. Toute la jeunesse est ainsi l'objet d'un travail idéologique officiel ${ }^{180}$, qui semble tout à fait légitime aux intéressés dès lors qu'il s'agit de soutenir le développement de la nation. Cela commence dès la petite enfance, car les familles qui le peuvent soumettent leurs enfants à une discipline draconienne pour qu'ils réussissent ${ }^{181}$. Le développement personnel qu'ils poursuivent de manière individualiste devient dès lors un devoir envers la nation chinoise, en particulier pour ceux qui profitent de l'ascenseur social ${ }^{182}$. Hoffman analyse cet effort de "civilisation" comme une forme de biopolitique $^{183}$. Pour elle, l'enjeu est de faire entrer la population dans un modèle qui assure à la fois le développement du pays et la reconduction de l'ordre social.

De plus, la notion de "qualité" sert de fondement à une hiérarchisation des groupes sociaux et justifie ainsi certaines inégalités qui touchent la société chinoise en les présentant comme naturelles $^{184}$. En effet la notion de qualité est tout aussi exclusive qu'elle est inclusive. Toutes les catégories de la population qui ne ressemblent pas à cette classe moyenne idéalisée sont ainsi classées dans la catégorie des "moins avancées", voire des "arriérés", vocabulaire péjoratif en Europe, mais qui est enraciné dans le vocabulaire maoïste. C'est le cas de tout le monde rural, qui est désigné comme non "moderne" comme en témoigne l'effort spécifique de

${ }^{178}$ TOMBA Luigi. "Of quality, harmony and community: civilization and the middle class in urban China", Positions: East Asia cultures critique, 2009.

${ }^{179}$ ARSENE Séverine. L'enseignement idéologique dans les universités chinoises, mémoire de Master Recherche, Paris: Institut d'études politiques, 2005.

${ }^{180}$ KWONG Julia. "Ideological crisis among China's youths: values and official ideology", The British journal of sociology, vol. 45, n², juin 1994, p. 247-264.

${ }^{181}$ FRENCH Howard. "In China, the upper-class quest starts low - at age 5", The New York Times, 22/09/2006.

${ }^{182}$ HOFFMAN Lisa. "Autonomous choices and patriotic professionalism: on governmentality in latesocialist China", Economy and society, vol. 35, n 4 , novembre 2006, p. 550-570.

183 au sens de FOUCAULT Michel. Histoire de la sexualité. 1 La volonté de savoir, Paris: Gallimard, 1976.

184 KIPNIS Andrew. "Neoliberalism reified: suzhi discourse and tropes of neoliberalism in the People's Republic of China", Journal of the Royal Anthropological Institute, n¹3, 2007, p. 383-400. 
planification des naissances et l'enseignement primaire qui y est dispensé ${ }^{185}$. Plus flagrant encore est le discours qui entoure la politique de l'enfant unique de ce point de vue ${ }^{186}$. En effet elle a été mise en place à l'aide de slogans qui associent directement la diminution de la population en nombre à son amélioration en qualité.

Le monde rural est stigmatisé jusque dans les villes au travers des ouvriers migrants. Ceux-ci en effet ne remplissent pas les conditions d'accession à la modernité aux yeux des citadins. Ils ne leur ressemblent certes pas. Ils ont souvent la peau tannée par le soleil et les températures extrêmes, ils sont modestement vêtus, parlent avec l'accent de leur région d'origine, et, comble de "l'arriération", ils se comportent souvent sans retenue, parlant fort et crachant par terre. Immédiatement désignés par leur apparence extérieure et leur comportement, les migrants sont victimes d'une discrimination qui est même institutionnalisée au travers du système d'enregistrement des citoyens dans leur localité d'origine, le "hukou". La distinction entre villes et campagnes et a fortiori entre plusieurs catégories de citoyens peut en ce sens être considérée comme un pilier de la politique du gouvernement chinois ${ }^{187}$.

J'ai moi-même eu l'occasion d'entendre ce discours sur l'arriération à de nombreuses reprises lorsque je résidais à Pékin. Par exemple, une serveuse de restaurant me déclara qu'elle était heureuse de vivre à Pékin car en-dehors de cette ville les gens étaient de "mauvaise qualité" (suzhi hen di). J'ai encore été plus marquée d'entendre ce discours chez des paysans à propos d'eux-mêmes, me demandant: "tu dois nous trouver arriérés, non?". Le discours sur la qualité fait l'objet d'une appropriation très ancrée dans toutes les catégories de la population chinoise, dans les contextes les plus divers. Il est parfois critiqué, souvent encensé, mais il reste une donnée de référence par rapport à laquelle tout le monde se positionne. En ce sens il

\footnotetext{
${ }^{185}$ MURPHY Rachel. "Turning peasants into modern Chinese citizens: 'population quality' discourse, demographic transition and primary education", The China Quarterly, n¹77, mars 2004, p. 1-20.

186 SIGLEY Gary. "Liberal despotism: population planning, subjectivity, and government in contemporary China", Alternatives, n²9, 2004, p. 557-575.

${ }^{187}$ En effet les personnes d'origine rurale ne peuvent avoir accès aux mêmes services que les urbains en termes d'éducation ou de soins par exemple, à cause du système d'enregistrement de la résidence, le hukou, qui est censé limiter l'exode rural. FROISSART Chloé. "Le système du 'hukou': pilier de la croissance chinoise et du maintien du PCC au pouvoir", Etudes du CERI, n 149 , septembre 2008, p.147.
} 
peut donc être vu comme une forme de représentation "hégémonique"188, qui s'impose à de nombreux niveaux de la société.

Cependant cette représentation circule au sein de la population et est utilisée de diverses manières par différents types d'individus. Par exemple, Lisa Rofel décrit la manière dont les différentes générations de femmes chinoises ont pu utiliser les stéréotypes de la femme moderne qui ont changé avec le temps. Elles se sont ainsi mises en valeur tantôt comme "travailleuse", tantôt comme "mère", pour se positionner dans la hiérarchie sociale de leur époque $^{189}$. Il est possible, également, d'utiliser ces représentations comme une ressource, pour tricher avec les préjugés et apparaître plus "moderne" que les moyens financiers ne le permettent en réalité. Il en est ainsi, par exemple, des jeunes ouvrières dans les usines, qui changent de vêtements à la sortie de l'atelier pour marquer leur accession à une classe sociale supérieure $^{190}$. Carolyn Hsu montre aussi très bien la variété des interprétations et des utilisations du récit sur la qualité parmi les travailleurs de la ville de Harbin ${ }^{191}$. Les homosexuels également ont revendiqué le fait d'incarner une culture "moderne", plus ouverte et cosmopolite, retournant ainsi la rhétorique sur la modernisation en leur faveur ${ }^{192}$.

Si hégémonie il y a, il faut donc également considérer la part d'appropriation, de réinterprétation et d'utilisation tactique par les personnes qui se sentent concernées par elles de manières très diverses. Les individus ordinaires, en réagissant de manière ad hoc, non seulement par rapport aux récits proposés par les autorités, mais aussi par rapport à certains contre-récits, contribuent ainsi à créer ces représentations collectives. Rofel propose donc une vision plus nuancée de cette normalisation. En étudiant la manière dont des homosexuels construisent leur identité sociale, elle montre que l'expression des désirs des individus

${ }^{188}$ GRAMSCI Antonio. Cahiers de prison. 1, Cahiers 1, 2, 3, 4, 5, Paris : Gallimard, 1996.

189 ROFEL Lisa. "Liberation nostalgia and yearning for modernity", in GILMARTIN Christina, HERSHATTER Gail, ROFEL Lisa et WHITE Tyrene. Engendering China, Cambridge (Mass.): Harvard University Press, 1994, p. 226-249.

${ }^{190}$ FRENCH Howard. "Dynamic young engines driving China's epic boom", The New York Times, 22/10/2008, à propos de CHANG Leslie. Factory girls. From village to city in a changing China, Spiegel \& Grau, 2008.

${ }^{191}$ HSU Carolyn. Creating market socialism. How ordinary people are shaping class and status in China, Durham et Londres: Duke University Press, 2007.

${ }^{192}$ ROFEL Lisa. Desiring China. Experiments in neoliberalism, sexuality, and public culture, Durham et Londres: Duke University Press, 2007. 
s'articule le long des grands traits du discours sur la modernité. Cependant elle montre aussi que loin d'être un modèle total et préconstruit qui s'impose aux individus, il est inventé, interprété de manière renouvelée jour après jour par les différents acteurs. C'est le produit d'une conjonction complexe entre le discours gouvernemental, le discours de modernisation des instances internationales ${ }^{193}$ et les aspirations variées des individus, qui s'actualise dans leurs actions quotidiennes.

Surtout, Rofel montre que les désirs des individus ont moins changé que les modalités de leur expression, ou de leur "narration", dans les "arènes culturelles publiques". Elle parle de "jeu" et de "représentation" publics (public enactment, public representations) ${ }^{194}$, et elle montre bien la distance que certains acteurs peuvent prendre par rapport à ces représentations publiques. Même si elle n'utilise pas ces termes, on peut donc considérer que la manière dont les individus expriment leur subjectivité en public relève de stratégies de présentation de soi, ou de mises en scène de soi en public. Il me semble donc fructueux d'emprunter à Goffman les idées de mise en scène ${ }^{195}$ et de "cadrage" ${ }^{196}$ de la parole publique, qu'il a en particulier développées dans Façons de parler ${ }^{197}$. Cette approche permet de montrer comment la norme sociale s'impose à tous sous la forme de rôles que l'on tient en public, tout en prenant en compte les nuances, les malentendus, les transgressions, et la distance que les individus prennent par rapport à elle. Cette piste me semble la plus intéressante pour éclairer la place toute particulière qu'occupe Internet dans cette idéologie, et pour en analyser les conséquences politiques.

\section{Prendre la parole sur Internet: la question du cadrage}

Internet est tout d'abord un média de masse qui véhicule de nombreux éléments de ce que Rofel appelle la "culture publique", c'est à dire la musique, les modes, la publicité, les

\footnotetext{
${ }^{193}$ Pour elle, cette idéologie est un avatar de la "globalisation néolibérale", qui prend forme de manière spécifique dans le contexte chinois. Si cette hypothèse est intéressante, elle n'est pas au cœur du présent travail.

${ }^{194}$ ROFEL Lisa. Desiring China, op.cit., p.21-22.

195 GOFFMAN Erving. La mise en scène de la vie quotidienne, Paris: Minuit, 1979, 2 vol.

${ }^{196}$ GOFFMAN Erving. Les cadres de l'expérience, Paris: Minuit, 1991.

${ }^{197}$ GOFFMAN Erving. Façons de parler, Paris: Minuit, 1987.
} 
séries télévisées par exemple. Sur les forums, on discute de voitures, de logement, d'éducation des enfants, de mariage et d'une foule d'autres sujets qui ont souvent un rapport avec la définition du style de vie idéal. Tous ces contenus fournissent donc une matière importante pour analyser les différentes représentations de la modernité et les débats que sa définition suscite.

La vraie spécificité d'Internet se trouve cependant dans la prise de parole publique des individus en elle-même. Elle est le signe de la transformation importante du statut de l'individu qui est associée à l'idée de modernité. En effet, se connecter sur Internet, c'est être moderne, "branché". Tenir son blog ou exprimer son avis sur un forum, c'est affirmer publiquement sa personnalité. Certeau ${ }^{198}$ a analysé cette valeur performative de la prise de parole et montré son importance symbolique. S'exprimer, c'est affirmer une certaine position dans la société, et montrer, voire déplacer la ligne de démarcation de certaines formes d'autorité. Les étudiants français de 1968 ont d'abord pris la parole là où les professeurs, les parents, les autorités en avaient toujours eu le monopole. De même, la prise de parole des internautes chinois peut s'analyser comme l'affirmation du droit de cité des individus, là où la parole publique avait longtemps été le monopole du Parti.

Il faut noter que dans ce contexte, prendre la parole sur Internet n'est pas a priori un acte subversif. En effet, dans le cas qui nous intéresse, cela relève d'une idéologie promue par les autorités et largement partagée au sein de la société. Prendre la parole sur Internet est pour bon nombre d'individus une manière de se mettre en scène comme des membres à part entière de la société chinoise moderne. La profusion des blogs peut par exemple se lire comme un effort de mise en valeur d'une identité personnelle originale dans une société qui récompense l'individualité et la personnalisation. De même, la multiplication des contacts éphémères et des groupes d'amis en ligne peut témoigner d'une certaine affirmation de l'autonomie individuelle par opposition aux obligations sociales fortes traditionnellement liées à la famille.

${ }^{198}$ CERTEAU Michel. La prise de parole, Paris: Seuil, 1994. 
Cette approche permet également de comprendre le désintérêt apparemment paradoxal de la plupart des internautes pour la politique ${ }^{199}$. Celle-ci est généralement présentée par les internautes comme une question d'un autre âge, appartenant à un univers qui ne les concerne pas, et qu'ils opposent dans leurs discours aux questions économiques et aux sujets qui les concernent directement. Pourtant les mêmes internautes manifestent une certaine curiosité pour des sujets "de société". En effet un bon nombre des sujets qui les préoccupent dans leur vie quotidienne touchent en réalité à la chose publique. Cette "évaporation" 200 de l'intérêt des internautes pour la politique peut s'expliquer par une conception de la modernité qui exclut la politique de la vie publique des individus. En effet, si l'idéologie dominante encourage les internautes à affirmer leur personnalité en ligne, elle les incite aussi à se préoccuper prioritairement de leur destin personnel et à confier le domaine politique au seul Parti. Surtout, le débat politique est considéré comme une source de conflits entre les différentes "couches" de la société. Elle représente donc une menace potentielle pour l'unité et la stabilité du pays, et a fortiori pour le développement économique que tout un chacun se doit de soutenir en public.

A ce titre, la prise de parole, en tant qu'affirmation d'une personnalité individuelle tournée vers la recherche de la prospérité et détournée de toute préoccupation politique, s'inscrit dans une conformité relative par rapport aux injonctions du pouvoir politique.

On peut voir une forme de sujétion ${ }^{201}$ dans cette manière d'affirmer son individualité de façon relativement normalisée. Cependant il me semble plus fructueux de l'analyser comme une forme de "civilité", une manière de se comporter en public qui garantit le maintien de l'ordre social $^{202}$. J'envisage cette civilité, à la suite de Ferrié ${ }^{203}$, comme une convention ${ }^{204}$, qui

${ }^{199}$ Les sondages mesurent en effet l'affirmation d'un désintérêt croissant pour ces questions parmi les internautes. GUO Liang. Surveying Internet usage and its impact in seven Chinese cities, Markle Foundation, 2007.

${ }^{200}$ ELIASOPH Nina. Avoiding politics. How Americans produce apathy in everyday life, Cambridge University Press, 1998.

${ }^{201}$ FOUCAULT Michel. Histoire de la sexualité. 1 La volonté de savoir, Paris: Gallimard, 1976. Voir aussi FOUCAULT Michel. "L'écriture de soi", in Correspondance Dits et Ecrits : tome IV, texte n ${ }^{\circ}$ 329.

${ }^{202}$ Il est donc important de ne pas réduire cette civilité à la notion de politesse. PAPACHARISSI Zizi. "Democracy online: civility, politeness, and the democratic potential online political discussion groups", New media and society, vol.6, $\mathrm{n}^{\circ} 2,2004$, p. 259-283. Bien que Papacharissi envisage la 
s'impose à tous dans la mesure où elle pose les règles de l'interaction publique, mais qui n'emporte pas nécessairement une adhésion très forte de la part de chaque individu. Pour la majorité des internautes, c'est l'image d'un comportement "civilisé" et "moderne" qui fixe les règles de ce qui se dit et de ce qui ne se dit pas. Toute opinion ne peut donc pas devenir publique. La norme opère un fort effet de cadrage qui ne laisse émerger que les thématiques qui s'insèrent dans une certaine conception du politiquement correct.

Certains des internautes que j'ai interrogés critiquent ce principe, d'autres trouvent cela légitime, mais tous en sont conscients et la plupart s'y conforment, que ce soit par conviction ou par commodité. En effet, il est compliqué d'adopter un comportement contraire à la norme sur la scène publique, car les autres internautes se chargent de rappeler les règles communément admises et pratiquées par la majorité. On voit souvent des messages agressifs, antipatriotiques ou trop engagés politiquement recevoir une large désapprobation de la part des internautes eux-mêmes. De ce point de vue, la norme sociale que représente l'adoption d'un comportement "civilisé" est plus contraignante que la censure effectuée par les autorités, car elle couvre un champ bien plus vaste.

Si les internautes ne s'autorisent pas toujours à transgresser ces codes, et même s'ils peuvent rarement les remettre en question, ils osent cependant les contourner, les détourner ou les bricoler ${ }^{205}$. Les travaux de Certeau et de $\operatorname{Scott}^{206}$ sont particulièrement utiles pour attirer l'attention sur les diverses manières dont des individus "dominés" sont capables de détourner les codes en utilisant le double langage, l'allusion, l'ironie et bien d'autres tactiques encore. Le Web chinois est truffé de caractères remplacés par leurs homonymes, et de phrases formulées à double sens. Les cas d'internautes qui écrivent des opinions tout en s'en dédouanant au début

civilité comme un comportement respectueux des principes de la démocratie en particulier, on peut l'appliquer de manière plus générale à tout comportement respectueux de l'ordre social, y compris dans des régimes politiques non démocratiques, comme le montre Ferrié.

${ }^{203}$ FERRIE Jean-Noël. Le régime de la civilité en Egypte, Paris: CNRS Editions, 2004.

${ }^{204}$ On trouve déjà ce terme chez WEBER Max. Economie et société, Paris: Pocket, 1995, t.1, p. 68s.

${ }^{205}$ CERTEAU Michel. L'invention du quotidien 1. Arts de faire, Paris: Gallimard, 1990.

${ }^{206}$ SCOTT James. Domination and the arts of resistance: hidden transcripts, Yale University Press, 1990. 
de leurs billets, ou qui font de l'ironie sur l'actualité entrent également dans cette catégorie ${ }^{207}$. Dans d'autres cas, ils gardent leurs critiques pour des espaces d'expression aux règles plus souples.

En effet les règles de civilité qui encadrent la parole publique ne sont applicables que sur certaines scènes publiques particulières. Les contraintes diffèrent en fonction du type d'espace, de son degré de publicité, ou encore du type de personnes qui le fréquentent, et bien évidemment du sujet autour duquel l'espace a été bâti. Des conversations informelles ou des espaces plus privés ${ }^{208}$, par exemple des groupes de discussion accessibles sur invitation, ou des espaces moins fréquentés, autorisent notamment un discours beaucoup plus critique sur la société ou la politique. On peut y lire la distance que les acteurs entretiennent par rapport aux rôles qu'ils endossent dans les espaces les plus publics. Il faut donc porter une attention particulière à la manière dont les acteurs conçoivent la séparation entre espaces publics et espaces privés, et les rôles qu'ils jouent sur ces différents espaces.

Or sur Internet, cette séparation est bien souvent ténue et ambivalente. Il y a des espaces considérés comme particulièrement exposés, comme les portails Sina ou Sohu ${ }^{209}$, et d'autres qui sont considérés comme tout à fait privés, comme les groupes de discussion que les internautes créent entre amis et ferment au public. Entre les deux, la plupart des blogs, ou encore les petits fils de discussion éphémères se situent dans une sorte de "clair-obscur" ${ }^{210}$ qui permet la tenue de discours moins conformes à la norme et leur circulation à grande échelle. En effet ils sont à la fois privés par leur fonction d'échange entre proches et publics par leur visibilité potentiellement infinie. Si les espaces les plus exposés conduisent à l"'évaporation" du politique, je suggère que ces espaces en clair-obscur sont propices à une diffusion des idées politiques de manière informelle mais massive. En d'autres termes, beaucoup de choses ne se disent pas publiquement, mais elles se savent, se racontent et se commentent, sur le

${ }^{207}$ ESAREY Ashley et XIAO Qiang. "Political expression in the Chinese blogosphere. Below the radar", Asian Survey, vol. 48, n 5, 09/10 2008, p. 752-772.

${ }^{208}$ FERRIE Jean-Noël. "Lieux intérieurs et culture publique au Maroc", Politix, vol. 8, n 31, 1995, p. 187-202.

${ }^{209}$ Ce sont les plus grands portails généralistes chinois, qui comprennent d'innombrables catégories, depuis les informations jusqu'aux forums en passant par les blogs ou les vidéos.

${ }^{210}$ CARDON Dominique. "Le design de la visibilité. Un essai de cartographie du Web 2.0", Réseaux, n ${ }^{\circ} 152,2008$, p.93-137. 
mode de la rumeur ${ }^{211}$. Peut-on cependant parler de mobilisations publiques pour ce qui n'est encore qu'une somme d'échanges et de conversations informels?

Cette présentation pourrait laisser entendre que l'idéologie dominante conduit essentiellement les internautes à s'autocensurer, et à ne s'exprimer que dans les espaces publics, pour réserver leurs critiques aux conversations privées. Or cette idéologie peut aussi fournir des registres de justification ${ }^{212}$ qui permettent de porter des causes sur la place publique ou de défendre des revendications. C'est le cas lorsqu'un événement vient heurter les valeurs liées à l'idéal d'une nation chinoise forte et moderne. On voit alors des internautes prendre la parole pour défendre l'honneur national, comme lors des émeutes de Lhassa en mars 2008, ou fondre sur des individus dont les mœurs ne sont, selon eux, pas dignes d'une Chine "moderne". Ce registre laisse généralement émerger des sujets relativement conformistes, ce qui n'empêche pas une certaine imprévisibilité.

En effet, si la tension vers la modernité est partagée par tous, et qu'elle fonde les principes de la civilité en ligne, il ne faut pas pour autant la considérer comme un principe immuable et non susceptible de discussions. Ferrié précise très bien qu'une convention, même appliquée par tous en apparence, peut en réalité faire l'objet d'interprétations différentes ${ }^{213}$, ce qu'il résume dans la notion de "solidarité sans consensus" empruntée à Kertzer ${ }^{214}$. Dans le cas chinois, le style de vie idéal qui sert de repère à l'ensemble de la société est une fiction complexe qui résulte notamment de la conjonction entre le discours gouvernemental, la publicité, les pratiques et les imaginaires de différentes catégories de la population. Ces discours et représentations se cristallisent au quotidien dans des images et des valeurs qui sont sans cesse renégociées au fil de l'actualité.

211 Sur l'importance politique de l'expression du mécontentement populaire, sous la forme du chuchotement et de la rumeur, voir FARGE Arlette. Dire et mal dire : l'opinion publique au 18 e siècle, Paris: Seuil, 1992.

${ }^{212}$ BOLTANSKI Luc et THEVENOT Laurent. De la justification. Les économies de la grandeur, Paris: PUF, 1987.

${ }^{213}$ FERRIE Jean-Noël. Le régime de la civilité en Egypte, Paris: CNRS Editions, 2004.

${ }^{214}$ KERTZER David. "Rituel et symbolismes politiques des sociétés occidentales", L'Homme, vol. 32 , $\mathrm{n}^{\circ} 121,1992$, p. $79-89$. 
Certains événements ou scandales jouent ainsi un rôle d'épreuves ${ }^{215}$ au travers desquelles se stabilisent momentanément des définitions de la norme. On lit chaque jour sur le Web chinois des controverses sans fin sur la manière dont il faut concevoir la modernité chinoise. Alors que certains internautes publient le récit de leurs nombreuses aventures amoureuses, ce qu'ils voient comme une preuve de leur émancipation, d'autres les traquent dans de véritables chasses à l'homme pour les punir de mettre à mal l'image d'une société civilisée en étant trop volages. Là où des jeunes femmes affichent tous les signes de leur appartenance aux couches les plus "avancées" de la société, d'autres y voient un mépris des plus pauvres contraire à la doctrine de l'harmonie.

La diversité même des définitions dont la modernité fait l'objet montre l'intensité des efforts que font les internautes pour se l'approprier et la mettre en actes. Au terme de ces épreuves et de ces ajustements, la définition des normes liées au modèle de modernité déborde largement du champ d'influence des autorités, et peut même se retourner contre elles. C'est précisément ce qui s'est passé dans le cas de l'affaire Sun Zhigang. Thireau a montré que ce scandale a pu être rendu public parce qu'un grand nombre de personnes partageaient l'impression que cette cause était légitime, même au regard de la doctrine officielle ${ }^{216}$. Les autorités nationales se sont ainsi trouvées "piégées" par leur propre rhétorique (entrapped) $)^{217}$ et obligées d'intervenir en faveur des plaignants.

Aborder la prise de parole des internautes chinois par ces normes sociales permet ainsi de comprendre l'irruption de mobilisations qui ne sont pas expliquées par un dysfonctionnement de la censure. En effet ces normes sociales peuvent parfois s'avérer plus contraignantes que la censure, mais elle peuvent aussi devenir de véritables "opportunités discursives" $^{218}$ qui permettent de rendre publiques des causes qui n'auraient pas vu le jour

${ }^{215}$ DE BLIC Damien et LEMIEUX Cyril. "Le scandale comme épreuve", dossier de Politix, vol.18 $\mathrm{n}^{\circ} 71,2005$.

${ }^{216}$ THIREAU Isabelle et HUA Linshan. "De l'épreuve publique à la reconnaissance d'un public: le scandale Sun Zhigang", Politix, vol.18, nº71, 2005, p 137-164.

${ }^{217}$ LYALL Jason. "Pocket protests. Rhetorical coertion and the micropolitics of collective action in semiauthoritarian regimes", World politics, vol. 58, n³, 2006, p. 378-412.

218 GAMSON William et MEYER David. "Framing political opportunity", in MCADAM Doug, MCCARTHY John et ZALD Mayer (dir.). Comparative perspectives on social movements, Cambridge University Press, 1996, p. 275-290. 
autrement. On voit ainsi que les imprécisions, les malentendus, les divergences d'interprétations contenus dans ce système normatif, créent un jeu, fournissent des marges de manœuvre pour des individus qui souhaiteraient faire passer un message spécifique. Je m'attacherai donc à montrer que certains individus procèdent à de véritables mises en récit stratégiques qui leur permettent de rassembler un public unanime autour de la cause qu'ils défendent.

Tous les internautes ne sont cependant pas susceptibles d'adopter ce type de comportement stratégique. Le degré de participation des internautes chinois aux discussions en ligne varie beaucoup en fonction de la manière dont ils se sentent concernés par les différents problèmes, de leur aisance dans l'argumentation et dans la maîtrise des différentes plates-formes d'échanges en ligne par exemple. J'ai rencontré de nombreux internautes qui ne se sentent pas capables de donner leur avis en ligne, et qui se contentent d'acquiescer de temps à autres aux commentaires des autres, lorsqu'ils ne restent pas complètement silencieux. A l'opposé, certains internautes célèbres disposent d'une crédibilité particulière qui leur donne une marge de manœuvre plus grande vis-à-vis de la censure et un public plus large parmi les autres internautes. Entre ces deux cas extrêmes, on peut distinguer une foule de comportements différents.

Une approche goffmanienne de la question permet de tenir compte de la diversité des positions (footing) ${ }^{219}$ tenues par les acteurs sur les différentes scènes que constitue Internet. Ce cadre théorique ne tient pas les acteurs pour égaux a priori, mais tient compte des différences de statut, d'autorité, ou encore de compétences, qui font que les différents individus ne prennent pas la parole de la même manière ni avec les mêmes chances de réussite. Comme le montre également Bourdieu ${ }^{220}$, les "conditions de félicité" de la prise de parole sont inégalement réparties entre les participants à une conversation ou, par exemple, à une conférence ${ }^{221}$. Les différents acteurs en sont conscients et adaptent leur comportement en

${ }^{219}$ GOFFMAN Erving. Façons de parler, Paris: Minuit, 1987.

${ }^{220}$ BOURDIEU Pierre. Ce que parler veut dire : l'économie des échanges linguistiques, Paris: Fayard, 1982.

${ }^{221}$ Par exemple, si quelqu'un demande à l'assistance d'une conférence de s'asseoir et de faire le silence, sa demande n'a pas les mêmes chances d'aboutir selon qu'il s'agit du conférencier ou d'un auditeur anonyme. 
conséquence. Gamson a par exemple montré que les individus prennent la décision de s'exprimer sur certains sujets en fonction de leur perception des effets possibles de leur parole, ce qu'il appelle "agency"222. La mobilisation qui en découle est donc fortement biaisée, cadrée à nouveau, par ces différences de positions entre les acteurs.

Enfin, partir des normes sociales permet d'éviter l'écueil qui consiste à traiter Internet comme un espace autonome, indépendant des autres médias, indépendant également de la vie hors ligne des internautes, et qui serait la cause unique des phénomènes de mobilisation. L'établissement de l'agenda des problèmes publics résulte de multiples formes d'interaction entre différents acteurs, qui ont lieu sur les différents supports d'expression et de publication, et qui se manifestent par la concurrence entre les titres de presse, des tentatives de manipulation et d'influence réciproques etc $^{223}$. Le seul exemple de l'affaire Sun Zhigang ${ }^{224}$ montre l'interdépendance entre les différents supports de publication qui ont donné lieu à cette mobilisation. L'affaire a été relayée par de nombreux médias comme des magazines ou des chaînes de télévision, et également par des individus possédant un statut spécifique, comme des juristes ou des professeurs d'université. La mise en récit de l'actualité n'est donc pas l'apanage des seuls individus qui ont des causes personnelles à porter. Elle est aussi un enjeu important pour les autorités d'une part et pour les médias d'autre part, qui se livrent parfois à de véritables courses de vitesse pour proposer une interprétation de l'actualité qui leur soit la plus favorable possible. La position gouvernementale sur le traitement de l'opinion publique, qui oscille entre sa reconnaissance et son guidage, peut s'analyser à la lumière de ces relations complexes entre les différents acteurs.

Ce qui se dessine au travers de ce cadre d'analyse ne ressemble donc ni à la masse manipulable présentée par les autorités, ni à l'expression d'une volonté populaire autonome qui émergerait à l'issue d'un processus de délibération. Cela ne constitue pas non plus une forme de représentation des aspirations de l'ensemble de la population, ni même de la population internaute. Envisager la multiplication des prises de parole publiques comme un

\footnotetext{
${ }^{222}$ GAMSON William. Talking Politics, Cambridge: Cambridge University Press, 1992.

${ }^{223}$ NEVEU Erik. "Médias, mouvements sociaux, espaces publics", Réseaux, n98, 1999, p.17-85.

${ }^{224}$ THIREAU Isabelle et HUA Linshan. "Liens personnels, expressions, repères d'identification. Actions expressives et nouveaux supports de communication en Chine", Réseaux, n¹33, 2005, p. 69100 .
} 
produit de la norme sociale dominante en Chine permet d'éclairer à la fois les multiples formes de cadrage qui en font une expression restreinte à un champ thématique et à des formes spécifiques, et les polysémies, ambivalences, marges de manœuvre qui en font l'objet de luttes d'influences complexes. Cette construction collective et conflictuelle de la prise de parole publique peut ainsi se concevoir comme l'affirmation et la négociation permanente d'un projet de société partagé, négocié (et parfois subi) tout à la fois par la population chinoise et par l'Etat.

Ce parti pris suppose donc une conception interactionniste du pouvoir. En effet le pouvoir est ici envisagé comme une relation, à laquelle participent tant les dominants que les dominés. Il ne s'agit donc plus tant de comprendre les logiques de domination ou les stratégies de résistance ou de transgression des individus face à l'Etat (même si elles existent), que de saisir les conditions d'adhésion à l'ordre public des diverses catégories d'individus, leur participation quotidienne à la définition des règles et des normes, à leur maintien, à leur négociation, ou le cas échéant à leur contournement ou leur contestation ${ }^{225}$. Les institutions, les contraintes, le contexte politique ne sont pas laissés de côté mais saisis au travers des pratiques et des représentations des intéressés, pour ainsi dire "par le bas" ${ }^{226}$.

\section{3 - Méthodologie}

Il existe deux sources principales de statistiques sur les usages d'Internet en Chine. Le CNNIC, Chinese Internet Nework Information Center ${ }^{227}$, fournit un rapport semestriel sur les chiffres d'utilisation d'Internet. Ce centre de recherche fondé par le Ministère de l'Information (MII, désormais MIIT), dispose de plusieurs techniques pour collecter des données, notamment des questionnaires téléphoniques (auprès d'abonnés au fixe et au mobile), questionnaires en ligne, extraction de données en ligne (noms de domaines, nombre de sites etc) entre autres. A titre d'exemple, environ 30000 personnes ont été interrogées pour le

\footnotetext{
${ }^{225}$ GOFFMAN Erving. "L'ordre social et l'interaction", et "L'ordre de l'interaction", in Les moments et leurs hommes, Paris: Seuil, 1988, p. 95-102 et p. 186-230.

${ }^{226}$ BAYART Jean-François, MBEMBE Joseph-Achille et TOULABOR Comi. Le politique par le bas en Afrique noire, Paris, Karthala, 1992, p. 33s.

${ }^{227}$ Statistical survey report on the Internet development in China, Pékin: CNNIC, juillet 2010, consulté sur www.cnnic.net.cn.
} 
rapport de juillet 2010. Les informations publiées par le CNNIC sont généralement considérées comme les plus fiables pour l'Internet chinois. Ce sont surtout les seules données chiffrées de cette ampleur qui soient disponibles. Il ne faut pas ignorer leur caractère officiel et garder en tête le fait qu'elles passent nécessairement par le filtre des autorités.

Une autre source de données chiffrées provient de l'Académie des Sciences Sociales de Chine ${ }^{228}$, où Guo Liang effectue régulièrement des sondages dans un certain nombre de villes chinoises - sept pour le rapport de 2007. Les statistiques principales comme le nombre global d'internautes, leur profil moyen, le type de connexion utilisée sont des informations reprises dans les données du CNNIC par exemple. En revanche les rapports de la CASS fournissent des données originales sur l'évolution dans le temps au plan des attitudes et des opinions. Chaque année sont notamment posées des questions concernant l'usage politique d'Internet par les individus, la place de l'information, le degré de confiance accordé aux informations publiées sur Internet par exemple.

Il est extrêmement difficile d'évaluer la fiabilité et la sincérité des réponses à ce style d'enquêtes. La qualité des enquêtes quantitatives en Chine est toujours problématique du fait de la faible qualification moyenne des enquêteurs, de la taille des échantillons nécessaires et a fortiori de la difficulté à vérifier les résultats sur le terrain. Néanmoins les questionnaires sont intéressants dans la mesure où ils donnent une estimation approximative des activités les plus courantes des internautes.

D'autre part, une attention particulière sera donnée à des faits d'actualité pour esquisser le paysage que constitue la toile chinoise. Je renonce d'emblée à choisir des sujets qui seraient "représentatifs", ce qui demanderait un travail de recherche à part entière. Les contenus publiés ne sont pas l'objet de cette recherche puisque c'est bien la prise de parole qui est au centre de ma problématique. En revanche il me semble impossible de comprendre cette dernière sans avoir une idée de ses objets, ses contenus, sans dresser un panorama de l'ambiance générale et du type de problèmes qui retiennent l'attention des internautes.

${ }^{228}$ GUO Liang. Surveying Internet usage and its impact in seven Chinese cities, Markle Foundation, 2007. 
La sélection de certains exemples a été facilitée tout au long de mon travail de terrain par plusieurs sites Internet ${ }^{229}$ qui relaient pour le public occidental un certain nombre de ces sujets et qui vont jusqu'à traduire des passages entiers de blogs ou de forums. Ils sont tenus soit par des acteurs du Web en Chine (activistes, propriétaires de start-ups), soit par des départements d'universités comme c'est le cas du "China Media Project" de l'université de Hong Kong. Il va de soi que ces sites ne font pas non plus une sélection statistiquement représentative du paysage Internet chinois dans sa globalité. En revanche ils effectuent une sélection très judicieuse parmi des controverses qui ont recueilli un très grand nombre de réactions en ligne, et sur des sujets de société ayant souvent des conséquences politiques importantes, ce qui est pertinent au regard de ma recherche. De manière générale, il m'a été facile de tester l'intérêt de ces sujets auprès de quelques personnes de mon entourage - voisins ou collègues chinois - pour vérifier la popularité réelle de chaque histoire mentionnée, en général avec succès.

De ce travail de défrichage et de vérification au quotidien, j'ai tiré une sélection d'articles de presse et des notes personnelles, relevant aussi les réactions constatées dans mon entourage, afin de mieux comprendre l'ambiance globale qui entoure le développement d'Internet. Sans cette approche qui s'est effectuée sur un temps assez long - sur place d'avril 2006 à novembre 2007 et à distance jusqu'au printemps 2009, il aurait été difficile de comprendre les discours tenus par les individus au cours des entretiens.

Pour travailler sur la prise de parole, il faut notamment comprendre ce qui peut la freiner ou l'empêcher. Il faut aussi comprendre les motivations individuelles qui la déclenchent et qui ne sont pas forcément explicitées dans un contenu publié. Tout d'abord les phénomènes d'autocensure, de représentation et de codage que j'ai déjà évoqués empêcheraient une analyse véritablement fiable des conversations en ligne ${ }^{230}$. Il faut compter aussi le fait que bon nombre d'échanges importants ont lieu sur des plates-formes fermées au public, comme QQ ou des forums réservés à des petits groupes d'amis.

229 China Digital Times (http://chinadigitaltimes.net), China Media Project (http://cmp.hku.hk), Danwei (www.danwei.org), East South West North (http://www.zonaeuropa.com/weblog.htm), entre autres.

${ }^{230}$ Même si cette technique est parfois utilisée. WU Mei. "E-publics: political participation in Chinese discussion forums", communication à la International Conference on Media and Democracy in the Knowledge Society, Séoul, 2006. 
Une raison plus importante est cependant la nécessité de comprendre tout autant les silences des internautes que leurs prises de parole, et les conditions dans lesquelles ils décident de s'exprimer. Ces décisions se prennent hors ligne, devant l'écran, et elles relèvent de décisions personnelles pour chacun. Elles s'expliquent par des perceptions, des représentations, des motivations individuelles. Cela suppose donc de trouver un accès aux internautes qui prennent la parole tout comme à ceux qui ne la prennent pas, pour les interroger sur leurs habitudes, leur en demander les raisons, et plus généralement pour tenter de saisir les représentations et les normes sociales sur lesquelles reposent ces comportements. C'est pourquoi j'ai décidé de procéder à une enquête par entretiens approfondis.

Dans le paysage très complexe de l'Internet chinois, on connaît de mieux en mieux le profil des blogueurs les plus célèbres, les contraintes des journalistes ou les motivations des journalistes-citoyens, comme je l'ai mentionné précédemment. D'autre part les utilisations des technologies de l'information par les fragments les plus défavorisés de la population chinoise, et en particulier les migrants ${ }^{231}$, ont fait l'objet d'études approfondies. En particulier, l'ouvrage de Qiu sur la "société ouvrière en réseau" a retenu mon attention. Il suggère que les usages des nouvelles technologies de l'information ont un caractère discriminant, qui incite les personnes les plus défavorisées à privilégier des usages qui leur sont les plus appropriés, mais qui en même temps les stigmatisent ${ }^{232}$. Il se dessine en creux l'image d'une norme dominante dont ils sont exclus, et qui est incarnée par les comportements banals des "internautes ordinaires": entre autres, s'informer, se divertir, s'exprimer en ligne.

J'appelle "internautes ordinaires" les personnes dont les profils sont les plus fréquemment représentés dans les sondages sur les usages d'Internet, à savoir des personnes relativement jeunes, d'origine urbaine, et plutôt éduquées ${ }^{233}$. Il est significatif que ce profil

${ }^{231}$ Cela ne concerne pas seulement Internet, mais aussi le téléphone mobile. THIREAU Isabelle et HUA Linshan. "Liens personnels, expressions, repères d'identification. Actions expressives et nouveaux supports de communication en Chine", Réseaux, n¹33, 2005, p. 69-100.

${ }^{232}$ Par exemple, jouer en ligne dans des cybercafés, ou recourir à des formules bon marché de téléphonie mobile. QIU Jack. Working-class network society. Communication technology and the information have-less in urban China, Cambridge (Mass.): MIT Press, 2009.

${ }^{233}$ La proportion de ruraux tend à augmenter avec le temps et elle n'est pas négligeable, mais ils ne forment pas encore la majorité des internautes. Ils sont donc largement sous-représentés sur Internet. Pour la répartition des profils à l'échelle du pays, voir Statistical survey report on the Internet development in China, Pékin: CNNIC, 2009, consulté sur www.cnnic.net.cn. Au sein de la population 
moyen corresponde aussi au stéréotype des membres de la classe moyenne idéale, ainsi qu'à la génération des premiers enfants uniques nés après 1980. C'est à ce titre une catégorie particulièrement concernée par les normes sociales véhiculées dans ce modèle.

Pourtant, les internautes chinois ordinaires n'ont pas fait l'objet d'investigations sous cet angle $^{234}$. On en sait peu sur les représentations qui encadrent leurs comportements en ligne, et a fortiori sur les raisons qui les poussent à prendre part à une mobilisation ou à donner leur avis sur un scandale qui ne les concerne pas toujours personnellement. Or on peut supposer que ce sont les internautes qui fournissent la plus grande masse des réactions en ligne, qui donnent du poids à certains mouvements plutôt qu'à d'autres, et qui font que l'on passe de prises de position individuelles à un mouvement collectif. C'est donc sur eux que porte ce travail.

Devant l'impossibilité de faire une étude qualitative à l'échelle nationale dans le cadre de ce travail, j'ai décidé de me focaliser sur un groupe bien spécifique de cinquante personnes $^{235}$, toutes recrutées à Pékin, ce qui introduit une spécificité particulière dans mon échantillon. Pékin, capitale politique du pays, est la ville où les individus ressentent au plus près l'existence du pouvoir politique. Ils y sont également plus souvent confrontés aux étrangers résidents et aux touristes, et ils bénéficient d'infrastructures plus sophistiquées que dans le reste du pays. Beaucoup sont par exemple équipés très correctement à la maison avec des connexions ADSL.

Je renonce d'emblée à construire un échantillon représentatif de la population des internautes pékinois ordinaires pour seulement cinquante entretiens. Les profils des personnes interrogées sont donc les plus diversifiés possible. Il y a autant d'hommes que de femmes. Leurs métiers sont très variés, du designer au cuisinier en passant par le chef d'entreprise et la femme au foyer. De même j'ai souhaité une répartition la plus éclatée possible entre les

urbaine, voir GUO Liang. Surveying Internet usage and its impact in seven Chinese cities, Markle Foundation, 2007.

${ }^{234}$ Sauf dans de rares cas où la question est envisagée sous l'angle du mode de vie effectif de la classe moyenne. SUN Sun Lim. "From cultural to information revolution. ICT domestication by middle-class Chinese families", in BERKER Thomas, HARTMANN Maren, PUNIE Yves et WARD Katie. Domestication of media and technology, Maidenhead: Open University Press, 2007.

${ }^{235}$ Voir la grille des profils en annexe. 
différents niveaux de revenus, qui s'étalent donc de zéro (femmes au foyer) à plus de 20000 RMB mensuels. Les seuls éléments retenus à l'origine pour sélectionner ces répondants sont une utilisation moyenne d'Internet de une heure par jour, et un intérêt déclaré pour la lecture des informations en ligne (pour des raisons détaillées ultérieurement).

En revanche, j'ai éprouvé le besoin de faire évoluer cet échantillon au fur et à mesure que les entretiens apportaient de nouveaux éléments à ma réflexion. A une première série de trente entretiens, j'ai souhaité ajouter deux autres groupes de dix personnes afin de répondre à ces nouveaux questionnements. Les personnes interrogées se regroupent donc en trois catégories.

Le groupe central, qui forme le cœur de l'étude, est constitué de trente personnes de vingt à trente ans, c'est à dire appartenant à la génération "post 80 " ou plus précisément étant nés après 1978, année de l'ouverture de la Chine à une nouvelle forme de régime et de l'instauration de la politique de l'enfant unique. Ce sont statistiquement les internautes les plus nombreux et d'une manière générale il est possible d'affirmer que le développement d'Internet est concomitant avec la période de socialisation des personnes de cette génération. Certains l'ont découvert durant leurs études supérieures, d'autres dès le collège. La grande majorité n'ont pas attendu d'entrer dans la vie active pour l'utiliser.

Pour former ce groupe, j'ai choisi des personnes qui étaient déjà entrées dans la vie active, afin de recueillir les témoignages de ceux qui sont concernés par différents problèmes de société, à la fois au travers de leurs centres d'intérêts et de leurs expériences personnelles. C'est en entrant dans la vie active que les individus rencontrent de manière encore plus accrue des difficultés comme le logement, ou l'avancement dans le travail, qu'ils sont confrontés à des choix de vie importants comme le mariage, avoir des enfants, et qu'ils doivent, pour certains, assumer la charge d'autres personnes. Les trente personnes du groupe central sont donc toutes dans la vie active.

Par ailleurs, les statistiques faisant des étudiants un groupe d'utilisateurs d'Internet nombreux et très actifs, j'ai voulu disposer d'un échantillon de dix étudiants. Cet échantillon serait intéressant à traiter en soi pour ses caractéristiques propres. Les étudiants se distinguent parfois du groupe que j'ai choisi d'étudier dans la mesure où d'une part ils sont globalement plus privilégiés que les autres internautes dont beaucoup n'ont pas suivi d'études supérieures, 
et d'autre part ils ne sont pas tous d'origine pékinoise ou citadine, et ils vivent sur le campus dans un monde relativement fermé sur lui-même avec des codes propres à l'université. Ils sont à la fois moins impliqués dans certaines problématiques (logement, voiture, animaux domestiques), et plus impliqués dans certaines pétitions de principe (éducation, moralité, nationalisme). Cependant ils apportent un éclairage intéressant au plan de la norme sociale, car ils bénéficient à plein de l'ascenseur social, et ont donc des attentes particulières envers l'Etat et la société.

D'autre part, les rythmes de vie s'allongeant avec la politique de l'enfant unique, j'ai constaté que presque toutes les personnes du groupe principal étaient sans enfant. La plupart retardent l'arrivée de l'enfant jusqu'à trente ans, pour bénéficier d'avantages sociaux plus importants (quelques jours de congés supplémentaires et une prime de l'employeur si l'enfant vient tard) et pour assurer à la famille un confort matériel stabilisé dont le point de départ est l'achat de l'appartement. Aussi mon échantillon ne comprenait-il pas de parents. Je l'ai donc élargi avec un autre groupe de dix personnes de trente à trente-neuf ans.

Ce groupe s'est révélé très intéressant pour deux raisons principales. D'une part leurs positions sociales se sont généralement stabilisées et ils sont en mesure de faire le bilan de leur parcours avec des réussites et des déceptions. Le fait d'avoir un enfant leur permet de se projeter dans l'avenir et de formuler des attentes fortes non seulement pour eux-mêmes mais également pour leur enfant, et d'exprimer un regard plus expérimenté sur la société chinoise. D'autre part ils ont une vision très particulière de ce qu'ils appellent eux-mêmes la génération "post 80", et dont ils se différencient nettement. Il devient ainsi possible de recueillir un discours sur le changement social qui accompagne cette rupture générationnelle.

Si ces trois groupes sont construits différemment dans le but de diversifier au maximum l'échantillon, je n'en tirerai pourtant pas de conséquences particulières des différents profils des répondants. Il aurait certes été intéressant d'étudier plus en détails que je ne l'ai fait la composition des facteurs qui conduisent tel ou tel internaute à s'investir particulièrement dans une discussion en ligne. Certains internautes sont peut-être plus concernés que d'autres par certains sujets en fonction de leur âge, du niveau d'éducation ou de revenus par exemple. J'ai notamment observé que les femmes de l'échantillon que j'ai interrogé s'intéressaient moins souvent aux mobilisations en ligne. Des facteurs biographiques jouent aussi très certainement. 
Cependant il ne serait pas sérieux d'envisager une telle analyse à partir d'un échantillon aussi petit, construit pour un travail de type qualitatif.

De plus une telle approche aurait pour défaut de figer dans un instantané des comportements qui peuvent changer dans le temps. En effet chacun peut se trouver dans une position différente pour chaque type de mobilisation, selon qu'il se sent plus ou moins concerné, selon le moment particulier de sa vie ou sa connaissance du sujet en question. Il y a donc un aspect très contingent dans la participation des individus aux mouvements, comme on pourra peut-être le voir au travers de certains exemples. S'il est possible d'identifier une ou deux personnes vraiment très critiques et quelques autres qui sont vraiment très peu politisées, ces attitudes peuvent changer à tout instant car la frontière est ténue entre le désintérêt, le petit commentaire posté en passant et l'engagement plus poussé. La plupart des internautes se trouvent dans l'entre-deux, ils réagissent sur certains sujets plutôt que d'autres selon qu'ils ont des enfants, un chien, des difficultés professionnelles, mais aussi selon qu'ils ont plus ou moins de temps libre à un moment donné par exemple.

C'est pourquoi on ne trouvera pas de typologie générale des internautes interrogés dans ce travail, et que je n'ai pas particulièrement mis en valeur les différents profils au cours de la démonstration. On pourra constater que certains internautes sont plus fréquemment cités dans les premiers chapitres, consacrés à l'adoption d'Internet en général et à la description de leur représentation de la norme dominante. C'est par exemple le cas de certaines femmes dont le discours est particulièrement stéréotypé. Pour autant, elles ne sont pas les seules personnes concernées par cette norme et j'ai trouvé des commentaires, enthousiastes ou critiques, sur ce sujet dans la presque totalité des entretiens. Les derniers chapitres, qui traitent de sujets plus sensibles ou de mobilisations, concernent directement un nombre plus faible de répondants, mais à nouveau, tous ont un discours sur ces mouvements, et chaque internaute leur donne une signification particulière. Si la lecture des citations peut parfois donner l'impression d'une unanimité quelque peu artificielle, c'est que les répondants parlent d'une norme qui s'impose à tous, mais il ne faut pas oublier qu'ils ne la vivent pas nécessairement de la même manière.

Les entretiens semi-directifs ont été menés dans des cafés, ont duré entre une heure trente et deux heures chacun et se sont répartis sur plus d'une année. Il en résulte qu'il était impossible d'interroger les différentes personnes sur les mêmes faits d'actualité, quand bien 
même ils auraient prêté attention aux mêmes thèmes. Je me suis donc appuyée sur les déclarations des répondants eux-mêmes pour trouver des thèmes à partir desquels je pouvais introduire mon questionnement.

Le questionnaire est très progressif et comporte tout d'abord une série de questions destinées à faire connaissance, sur l'âge, la profession, la famille, la réussite professionnelle par exemple. Certaines remarques importantes apparaissent déjà à ce stade, car les répondants se positionnent par rapport au reste de la société et parlent de leurs ambitions. Ensuite vient un groupe de questions sur leurs habitudes sur Internet, qui reste très général et qui suit le déroulement de la pensée de l'internaute. En plus des habitudes proprement dites - temps passé, type de sites visités, lieux de connexion etc - j'ai toujours interrogé les personnes sur leurs goûts, leurs centres d'intérêts. Ce sont eux qui guident la navigation des internautes sur le Web, et qui sont l'objet des conversations en ligne. Mon objectif n'est pas tant de juger de leurs goûts en tant que tels, mais de disposer de points d'entrée vers des questions plus complexes sur la manière dont les internautes en parlent entre eux et sur les jugements qu'ils attribuent à ces échanges.

Un troisième train de questions concerne la manière dont les personnes s'informent sur ces questions, les médias choisis pour s'informer et la spécificité d'Internet. Je les interroge ensuite sur la manière dont ils en discutent avec leur entourage et s'ils sont amenés à choisir particulièrement leurs interlocuteurs. A ce stade apparaissent des réponses intéressantes sur la sociabilité des individus, leur rapport à la vie privée, leur attachement à des réseaux sociaux plus ou moins choisis. Beaucoup de ces réponses comportent un caractère normatif de type "il faut" ou "il ne faut pas", "j'aime" ou "je n'aime pas", qui fournissent beaucoup d'informations sur les normes sociales en mutation.

Les centres d'intérêt explorés sont de natures très variées, depuis les émissions de téléréalité jusqu'aux manifestations anti-japonaises. On l'a compris, mon questionnement s'étend bien au-delà du domaine de la politique au sens restreint. En posant de multiples questions sur la manière dont les personnes reçoivent et échangent sur divers types de sujets, j'ai tenté de délimiter la frontière entre ce qui est de l'ordre du particulier et de l'ordre du général, et a fortiori d'identifier ce qui, dans ces domaines, est selon eux légitimement accessible à la prise de parole publique, et sous quelles conditions. 
Avec ces questions, l'entretien peut donc évoluer vers une dimension plus générale sur le rôle d'Internet et de la parole publique. A partir de faits divers, ou en rebondissant sur une remarque, il est possible de demander dans quelle mesure la personne serait capable de s'investir en ligne, de publier des commentaires sur des forums, pour faire valoir un point de vue. En décrivant le comportement des autres, la personne est aussi amenée à faire des commentaires sur ce qu'est un comportement approprié, sur l'utilité de l'action collective, et surtout sa légitimité.

A chaque instant de l'entretien il s'agit de faire ressortir des cas limites, de demander des précisions, faire définir tous les termes utilisés (responsable, opinion publique, politique, contrôle etc), et de faire expliquer toutes les règles implicites qui déterminent la définition des normes sociales dont il est question.

Une place est toujours laissée à la fin pour quelques minutes d'entretien non directif avec la question: "y a-t-il des aspects importants à vos yeux et dont nous n'avons pas parlé"? Cette opportunité était souvent saisie par les internautes pour procéder à un récapitulatif de toutes les qualités pratiques d'Internet, qui facilite la vie grâce aux achats en ligne, à l'information sur la bourse et le trafic par exemple. Les discussions sur les sujets de société, quoi qu'il en soit, restent un sujet marginal.

Les entretiens ont ceci d'artificiel que nous amenons des personnes à répondre à des questions auxquelles elles n'avaient peut-être jamais réfléchi, les conduisant dans certains cas à monter des réponses de toutes pièces, faussant ainsi les résultats de l'enquête. Cependant la spontanéité des réponses qui est généralement palpable au moment de l'entretien, ainsi que leur longueur donnent une idée relativement bonne de la qualité des résultats avec chaque personne. Parfois il faut passer un moment à parler de tout et de rien avec une personne, et trouver dans ses goûts personnels le point d'entrée vers les questionnements qui touchent plus particulièrement mon enquête. Il arrive que ce soit un échec, et deux ou trois entretiens se sont terminés prématurément, faute de sujets de conversations, le répondant déclarant systématiquement "je ne pense rien de particulier là-dessus". C'est la raison pour laquelle j'ai fait introduire dans le questionnaire de sélection des questions sur l'attitude des personnes visà-vis de la lecture de l'information. Le but était de choisir des personnes qui s'informent et qui 
ont tendance à en discuter, afin de disposer au minimum de quelques sujets d'actualité sur lesquels les faire réagir.

Ces précautions n'empêchent pas de tomber dans d'autres ornières. Deux heures passées avec une personne ne doivent pas donner l'illusion de sa sincérité. Comme dans tous les rapports sociaux, une enquête est un jeu où chacun joue son rôle. A ce titre je dois mentionner les conditions dans lesquelles je me suis présentée aux enquêtés.

Tout d'abord les contacts ont été pris par l'intermédiaire d'une société spécialisée dans les sondages. Chargée du recrutement et de la prise de rendez-vous, l'entreprise disposait d'une liste de critères - âge, profils, niveaux de revenus, éducation - ainsi que d'une liste de questions à poser afin de ne retenir que des personnes adéquates. Les personnes qui ont accepté de se soumettre aux entretiens ont reçu une somme d'argent aux alentours de 150 RMB (environ 15 euros) pour les dédommager du déplacement et du temps passé. Selon les personnes, cette somme représentait entre une heure et une journée de travail, voire plus, ce qui signifie qu'elle semble dérisoire pour certains et très importante pour d'autres.

Une critique habituelle de cette pratique est qu'elle peut biaiser la relation entre intervieweur et interviewé, faussant les réponses dans la mesure où une personne payée a tendance à répondre selon les attentes qu'elle imagine être celles de l'enquêteur (ce qui ne signifie pas qu'un entretien non rémunéré soit exempt du même biais). Pour atténuer les effets du recrutement financé et effectué par le biais d'une entreprise, j'ai toujours pris la peine d'expliquer moi-même, en trois mots, le contexte et les objectifs de ma recherche au début de l'entretien. J'insiste toujours sur le fait que je suis étudiante en doctorat de sociologie (la science politique a une définition très différente en Chine et en France, ce qui me conduit à éviter ce terme). J'indique ensuite que je profite d'un financement pour recruter des répondants, car cela me serait très difficile par mes propres moyens. J'explique qu'il ne s'agit donc pas d'une enquête à visée commerciale, mais à visée universitaire. Ce qui m'intéresse principalement, c'est donc le mode de vie de la personne, son attitude et les commentaires qu'elle peut me donner sur ses habitudes quotidiennes. Ce discours est généralement bien reçu et a parfois contribué à renverser la situation, avec des individus qui m'ont prise en pitié, insistant pour payer mon verre de coca-cola! 
Le biais introduit par l'argent est probablement réel même si la médiation de l'argent est bien plus banale et moins mal considérée en Chine qu'elle ne l'est en France. Cependant je crois qu'il est difficile de biaiser les entretiens plus qu'ils ne le sont déjà dans le cas d'une enquêtrice française, qui représente pour de nombreuses personnes interrogées la première vraie conversation avec une personne étrangère. La question des rôles joue à plein et une immense majorité des personnes interrogées déclame un discours extrêmement stéréotypé et patriotique. Quoi de plus naturel que de vouloir donner une bonne image de son pays face à une personne qui est venue de loin pour le découvrir? Cette attitude doit être analysée pour sa valeur performative. Les internautes illustrent ainsi parfaitement la question de la représentation de la norme dominante.

Il n'est cependant pas impossible de sortir de ce discours. Si certaines personnes y tiennent jusqu'au bout, avec un discours très rôdé, d'autres au contraire le prononcent avec un peu moins de conviction, et quelques exemples concrets opposés à ce discours sur le ton de l'étonnement suffisent à déchirer le voile. En effet quelques personnes ont véritablement effectué une volte-face au cours de l'entretien, déclarant tout à trac "bon, ça c'est la théorie, mais on sait bien que ça se passe différemment". Ces discours sont particulièrement intéressants car ils comportent non seulement l'attitude officielle, mais aussi quelques phrases qui semblent plus sincères, et en sus un discours sur ces deux attitudes. Malheureusement j'ai pu réunir peu d'enregistrements de ces remarques, qui interviennent parfois tout à la fin d'un entretien, quand la personne se détend et que le dictaphone est éteint.

Les entretiens ont tous été enregistrés avec l'accord des personnes interrogées, dans le but d'être transcrits systématiquement. Le dictaphone numérique, qui ne nécessite aucune manipulation, était posé sur la table mais il attirait souvent moins l'attention du répondant que son propre téléphone mobile qui sonnait souvent et affichait dans plusieurs cas les cours de la bourse en temps réel. Le fait d'enregistrer les entretiens peut être la source du double discours que j'évoquais plus haut, ou du moins il peut l'accentuer. Faut-il en conclure pour autant qu'il fausse totalement les résultats? Personnellement je crois que non. Lorsqu'une personne était véritablement gênée par un sujet ou une question, il est arrivé qu'elle me demande de ne pas la citer, mais plus rarement de couper l'enregistrement. Dans tous les cas je me conforme à la demande de l'interviewé et je m'appuie sur une prise de notes manuelle. 
Enfin, j'ai toujours insisté pour situer les entretiens dans des cafés afin de proposer une ambiance décontractée et de rompre avec l'image de l'entreprise qui a procédé au recrutement. Seuls les cafés à l'occidentale - UBC coffee ou New Island Coffee par exemple - offrent un cadre suffisamment calme et un niveau sonore assez faible pour pouvoir discuter tranquillement et enregistrer l'entretien. Ces cadres, s'ils ne sont pas toujours très habituels pour les personnes interrogées, créent tout de même une atmosphère informelle, et mettent les deux personnes sur un pied d'égalité qui permet l'instauration d'un certain climat de confiance. On jugera de la réussite de cette stratégie au travers de la remarque d'un répondant.

"Par exemple on peut discuter tous les deux, mais tu as un dictaphone. Quand on aura fini, tu peux aller voir la police, mais ils s'en fichent, de ce genre de choses. On est dans un café, on est des amis, on peut dire ce qu'on veut, il n'y a pas de tabous (wuhuabutan)." (Dong) ${ }^{236}$

${ }^{236}$ Les noms des répondants ont été modifiés. Les pseudonymes entre parenthèses renvoient à la liste des profils qui figure en annexe. 


\section{Incarner l'image d'une Chine "moderne"}


Sur les 420 millions d'internautes chinois, il y a un gros contingent de jeunes gens nés après 1980, urbains, et mieux éduqués que la moyenne du pays. Ils se servent d'Internet pour s'informer, communiquer avec leurs amis, échanger des vidéos, faire des achats, ou encore écrire leur blog, avec un enthousiasme frappant. Leur génération, leurs profils, leur connectivité, tout contribue à faire d'eux des ambassadeurs d'une classe moyenne chinoise idéale et de ses valeurs.

La classe moyenne n'existe en fait qu'au travers d'un projet de société, porté par les autorités et relayé dans les diverses couches de la population. La publicité, la presse, les discours des individus, évoquent tous la nécessaire "modernisation" de la nation, qui doit passer par une "élévation de la qualité" des individus, et donc par la formation de la classe moyenne. Tournée essentiellement vers la recherche du confort matériel, éduquée, et dotée d'un fort esprit d'entreprise, cette population tirerait le pays vers la prospérité sans remettre en question les fondements politiques du pays.

Etre internaute, c'est en quelque sorte participer à ce projet de modernisation de la société. Qu'est-ce qu'Internet, sinon la preuve matérielle du développement des nouvelles technologies dans le pays? Aux yeux des internautes, la connexion facilite les opportunités commerciales, accélère les transactions, permet l'entreprise. En somme, Internet est une source de développement économique. Quelle que soit la diversité de leurs situations individuelles, les internautes participent à ce mouvement très symbolique vers ce qu'ils considèrent comme le "progrès" et la "modernité".

En effet, Internet est aussi un facteur de développement personnel et donc de transformation de la société. La tension vers la modernité se traduit par une sorte d'injonction à l'autonomie et à la singularisation des parcours individuels, qui ressort très fortement dans les modalités de prise de parole des internautes sur le Net. Comme celle du téléphone mobile, l'utilisation de l'ordinateur est essentiellement personnelle. On s'y informe, on s'y instruit, on y développe une identité et des contacts. Les sites de réseaux sociaux offrent des modes de socialisation alternatifs, qui permettent d'ajouter des liens plus sélectifs aux liens traditionnels que sont la famille ou l'entourage professionnel par exemple. A de nombreux égards, Internet est donc le vecteur d'un individualisme très affirmé, qui tranche singulièrement avec la culture dominante de la génération précédente. Cependant il est aussi le vecteur par lequel les 
individus s'assujettissent à de nouvelles normes sociales. En effet cette singularisation révèle aussi la prise en charge par les individus de leur "développement personnel" dans le cadre du processus de modernisation. Ils ont comme référence un mode de vie "moderne", sur le modèle duquel ils peuvent affirmer une certaine "distinction".

Les internautes chinois vivent donc d'importantes transformations dans leurs rapports sociaux. Ces transformations affectent aussi leurs rapports à la chose publique et aux questions politiques. L'individualisme croissant est-il dans ce cas une première étape dans un processus d'émancipation individuelle, ou au contraire une source d'atomisation des rapports sociaux, signe de l'adoption d'une forme de "postmodernité" chinoise?

D'après les premières déclarations des internautes, on observe un phénomène proche de ce que décrit Eliasoph ${ }^{1}$ à propos des associations d'une petite ville américaine. Les internautes se présentent eux-mêmes comme très préoccupés par des sujets qui les concernent personnellement, et très peu intéressés par les questions de politique en général. Ils sont pourtant sensibles à ces questions sous certaines conditions. L'individualisme porté par les internautes chinois n'induit pas une indifférence complète à la chose publique, mais il semble conduire à son "évaporation". Les internautes la renvoient inexorablement vers un monde qui ne leur appartient pas.

\footnotetext{
${ }^{1}$ ELIASOPH Nina. Avoiding politics. How Americans produce apathy in everyday life, Cambridge University Press, 1998.
} 


\section{Chapitre I - La quête de "modernité" des internautes chinois}

Pour entamer cette étude de la prise de parole des internautes chinois, un tour d'horizon s'impose. Il y a plus de 420 millions d'internautes chinois, qui se livrent à des activités aussi diverses que nombreuses et entremêlées. Parmi eux cependant, les jeunes, les urbains et les personnes les mieux éduquées sont les plus représentés. En consommant, en publiant leurs états d'âme, en discutant de leurs choix de vie, et dans le fait même d'être connectés, ils mettent en scène publiquement des styles de vie qui leur sont propres, et qu'ils associent à des valeurs particulières. Les usages les plus répandus d'Internet en Chine, mais également les divers "imaginaires" ${ }^{2}$ auxquels ils sont liés, et qui percent dans les entretiens menés avec cinquante internautes pékinois, montrent à quel point le développement d'Internet est intrinsèquement lié à la quête de "modernité" dans laquelle est engagée la société chinoise.

\section{1 - Etre un internaute en Chine}

\section{Des internautes jeunes, urbains, et éduqués}

La croissance du nombre d'internautes chinois est exponentielle. Désormais plus d'un Chinois sur cinq est un internaute, ce qui représente 420 millions de personnes ${ }^{3}$ ayant un accès à Internet c'est à dire plus que les 239 millions d'internautes américains ${ }^{4}$ et, en fait, plus que l'intégralité de la population américaine. Cependant il y a aux Etats-Unis un taux de pénétration de près de $77 \%$ alors qu'il n'est que d'un peu plus de $31 \%$ pour la Chine. Cela situe le pays loin derrière des pays développés comme la Corée ou le Japon qui ont des taux de pénétration proches de $80 \%$, mais dans la moyenne mondiale qui est de presque $29 \% 5$.

Ce taux de pénétration de l'usage d'Internet en Chine cache en fait d'importantes disparités géographiques et sociales. Au plan géographique tout d'abord, il y a une différence

\footnotetext{
${ }^{2}$ FLICHY Patrice. L'imaginaire d'Internet, Paris: La Découverte, 2001.

3 Source CNNIC, Statistical Report on Internet Development in China, juillet 2010, p.12.

${ }^{4}$ A la date du 30 juin 2010. Source http://www.internetworldstats.com/

${ }^{5}$ Le taux de pénétration pour la France est de 68,9\% selon les mêmes sources.
} 
marquante entre l'Internet dans le monde rural et dans le monde urbain. Les internautes classés comme "habitant en zones rurales" par le CNNIC ne représentent que 27,4\% de l'ensemble des internautes chinois en juillet 2010, et la croissance semble moins rapide dans ces zones que dans les zones urbaines ${ }^{6}$. De même le sondage effectué dans sept villes chinoises par l'équipe de Guo Liang en 2007 révèle un taux de pénétration d'Internet supérieur à $50 \%$ dans six villes étudiées sur sept ${ }^{7}$. Cela signifie que les campagnes sont pour le moment beaucoup moins bien connectées que les villes, même si le taux de connexion y augmente tout de même. Elles le sont aussi différemment. Il est souvent beaucoup plus difficile de connecter individuellement des foyers ruraux à Internet, ce qui conduit les internautes ruraux à fréquenter plus souvent les cybercafés, alors que les connexions à haut débit individuelles sont presque devenues la norme dans les plus grandes villes. Les immeubles neufs sont systématiquement équipés de câblage compatible avec l'ADSL. Les internautes ruraux et les internautes urbains ne sont donc pas les mêmes. Ils ne disposent pas du même temps, ni des mêmes conditions matérielles pour faire usage des différents services proposés sur Internet. Il en résulte qu'Internet est avant tout un outil de communication urbain pour le moment, en dépit des efforts de développement qui peuvent être faits dans de nombreuses régions du pays.

Si Internet est l'objet de disparités géographiques, il est aussi inégalement réparti dans les différents groupes de la population. Il semblerait que les hommes soient légèrement plus nombreux que les femmes sur la toile chinoise, soit $54,8 \%{ }^{8}$. Deux ans plus tôt, le rapport de Guo Liang indiquait que dans son échantillon urbain, $73 \%$ des hommes interrogés étaient internautes, alors que seules $57.6 \%$ des femmes interrogées l'étaient.

Internet est donc un phénomène essentiellement urbain et plutôt masculin en Chine. C'est également l'apanage des plus jeunes générations. Plus de $80 \%$ des moins de 30 ans ont déjà été sur Internet selon le sondage de Guo Liang (pour les villes). Le même type de chiffres ressort du sondage national bien que le découpage des âges ait été fait différemment (figure 1). Ainsi, près de $71 \%$ des internautes ont moins de 30 ans selon le CNNIC. Les 20-30 ans, dont beaucoup sont étudiants, forment un gros contingent. Ce contingent évolue à mesure que

\footnotetext{
${ }^{6}$ CNNIC, op.cit., p. 19.

${ }^{7}$ GUO Liang. Surveying Internet usage and its impact in seven Chinese cities, Markle Foundation, 2007, p. 17.

${ }^{8}$ CNNIC, op.cit., p. 16.
} 
les internautes vieillissent et le groupe des 30-39 ans tend à prendre une importance de plus en plus prépondérante d'année en année (19,6\% en 2010).

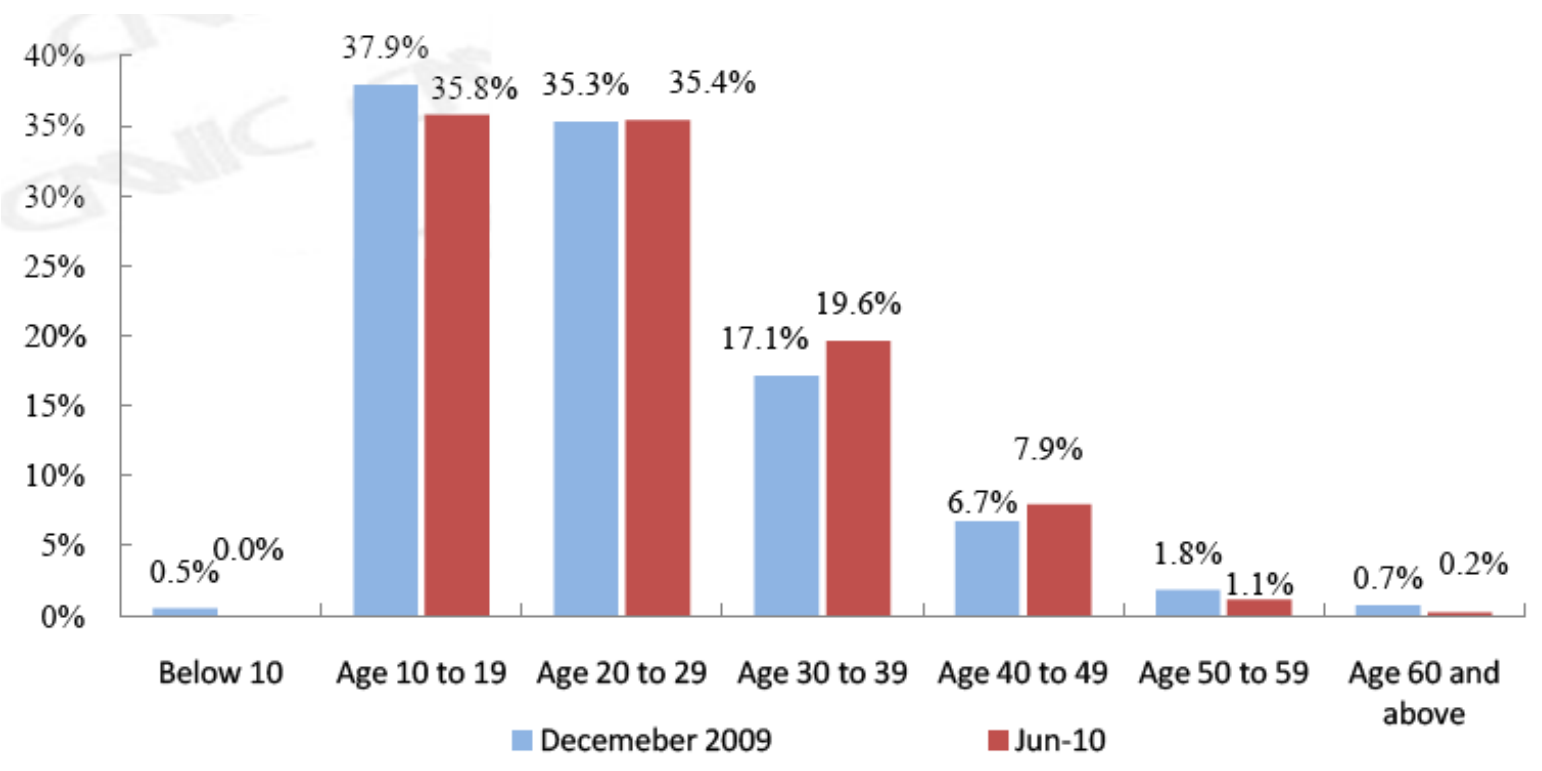

Fig. 6 Comparison on age structure of net citizens from December 2009 to June 2010

Figure 1 Répartition des internautes chinois par âge. Source: CNNIC, p.17.

Sur la figure 2 sont indiqués les niveaux d'études des internautes interrogés par le CNNIC. Il ressort de ces statistiques que seuls $37 \%$ des internautes n'ont pas atteint le diplôme de fin d'études secondaires. $40 \%$ ont atteint ce diplôme, et $23 \%$ ont même effectué des études supérieures. Cela constitue une forte sur-représentation des diplômés de l'enseignement supérieur, qui ne formaient que $12 \%$ de la population totale en $2006^{9}$. Envisageant la question dans l'autre sens, Guo Liang révèle également une forte corrélation entre le niveau d'études et l'utilisation d'Internet. Ainsi, dans son enquête, $90.5 \%$ des diplômés d'un Master sont des internautes, alors que c'est le cas de seulement $43 \%$ des personnes qui n'ont pas de diplôme.

${ }^{9}$ UNESCO, Institut de statistique. Education counts. Benchmarking progress in 19 WEI countries, World Education Indicators, 2007. Avec 2.4 millions de diplômés du supérieur en 2006, la Chine n'en était pas moins le pays comptant le plus grand nombre de diplômés au monde selon l'Unesco. Par ailleurs, le taux d'admission du gaokao, l'examen qui sanctionne la fin des études secondaires et tient lieu de concours d'entrée dans le supérieur, a fortement augmenté ces dernières années. En 2008 il était de $23 \%$ d'une classe d'âge. 


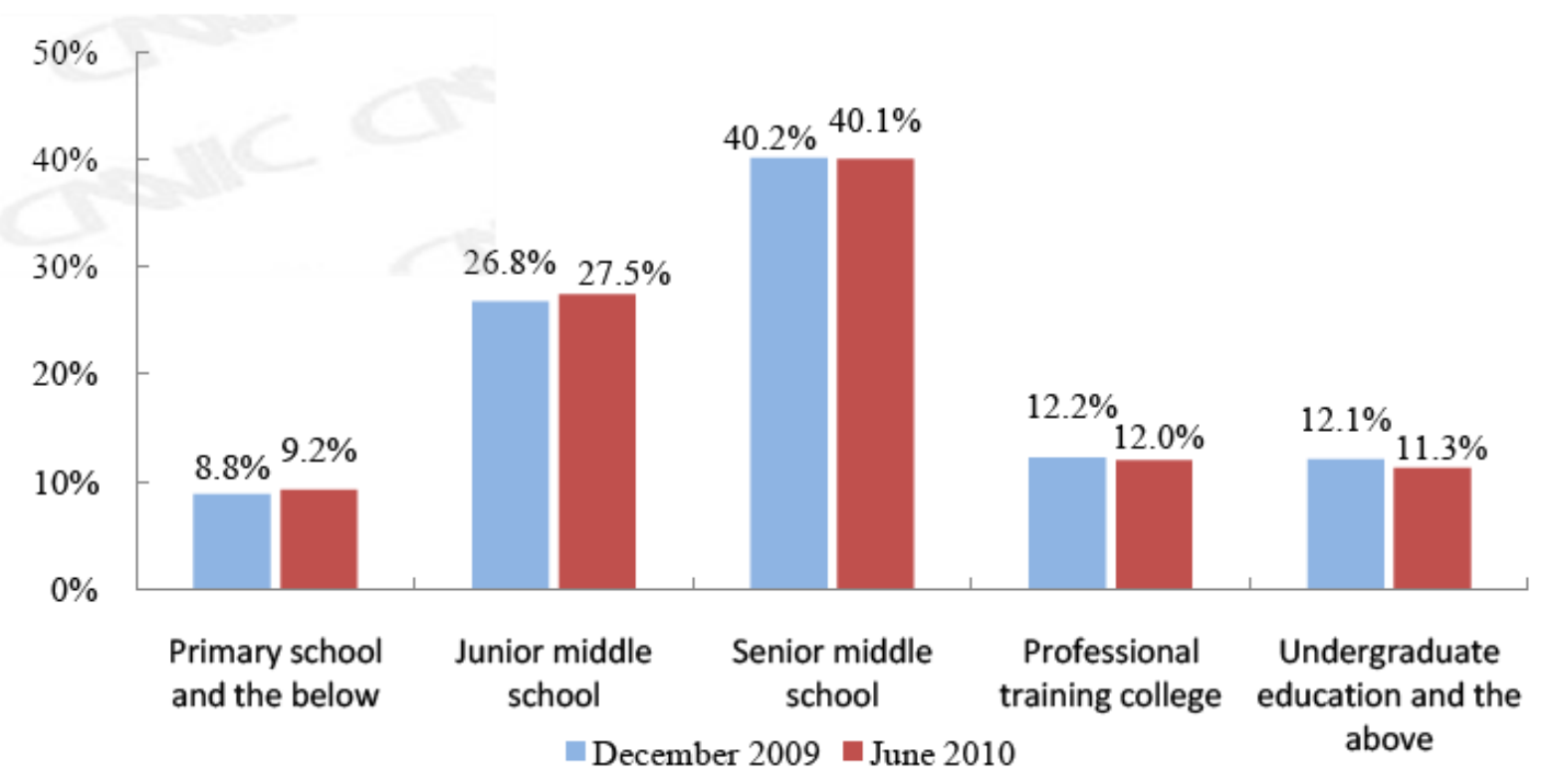

Figure 2 Niveaux de diplôme des internautes chinois. Source: CNNIC, p. 17.

Le niveau de diplôme montre une certaine inégalité sociale dans l'accès à Internet. Celle-ci est également visible dans le type d'activité professionnelle exercée par les internautes. La figure 3 montre que les individus exerçant des activités dirigeantes ou les "cols blancs" sont beaucoup plus souvent internautes que les autres. Il n'est donc pas étonnant que le niveau de revenus soit aussi discriminant, comme le montre la figure 4.

Il faut noter cependant que la comparaison de ces chiffres d'année en année révèle une évolution de la population des internautes chinois. La massification progressive de l'accès à Internet entraîne une augmentation régulière de la proportion des bas salaires ainsi que l'abaissement progressif du niveau d'études moyen des internautes. Si cette tendance n'a pas encore radicalement transformé le profil typique de l'internaute moyen, elle sera à prendre en compte de manière croissante dans les prochaines années. 
Figure 3-6 Different occupations accessing the Internet $(\mathrm{N}=1959$, Sig. $=.000)$

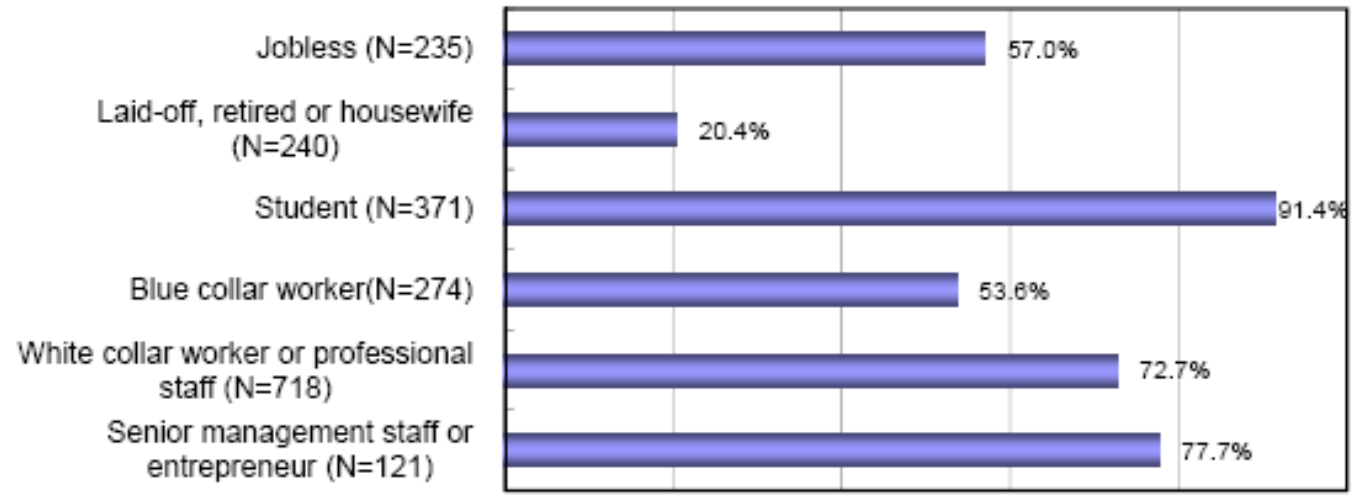

Figure 3 Pourcentage d'internautes selon le type d'activité professionnelle. Source: GUO Liang. Surveying Internet usage and its impact in seven Chinese cities.

Figure 3-7 Users distribution within income groups ( $\mathrm{N}=1203$, Sig. $=.000)$

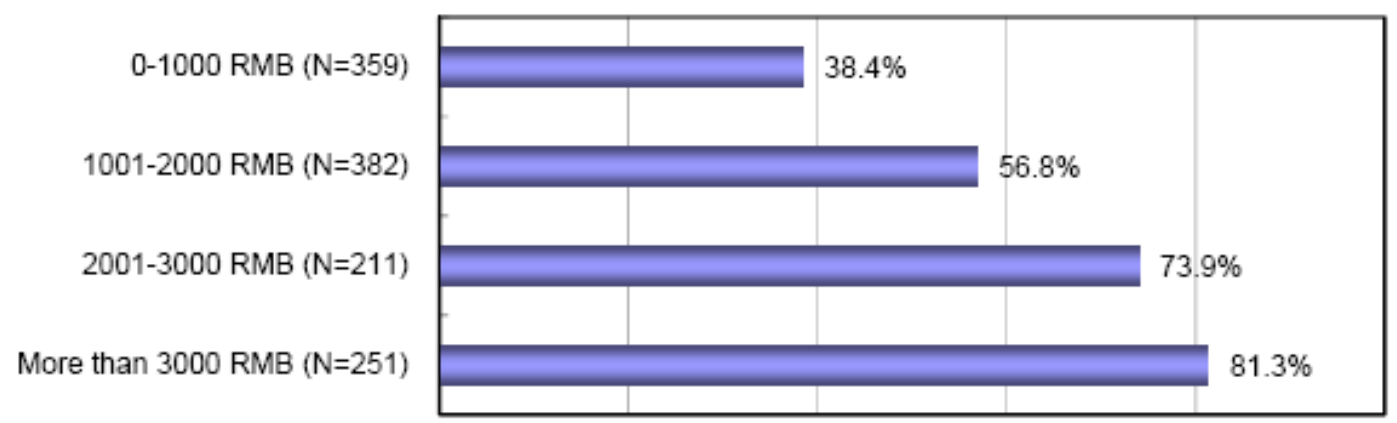

Figure 4 Pourcentage d'internautes selon le niveau de revenus. Source: GUO Liang. Surveying Internet usage and its impact in seven Chinese cities.

Internet ne peut donc pas être considéré comme uniformément réparti dans la population chinoise. Il est peut-être facile d'y accéder depuis n'importe quel endroit de Chine grâce aux cybercafés, mais les utilisateurs les plus fréquents et les plus nombreux restent des jeunes de moins de 35 ans, plutôt des hommes, citadins, relativement éduqués et exerçant des activités de "cols blancs". Voilà donc le portrait "moyen" de l'internaute chinois, qui se livre, à en juger par les statistiques, à une pléthore d'activités en ligne. 


\section{Des services pléthoriques}

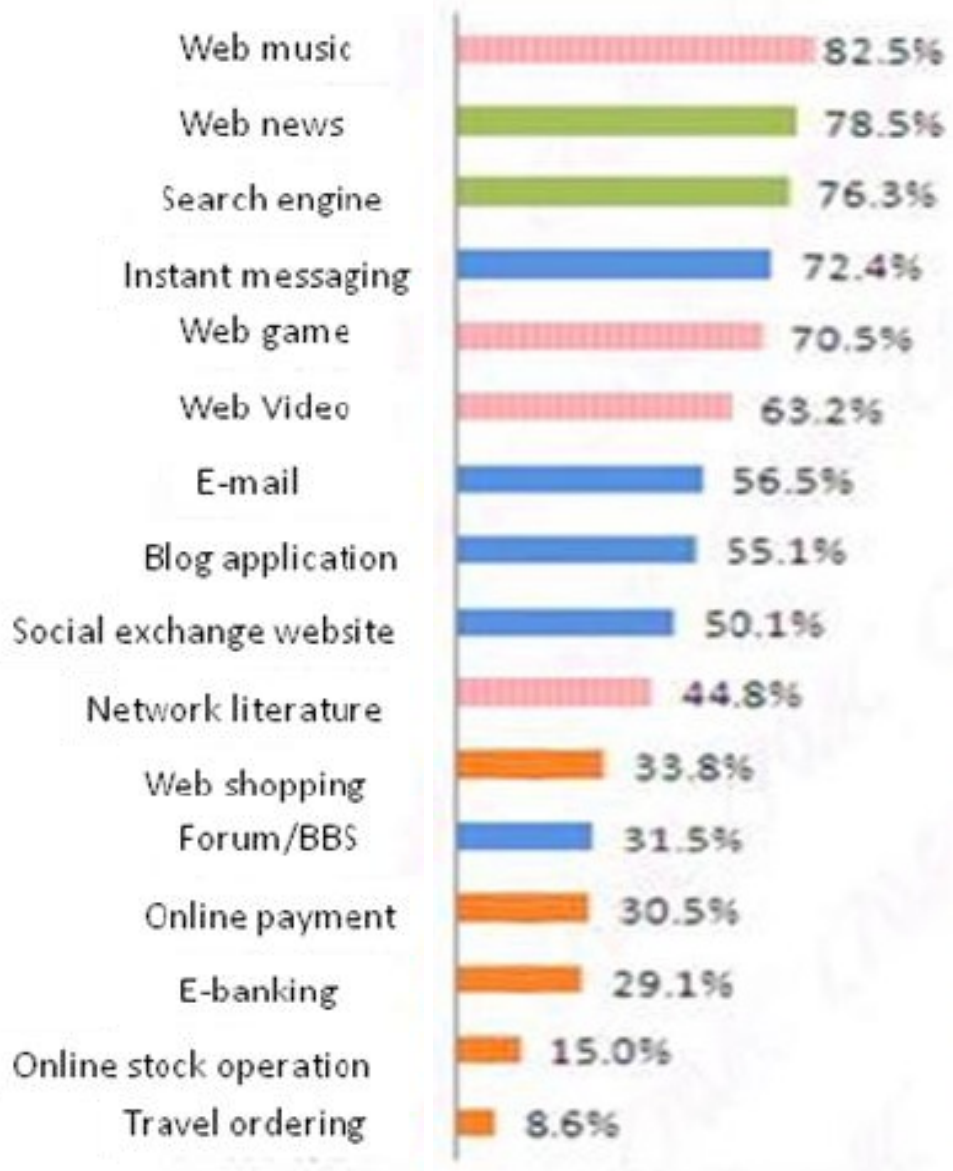

Figure 5

Les usages les plus fréquents d'Internet en Chine.

Source: CNNIC

L'une des premières catégories d'usages d'Internet est l'accès à l'information en ligne. Cela comprend la consultation de sites d'actualité (souvent adossés à des journaux papier) ou à des portails généralistes qui relaient ces sources d'information, comme Sohu, Sina ou le portail QQ entre autres. Des formes plus spécifiques de recherche d'information peuvent se lire dans le fort usage des moteurs de recherche. On s'informe de plus en plus fréquemment en ligne sur tous les sujets de la vie quotidienne. Ces usages viennent compléter, voire concurrencer, la consultation d'informations dans les "médias traditionnels" sans la surpasser. La télévision reste la source principale d'informations pour ceux qui souhaitent suivre l'actualité.

Les chiffres sur les usages d'Internet témoignent également du fait que le divertissement est central dans les motivations des chinois pour se connecter à Internet. Le téléchargement de 
musique et de films est massif, ce qui a fait le succès du principal moteur de recherche chinois, Baidu, qui propose cette fonctionnalité. Les Chinois lisent aussi beaucoup de livres en ligne. De plus les jeux vidéo représentent une très large proportion des usages d'Internet avec notamment le succès de jeux comme World of Warcraft. 70\% des internautes chinois jouent en ligne ${ }^{10}$. Cet usage est particulièrement visible dans les cybercafés où les jeunes se retrouvent pour jouer les uns avec ou contre les autres, et en tous cas, les uns à côté des autres.

Les internautes chinois ne se contentent pas de consommer des divertissements et des contenus culturels. Ils sont également très vite devenus créateurs de contenus, que ce soit au travers des fils de discussion, des (micro)blogs ou de leurs avatars. Ils disposent d'outils d'expression extrêmement variés. Pensons simplement au partage de vidéo avec le site Tudou, qui rappelle très fortement le fonctionnement de Youtube. La vidéo a l'avantage de favoriser toutes les formes d'expression, comme par exemple la parodie, dont les "Back Dormitory Boys" ${ }^{11}$ sont devenus l'icône.

Une troisième tendance notable est le développement progressif du commerce en ligne ainsi que du commerce de consommateur à consommateur. Sur Taobao par exemple, les internautes sont invités à fonder des boutiques où ils revendent leurs effets, neufs ou d'occasion. Beaucoup s'organisent même pour acheter en groupe sur Internet. Les consommateurs s'expriment aussi sur les produits de consommation qu'ils y trouvent, avec par exemple la notation de services commerciaux. Sur Dianping, fondé en 2004, les internautes échangent des idées de sorties et des commentaires de restaurants par exemple.

Enfin le développement des réseaux sociaux est une dimension très importante de l'expérience du Net pour les internautes chinois. Les forums, ou Bulletin Board Systems, représentent de loin le service le plus ancien dans cette catégorie en Chine. Ils comptabilisaient en 2009 plus de 102 millions d'utilisateurs sur des plates-formes telles que Sina, Tianya ou Xici Hutong. Les forums ont d'abord été un moyen de s'informer au sein de communautés restreintes. Beaucoup ont été développés dans des universités ${ }^{12}$, et ils servaient

${ }^{10}$ CNNIC. Statistical survey report on the Internet development in China, 2010, p. 38.

${ }^{11}$ Voir leur vidéo: http://www.youtube.com/watch?v=UrwwdtO_SD4. Eux aussi ont l'honneur d'un article sur Wikipedia. http://en.wikipedia.org/wiki/Back_Dorm_Boys. Consulté le 16/10/2010.

${ }^{12}$ L'un des plus célèbres est celui de Tsinghua, http://smth.org/, qui a même les honneurs d'une entrée dans Wikipedia: http://en.wikipedia.org/wiki/SMTH_BBS (consulté le 16/10/2010). 
au partage des informations sur le calendrier des examens tout autant qu'à la diffusion d'informations et de réflexions sur la société. La liberté de parole qui régnait au sein de ces forums universitaires en a fait des supports de publication très surveillés, voire interdits pour certains. Aujourd'hui, les grands portails qui hébergent les forums ont un statut bien différent. Tenus par de grandes sociétés commerciales et soumis à autorisation, ils concentrent des flux d'informations à la fois plus contrôlés et plus diversifiés. Leur popularité est néanmoins plus grande car ils s'adressent au grand public et non plus aux seuls étudiants des universités.

Peu de temps après les forums, sont apparues les messageries instantanées ICQ puis QQ qui rassemblent près de 304 millions d'utilisateurs ${ }^{13}$. QQ à lui seul réunit environ $80 \%$ des utilisateurs de messageries instantanées en Chine. Compte tenu des changements de numéros de comptes QQ, et des inscriptions multiples, il existait en 2008341 millions de comptes QQ activés, c'est à dire plus que d'internautes en Chine. Ciblé sur une clientèle jeune, avec un graphisme très inspiré des mangas japonais, il propose des avatars amusants, des pages de blogs, des éléments de décoration pour les espaces virtuels développés par les utilisateurs. QQ a même développé une monnaie virtuelle, destinée à l'échange de ces biens virtuels. La plateforme peut également servir de site de rencontre grâce à son moteur de recherches et à la grande témérité de ses utilisateurs, dont beaucoup acceptent facilement les mises en relation spontanées. QQ combine ainsi plusieurs facettes de l'expression individuelle sur un mode extrêmement ludique.

Dans le début des années 2000, s'est progressivement développée la pratique du blog, rendue célèbre par des personnages marquants comme la sulfureuse $\mathrm{Mu} \mathrm{Zimei}^{14}$ qui décrivait en ligne sa vie sexuelle. Il y aurait aujourd'hui plus de 100 millions de blogueurs actifs en Chine, un chiffre en rapide augmentation. Bien sûr ces chiffres sont difficiles à confirmer en raison du nombre de pages dormantes, mais ces usages sont tout de même pléthoriques et ils témoignent d'un engouement certain pour la tenue de pages personnelles, sous quelque forme que ce soit. Ce modèle évolue de jour en jour et se développe désormais sous la forme du "micro-blogging" qui permet de poster, phrase après phrase, de courtes informations sur ses

${ }^{13}$ CNNIC, op. cit., p.34.

${ }^{14}$ Voir son journal, adaptation de son blog, traduit en français. MU Zimei. Journal sexuel d'une jeune Chinoise sur le Net. Paris: Albin Michel, 2005. 
activités, y compris depuis un téléphone mobile. Si Twitter et plusieurs sites chinois fonctionnant sur le même principe, comme Fanfou, ont été bloqués à cause de leur rôle dans la coordination de certains mouvement sociaux, l'utilisation de proxys permet d'y accéder malgré tout, et de nouveaux services similaires sont créés régulièrement, comme Zuosa, Taotao ou encore Weibo, le service de microblog du portail Sina (littéralement: microblog).

Tous ces services sont bien entendu l'occasion de bâtir des réseaux sociaux en ligne. Rapidement, des sites exclusivement dédiés à cette fonction se sont créés. Le Web a rapidement donné naissance à la copie conforme chinoise de Facebook, Xiaonei, réservé aux étudiants des plus grandes universités comme l'était ce service dans ses premiers temps. Xiaonei a par la suite été ouvert au plus grand nombre, et rebaptisé Renren. Il comptabilise aujourd'hui plus de 70 millions de membres ${ }^{15}$. D'autres services concurrents s'adressent à des segments plus restreints de la population internaute, comme Kaixin001, ou 51.com ${ }^{16}$.

Il se dégage globalement de cet Internet chinois une impression pléthorique, foisonnante, dynamique, presque euphorique. Les modes se font et se défont, les sites attirent les internautes par millions, ce qui ne manque pas de fasciner de nombreux investisseurs. $\mathrm{Au}$ premier abord, l'Internet chinois est tout le contraire du portrait qui en est généralement tiré en Europe par les associations de défense de la liberté d'expression: un Web bloqué en permanence, où règne la peur de s'exprimer, où les contenus seraient inaccessibles et l'information bridée à chaque instant ${ }^{17}$. Bien au contraire, les utilisateurs d'Internet montrent souvent le plus grand enthousiasme pour cet outil dont ils disent souvent ne pas voir les limites. On s'y informe, on y joue, on y achète des biens, on y rencontre de nouveaux amis, on y discute avec son patron sur QQ...

${ }^{15}$ MACMANUS Richard. China's top 3 social network sites, 04/03/2010, consulté le 22/10/2010 sur http://www.readwriteweb.com/archives/china_top_3_social_network_sites.php

${ }^{16}$ Au moment où l'enquête par entretiens a été menée, en 2006 et 2007, le micro-blogging et les sites de réseaux sociaux existaient déjà, mais n'étaient pas encore aussi développés qu'aujourd'hui. A ce moment-là, les messageries instantanées, blogs et autres partages de vidéos en ligne battaient leur plein.

${ }^{17}$ Cela ne signifie pas que ces aspects n'existent pas, bien au contraire, mais il faut souligner qu'ils ne sont pas perceptibles au premier abord pour qui aurait une utilisation des plus banales des différents services disponibles. 
Ce résumé des utilisations d'Internet en Chine gomme quelque peu artificiellement la grande diversité des internautes. Il ne faut certes pas ignorer l'existence d'un grand nombre de cas qui ne correspondent pas à ce portrait de l'internaute moyen, surtout en ce qui concerne les usages ruraux. Tous les utilisateurs ne fréquentent pas l'ensemble des sites mentionnés, et beaucoup fréquentent des sites trop petits pour être visibles dans les statistiques. Jack Qiu, en particulier, a montré dans une étude détaillée que les ouvriers migrants ou encore les ruraux ont des usages très spécifiques du Net et du téléphone mobile. Cela leur permet de répondre à des besoins qui leur sont propres, en particulier dans des situations de mobilité ou d'éloignement très contraignantes. Les technologies de l'information deviennent ainsi de véritables ressources pour des catégories entières de la population (y compris pour défendre leurs droits). Cependant Qiu remarque que ces usages très spécifiques, qui se démarquent de ceux des internautes urbains, contribuent à stigmatiser ces catégories, et en tous cas les laissent quelque peu à l'écart d'une certaine culture dominante qui caractérise les espaces les plus fréquentés du $\mathrm{Web}^{18}$.

En effet, les pratiques des internautes en ligne, même les plus banales, ne sont pas si anodines qu'on pourrait l'imaginer. Elles sont le reflet de "styles de vie" adoptés, et parfois revendiqués par les internautes. S'informer, consommer, s'exprimer en ligne sont autant d'actions quotidiennes qui illustrent et mettent en actes des identités et des valeurs spécifiques.

\section{2 - Internet, l'attribut d'une génération "moderne"}

Urbain, éduqué, jeune: l'internaute chinois moyen semble incarner parfaitement une certaine image de la "classe moyenne", cette promesse d'une société moderne qui est devenue une référence dans le discours officiel, mais aussi dans la publicité et qui est relayée de diverses manières dans l'imaginaire de bon nombre de citoyens. Or, si les internautes semblent indistinctement appartenir à la classe moyenne par leur profil démographique, ils lui appartiennent bien plus nettement par le fait même d'être "connectés". En effet il se trouve que les nouvelles technologies sont au cœur des discours ambiants (gouvernemental, publicitaire, populaire) sur l'ascension sociale et la modernité en Chine. Internet, de même que

${ }^{18}$ QIU Jack. Working-class network society. Communication technology and the information have-less in urban China, Cambridge (Mass.): MIT Press, 2009. 
le téléphone mobile, fait partie de ces technologies "grand public" qu'il est indispensable de maîtriser. Pour être moderne, il faut être "branché".

Tout d'abord Internet fait partie des priorités gouvernementales dans la politique de modernisation du pays. En effet l'accès au réseau transforme les industries, améliore les systèmes d'information, et, en somme, il est source de croissance ${ }^{19}$. Internet est même devenu un outil de mesure de la modernisation du pays pour les autorités, comme en témoigne un article du China Daily en 2001.

Le degré de développement des technologies de l'information est devenu un critère important dans la mesure du niveau de développement d'un pays et de sa puissance nationale en général. ${ }^{20}$

Le discours officiel sur la question ne laisse aucun doute. Au-delà du seul réseau, les internautes eux-mêmes sont présentés dans la presse officielle comme le fer de lance du développement économique et de la modernité. A ce titre, son développement rapide est une fierté nationale.

Le fait de surfer en ligne est devenu indispensable à la vie quotidienne de $\mathrm{Lu} L \mathrm{Li}$, 23 ans, qui travaille dans une entreprise étrangère à Shanghai dans l'Est de la Chine, depuis qu'elle a eu son premier accès à Internet il y a sept ans. "Cela a ouvert un nouveau chapitre de ma vie", dit-elle, ajoutant qu'elle peut difficilement imaginer vivre sans Internet. Lu fait partie des gens de cette nouvelle génération qui émerge dans le pays depuis une dizaine d'années, qui apprennent, se distraient et font leurs achats en ligne. ${ }^{21}$

En quelques lignes, tous les éléments sont présentés. Le personnage central est emblématique. En tant que salariée d'une entreprise étrangère, dans la ville la plus développée de Chine, Lu Li représente un modèle de réussite. Elle ne serait pas un cas exceptionnel à en

${ }^{19}$ KANG Xie. "Industrialization supported by informatization. The economic effects of the Internet in China", in DAMM Jens et THOMAS Simona, Chinese cyberspaces. Technological changes and political effects, Londres et New York: Routledge, 2006, p. 132-147.

20 "Using legal means to guarantee and promote sound development of information network", People's daily, $12 / 07 / 2001, \quad$ consulté $\quad$ le $\quad 16 / 10 / 2010 \quad$ sur http://english.peopledaily.com.cn/200107/12/eng20010712_74810.html.

21 "Internet becoming part of life for Chinese", Xinhua via China Daily, 07/10/2005, consulté le 16/10/2010 sur http://www.chinadaily.com.cn/english/doc/2005-10/07/content_483019.htm. 
croire l'article. Au contraire, son profil est présenté comme représentatif d'une génération entière, génération qui doit son succès à l'adoption d'Internet. Cette image de la modernité du Net et a fortiori de ses utilisateurs semble faire l'objet d'une forte appropriation chez les internautes, et elle se retrouve nettement dans le discours des personnes que j'ai interrogées.

J'ai pris l'habitude de conclure les entretiens par une question très générale, pour faire le bilan en quelque sorte, et qui était le plus souvent formulée ainsi: "D'après toi, quel(s) changement(s) est-ce qu'Internet peut apporter en Chine?". La formulation de cette question a pu souffrir quelques modifications selon le contexte, ou être remplacée par "quels sont les avantages et les inconvénients d'Internet en général?". Une très grande majorité des réponses dresse un bilan positif et très optimiste $\mathrm{du} \mathrm{Web}$, assorti de quelques mises en garde sur les risques pour la jeunesse. Aucun internaute n'a montré de pessimisme à propos des évolutions possibles du Net, et il se dégage une forte unanimité sur l'idée qu'Internet est un vecteur de développement du pays. Les divergences éventuelles des internautes interrogés ne portent que sur les modalités spécifiques de ce développement, qui peut passer par la consommation, par l'amélioration du niveau "culturel" de la population, ou encore par une puissance de frappe plus importante du pays au niveau international. Au-delà de la diversité des sens qu'ils donnent à ce mot, tous les internautes interrogés s'accordent à dire qu'Internet est, pour eux, l'atout majeur d'une génération "moderne".

\section{Un outil pour consommer mieux et plus.}

En premier lieu, Internet est conçu comme un outil facilitant la vie, permettant une meilleure efficacité dans de nombreuses actions du quotidien. Internet est d'abord caractérisé par la rapidité de circulation de l'information. Cela le rend bien plus efficace que la télévision pour ce qui est de l'information pure, et cette ressource est également mise au service de la vie quotidienne des internautes.

"Le plus important c'est l'information. Par exemple maintenant on peut trouver $\mathrm{du}$ travail, acheter des choses, et puis on peut faire une recherche des objets qu'on a envie d'acheter, on trouve presque tout. Et puis j'ai un ami qui veut écrire un roman sur Internet, il vient de commencer. En-dehors de ça, Internet peut changer la société, on ne peut pas dire que ça changera tout d'un seul coup, mais sûrement à $60 \%$. Par exemple avant, quand il n'y avait pas Internet, si on voulait acheter quelque chose, on 
pouvait ne pas le trouver du tout, ou bien on n'avait même pas entendu parler de cet objet. Mais maintenant si on va sur Internet et qu'on cherche un peu, on peut tout de suite savoir quelle maison fabrique cet objet, combien ça coûte, et si on n'a pas tout de suite le prix, on trouvera un numéro de téléphone, on peut prendre contact. Je trouve qu'Internet c'est quand même très bien." (Han)

Grâce aux bénéfices matériels qu'il apporte dans la vie quotidienne, Internet est systématiquement associé à des valeurs comme l'efficacité et la rapidité, qui sont au cœur des nécessités du développement économique et de la définition de la modernité.

"Je trouve simplement que c'est très pratique, ça permet de tout faire, tout est plus pratique, plus rapide, plus efficace. Par exemple je peux commander des billets d'avion sur Internet." (Deng)

L'exemple proposé le plus souvent par les internautes lorsqu'ils parlent des aspects pratiques du Net est l'achat de billets d'avion en ligne. Il est vrai que ce service de réservation de billets a été développé très tôt en Chine. Il est peu probable que ce soit un usage extrêmement fréquent par rapport à d'autres comme la prise d'informations sur des produits de plus grande consommation par exemple. En revanche l'exemple de l'avion permet aux internautes de donner corps à l'impression de vitesse et de modernité qu'ils essaient d'exprimer. Il en va de même pour les produits de haute technologie, comme les téléphones portables ou les appareils photos numériques, qui sont également l'objet de l'attention de beaucoup d'internautes. Les hommes en particulier déclarent ainsi consulter de nombreux sites de comparaison de produits numériques.

Au-delà, Internet facilite l'accès aux biens de consommation en général. Certains sont parfois difficiles à se procurer, et la qualité et les prix sont l'objet de grandes incertitudes. Aussi utilise-t-on le Net pour s'informer sur les produits, les comparer et de plus en plus souvent, les commander.

"Si on est quelqu'un de moderne, on est obligé de passer par Internet. On passe par Internet pour acheter beaucoup de choses, c'est une tendance, c'est plutôt pratique d'acheter des choses sur Internet. Il y a Alibaba qui est spécialisé dans le commerce en ligne, c'est très pratique pour beaucoup de choses. Il suffit de chercher, on peut comparer, on peut acheter, on peut discuter le prix directement sur Internet. On gagne du temps et de l'énergie, c'est plutôt bien. Et puis quand il y a de nouvelles choses, 
elles sont présentées plus rapidement, on peut en avoir une vision plus complète, alors que dans la vie réelle il y a beaucoup de choses auxquelles on n'a pas accès. Si on est à Pékin on a accès à beaucoup de choses, mais si on est à la campagne il y a beaucoup de choses auxquelles on n'a pas accès et on est obligé de passer par Internet. Par exemple si tu veux acheter un ordinateur à Pékin il suffit d'aller voir à Zhongguancun, tu peux demander les prix, mais dans d'autres villes tu ne peux pas, et encore moins à la campagne. Tu peux aller sur Internet pour voir comment sont les équipements informatiques ou les prix, tu peux voir les marques. Tu peux voir ça sur des sites ou des forums, des fois ils font des forums spécialisés sur les ordinateurs et tu peux lire leurs commentaires sur les ordinateurs. Ca c'est très utile, je trouve." (Luo)

Internet est donc considéré comme un outil qui facilite le développement de la consommation, notamment pour ceux qui n'ont pas les moyens de consommer à des prix plus élevés. Internet ne fait pas seulement économiser de l'argent, mais aussi du temps et de l'énergie. La productivité et l'efficacité de l'internaute en sont donc décuplées. Aux yeux de cette pékinoise, Internet désenclave les gens des petites villes, leur permettant de se rapprocher du modèle des grandes villes.

Il faut d'autre part remarquer que la prise d'information ne débouche pas forcément sur des achats en ligne. Cette remarque vaut particulièrement pour les automobiles. Presque tous les hommes interrogés ont déclaré s'intéresser aux sites sur les voitures, indépendamment du fait qu'ils en possèdent une ou comptent en posséder. Il semblerait même que l'intérêt de ceux qui n'en possèdent pas est d'autant plus fort qu'ils se servent d'Internet pour imaginer la voiture de leurs rêves. Sans être propriétaires d'une voiture, ils se positionnent en "amateurs".

"On aime bien les voitures, donc on discute beaucoup des infos sur les voitures, sur la qualité des voitures allemandes, sur l'élégance des voitures américaines, sur la faible consommation des voitures japonaises. On parle de leurs avantages à toutes, et puis il faut bien dire que les voitures chinoises n'ont rien de tout ça." (Song)

Dong, lui, possède déjà trois voitures dont un petit utilitaire qui est garé à côté du lieu de l'entretien. Il compte en acheter encore une pour sa mère. Il a très bien conscience que cela fait de lui un privilégié et il en est plutôt fier ("tu connais beaucoup de gens qui ont une voiture à Pékin? Quand j'ai acheté la première, on était peut-être 1\%."). Pour lui aussi, Internet est un outil central dans la sélection des voitures. 
"Je cherche des informations sur Internet, par exemple où je peux trouver des promotions sur une voiture. Il y a des comparateurs. Et puis je ne peux pas passer mon temps à toutes les essayer, donc je compare tous les modèles pour les classer, pour savoir lequel me correspond le plus, et je ne vais essayer que celui-là." (Dong)

Ces pratiques de comparaison et de classement s'appliquent à tous les domaines. Les voitures, les produits numériques sont surtout des centres d'intérêts masculins, alors que le maquillage ou les vêtements attirent plus souvent les femmes. Dans les deux cas, Internet est utile pour rester au courant des dernières sorties, des modes. Le système des promotions commerciales est tellement utile à cette fin que les internautes sont vite prêts à se laisser pister par les marques, en dépit des risques liés à la protection des données personnelles.

"C'est facile de savoir ce que fait quelqu'un sur Internet. Et puis sur Internet il y a quelque chose de bien, par exemple si tu achètes souvent des sacs, les gens peuvent le savoir, et en fait ce genre de système est très bien, parce qu'ils t'envoient des informations, et comme ça on peut tout trouver sur Internet. Il y a les prix des produits de maquillage, les discounts sur les vêtements, tout est mentionné. Ces derniers temps c'était le livre 'Les voyages en vogue', pour un livre acheté on vous offre un produit de la marque Clarins, c'est pas mal, c'est un peu comme une crème protectrice contre le soleil." (Luo)

Cette internaute se rend carrément disponible aux annonceurs et elle attend d'eux qu'ils lui envoient de la publicité. En quelque sorte, Internet permet d'être à la mode à bon marché, et la mode permet de se sentir moderne. D'ailleurs, pour certains, Internet en soi est une mode, en tous cas par certains de ses aspects.

"Je trouve que ça correspond à une mode, tout le monde ouvre son blog, c'est à la mode." (Zhou)

Le vocabulaire utilisé par les internautes pour parler des améliorations qu'Internet apporte à leur vie semble parfois exagérément laudateur. Pour certains, le simple fait d'utiliser des annuaires en ligne change fondamentalement les couleurs de la vie.

"Je trouve que ça a accéléré le rythme de ma vie, ça fait monter la fréquence de ma vie, et puis ça a rendu ma vie un peu plus riche et colorée. Par exemple je ne savais pas où se trouvait le Shangdao Kafei où tu m'as donné rendez-vous, alors j'ai utilisé 
Internet pour faire une recherche. Comme ça, ça rend plus faciles ma vie et mon travail. Donc ça donne le sentiment que la vie est plus gaie." (Gao)

Cet enthousiasme pour la consommation facilitée par Internet n'aurait rien de fondamentalement spécifique à la Chine s'il n'était associé au processus de développement du pays dans les représentations des individus. Les achats en ligne, les comparateurs de prix, les conversations sur les prix sont des éléments banals du Web dans tous les pays. Dans ce cas cependant, ils représentent ce qui ressort le plus à propos d'Internet dans l'esprit des utilisateurs interrogés, ce dont ils souhaitent faire la conclusion de l'entretien. En effet les remarques sur la rapidité et le côté pratique du Net sont faites le plus souvent au moment du bilan, après une longue conversation sur bien d'autres aspects du Web, en particulier l'information et la discussion.

Ce n'est probablement pas anodin. Les internautes ne recherchent pas seulement des informations sur les meilleurs produits. La recherche de la meilleure consommation possible ressort d'une aspiration plus générale, d'une recherche de la meilleure vie possible. Les internautes échangent des questionnements sur les comportements à adopter ou les choix de vie qu'il faut faire. En somme, ils utilisent Internet pour construire ensemble des "styles de vie" qui doivent illustrer les progrès de la société.

\section{Progrès des individus et de la société}

Pour beaucoup d'internautes, le Web fait partie des ressources qui permettent de se hisser vers les catégories les plus "élevées" de la population. En effet, la conception d'une société en "couches" est largement partagée au sein de l'échantillon, et les internautes ne se différencient en fait que par le type de jugement qu'ils portent sur les catégories les moins favorisées de la population. Tantôt leur discours reflète le mépris, tantôt ils expriment une certaine pitié. Dans de nombreuses descriptions recueillies, les positions reflètent par exemple la "valeur" des individus dans la société, au sens économique du terme.

"Si tu leur donnes ce genre d'informations, si tu es un "rmiste" (dibaohu) qui touche 500 RMB par mois, ça veut dire que tu es du mauvais côté de la barrière (pimin jieceng), tu es quelqu'un de trop ordinaire, si tu es tout en bas de la hiérarchie en ligne, alors tes données personnelles n'ont pas de valeur. A la base ils n'ont rien à faire de cette catégorie. Mais si tu gagnes plus de dix mille yuans, alors tes données vont avoir 
beaucoup de valeur, et donc si tu écris que tu gagnes plus de dix mille RMB ou entre dix et cinquante mille RMB, et c'est sans parler des gros riches hein, eux ils ne s'inscriraient pas comme ça, je parle des gens ordinaires (putong de laobaixing). Quand mes revenus dépassent dix mille, toutes mes infos, mon adresse, mon numéro de téléphone, mon nom, ma date de naissance, et mon âge, c'est très précieux pour ces entreprises d'information, parce qu'il y a trop de choses qu'ils peuvent essayer de me vendre." (Zhang)

L'idée selon laquelle les internautes n'ont pas la même valeur aux yeux des marketeurs est ici entérinée comme un principe de hiérarchisation de la société en général. La reprise du discours sur la stratification de la société repose en premier lieu sur une distinction des modes de vie et des règles auxquelles sont soumises différentes catégories de la population. Un internaute décrit par exemple deux catégories particulières, les pauvres, et les riches, constatant simplement l'existence d'une inégalité entre ces deux catégories et une troisième qui apparaît en creux, celle de la classe moyenne.

"En Chine c'est très intéressant, chez les pauvres, c'est à dire dans les foyers paysans de la couche la plus basse, il y a beaucoup d'enfants. Les riches et les gens célèbres, eux aussi ils peuvent avoir beaucoup d'enfants. C'est parce que les pauvres sont comme ça, parce que le contrôle de la planification des naissances n'est pas très sévère dans les campagnes chinoises. Parce qu'à la campagne, les concepts traditionnels sont plus profondément ancrés, ils veulent absolument avoir beaucoup d'enfants. Comme ils ne savent pas ce que deviendra chaque enfant, ils en font d'autres. Pour les couches plus élevées qui ont de l'argent, si tu veux deux, trois enfants, tu as une amende, mais il suffit de payer l'amende, pour obtenir des hukou. Donc maintenant il y a ces deux groupes aux extrémités, ils ont plus d'enfants chez eux. Personnellement je suis d'accord, avoir deux ou trois enfants à la maison c'est assez convenable, si on a de l'argent, moi aussi je ferais pareil, en fait je ne trouve pas que ce soit anormal ou que ce soit très grave." (Cai)

Les deux catégories qui sont présentées ici sont séparées en "couches" (jieceng) superposées, dont certaines sont considérées comme inférieures (les paysans) et d'autres comme supérieures. Entre les lignes, se détache le profil de la classe moyenne dont cette personne pense visiblement faire partie. Ce sont pour lui les individus urbains ordinaires, qui ne sont ni aussi pauvres et attachés aux valeurs traditionnelles comme les paysans, ni en 
position de contourner les lois comme les riches. En somme, pour lui, la classe moyenne est une catégorie de gens à qui la loi s'applique, ce qui fait partie de ses qualités.

Outre le fait de reproduire un certain discours sur la stratification sociale, plusieurs personnes que j'ai interrogées ont entériné l'idée d'une formation, d'un effort actif pour améliorer les qualités de chacun. Cela peut se faire au travers des médias de masse, comme par exemple la télévision, et cela peut s'appliquer à soi-même.

"Je regarde des programmes plutôt généralistes, et puis des séries télé, j'aime bien regarder Hunan TV, bien que ce soit surtout du divertissement. Hier soir il y avait l'émission de He Gui, c'est du divertissement qui encourage l'amour. Dans le programme ils invitent des stars, mais ils utilisent les stars pour développer l'amour chez les gens, pour construire des écoles dans les zones montagneuses misérables, pour aider des élèves en grande difficulté à aller à l'école. Ce n'est pas que du divertissement, il y a aussi un sens de la responsabilité sociale. En même temps je trouve qu'on peut connaître les stars beaucoup mieux. Avant je trouvais que ces stars étaient des gens très différents de nous, mais avec ces programmes, je trouve qu'il n'y a pas beaucoup de différence avec des gens ordinaires. On s'intéresse beaucoup à leur vie privée, mais avec ces programmes, je trouve qu'ils nous enseignent des clés pour la vie. Il y a aussi la cuisine, ils invitent des gens de familles différentes à montrer leurs talents, j'aime faire la cuisine, donc là-dedans on peut apprendre des choses en cuisine. Et puis en-dehors de ça il y a aussi des émissions de mode, qui t'apprennent comment te maquiller, comment t'habiller, ça j'aime beaucoup. Sinon je regarde les informations de Pékin, les infos nationales." (Yang)

J'ai mis en italique les nombreuses expressions qui montrent que cette personne se prête à une véritable formation comportementale, qui touche tous les pans de sa vie. Au travers des émissions de divertissement, elle apprend à devenir une bonne ménagère (la cuisine), une femme séduisante (le maquillage et la mode), et surtout une femme compatissante, qui remplit son rôle, sa "responsabilité" sociale. Elle semble beaucoup apprécier tous ces conseils qui lui sont prodigués par les émissions de télévision, car ils l'aident à se positionner, à adopter le meilleur comportement possible. 
De même, cet autre internaute parle de son métier de coach sportif comme d'une amélioration de la santé de ses concitoyens. Sa vision de la hiérarchisation de la société transparaît lorsqu'il évoque "toutes les couches" de la société.

"Je suis chargé de promouvoir une certaine prise de conscience sur la santé, faire en sorte que les gens (dazhong), les étudiants, les gens de toutes les couches (jieceng) comprennent ce que c'est que la santé, qu'ils aient conscience de leur santé, et puis qu'ils développent spontanément des exercices pour leur santé." (Song)

Au-delà de ces premiers indices qui soulignent une perception très répandue de la stratification de la société, le discours que les internautes portent sur Internet en général est particulièrement révélateur d'une appropriation très forte des idées de progrès et de modernité, telles qu'elles sont articulées dans le discours sur la classe moyenne. Beaucoup d'internautes y voient le vecteur d'une amélioration culturelle de la société chinoise dans son ensemble. Confrontés sur Internet à plus d'informations, qui viennent d'horizons plus diversifiés (notamment de l'étranger), les internautes seraient invités à "s'ouvrir".

"Internet permet de diffuser l'information plus vite, et puis ça augmente la culture, enfin, ça fait progresser la culture de l'humanité (zujin renwen wenhua). Et puis en passant par Internet, on peut comprendre immédiatement les informations internationales à l'étranger, ça aide sûrement la Chine à se développer.

- Dans quel sens?

- Par exemple, élever la qualité des gens, et puis se rapprocher plus vite de l'international, tout cela est favorable au développement de la Chine. Parce que si un pays reste fermé, il ne pourra jamais se développer." (Zhu)

Les progrès techniques apportés par Internet changent la vie quotidienne, mais au-delà, c'est la société tout entière qui progresse, et ce mouvement invite les individus à changer eux aussi.

"Ce qu'Internet peut apporter à la Chine? Cela peut accélérer le progrès de la société et le développement technologique. Mais je crois que c'est aussi une plateforme où tout le monde peut échanger ses points de vue. En fait je pense que c'est une très bonne chose." (Shao) 
On retrouve le terme de "qualité" qui parsème le discours sur la classe moyenne et la modernisation. Aux yeux des internautes eux-mêmes, Internet est donc un outil de l'ingénierie sociale qui consiste à promouvoir la formation d'une catégorie plus "avancée" de la population. Cette catégorie, à l'image de la classe moyenne idéale, est présentée comme mieux informée et mieux éduquée que le reste de la population, et elle manifesterait sa "qualité" tant dans ses modes de consommation que dans son comportement quotidien. Ce progrès social, dans la mesure où il doit changer les individus pour les rendre meilleurs, peut toucher les aspects les plus intimes de la vie, comme la décision de faire un enfant.

"On est tous les deux assez occupés par notre travail, on est tous les deux en train de développer notre activité. Donc on va attendre encore un peu pour avoir un enfant. Maintenant à Pékin il y a beaucoup de gens dans cette situation, il y en a même qui n'ont pas l'intention d'avoir un enfant du tout, qui restent des familles sans enfants, autour de moi il y a beaucoup de gens qui pensent comme ça. Ca doit sûrement correspondre à un progrès de la société (shehui de jinbu)...

- Tu es satisfaite de ton travail maintenant?

- Je peux seulement dire pas très satisfaite, parce qu'ils nous en demandent beaucoup, il faut tout le temps s'améliorer, il faut se donner des objectifs, les gens doivent aller vers le haut, personne ne veut redescendre de position, ni rester au niveau de maintenant, que ce soit dans la vie ou dans le travail, il faut toujours s'élever, donc je ne suis pas satisfaite." (Yang)

Ici, le progrès de la société s'accompagne d'une conception très malthusienne de la vie et de la famille, qui s'accorde (nécessairement) avec la politique de l'enfant unique. Les doutes de cette internaute sont palpables dans sa façon de présenter cette évolution. D'un côté, elle présente la nécessité du progrès sous cette forme comme s'il s'agissait d'une évidence, et en même temps elle exprime les frustrations que cela lui inflige. Elle a du mal à suivre le rythme, et elle retarde la naissance de son enfant autant par nécessité que par conviction, puisqu'elle n'en a tout simplement pas les moyens. Parfois, cette évolution de la société peut soulever une franche désapprobation, par exemple lorsqu'elle signifie que désormais l'argent est au cœur des préoccupations des individus, au détriment de la solidarité.

"Ma mère c'est plutôt le genre à parler de morale, des règles, à dire qu'elle s'est occupée de sa mère, de ma grand-mère, et que peu importe combien ça lui coûte, elle 
ne se plaint pas. C'est peut-être par elle que j'ai appris cette manière de réfléchir, parce que les jeunes de maintenant, ou ceux qui sont un peu plus jeunes, on dirait qu'ils ont beaucoup moins ce sens-là. On dirait qu'ils pensent beaucoup plus à l'argent, ils lui donnent beaucoup d'importance. Ils n'apprennent pas notre morale à nous." (Deng)

Cela souligne le fait que le "progrès" auquel une grande majorité des internautes se réfèrent dans des termes identiques ne correspond pas forcément à des expériences identiques ni à des évaluations personnelles identiques. Dans ce contexte, Internet peut servir d'outil pour partager les doutes, les craintes, les questionnements liés aux transformations du mode de vie contemporain et au modèle de société proposé dans le discours dominant. C'est ainsi que se pose sur Internet la question de l'adultère et du mariage.

"Des fois, par exemple, je réponds à des questionnaires en ligne, surtout à des enquêtes sur les femmes, comme sur Sohu ou Sina, sur la "Chaîne des femmes". Ils posent des questions, par exemple sur la situation du mariage, comment on considère l'adultère, ce genre de choses. Je réponds toujours, pour que tout le monde sache que cette habitude et le mode de vie sont en train de changer. L'adultère n'est plus aussi toléré qu'avant, quand il n'y avait pas de communication possible entre les hommes et les femmes. Mais la technologie se développe, notre champ de vision s'élargit sans arrêt, par exemple il y a plein de gens qui partent voyager à l'étranger, ils ont les moyens. Ils ont gagné le droit de chercher à profiter d'une belle vie. Je participe souvent à des activités sur Internet, parce qu'en participant on montre le comportement des gens dans la vie, et je dis mes vrais sentiments et ma manière de penser. Ce n'est pas parce que je me trouve super intéressante, mais c'est marrant." (Yang)

Cette internaute répond à des sondages pour témoigner des changements de la société. Elle associe le progrès technique à un progrès des mœurs qui améliore la communication dans le couple. Paradoxalement, ce modèle permet moins la transgression, comme par exemple dans le couple, car les individus plus autonomes sont aussi plus responsables de leur vie, rendant les attaches plus contraignantes encore parce qu'elles sont désormais choisies. Cette jeune femme, en répondant aux sondages, veut rendre visible le changement culturel que représente cette nouvelle conception de la vie et du mariage. Elle veut le faire entériner comme une norme. Ce faisant, elle se pose en pionnière du mode de vie moderne et elle endosse en fait le rôle de modèle social qui est attribué à la classe moyenne. 
Il semble que pour elle, comme pour beaucoup d'internautes, la référence à l'étranger soit centrale. Pour s'instruire et changer leur perception de la vie, les Chinois devraient partir en voyage. Précédemment, Zhu mentionnait l'importance de s'informer sur l'international. De nombreuses personnes interrogées mentionnent ainsi "l'étranger" lorsqu'ils parlent du progrès, non sans faire ressortir une certaine ambiguïté, dans laquelle Internet se voit également attribuer un rôle important.

\section{Un levier de la puissance nationale}

"L'étranger" (guowai dans les entretiens) semble désigner de manière globale le monde occidental, et plus particulièrement les Etats-Unis, parfois la France puisque les personnes interrogées s'adressent à une Française. Ce terme est évoqué comme un modèle de développement, sur lequel il faut encore aujourd'hui prendre exemple pour accélérer l'évolution de la Chine. C'est par exemple le cas dans des domaines spécialisés comme le design.

"Je trouve qu'il y a beaucoup d'avantages [à l'utilisation d'Internet], comment dire, ça ouvre les yeux, on peut voir les choses très en avance (xianjin) qui se font à l'étranger. Par exemple, nous faisons du design, on peut s'inscrire sur des sites étrangers, on peut voir les nouveautés, les méthodes de finition les plus récentes, les documents d'exposition etc. On n'est plus aussi ignorants qu'avant Internet. Ca aide beaucoup le développement économique." (Guo)

Il semble que l'innovation en termes de design se fasse encore à l'étranger aux yeux de cet internaute. Pour lui, l'avantage d'Internet est donc de donner aux citoyens chinois un accès direct aux techniques, aux tendances qui se développent à l'étranger, afin qu'ils puissent se les approprier et faire eux aussi un meilleur travail. Il ne s'agit pas seulement d'importer des tendances de l'étranger, mais de hisser la Chine au meilleur niveau et de l'insérer dans le "village global", comme le pense un autre internaute.

"C'est un changement sur tous les aspects, certainement. Y compris la culture tout entière, parce que s'il n'y avait pas Internet, je ne saurais pas comment on vit à l'étranger, quelles sont leurs habitudes. Grâce à Internet maintenant je connais certaines habitudes de l'étranger, certaines cultures etc. Grâce à Internet on a pu comprendre beaucoup de choses là-dessus. Maintenant on utilise la métaphore du 
'village global' n'est-ce pas, le monde est un village. Y compris pour la mode, pour les vêtements, les voyages, tout ça est en train de s'unifier peu à peu. Par exemple pour la mode, s'il y a quelque chose de tendance à Paris, il y a tout de suite des fabricants de vêtements qui le sauront grâce à Internet, ils connaissent les couleurs, ils connaissent les tissus, et ça devient très vite populaire. Et puis il y a les styles par exemple, les sacs à main, on se rapproche peu à peu de l'international, et ça change vraiment la vie des gens petit à petit. De ce côté-là, l'économie est en train de s'unifier, et Internet a accéléré l'unification du monde." (Tian)

Internet est clairement considéré comme un outil qui permet aux citoyens chinois de participer pleinement à la globalisation. On voit ici le sentiment de s'aligner sur des standards mondiaux, définis un peu par la circulation des marchandises, mais surtout par celle des styles, des modes, de la consommation, circulation qui est facilitée, il faut le dire, par la contrefaçon et le piratage des créations audiovisuelles.

"Tu ne connais pas Prison Break? [...] Dès qu'il sort un épisode aux Etats-Unis, on le met tout de suite sur Internet, et moi je le télécharge, donc je peux les voir tout de suite. Les films américains, comme les grands films hollywoodiens, c'est bien, ils arrivent très vite en Chine. Mais les séries comme Prison Break, ça dure longtemps, d'épisode en épisode, et pour arriver en Chine elles doivent passer des contrôles, obtenir des autorisations, c'est très compliqué et elles n'arrivent jamais à temps. Tout ce qu'on regarde [à la télé] a peut-être été filmé il y a dix ans. Quand on peut les voir tout de suite, on a l'impression de regarder ça en même temps que tout le monde, en temps réel." (Liu)

De toute évidence Internet permet de contourner massivement la censure qui concerne le cinéma, de la même façon que le faisait le trafic de DVD et de VCD auparavant, et à une échelle décuplée. Cette internaute s'en félicite car elle a désormais l'impression de faire partie d'un véritable public globalisé, qui prête attention aux mêmes œuvres que le public américain, en même temps.

"Comment dire, c'est un changement tellement grand que je ne sais pas par où commencer. D'abord, c'est un changement dans le domaine de l'information, c'est le partage. Ca permet de partager l'information très rapidement, instantanément. Par exemple s'il se passe quelque chose n'importe où, tout le monde le sait partout. $\mathrm{Ca}$ rapproche les gens, y compris là où il n'y a pas Internet, ça permet de ressentir le fait 
que le monde est grand. Par exemple, la France est très loin de moi, mais je n'ai pas l'impression qu'elle soit très loin. Par exemple j'ai un ami en Tanzanie, il est en Afrique, il y travaille, et on discute sur MSN quand on veut, comme si on était à une très faible distance. Et puis, plus la communication est facile, plus on peut se développer. Internet permet le développement de beaucoup de choses comme les traditions, les échanges linguistiques, et le progrès réciproque." (Chen)

Or "l'unification du monde" dont parle Tian et les séries que regarde Liu trouvent essentiellement leur origine aux Etats-Unis ou en Europe. Le développement, dans ces conditions, se ferait par le rattrapage, l'imitation ou l'importation. Il semble que tous deux expriment, en même temps que de la fierté devant les progrès de leur pays, une certaine fascination pour l'étranger, qui n'est pas partagée par tous. Pour d'autres en effet, la Chine ne peut retrouver son rang de grande puissance, et les Chinois leur fierté, qu'en abandonnant ce complexe d'infériorité. Ainsi en témoigne cette digression spontanée au cœur d'un entretien.

"Je me souviens que quand j'étais petit, quand j'étais au Xinjiang, nous avons étudié un article qui s'appelait "je suis chinois". C'était l'histoire d'un étudiant chinois aux Etats-Unis. Il était victime de discriminations, parce que pendant les années 1980, la Chine était encore assez arriérée, donc ses camarades de classe lui ont dit que s'il participait à des conférences de haut niveau aux Etats-Unis, il n'avait qu'à dire à tout le monde qu'il était japonais, parce que les Japonais ont de l'argent. Mais cet étudiant a demandé "pourquoi est-ce que je devrais dire que je suis japonais, je suis clairement un Chinois". Et puis il s'est accroché une pancarte devant la figure, qui disait "je suis chinois". Cet article, beaucoup de gens l'ont lu quand j'étais petit, et ça nous a influencés. Mais maintenant c'est différent, maintenant on est après l'an 2000, nous les jeunes on a rattrapé notre retard, notre pays grandit, et on est aussi plus forts, il y a une sorte de sentiment de fierté, on est très contents. On ne pourrait pas avoir encore le même sentiment qu'avant, selon lequel nous les Chinois serions moins bien que les autres, ce sentiment c'est fini." (Zeng)

L'internaute poursuit en disant que la fierté d'être chinois ne justifie pas d'être agressif sur les forums Internet, comme c'est parfois le cas sur le forum Zhonghuawang qu'il visite souvent. Il prône un sentiment patriotique qui repose sur la fierté et non la frustration, mais il n'est pas surpris que les humiliations du passé donnent lieu à des propos excessifs sur Internet. Cependant il fait une remarque intéressante sur le changement de génération. En disant "nous 
les jeunes" et "après l'an 2000", il situe clairement son propos dans ce projet de société moderne, en rupture avec les frustrations et les humiliations de la Chine du XXème siècle. Internet, pour lui, doit donc être l'occasion d'exprimer une fierté et une puissance nationale sans complexes. Pour lui, seule la jeune génération est capable d'assumer sereinement cette position.

Dans ces discours, Internet est donc essentiellement présenté comme un instrument au service d'un projet de modernisation de la société chinoise et de son rétablissement comme grande nation sur la scène internationale. Au-delà de leurs statuts sociaux très différents, les internautes que j'ai rencontrés manifestent tous l'ambition de participer à ce mouvement de modernisation, même s'ils l'interprètent de manières variées. Outil de consommation, d'éducation ou d'affirmation de la puissance nationale, Internet est, aux yeux de tous, un instrument de modernisation aux mains de la jeune génération.

La position de l'individu dans la société s'en trouve redessinée, comme en témoignent les différentes voies d'expression en ligne investies par les internautes. 


\section{Chapitre II - Vers une forme singulière d'individualisme}

Le travail d'ingénierie sociale dont fait l'objet la société chinoise, et dont Internet est l'un des outils et révélateurs, se décline dans une conception renouvelée de la place de l'individu au sein de la société. Dans la perspective de modernisation de la nation, l'individu est sommé de se construire, se développer, de manière très individualiste et dans un esprit de compétition. En même temps le sentiment d'identité nationale est plus encouragé que jamais.

Une illustration très complète de cet état d'esprit m'a été donnée par Yang. Elle envie beaucoup toutes les qualités qu'elle attribue aux jeunes de la génération "post 80", et qu'elle décrit longuement.

"Je suis très curieuse, je veux vraiment discuter avec les jeunes, comprendre ce qu'on appelle chez eux le point de vue des "post 80 " sur la société.

- Ils ont un point de vue particulier sur la société?

- Leur pensée est forcément plus ouverte que la nôtre, leur capacité de discernement est très forte, et puis ils s'expriment avec beaucoup de lucidité, ils savent très bien défendre leurs avis, et ils ont une opinion personnelle. Par exemple ils aiment bien Superboy ${ }^{1}$, mais ils ont une attitude différente de la nôtre, ils votent sur Internet, en espérant que tout le monde vote pour le candidat qu'ils aiment. Surtout ce qui m'a surprise, c'est au KFC de Chongwenmen l'autre jour, quand je suis allée déjeuner, j'ai vu des étudiants. A ce moment-là dans Superboy, Zhang Jie n'était pas encore sorti. Il y avait environ une dizaine de garçons et filles, qui discutaient, ils soutenaient Zhang Jie. J'ai dit que j'aimais bien Zhang Jie aussi, ils étaient très touchés, ils étaient contents de recevoir mon avis. Ils ne me connaissaient pas, mais ils parlaient avec moi. Dire que j'avais 31 ans, et qu'ils avaient la vingtaine, mais comme je soutenais Zhang Jie, on avait des choses en commun (gongtong yuyan), et ils étaient là à discuter avec moi. Et puis à la fin ils m'ont dit 'tu dois voter pour lui, nous aimons tous

\footnotetext{
${ }^{1}$ Superboy est un divertissement télévisé extrêmement populaire de la chaîne Hunan TV, dont le principe est inspiré de l'émission américaine American Idol. Il s'agit d'un concours de chant, d'abord conçu pour les jeunes filles sous le nom de Supergirl, et organisé en 2007 pour les garçons. Les téléspectateurs sont appelés chaque semaine à voter par SMS pour éviter l'élimination de leur candidat préféré, ce qui en a fait une expérience de "démocratie" très commentée.
} 
Zhang Jie, si on l'aime bien il faut le faire rester, s'il te plait vote pour lui'. Je trouve que la pensée des post 80 est particulièrement flexible, ils sont capables d'aller voter pour les gens qu'ils aiment bien, moi sur Internet je n'ai pas du tout cette attitude. Chaque type de personnes traite vraiment les questions d'une manière différente.

- Pour quelle raison à ton avis?

- C'est sûrement à cause de leur pensée, ils aiment bien être à la mode, ils traitent les choses avec plus d'enthousiasme, ils traitent les choses plus directement.

- Quel est le rapport?

- Il y a un rapport, par exemple je peux discuter avec eux de Superboy, ça me donne un sentiment de fraîcheur, ça permet de connaître certains de leurs points de vue. Mais avec les gens de mon âge, ce genre de choses passe très vite, ils ne pourraient pas en discuter comme ça avec moi. Quand je discute de Superboy avec des collègues, j'aime bien Xu Jie. Xu Jie est le patron d'une entreprise de 500 personnes, il a déjà 30 ans, et à côté des jeunes garçons, il a un charme fou. Et puis il connaît bien la musique, et il joue très bien, je l'admire beaucoup. Mais quand j'en discute avec des gens de mon âge, ils me disent 'où est l'intérêt?', alors que si j'en parle avec des 'post 80 ', on a de quoi discuter ensemble. Même s'ils n'aiment pas Xu Jie, ils me disent qui est leur favori, et ils en discutent avec moi, donc ça me fait très plaisir. Donc je trouve qu'il faut contacter des gens de toutes les générations différentes, parce que même s'ils n'ont pas autant d'expérience que moi, je suis touchée par leur manière de rechercher un plaisir immédiat (shishang de kuaile), ça a beaucoup de conséquences sur moi.

- Tu trouves ça important?

- Assez important.

- En quoi est-ce important?

- Si tu veux comprendre des problèmes de société, je pense qu'il faut discuter avec des gens un peu plus âgés, ils considèrent ces questions-là plus sérieusement. Mais les plus jeunes, ça les effleure seulement, ils ne vont pas discuter de choses très profondes avec toi, comme des questions nationales, et ça n'a pas d'impact sur eux. Ces jeunes recherchent une sorte de nouveauté, ils recherchent une sorte d'expérience du plaisir. Mais le sens de la responsabilité sociale est plus fort chez les plus vieux.

- Pourquoi? 
- Parce qu'ils ont eu plus d'expériences, comme quand je demande à mes parents, ils sont très nostalgiques, ils se rappellent de l'époque juste après la Libération, l'époque de Mao Zedong, parce qu'ils l'ont vécue. Quand on discute de choses trop anciennes, ça ne nous intéresse pas beaucoup, les 'post 80' c'est pareil, si on leur demandait d'aller discuter des choses du passé avec leurs grands-parents, c'est sûr que ça les impatienterait. Si ton grand-père te raconte 'on ne mangeait pas à notre faim, et la vie était difficile, regardez tout ce que vous dépensez en si peu de temps', ceci cela, ça les énerve. La société est différente, parce que la société progresse, les gens ne peuvent pas s'arrêter dans le passé, les jeunes pensent que chaque famille progresse, toute la société chinoise progresse, se développe." (Yang)

Ce long portrait de la génération des "post 80" est tiré par une jeune femme de 32 ans qui pense ne pas appartenir à cette génération, bien qu'elle ne soit née que quatre ans plus tôt. Elle fait partie de ces personnes qui ont l'impression d'avoir manqué l'ouverture à quelques années près. Ce portrait, qui est vraisemblablement très idéalisé, en dit en fait plus long sur elle-même que sur ceux qui sont représentés. Cette jeune femme constate simplement que de nouvelles pratiques et valeurs sont à l'honneur, et elle fait une nouvelle évaluation de sa propre expérience en fonction de ce modèle. Très symboliquement, la scène se passe dans un fast-food américain de la chaîne KFC, ce qui d'emblée marque une forme de rupture avec les générations précédentes qui auraient probablement choisi d'autres lieux de rencontre. Comme le dit Davis, "Manger un Big Mac ne renversera pas un dictateur, mais cela peut envoyer un million de fois le message quotidien que les temps ont changé" ${ }^{2}$.

J'ai souligné dans le texte quelques éléments importants qui se détachent de ce portrait et qui permettent de comprendre à quel point les changements sont profonds aux yeux de Yang. Son discours laisse penser que l'idéologie moderniste ne se limite pas à la valorisation de la consommation et de l'efficacité, même si elle comporte aussi ces aspects. Si les jeunes ont une manière si spéciale de rechercher le plaisir, c'est qu'ils pensent complètement différemment.

Cette jeune femme attribue à ses cadets une grande liberté d'esprit, et une capacité à développer une opinion personnelle qu'ils expriment en votant sur Internet. Cependant cette

${ }^{2}$ DAVIS Deborah (dir.). The consumer revolution in urban China, Berkeley: California University Press, 2000, p.22. 
disposition d'esprit plus indépendante n'est absolument pas tournée vers la politique, qu'elle décrit presque comme un archaïsme ("les histoires du passé"). Bien au contraire, elle décrit des jeunes très individualistes, ostensiblement tournés vers le matériel, l'apparence, la superficialité, la mode, empreints d'une forme de légèreté ou d'insouciance. Elle rassemble ces éléments dans la catégorie "recherche du plaisir". C'est lié, selon elle, à une évolution inéluctable de la société dans la direction du développement et du progrès. Dans son enthousiasme un peu jaloux, Yang signale en fait l'impact du discours sur la modernisation dans la redéfinition de la place des individus en Chine ${ }^{3}$.

Cette redéfinition est particulièrement visible dans les usages qui sont faits d'Internet. Généralement utilisé de manière personnalisée, l'ordinateur devient, avec Internet, un outil d'expression de l'individualisme presque forcené qui accompagne la modernisation des mœurs. Sur Internet, on se montre, on se raconte, individuellement mais aussi sous le regard attentif des autres. D'autres formes de lien social se nouent, qui viennent se superposer aux liens plus traditionnels, lesquels trouvent aussi d'autres manières de se gérer au quotidien. Les individus semblent bien plus autonomes dans leur manière de se lier en société. Cependant, comme le dit Yang, cette autonomie ne se déploie pas dans des préoccupations politiques accrues, mais au contraire, semble conduire à une recherche d'engagement minimal, qui est particulièrement facilitée par les formes de participation sur le Net.

\section{1 - L'expression individuelle au cour des contradictions de la modernité}

\section{Singularisation de l'individu}

Les personnes que j'ai rencontrées se connectent à Internet depuis leur domicile, et parfois leur bureau. C'est le cas de la majorité des internautes urbains, et a fortiori de presque l'ensemble des internautes qui restent en ligne un grand nombre d'heures dans la semaine. Dans la mesure où les moyens financiers le permettent, chaque personne possède son propre ordinateur. Les couples ont même fréquemment deux ordinateurs, le plus souvent un fixe

\footnotetext{
${ }^{3}$ HOOK Brian (dir). The individual and the state in China, Oxford: Clarendon Press, 1996.
} 
utilisé par la femme et un portable utilisé par l'homme, qui lui sert aussi dans le cadre du travail s'il a un métier mobile.

Il s'agit là de jeunes, qui sont parfois encore célibataires ou plus souvent en couples, mais qui pour la plupart n'ont pas encore la charge d'une famille. Ils ont le plus souvent un usage très personnel de l'ordinateur. Cet usage n'est pas jaloux ni exclusif et ils partagent tous leur ordinateur très volontiers avec d'autres personnes sans leur en cacher le contenu. Néanmoins, tous se sentent plus libres dans leur utilisation avec un ordinateur par personne, d'autant que cela répond aussi à des contraintes nouvelles en termes professionnels. Les individus, soumis à une plus grande flexibilité qu'auparavant, sont amenés à travailler à domicile, le soir, ou bien en déplacement, et doivent trouver un équilibre nouveau dans la répartition des ressources à la maison.

"Quand mon mari est à la maison, et que je suis aussi à la maison, ce n'est pas très pratique pour surfer sur Internet. Parce que quand il fait des trucs sur Internet, il fait de la photo, des fois il travaille sur des images ou des choses comme ça, eh bien je ne peux pas monopoliser l'ordinateur, s'il l'utilise je ne peux pas l'utiliser. Donc il a installé un autre ordinateur, comme ça c'est pratique, on peut utiliser les deux en même temps, il n'y a pas de conflit." (Deng)

Cette remarque tranche nettement avec les premiers développements de la télévision en Chine par exemple. Le poste de télévision est d'ordinaire en bonne place dans le salon, et regarder les émissions du soir a longtemps été un rituel familial important. Les premiers ordinateurs ont parfois pris un statut similaire dans les familles chinoises. Souvent, dans les familles, l'ordinateur est mutualisé et placé dans le salon. Cependant, l'ordinateur étant perçu comme un moyen de donner toutes ses chances de réussite à l'enfant unique, ce dernier peut y avoir un accès privilégié. Dans ce cas, l'ordinateur est souvent positionné dans sa chambre ${ }^{4}$. Les jeunes individus qui composent mon échantillon sont en quelque sorte les enfants de ces familles qui ont grandi, et qui ont gardé l'habitude d'utiliser un ordinateur personnel, même lorsqu'ils vivent en couple.

En ce sens, l'ordinateur s'insère dans une série de changements qui affectent l'ensemble des foyers chinois urbains et qui va dans le sens d'une plus grande privatisation du lieu de vie

${ }^{4}$ TONG Hui. Family study. Rapport interne non publié, Orange Labs Beijing, 2006. 
et de l'équipement. Les logements deviennent plus privatifs avec la construction massive d'appartements dans des immeubles pour remplacer les habitats traditionnels ${ }^{5}$. Les jeunes y trouvent leur compte lorsqu'ils ne souhaitent pas cohabiter avec leurs parents ${ }^{6}$. Ils peuvent éventuellement leur fournir un appartement à proximité pour leurs vieux jours, mais dont ils seront séparés par une porte, un étage, une rue... Dans le même ordre d'idées, le téléphone mobile, qui accompagne désormais des millions de chinois, est par définition un outil personnel, qui permet de gérer individuellement sa disponibilité et sa liste de contacts. L'appartement, l'ordinateur, le téléphone mobile et bien d'autres objets fournissent aux individus les moyens matériels d'une plus grande autonomie.

Cette autonomie de l'individu se décline de nombreuses manières sur Internet, et tout d'abord dans la manière d'aborder l'information qui y est diffusée. Contrairement à la télévision, Internet permet à l'individu de décider lui-même de la programmation. Parmi tous les changements qu'apporte Internet dans la vie des internautes chinois, celui-là en particulier est parfois vécu comme "révolutionnaire".

"Je n'y avais jamais vraiment réfléchi, mais je pense que ça change forcément beaucoup de choses. Si on pense d'abord du point de vue de l'économie, les start-ups ont pris les devants, elles gagnent de l'argent par Internet, ça, c'est sûr. Mais du point de vue des gens ordinaires, ça a vraiment des avantages, par exemple on peut discuter sur Internet, même avec la vidéo, et on n'a pas besoin de dépenser de l'argent. Et puis on peut regarder sur Internet l'argent qu'on a mis en bourse, et on peut aller sur Internet pour lire plus d'informations, y compris des informations qui ne peuvent pas

\footnotetext{
${ }^{5}$ LI Jingyu. "Le rêve de résidence individuelle fait recette chez les nantis", Zhongguo xinwen zhoukan, via Courrier International, Hors série, automne 2004. Le chapeau est révélateur: "Sortis du cocon de la vie collective, les Chinois veulent désormais des logements qui reflètent leur changement de statut. Le marché de l'immobilier s'empare de ce désir." On précise dans l'article que les nouveaux programmes immobiliers s'adressent à des acheteurs qui ont, certes, du pouvoir d'achat, mais aussi, et surtout, du savoir-vivre. "L'objectif est clair: un habitat de qualité doit promouvoir l'ascension sociale de son propriétaire".

${ }^{6}$ LOGAN John, BIAN Fujin et BIAN Yanjie. "Tradition and change in the urban Chinese families: the case of living arrangements", Social Forces, vol. 76, n³, mars 1998, p. 851-882. Si la décohabitation n'est pas toujours possible, faute de financement et d'infrastructures adéquates, par exemple pour garder les enfants, celle-ci est désormais affirmée comme un souhait par de nombreux habitants des villes chinoises.
} 
passer à la télé. A la télé on subit l'information, alors que sur Internet on choisit soimême, c'est quand même un grand changement, c'est un changement révolutionnaire." (Zeng)

Pas un répondant n'a omis de souligner la grande liberté pratique que constitue cet accès à l'information illimité dans le temps. Alors que la télévision ne propose qu'un journal télévisé d'une trentaine de minutes, diffusé à heure fixe, complété de quelques magazines d'information, Internet, au contraire, fournit une profusion de contenus, beaucoup moins conventionnels. En ce sens, il est utilisé par certains comme un accès à des connaissances qui sont habituellement difficiles à trouver ou à publier.

"Si le gouvernement chinois ne veut pas diffuser le savoir jusqu'à moi, alors je le diffuse moi-même, j'apprends par moi-même." (Tian)

Cette confrontation avec des sources d'informations plus diversifiées suppose une plus grande curiosité et une certaine ouverture d'esprit de la part des internautes.

"Je trouve que c'est plus diversifié. Les jeunes, maintenant ils sont plus ouverts, ils peuvent tout accepter (neng jieshou), c'est ça le plus important, ils peuvent tout accepter. Il ne suffit pas de dire qu'on veut voir beaucoup de choses, mais après il faut les accepter." (Chu)

Une certaine indépendance d'esprit se développerait donc au travers de la diversification des sources d'information. Les jeunes, plus curieux, seraient mieux capables de rechercher les données qui les intéressent et de s'en faire une opinion personnelle, ce qui est vécu comme une rupture majeure. Il n'est pas dit cependant que les internautes mettent effectivement cette capacité à profit pour se donner une information très pluraliste ou très critique, mais simplement qu'ils trouvent positif le fait d'en avoir la potentialité.

Si la faculté de recevoir des informations plus nombreuses et diversifiées est censée développer une certaine indépendance d'esprit, les possibilités d'expression rendent cette impression encore plus forte. Les nombreuses fonctions qui permettent aux internautes de prendre la parole sont d'abord présentées comme des vecteurs d'expression de nouvelles individualités. Le principe de la personnalisation est désormais au cœur du discours sur les 
jeunes générations. Alors que la conformité était la règle dans la Chine maoïste, il faut au contraire se démarquer, exprimer ses propres opinions, sa personnalité.

"Maintenant [...] les jeunes ont leurs propres opinions, ils ont leur propre personnalité. En Chine on les appelle les "post 80 ", tout le monde utilise cette phrase pour parler de notre génération, on nous appelle "post 80 ", nous sommes nés après 1980. La définition de cette génération c'est qu'ils ont de fortes personnalités (you gexing), et puis ils sont très rebelles, ils ont leurs propres opinions. Je veux dire, je ne pense pas que les gens de mon entourage soient comme moi, mais je pense que ça ne fait rien, chaque personne doit avoir ses propres goûts, ses particularités." (Zeng)

Ce constat, s'il est majoritaire, n'est pas unanimement partagé. Pour un autre internaute, les jeunes se distinguent au contraire par leur absence de personnalité.

"Les Chinois n'ont pas de personnalité (gexing), je veux dire en général, car ils ont certainement une petite personnalité, mais depuis tout petits, et jusqu'à l'université, leur pensée est enfermée dans un grand cadre, où il n'y a que les [cours de] sciences et [de] lettres. Les sujets de conversation des étudiants entre eux ne sortent pas de ça, c'est très rare d'entendre un collégien de quatrième discuter de musique ou d'art, ou quelque chose qui s'éloigne de ses cours". (Tian)

Si le constat diffère, c'est peut-être que la définition de ce que doit être la personnalité est sujette à des interprétations diverses. La citation ci-dessus est d'un internaute très critique qui se positionne facilement en porte-à-faux par rapport à ses concitoyens. En revanche, l'idée selon laquelle les individus devraient affirmer leur personnalité (sous une forme ou sous une autre) est partagée. En effet, ce constat est fait sur le ton du regret. Pour l'internaute concerné, il faudrait que les jeunes aient plus de personnalité.

Le cas de la messagerie instantanée QQ est emblématique de cette tendance à l'individualisation, mais aussi à la personnalisation. La personnalisation en tant que telle n'est pas du tout spécifique à la $\mathrm{Chine}^{7}$, ni même à Internet, mais ce service est archétypique de la

\footnotetext{
${ }^{7}$ Internet fournit de nombreuses occasions de construire et d'exprimer son identité partout dans le monde, par exemple dans le cas des jeunes ou adolescents, chez qui l'utilisation d'Internet peut véritablement servir à construire l'identité. AMIR-EBRAHIMI Masserat. "La jeunesse iranienne dans le miroir du blog", Les Cahiers de l'Orient, n79, 2005, p. 43-56. FLUCKIGER Cédric. "La sociabilité juvénile instrumentée. L'appropriation des blogs dans un groupe de collégiens", Réseaux, vol.24, $\mathrm{n}^{\circ} 138,2006$, p. 109-138.
} 
proclamation dont elle fait l'objet pour un certain nombre d'internautes. Les utilisateurs sont représentés par un avatar qu'ils peuvent choisir dans un catalogue lors de leur inscription. Ensuite il leur est proposé de modifier cet avatar avec des accessoires payants, c'est à dire des éléments de dessin qu'ils pourront intégrer à l'image qui les représente. Il peut s'agir de vêtements virtuels, de bijoux, voire d'animaux familiers qui viendront se blottir dans les bras de leur avatar. Ce service est massivement utilisé. De nombreuses personnes sont prêtes à dépenser de l'argent réel pour personnaliser leur image virtuelle. Cette personnalisation est même l'avantage de QQ sur ses concurrents, et elle s'applique à tous les aspects du service, par exemple aux "émoticons", petites images que les utilisateurs peuvent insérer dans la conversation pour rendre plus vivante une expression ou illustrer une émotion.

"Et puis sur QQ il y a beaucoup d'émoticons, pas comme sur MSN où il faut les acheter, ou bien les faire soi-même. La plupart des gens utilisent QQ. Comme ils ont développé beaucoup d'émoticons, c'est très pratique à utiliser." (Yang)

Cela s'accompagne d'une recherche de valorisation très forte. Sur QQ, des petites lunes accolées au pseudonyme des utilisateurs témoignent de leur ancienneté sur le service et jouent un rôle important dans le choix d'accepter ou non un nouveau contact. Il n'existe plus un fournisseur de messagerie ou un hébergeur de blogs qui ne propose des inscriptions payantes au titre de "VIP". Cela permet d'accéder à des services améliorés, comme de plus grands espaces de stockage, mais surtout de disposer d'une adresse construite sur le modèle: pseudonyme@vip.fournisseur.com.cn. Cette adresse devient ainsi un signe extérieur d'une certaine valorisation sociale, en montrant un certain niveau de consommation de service. Internet fournit donc, comme le téléphone portable fixé à la ceinture (habitude déjà presque passée de mode, car trop répandue dans des "couches plus basses" de la société), des moyens de "se distinguer" ${ }^{8}$, comme des signes extérieurs de richesse qui sont, en fait, accessibles à tous ou presque. Ce ne sont que quelques exemples de l'importance de se démarquer, qui ressort tant de la manière dont sont conçus les services que du discours des personnes interrogées.

${ }^{8}$ BOURDIEU Pierre. La distinction : critique sociale du jugement, Paris: Minuit, 1979. 


\section{Le blog comme récit de soi et intériorisation de la norme}

De la même manière, de nombreux internautes tiennent des blogs personnels qui leur permettent de se mettre en scène individuellement.

"Je trouve que le blog c'est à toi, on dirait que c'est quelque chose de personnel.

$\mathrm{Tu}$ ne peux pas te réserver un forum entier, mais tu peux avoir un blog entièrement à toi, tout ce qui y est écrit est à toi, c'est ton point de vue. Surtout sur ces sujets de société, par exemple si une star a dit quelque chose, et que tu as ton idée dessus, ou s'il s'est passé quelque chose dans le pays, après tu peux aussi dire comment tu trouves ça, ce que tu en penses, peu importe. Tu peux aussi le mettre sur un post, mais ça ne reste pas longtemps, c'est mieux de le mettre sur ton blog." (Luo)

La plupart des internautes interrogés présentent leurs activités d'écriture sur Internet comme une démarche de "récit de soi". Prenons simplement le cas archétypique de cette jeune femme, urbaine, relativement éduquée, ayant quitté son travail d'institutrice momentanément, et qui consacre son blog à la naissance de son bébé.

"En fait le plus important sur le blog, le plus important, c'est principalement moi et mon bébé. [...] Je n'avais encore jamais rien écrit sur moi, ou alors juste en passant. $\mathrm{C}^{\prime}$ est à dire que je pense que ce blog, c'est un journal sur Internet. Je trouve que si je l'écrivais au stylo, si je l'écrivais sur un journal papier ça laisserait une trace aussi, c'est à dire que si on le regarde plus tard on saura quand je suis tombée malade, quelle maladie j'ai attrapée. [...] J'ai aussi mis un album photo, j'en ai mis un pour mon bébé, et aussi un pour moi. [...] J'ai d'abord fait un album pour moi-même, et puis un pour mon bébé, ce qui fait qu'on a chacun notre album sur le site, et il y a des liens sur mon blog, il suffit de cliquer et on peut voir les photos. J'ai mis toutes les photos de mon bébé sur l'album, pour lui faire un... un quoi? C'est un souvenir en fait. Comme à la maison on a beaucoup de photos, on a un appareil numérique donc on en prend beaucoup, si on devait les imprimer ça nous coûterait un paquet d'argent, donc comme ça, il suffit de les mettre sur Internet et si on veut les voir on n'a qu'à cliquer. Si on les mettait sur notre ordinateur à la maison, on pourrait les regarder en famille, mais si on les met en ligne dans un album comme ça, les amis et la famille éloignée peuvent les voir." (Shao)

Trois points en particulier retiennent mon attention dans cet extrait. D'une part, la construction du blog est d'une personnalisation extrême. Cette jeune femme écrit un blog, 
mais elle met en ligne deux albums photos différents, l'un pour son enfant et l'autre pour ellemême. Elle donne ainsi à son enfant un journal personnel par procuration, tant elle conçoit cet outil de mémorisation comme quelque chose d'individuel. Deuxièmement, il s'agit d'un véritable journal en ligne, dont l'objectif est de construire un récit de vie, d'enregistrer tous les événements un peu significatifs qui marquent la vie de la mère et de l'enfant. Enfin, il faut noter la volonté de prendre les amis et la famille élargie à témoin de cette mise en récit.

Cette manière de concevoir l'écriture comme un journal intime illustre la dimension du "soi comme projet et comme récit", du "partage des intériorités" qui caractérise un certain type de blogs. En France, ce type d'utilisation est plus souvent représenté chez les femmes ${ }^{9}$, comme c'est également le cas dans mon échantillon. Il est vrai qu'au cours de mon enquête, les femmes sont le plus souvent tournées vers ce type d'expression très autobiographique. Les hommes ont plus souvent tendance à parler de leurs activités de loisirs, comme le sport, la musique ou les voitures par exemple. Cependant dans les deux cas ces divers centres d'intérêts prennent la forme d'un journal personnel. Certains blogs sont construits avec un groupe d'amis, mais ils sont dissociés des blogs personnels et remplissent des fonctions différentes. $\mathrm{Au}$ contraire, dans les blogs personnels la notion de "traces" et "d'enregistrer" le parcours de l'individu reste centrale. Pour autant, cela ne signifie pas que les individus créent en ligne une identité unique et monolithique. Dans certains cas, ils construisent plusieurs blogs, dont chacun aborde une facette particulière de leur personnalité.

"Ca dépend du sujet du blog, par exemple moi j'en ai trois, un pour les camarades de classe, c'est un groupe de camarades de classe, c'est un groupe assez fixe. Ensuite j'en ai aussi un à moi, avec mes œuvres, et avec des traces de ma vie, mes humeurs. Celui-là, ce sont des amis très proches qui le lisent, et aussi beaucoup de gens que je ne connais pas, parce qu'il est ouvert, donc il y a beaucoup d'inconnus qui le lisent, et tout le monde peut devenir amis comme ça, c'est possible. Et puis il y en a un que je fais pour notre chien, c'est surtout pour enregistrer sa croissance, et sa santé et tout." (Zhang)

Ce type de blogs est également conçu comme un outil de développement personnel. L'écriture fonctionne comme une formation de soi et de ses compétences.

${ }^{9}$ CARDON Dominique et DELAUNAY-TETEREL Hélène. "La production de soi comme technique relationnelle. Un essai de typologie des blogs par leurs publics", Réseaux, ${ }^{\circ} 138,2006$, p. 15-72, p. 32. 
"C'est comme quand le professeur nous demandait d'écrire notre journal tous les jours, à cette époque-là je n'avais rien à écrire, j'écrivais ce qui m'arrivait tout au long de la journée. Peut-être que le premier objectif de nous faire écrire notre journal n'était pas de garder une trace de notre parcours psychologique, même si on écrivait aussi nos impressions personnelles. Mais c'était plutôt une obligation entre le professeur et la famille, pour s'entraîner à écrire, comme ça on écrivait des compositions. D'abord on s'entraînait à écrire des caractères, pour qu'après, en société, quand on doit écrire quelque chose, on n'ait pas peur de le montrer à des gens. Et puis d'autre part ça permettait de mettre notre parole par écrit, pour qu'on puisse écrire plus facilement ensuite. Il y a peut-être des gens qui peu à peu ont fini par trouver que c'était comme une plate-forme où ils pouvaient épancher leurs sentiments intimes. Aujourd'hui comme je ne vais plus à l'école je n'écris plus de journal, je n'ai rien à écrire, et je trouve que si j'écrivais chaque petite chose qui m'arrive ce serait comme tout le monde, donc ça ne sert à rien de l'écrire. Je suis comme tous les gens normaux, faire des photos, vivre, manger, la plupart des gens s'intéressent à leur propre vie." (Lin)

Il faut souligner le fait que la tenue imposée d'un journal personnel est un élément constant de la politique du Parti pour inciter les citoyens à intérioriser les grands principes de la politique nationale. Internet permet donc paradoxalement de perpétuer ces pratiques sous une autre forme, plus spontanée et peut-être plus efficace. L'analogie avec l'école souligne le fait que le vecteur de l'écrit permet plus de réflexion que le dialogue oral. Il ne contraint pas les locuteurs à l'immédiateté mais permet au contraire l'approfondissement et la réflexion.

"Mais dans une discussion en face à face, on ne parle que du sujet de conversation. Quand on écrit, si c'est toi qui écris directement, en écrivant sur quelque chose peut-être que tu peux être amené à penser à autre chose, tu peux fouiller, tu peux réfléchir avec plus d'ouverture. En plus je trouve qu'il y a des gens qui sont forts pour parler, et des gens qui sont forts pour écrire. Si tu fais partie des gens qui ne s'expriment pas très bien à l'oral, tu peux aussi exprimer tes commentaires à l'écrit. Tu peux écrire, et après avoir écrit tu peux modifier, ce n'est pas pareil à l'oral. Si on parle d'un sujet, je ne sais pas, si ça ne me vient pas, alors je ne dis rien, c'est une limite aussi. Donc je trouve qu'Internet permet de se détendre, et on ne peut absolument plus s'en passer." (Yang) 
Les internautes parviennent ainsi à développer une réflexivité très marquée, qui s'accompagne d'une démarche d'ordre psychologique, comme le suggère un peu le terme de "se détendre", mais plus encore chez de nombreux individus, celui de "psychologie" (xinli).

"D'une part ça m'amuse, et d'autre part j'écris mon parcours psychologique, et puis j'écris aussi mes sentiments." (Sun)

Cette réflexivité ne se construit pas nécessairement de manière solitaire. Au contraire, elle se fait sous le regard des autres (inconnus le plus souvent), mais elle apporte un certain bien-être psychologique, notamment dans des situations de difficultés personnelles. Surtout, ce bienfait très personnel, voire narcissique, est indissociable d'un certain rapport à l'autre et elle prend tout son sens avec les réactions des lecteurs anonymes, ce qui marque une différence fondamentale par rapport à l'écriture du journal intime sur un cahier ${ }^{10}$. En effet, il est plus facile de parler de choses très intimes avec des inconnus qu'avec ses proches. Bien sûr, l'amour, l'adultère, et les sujets très délicats sont plus propices à une expression hors du cadre familial.

"Par exemple on discute des phénomènes comme l'amour sur Internet, comment on considère l'amour sur Internet, ou fréquenter les amis d'Internet après les avoir rencontrés, ou peut-être qu'on trouve que se fréquenter comme ça n'est pas correct, et puis on se sépare... donc on discute un peu de comment tout le monde considère l'amour par Internet. Et puis on parle aussi de notre opinion sur l'amour en-dehors du mariage. Parce qu'avec les amis qu'on connaît, on connaît aussi leur femme, ce n'est pas pratique de parler de ces choses-là. Mais il n'y a plus ce problème-là sur Internet, on ne connaît pas les gens, on ne fait que discuter par Internet, donc on peut se débarrasser de toute hypocrisie pour discuter de ces choses-là. Mais discuter de sujets sensibles avec les amis, il faut absolument éviter." (Yang)

Le travail, et surtout les situations d'échec, sont aussi l'objet d'un certain nombre de conversations.

"Pour prendre un exemple, si tu as un échec en amour, ou dans le travail, et qu'en même temps tu ne veux pas que ta famille ou que tes amis le sachent, mais que tu es très triste, tu peux chercher quelqu'un que tu ne connais pas, et qui ne te connaît

\footnotetext{
${ }^{10}$ LEJEUNE Philippe. "Cher écran..." journal personnel, ordinateur, Internet, Paris: Seuil, 2001.
} 
pas non plus, et tu lui parles de tout ça sur QQ, et tu lui demandes de te dire ce que tu dois faire dans ce cas-là. Comme ça tu as dit ce que tu avais sur le cœur, mais ta famille et tes amis ne le savent pas, et tu te sens mieux psychologiquement." (Song)

L'intérêt de ces échanges repose, pour beaucoup d'internautes, sur une forme de réconfort ou de libération des sentiments dans les moments difficiles.

"Il y a aussi des gens que je ne connais pas, certains blogs on dirait que c'est des portraits d'un peu tout le monde, des gens qui ne sont pas heureux, ou qui ont un problème psychologique. Si tu as un problème, d'autres gens peuvent lire ton blog, quand ça ne va pas, et ils peuvent te réconforter, libérer tes sentiments, ça permet aux gens d'aller sur Internet pour apporter un peu d'amour et d'attention. Et puis le mieux de tout sur Internet c'est que ça rapproche les gens, peu importe que tu sois de Tianjin, ou de Shanghai, ou surtout de l'étranger, ça peut nous rapprocher tous. La communication sur Internet, c'est bon pour la santé mentale et physique, parce qu'on peut se libérer. Si quelqu'un garde tous ses problèmes pour lui, il peut déprimer beaucoup, donc se libérer en sortant tout ça, ça détend. En plus si tu écris beaucoup sur ton blog c'est bien, mais les forums aussi c'est bien. Je trouve que quand on est sur Internet, ça revient à se faire accepter par tout le monde, on dirait que ça influence la société, et ça en général c'est irremplaçable." (Yang)

L'écriture, mais aussi l'échange avec d'autres internautes, permet de soulager le stress, de se sentir mieux. Ce développement de préoccupations d'ordre psychologique chez les internautes est le reflet d'un succès grandissant de la psychologie en Chine au sens large. Des psychanalystes ouvrent des cabinets à Pékin ${ }^{11}$, des magazines de développement personnel font florès, au premier rang desquels le magazine Psychologies, dont la couverture, reproduite sur d'immenses affiches publicitaires, titrait "Connais-toi toi-même" (renshi ni ziji) en septembre $2007^{12}$. Il faut également mentionner la sortie en Chine du magazine Self en $2007^{13}$.

\footnotetext{
${ }^{11}$ HASKI Pierre."En Chine, le divan n'est plus un rêve", Libération, 05/05/2004.

${ }^{12}$ Voir la couverture sur le site: http://www.psychologies.com.cn/magazine, consulté le 16/10/2010.

13 GOLDKORN Jeremy. "Self China magazine launch", Danwei.org, 04/04/2007, consulté le 16/10/2010 sur http://www.danwei.org/magazines/self_magazine_launch_and_other.php.
} 
Cet engouement pour la psychologie peut être interprété comme un aspect et une conséquence de l'appel à la modernisation ${ }^{14}$. La concurrence accrue, la responsabilisation des individus dans la maîtrise de leur destin par exemple, sont souvent vécues comme des sources de pression sociale. Dans ce contexte, la tenue de blogs personnels semble apporter un soulagement face à la pression croissante que les internautes ressentent dans la vie réelle. Il semble que la modernisation elle-même soit porteuse de tensions, de difficultés pour les citoyens. Celles-ci sont toujours présentées sur un mode psychologique.

"En-dehors de l'information, il y a aussi un aspect psychologique. En Chine on ne commence à s'intéresser à ça que depuis quelques années, et puis maintenant on fait attention aux informations sur la santé, et sur... Par exemple maintenant il y a des étudiants de haut niveau, très qualifiés, pour qui la pression est trop forte, et il y en a beaucoup qui se jettent par la fenêtre. Je regarde ce genre de choses. [...]

C'est pour alléger sa charge psychologique, être un peu plus heureux, parce que la pression sociale est trop forte. Si on n'est pas heureux, on ne peut pas vivre. Et puis en Chine maintenant la pression est de plus en plus forte, surtout à Pékin, parce qu'il y a beaucoup de gens qui viennent d'ailleurs (wai lai renkou), et ça crée une pression sur le travail, et aussi une pression psychologique très forte." (Ye)

Il est vrai que les étudiants diplômés qui ne trouvent pas de travail, ainsi que les millions de personnes qui constituent la population flottante sont devenus des préoccupations majeures pour le gouvernement. Les deux catégories citées, les étudiants et les travailleurs migrants, sont donc symptomatiques des paradoxes de l'eldorado de la modernisation, qui est plus une norme qu'une réalité pour beaucoup. Peut-on considérer pour autant que les difficultés soient plus grandes qu'avant, pour les internautes qui sont souvent parmi les groupes les plus favorisés? Le sentiment de pression qu'ils ressentent est certainement réel, mais il est surtout révélateur du fait qu'ils ont recours à un travail réflexif et psychologique pour le surmonter. Dans leur manière d'évoquer ces deux catégories, les internautes semblent en fait intérioriser la notion de concurrence entre les couches sociales, et il est notable que les individus interrogés présentent moins ces problèmes comme des récriminations que comme un défi à relever personnellement. Ils "prennent sur eux", ce qui peut expliquer le recours aux

${ }^{14}$ GIDDENS Anthony. Modernity and self-identity: self and society in the late modern age, Stanford University Press, 1991. 
termes liés à la psychologie. C'est pourquoi de nombreux internautes considèrent Internet comme un exutoire où il est possible d'évacuer cette tension.

"La plupart des gens veulent se soulager de tout ce qui ne les satisfait pas dans la société, ou ce qui les déçoit dans d'autres choses. La plupart veulent juste se défouler." (Zeng)

"Ca permet d'atténuer la pression psychologique. Dans la vraie vie, la pression psychologique est très forte, et sur Internet on peut s'en débarrasser pour s'alléger un peu." (Luo)

Les internautes mentionnent encore et toujours la pression qu'ils vivent au quotidien, dans des termes presque identiques.

"Je trouve qu'aujourd'hui il y a beaucoup de pression au travail, par exemple moi, en tant que professeur j'ai une très grande pression. Surtout quand mon mari travaille, et qu'il ne rentre pas, quand il me laisse toute seule, je vais sur Internet, et je dis ce que j'ai sur le cœur, c'est une façon de relâcher la pression." (Lei)

Le défoulement est devenu tellement constitutif du mode de vie moderne qu'il en vient à s'institutionnaliser au travers de services rendus par des entreprises. Il est présenté comme un "besoin physiologique" de l'individu moderne, auquel l'expression sur Internet répond très bien.

"Je trouve qu'Internet est surtout un moyen pour les gens de se défouler. En Chine aujourd'hui on manque de moyens pour se défouler. Il y a même des entreprises qui font ça, ils fournissent des équipements ou bien des employés pour qu'on se défoule. Par exemple il y a des poupées qu'on peut détruire. C'est comme les animaux, quand ils détruisent des objets, c'est premièrement par besoin physiologique, et deuxièmement c'est une façon d'évacuer leur bile. Si tu ne joues pas avec un animal qui aime bien bouger, il va se défouler, détruire des choses. Aujourd'hui les points de vue très indépendants sur Internet, je trouve que c'est une sorte de défoulement émotionnel, c'est un moyen de se laisser aller." (Lin)

Internet semble en effet apporter un soulagement, en partie parce qu'il libère d'un certain nombre de conventions sociales qui peuvent sembler pesantes, comme la nécessité de garder son calme ou d'être poli en toutes circonstances. 
"Dans les salons de discussion en fait on peut dire franchement ce qu'on a sur le cœur à des gens qu'on ne connaît pas, si ça se passe bien, ils peuvent t'aider à résoudre ton problème de pression psychologique, et si vous ne vous entendez pas, ils se mettent à t'insulter au bout de deux phrases, c'est une sorte de défoulement mutuel [faxie]. Ce n'est pas vraiment pour rire non plus, chacun recherche un endroit pour s'épancher un peu. Les salons de discussion c'est assez libre, le point principal c'est premièrement qu'on peut y dire n'importe quoi, et deuxièmement qu'on peut être sympa ou attaquer les gens, on peut vraiment se lâcher librement (fangsong ziyou). Les gens ne peuvent pas être toujours au juste milieu, toujours parfaits. Je te donne un exemple, s'il y a une fille et que j'ai oublié comment elle s'appelle, je peux l'appeler comme ça 'hey beauté!'. Est-ce qu'on pourrait appeler les gens comme ça sur QQ? Les gens s'énerveraient." (Chu)

Le même vocabulaire revient systématiquement à ce propos, comme un message appris par cœur. Internet est un outil formidable pour se détendre, se défouler, vider son sac, et surtout il permet de le faire sans se soucier des conventions sociales ni avoir peur de blesser les autres. Internet est explicitement le lieu où les individus peuvent s'exprimer individuellement, sans être obligés de tenir compte des autres. De plus, par son anonymat, il permet de trouver un exutoire où livrer des préoccupations personnelles hors du carcan des relations sociales de la vie réelle. Internet remplace le journal intime dans le rôle du tiers sans visage, à qui l'on peut livrer des sentiments intimes.

"Discuter avec des inconnus en ligne, ça ne te fait pas peur?

- Non, parce qu'aujourd'hui tout le monde est très ouvert. Pourquoi c'est bien de discuter en ligne? Tout le monde vide son sac (changsuoyuyan), sans façons (mei you shenme tebie de). Si on venait de faire connaissance toutes les deux, des fois on parle un peu timidement (hanxu), on réfléchit à ce que ressent l'autre, je trouve que sur Internet ce n'est pas obligé, Internet c'est une plate-forme où tout le monde peut vider son sac, une plate-forme où on libère ses sentiments (jiefang xinqing), on peut dire ce qu'on a sur le cœur (suixinsuoyu)." (Yang)

Les différents répondants sont unanimes pour affirmer que la grande liberté d'expression est une bonne chose et qu'elle est l'atout principal d'Internet. Elle peut même donner lieu à certains excès, car les individus s'expriment sur des sujets qui les touchent personnellement et qui peuvent être très émotionnels. 
"Oui, parce que je trouve que sur Internet il n'y a pas de scrupules, on ne peut pas dire que sous prétexte que je fais un commentaire je dois porter la responsabilité de mes paroles, donc on dit plutôt la vérité, on parle avec audace, et parfois ça dépasse peut-être un peu les bornes ou c'est violent, mais c'est Internet, c'est sûrement l'endroit qui peut incarner le plus la liberté d'expression de tout le monde." (Liu)

L'uniformité même de ces remarques montre qu'en même temps qu'Internet apporte des modes d'expression moins contraints par la politesse, il contribue à ériger en norme le fait d'exprimer les difficultés rencontrées dans la vie quotidienne sur le mode psychologique. Internet permet d'une part de faciliter de nombreuses actions (de consommation, de communication) et ainsi de se rendre plus efficace, et d'autre part il se présente comme une soupape pour atténuer le poids de la pression sociale. Or l'autonomisation et la responsabilisation individuelle sont constitutives des contraintes de la modernisation ellemême, car celle-ci fait peser sur l'individu le poids de son propre destin, dans une société dont il ne maîtrise que peu d'éléments et qu'il ne peut pas remettre en question ${ }^{15}$. En leur permettant de construire leur identité de manière toujours plus réflexive, les espaces d'expression donnent les moyens aux internautes de se conformer au modèle de la modernité, qui suppose de leur part un intense travail de construction personnelle.

La liberté d'exprimer une identité particulière est donc aussi le produit de l'injonction gouvernementale à s'engager comme individu dans le processus de la modernisation nationale. Le fait que les magazines ou la publicité encouragent le développement de la psychologie et la recherche de "soi" en témoigne. Pour trouver sa place et échapper au stress, l'individu doit en permanence s'interroger sur lui-même et construire le récit de son parcours et de sa personnalité, dans une attitude de réflexivité permanente.

\section{2 - Le triomphe des liens faibles}

Or ce phénomène se décline également dans une certaine recomposition des appartenances sociales. J'ai déjà mentionné que les blogs, ou les avatars choisis par les internautes peuvent exprimer différentes facettes de la personnalité d'un internaute. L'identité peut s'exprimer de manière plurielle, à la faveur des environnements (de plus en plus) variés

\footnotetext{
${ }^{15}$ BECK Ulrich. Risk society: towards a new modernity, London : Sage, 1997.
} 
dans lesquels les individus évoluent ${ }^{16}$. En effet, les internautes chinois sont en mesure - et ils sont obligés - de moduler leur implication dans les divers groupes auxquels ils appartiennent.

\section{Une sociabilité (plus) choisie}

Les contacts rassemblés dans les messageries instantanées illustrent bien la superposition des types de sociabilités que permet Internet. Les internautes que j'ai rencontrés ont généralement entre trente et cent contacts dans leur liste "d'amis". Lorsque je leur demande s'ils subdivisent ces contacts en catégories et lesquelles, ils répondent invariablement "les camarades de classe, les collègues, les amis et les amis d'Internet". La famille en revanche est très souvent absente de ce panorama, à l'exception parfois de cousins, surnommés "petit frère" ou "grande sœur" selon les cas, et qui semblent s'intégrer plus ou moins au groupe des "amis".

Il arrive que l'utilisation de deux messageries différentes permette de différencier entre des catégories de contacts, vis-à-vis desquels la disponibilité et l'identité affichée sont différentes. MSN est le plus souvent utilisé pour des contacts professionnels, alors que QQ rassemble essentiellement des contacts amicaux, plus nombreux et plus disparates, ainsi que des contacts purement virtuels.

"Ce qu'on utilise le plus c'est QQ, c'est une messagerie instantanée très rapide. MSN en général on l'utilise quand on travaille. En général je trouve que MSN est un outil assez formel, parce que ça n'a pas du tout été adapté pour la Chine. QQ a beaucoup de fonctions, et même si beaucoup d'options et de fonctionnalités de QQ ont été copiées sur MSN, les infimes adaptations de MSN pour s'adapter à la Chine sont trop mauvaises. On ne peut pas dire que c'est mauvais, mais ça n'est pas suffisant, et donc très peu de gens comprennent comment ça marche. Donc il n'y a que des gens comme nous, qui avons besoin de la messagerie instantanée tous les jours pour travailler, qui utilisons MSN." (Zhang)

Il semble que la distinction entre les deux messageries corresponde plus à un impératif pratique qu'à une réelle volonté de séparer les contacts. Les internautes s'adaptent

\footnotetext{
${ }^{16}$ LAHIRE Bernard. L'homme pluriel : les ressorts de l'action, Paris: Nathan,1998.
} 
essentiellement aux modes de connexion de leurs contacts, ou aux outils qui sont disponibles, en l'occurrence, sur leur lieu de travail.

"D'après moi la différence, c'est qu'au bureau il n'y a que MSN sur les ordinateurs, donc tout le monde utilise MSN. Mais à la maison, avec les amis on utilise tous QQ. [...] Sur QQ j'ai en majorité des amis qui viennent de ma vraie vie, et j'en ai connu une partie dans des jeux en ligne.

- Ceux de la vraie vie, est-ce que c'est un groupe d'amis, ou est-ce qu'il y a plusieurs catégories?

- On peut séparer les anciens camarades de classe, les collègues, et puis mes voisins.

- Il y a des gens de ta famille? Des cousins, par exemple?

- Oui.

- Et des inconnus?

- Non, pas d'inconnus.

- Pourquoi?

- Les inconnus peuvent te demander d'entrer en contact par QQ. Ils doivent te donner des informations, mais n'importe qui peut t'ajouter dans sa liste d'amis. Moi je l'ai configuré pour que n'importe qui puisse devenir mon ami. Quand quelqu'un me parle, des fois je lui parle aussi, mais s'il ne m'a pas ajouté, je ne lui parle pas." (Wang)

La notion d'amis et de contacts est parfois relative. Ainsi les "inconnus" ne sont-ils plus inconnus s'ils se font ajouter dans la liste des contacts, autorisant ainsi l'affichage d'un certain nombre de données personnelles. Bien sûr d'autres internautes sont beaucoup plus circonspects.

"Avec un inconnu, on ne se connaît pas, donc on a moins de choses à se dire. Si je discute avec un ami, on se connait mutuellement, et il y a plus de points communs entre nous.

- En général, tu n'acceptes pas de discuter avec des inconnus?

- Non, très peu. Parce qu'Internet est virtuel, beaucoup de choses ne sont pas comme on les imagine." (Xie) 
Internet suscite donc les mêmes méfiances que partout ailleurs dans le monde, et n'implique pas nécessairement la multiplication des contacts sans conditions. Cependant il semble que le rejet complet soit relativement minoritaire. Pour la plupart des internautes, il est possible d'établir le contact avec des inconnus en ligne, à condition de garder une certaine distance et surtout de conserver à tout moment la possibilité de rompre le contact, ce qui, sur une messagerie instantanée, est possible en un clic.

"Je n'ai pas beaucoup d'amis, et en général je n'ai pas grand chose à dire non plus. En plus quand je discute avec des inconnus, en général c'est pour dire 'quel âge as-tu, qu'est-ce que tu fais, je fais ça', c'est assez ennuyeux. Donc je préfère discuter avec des amis.

- Tu n'aimes pas discuter avec les inconnus?

- Ca dépend si la personne est intéressante ou non.

- Tu as déjà ajouté des gens comme ça dans ta liste d'amis?

- Avant, quand je n'avais pas encore mon petit copain, avant qu'on se marie, j'en avais ajouté beaucoup, mais après ça n'était pas intéressant donc je les ai tous supprimés."

$\mathrm{Au}$ travers du moteur de recherche de QQ, il semble que les internautes ont la possibilité de multiplier les contacts éphémères et, le plus souvent, très superficiels. Il est rare en effet que ces conversations soient très approfondies, et elles servent surtout à tuer le temps. Si l'emploi du temps de l'internaute devient plus serré, on consacrera moins de temps à l'établissement de contacts qui semblent peu utiles. Si la sociabilité dans la vie réelle se transforme, comme c'est le cas avec le mariage de cette jeune femme, alors les contacts virtuels peuvent devenir indésirables ${ }^{17}$.

Ces contacts sont acceptés facilement, semble-t-il, parce qu'ils ne comportent que très peu d'obligations mutuelles. Ils sont faciles à rompre, ne demandent presque aucun engagement, ni investissement émotionnel. Ce sont des "liens faibles" ${ }^{18}$. Or, dans le cas

${ }^{17}$ C'est un cas classique d'évolution de la sociabilité au cours de la vie. FORSE Michel. "Les réseaux de sociabilité: un état des lieux", L'année sociologique, n 41, 1991, p.247-264.

${ }^{18}$ Pour Granovetter, la force d'un lien dépend du "temps, de l'intensité émotionnelle, de l'intimité (la confiance mutuelle) et des services réciproques qui caractérisent ce lien". GRANOVETTER Mark. "The strength of weak ties", The American Journal of Sociology, vol. 78, n 6, mai 1973, p. 1360- 
chinois comme dans les cas occidentaux, ces liens faibles jouent un rôle important dans la construction d'une sociabilité de plus en plus choisie.

La multiplication de ces liens faibles peut se faire par différents canaux, selon les préférences de chaque individu. Parfois, les forums peuvent réunir des individus qui ne se connaissent pas autour de sujets de conversations. La plupart des contacts se font ainsi autour de centres d'intérêts partagés. Ces centres d'intérêt, qui passent par certains modes de consommation, de présentation de soi, de pratiques musicales ou littéraires, sont autant de "goûts" (aihao), par lesquels les individus se définissent et se reconnaissent, et fondent des groupes.

"Ca dépend des goûts personnels, quand j'aime un forum je vais dessus. Par exemple si en ce moment c'est la mode de faire partie de tel ou tel groupe, [...] par exemple j'aime les cheveux colorés, alors je vais dans un groupe sur les cheveux colorés. Et comme tout le monde a des goûts en commun (gongtong aihao), ils vont tous sur ce groupe. Donc s'il y a un groupe sur le badminton, et que j'aime le badminton, je rentre dans le groupe. Et après on peut choisir différentes catégories, des groupes dans différentes catégories, et quand tu rentres dans un groupe d'une certaine catégorie, tout le monde aime une chose en particulier." (Huang)

Tous ces centres d'intérêts ne sont pas seulement de pures considérations esthétiques et superficielles. Au contraire, les goûts catalysent les points communs entre les gens, et sont des points d'appui essentiels pour bâtir des relations. On voit là ressortir le stéréotype des "relations" (guanxi), qui apparaît de manière saillante dans ce mode de fonctionnement.

"Oui, là-dessus je ne sais pas si tu comprends ça, en Chine que tu fasses quoi que ce soit, on met toujours les relations en premier. Par exemple avec nos clients, et aussi avec nos dirigeants, nos subordonnés, quand on fait beaucoup de choses, on met les sentiments en premier, en espérant qu'on échangera encore plus. On échange nos avis sur d'autres sujets, ou on parle de nos goûts, et comme ça on améliore notre relation, et après dans le travail c'est plus fluide. Ce n'est pas moi qui l'invente, tout le monde fait comme ça, et moi je fais comme tout le monde." (Cai)

1380, repris dans MERCKLE Pierre. Sociologie des réseaux sociaux, Paris: La Découverte, 2004, p.46-47. 
Les goûts partagés permettent d'améliorer les relations professionnelles en les rendant plus humaines, moins formelles. Ils permettent aussi de trouver de nouveaux amis, des gens qui pensent de la même façon, qui, en bref, se ressemblent.

"Les amis que j'ai connus sur Internet au tout début, c'était en écrivant des commentaires sur une page d'informations. On a tous plus ou moins le même avis, et quand on a le même avis ça nous donne une impression de, enfin au minimum on a l'impression qu'on est à peu près pareils psychologiquement, non? Avec les gens qui pensent comme moi, on a des atomes crochus (gongtong yuyan), et après on commence à discuter, et puis en discutant, si on a une discussion spécialement sur un sujet, plus on discute plus on devient proches, et quand on est proches on finit par connaître des choses personnelles sur la personne, comme le sexe, l'âge, le métier, des informations personnelles. (Wu)

La tendance à "l'homophilie" est une constante ${ }^{19}$ de la construction de l'amitié en France, même si elle peut souffrir des exceptions. Elle semble l'être également en Chine. Les internautes déclarent volontiers préférer les contacts avec les gens qui leur ressemblent. A mesure qu'ils évoluent dans la vie, ils peuvent ainsi être amenés à privilégier divers types de contacts.

"Il n'y a pas seulement mes [anciens] camarades de classe, il y a aussi des collègues, toutes sortes de gens. Regarde mon groupe de femmes enceintes, ce sont celles que j'ai rencontrées le plus récemment, mais je parle peut-être plus avec elles qu'avec mes amis plus anciens. Je discute encore beaucoup avec mes anciens camarades de classe. Je pense qu'on ne peut pas toujours dire que quand on arrive à une position on ne fréquente qu'un certain type de personnes, donc je reste en contact avec eux. Peut-être que ma position aujourd'hui, vu que je suis devenue maman, bien sûr ça me conduit à discuter plus souvent avec des mamans, et mon entourage aujourd'hui c'est surtout ces personnes-là. Mais si je recommence à travailler, alors les affaires du jardin d'enfant vont reprendre, il faudra que je m'intéresse à nouveau aux questions d'éducation et tout. Je vais peut-être télécharger des choses, aller sur des forums, lire des commentaires sur les jardins d'enfants, des commentaires sur les professeurs du jardin d'enfants, je ferai peut-être plus attention à ces choses-là. C'est comme ce que je disais à propos de ma période de grossesse, à ce moment-là je

${ }^{19}$ BIDART Claire. L'amitié. Un lien social, Paris: La Découverte, 1997. 
m'intéressais à la situation de l'intérieur de mon ventre, la situation du bébé. Maintenant je suis une maman, donc ce qui m'intéresse maintenant c'est est-ce qu'il y a une réunion, est-ce qu'il y a une activité, ou quelque chose, par exemple est-ce qu'on recherche des petites stars, pour que nos bébés deviennent des stars, je cherche ce genre de petites infos. Quand je retournerai travailler au jardin d'enfants je m'intéresserai à nouveau à ces, eh bien disons des choses du moment, c'est à dire que je déciderai des sites que je lis en fonction de ma situation du moment, voilà. " (Shao)

Les centres d'intérêts évoluant, différents types de contacts peuvent être activés, ce qui peut donner une certaine impression de volatilité. On dirait que cette jeune femme papillonne. Pourtant, son témoignage révèle aussi la persistance des liens auxquels elle ne fait pas appel, et la possibilité de les réactiver à tout moment. Elle se réserve seulement la possibilité de choisir en fonction de sa situation. Ainsi, les liens préexistants, ou ceux qui se construisent dans d'autres sphères de la vie quotidienne, ne sont-ils pas évincés. Au contraire, les forums, les blogs facilitent aussi le développement et l'entretien de ces liens ou de ces groupes, qui viennent ainsi se juxtaposer les uns aux autres.

"Comme je n'ai pas beaucoup de temps pour surfer sur Internet, j'ai découvert un BBS qui m'intéresse beaucoup et que je télécharge souvent. Quand j'ai l'occasion, je jette un œil sur ce BBS, et avec le temps je me suis fait des connaissances. On a des goûts en communs, on a les mêmes habitudes sur Internet, on est sur Internet en même temps. Quand on s'est croisés plusieurs fois, après on se salue, et peu à peu on devient proches. Comme on s'entend très bien, des fois on se met d'accord pour se retrouver sur un BBS et pour discuter. Il arrive que d'autres personnes se mêlent à la conversation, mais comme ils ne connaissent pas les tenants et les aboutissants, ça les ennuie. Sur ce genre de BBS il y a un ou des sujets bien particuliers, par exemple le thé, les films, l'anti-japonisme, la science, etc. Et puis il y a quelques BBS qui sont faits pour un groupe particulier dès le départ, par exemple pour une école, une classe, une unité de travail... Les gens qui participent se connaissaient déjà avant, ils ont peut-être du mal à se réunir d'habitude, alors ils se donnent rendez-vous à un moment précis sur le BBS. Ca leur sert à garder le contact, parler de leurs sentiments, et il n'y a pas beaucoup d'autres internautes là-dessus non plus." (jeune femme, 25 ans, enseignante, $5000 \mathrm{RMB} / \mathrm{m}$, entretien exploratoire). 
Les facteurs qui peuvent réunir des individus en ligne sont donc nombreux. Ils s'étendent des liens traditionnels comme la famille, pour laquelle on peut publier un blog, à des contacts tout à fait éphémères, qui peuvent permettre d'évacuer un souci passager, en passant par la constitution de groupes et de forums autour de centres d'intérêts. Les individus sont donc amenés à vivre leur sociabilité d'une manière très diversifiée et plus choisie que jamais.

Or les groupes qui se forment autour de centres d'intérêts ont ceci de particulier qu'ils sont le plus souvent structurés autour de "liens faibles", de contacts très informels, qui ne comportent que très peu d'engagement, mais qui ne sont pas pour autant dépourvus de toute notion d'intérêt.

\section{Gratuité et intérêt}

Spontanément, les personnes interrogées font une distinction nette entre ce qui les attire pour des raisons d'intérêts, et ce qui les attire par goût, au titre de loisirs.

"Par exemple en ce moment je m'intéresse à la ruée sur les actions (en bourse), et au concours du Rêve dans le Pavillon Rouge, là j'attends de savoir qui sera choisi à la neuvième étape finalement, qui sera le champion.

- Pourquoi ça t'intéresse?

- C'est parce que j'aime ça, parce que les actions c'est en rapport avec nos intérêts économiques, donc je m'y intéresse forcément, et le Rêve dans le Pavillon Rouge c'est un loisir (yeyu aihao)." (Ma)

La gratuité de ces goûts, de ces loisirs ou hobbys, est perçue comme une qualité. Elle fait ici partie de la dynamique d'autonomisation des individus dans la mesure où elle permet une certaine émancipation par rapport aux réseaux sociaux traditionnels. Cette apparente gratuité soulève un certain enthousiasme car elle libère les individus des liens de dépendance entre les gens. Deng, par exemple, préfère de loin les contacts avec ses amis sur Internet par rapport à sa famille, avec laquelle elle entretient une relation assez mauvaise. 
"[Je ne contacte pas ma famille par Internet] parce que ma mère ne va pas sur Internet, elle ne sait pas utiliser Internet, et puis sinon mes frères et sœurs ${ }^{20}$ sont tous à Pékin. Ceux qui sont en province, je ne connais pas leur numéro QQ. Et puis franchement, je ne discute pas autant avec ma famille qu'avec mes amis, et on ne se voit que quand ceux de province rentrent pour le nouvel an et pour les fêtes. Mon lien avec ma famille est certainement moins bon que mon lien avec mes amis. Honnêtement, côté amitié, la famille ne vaut pas les gens de l'extérieur. C'est peut-être dû à notre famille, en fait dans notre famille on ne s'entend pas trop, c'est à peu près aussi douloureux qu'une rage de dents, voilà comment sont nos relations. Il y a quelques jours ma grand-mère était malade. De toutes ses filles aucune n'est venue s'occuper d'elle. Ma mère a payé et fait des efforts, elles l'insultaient encore. Rien que pour que leur grand-mère voie un médecin ils se disputent. La génération suivante, on n'a pas beaucoup de contacts. On n'est pas comme avec des amis, très proches (jinjin mimi), sur la même longueur d'ondes (hehe xiexie). Dans ma famille, quand on a un problème on demande et c'est toujours les amis qui nous aident, mais pas la famille. On dirait que l'amitié entre les amis, c'est différent de l'amitié avec les gens sur Internet, mais je crois que l'amitié, franchement, c'est beaucoup mieux que la famille." (Deng)

Evidemment, tous les internautes n'ont pas ces relations déplorables avec leur famille. Pour la plupart, ils entretiennent même des rapports très étroits, soit qu'ils logent ensemble, soit qu'ils dépendent de leurs parents ou au contraire qu'ils subviennent à leurs besoins. Ce qu'il faut souligner cependant, c'est l'apparition d'un réseau de relations qui fournissent une ou des alternatives aux liens préexistants et qui sont utiles dans la résolution de problèmes matériels. Plus que jamais il est possible, en cas de tension dans ces relations, de les distendre, de réduire la fréquence des contacts, et de privilégier d'autres groupes de contacts qui pourront être mobilisés alternativement selon les périodes de la vie. Notre internaute va même plus loin à ce sujet.

"Avec les amis, on peut avoir des relations désintéressées. Dès qu'on implique des intérêts, ça devient très difficile de se faire des amis. C'est comme les relations familiales. Pourquoi je ne m'entends pas avec ma famille, c'est parce qu'on a des intérêts en jeu, parce que ma grand-mère ne travaillait pas, c'est une vieille dame, elle

\footnotetext{
${ }^{20}$ Elle veut dire ses cousins.
} 
n'a pas d'assurance maladie, elle doit tout payer elle-même. Aujourd'hui quand elle tombe malade elle paie tout de sa poche, c'est une vieille dame qui n'a pas d'argent. Là où ça cloche dans la famille, c'est que quand elle tombe malade tout le monde doit payer. Tu paies, tu fais des efforts et tu n'es même pas récompensé. Donc il vaut mieux ne pas impliquer des relations d'argent, je trouve que ça empêche de se faire des amis. Je préfère me faire des amis sur Internet avec qui il n'y a pas de question d'intérêts, ce sont des amis à qui on peut tout dire." (Deng)

Ici notre internaute souligne le caractère désintéressé de l'amitié et plus particulièrement de l'amitié construite sur Internet. Du même coup, elle se plaint du caractère particulièrement intéressé des relations familiales, contredisant ainsi certaines idées reçues sur les relations sociales en Chine. La relation de dépendance familiale peut en effet être vécue de manière très pesante, surtout dans les jeunes générations, et en particulier dans sa dimension financière. Aussi l'émancipation proposée par Internet n'est-elle pas restreinte au plan des loisirs. Elle offre aussi une autre expérience des relations sociales, une expérience dans laquelle la relation de dépendance financière est (presque) absente.

Cela ne signifie pas pour autant que cette relation soit parfaitement gratuite. En effet, comme le don ${ }^{21}$, l'amitié ${ }^{22}$ peut remplir un certain nombre de fonctions qui ne ressortissent pas forcément de la subsistance matérielle mais n'en sont pas moins importantes. Il en va ainsi du soutien moral, des conseils pratiques, et tout simplement du sentiment d'appartenance à un groupe spécifique, qui peut valoriser symboliquement un individu. Il n'est donc pas à exclure que les internautes puissent trouver une forme d'intérêt dans les nouvelles relations sociales qu'ils construisent.

"Maintenant je suis maman, donc les gens avec qui je discute sont surtout autour de la question de la maternité, et puis on parle ensemble de la situation des enfants. [...] Quand j'étais enceinte, je suis allée sur les forums Sohu, là-dessus il y a un espace maternité, et à l'intérieur il y a aussi un forum, hein. Donc je suis allée dessus et j'ai lu des posts, et comme j'étais enceinte je me posais des questions sur la situation du bébé dans mon ventre. En cherchant dans le forum j'ai découvert qu'il y

\footnotetext{
${ }^{21}$ MAUSS Marcel. Essai sur le don : forme et raison de l'échange dans les sociétés archaïques, Paris: PUF, 2007.

${ }^{22}$ BIDART Claire. L'amitié. Un lien social, Paris: La Découverte, 1997.
} 
avait un groupe de mamans de l'hôpital Xuanwu. Ce post disait qu'il y avait un groupe de mamans qui avaient eu leur enfant à l'hôpital Xuanwu. Comme j'avais décidé d'accoucher à l'hôpital Xuanwu, ce post m'a beaucoup intéressée bien sûr, donc je suis entrée dans le forum, et je me suis rendue compte que la personne qui avait écrit le post avait eu son bébé à l'hôpital Xuanwu. Et puis j'ai aussi découvert un numéro de groupe QQ, alors je me suis inscrite dans le groupe QQ, c'est un groupe de personnes qui ont eu leur enfant à l'hôpital Xuanwu. Donc on a discuté ensemble, c'est à dire qu'on a commencé à discuter alors qu'on était enceintes. Et puis quand nous sommes allées à l'hôpital pour la visite médicale, on s'est dit mutuellement avec quel médecin on avait rendez-vous, et on est allées ensemble à la visite. C'est comme ça qu'on a fait connaissance à l'hôpital, quand on était enceintes." (Shao)

Ces groupes sont bien plus qu'une façon de partager des préoccupations communes et des conseils pratiques. De même que l'expression personnelle peut offrir un certain soulagement psychologique, les relations sociales bâties sur Internet peuvent servir à partager des informations dans des moments clés de la vie des internautes. Ces relations sont utilisées comme de véritables ressources, non seulement d'informations, mais aussi de soutien moral et de solidarité. Il arrive même que ces relations soient utilisées pour des objectifs pécuniaires.

"Sur Taobao il y a beaucoup de mamans qui ont ouvert leur boutique. Par exemple si je vais dans un magasin de vêtements, et que j'achète un vêtement à mon bébé, peut-être qu'ils me feront un discount de $20 \%$, mais si j'en achète une poignée, pour eux ça représente une poignée, si j'achète au prix de gros, peut-être trois ou quatre à la fois, si j'achète tout à la fois, peut-être qu'ils me feront $60 \%$ de réduction. [Donc je peux en acheter plusieurs, dont un seul pour mon bébé], et je peux passer par une boutique Taobao ou par mon groupe QQ ou par notre site "les enfants rouges" où il y a une plate-forme d'achat en groupe. Je revends mon surplus, comme ça j'achète des vêtements moins chers pour mon enfant, et je peux revendre le surplus un peu plus cher, comme ça c'est comme si le vêtement de mon enfant était gratuit." (Shao)

Le même type d'utilisation du Net, ainsi que du téléphone mobile, a été remarqué par Shang Dan dans son étude des groupes de propriétaires de voitures. Certains groupes s'organisent en ligne autour d'un modèle spécifique de voitures (Audi, Polo, etc...). Les membres se réunissent de temps en temps pour parader ensemble avec leurs véhicules. Internet sert donc, dans un premier temps, à publiciser l'appartenance à la catégorie favorisée 
des propriétaires de voitures, en d'autres termes à faire connaître le niveau de "distinction" de ces personnes. Au travers de ces activités, des communautés se forment, et les membres du groupe sont amenés à se rendre des services mutuellement, par exemple en indiquant des accidents de la route, ou en conseillant un bon fournisseur de pièces détachées. Chaque participant y trouve finalement un avantage matériel conséquent ${ }^{23}$.

Les liens faibles construits sur Internet sont donc aussi utilisés pour répondre à certains besoins pratiques directement liés au développement de la société de consommation et aux contraintes du mode de vie moderne. D'une part, l'adhésion à certains groupes signale un style de vie. Les goûts particuliers exprimés en ligne dénotent un niveau de vie, d'éducation, et de consommation qui sont propres à un certain groupe social et qui distinguent des gens de même "niveau". D'autre part, ces groupes peuvent être utilisés comme des ressources matérielles, qui favorisent la réalisation de ce style de vie en dépit des contraintes matérielles. Si le temps ou l'argent viennent à manquer, Internet permet d'y suppléer, en passant des commandes groupées et à distance, ou en s'informant sur les "bons plans" et autres combines. En ce sens, les nouveaux liens peuvent s'apparenter à des groupes d'intérêts informels et ad hoc, bien que cette expression ait au premier abord quelque chose d'un oxymore.

On retrouve là l'efficacité des liens faibles en termes de capital social identifiée par Granovetter $^{24}$ et que Cardon applique au cas des "coopérations faibles" ${ }^{25}$ que sont les sites dits "collaboratifs" comme Wikipedia ou FlickR ${ }^{26}$. Ces liens naissent essentiellement d'une forme d'individualisme, d'opportunisme, qui entraîne les internautes dans une dynamique de coopération.

${ }^{23}$ SHANG Dan, DOULET Jean-François et KEANE Michael. " Urban informatics in China, exploring the Emergence of the Chinese City 2.0", in FOTH Marcus (Ed.). Handbook of research on urban informatics: the practice and promise of the real-time city. Hershey, PA, 2009.

${ }^{24}$ GRANOVETTER Mark. "The strength of weak ties", The American Journal of Sociology, vol. 78, $\mathrm{n}^{\circ}$ 6, mai 1973, p. 1360-1380.

${ }^{25}$ AGUITON Christophe et CARDON Dominique. "The strength of weak cooperation : an attempt to understand the meaning of Web2.0 ", Communications \& Strategies, n ${ }^{\circ} 65,2007$, p. 51-65.

${ }^{26}$ PRIEUR Christophe, BEUSCART Jean-Samuel, CARDON Dominique, PONS Pascal et PISSARD Nicolas. "The strength of weak cooperation : A case study on Flickr", 2007, consulté le 16/10/2010 sur http://arxiv.org/ftp/arxiv/papers/0802/0802.2317.pdf. Par exemple, la volonté de se créer une notoriété peut conduire un photographe amateur à proposer ses œuvres en ligne sur FlickR, mais aussi à s'engager dans des activités sociales très intensives, comme écrire des commentaires des photographies des autres ou attribuer des mots-clés à de nombreuses images. 
Cardon $^{27}$ nous encourage cependant à ne pas concevoir cet individualisme comme une forme de dissolution postmoderne des relations sociales au sens de Bauman ${ }^{28}$. Il montre que de nombreuses et fructueuses coopérations peuvent naître de ces diverses formes d'expressivité individuelle. De même, on voit bien comment les nombreuses interactions entre les internautes chinois sont variées et plastiques, tout en donnant des fruits concrets. Certaines formes de coopération trouvent leurs racines dans des préoccupations qui peuvent être matérielles alors qu'elles sont vécues comme gratuites, et des liens se tissent au travers même de l'expression la plus intimiste.

Pour autant, il faut remarquer que ces liens alternatifs ne donnent pas souvent lieu à une consolidation. La pérennisation des liens, si elle peut exister, n'est pas un objectif, et bien au contraire, la fluidité est justement l'élément apprécié par les internautes. A la limite, un groupe ou un ensemble de liens ont d'autant plus de chances d'être pérennes que leur dissolution semble possible. Est-ce à dire que l'intérêt pour ce qui relève du collectif ne peut naître que dans la mesure où il est un outil de la construction de soi?

On le voit, les internautes chinois investissent avec enthousiasme tous les outils de présentation de soi proposés sur Internet. En racontant par exemple leur histoire personnelle sur leur blog, en se présentant au travers d'un avatar sur la messagerie instantanée QQ, et en commentant sur les forums les embûches qu'ils rencontrent dans la vie quotidienne, ils ont la possibilité d'affirmer leur identité et leurs goûts de manière extrêmement personnalisée. Sur la base de ces goûts, ils s'insèrent dans des réseaux sociaux choisis, beaucoup plus souples que leurs attaches familiales ou professionnelles, et dans lesquels l'individu est central. Désormais, l'individu devient un acteur central et narcissique, au sein d'une société qui valorise la singularité. Ce phénomène est l'objet de critiques en Chine et parmi les internautes euxmêmes. Certains refusent d'ouvrir leur blog ou d'y écrire des choses trop personnelles par exemple, mais tous confirment être témoins de son expansion. L'injonction sociale est maintenant de se distinguer des autres, de montrer une personnalité originale, et ainsi de se

${ }^{27}$ CARDON Dominique. "Présentation", dossier "Réseaux sociaux de l'Internet", Réseaux, n¹52, 2008, p.7-17.

${ }^{28}$ BAUMAN Zygmunt. Liquid Modernity, Cambridge: Polity, 2000. 
ménager une place de choix dans la société. Il faut mesurer le renversement complet de l'économie morale que cela représente dans le contexte de la Chine. Il y a à peine trente ans, les discours publics valorisaient essentiellement la conformité culturelle et politique. Sur ce plan, la Chine semblerait rejoindre les pays occidentaux sur la voie d'une forme de postmodernisme.

Il ne s'agit pas pour autant d'une forme d"'émancipation individuelle" sans contrepartie. En effet il faut noter que ce processus est le pendant d'une responsabilisation des individus dans un système de plus en plus compétitif et qui crée des tensions. En participant à la diffusion d'informations sur les styles de vie considérés comme modernes, Internet conduit les individus à s'assujettir à de nouvelles normes très contraignantes ${ }^{29}$. Les nombreux questionnements partagés en ligne montrent enfin que les internautes se saisissent de ces normes avec plus ou moins de recul. Parfois ils les embrassent, d'autres fois ils les rejettent, les détournent ou les bricolent ${ }^{30}$, mais ils ne peuvent les ignorer.

Cette forme singulière d'individualisme se déploie dans tous les aspects des usages des internautes, et tout particulièrement dans leur rapport à l'information et à la politique.

${ }^{29}$ FOUCAULT Michel. Histoire de la sexualité. 1 La volonté de savoir, Paris: Gallimard, 1976.

${ }^{30}$ CERTEAU Michel. L'invention du quotidien 1. Arts de faire, Paris: Gallimard, 1990. 


\section{Chapitre III - L'évaporation de la politique}

Dans le portrait que Yang dresse de la jeune génération ${ }^{1}$, les "post 80 " se caractérisent par leur goût de la personnalisation, leur faculté à exprimer une opinion personnelle, leur aisance dans la création de nouveaux liens sociaux, mais aussi par leur désintérêt pour la politique et les sujets de société en général. Toute mention des "souffrances du passé", par exemple, est présentée comme rétrograde, et le présent, incarné par le plaisir, la mode et la superficialité, représente une valeur centrale.

Cette manière d'envisager la politique est plutôt contre-intuitive pour les lecteurs des théories normatives de l'espace public ${ }^{2}$. Celles-ci suggèrent en effet que l'émancipation individuelle, ainsi que l'établissement de liens électifs, peut conduire à un investissement plus fort dans la sphère publique, et a fortiori dans des engagements de nature plus politique. Les internautes chinois ne sont pas complètement isolés et ils n'ont peut-être jamais eu autant de faculté à s'associer sur la base d'intérêts individuels et collectifs. Pourtant, le portrait tiré ici semblerait plus proche d'une certaine vision de la postmodernité, voire de l'hypermodernité ${ }^{3}$, avec des individus complètement centrés sur eux-mêmes, et a fortiori coincés dans une attitude anti-politique. Le Bart remarque que "l'individualisation des trajectoires met à mal l'idée d'un engagement unique et définitif qui refléterait une identité elle-même solide et unifiée. En termes hirschmaniens, l'exit l'emporte sur la prise de parole et a fortiori sur la loyauté." ${ }^{4}$ Comme le montre Putnam aux Etats-Unis, des engagements politiques plus fragiles et plus volatiles peuvent représenter un affaiblissement relatif du capital social, et surtout entraîner une certaine désaffection pour l'action politique proprement dite ${ }^{5}$. En d'autres termes, les individus hyper-modernes, à cause de leur identité plurielle, deviendraient indifférents politiquement. Qu'en est-il ici?

\footnotetext{
${ }^{1}$ Voir chapitre précédent.

${ }^{2}$ Notamment avec l'héritage de HABERMAS Jürgen. L'espace public, Paris, Payot, 1992.

${ }^{3}$ LIPOVETSKY Gilles. L'ère du vide : essais sur l'individualisme contemporain, Paris : Gallimard, 1983.

${ }^{4}$ LE BART Christian. L'individualisation, Paris: Presses de Sciences-po, 2008, p. 183.

${ }^{5}$ PUTNAM Robert. "Bowling alone : America's declining social capital", Journal of Democracy, vol. $6, \mathrm{n}^{\circ} 1,1995$, p. $65-78$.
} 
La spécificité de ce rapport aux enjeux de société peut se lire dans l'intérêt déclaré des internautes pour certains sujets d'actualité plutôt que d'autres. C'est pourquoi je les ai systématiquement interrogés sur leurs habitudes de consultation des informations, non seulement en ligne, mais également au travers de la presse écrite et de la télévision ${ }^{6}$. Dans son rapport de 2007, Guo Liang révèle que la grande majorité (jusqu'à 85\%) des internautes urbains lisent des informations en ligne ${ }^{7}$. J'ai mentionné précédemment les nombreux avantages que les internautes trouvent à s'informer sur Internet plutôt qu'à la télévision ou dans les journaux, dont les horaires ou les contenus sont plus restreints. Il ne faut pas supposer que les internautes qui déclarent un intérêt particulier pour un sujet passent effectivement beaucoup de temps à s'informer sur ce sujet. Les usages réels peuvent différer largement des usages déclarés. De plus, il ne faut pas surestimer l'importance prise par ces informations dans la vie quotidienne des internautes, ni les efforts qu'ils déploient pour les obtenir. Le plus souvent, ils se contentent de jeter un coup d'œil distrait à la page d'accueil de leur portail favori, et éventuellement de cliquer sur un ou deux titres qui leur auront semblé plus séduisants que les autres.

"Je fais un tour à partir de la page d'accueil, il y a toutes sortes d'informations." (Wang)

"En fait après avoir cliqué, je parcours très rapidement le site. Je lis beaucoup de titres importants, mais le plus souvent ça ne me marque pas très fortement. Je ne fais que surfer." (Li)

Il est vrai que les internautes sont plus souvent intéressés par les informations sur les célébrités, les insolites, la mode etc... J'écarterai pourtant de mon analyse ces catégories que je range dans le domaine du divertissement, pour me pencher sur ce qui concerne plus précisément l'actualité: locale, nationale, mondiale. Encore une fois, ce qui est important ici n'est pas de savoir à quel point les internautes lisent vraiment l'actualité, mais comment ils la perçoivent et quel est l'intérêt qu'ils lui manifestent dans leur discours. La plupart des

${ }^{6}$ Rappelons que cette enquete est biaisée volontairement par le mode de recrutement: seules les personnes qui déclarent "lire les informations en ligne" ont été retenues pour l'enquête. C'est donc la dispersion entre les sujets, et non la lecture en soi des informations, qui pourra être analysée ici.

${ }^{7}$ GUO Liang. Surveying Internet usage and its impact in seven Chinese cities, Markle Foundation, 2007 , p. $34.85 \%$ des internautes lisent souvent des informations locales, $84 \%$ lisent des informations nationales, et $71 \%$ lisent des informations internationales. 
internautes affirment bel et bien se désintéresser de la politique, et se concentrer sur des enjeux liés à leur vie quotidienne. Cependant sur d'autres questions, ils manifestent une grande sensibilité pour des enjeux à teneur politique, par exemple dans leur environnement proche ou sur des questions d'honneur national. La politique nationale, en revanche, est affublée de divers qualificatifs dépréciateurs. La grande majorité des internautes s'en détourne ou l'évite, et l'intérêt pour la politique finit par "s'évaporer"8.

\section{1 - La politique confisquée}

\section{Le quotidien, lieu de naissance de l'intérêt politique}

$\mathrm{Au}$ premier abord, les internautes interrogés présentent une vision sexuée de leurs centres d'intérêts. Les hommes s'intéresseraient plus souvent à la politique, aux relations internationales ou encore aux affaires militaires, alors que les femmes seraient plus préoccupées par des choses superficielles comme la beauté ou la mode.

"Depuis que je suis petite, je n'ai jamais aimé lire les infos, je ne suis pas quelqu'un qui s'intéresse beaucoup aux affaires nationales. Je trouve qu'en tant que femme, je ne m'intéresse pas trop à ces choses-là. Mon petit ami n'est pas comme ça, lui il aime bien ces choses-là. Quand il y a un mouvement à Pékin, ou bien un match de foot, ou de basket, il aime bien tout ça, mais pas moi.

- Est-ce que le foot est une affaire nationale?

- $\mathrm{Ca}$, je n'y comprends rien, franchement je ne comprends rien au foot, ni au basket, et des fois quand mon copain regarde le foot, moi je surfe sur Internet.

- Tu lis les journaux papier?

- Oui, mais je lis des journaux comme Xiandai Nü Bao ["Femme moderne"], des trucs sur les sentiments, parce que les femmes aiment bien ce genre de choses. Sinon je lis aussi un peu "Jinghua Shibao", et "Xinjing Bao", mais très rarement." (Ma)

${ }^{8}$ ELIASOPH Nina. Avoiding politics. How Americans produce apathy in everyday life, Cambridge University Press, 1998. 
Cette différence tient lieu d'explication pour beaucoup d'internautes (on fait ses choix "en tant que femme"). Mettre leurs goûts personnels sur le compte de leur position sociale permet de contourner la demande de justification, à laquelle il est parfois difficile de répondre. De fait, il est vrai qu'un nombre plus important de jeunes femmes déclarent se désintéresser des informations nationales ou internationales.

"Les hommes s'intéressent plus aux informations que les femmes, ils s'intéressent plus aux affaires importantes du pays, et puis au sport, aux voitures et ce genre de choses. Les femmes s'intéressent à la mode, il y a des sites spécialement pour ça, sur la mode, les sujets sont plus compliqués. Et puis il y a aussi des sites sur les vêtements, la beauté, et la cuisine. La culture culinaire chinoise est très prestigieuse. Et puis il y a des sites sur la vie privée, les femmes s'y intéressent un peu plus. Et puis il y a aussi la beauté et le maquillage, ou la santé, ce genre de choses. Et puis les produits numériques, ça, les hommes et les femmes s’y intéressent autant." (Zhao)

Cela influence aussi le contenu des conversations que les internautes tiennent entre eux.

"En général ça dépend de la relation entre les deux personnes, et puis les goûts des deux personnes influencent les sujets de conversation, par exemple si la personne aime bien quelque chose et moi aussi, on en discute. Et puis si c'est une fille, en général avec les filles c'est très rare que je discute en détails des informations internationales, mais ça m'arrive avec les garçons." (Sun)

Le clivage sexué semble donc très structurant au premier abord. Pourtant, un questionnaire plus poussé montre que dans le fond, les deux profils se rejoignent plus souvent qu'il n'y paraît. En effet, les hommes comme les femmes ont pour motivation première d'éclairer leur vie quotidienne en s'informant. Tous les internautes interrogés déclarent s'intéresser en priorité aux thèmes qui les concernent personnellement. Ceux-ci, en effet, peuvent être différents entre les hommes et les femmes, comme ils sont différents aussi entre les générations, comme je l'ai déjà montré. Cependant le lien avec la vie personnelle est un élément structurant des choix de tous les internautes. Tous les types d'informations peuvent attirer l'attention des internautes à partir du moment où ils perçoivent le lien avec leur vie personnelle ou leur environnement direct. 
"Mais sinon, on s'intéresse aux sujets de société qui nous concernent directement, c'est peut-être des choses qui nous sont déjà arrivées, et du coup on lit ces informations-là." (Wang)

On s'intéresse par exemple aux animaux domestiques, qui font régulièrement l'objet de reportages à cause de politiques publiques restrictives (restriction de la taille des chiens dans Pékin, rafles de chiens errants, lutte contre la rage). Or beaucoup de personnes se sentent concernées car elles ont des animaux chez elles.

"Je regarde surtout des sites sur les animaux familiers, parce que dans ma famille on a un petit chien, un petit Yorkshire, et on aime bien les animaux. Donc je lis des choses là-dessus sur Internet. En fait je suis assez directe sur Internet, quand je veux lire quelque chose je le lis. En-dehors de ça, je lis aussi des choses sans but particulier, mais c'est surtout quand je n'ai pas grand chose à faire." (Du)

Certains sujets à portée plus générale peuvent attirer l'attention, si leur impact sur la vie quotidienne est clairement perçu. Les questions économiques tiennent ainsi une place particulière, car elles semblent toucher plus directement les intérêts matériels des internautes. C'est surtout le cas de ceux qui ont investi de l'argent en bourse.

"Surtout les choses qui sont très proches de ma vie. Par exemple récemment il y avait la fièvre de la bourse, donc je lisais souvent des informations sur la bourse, sur les mouvements, le développement du marché, et le comportement des finances chinoises. Et puis sur les forums il y a des gens qui racontent que les banques augmentent les taux d'intérêts pendant le week-end, qui discutent des hausses et des baisses de la bourse. Je peux poser des questions, et puis il y a des gens qui répondent à mes questions. Est-ce que la bourse peut s'effondrer, est-ce que je dois investir ou me retirer, ce genre de questions." (Liang)

J'ai pu constater cette préoccupation très directement, puisque l'internaute consultait les cours de la bourse sur son téléphone en même temps qu'il me parlait, pendant toute la durée de l'entretien. La bourse est un sujet partagé aussi par ceux qui n'ont pas d'actions eux-mêmes, mais qui peuvent penser que l'évolution des cours a une influence directe sur les prix des produits de consommation. Il faut rappeler que la période pendant laquelle les entretiens ont été menés a vu une augmentation spectaculaire des prix des produits alimentaires de base, en particulier des céréales et de la viande de porc. 
Les questions financières sont très souvent citées, par exemple avec le taux de change du RMB.

"Les informations sur la société, bof.

- Pour toi, c'est quoi les informations sur la société?

- Les informations sur la société sont celles qui influencent l'intérêt public ou les relations entre les pays.

- Pourquoi est-ce que tu trouves ça pas terrible?

- Parce que ce qui m'intéresse, ce sont les informations qui sont directement liées à ma vie. Des fois je m'intéresse par exemple au cours du RMB, ce genre de choses." (Guo)

Le logement est aussi en bonne place parmi les préoccupations matérielles qui incitent à lire la presse.

[Sur les Jeux Olympiques et les sujets de société]. "J'y fais attention, parce que dès qu'on va sur Internet c'est inondé d'informations là-dessus, donc on ne peut pas faire autrement que d'y faire attention, mais on ne peut pas dire non plus que ça m'intéresse beaucoup.

- Il y a eu beaucoup de destructions dans la ville pour les Jeux Olympiques, surtout l'année dernière.

- Oui.

- Ca t'a intéressé, ce sujet-là?

- Beaucoup, j'ai lu des choses spécialement là-dessus, et j'ai même acheté des livres à propos des lois sur les destructions. Je voulais comprendre la loi et les règles là-dessus. Et puis dans la vie de tous les jours, tout le monde en parle. On en discute sur le moment, par exemple maintenant on discute des Jeux Olympiques, mais à ce moment-là on parlait des destructions de logements, et des réformes." (Gao)

Les expropriations sont particulièrement présentes à l'esprit de la plupart des citoyens de Pékin, qui connaissent tous des habitants des vieux quartiers rasés avant les Jeux Olympiques, ou qui ont parfois été expropriés eux-mêmes. Cette thématique a une résonance très forte pour plusieurs internautes interrogés. 
"Sur Sina, en général je jette juste un coup d'œil aux informations, le reste je ne le regarde pas. Je regarde des choses amusantes, et puis quand je voulais acheter un appartement l'année dernière, je lisais des choses sur la propriété du logement. Donc je choisis ce que je lis en fonction de mes besoins du moment.

- Mais ce que tu préfères, en fait c'est le divertissement?

- Non, pas forcément, je m'intéresse surtout aux informations qui sont en rapport avec ma vie.

- Comme quoi par exemple?

- Comme des politiques du gouvernement, par exemple pour acheter un appartement, le gouvernement a augmenté les taxes, ça m'intéresse. Par exemple il y a eu le sommet Chine-Afrique ces derniers jours, et la circulation était très perturbée, ça aussi ça m'intéresse. Surtout, je lis avec attention les informations qui sont intimement liées à ma vie quotidienne." (Guo)

Les informations qui intéressent les internautes sont donc presque toujours en lien direct avec leur vie personnelle. Ces centres d'intérêts sont variables entre les différentes personnes, mais aussi variables dans le temps pour chaque personne. Chacune de ces thématiques est d'un intérêt direct pour certains internautes, à un moment donné de leur vie, mais elles ne suscitent pas forcément une attention pérenne. Au moment d'investir en bourse, ils s'informent sur les modalités de taxation et sur l'état de l'économie. Au moment d'acheter un logement, ils sont plus attentifs aux annonces sur les expropriations. Au moment de prendre le volant, ils sont plus concernés par les entraves à la circulation provoquées par une conférence internationale. Ces derniers exemples montrent que ce critère peut conduire à s'informer sur des questions très politiques, même si c'est sous l'angle de leur impact sur la vie personnelle des internautes. Pourtant, ceux-ci affirment unanimement ne pas s'intéresser à la politique en elle-même.

"Par exemple les accidents de voiture, et puis les déclarations sur l'économie du pays, et puis les informations sur la vie quotidienne qui me correspondent, comme la hausse des taux d'intérêts, les prix des logements, ou la médecine.

- Tout ça, c'est des informations sur la société?

- Oui, on est obligés de les lire. 
- Pourquoi obligés?

- On doit s'intéresser à ce qui touche notre propre intérêt.

[...] - Tu as dit que tu lisais des informations sur la société, et sur les loisirs. Et sur la politique?

- La politique ne m'intéresse pas.

- Pourquoi?

- Parce que je trouve que la politique c'est trop éloigné de moi." (Chu)

En somme, les internautes ont des centres d'intérêts relativement égocentrés, mais aussi très diversifiés, et qui peuvent changer au fur et à mesure des étapes de leur vie.

"Donc on parle seulement de divertissement ou de sport?

- Oui, les films, la musique, les histoires de cœur... Sinon les gens ne discutent pas trop de tout ça. Ou alors on parle des vacances.

- Et des sujets comme les taux d'intérêts qui augmentent?

- Par exemple les taxis on en a parlé, car il y a une influence très directe sur notre vie. Mais les questions de stationnement on n'en discute pas tant qu'on n'a pas de voiture à nous." (Ren)

Le désintérêt affirmé des internautes pour les questions politiques se traduit donc souvent par une certaine volatilité de leur attention, au gré de leurs changements de situations, mais ce n'est pas un désintérêt absolu.

"Comment dire, quand j'étais au lycée je réfléchissais beaucoup à ces questions, mais maintenant je travaille, je n'ai plus assez de temps pour ces questions, parce que ça ne concerne déjà plus ma génération. Ca m'intéresse dans le sens où je peux en parler comme ça, j'ai mon avis, mais si tu me demandes d'aller exprès là-dessus, c'est impossible. Parce que j'ai mis toute mon énergie dans mon travail, c'est comme ça." (Ye)

Les internautes peuvent ainsi être amenés à s'informer sur de nombreuses questions de société, qu'ils appellent alternativement des questions économiques, juridiques, ou sociales, faisant en sorte de les distinguer de ce qu'ils appellent la politique. Cela peut sembler paradoxal car chacune de ces questions a indéniablement des dimensions politiques, au sens 
où elles concernent la définition de l'intérêt général. Or la politique, au contraire, est unanimement désignée comme un domaine qui ne les concerne pas, un domaine inintéressant. Cela concerne même les internautes les plus investis dans des causes diverses, car ils ne considèrent pas ces actions comme politiques. Que recouvre donc cette "politique" dont les internautes se détournent? Comment la définissent-ils?

\section{Une séparation virtuelle entre le social et le politique}

La politique est toujours désignée comme éloignée des individus, trop compliquée, ou pas intéressante. Elle passe toujours en dernier dans les centres d'intérêts des internautes, mais elle peut désigner des objets très divers.

"Je lis tous les jours les news sur Internet, maintenant je m'informe essentiellement sur Internet, quand il y a un tremblement de terre quelque part, ou une hausse du prix d'un produit, ou bien si une conférence importante s'ouvre quelque part, ou bien s'il y a un document important. Et puis je lis aussi des informations sur le sport ou le divertissement.

- Ce que tu préfères lire, c'est quoi, c'est le divertissement?

- Le sport, et aussi les technologies informatiques.

- Et quoi d'autre?

- En troisième, il y a les films, et encore ensuite il y a la société.

- La société, c'est quoi?

- Les informations nationales et internationales, des informations sur la société un peu partout, quand il se passe quelque chose quelque part, et y compris le sport, les affaires militaires...[...]

- Pourquoi est-ce que tu mets la société en troisième position?

- Parce que je ne fais pas des études de sociologie, je ne m'intéresse pas beaucoup à ces choses-là. Je trouve que je suis quelqu'un de naturel à la maison, et je suis quelqu'un de social quand je travaille, mais je n'ai pas beaucoup de liens avec la société.

- Pourquoi ça, ça t'ennuie? 
- Il y a un poème qui dit: "je me réfugie dans un petit grenier qui devient mon monde" ("duo jin xiao lou cheng yi tong"), tu le connais?

- Non.

- C'est de Lu Xun, il s'enferme dans ses terres et n'a plus aucun contact avec la société. Parce que je ne m'intéresse pas aux questions de société. Ou plutôt je ne m'intéresse pas beaucoup aux questions de société très courantes, comme la politique, ou l'économie. La politique, l'économie, les affaires militaires, les lois sont des domaines qui ne m'intéressent vraiment pas du tout. Je donne plus d'importance aux domaines de la culture et du sport. Il y a des choses trop compliquées, qui ne m'intéressent pas beaucoup, comme la politique, l'économie, et les lois, c'est trop compliqué." (Zhou)

Cet internaute commence par désigner ce qu'il n'aime pas par le terme "société". Il dit même qu'il ne se sent pas fondamentalement solidaire de la société en-dehors de ses obligations de travail. Pourtant il s'intéresse aux hausses de prix ou aux catastrophes naturelles. Le domaine qu'il désigne comme inintéressant est en fait restreint au domaine de la politique "politicienne", de l'économie, et des armées. Ce sont en somme des domaines dont il pense qu'ils ne le touchent pas personnellement. Il en est de même chez cet autre internaute.

"Ca ne m'intéresse pas trop, par exemple les aspects politiques ou militaires ne m'intéressent pas trop. La politique c'est vraiment le truc qui ne m'intéresse pas du tout.

- Pourquoi?

- Ca n'arrive pas à soulever mon intérêt (xingqu aihao).

- Quelles sont les informations qui ne t'intéressent pas exactement? Il y a quoi dans cette catégorie?

- Les affaires militaires, la politique, et les guerres, les batailles ne m'intéressent pas. Je m'intéresse plutôt aux choses plutôt sensibles (ganxing), comme la vie quotidienne, les sentiments, le shopping entre autres, ou un peu de culture générale, des exercices d'adresse, ou la littérature." (Gao)

Parfois, le domaine de la politique est étendu au-delà des questions militaires, et s'étend à la diplomatie. Dans ce cas aussi, c'est un domaine qui ne concerne pas les individus. 
"Donc tu veux dire que la plupart des gens s'intéressent surtout au shopping, à des choses liées à la vie quotidienne?

- Oui, des choses liées à notre propre vie.

- Et les informations nationales, ou internationales?

- Ces choses-là, beaucoup moins, il n'y a presque rien à en dire, parce que je ne comprends pas grand chose à tout ça, surtout les relations internationales. Par exemple il va y avoir les élections présidentielles aux Etats-Unis, en 2008, si tu me demandes quel est le rapport avec moi, eh bien aucun, en quoi ça me concernerait les élections aux Etats-Unis? Donc je ne m'y intéresse pas du tout, et je n'ai pas d'opinion là-dessus. Par exemple si le premier ministre chinois va rendre visite à quelqu'un aux Etats-Unis, et qu'on écrit 'aujourd'hui il a vu untel, et demain il verra untel', je jette un coup d'œil et puis voilà, je lis le titre principal et puis voilà, mais je ne lis pas le contenu." (Huang)

L'aspect le plus stigmatisé est certainement la profusion de réunions qui se tiennent au Parti et qui laissent tout le monde dans l'indifférence. Le choix des dirigeants et les grandes déclarations politiques sont aussi écartés de manière unanime.

Au-delà de la diversité des objets placés par les internautes dans la catégorie "politique", il se dessine en fait une définition générale commune. C'est invariablement le domaine des décisions qui sont prises loin des individus, et qui selon eux ont un impact très faible sur leur vie quotidienne. Il peut y avoir des divergences de vues sur ce qui relève du domaine politique, pour un certain nombre de sujets qui concernent l'économie ou la société. Selon que ces sujets touchent plus ou moins personnellement un internaute, sa vision sera plus ou moins nuancée. De nombreuses questions qui ont un rapport de près ou de loin avec la politique peuvent ainsi intéresser les individus parce qu'elles se trouvent dans un domaine qui les touche au quotidien. Quelques internautes, notamment ceux qui sont porteurs d'une revendication particulière à ce moment-là, font preuve d'une certaine difficulté à délimiter le domaine de la politique, car il se confond bien souvent avec des questions qui les touchent de plus près. C'est le cas de Zhang qui, nous le verrons en dernière partie, milite pour la défense des chiens dans la ville de Pékin.

"Il faut distinguer plusieurs choses, c'est comme ce sentiment que je viens de t'expliquer, je m'intéresse beaucoup aux infos, parce que les infos, surtout les infos 
nationales, qui sont plus en rapport avec moi, c'est sûrement ce qui m'intéresse le plus sérieusement. Mais je ne m'intéresse vraiment pas beaucoup à la politique, parce que cela n'a pas de rapport avec ma vie, mais souvent on ne peut pas séparer les deux choses très clairement, au fond qu'est-ce que c'est que la politique, qu'est-ce que la vie, en fait très souvent ces deux choses vont ensemble, on ne peut pas les séparer complètement. Donc pour en revenir à mon point de départ, est-ce que je m'intéresse aux infos, oui, j'y fais attention." (Zhang)

Ce n'est que dans les cas où les internautes identifient clairement les liens directs entre la politique et les questions de société qui les concernent, que les internautes déclarent s'y intéresser. C'est une position extrêmement minoritaire, partagée par deux ou trois internautes tout au plus.

"Des questions politiques en Chine, je ne m'y suis intéressé que récemment, parce que les questions politiques et les questions sociales en fait c'est la même chose, donc je m'y intéresse assez. Par exemple en Chine il y a un slogan en ce moment qui dit rien de moins que 'construire une nouvelle campagne socialiste'. Ca m'intéresse beaucoup, mais je n'ai pas de point de vue spécifique là-dessus, je ne peux que me contenter de lire les avis des spécialistes là-dessus.

- Qu'est-ce que tu trouves d'intéressant dans cette question?

- Je trouve que c'est très important pour la Chine, et parce que j'ai passé du temps à la campagne quand j'étais petit." (Jiang)

L'intérêt potentiel des internautes pour des sujets d'intérêt général, qui ressort assez clairement de leurs préoccupations quotidiennes, semble donc se heurter à l'idée d'une séparation entre les problème de société et le monde de la politique, idée qui est partagée par une grande majorité des internautes interrogés. Cette séparation est essentiellement virtuelle, ce qui explique que les internautes éprouvent des difficultés certaines à la cerner. Pourtant, rares sont ceux qui montrent explicitement les liens entre les questions qui les concernent personnellement et les décisions politiques auxquelles elles sont liées. Pour tous les autres, la séparation fictive entre le domaine du social et du politique relève presque d'une confiscation de la politique au profit d'un monde séparé, hors du domaine des gens ordinaires. 


\section{La politique hors du domaine des gens ordinaires}

En effet, ce qui ressort véritablement des définitions variables du domaine politique est l'idée que la politique politicienne relève presque d'un autre monde, complètement étranger aux individus qui se perçoivent comme des gens ordinaires.

"Et puis franchement, les questions vraiment majeures n'ont pas beaucoup d'influence sur les gens de notre couche [sociale]. On est au courant des réunions qui se tiennent, des changements de dirigeants, les gens en parlent, mais il suffit de le savoir et puis voilà. Mais pour notre vie, peu importe qui dirige, je vis comme je dois vivre.

- Alors quel est l'intérêt de savoir?

- Le savoir, ça sert seulement à améliorer ses connaissances, et après on peut en discuter avec les amis. Il y a aussi des choses qui ont un impact sur nous, et à ce moment-là on en discute avec d'autres personnes, et si on n'est pas au courant les gens nous traitent d'imbéciles.

- Quel type d'informations a un impact sur les gens ordinaires?

- La hausse des prix, je crois, et aussi les prises de position importantes des pays, et sinon ce sont des choses importantes pour le monde." (Xie)

Il faut souligner le fait que cet internaute mentionne sa "couche" sociale. La plupart des internautes font également référence aux "gens ordinaires" (laobaixing), terme qui s'utilise le plus souvent par opposition aux postes officiels, au Parti, dans l'administration ou dans des grandes entreprises. Le laobaixing, parce qu'il ne tient pas une fonction particulière, n'est pas concerné par les questions politiques.

"Je ne m'intéresse pas à la politique.

- Pourquoi?

- Parce que je trouve que c'est vraiment trop éloigné de moi.

- Pourquoi est-ce que tu trouves que c'est éloigné de toi?

- La politique, c'est difficile à cerner (zhuomo), et puis ce n'est pas bien de se rapprocher de trop de la politique, donc je n'y fais pas trop attention.

- Cela n'a pas d'impact sur ta vie? 
- Non. $[\ldots]$

- Pour toi, c'est quoi la politique en fait?

- C'est par exemple l'élection des dirigeants, les promotions, les dirigeants qui vont et viennent au pouvoir, et aussi quelques changements et adaptations concernant la direction générale du pays, et aussi des mouvements et actions du gouvernement, tout ça c'est de l'information politique. L'information sur la société, ça concerne les gens ordinaires (laobaixing), l'information économique c'est sur l'argent, l'information politique concerne les dirigeants." (Chu)

Dans cette phrase se dessine donc une frontière entre le domaine des laobaixing, les gens du peuple, pris dans leur vie quotidienne, et la vie des dirigeants, pris dans leur rôle de gestionnaires du pays. Les internautes déclarent se situer très loin de la politique. Selon eux, elle n'a qu'un impact minime sur leur vie et ils n'ont pas d'influence possible sur elle. Il s'agit véritablement pour eux de deux mondes séparés, qui n'ont que très peu d'influence l'un sur l'autre. A la manière des paysans italiens de Carlo Levi ${ }^{9}$, les internautes chinois déclarent que la politique est loin d'eux et ne les atteint pas.

"Au Comité Central encore je sais un peu, qui change de poste et tout, mais au niveau de la province, je n'en sais rien, à part s'il y avait un cas de corruption ou un pot de vin énorme, là par contre je m'en rappellerais très bien. Par exemple les inondations, ce genre d'informations, quelles qu'elles soient, ça me marquerait. Mais d'autres choses, par exemple qui va faire un discours, ça, ça ne m'intéresse pas. Mais par exemple les inondations qui ont eu lieu cet été, les catastrophes naturelles, ou bien ce genre d'infos nationales, de politique intérieure, on s'y intéresse forcément. Comme les conséquences des inondations cette année en Chine, c'est la première fois qu'il y en a autant en une année, ça je m'y intéresse.

- Je ne comprends pas encore bien ce qui est intéressant pour toi et ce qui ne l'est pas?

- La politique en fait ce n'est pas intéressant du tout. Si tu n'es pas fonctionnaire, sauf si tu es un businessman très riche et que tu veux utiliser la politique pour faire

${ }^{9}$ Dans un compte-rendu de son exil forcé dans le Sud de l'Italie, cet opposant au régime fasciste décrivait une population très pauvre et éloignée de tout, qui vivait les décisions politiques comme une sorte de fatalité contre laquelle nul ne pouvait rien. LEVI Carlo. Le Christ s'est arrêté à Eboli, Paris: Gallimard, 1948. 
fortune, mais si tu n'es qu'un petit employé, ça a très, très peu d'influence sur ta vie quotidienne. Qui va être président de ci ou de ça a un impact vraiment minuscule pour toi." (Ren)

Le domaine de la politique semble donc restreint aux arcanes du pouvoir et au domaine des riches et des puissants, qui s'en servent ici à des fins d'enrichissement personnel. Pour tous les autres, qui ne sont "que des individus", la politique est sans intérêt.

"J'aime bien regarder les infos à la télévision, par exemple les accidents qui arrivent aux gens ordinaires, je trouve ça intéressant. Mais les informations centrales, les Informations de Pékin, les informations de chaque province, je regarde très peu. Je ne regarde pas d'informations très officielles.

- Pourquoi est-ce que tu ne regardes pas les informations centrales?

- La raison principale, c'est que ça ne m'intéresse pas. Apparemment il y a encore eu je ne sais quelle réunion du gouvernement, ça passe dans l'actualité, mais ça ne m'intéresse pas, pas du tout.

- Est-ce que c'est important?

- C'est peut-être important pour le pays, mais je ne suis qu'une personne, en ce qui me concerne ce n'est certainement pas important. Mon cadre de vie est très petit, c'est une vie très linéaire, donc tout ça n'est pas très important pour moi." (Luo)

Non seulement la politique est présentée comme appartenant à un monde séparé, comme si elle était confisquée aux individus ordinaires, mais elle est souvent affublée de qualificatifs très péjoratifs, qui semblent associés, eux aussi, à ce monde des puissants et des dirigeants.

\section{2 - La politique dévoyée}

\section{Un archaïsme hérité du maoïsme}

J'ai déjà noté dans des recherches précédentes le fait que les étudiants des universités montraient souvent un désintérêt pour la politique qui pouvait aller jusqu'au dégoût ${ }^{10}$. Les

${ }^{10}$ ARSENE Séverine. L'enseignement idéologique dans les universités chinoises, mémoire de Master Recherche, Paris: Institut d'études politiques, 2005. 
cours de politiques auxquels ils sont astreints tout au long de leur scolarité contribuent à leur faire associer implicitement le terme de "politique" avec les slogans des grandes campagnes qui ont marqué l'histoire récente du pays. Cette tendance se retrouve dans un nombre non négligeable des entretiens de l'enquête qui nous occupe. L'association de la politique et des "mouvements politiques" conduit certains internautes à considérer que la politique appartient aux héritages archaïques de la Chine maoïste.

"Parce qu'on est jeunes, on aime bien le divertissement, mais les gens de quarante, cinquante ans s'intéressent plus aux informations nationales, aux tendances du pays, ça les intéresse.

- Pourquoi est-ce qu'ils aiment ces informations?

- Parce qu'ils ont quarante, cinquante ans. Ils s'intéressent à la politique, mais pas les jeunes, certainement parce que c'est la génération de la guerre.

- La génération de la guerre?

- La génération d'avant, parce que maintenant, les gens qui ont cinquante, soixante ans, sont de cette génération.

- Quelle guerre?

- Eh bien, il y a eu des révolutions et des trucs comme ça, il y a très, très longtemps. A cause de ça ils s'intéressent aux politiques du pays, aux tendances du pays.

- Et pas les jeunes?

- Il y en a que ça intéresse, mais pas moi.

- Quelle est la différence entre toi et ces jeunes?

- Je pense que la plupart des jeunes ne s'y intéressent pas, aujourd'hui les jeunes s'intéressent surtout au divertissement, il y en a très peu qui s'intéressent à des infos nationales.

- Pourquoi?

- Parmi les gens que je connais, tout le monde s'intéresse au divertissement, et très peu aux affaires nationales. Peut-être que c'est parce qu'ils n'ont pas vécu les agitations du pays, ça ne les touche pas." (Ma) 
Les jeunes aussi sont confrontés à des questions politiques, qui font malgré tout partie de leur environnement, mais au contraire de leurs aînés, ils semblent considérer ces contacts avec la politique comme une obligation quelque peu ennuyeuse. Pour cet internaute, prêter attention aux enjeux du monde et du pays est l'apanage des gens des "couches élevées".

"La société d'aujourd'hui se mondialise, il y a le développement économique, donc on ne peut pas s'intéresser seulement à la Chine, il faut aussi regarder à l'extérieur, et prêter attention à des choses internationales, y compris le développement économique de certains pays étrangers, et y compris des événements internationaux etc. Je m'intéresse à tout ça. Il y a la question nucléaire nord-coréenne, la question nucléaire iranienne, tout ça.

- Est-ce que tu trouves que ces choses-là ont un rapport avec ta vie?

- Pas pour ma vie de tous les jours, mais c'est une question de vision, je ne sais pas comment exprimer ça. Parce que même le développement économique, et la question de l'environnement et des ressources du monde, ce n'est peut-être pas un problème qui ne concerne que nous en Chine, peut-être que tout le monde s'y intéresse. [...] Tout le monde doit participer au niveau international. Il y a ces problèmes-là, et puis il y a la question de la guerre, où que ce soit, même si ça nous semble très éloigné. Les gens des couches élevées regardent peut-être un peu plus loin [que les autres]." (Chen)

Cette déclaration sur les "couches élevées" fait écho au discours sur les classes moyennes et la modernisation du pays. Pour être quelqu'un de moderne, ou de plus élevé dans la société, il faudrait donc montrer de l'engouement pour des questions politiques. Cet état d'esprit conduit certaines personnes à faire des efforts particuliers pour montrer qu'ils ne s'intéressent pas uniquement à leur vie personnelle, même s'ils peuvent par ailleurs réaffirmer qu'ils lui donnent la priorité.

Cela reste donc un effort pour le plus grand nombre des internautes que j'ai rencontrés, voire une corvée pénible. Le cas le plus flagrant est celui des étudiants, qui sont interrogés sur des questions de société dans certains de leurs examens.

"En général ce qui m'intéresse personnellement, c'est la beauté, les vêtements, la coiffure, et d'autres choses sur la vie des femmes. Sinon, je veux passer l'examen de fonctionnaire d'Etat, donc je m'intéresse un peu à l'actualité, je regarde aussi un peu 
l'actualité internationale, c'est à dire que je m'oblige (qiangpo) à la regarder, parce qu'ils pourraient m'interroger sur des questions de société. Par exemple, on peut être concernés par des problèmes de corruption dans le commerce, ou par les destructions de logements. Par exemple, en Chine on construit des logements à grande échelle, et beaucoup d'appartements détruits ne donnent pas lieu à des indemnisations, c'est à dire que quand le gouvernement ordonne des destructions au nom de l'intérêt public, il ne donne pas assez d'argent pour compenser. Je me force (yingyi) à faire attention à ces questions-là, mais après mon examen je ne le ferai peut-être plus. Mais en regardant ça, je comprends quand même de quoi il s'agit, ça m'est sûrement utile quand même, peut-être qu'on ne s'intéresse aux choses que quand on y a déjà un peu touché. Avant je ne pensais pas ça, avant l'actualité, les réunions, les trois représentations, je n'y connaissais vraiment rien quand j'étais étudiante. Mais comme je vais passer un examen là-dessus, je me familiarise avec ça peu à peu, parce que pour bien comprendre ce qu'il y a dedans, je trouve qu'il faut réfléchir. De ce côté-là c'est utile, Internet est un canal très rapide pour obtenir des connaissances, je trouve." (Liu)

Qui eût cru que l'Etat serait amené à obliger les citoyens à s'intéresser à la politique? De la même façon, consulter les informations internationales sur des sites anglophones peut être un bon moyen d'améliorer son anglais pour les étudiants.

"Ce que je regarde le plus sur Internet, c'est les informations, et aussi les informations internationales. Je lis beaucoup les informations internationales, parce que je suis étudiant en anglais. La plupart du temps, ça fait partie des devoirs que le prof nous donne. Donc je lis beaucoup les informations internationales, mais sinon je lis aussi des informations nationales." (Sun)

Pour certains internautes, s'informer est en quelque sorte une obligation vis-à-vis de la société. Il en ressort un champ lexical de la contrainte que l'on s'inflige à soi-même: "s'obliger à" (qiangpo), plus haut, ou "se forcer à" (yingyi), ci-dessous.

"Des fois je me force (yingyi) à lire la page des informations, parce que je dois comprendre ce qui se passe dans la société. Après je lis les pages de divertissement, de littérature et aussi les pages féminines, il y a des informations sur la mode, la beauté. Je lis aussi souvent les pages culturelles, ce n'est pas que j'aime beaucoup, mais je trouve que je dois lire un peu ce genre de choses, comprendre un peu." (Li) 
Beaucoup d'internautes s'astreignent à suivre l'actualité nationale et internationale, dans le but d'en savoir autant que la majorité de la population, comme pour maintenir un certain niveau de culture générale.

"En fait, je lis assez peu les informations internationales. Bien sûr je lis forcément les choses très importantes, par exemple je lis forcément les trucs sur Saddam Hussein, mais je n'irais pas lire de choses plus précises.

- Pourquoi?

- Parce que je n'ai pas besoin de comprendre plus précisément, je me contente de savoir à peu près ce qui se passe et c'est tout. Par exemple hier j'ai vu que le petit frère de Saddam Hussein avait été condamné à mort, je pense que tout le monde le sait, donc j'ai cliqué pour lire un peu. Mais je trouve qu'il suffit de comprendre de quoi il s'agit, ça ne sert à rien de chercher à comprendre plus précisément, je le sais et voilà." (Cao)

Certains comptent même sur les informations importantes pour les atteindre sans qu'ils aient à faire d'efforts particuliers.

"Si ça n'a pas de rapport avec moi, je ne vais pas lire l'info bien sûr. Des fois peu importe ce que dit la loi, ça n'a aucun impact réel sur toi, ça n'est pas intéressant.

- Même les lois sur l'université par exemple?

- Là oui, mais je lirais juste un peu, je jetterais un tout petit coup d'œil.

- Donc tu trouves que les infos nationales n'ont pas d'impact sur la vie des gens ordinaires?

- Si, mais cet impact ne nous atteint peut-être pas.

- Pourquoi?

- Je veux dire que tout n'a pas d'impact. Ce qui ne m'intéresse pas, c'est la politique, mais les choses qui nous atteignent, ils en parlent toujours, et on finit toujours par être au courant. Ce n'est pas la peine d'aller chercher ces informations spécialement sur Internet." (Deng) 


\section{Une frustration à la hauteur des attentes}

Le modèle d'une société archaïque, sollicitée en permanence pour des mouvements politiques et celui d'une jeunesse moderne, recherchant le plaisir sont renvoyées dos à dos en permanence. Contrairement à leurs aînés, les jeunes ne seraient préoccupés que par la recherche permanente de divertissements. Dans cette optique, la politique est perçue comme décevante, décourageante, voire déprimante.

"Quand on surfe sur Internet c'est pour se détendre, c'est un environnement qui détend. Dans cette atmosphère-là, les sujets sérieux ne rentrent pas. En général, pour beaucoup de gens, et surtout pour les plus jeunes, Internet sert surtout à obtenir des informations amusantes, on se sert d'Internet pour se divertir. Donc je trouve que les informations, les affaires militaires et ce genre de questions sérieuses ne vont pas avec ça, donc ça ne peut pas susciter mon intérêt." (Gao)

Les informations "sérieuses" en général, pas seulement le domaine politique, sont ainsi stigmatisées comme trop sérieuses, presque ringardes. Surtout, lorsque les nouvelles sont mauvaises, elles peuvent être perçues comme beaucoup trop contrariantes, voire difficiles à supporter.

"Ces choses-là, sur le pays, n'ont pas beaucoup d'impact sur ma vie. Des fois quand je lis des nouvelles très mauvaises, ça peut me rendre triste pendant deux jours." (Sun)

C'est peut-être la raison pour laquelle on se tourne plus facilement vers des sujets plus amusants.

"Quand je regarde les infos, sur Sohu je lis surtout les affaires nationales, puis la société, puis l'économie, et puis les téléphones mobiles, les présentations de produits numériques, et puis les voitures. Après les voitures il y a aussi les appartements, et ensuite les loisirs, l'un après l'autre. Je lis très bien les infos.

- Alors tu lis beaucoup de choses?

- Oui, c'est un site qui renouvelle beaucoup ses infos. Quand on a fini de lire les infos sur la société, on peut être un peu énervé, par exemple les taux d'intérêts ont monté, ça peut nous concerner directement. On lit que les taux d'intérêts ont encore 
monté, et après on regarde d'autres choses, comme les téléphones mobiles, ou les voitures, des infos divertissantes qui nous intéressent, et ça détourne l'attention." (Chu)

Nombreux sont ceux pour qui les divertissements offerts par Internet permettent visiblement de fuir les questions contrariantes.

"En général, je commence par regarder ce qui s'est passé dans le pays, je pense que tout le monde aime bien ça. C'est assez triste, je commence par ça, ils mettent beaucoup de problèmes sérieux dans les premières rubriques, et je les regarde dans l'ordre. Mais j'ai aussi envie de savoir ces choses-là, donc je choisis de les lire, mais à la fin, je choisis des choses sur le sport ou sur le cinéma." (Sun)

Ce rejet fréquent des nouvelles tristes serait-il un signe de la postmodernité des internautes chinois, de leur superficialité consumériste? Il semble en fait que les internautes ne rejettent pas la politique en tant que telle, ce que suggérait déjà leur sensibilité à de nombreuses questions sociales. La politique qu'ils décrivent les déçoit car elle ne répond pas à leurs attentes. De nombreuses frustrations peuvent expliquer le fait qu'ils finissent par s'en détourner durablement.

En premier lieu, de nombreux internautes expriment un grand sentiment d'impuissance devant l'impossibilité de participer vraiment à la résolution des questions politiques. Un petit nombre d'internautes rendent cet aspect plus explicite.

"Ca n'a pas beaucoup de rapport direct avec la vie des gens. Peut-être qu'il y en a un tout petit peu, mais ça ne saute pas aux yeux. Et puis ces choses-là, ça ne sert à rien de les commenter, on ne peut pas participer personnellement à des choses politiques. Ils disent que nous, tous les citoyens, sommes les maîtres du socialisme, mais pour toutes les grandes questions nationales on ne peut pas participer, personne ne participe. Les choses auxquelles le peuple participe sont déjà décidées depuis longtemps par l'Etat. Il n'y a aucune autonomie. Le plus souvent, c'est le gouvernement central qui définit une ligne pour les questions politiques, et il faut suivre cette ligne. Du moment qu'on ne s'en éloigne pas ça va. Les autres opinions doivent au moins rester dans des espaces séparés (duideng de diwei), sinon il n'y a pas d'autres opinions, en fait on ne parle pas de politique. Ca ne sert à rien de s'opposer, et ça ne sert à rien d'exprimer son point de vue, il suffit de s'informer et puis voilà. C'est surtout une habitude qu'on apprend quand on est étudiant, on a des examens de 
politique. Il faut s'intéresser à la politique, mais de façon limitée. Il ne faut pas faire de commentaires, il suffit de remplir des trous ou de cocher des réponses. Il suffit de comprendre un peu l'orientation politique du moment, ça suffit. Ca se limite à quelques grandes questions d'actualité. Comme individus, on n'est pas capables de commenter la politique du gouvernement, et puis de toute façon ça ne sert à rien." (Lin)

Dans ces conditions, l'information ne peut que créer un sentiment de tristesse. Il peut en effet devenir frustrant de s'intéresser à de tels thèmes tout en sachant qu'il n'est pas possible d'y changer quoi que ce soit. L'impression d'impuissance démotive un grand nombre d'internautes.

"Quand on discute de questions internationales on ne s'attend à rien, ça ne peut pas évoluer d'après notre opinion, on se contente d'exprimer notre point de vue sur le sujet. Au contraire sur les questions domestiques, d'abord on veut que le pays aille de mieux en mieux, on veut résoudre les problèmes. Mais on a toutes ces attentes, et si la situation ne correspond pas à nos attentes ça nous fait mal." (Wu)

Cette frustration s'accompagne de la conscience assez diffuse de la manipulation de l'information politique. Quelques témoignages vont même dans le sens d'un véritable dégoût de l'information telle qu'elle est présentée aux citoyens par les médias officiels. Le cas le plus caricatural est celui de la télévision, ici décrit par un internaute particulièrement critique.

"Cela fait déjà plus de dix ans que je n'ai pas regardé le journal de CCTV. En général même si des gens le regardent autour de moi, j'éteins la télé. Je trouve que c'est une pollution de la vue et de l'ouïe, vraiment. Je voue une véritable haine aux fausses informations, aux fausses nouvelles. Et puis je suis sûr que tu as déjà vu le journal de CCTV, il n'y a aucun contenu, ça ne fait que dire notre Parti est bien parce que ceci cela, nous avons encore fait toutes ces choses bien pour les gens, nos ancêtres ont fait telle ou telle bataille historique, nos dirigeants aujourd'hui sont tellement grands, ils ont sorti telle ou telle pensée, bla bla bla, il n'y a pas une phrase utile. S'il y a un accident en Chine, une catastrophe, tous les reportages un peu complets sont sur l'étranger. Je suis sûr qu'il n'y a que les cinq dernières minutes qui sont vraies, et encore ils enlèvent tout ce qui n'est pas favorable à la Chine." (Tian) 
Il est relativement difficile de savoir si d'autres internautes partagent cet avis. La censure et la fabrication d'une information politique biaisée contribuent certainement à dégoûter un certain nombre d'internautes qui voient la politique comme dévoyée, ce qui lui enlève tout intérêt. D'autres travaux rejoignent cette conclusion et montrent que les sujets politiques intéressent les gens a priori, mais qu'ils sont vécus comme "déconnectés" ${ }^{11}$ de la réalité à cause de la censure et du discours officiel.

L'information politique est donc affublée de stigmates qui en détournent de nombreux internautes: selon les cas, elle serait trop censurée, inutile, frustrante, sans intérêt direct pour les individus ordinaires. Ces seuls adjectifs montrent en creux que les internautes attendent une information plus transparente, plus pertinente par rapport aux enjeux qui les concernent, et dont ils pourraient se sentir partie prenante. L'affirmation du désintérêt pour la politique ne peut donc pas se comprendre comme résultant uniquement du caractère postmoderne des jeunes chinois. Bien au contraire, elle montre qu'ils ont des attentes très fortes, et une idée exigeante de ce que pourrait être une politique intéressante et à leur portée. Là où les dimensions politiques de l'information sont plus conformes à leurs attentes, l'intérêt renaît.

\section{3 - Les voies détournées de la curiosité politique}

\section{Les relations internationales}

Dans la catégorie des informations qu'il est important de connaître, les questions internationales semblent figurer en bonne place. En effet de nombreux internautes déclarent se désintéresser de la politique en général, mais faire exception pour les informations internationales.

"Oui, en général je regarde d'abord l'international, et puis s'il y a des choses qui m'intéressent dans les informations nationales, je les lis." (Zhu)

Ces informations peuvent sembler divertissantes, ou intéressantes au titre d'une certaine curiosité géographique. Dans cette catégorie on trouve des gens qui semblent simplement curieux du monde, et intéressés par la question de la paix dans le monde. De plus les

${ }^{11}$ LEE Hsiao-Wen. "The popular press and its public in contemporary China", article présenté au Congrès annuel de l'International Communication Association - ICA, Montréal, mai 2008. 
événements d'ampleur internationale, entre les conflits et les catastrophes naturelles, ont souvent un côté spectaculaire.

"En général dès que je me connecte je lis les dernières informations sportives, surtout le foot. En général je jette un oeil aux grandes affaires internationales, et puis après je lis des trucs sur les jeux vidéo, et tout le reste, je le lis très peu. Voilà ce que je lis.

- Pourquoi est-ce que tu préfères ces thèmes-là?

- Ce que je préfère, c'est surtout le foot.

- Tu as aussi parlé des affaires internationales?

- Ce que je préfère, c'est le foot, et ensuite les affaires internationales. Je m'y intéresse surtout quand la sécurité d'une région est déstabilisée, en Afghanistan, ce genre de choses, ça m'intéresse et en général je le lis. Les jeux aussi, j'y jette un oeil, mais pas autant que les deux précédents.

- Et est-ce que tu aimes les questions économiques ou sociales?

- Je n'aime pas trop l'économie, mais je lis un peu des trucs sur la société. Je suis complètement hermétique à la finance." (Fang)

Lorsque les aspects les plus politiciens réapparaissent, l'intérêt s'évanouit.

"Les relations internationales ça m'intéresse en général, sauf quand c'est des réunions, parce que quand les Chinois font des réunions, il y a peu de contenu pour beaucoup de temps, c'est assez ennuyeux." (Gu)

Qu'est-ce qui fait donc l'intérêt des questions internationales? En fait, il semblerait que le patriotisme soit à l'origine de nombreux clics sur les pages de relations internationales des journaux en ligne. Beaucoup d'informations traitent en effet, directement ou indirectement, des relations de la Chine avec ses voisins et partenaires, et plus généralement de sa nouvelle place dans le monde. C'est pourquoi certains événements très politiques peuvent être considérés comme importants, même par les personnes qui n'aiment pas ces questions a priori.

"Je ne m'intéresse pas à tout ce qui touche à la société.

- C'est parce que tu n'aimes pas ça, ou bien parce que tu ne comprends pas? 
- Ca ne m'intéresse pas, parce que si ça m'intéressait, je trouverais un moyen pour comprendre. Tout ce qui touche à la société, c'est trop compliqué, psychologiquement je ne peux pas supporter.

- Donc tu ignores absolument tout, ou bien est-ce que tu as l'occasion de voir certaines choses?

- A la base, je ne lis rien du tout là-dessus. Mais des fois il y a des sujets assez sensibles, alors des fois je regarde, comme les relations entre la Chine et d'autres pays. Ca m'arrive aussi de lire d'autres choses, je m'intéresse un peu aux questions de souveraineté.

- Comme les relations entre la Chine et le Japon?

- Non. Je veux dire: l'expression 'l'économie est chaude, et la politique est froide', résume bien ma pensée.

- Tu as dit que des fois tu t'intéressais à des sujets sensibles. Quel genre de sujet peut attirer ton attention comme cela?

- Il y a des sujets qui ont un rapport avec le pays, les dirigeants, la souveraineté nationale, ça, j'y fais attention.

- Pourquoi?

- Ces choses-là concernent les intérêts vitaux de ma patrie, la patrie c'est le pays, c'est comme une famille. Pourquoi est-ce qu'on dit "pays" (guojia) c'est parce que le pays (guo) c'est aussi la famille (jia).

- Donc pour toi, ce qui est le plus important, c'est ce qui arrive à ton pays, et ce qui se passe dans la vie quotidienne?

- Oui. Ce qui se passe dans la société, c'est moins important que ce qui se passe entre les pays. Par exemple quand il se passe quelque chose dans le pays, un changement de politique, ou la promulgation d'une nouvelle loi, ça ne m'intéresse pas. Mais les relations internationales, et surtout les questions de souveraineté, ce qui concerne l'intérêt du pays, l'intérêt de la nation, ça m'intéresse plus." (Zhou)

Les internautes prennent souvent la peine de s'informer de la position de leur nation sur la scène internationale, car il s'agit presque d'un devoir patriotique. Certains aspects de la politique nationale et internationale touchent donc les internautes non pas en tant qu'individus, 
mais en tant que membre de la nation chinoise, et plus largement en tant qu'Asiatiques, comme ci-dessous.

"Sur certaines infos, comme en ce moment par exemple, récemment on parle beaucoup de la question nucléaire nord-coréenne, n'est-ce pas? La conférence sur la paix vient de sortir une décision, pour des sanctions, c'est ça? Cette question, dans la mesure où elle a un impact sur notre système de sécurité à nous, les Asiatiques, même si on ne comprend pas très bien, on s'y intéresse . Et puis il y a aussi les relations avec Taiwan, les communications de chaque côté du détroit, comme hier: Chen Shuibian a annoncé qu'il voulait une deuxième république. Voilà, c'est ce genre de choses qui peut m'intéresser. Parce que ça a un rapport avec notre vie, même si ce n'est pas un rapport étroit au moins ça a une influence, non?"

Ici il devient clair que la politique n'est pas inintéressante en soi. Elle représente seulement un domaine de compétences hors du champ des individus, qui leur est interdit et où très peu s'aventurent. Au contraire, les individus se sentent concernés par la question de la puissance de leur pays sur la scène internationale, d'où, chez certains, un intérêt particulier pour les questions militaires et l'armement, qui fait l'objet d'un traitement spécifique sur de nombreux sites.

"En général, mon habitude c'est de regarder d'abord le sport sur Sohu, parce qu'ils font très rapidement les mises à jour. Je regarde aussi les informations nationales et internationales, et le basket et le foot, et après je regarde des sites sur des trucs comme les ordinateurs, pour voir ce qui est sorti comme nouveaux produits ou nouvelles technologies. Ensuite j'ouvre QQ et je me connecte, et puis je cherche des romans à lire, voilà ce que je fais. Et il y a aussi les affaires militaires.

- Les affaires militaires?

- Oui, il y a des reportages sur les armements que le pays choisit, et j'aime bien regarder ce genre de choses." (Song)

Cette curiosité pour des questions d'ordre international s'accompagne d'un travail identitaire très intense, qui consiste à retrouver l'honneur bafoué de la Chine, et qui se reflète aussi sur de nombreux sites d'informations. 


\section{L'histoire}

L'abondance des sites historiques sur Internet montre une volonté certaine de renouer avec les racines, de mieux comprendre et surtout de valoriser l'identité nationale chinoise. La plupart de ces sites en effet reviennent sur divers éléments de ce qui est censé fonder la culture nationale, avec par exemple la philosophie de Confucius. Cela fait écho à la relance des études confucéennes depuis les années $1980^{12}$. D'autres articles en ligne sont l'occasion de réinterpréter comme des actions héroïques à caractère patriotique un certain nombre d'événements qui ont pu auparavant être analysés comme des échecs, comme la révolte des boxers $^{13}$, et plus encore divers épisodes de l'invasion japonaise. Internet est un vecteur central dans la diffusion de ces questions. Les sites Internet historiques peuvent être considérés comme faisant partie des sites d'actualité, car certaines questions historiques qui font l'objet de débats ont une influence directe sur l'actualité politique contemporaine et les relations internationales de la Chine, surtout dans le cas des relations sino-japonaises.

"D'abord j'aime bien lire différents points de vue sur un phénomène social, et puis j'évalue si mon propre point de vue est correct ou si je peux adopter des éléments qui sont plus intéressants (youyi). Et puis il y a de nouvelles études historiques, je lis un peu ça, c'est un miroir de l'actualité (yishiweijian), pour voir comment les générations suivantes ont évalué les choses du passé et les personnages historiques, et j'essaie de voir comment je peux en tirer une ouverture, si ça peut m'aider dans mon travail ou dans ma vie.

- Donc ton but est de trouver une aide dans ta propre vie?

- Oui, c'est vrai, c'est d'abord pour répondre à mes intérêts et à mes goûts (xingqu he aihao), et deuxièmement il y a des choses touchantes là-dedans, et puis je peux en faire un usage positif dans ma propre vie." (Lin)

Non seulement l'histoire permet de donner un éclairage intéressant sur certains faits d'actualité, mais elle donne aussi des clés, des modèles, pour le développement personnel de

12 BILLIOUD Sébastien et THORAVAL Joël. "Jiaohua: le renouveau confucéen en Chine comme projet éducatif", Perspectives Chinoises, n¹01, 2007/4, p. 4-21.

${ }^{13}$ WU Xu. Chinese Cyber Nationalism: Evolution, Characteristics and Implications, Plymouth: Lexington Books, 2007, p.64. 
chaque individu. Elle s'insère ainsi très bien dans le modèle d'accès à la modernité qui est promu à de nombreux échelons de la société.

Il ne faut pas négliger non plus le fait que l'histoire permet aussi de parler du pays d'une manière plus libre, et de rétablir des faits qui ont pu être cachés à l'époque où ils se sont produits. En somme, l'histoire est une façon de contourner la censure a posteriori.

"Je ne sais pas si tu as déjà vu le site 163, c'est à dire Wangyi. Dans les rubriques info il y a une catégorie 'histoire', un menu 'histoire'. Quand tu cliques dessus, il y a la liste de toutes les périodes des plus anciennes jusqu'à maintenant, ça fait beaucoup de choses. Pourquoi est-ce que j'aime bien lire la partie histoire? Parce que ça parle de l'histoire du gouvernement depuis la guerre contre le Japon jusqu'à la Libération. Ils publient toute l'histoire de Mao Zedong sous la forme de petites histoires. A l'époque le blocage de l'information était très dur en Chine. Ils révèlent des informations, des histoires qu'on n'avait pas le droit de rapporter à l'époque." (Zhao)

Surtout, s'intéresser à l'histoire de la Chine et de sa position dans le monde est une manière pour beaucoup d'internautes de prendre une revanche sur les humiliations subies par leur pays dans l'histoire récente. C'est ainsi que de nombreux internautes ne s'intéressent à des questions d'ordre politique que lorsqu'elles concernent les relations entre le Japon et la Chine.

- Pourquoi est-ce que tu t'intéresses aux questions sur le Japon, mais pas aux affaires nationales, à la politique, ni aux informations internationales?

- Je lis un peu tout ça, mais en fait je ne m'intéresse pas aux informations nationales, ni au côté politique.

- Et international?

- Moyen.

- Tu penses que le Japon ne fait pas partie de l'international?

- Pas vraiment, je trouve que les aspects politiques sont moins attirants que l'histoire." (Gao)

En lisant des sites historiques, les internautes affirment le statut de grande puissance de la Chine. Ils trouvent des éléments qui prouvent la puissance passée du pays, et qui pourraient ainsi justifier une "remise à zéro" des compteurs et un rétablissement de l'honneur perdu. 
"Pratiquement tout le monde pense comme moi. Tout le monde pense que nous avons trop été outragés (qinfan). Tout le monde trouve que comme nous n'irions pas violer les possessions des autres, les autres ne doivent pas non plus emporter nos biens. On pense comme ça, et on trouve que la Chine devrait repartir de zéro (fu qing), tu comprends? Historiquement la Chine a toujours été brillante, sa culture est très profonde, on veut que la Chine devienne meilleure, qu'elle redevienne une grande puissance mondiale comme avant, et qu'elle obtienne le respect des autres peuples du monde. Ca, je l'ai lu il n'y a pas longtemps dans un article qui parlait de la Corée. Ils ont beaucoup d'idées fausses sur la Chine, c'est à dire qu'ils pensent que la société chinoise en est encore à la période de la Libération, dans les années 60 ou 70, qu'elle est très pauvre, très arriérée, et ils pensent qu'ils sont supérieurs. Ils pensent que la Chine absorbe leur culture. Ils se sont emparés des fruits de notre culture historique chinoise, et ça me met très en colère." (Gu)

Loin d'être justifié par un désintérêt intrinsèque et "postmoderne", le rejet de la politique manifesté par les internautes s'explique donc par un véritable sentiment de confiscation et de dévoiement de la politique. En effet la grande majorité des internautes interrogés affirment ne s'intéresser qu'à des questions très concrètes et étroitement liées à leur vie personnelle, qu'ils opposent aux sujets politiques. Par exemple, les questions d'éducation, avec les interrogations sur l'équité à l'examen d'entrée à l'université (le gaokao) préoccupent beaucoup les plus jeunes internautes et leurs parents. Les questions d'emploi à la sortie de l'université sont un véritable souci pour les étudiants. Les plus âgés sont amenés à se renseigner sur les conditions de protection de la propriété lorsqu'ils achètent leur premier appartement, sur l'évolution des prix des produits de grande consommation etc... Toutes ces questions sont le plus souvent rangées par les internautes dans la catégorie des questions "sociales".

Or ces questions, qui intéressent de nombreux internautes, peuvent être considérées comme politiques au sens où elles impliquent une définition de l'intérêt général et supposent bel et bien des arbitrages politiques. Les internautes semblent donc procéder à une séparation quelque peu artificielle entre le domaine de la société et celui de la politique, l'un étant le domaine des problèmes quotidiens rencontrés par les gens ordinaires, et l'autre étant le lieu 
des luttes de pouvoir entre des gens d'une caste politique à part. Cela n'est pas sans rappeler 1"'évaporation du politique" décrite par Eliasoph dans les associations américaines ${ }^{14}$.

Ainsi les internautes ne rejettent-t-ils pas la politique au sens d'esprit public, mais une conception à leurs yeux dévoyée et archaïque de la politique qui leur est imposée dans bien des aspects de la vie quotidienne, dans le fonctionnement des institutions, au travers de l'éducation ou dans les médias. Ce rejet en lui-même montre que les internautes interrogés ont des attentes relativement précises et exigeantes envers la politique, attentes qui ne sont pour l'instant pas remplies, sauf au travers de sujets relativement périphériques comme les relations internationales ou l'histoire.

Ainsi, l'idée d'un individualisme excessif n'explique pas le désintérêt relatif affiché par les internautes pour la politique, même si eux-mêmes le décrivent comme un élément de la nouvelle modernité chinoise qui caractérise la jeune génération. En fait, les principes portés par cette idée de modernité contribuent moins à dissoudre l'intérêt pour le bien public qu'à le renvoyer dans un univers virtuellement hors d'atteinte des citoyens ordinaires. C'est cette frontière, vécue comme un élément de l'ordre public moderne, qui préside à la prise de parole publique des internautes ou, le cas échéant, à son interdiction.

${ }^{14}$ ELIASOPH Nina. Avoiding politics. How Americans produce apathy in everyday life, Cambridge University Press, 1998. 
Les contraintes d'une civilité "moderne" 
Les internautes chinois semblent sujets à la même "évaporation du politique" que certains citoyens américains ${ }^{1}$. Ils sont sensibles à de nombreuses questions d'intérêt public comme l'école, la pollution, ou l'évolution des prix, mais en même temps ils affirment ne jamais s'intéresser à des questions qui ne leur sont pas directement liées, et ils rejettent explicitement tout intérêt pour la politique.

Est-ce seulement un effet de la censure qui touche Internet, et de l'autocensure dont peuvent faire preuve les répondants lors d'un entretien? Je ne peux pas écarter complètement cette hypothèse. Il me semble cependant pouvoir trouver des éléments de réponse dans le modèle de modernité auquel adhèrent la plupart des internautes que j'ai interrogés, et dans les règles de civilité qui lui sont attachées.

En effet, j'ai montré qu'Internet est adopté par les individus en même temps qu'une certaine idée de la modernité, qui est largement promue par les autorités, mais aussi relayée sous des formes diverses dans toutes les sphères de la société: publicité, magazines, imaginaires des individus etc... Cette idéologie moderniste est porteuse d'une forme d'individualisme qui s'épanouit pleinement avec Internet, et qui se décline dans une différentiation très forte des parcours individuels. Les internautes investissent notamment le Web pour affirmer leur identité, trouver de nouvelles opportunités, construire des liens plus flexibles qui sont aussi de nouvelles ressources.

Il est facile pour les internautes eux-mêmes de présenter cet individualisme comme une forme d'égoïsme, comme chez les citoyens américains interrogés par Eliasoph. C'est ainsi qu'ils affichent publiquement un grand désintérêt pour la politique en-dehors de ce qui les concerne personnellement. Pourtant, un examen plus précis des mécanismes de "l'évaporation du politique" montre qu'il n'en est rien. Je souhaite montrer ici que si l'idéologie moderniste contribue à écarter les internautes de certaines questions politiques, ce n'est pas en favorisant un égoïsme forcené et "postmoderne", mais en posant les règles d'une civilité qui n'autorise pas l'expression des dissensions en public, et les renvoie vers les conversations privées. ${ }^{2}$

\footnotetext{
${ }^{1}$ ELIASOPH Nina. Avoiding politics. How Americans produce apathy in everyday life, Cambridge University Press, 1998.

${ }^{2}$ Sur le modèle de ce que décrit Ferrié dans le cas de l'Egypte. FERRIE Jean-Noël. Le régime de la civilité en Egypte, Paris: CNRS Editions, 2004.
} 
Il faut d'abord écarter l'idée selon laquelle la surveillance de l'information par la police serait une source de coercition unique et infaillible. Il est vrai que l'architecture de ce contrôle est particulièrement sophistiquée. Elle associe plusieurs niveaux de filtrage automatique des données à des moyens humains importants. Cependant les règles de la censure sont très floues et relèvent le plus souvent d'un arbitrage individuel, qui est toujours contingent. Surtout, les internautes se sentent peu concernés par une surveillance politique qui selon eux ne concerne qu'un petit nombre d'individus hostiles au régime, ce en quoi ils ne se reconnaissent pas. En revanche, la plupart des internautes interrogés sont très sensibles à des questions de civilité, qui comprennent aussi bien la politesse ou les insultes, que l'évitement des conflits dans les forums et sur les blogs. Ils établissent un lien très net entre le respect de ces règles de civilité et l'idée d'adopter un comportement "civilisé" et "moderne".

Or la modernisation du pays se fait aussi dans un contexte de différenciation et de hiérarchisation assumée entre les individus, qui ne trouvent de cohésion sociale que dans l'affichage d'un patriotisme unanime. Cette "solidarité négative", pour reprendre les termes de Ferrié, ne permet pas l'expression du pluralisme et des désaccords en public. Les internautes décrivent comme une preuve de civilité le fait de ne pas porter atteinte publiquement à l'image de la cohésion nationale. C'est pourquoi ils renvoient vers le domaine privé l'expression de la diversité, et qu'ils déclarent juger très mal les situations conflictuelles qui éclatent souvent en ligne. Ils s'écartent ainsi, en principe, de toute prise de parole politique.

Cependant ils ne font que renvoyer la prise de parole hétérodoxe, ou critique, vers des espaces plus privés. Or ces espaces, qui foisonnent sur Internet sous des formes très diverses, n'en sont pas moins visibles à qui veut les visiter. Ils sont donc en "clair-obscur" ${ }^{3}$ de diverses manières. Ce sont des forums utilisés de manière privative par des groupes d'individus, des blogs revendiqués comme des voix individuelles, ou encore des prises de positions ironiques qu'il faut lire entre les lignes. Ces nombreuses formes de prise de parole sont ambivalentes: elles réaffirment la frontière entre le public et le privé en même temps qu'elles s'en affranchissent.

\footnotetext{
${ }^{3}$ CARDON Dominique. "Le design de la visibilité. Un essai de cartographie du Web 2.0", Réseaux, n 152,2008 , p.93-137.
} 


\section{Chapitre IV - La responsabilisation de l'individu}

Bien souvent, le fait que les internautes chinois ne s'expriment pas beaucoup sur des questions politiques est attribué à la finesse du contrôle mis en place par les autorités chinoises sur le Web. Il est vrai que ce contrôle est relativement efficace dans certains cas, et ce serait faire affront aux internautes emprisonnés pour leurs écrits sur Internet que de prétendre le contraire. Cependant il faut pouvoir évaluer l'impact de ce contrôle sur le comportement des internautes en général, et en évaluer l'importance de manière équilibrée. Si la surveillance mise en place a une réelle force répressive sur les activités militantes proprement dites, elle n'est pas forcément vécue de manière aussi coercitive par des internautes ordinaires, lorsqu'ils n'ont pas d'engagement particulier.

Pour la plupart des internautes, la police du Web n'a qu'une existence théorique tant qu'ils n'y sont pas confrontés directement. Les limites politiques de l'utilisation du Web ne font pas l'objet d'une publication explicite. Bien au contraire, ce sont les diverses atteintes "ordinaires" à l'ordre public qui attirent l'attention des internautes lors de leur utilisation quotidienne du Web. Il faut donc comprendre de quelle manière et selon quels critères l'ordre public est effectivement maintenu et surtout perçu. Alors que les médias officiels insistent sur les nombreuses formes de criminalité qui envahiraient le Web chinois, les internautes sont beaucoup plus préoccupés par des questions de civilité, c'est à dire de politesse, de bonne gestion des échanges, de modération dans les relations interpersonnelles en ligne. Ils semblent ainsi beaucoup plus sensibles à un cadre normatif basé sur leur conception de la modernité et qui s'impose dans les relations interpersonnelles, qu'à la surveillance politique qui semble les concerner relativement peu.

\section{1 - Les gardiens de l'ordre public: de la police aux individus}

\section{Les outils techniques et juridiques du contrôle}

La mise en place des réseaux de télécommunication ainsi que la mise au point des règlements, directives et autres circulaires qui encadrent ce secteur relèvent de la responsabilité du Ministère de l'Industrie de l'Information (MII), qui a été rebaptisé en 2008 Ministère de l'Industrie et des Technologies de l'Information (MIIT). Sous l'égide de ce 
ministère, les outils techniques qui permettent le contrôle de l'Internet chinois ont été conçus dès la mise en place du réseau et intégrés à son architecture même. Une description détaillée en est faite sur le site de Opennet Initiative ${ }^{1}$, aussi me contenterai-je d'en résumer brièvement les grands principes, sans aborder les questions les plus techniques.

L'infrastructure du réseau est conçue de telle sorte que toutes les informations qui entrent et sortent de Chine passent par un petit nombre de serveurs qui filtrent les paquets d'informations. Ce filtrage se fait par mots-clés ${ }^{2}$ et par URL. Par exemple, un certain nombre de sites sont inaccessibles depuis le territoire chinois à partir de connexions Internet ordinaires. Parmi ces sites figurent notamment les associations qui militent pour l'indépendance du Tibet, des associations de défense des Droits de l'Homme (Human Rights Watch notamment) ou encore certains médias occidentaux. La liste des adresses de sites inaccessibles change en fonction de l'actualité, comme en témoignent des blocages ad hoc. Wikipedia par exemple a été successivement inaccessible, accessible partiellement ou complètement selon les périodes (et selon l'évolution des techniques de filtrage plus ou moins précises). Il arrive aussi que les sites des quotidiens nationaux français, ou plus souvent américains ou anglais, soient inaccessibles mais ce n'est pas toujours le cas. De même, un certain nombre de mots-clés - pensons à $\mathrm{Hu}$ Jintao, ou à l'association de mots Tiananmen/1989 par exemple - conduisent au signalement des contenus concernés, voire à leur perte complète. Ce système centralisé et automatisé de filtrage et de blocage des contenus est surnommé le "Grand Pare-feu" (Great Firewall) ou "Bouclier d'Or" (Golden Shield) 3 , à l'image d'un immense paravent technologique qui ne laisserait passer que quelques informations, et qui enfermerait le pays à l'intérieur d'une sorte de cordon, comme coupé du monde ${ }^{4}$.

1 Internet Filtering in China, Opennet Initiative, consulté le 25/06/2009 sur http://opennet.net/sites/opennet.net/files/ONI_China_2009.pdf. ainsi que le profil "Chine" sur le même site. Voir aussi TSUI Lokman. Internet in china: big mama is watching you.

${ }^{2}$ SOONG Roland. "The List of Banned Terms in Xinhua News Reporting", East South West North, 28/12/2006, consulté le 28/12/2006 sur http://www.zonaeuropa.com/20061228_1.htm.

3 GREENBERG Andy. "China's Golden cyber-shield", Forbes.com, 31/07/2007, consulté le 18/11/2008 sur http://www.forbes.com/security/2007/07/30/china-cybercrime-war-techcx_ag_0730internet.html.

${ }^{4}$ Plusieurs entreprises privées occidentales ont joué un rôle non négligeable dans la mise en place de ces technologies de contrôle. Voir Internet Filtering in China, Opennet Initiative, op.cit. 
Pour assurer la force de dissuasion de ce système, le MII a progressivement imposé une plus grande sophistication des systèmes d'identification ${ }^{5}$ des internautes, dans tous les lieux où ils pouvaient auparavant se connecter anonymement. Aussi doit-on désormais fournir une carte d'identité dans les hôtels ${ }^{6}$, les cybercafés ${ }^{7}$, pour ouvrir un blog ${ }^{8}$ etc... Ces procédures sont en réalité rarement appliquées (de plus en plus cependant) et largement contournées mais elles restent comme une épée de Damoclès au-dessus de tous les établissements qui laisseraient leurs clients dépasser les bornes 9 .

Les systèmes de surveillance ont ainsi tendance à être installés de plus en plus près des utilisateurs finaux. Fin mai 2009, le gouvernement a annoncé son intention de rendre obligatoire la livraison d'un logiciel de filtrage avec tout nouvel ordinateur vendu à la date du premier juillet 2009. Ce logiciel, appelé "Green Dam Youth Escort", et présenté comme un logiciel de contrôle parental, filtre de manière relativement incohérente les sites pornographiques, mais il filtre aussi des sites politiques. Ses défauts techniques graves et sa très faible acceptabilité auprès des internautes chinois en ont fait un échec. Les autorités ont finalement dû retirer le projet. Cependant le fait que les autorités cherchent à déconcentrer le travail de filtrage en le faisant porter sur les ordinateurs individuels montre bien que

${ }^{5}$ BANDURSKI David. "Chongqing police say all Internet users must register by October 30 or face fines and denial of web access", China Media Project, 07/06/2006, consulté le 24/10/2010 sur http://cmp.hku.hk/2006/07/07/90/; ANSFIELD Jonathan. "China web sites seeking users' names", 06/09/2009, The New York Times, p. A4. En juillet 2009, les autorités auraient demandé aux fournisseurs de services en ligne d'exiger l'identité réelle des utilisateurs pour toute ouverture de compte.

${ }^{6}$ ZHENG Caixiong. "Police have easier access to data on hotel guests", China Daily, 18/08/2006.

${ }^{7}$ BONNASSIEUX Mathilde. "Clients des cafés Internet de Pékin, souriez vous êtes photographiés", Aujourd'hui la Chine, 18/10/2008.

8 "China orders bloggers to register with government", The Guardian, 07/06/2005.

${ }^{9}$ Les fermetures massives de cybercafés "clandestins" en 2003 suite à des incendies montrent bien la manière dont ces règles s'appliquent. En 2002, presque aucun cybercafé n'était entièrement dans les règles. Suite à une série d'incendies dans des cybercafés tout à fait clandestins, ce sont des dizaines d'établissements jugés insalubres qui ont été fermés dans une vague de régularisation. Ce durcissement de la politique à l'égard des cybercafés a eu lieu peu après l'épidémie de SRAS, au cours de laquelle Internet était devenu un mode privilégié de partage de l'information. Ainsi, des règles aussi légitimes que la sécurité et la salubrité ne sont-elles en fait appliquées et sanctionnées que dans des circonstances très précises, et elles servent en même temps d'outil de pression en cas de problème politique. 
l'ambition d'un contrôle automatique généralisé pose d'importants problèmes de faisabilité et d'efficacité $^{10}$.

Parce qu'il ressemble à un "panoptique" ${ }^{11}$, ce système de surveillance automatisé semble omnipotent. Il ne l'est pourtant pas ${ }^{12}$ dans la mesure où tout filtrage repose sur la capacité d'analyser qualitativement l'information et suppose d'y consacrer des ressources considérables. Ce système n'empêche pas complètement les internautes d'atteindre les informations qui les intéressent vraiment ${ }^{13}$, et il a le défaut de ne pas faire la différence a priori entre des phrases anodines et des phrases véritablement contestataires. En fait sa principale efficacité réside dans le fait d'attirer l'attention, et ainsi d'intimider les utilisateurs. N'est-ce pas le principe même du panoptique que de provoquer l'autocensure ${ }^{14}$ ? En fait, le concept même de "Grand Pare-feu" donne une importance démesurée aux dimensions techniques du contrôle, et il tend à dissimuler le facteur humain qui est fondamental dans le contrôle du Web chinois ${ }^{15}$.

Derrière les filtres, qui contrôle les comportements illicites des utilisateurs? Nul ne sait exactement qui sont les véritables contrôleurs du Web chinois. Selon les sources, la police d'Internet comprendrait des dizaines de milliers de fonctionnaires affectés à la surveillance du $\mathrm{Web}^{16}$, mais personne ne sait combien ils sont précisément, ni quelle est leur mission. Ils ont pourtant deux représentants ultra-célèbres.

10 "China's Green Dam: the implications of government control encroaching on the home PC", Opennet Initiative, consulté le 24/10/2010 sur http://opennet.net/chinas-green-dam-the-implicationsgovernment-control-encroaching-home-pc.

11 GIESE Karsten. "Speakers corner or virtual panopticon: discursive construction of Chinese identities online", in MENGIN Françoise (dir). Cyber China, Reshaping national identities in the age of information, New York: Palgrave Macmillan, 2004, p. 19-36.

12 VAAS Lisa. "Toppling the Great Firewall of China", Eweek.com, 12/09/2007, consulté le 24/10/2010 sur http://www.eweek.com/c/a/Security/Toppling-the-Great-Firewall-of-China/.

${ }^{13}$ Il est techniquement possible de les consulter en ayant recours à des "proxys" qui permettent de contourner les filtres en rendant les paquets d'information plus difficiles à tracer, mais cela demande de bonnes connaissances informatiques et une certaine ténacité.

${ }^{14}$ BENTHAM Jeremy et FOUCAULT Michel. Le Panoptique, Paris: Belfond, 1977.

15 TSUI Lokman. "An inadequate metaphor: the Great Firewall and Chinese Internet censorship", Global Dialogue, vol.9, $n^{\circ} 1-2,2007$, p.60-68.

${ }^{16}$ XIAO Qiang. "Who are China's top Internet cops", China Digital Times, 28/09/2006, consulté le $24 / 10 / 2010$ sur http://chinadigitaltimes.net/2006/09/0\%C2\%BA\%E2\%88\%91ho-are-chinas-topinternet-cops/. 


\section{Les "cyberflics": une mosaïque aux agendas diveregnts}

Depuis 2006, deux policiers virtuels, tout droit sortis d'un film de Tex Avery, "patrouillent" sur les portails à large audience de Shenzhen ${ }^{17}$. Il s'agit d'images "pop-up" représentant des policiers en uniforme très souriants, qui s'affichent spontanément lorsque les internautes visitent les pages des plus grands portails d'information comme Sina, Sohu ou QQ. Leurs noms, "Jingjing et Chacha", sont formés à partir des deux caractères du mot "police" (Jingcha). Le redoublement des caractères est généralement une façon d'exprimer l'atténuation, et son utilisation ici semble montrer une volonté de rendre ces personnages sympathiques et proches du public. Leur exemple a été imité par d'autres municipalités comme Pékin ${ }^{18}$, preuve de leur succès. Immédiatement décriés par la presse internationale comme un coup de massue supplémentaire sur la liberté d'expression des internautes chinois, ils leur rappellent que la police est là qui les surveille. Ils rappellent surtout le caractère public des prises de parole qui sont faites sur Internet, et le fait que chacun ne peut pas y faire ni y dire ce qu'il veut.

$\mathrm{Du}$ point de vue des services de communication qui en font la promotion, le développement de ces deux avatars relève d'une opération de protection de l'ordre public ${ }^{19}$ et plus généralement d'un outil de e-gouvernement. Ces policiers possèdent une page sur QQ avec plusieurs fonctions (blog, ressources, photos etc). Une partie interactive est ouverte pour les questions des internautes. Ceux-ci peuvent y trouver des indications sur le comportement à adopter sur le Net, des règlements, des informations diverses. En somme Jingjing et Chacha se présentent comme "au service des internautes", pour les protéger en cas de difficulté, leur fournir assistance et conseil, en bref: les aider, et même protéger la liberté d'expression.

De fait les deux fonctions d'aide et de surveillance s'entrecroisent dans le discours des personnes en charge du service. Ces deux personnages sont à la fois une menace de sanction et la promesse d'une assistance.

'La principale fonction de Jingjing et Chacha est d'intimider, pas de répondre aux questions', ont répondu les officiels en charge de la division de surveillance et de

\footnotetext{
${ }^{17}$ DICKIE Mure. "China's virtual cops pinpoint web dissent", The Financial Times, 17/02/2006.

${ }^{18}$ BEN GHARBIA Sami. "Chinese cartoon cops patrolling the Web", Global Voices Online, consulté le 24/10/2010 sur http://globalvoicesonline.org/2007/08/29/chinese-cartoon-cops-patrolling-the-web/.

19 "Cyber-surveillance. Jingjing et Chacha veillent sur vous", L'Atelier, consulté en ligne le 16 juin 2007. http://asie.atelier.fr/internet/article/cyber-surveillance-jingjing-et-chacha-veillent-sur-vous.
} 
sécurité d'Internet du bureau de sécurité publique de Shenzhen. [...] la signification de l'apparition de Jingjing et Chacha est de rappeler publiquement tous les internautes à la nécessité d'être conscients de l'usage sain et sûr d'Internet, d'autoréguler leur comportement en ligne, et de maintenir ensemble l'ordre harmonieux d'Internet.

Maintenant, six employés de police ont commencé à travailler sur Jingjing et Chacha. Quand la charge de travail augmentera, il y aura plus de policiers impliqués dans le travail. Au travers d'Internet, les internautes peuvent entrer en contact avec eux et obtenir de l'aide de leur part. ${ }^{20}$

Ces deux personnages n'étaient pas encore visibles à Pékin au moment des entretiens et je n'ai donc pas pu interroger les répondants sur ce sujet. Cependant ils perçoivent de manière relativement positive l'intervention de la police sur les questions de cyber-délinquance en général. Compte tenu des risques et dangers réels encourus par les internautes, la police peut facilement se dire investie d'une mission de service public dans ce domaine. Il semble aller de soi qu'elle intervienne dans la régulation des cybercafés insalubres, mette en place des contrôles d'identité pour éviter que des mineurs n'accèdent à des contenus pornographiques par exemple. Ces remarques ne doivent être interprétées ni comme un aveuglement naïf devant ces justifications, ni comme une accusation radicale de ce qui ne pourrait être qu'une façade derrière laquelle se cache la censure. Les deux aspects existent à part entière. Les autorités exercent, et les usagers perçoivent, tout autant le service public de protection des individus que la surveillance politique des échanges d'opinions.

La fonction principale de Jingjing et Chacha est justement de faire ressentir l'omniprésence de la surveillance, mais pas de donner une idée précise de sa nature. Or cette surveillance, bien qu'elle existe réellement, se présente sous des traits tout aussi virtuels que ceux de Jingjing et Chacha. En fait presque tous les ministères, toutes les administrations, tous les bureaux, ont créé leur cellule de surveillance des contenus diffusés sur Internet, et ce à tous les échelons de l'appareil administratif et politique du pays. Chacune de ces cellules effectue un contrôle des discussions en ligne en fonction de ses préoccupations particulières, qu'elles soient thématiques ou géographiques. Ainsi le contrôle n'est-il pas le même selon qu'une discussion porte sur le développement des transports urbains ou sur la protection des

${ }^{20}$ HONG Yan. "Image of Internet police: Jingjing and Chacha online", China Digital Times, consulté le 24/10/2010 sur http://chinadigitaltimes.net/2006/01/image-of-internet-police-jingjing-and-chachaonline-hong-yan-o \%c2\%baae \%c2\%a5\%e2\%84\%a2aaio\%c2\%baa/. 
terres agricoles, et selon qu'elle traite d'un cas ayant eu lieu à Urumqi ou à Xi'an. La variabilité du contrôle constitue autant une marge de manœuvre qu'une source d'incertitude pour les internautes désireux de discuter de sujets politiquement sensibles en ligne. Les agendas de ces différentes administrations peuvent même parfois être contradictoires, ce qui ajoute à cette source d'incertitude.

De plus, les autorités auraient recruté des milliers de petites mains à bon marché dont le rôle ne serait pas tant de dénoncer les actions des internautes que de les noyer dans une propagande permanente et minutieuse sur les forums. Une armée de personnes payées pour poster des commentaires favorables à la politique du gouvernement sur les forums les plus populaires formerait ainsi ce qu'on appelle le "Parti à cinq maos" (wu mao dang), cinq maos étant le prix payé pour chaque commentaire posté, soit environ cinq centimes d'euro ${ }^{21}$. Il devient ainsi impossible de savoir si les avis postés en ligne le sont par des utilisateurs ordinaires ou par les organes de propagande du régime, qui eux aussi semblent démultipliés par le réseau.

En fait les ressources humaines les plus importantes ne sont même pas à compter parmi les fonctionnaires de police ni dans ce wu mao dang. Les censeurs les plus prolifiques sont dans les rangs des modérateurs au sein des fournisseurs d'accès à Internet et des fournisseurs d'espaces de publication.

\section{Le rôle ambigu des fournisseurs de contenus et des modérateurs}

En effet, il est notable que le contrôle par les autorités est effectué a posteriori. Il sanctionne en fait le contrôle effectué en amont par les fournisseurs de services sur Internet. Ces entreprises sont soumises à un régime de licence par le MII, qui impose des contraintes très strictes concernant les modalités de publication des contenus en ligne ${ }^{22}$.

Tout site qui propose de l'information doit se soumettre à des contraintes très strictes. Soit il est un organe de presse classique qui met en ligne ses contenus, et dans ce cas il est soumis comme toute publication aux règles édictées pour la presse écrite par la

\footnotetext{
${ }^{21}$ BANDURSKI David. "China's Guerrilla War for the Web", Far Eastern Economic Review, Juillet 2008, http://www.feer.com/essays/2008/august/chinas-guerrilla-war-for-the-web, consulté le 15 juillet 2008.

${ }^{22}$ Pour une analyse de l'économie politique des médias en Chine, voir ZHAO Yuezhi. Communication in China : political economy, power, and conflict, Lanham: Rowman \& Littlefield, 2008.
} 
Administration Générale de la Presse et des Publications (GAPP) et pour la presse audiovisuelle par l'Administration d'Etat pour la Radio, les Films et la Télévision (SARFT), ce qui inclut la censure. Une autre possibilité est de se positionner comme un agrégateur de contenus et de ne proposer que des liens vers des articles d'autres organes de presse, ou des copies de ceux-ci. En tout état de cause, il est conseillé de ne pas relayer d'informations qui pourraient être trop défavorables au gouvernement. De plus, les sujets que les services de communication du gouvernement et du Parti (notamment le SCIO, State Council Information Office) souhaitent voir mis en valeur le sont sur la page d'accueil, avec des titres en couleurs ou par le format des caractères ${ }^{23}$.

Ces sites proposent généralement des services interactifs, parmi lesquels la possibilité de commenter les informations ou d'écrire son propre blog par exemple. Cette fonction place les fournisseurs de tels espaces dans une situation très délicate car ils sont légalement responsables des contenus publiés par les internautes. Afin de garantir la surveillance effective des contenus par les fournisseurs d'espaces, la loi les oblige à engager des employés dont la seule mission est de surveiller la qualité des contenus, ou bien de mettre en place des systèmes de filtrage automatique ${ }^{24}$. Sur certains sujets sensibles, les forums de discussion peuvent être fermés, temporairement ou définitivement. Outre le tri des contenus proprement dits, les fournisseurs de services sont également tenus de communiquer les coordonnées de leurs utilisateurs à la demande des autorités ${ }^{25}$. De plus, ces entreprises sont invitées fermement à adhérer à des associations comme la Beijing Association of Online Media, dont

23 "CHINE. Voyage au cour de la censure d'Internet", Reporters sans frontières, octobre 2007, consulté le 20/01/2009 sur:

http://www.rsf.org/IMG/pdf/Voyage_au_coeur_de_la_censure_FR.pdf.

24 "Analysis of China's non-commercial web site registration regulation", OpenNet Initiative: Bulletin 011, 22/02/2006, consulté le 12/05/2009 sur http://opennet.net/bulletins/011/.

${ }^{25}$ Les exemples ne manquent pas d'entreprises occidentales critiquées pour s'être soumises à la loi chinoise. GUERIT Christophe, "Yahoo rattrapé par son ambiguité en Chine", Ecrans.fr, consulté le 24/10/2010 sur http://www.ecrans.fr/Yahoo-rattrape-par-son-ambiguite,1871.html; CORMIER Bruno. "Skype se soumet volontiers à la censure chinoise", www.pcinpact.com, 19/04/2006, consulté le 24/10/2010 sur http://www.pcinpact.com/actu/news/28155-Skype-se-soumet-volontiers-a-la-censurechin.htm? $\mathrm{vc}=1 \& \mathrm{cid}=641316$; EWING Kent. "China flap turns up heat on US tech giants", Asia Times Online, 14/02/2006, consulté le $24 / 10 / 2010$ sur http://www.atimes.com/atimes/China_Business/HB14Cb01.html; GRANGEREAU Philippe. "Google ferme des fenêtres pour s'ouvrir au marché chinois", Libération, 26/01/06; WATERS Richard, DICKIE Mure et KIRCHGAESSNER Stephanie. "How western web groups survive in China", The Financial Times, 14/02/2006. 
l'objectif officiel est de promouvoir des bonnes pratiques, mais qui sont directement gérées par le MII et qui sont le plus souvent une courroie de transmission des consignes politiques ${ }^{26}$.

Cette configuration fait peser une lourde responsabilité sur les entreprises concernées. Trop censurer les informations les mènerait probablement à perdre des clients. En même temps, laisser passer trop d'informations ou de commentaires critiques les mettrait en porte-àfaux vis-à-vis des autorités ${ }^{27}$. Dans le pire des cas, un site Internet peut être menacé de fermeture et ses dirigeants peuvent être licenciés et/ou poursuivis pénalement. Les entreprises importantes doivent donc ménager la chèvre et le chou, en laissant se développer des sujets suffisamment attractifs, tout en surveillant de près les contenus publiés par leurs utilisateurs. Une telle surveillance est très qualitative et elle ne peut pas être faite seulement par des automates, même si ceux-ci peuvent être utilisés en soutien. La majeure partie de ce contrôle repose sur une vigilance humaine. C'est d'abord celle des modérateurs des sites Internet.

Je désigne par le terme de modérateur toute personne qui est en charge de surveiller les contenus d'un site ou d'une partie d'un site, quel que soit son statut et le moment où il intervient dans le processus de publication. Les statuts peuvent varier grandement entre la personne qui effectue un balayage quotidien d'un grand portail Internet pour le compte de ce dernier et la personne bénévole qui ouvre son propre fil de discussion sur un forum et sera considérée comme responsable de ce forum. Néanmoins les deux effectuent une relecture des écrits des autres internautes et sont amenés à signaler des comportements illicites.

Les modérateurs sont toujours responsables des contenus publiés sur les différents sites. Ils ont la possibilité de censurer tous les messages qu'ils jugent excessifs pour une raison ou pour une autre. Ils peuvent, inversement, donner plus de visibilité à des messages qui leur semblent plus intéressants que la moyenne, en les faisant passer en début de liste. C'est également leur rôle de rappeler les règles de bonne conduite aux contributeurs de leur fil, voire de parlementer avec eux pour leur expliquer les raisons de la suppression de leur article le cas échéant. Or les exigences légales auxquelles répondent les modérateurs sont le plus souvent floues. Les plus grands portails disposeraient de listes de mots-clés à surveiller

\footnotetext{
${ }^{26}$ BANDURSKI David. "Pulling the strings of China's Internet", Far Eastern Economic Review, décembre 2007.

${ }^{27}$ XIAO Qiang. "Blogger: how headlines get written in China" China Digital Times, 02/11/2008, consulté le 24/10/2010 sur http://chinadigitaltimes.net/2008/11/blogger-an-editor-was-sacked-for-aheadline/.
} 
particulièrement en fonction de l'actualité, mais pour la plupart des petites mains qui effectuent ce travail de vigilance, seule l'intuition permet d'établir des critères de bon comportement $^{28}$. Aussi ces critères sont-ils le plus souvent des critères de politesse et d'honnêteté, mais il arrive également que ces critères soient politiques.

"J'ai eu des messages effacés, beaucoup, parce que ceux qui font office d'administrateurs [d'un fil de discussion] (banzhu), ils trouvent que c'est une menace pour eux, parce que tous les sites sont surveillés par le gouvernement, et c'est pareil pour les administrateurs, si on se rend compte que sur leur site il y a eu beaucoup de choses négatives, y compris des choses défavorables au gouvernement, alors ils peuvent forcer l'administrateur à les effacer, ça m'est arrivé souvent, parce que j'ai dit beaucoup de choses vraies." (Tian)

Ce rôle est souvent décrit comme une censure pure et simple par les observateurs occidentaux, qui tendent à oublier le fait que cette fonction est également cruciale dans le cas des sites Internet en régimes démocratiques ${ }^{29}$. En effet, le rôle des modérateurs chinois est très ambigu, car ils doivent garantir que l'espace virtuel sous leur responsabilité reste accueillant et permette de bons échanges. Ils n'ont donc pas intérêt à laisser se développer des controverses trop violentes ni à se faire remarquer par les autorités politiques. Ce contrôle peut être l'objet de négociation entre les responsables de certains fils de discussion, qui sont souvent des particuliers, et les responsables des rubriques au sein des plates-formes qui les hébergent.

"J'ai monté mon propre forum un jour, je veux dire mon forum personnel, donc je comprends un peu comment ça se passe de l'intérieur, parce que j'avais besoin de me protéger contre des attaques. Il y a des commentaires mal intentionnés, et il y a des gens qui laissent des messages mal intentionnés sur les forums, et après ils te dénoncent, ça arrive souvent. Donc si tu veux éviter ce genre de situations, tu dois discuter avec des gérants de grandes plates-formes à propos de ça. Bien sûr nous avons des sujets de discussion qui sont assez spécialisés, comme comment éviter cette situation, comment trouver des méthodes de contrôle quand je veux contrôler quelque chose." (Zhang)

\footnotetext{
${ }^{28}$ WU Vivian. "Internet police keep tight grip on blogs", South China Morning Post, 08/03/2007.

29 WOJCIK Stéphanie. "The Three key roles of moderator in municipal online forums", communication à Politics: Web 2.0: An international conference, Royal Holloway, University of London, 2008.
} 
Parfois, les modérateurs se trouvent donc en situation de supprimer des messages qui sont contradictoires avec les positions de l'Etat. Ils le font bien plus pour se protéger euxmêmes et pour préserver la possibilité même de s'exprimer, que pour ennuyer l'auteur de ces commentaires. Le Japon est certainement l'un des exemples les plus significatifs sur ce point.

"Chaque site Internet qui a une page de commentaires a un manager, son rôle est de nettoyer les saletés, les commentaires pourris. Donc ils pensent que si tu es un peu contre le Japon, si tu critiques le Japon, à la base le problème qu'ils veulent évoquer c'est cet aspect-là, ils veulent répandre l'idée que le Japon est mauvais. Donc si tu dis quelque chose d'autre sur le site ils vont l'enlever, ça ne sert à rien." (Liu)

C'est bien évidemment sur les sujets les plus sensibles que les modérateurs prennent le moins de risques, et en particulier sur tout ce qui touche la souveraineté nationale. Cependant il est important de garder en tête toute la dualité de ce contrôle, effectué par des modérateurs le plus souvent amateurs, qui sont eux-mêmes utilisateurs, friands de cette liberté d'expression et qui doivent eux-mêmes mettre en place les cadres les plus à mêmes de garantir la pérennité de cette liberté d'expression. Un site trop conformiste n'attire pas les utilisateurs et n'est pas non plus dans l'intérêt des modérateurs, qui doivent donc trouver un équilibre entre ouverture et respect des règles. En fonction de ces arbitrages délicats, le destin d'un commentaire posté en ligne peut varier énormément ${ }^{30}$. Paradoxalement, la vigilance des modérateurs sur ces points entraîne un relâchement des contrôles effectués en plus haut lieu, et autorise donc une plus grande liberté de ton pour les utilisateurs. Ce n'est que lorsque ce contrôle est défaillant que les autorités interviennent, si véritablement des écarts sont observés. De fait, la police n'a bien souvent pas grand-chose à sanctionner après le passage des modérateurs, et compte tenu de l'autocensure effectuée par les individus eux-mêmes.

\section{Le contrôle pour et par les individus}

En effet, les individus savent parfaitement que le contrôle du Web existe et qu'il ne concerne pas que les comportements délictueux. Ils en sont très conscients, même s'ils ne savent pas toujours jusqu'où s'étendent précisément les pouvoirs des autorités dans ce domaine.

${ }^{30}$ CHEN Ding. "Yi ge tiezi de 7 zhong mingyun" ["Les sept destins d'un post"], Nandu Zhoukan, 05/03/2009, consulté le 24/10/2010 sur http://nbweekly.oeeee.com/Print/Article/7325_0.shtml. 
"Aujourd'hui on dirait que si on écrit des choses qui dépassent vraiment les bornes c'est effacé directement, donc on peut dire que le contrôle est bien fait, ça rend le Web un peu plus clair." (Yuan)

Il faut parfois insister pour obtenir des précisions sur "ce qui dépasse les bornes", mais tout le monde sait que l'on ne peut pas tout publier sur un forum, sous peine de voir ses contributions effacées.

"Est-ce qu'on peut effacer des posts en ligne?

- Il y en a qui sont insultants, c'est sûr qu'ils sont effacés, parce que la police d'Internet les efface forcément.

- Dans quels cas cela arrive?

- Ca n'arrive pas souvent, ils ne peuvent pas te punir sous prétexte que tu as insulté quelqu'un ou dit des grossièretés, je ne crois pas que ça puisse arriver, sur Internet la parole est libre.

- Si elle est libre, alors pourquoi est-ce qu'il y a des posts effacés?

- Ils trouvent que les insultes sont moches, il doit y avoir différents aspects.

- Donc tu penses qu'on peut à peu près tout dire?

- Oui, je pense." (Cao)

Cette censure n'est pas ignorée des internautes, même si elle est parfois euphémisée (c'est peut-être un effet de l'entretien). Si certains s'abstiennent de la mentionner, c'est aussi qu'ils font en sorte de ne pas en être l'objet. Mais cela n'est pas un tabou très difficile à percer. La plupart des internautes parlent de cette question de manière relativement directe.

"Mes propres posts n'ont jamais été effacés, ceux des autres le sont parfois, c'est peut-être parce qu'ils écrivent des choses malsaines ou assez sensibles, comme des choses violentes, ou pornographiques, peut être que ça peut être effacé.

- Tu trouves qu'il y a beaucoup de choses effacées?

- Il faut séparer les sites et les forums, parce que sur certains sites de rencontres il y a beaucoup de publicités avec de la violence, de la pornographie, mais pour des forums normaux il n'y en a pas ou très peu.

- Tu trouves que le Web chinois est très contrôlé? 
- Il n'y a pas d'interférences avec les paroles normales, mais si on enfreint les lois du pays ou si on dit sur Internet des choses qu'il est interdit de diffuser, si quelqu'un s'en rend compte alors c'est effacé, et je pense qu'il y a beaucoup de contrôleurs, et qu'ils sont très compétents.

- En général tu te permets de dire ce que tu veux en ligne?

- Si c'est des choses normales, oui." (Pan)

Cette personne ne se sent pas vraiment concernée par la question de la censure, probablement parce qu'elle s'autocensure elle-même suffisamment pour ne pas avoir à être inquiétée. En revanche, sa manière d'aborder la question des sujets "sensibles" en évoquant de manière générale les "lois du pays", tout en circonscrivant ses exemples à la sphère de la violence et de la pornographie, laisse supposer qu'elle a en tête l'ambivalence du contrôle.

Les internautes sont donc généralement très conscients du système de surveillance dont ils sont l'objet. De toute évidence, cette idée même peut encourager une très forte autocensure chez les individus dans leur comportement quotidien. Cela revient à faire peser le contrôle sur les individus eux-mêmes. En effet, les autorités ne manquent pas une occasion de rappeler aux internautes qu'ils portent eux-mêmes la responsabilité de se comporter correctement en ligne. Ils disposent pour cela des chroniques de M. Liu Shinan, dans le très officiel China Daily et qui traite le plus souvent de l'actualité du Web. Pour lui la solution aux incivilités prend son point de départ dans l'éducation des enfants.

Les jeunes sont curieux de tout ce qui est nouveau et ils sont très curieux de tout essayer. Les adultes ne devraient pas essayer de les empêcher d'avoir accès à ces choses sous prétexte que cela pourrait être dangereux, car les interdictions ne peuvent que créer un intérêt plus grand et un désir d'y accéder secrètement. Au lieu de cela, ils devraient autoriser une certaine exposition à ces choses et donner des conseils en même temps. Nous devrions croire en la capacité des jeunes gens à tirer des leçons de la réalité. ${ }^{31}$

Les mauvaises influences dont il parle sont illustrées dans son article par les réponses nationalistes excessives des adolescents suite aux événements tibétains de mars 2008 et au passage de la flamme olympique en Europe et aux Etats Unis. Il trouve que le réflexe nationaliste est un très bon signe, mais que les manifestations ont été mal canalisées. Sa vision

\footnotetext{
${ }^{31}$ LIU Shinan. "Letting the young learn from life", China Daily, 07/05/2008.
} 
est très pragmatique en somme. Puisqu'il est impossible d'empêcher l'accès à tous types de contenus, mieux vaut canaliser, éduquer, rappeler les bonnes règles de conduite, et ce en responsabilisant les utilisateurs eux-mêmes.

En effet Liu Shinan, dans sa conclusion, exprime bien l'idée que le contrôle n'est pas seulement l'affaire des autorités. S'il y a un ordre à maintenir, ce sont les utilisateurs euxmêmes, et en premier lieu les parents, qui doivent s'en charger. La voix officielle met ainsi la question de la civilité en ligne entre les mains des citoyens, faisant d'eux les principaux gardiens d'Internet. Ils ont donc en charge la définition, la transmission, et l'application des bonnes règles de comportement, sur le fond comme sur la forme. C'est en effet chez les internautes que l'on peut détecter la mise en place des règles de fonctionnement les plus opérationnelles. En tant qu'utilisateurs, ils n'attendent pas l'intervention des autorités pour développer des lignes de conduite et se prémunir contre les différents risques qui existent sur Internet.

Les internautes interrogés font très bien écho à cette responsabilisation des individus. Il faut se contrôler soi-même pour pallier les insuffisances du gouvernement.

"Alors il faut te contrôler toi-même, si tu exprimes tes propres opinions sur Internet $[\ldots]$. Là où le gouvernement exerce un contrôle, on ne peut pas dire certaines choses, et ce n'est pas parce qu'Internet n'a pas de limites qu'il faut dire n'importe quoi." (Yang)

La surveillance la plus serrée s'exerce en fait entre individus. Responsables d'euxmêmes, les internautes sont également les surveillants les uns des autres.

"Qui peut avoir accès à mes données personnelles? Je suppose que les administrateurs d'Internet (wangluo guanlizhe) doivent pouvoir atteindre mes données, parce qu'en fait c'est pour que tout le monde soit en sécurité. Aujourd'hui sur Internet il y a des choses comme des sites pornographiques, et si tu fournis tes données, tu ne peux pas propager ce genre de choses. Pour que tout le monde prenne ça en compte, une fois qu'on s'est donné mutuellement nos informations, nos données personnelles, ça crée une forme de contrôle, non?" (Wu)

Le contrôle dont parle cet internaute n'est plus, en fait, le contrôle des autorités. C'est celui effectué par les internautes, sous le regard les uns des autres, et qui deviennent ainsi les meilleurs garants de l'ordre public. Si l'Internet chinois peut être considéré comme un 
panoptique $^{32}$, ce n'est donc pas à cause de l'omnipotence du filtrage et de la surveillance, mais bel et bien à cause de l'omniprésence du regard des autres.

"En général, quand on a envie de s'exprimer, on le fait?

- Oui, en ce qui concerne les critiques sur le gouvernement, les Etats-Unis sont assez libres, et en fait en Chine c'est pareil. Si on n'avait pas cette liberté, on ne pourrait pas lire les informations négatives dont je te parlais tout à l'heure, et je ne pourrais pas faire de commentaires, donc c'est à peu près pareil dans tous les pays.

- Est-ce que le problème le plus important est d'avoir accès à l'information, ou de pouvoir la commenter?

- On peut faire les deux, ce que je veux dire c'est qu'on peut faire des commentaires sur tout. Moi, je ne commente pas ces informations-là, mais je pourrais le faire, personne ne m'en empêcherait. S'ils en parlent, ils ne peuvent pas nous interdire de faire des commentaires." (Zhao)

Ce jeune homme affirme donc que le contrôle par les autorités n'a que très peu d'impact sur sa prise de décision. Il s'attribue d'abord à lui-même la responsabilité de prendre la parole.

C'est cet aspect qui permet de parler d'une "censure 2.0"33 , par analogie avec les utilisations collaboratives de services Internet. L'expression "2.0" désigne la place prépondérante donnée aux utilisateurs dans la mise en ligne des contenus, l'organisation des liens, la hiérarchisation et la mise en relation des informations. Les fournisseurs ne s'occupent plus que de construire des plates-formes vides pourvues de fonctionnalités qui permettent les meilleures interactions possibles. Là où le terme "censure 2.0" est souvent utilisé pour décrire une censure ultra-technologique, il faudrait voir en fait une forme de contrôle de tous par tous, par le simple fait du regard des autres et des normes sociales. La simple interaction entre les individus a un pouvoir coercitif bien plus efficace que la technologie en elle-même.

${ }^{32}$ GIESE Karsten. "Speakers corner or virtual panopticon: discursive construction of Chinese identities online", in MENGIN Françoise (dir). Cyber China, Reshaping national identities in the age of information, New York: Palgrave Macmillan, 2004, p.19-36.

33 MCKINNON Rebecca. "China's censorship 2.0: how companies censor bloggers", First Monday, Vol.14, n², février 2009, consulté le 24/10/2010 sur http://www.uic.edu/htbin/cgiwrap/bin/ojs/index.php/fm/article/view/2378/2089. 
Or les individus, pas plus que les modérateurs, ne disposent de règles écrites et fermes pour savoir comment se comporter sur le Web. Si certains sites publient des chartes de bon comportement, ces chartes restent toujours vagues sur les règles de bienséance politique.

"Dans une dispute il est très facile de blesser quelqu'un, et ce n'est pas bien, que ce soit un proche ou un inconnu, blesser les autres c'est toujours une mauvaise action. Bien qu'on soit anonyme sur Internet, on accède à Internet par des moyens légaux, et ça suffit pour qu'on puisse nous retrouver. Ca peut être très embêtant si ça touche la réputation, ça arrive souvent, on publie toutes sortes d'opinions sur Internet, et ça conduit à des procès. D'après la loi chinoise, bien qu'il n'y ait pas de limite ferme làdessus, peut-être qu'on ne sera pas puni, mais peut-être que si. Pour ces choses-là, rien n'est clair, les conséquences ne sont pas claires. C'est une question d'opinion personnelle, en tant qu'individu il faut assumer les conséquences de nos opinions." (Lin)

L'incertitude sur les règles de droit est un aspect typique des pays autoritaires, car elle incite généralement les citoyens à se montrer plus prudents que nécessaire. En somme, elle rend l'autocensure plus efficace que n'importe quelle censure ne pourrait le faire. Il revient donc à chacun, selon sa sensibilité particulière, d'évaluer ce qu'il est possible ou non de dire ou de faire en ligne, en s'appuyant sur des indices flous que sont la communication gouvernementale d'une part, et les usages des autres internautes d'autre part.

\section{2 - Les règles de la civilité}

\section{L'épouvantail de la criminalité}

Les médias traditionnels ne cessent de mettre en garde les internautes contre de nombreux dangers liés à l'utilisation d'Internet. De fait, les internautes citent aisément ces nombreux dangers, mais ils se sentent personnellement peu concernés par la cybercriminalité, qui reste pour eux très virtuelle.

Pour se repérer, les internautes disposent de quelques règles très générales glanées dans leur environnement, dans la presse, ou sur les chartes d'utilisation des sites qu'ils utilisent. Par exemple les cybercafés affichent souvent de grands panneaux avec une liste d'interdictions à respecter: ne pas consulter de sites pornographiques ou parlant du Falungong par exemple. 
Ces règles restent très générales. Elles reflètent en cela les consignes données en plus haut lieu.

En septembre 2000, le Conseil des affaires d'Etat a établi la première liste formelle du type d'informations interdites:

'1. celles contraires aux principes fondamentaux de la Constitution; 2. celles qui portent atteinte à la sécurité nationale, divulguent des secrets d'Etat, constituent une menace pour le gouvernement; 3. celles qui nuisent à l'honneur et aux intérêts de l'Etat; 4. celles qui incitent à la haine ou à la discrimination ethnique, ou qui vont à l'encontre de l'unité nationale; 5. celles préjudiciables à la politique gouvernementale en matière de religion, qui contribuent à la promotion d'organisations hérétiques, de croyances féodales et de superstitions; 6. celles qui répandent des rumeurs susceptibles de provoquer des désordres sociaux ou de menacer la stabilité sociale; 7. les documents de type pornographique ou à caractère licencieux; ceux qui encouragent le jeu, la violence, l'homicide et la terreur; ou qui incitent à commettre des crimes; 8. les informations insultantes ou diffamatoires, ou qui portent atteinte aux droits et intérêts légitimes d'autres personnes; et 9. toutes autres informations prohibées par la loi ou les règlements administratifs'. ${ }^{34}$

Il y a là des règles de natures très différentes. Les premières, qui semblent prioritaires, sont presque toutes de nature politique, mais elles ne sont définies que de manière générale. En revanche, celles portant sur la pure et simple criminalité ne figurent pas parmi les plus importantes (points 7 et 8). Ce sont pourtant elles qui font l'objet de la communication la plus large dans le presse officielle, fonctionnant comme une sorte d'épouvantail.

A lire les médias chinois, par exemple dans la presse écrite, Internet est véritablement le lieu de tous les dangers. Chaque nouvelle année est l'occasion d'une campagne de communication destinée à rappeler à la fois les dangers que comporte Internet et les mesures sévères prises par les autorités pour y remédier, dans divers domaines ${ }^{35}$.

${ }^{34}$ DOUZET Frédérick. "Les frontières chinoises de l'Internet", Hérodote, ${ }^{\circ} 125$, 2007, p. 127-143.

35 "Chinese police close 598 pornographic websites", 03/01/2006, China Tech News, consulté le 24/10/2010 sur http://www.chinatechnews.com/2006/01/03/3363-chinese-police-close-598pornographic-websites/; "Beijing police: Chinese Internet services frequently used by thieves", China Tech News, 09/01/2008, consulté le 24/10/2010 sur http://www.chinatechnews.com/2008/01/09/6274beijing-police-chinese-internet-services-frequently-used-by-thieves/; BUCKLEY Chris. "China targets big websites in Internet crackdown", Reuters, 05/01/2009, consulté le 24/10/2010 sur http://uk.reuters.com/article/mediaNews/idUKSP36401920090105?sp=true. 
La première et la plus simple mise en garde concerne bien sûr la vie privée. Comme partout, les Chinois qui se font prendre au jeu des confidences sur Internet sont susceptibles de tomber sur des collègues ou amis qui peuvent les reconnaître ${ }^{36}$. La presse ne manque pas de rappeler qu'il est imprudent de trop se livrer en ligne. Bien sûr cela va de pair avec les risques liés aux détournements d'identité, vols de comptes d'utilisateurs par exemple.

"Internet n'est pas sûr du tout.

- Que veux-tu dire par 'sûr'? Qu'est-ce qui te fait dire ça?

- La sécurité c'est par rapport à des pirates informatiques, des gens qui ont des idées derrière la tête, pour le commerce, ou bien des intérêts dans d'autres domaines, et dont l'objectif est peut-être d'obtenir les données personnelles d'un utilisateur. Moi, je suis déjà tombé sur ce genre de situation, donc en général quand ce sont des petits sites, je laisse tomber, je ne donne pas toutes les informations qu'ils demandent." (Xu)

Au-delà de ces dangers qui semblent évidents, la presse regorge d'histoires spectaculaires liées à Internet. Par exemple il est fait grand cas des situations de dépendance aux jeux vidéo qui menacent les adolescents. Cela justifie la mise en place de véritables centres de redressement où les jeunes sont "sevrés" d'Internet comme d'une drogue dure avec des méthodes militaires ${ }^{37}$. Les enfants sont bien sûr une cible prioritaire pour cet effort de communication, car ils sont par définition plus vulnérables que le reste de la population ${ }^{38}$. Les internautes interrogés sont sensibles à ces mises en garde. D'ailleurs chacun connaît plus ou moins bien un adolescent qui délaisse ses études pour jouer en ligne, ce qui constitue pour eux la preuve que jouer en ligne est intrinsèquement dangereux.

"En fait, j'en connais trois ou quatre, qui ont dix-huit ans, ou bien entre dix-huit et vingt ans. Pour jouer à leurs jeux en ligne, ils ne vont plus à l'école, ils ne travaillent pas bien, et ils jouent, comme ça. Tous les mois ils dépensent jusqu'à mille yuans, parce qu'il faut payer la facture de téléphone, car Internet est facturé sur le téléphone, et puis il y a aussi un abonnement pour jouer, et tout ça c'est payant. Donc on peut dire que ça coûte beaucoup d'argent, et en plus, si tu veux atteindre un certain niveau et que tu ne joues pas pendant deux ou trois heures d'affilée, tu ne peux pas l'atteindre, mais

\footnotetext{
${ }^{36}$ CHEN Qimei. "Bie wen wo shi shei, qing ting wo qingsu", Qingnian Zhoukan, 07/09/2006.

${ }^{37}$ SEGRETIN Abel. "L'opium électronique du peuple en Chine", Libération, 22/08/2006.

${ }^{38}$ WANG Xing. "More Chinese children exposed to online danger", China Daily, 03/03/2008.
} 
tu ne peux pas non plus enregistrer ton parcours, donc tu dépenses plein d'argent, et du temps, et aussi du travail." (Deng)

Cette addiction est surtout dangereuse aux yeux de Deng car elle vient s'interposer dans le processus d'éducation des adolescents (qui ont déjà entre dix-huit et vingt ans). Elle leur fait perdre non seulement de l'argent, mais aussi du temps et du travail. Voilà résumées en trois mots les valeurs reines qui constituent l'ambition de cette catégorie de la population. Ce sont précisément les moteurs du développement du pays qui sont foulés aux pieds par ces adolescents. Il est donc d'autant plus justifiable pour cette internaute de lutter contre l'addiction.

C'est pourquoi les fabricants de jeux ont été rapidement sommés de mettre en place des mesures strictes pour empêcher les mineurs de rester trop longtemps devant l'ordinateur, et pour promouvoir une "utilisation responsable" d'Internet. Les utilisateurs doivent donner leur numéro de carte d'identité au début du jeu pour attester de leur âge. Au bout de trois heures d'utilisation, le jeu intime l'ordre à tout internaute mineur de cesser de jouer, faute de quoi les points acquis sont décomptés de leur résultat ${ }^{39}$.

Les jeux sont très souvent la cible des mises en garde concernant la question de la sécurité. Il est vrai qu'ils sont le théâtre de véritables arnaques, comme c'est le cas de tous les environnements virtuels (et réels). Les joueurs achètent par exemple des biens virtuels qui leur coûtent de l'argent réel, et qui leur sont subtilisés en ligne pour être revendus à d'autres ou utilisés sur des sites où ils sont d'une quelconque valeur. C'est le cas par exemple de la monnaie virtuelle QQ, qui sert à acheter des éléments de décoration pour son espace personnel, à offrir des cadeaux à ses amis ou à donner une nouvelle tête à son avatar. Cette monnaie, convertible en monnaie réelle, a fait l'objet de nombreux vols jusqu'à ce que les autorités interviennent ${ }^{40}$.

QQ, par son succès, est tout naturellement l'objet d'un très grand nombre d'actions malveillantes. Outre le vol de monnaie QQ, chaque utilisateur a eu l'occasion de se faire voler son identifiant QQ lui-même, et avec lui toute l'ancienneté qui lui donne des privilèges et du

${ }^{39}$ LEDUC Christian. "Chine: nouvelle loi pour freiner la dépendance à Internet", Branchez-vous! Techno, 18/07/2007, consulté le 24/10/2010 sur http://techno.branchezvous.com/actualite/2007/07/chine_nouvelle_loi_pour_freine.html

40 "Computer game operators unite to fight against online thieves"09/01/2007, China Tech News, consulté le 24/10/2010 sur http://www.chinatechnews.com/2007/01/09/4817-computer-gameoperators-unite-to-fight-against-online-thieves/. 
prestige sur le réseau ${ }^{41}$, cela sans parler des possibilités d'usurpation d'identité que cela donne au voleur.

Les arnaques sont donc nombreuses et les internautes en sont très conscients. La plupart en ont déjà subi et quand ce n'est pas le cas, ils connaissent quelqu'un qui en a été victime, ou alors ils procèdent par analogie avec d'autres types d'abus.

"Une fois j'ai reçu un appel d'un ami, il me dit "tu te souviens de moi?" Sur le coup, j'ai eu peur de lui poser la question, j'ai beaucoup d'amis qui me téléphonent, comment je pouvais savoir qui c'était? Il a dit qu'il était de Canton, il a dit 'qui c'est, devine un peu, je suis qui, de Canton'. Je me suis souvenu d'un ami de Canton. J'ai dit son nom, il a dit 'oui, c'est moi'. Après il a dit qu'il était à Shijiazhuang, juste à côté de Pékin, je lui ai demandé ce qu'il faisait là, et il m'a dit que le lendemain il avait l'intention de venir me voir, pour manger avec moi. Je lui ai dit 'd'accord, viens demain', et j'ai noté son numéro. Le deuxième jour au matin, il m'a rappelé, et il m'a dit qu'il se mettait en route, en voiture, j'ai dit 'd'accord', et une demi-heure plus tard il a rappelé en disant qu'il avait eu un accident sur la voie rapide de Shijiazhuang. Après il m'a encore rappelé, il a dit 'ma voiture a eu un accident, je vais perdre de l'argent, et la police est arrivée qui veut me faire payer, mais je n'ai pas assez d'argent, tu ne peux pas venir me donner un coup de main?' En fait pour ce genre de choses, j'ai dû avoir un réflexe de protection, je me demandais si c'était vrai ou faux, parce que depuis le début je ne l'avais pas reconnu. Heureusement, après j'ai retrouvé le numéro de cet ami de Canton, je l'ai rappelé, il m'a dit qu'il n'était jamais allé à Shijiazhuang, et que c'était un arnaqueur qui m'avait appelé. Ce genre de choses sur Internet il y en a encore plus, parce qu'on ne peut pas voir le visage des gens." (Zeng)

Internet est donc le lieu rêvé pour les arnaqueurs qui peuvent se livrer à toutes sortes de vols et manipulations sous le couvert de l'anonymat. Ils ont aussi dans leur boîte à outils toute une panoplie de virus ${ }^{42}$. Les virus, parce qu'ils sont véhiculés par de nombreux supports et en particulier par les contenus de divertissement, sont une source d'inquiétude très sérieuse. $85 \%$

${ }^{41}$ L'ancienneté d'un utilisateur sur QQ se voit à la longueur du numéro: les numéros très courts valent très chers car ils ont été attribués tout au début du développement du service. De plus le service attribue des statuts particuliers aux utilisateurs selon l'intensité de l'utilisation qu'ils font du service et l'argent qu'ils y ont dépensé. Ces statuts sont représentés par l'attribution de lunes et de soleils qui s'affichent sur le profil de l'utilisateur. Les internautes prennent volontiers contact avec d'autres internautes qui ont le même statut qu'eux ou au-dessus, mais rarement en dessous.

42 "China's anti-cyber virus authorities warn of new virus transmitted by MSN", Xinhua, 14/10/2007, consulté le 24/10/2010 sur http://english.sina.com/china/1/2007/1014/128278.html. 
des ordinateurs chinois seraient infectés par un virus ou un autre selon certaines sources ${ }^{43}$. Par exemple des films aussi controversés - et donc téléchargés - que Lust, Caution de Ang Lee seraient utilisés, selon l'agence officielle Xinhua, pour implanter des virus dans les ordinateurs des particuliers! $!^{44}$ Il est difficile de connaître le degré de véracité de ce type d'informations, ni de connaître véritablement la ou les sources de ces diverses menaces, mais il est facile en revanche de discerner l'influence anxiogène que cela peut avoir sur la population internaute.

Les risques que présente Internet sont en effet nombreux et en même temps difficiles à évaluer, à l'image des virus qui sont invisibles et presque omniprésents. L'utilisation d'Internet en devient presque un acte de foi...

"Donc je trouve que ce dont tu parles, donner son vrai nom ou pas, je trouve que ça s'impose. Peut-être que beaucoup de gens pensent le contraire, ils n'ont pas confiance dans Internet, ni dans les internautes, ils voient surtout le côté négatif, trop négatif? C'est ce que je disais tout à l'heure, les Chinois sont trop extrêmes, s'ils ne sont pas extrêmement radicaux ils sont extrêmement conservateurs, et les gens négatifs ne veulent pas avoir confiance. En fait je trouve que si on fait confiance à quelque chose, cette chose nous donne du vrai, mais si on ne lui fait pas confiance elle ne nous donne jamais que du faux." (Tian)

Comme le souligne l'internaute lui-même, il décide de faire confiance là où les autres sont plutôt inquiets et penchent plus facilement pour une meilleure régulation, pour contrer cette omniprésence (réelle ou imaginée) de la criminalité sur Internet, dont ils peuvent lire des échos quotidiennement dans la presse.

Cette criminalité justifierait donc un contrôle accru des comportements sur Internet, comme en témoigne le sondage de Guo Liang en $2007^{45}$. Plus de $80 \%$ d'internautes y déclarent qu'il est important que certains types de contenus soient contrôlés en ligne. La

${ }^{43}$ YANG Zhao. "Virus informatique: la Chine est la zone la plus sinistrée au monde", 168.fr, 26/07/2007, consulté le 24/10/2010 sur http://www.168.fr/2007/07/26/virus-informatique-la-chine-estla-zone-la-plus-sinistree-au-monde/; "Over $80 \%$ of computers hit by viruses: survey", Xinhuanet, 20/10/2008, consulté le 24/10/2010 sur http://news.xinhuanet.com/english/200310/20/content_1133111.htm.

44 "Lust, Caution utilisé pour diffuser un virus informatique", Xinhuanet, 18/11/2007, consulté le 14/11/2008 sur http://www.french.xinhuanet.com/french/2007-11/18/content_525822.htm. Cette information laisserait presque supposer que l'agence Xinhua tente de faire une mauvaise publicité à ce film par tous les moyens.

${ }^{45}$ GUO Liang. Surveying Internet usage and its impact in seven Chinese cities, Markle Foundation, 2007. 
pornographie, la violence et les spams sont le sujet principal de préoccupation des personnes interrogées. Cependant le nombre de personnes qui déclarent que les contenus politiques doivent être contrôlés a considérablement augmenté depuis 2005, passant de $8 \%$ à $41 \%$ entre les deux sondages, de même que le nombre de personnes qui ont déclaré qu'il faut contrôler plus efficacement les salons de discussion (de 8 à 28\%) et les jeux (de 33 à 60\%). 84\% des répondants ont déclaré que le gouvernement doit avoir la charge de ce contrôle, même si des acteurs privés comme les écoles et les parents doivent exercer une certaine responsabilité. Ces chiffres montrent la demande importante de régulation, ou peut-être l'intériorisation d'une norme collective qui fait de ce contrôle, du besoin de sécurité réel ou imaginaire, une donnée largement partagée.

\section{La civilité au cœur des préoccupations}

Cependant les préoccupations des internautes dépassent la seule question de la criminalité. Celle-ci en effet ne les touche que ponctuellement, marginalement. En revanche tous les internautes sont confrontés quotidiennement à des questions de civilité. Au travers de ces difficultés, la question qui se pose aux internautes est celle du maintien de l'ordre public d'une manière plus générale.

Tout conscients qu'ils sont des risques que comporte en principe la diffusion de données personnelles sur Internet, dans les faits les internautes ne semblent pas si inquiets que cela. La grande majorité des internautes interrogés ont par exemple déclaré acheter ou même vendre beaucoup de produits en ligne: des livres, des vêtements, des produits de beauté par exemple. Pour tous, le commerce en ligne n'est pas une source de danger. En effet, les paiements sont effectués en liquide et seulement à la réception du produit acheté s'il convient à l'acheteur.

De plus, le vol d'un numéro QQ, même s'il est fréquent, est vécu comme un incident sans gravité.

"Je n'ai jamais réfléchi aux questions de sécurité. C'est à dire que si quelqu'un passe par Internet pour obtenir une information sur moi, je ne sais pas, par exemple si on connaît mon vrai nom, eh bien ça ne fait rien, s'ils connaissent mon adresse e-mail ils peuvent m'envoyer plein de trucs, ou bien par mon numéro de téléphone ils peuvent m'appeler, mais sinon ça ne peut pas vraiment aller plus loin. En fait ils ne peuvent pas en savoir vraiment beaucoup sur moi, mais ça m'est déjà arrivé de me faire piquer mon numéro QQ. 
- C'est vrai?

- Oui, et puis j'en ai repris un autre, ça m'a coûté deux yuans depuis mon téléphone pour en avoir un autre, c'est rapide. Mais je me suis rendu compte que j'avais perdu tous mes amis sur QQ, donc j'en ai ajouté quelques uns, mais maintenant je ne vais plus trop sur QQ. Voilà, on me l'a piqué une fois." (Liu)

Il n'y a pas dans le discours de cet internaute de notion de danger, mais bien plutôt celle de désagrément. Il est ennuyeux de devoir reconstituer toute sa liste d'amis, mais cela ne justifie pas pour autant un discours particulièrement alarmiste. En effet, le fait que les personnes restent anonymes et à distance est perçu comme un avantage et un gage de sécurité. Il est toujours possible de se retirer d'une conversation, de s'effacer, pour que le danger disparaisse.

"Oh, il n'y a pas de danger, parce qu'on ne peut pas se rencontrer, il suffit de couper et c'est terminé." (Li)

De plus, lorsque de telles situations se présentent, les gens mal intentionnés sont considérés comme assez faciles à repérer.

"Non, je n'ai pas peur de discuter avec des inconnus sur Internet, parce que quand on discute, on discute assez bien sur Internet, c'est à dire qu'on ne s'entend pas trop mal, et je n'ai jamais ressenti de peur. Et puis en général, je trouve qu'à Pékin [...], quand on se fait de nouveaux amis, il suffit que tout le monde soit vigilant, et il ne se passe rien. Je n'ai jamais rien vu comme ce qu'on entend, comme quoi tous les gens sur QQ seraient des escrocs. Il suffit de les séparer clairement, en général on arrive à faire la différence. Et puis j'ai moi-même créé beaucoup de groupes, et dans ces groupes il y avait beaucoup de gens, tout le monde discute ensemble, et finalement tout le monde devient bons amis." (Deng)

La violence, la criminalité sur Internet sont donc vues comme relativement inévitables, mais non insurmontables. Elles font partie des risques inhérents à toute vie en société, à tout espace accessible à un public relativement vaste. Dans le fond, Internet n'est ni plus ni moins dangereux que la vie réelle.

"Je n'avais jamais pensé au fait qu'il puisse y avoir des risques, même si je sais que sur Internet il peut aussi y avoir des choses négatives, y compris de l'horreur, de la violence, des meurtres, de la pornographie, de la superstition et ce genre de choses, mais je trouve que c'est très difficile à éviter. Mais ça ne peut pas avoir un très 
mauvais impact, c'est à dire que l'impact négatif reste dans le domaine de ce que la société peut supporter. Je crois que pour chaque nouveau phénomène montant, il faut qu'il y ait de nouvelles lois pour le résoudre, et si on s'y prend trop tard, peut-être que les impacts négatifs peuvent être un peu plus nombreux. Mais ce genre de choses n'est pas propre à la Chine, ou au type de réseau qu'on a en Chine, peut-être que ça reste plus sûr que dans d'autres zones. En plus, peut-être qu'à l'étranger, où Internet est encore plus développé, et depuis plus longtemps, peut-être que l'impact est encore plus grand. Par exemple il y a des sites pour se donner rendez-vous pour se suicider, tu sais, on convient d'un moment précis et on se suicide, mais en Chine au moins je n'ai jamais entendu parler de ce genre de sites, pas encore jusqu'à aujourd'hui. Par exemple en Allemagne il y a un médecin qui a dit qu'il voulait manger des gens, il y a aussi des suicidaires, il y a ces suicides, et puis au Japon il y en a qui se sont réunis dans une voiture et ont mis du monoxyde de carbone. Il y a tout ce genre de choses, mais en Chine cela n'est pas encore arrivé." (Zhou)

Comme le fait remarquer L.G., la violence n'est pas propre à l'Internet chinois. Les autorités de tous les pays du monde ont bien des difficultés à contrôler les contenus de leurs parts respectives du Web, pour éviter la recrudescence de comportements violents en ligne, à commencer par la diffamation, les trafics ou vols d'identité par exemple, mais aussi des actes de barbarie facilités par la mise en relation discrète que permet le Web. Les internautes se sentent relativement à l'abri de tout cela car ils pensent qu'ils sont presque intouchables derrière leur écran d'ordinateur dès lors qu'ils ne laissent pas trop d'indications personnelles. Il $\mathrm{y}$ a des règles basiques de sécurité que tout le monde respecte, et qui commencent par ne pas donner son numéro de carte d'identité ou de carte bancaire. De ce point de vue, le fait de rester anonyme en ligne est très rassurant pour les internautes. En un clic il est possible de mettre fin à une conversation dérangeante ou de quitter un site déplaisant. En somme, ils pensent qu'Internet n'est pas une plate-forme si dangereuse que cela.

"Est-ce qu'en général tu trouves qu'Internet est un endroit sûr?

- Comment dire? Oui, ça va, on peut dire que c'est relativement sûr." (Zhu)

En fait, ce qui dérange le plus les internautes au quotidien ne relève pas véritablement du domaine de la criminalité, mais du domaine de la civilité ${ }^{46}$. Ils sont plus souvent dérangés

$46 \mathrm{~J}$ 'entends ici par civilité à la fois les règles de la politesse et plus généralement les règles de comportement qui garantissent la cohésion de la société. PAPACHARISSI Zizi. "Democracy online: 
par des comportements agressifs, du vocabulaire vulgaire ou des images choquantes. Les échanges violents, le vocabulaire cru, les insultes, sont plus préoccupants au quotidien que des questions comme la protection des données personnelles par exemple, sans doute parce que les marques d'incivilité sont présentes dans une grande part des discussions en ligne et qu'il est difficile de les ignorer. Il faut donc apprendre à les réguler, ou à faire avec.

"C'est à dire qu'il y a des gens sur les forums, quand ils font des commentaires, ils disent des vulgarités, moi je ne pourrais pas. Il y a des gens qui disent des gros mots, qui insultent les autres, moi je ne pourrais pas, parce que c'est trop violent, il faut se retenir un peu." (Huang)

Cette vulgarité justifie parfois le fait que les sites mettent en place des systèmes de filtrage du vocabulaire.

"Ce qu'on écrit doit être objectif, ça doit être positif, et sain. Peu importe que ton point de vue soit correct ou faux, je pense qu'il faut respecter les règles. C'est à dire que toutes les phrases moches, si tu postes des images ou des mots pornographiques, je trouve que ces gens-là doivent être effacés, c'est mon avis." (Fang)

De ce point de vue, les systèmes de filtrage automatique peuvent être jugés relativement efficaces.

"Par exemple, quand on dit 'ta mère' (ni ma), ou 'merde'(ta $m a$ ), on ne peut pas taper ces deux caractères ensemble, tu peux seulement les taper séparément, mais pas ensemble, ça ne s'afficherait pas sinon.

- Il n'y a que les gros mots qu'on ne peut pas taper comme ça?

- Pour d'autres choses, je ne sais pas, je n'ai jamais essayé, mais on dirait bien que c'est pour les gros mots, je n'ai rien essayé d'autre. Ça va beaucoup mieux, avant sur les salons de discussion dès que tu rentrais il y avait des insultes, des insultes du début à la fin, maintenant il n'y en a plus." (Zhu)

Cette question de la civilité est d'autant plus préoccupante qu'elle concerne chaque personne, car aucun internaute n'est épargné par l'envie momentanée d'insulter les autres. Chacun doit trouver le moyen de se réfréner afin de conserver des conditions de dialogue 
acceptables. Même la liberté d'expression peut se retourner contre les individus si elle est mal utilisée. Naturellement, être confronté à la grossièreté n'est pas sans faire réagir les internautes, mais il n'est pas si facile de conserver son flegme devant des idées choquantes.

"Tu trouves qu'il y a trop de liberté d'expression?

- Je ne sais pas si c'est bien ou pas, des fois je trouve que quand j'ai des problèmes avec mon école, bien sûr je peux en parler, avec mon propre point de vue. J'ai envie de parler d'eux, j'ai même envie de les insulter, de dire des choses très moches, je suis très en colère. Mais après je me dis que la liberté d'expression c'est quand même très bien, tout ce que j'ai envie de dire je peux l'exprimer." (Liu)

En fait les insultes ne seraient peut-être pas un problème si elles ne contribuaient pas à polluer la source d'informations que représente Internet. Aussi le maintien de l'ordre est-il nécessaire pour préserver le potentiel d'efficacité et de développement que représente Internet.

"Par limites, tu veux dire, se demander si on envoie un commentaire ou pas, si on doit faire quelque chose ou pas? Je trouve qu'il faut mettre des limites, je trouve que ça a quand même des avantages. Si on envoyait trop de choses, sans que ce soit géré, ce serait le désordre (luan), et donc comment dire? Tel que c'est maintenant, ça peut créer une mauvaise influence sur les gens, Internet peut encourager la fausseté (shizhenxing). Il n'y a pas d'information utile, ça fait perdre beaucoup de temps à tout le monde, donc il vaut encore mieux qu'il y ait des gens qui effacent ces choses-là immédiatement. Je crois qu'il faut effacer les posts inutiles, c'est ce que je pense. Les posts inutiles font perdre du temps de lecture à tout le monde. Et puis apparemment nos profs ont tous le wi-fi sur leur ordinateur portable, en général. Il y a des machines dans toutes les salles de classe. Quand les profs ont besoin de quelque chose ils peuvent le vérifier tout de suite. Mais quand on cherche sur Internet on n'est pas sûr du résultat. S'il n'y avait vraiment pas de contraintes, s'il y avait tout sur Internet, ça aurait de très mauvaises conséquences sur tout le monde. Mais en Chine aujourd'hui il y a certainement encore des contraintes, il y a des choses qu'on ne peut pas publier sur Internet, ces choses ne nous servent sûrement à rien. Tout le monde peut aller sur Internet, et ça nous économise du temps de lecture, et on trouve des documents plus efficacement. Je trouve que c'est bien, il n'y a rien à redire." (Liu)

Ici l'efficacité et le gain de temps sont prioritaires par rapport à d'autres fonctions de la communication, et cela justifie un contrôle qui peut aller jusqu'à l'effacement des contributions "inutiles". Dans ce discours, le désordre (luan) est plus préjudiciable que la 
censure. Il est vrai que ce désordre est généralement attribué aux catégories les "moins avancées" de la population.

Au-delà du vocabulaire et de la politesse, c'est ainsi toute une attitude, une forme de socialisation que les internautes souhaitent promouvoir. En dénonçant les mauvais comportements, ils tracent en fait le dessin en creux d'un mode de vie, d'une culture trop populaire, pas assez lissée, normée, en somme pas assez "civilisée". Par contraste, les normes qui devraient encadrer le comportement de tous sont directement liées à l'image de ce qu'est un citoyen "moderne" et "de qualité".

Tout d'abord l'incivilité, aux yeux de certains internautes interrogés, provient du fait de la popularisation d'Internet et de son ouverture à des publics moins bien sélectionnés, moins bien éduqués. En d'autres termes Internet, qui est l'apanage d'un certain mode de vie moderne, est menacé par l'ouverture à des catégories de la population qui ne partagent pas ce mode de vie ni les valeurs qui lui sont attachées. Ce mode de vie semble dépendre essentiellement du niveau d'éducation.

"Le développement d'Internet en Chine s'est fait selon un processus en étapes. Qu'est-ce que j'appelle un processus en étapes? C'est, disons, l'apport du haut débit. Il $\mathrm{y}$ a dix ans, plus de dix ans, quand je venais à peine de commencer à utiliser Internet, pour aller sur Internet on utilisait des modems analogiques, la vitesse était de 33,6k. Et à ce moment-là les ordinateurs n'étaient pas très répandus en Chine. A ce moment-là les gens qui avaient les moyens d'aller sur Internet (you tiaojian shang wang de ren), bon ce n'est pas une loi absolue, mais en gros c'était surtout les gens qui avaient de l'éducation, ou bien au minimum des familles éduquées, et les gens ordinaires n'en avaient pas les moyens. Sur Internet il n'y avait personne, il n'y avait pas ce, enfin les gens trop ordinaires, parce qu'ils ne pouvaient pas supporter le coût d'Internet et les dépenses sociales. A ce moment-là je trouvais que les gens discutaient de manière assez pure (chuncui), parce qu'il y avait peu de gens, peut-être qu'il n'y avait pas le genre de tromperie dont tu parles." (Zhang)

En même temps, Internet est lui-même un vecteur de diffusion de ce mode de vie "moderne", et il contribue à l'intégration progressive des normes par l'ensemble des utilisateurs.

"Donc dans ce contexte, tu me demandes si sur Internet on peut tomber sur des arnaques, c'est certain qu'il y en a. Mais après cette étape, depuis peut-être trois à cinq 
ans, je trouve que ça s'est amélioré à nouveau. Parce qu'Internet n'est plus une curiosité pour les gens, on ne le regarde plus avec l'attitude qu'on a devant les nouveautés, les gens s'y sont adaptés progressivement, en un peu plus de dix ans, et même en quelques années, Internet s'est diffusé dans tous les aspects de nos vies." (Zhang)

Les internautes chinois, pour Zhang, se sont donc habitués à cet outil, ils s'y sont adaptés. Ils se sont même "civilisés". Est-ce la raison pour laquelle les situations d'incivilité font émerger chez tous les répondants un champ lexical normatif très unifié? Anecdote après anecdote, les répondants font part des mêmes préoccupations, celles de trouver un environnement "civilisé" en ligne. Ce faisant ils tracent une ligne de séparation entre leur système normatif à eux, et celui des autres, qui sont moins éduqués, moins polis, moins "civilisés", en somme de "faible qualité" (suzhi di).

"Les gens l'insultent avec des mots très sales, ils ne regardent même pas la nature de cet homme, tu vois? La seule impression que ces gens m'ont donnée c'est de libérer leur colère violente, ils ne peuvent pas réussir à discuter vraiment de quelque chose. Pourquoi est-ce que je dis que je ne me fais pas d'amis sur Internet, c'est parce que je pense que si on a des émotions à libérer, ce n'est pas le bon moyen de le faire, il faut être un peu plus civilisé.

- Qu'est-ce qu'il faut faire alors?

- Tu peux exprimer ta propre opinion sur ce sujet, mais il ne faut pas attaquer la personnalité ou le physique de cette personne. Donc les gens qui répondent sur les forums aujourd'hui sont d'une qualité assez faible (suzhi bijiao di)." (Wu)

Autant que la vulgarité, les attaques personnelles font partie des comportements répréhensibles. C'est l'apanage d'une certaine catégorie de la population qui se trouve ainsi sévèrement stigmatisée. Il est courant que les gens soient ainsi classés en bons ou mauvais, de "bonne" ou de "mauvaise qualité", ce qui représente souvent le niveau d'éducation ou le niveau culturel qui leur est prêté. Il semble que la présence sur Internet de ces individus, dont les modes d'expression ne correspondent pas aux canons de ce comportement "civilisé", représente une source d'inquiétude bien plus quotidienne, plus diffuse et plus présente pour les personnes que j'ai rencontrées, que la criminalité en tant que telle.

"Il faut élever la qualité de la nation.

- C'est à dire? 
- Par exemple si on ouvre un nouveau site, on est obligé de le contrôler de manière très serrée. C'est à dire que pour la catégorie des commentaires, je pense qu'on peut contrôler complètement les posts. Il n'y a aucun contenu essentiel, ce n'est que du défoulement (xuanxie). On n'est pas obligé de les laisser commenter, on les passe directement, on n'est pas obligé de demander leur accord, on efface directement et puis c'est tout. S'il y a moins de choses, les gens feront spontanément plus attention. Ils se maîtriseront, ils n'enverront pas n'importe quoi, non? Si on les laissait sans aucune gestion, ils feraient ce qu'ils veulent, ils se laisseraient aller (fangren de xinli), non? Pour l'instant, quoi qu'on fasse il ne se passe rien, si j'insulte quelqu'un, eh bien je l'ai insulté. Je trouve que le site Internet se fait du tort en ne prenant pas ses responsabilités. Au bout du compte le site est très sale, et une fois qu'il est sale une certaine couche de gens (yi bufen cengci de ren) ne va certainement plus venir sur ce site. Ils vont penser que ce qu'ils voient ne leur correspond pas (bu shihe ziji), non? Il $\mathrm{y}$ a des gens qui vont y aller, et pour quoi faire? Parce qu'ils aiment voir ces choses-là, ils aiment voir des insultes, ils s'ennuient à ce point-là, dans les cybercafés. Dans les cybercafés, une partie des gens discutent, une autre partie des gens jouent en réseau, et tous ceux qui restent sont là pour insulter des gens, oui ou non? Leur niveau culturel est bas, ils n'ont même pas le brevet." (Wu)

Contrairement à Zhang cité précédemment, $\mathrm{Wu}$ fait partie des internautes les plus conformistes de l'échantillon. Son discours reprend très souvent les grandes lignes de la communication officielle. C'est également le cas de M.N, qui explique aussi les désordres d'Internet par la grande liberté qui y règne et par le mélange des couches sociales, ce qui a pour lui conséquences ambivalentes.

"L'avantage d'Internet c'est la quantité d'informations, c'est libre, c'est pratique, et si on dit des saletés, ils ne font pas la différence entre les âges, entre les couches, tout est possible. Ce n'est pas bien pour les enfants, ils sont fascinés par ces choses-là. S'ils ne sont pas capables de supporter tout ça, ils peuvent prendre de mauvaises habitudes, c'est très mauvais, et on ne peut pas le contrôler." (Chu)

Le fait que les répondants les plus conformistes partagent un même discours avec l'un des internautes les plus critiques est significatif. Tous identifient l'ordre aux valeurs de la classe moyenne, à un certain niveau d'éducation, à une maîtrise des règles de civilité, et, en somme, à une certaine capacité d'autodiscipline.

Il y a donc un effet d'imposition d'un cadre normatif par les internautes les uns sur les autres. Bien sûr, ce cadre n'est pas indépendant des contraintes imposées par les autorités, qui interdisent explicitement de nombreux sujets de conversations en ligne. Il est directement issu 
$\mathrm{du}$ discours modernisateur dont l'Etat contribue à fixer les grandes orientations. Cependant le discours des internautes sur les règles de civilité couvre en fait des comportements qui dépassent de très loin le champ d'application de la censure et de la répression proprement dites. Il s'appuie de manière générale sur une conception de la modernité, principe de justification $^{47}$ qui couvre presque toutes les dimensions des activités en ligne.

Cela contribue, en particulier, à circonscrire la parole critique des individus ordinaires à l'intérieur d'un champ considéré comme privé, tandis que la parole publique est réservée à une représentation consensuelle de la nation.

${ }^{47}$ BOLTANSKI Luc et THEVENOT Laurent. De la justification. Les économies de la grandeur, Paris: $P U F, 1987$. 


\section{Chapitre V - La clôture du domaine public}

Les contraintes qui s'imposent aux internautes au moment de prendre la parole sont le plus souvent présentées comme relevant du domaine de la civilité. Il incombe aux citoyens qui veulent se considérer comme "avancés" ou "modernes" de respecter certaines règles fondamentales pour le maintien de l'ordre public ou, pour reprendre le vocabulaire le plus souvent utilisé, d'adopter en public un comportement "responsable".

Ces règles consistent essentiellement à respecter une séparation très politique entre parole privée et parole publique. Arguant de l'idée que toute expression représente de manière partiale les intérêts de son émetteur, les internautes justifient un certain contrôle sur la représentation de l'intérêt collectif. Pour donner à l'extérieur une image d'unité, les individus s'abstiennent de rendre publiques les dissensions qui sont, selon eux, le propre d'une société plurielle, diversifiée et de plus en plus stratifiée.

Chacun est ainsi sommé de se cantonner à l'expression de ses sentiments personnels, sans empiéter sur ce qui peut toucher d'autres personnes, c'est à dire sans se mêler de questions d'intérêt général d'une part, et sans entrer en conflit avec d'autres internautes pour essayer de les convaincre d'autre part. Le politique se trouve ainsi exclu de la prise de parole publique.

\section{1 - Une fragile unité nationale}

\section{Le discours de la "société harmonieuse"}

Lorsque les internautes voient leurs commentaires effacés sur un forum, ils disent qu'ils "ont été harmonisés" ${ }^{1}$. Ils décrivent avec une pointe d'humour la censure bien réelle dont ils sont l'objet, en détournant le mot d'ordre de créer une "société harmonieuse" lancé par le gouvernement actuel. La dérision souligne ici la présence des contraintes imposées d'en haut dans l'esprit des internautes, et surtout la distance que certains prennent avec ce mot d'ordre officiel. En même temps, et non sans paradoxe, ces contraintes sont reprises, reformulées et

\footnotetext{
${ }^{1}$ XIAO Qiang. "Under the Internet police's radar", China Digital Times, 28/08/2007, consulté le 24/10/2010 sur http://chinadigitaltimes.net/2007/08/under-the-internet-polices-radar/.
} 
justifiées dans un discours laudateur sur les avantages de l'unanimité, surtout dans le domaine de la politique.

"En politique l'unanimité (yizhixing) c'est très bien, pas comme dans les pays occidentaux où peut-être que ce n'est pas la même chose." (Ren)

Plusieurs types d'explications sont proposés par les internautes interrogés pour justifier cette contrainte. En premier lieu on évoque le facteur culturel. Les personnes interrogées évoquent la nécessité de faire attention, de parler dans des termes autorisés par les autorités, c'est à dire en concordance avec le ton des médias officiels. Dans les domaines les plus sensibles politiquement, il faut "jouer la mélodie de l'harmonie".

"Les dirigeants du pays c'est sûr tu n'as pas le droit d'en parler, tu ne peux pas dire n'importe quoi sur les dirigeants nationaux, dans ton mémoire il ne faut surtout pas que tu impliques des choses sur les dirigeants du pays. Je trouve qu'en Chine ce n'est pas possible, en Chine on ne peut surtout pas parler de tout ce qu'on a envie de critiquer. Quand tu es en Chine il ne faut surtout pas en parler, ce n'est pas comme à l'étranger, si tu veux contredire tel ou tel thème (zhuzulu, mélodie, thème), ce n'est pas possible. C'est comme toi qui écris ta thèse, en Chine quand on écrit une thèse il faut être dans le ton, écrire les articles en accord avec le thème, il ne faut pas quitter le thème. La Chine cherche à atteindre la société harmonieuse, il faut que tu fasses bien attention à savoir si c'est l'harmonie d'Internet, l'harmonie de la Chine, il faut écrire en s'habillant des couleurs de l'harmonie. C'est ça la culture chinoise, on ne peut rien y changer, tout le monde est comme ça." (Chu)

La "culture chinoise" est ici utilisée comme justification pour ce qui est tout d'abord une règle politique posée par les autorités. Dans ce contexte, il est bien plus facile pour des utilisateurs d'Internet de publier des opinions conformistes que de tester des idées originales, ce qui pourrait représenter le risque de "jouer une autre mélodie". La métaphore musicale est utilisée ici pour donner un air naturel aux limites de la liberté d'expression. Dans l'explication de $\mathrm{Chu}$, ces limites font partie de la culture chinoise et ne peuvent pas être remises en question puisqu'elles sont inscrites dans une sorte de code génétique de la société chinoise. Quel que soit le degré de sincérité de cette affirmation, l'effort rhétorique fourni pour donner une légitimité à cet état de fait montre l'appropriation de ces règles par l'interviewé. Il semble appliquer lui-même le principe de l'unanimité dans son discours. 
Un élément frappant au cours des entretiens est justement l'impressionnante cohérence qui s'en dégage, pour ne pas dire la prévisibilité de certaines répliques comme le classique "les Chinois sont trop nombreux" ou "la Chine est un grand pays" par exemple. Ce modèle, qui valorise l'unanimité, semble être mis en application directement dans les entretiens, qui sont souvent vécus comme une autre façon de prendre la parole en public. En effet, répondant à une personne étrangère, pour des besoins de recherche, c'est à dire dans l'optique d'une diffusion même restreinte, les répondants ont conscience de s'adresser à un public extérieur.

Cette situation d'entretien est ambivalente. Il faut signaler ici la longue conversation que j'ai tenue avec l'un des répondants, après la fin de l'enregistrement et de l'entretien proprement dit. Poussant un soupir, ce dernier m'a confié sa satisfaction de pouvoir partager ses pensées avec une "sociologue étrangère", car à ses yeux ses paroles ne seront pas interprétées de la même manière que si elles étaient recueillies par un chercheur chinois. Quelque sentiment de proximité que je puisse créer, la plupart des internautes interrogés ont l'impression de parler publiquement, et ils répondent en tant que représentants de leur pays, en tant que Chinois. D'un côté, les internautes interrogés se sentent donc en devoir de donner une bonne image de leur pays, et d'un autre côté ils sont amenés à prendre un recul qu'ils ne s'autoriseraient peutêtre pas avec un concitoyen.

"En ce moment tu es une amie étrangère, je suis un Chinois, et je te parle des aspects sombres de la Chine, je peux te parler de ce point de vue là, ce qui montre que l'expression est libre en Chine." (Zhao)

Le soutien de la notion d'unanimité est donc presque unanime lui-même dans le discours public, et il est presque unanimement mis en actes dans la situation de l'entretien. Je n'ai aucun moyen de mesurer le degré d'adhésion profonde de tous ces individus à ce discours. Il est probable que nombre d'internautes prennent des distances par rapport à cela dans leur for intérieur. Cependant, dans la situation particulière que représente l'entretien, seules une ou deux personnes ont émis des réserves à des degrés divers. Si cette adhésion n'est pas forcément profonde, elle s'avère efficace, au moins de manière performative.

Or la répétition constante des bienfaits de l'unanimité se heurte constamment à l'affirmation inverse selon laquelle les Chinois, selon leur origine et leur catégorie sociale, ont très peu d'intérêts en commun. La contradiction semble d'autant plus paradoxale que ces affirmations sont juxtaposées au sein même des entretiens. 


\section{L'impossible représentation}

Comme je l'ai précisé plus tôt, le processus de modernisation de la nation dans son ensemble s'accompagne d'une stratification très forte de la société, dans les faits et surtout dans les mentalités. Il en résulte que les internautes conçoivent comme un fait évident que les différentes "couches" de la société aient des intérêts contradictoires. La société n'étant à leurs yeux que conflits et luttes de pouvoirs, il leur semble logique, presque naturel que chaque groupe ait des intérêts spécifiques.

"Non, on ne peut forcément pas parler de tout, je trouve que certaines personnes peuvent représenter un groupe, c'est à dire qu'ils représentent un groupe déterminé, comme une activité professionnelle, peut-être que quelques personnes peuvent représenter les ouvriers, d'autres peuvent représenter les paysans, et d'autres peuvent représenter les cols blancs, mais ceux qui parlent au nom de la couche des cols blancs ne peuvent pas représenter les citoyens des couches plus basses de la société, ça c'est sûr, et puis les citoyens de tout en bas ne peuvent pas représenter des couches élevées comme les cols blancs, c'est certain qu'ils ne peuvent pas se représenter mutuellement." (Zhang)

Les différents internautes n'utilisent pas toujours les mêmes critères de stratification. Les uns découpent la société en classes sociales d'inspiration marxiste (ouvriers / paysans / cols blancs), tandis que les autres choisissent des critères plus spécifiques, comme le style de vie, ou le niveau d'éducation par exemple, qui sont plus ou moins directement liés à la définition mouvante de la classe moyenne en construction.

"Vu que tout le monde a des points de vue différents sur chaque problème, et que tout le monde a des habitudes de vie, des niveaux d'éducation différents, on ne comprend pas les choses de la même façon, donc c'est difficile de dire qu'on va commenter, peut-être que des fois tu trouves que ce n'est pas vrai, tu trouves que ce n'est pas correct, et quelqu'un d'autre trouve que c'est correct, il trouve que c'est bien, tout est possible, je trouve que n'importe quelle chose a deux faces, il ne peut pas y avoir un seul avis sur une chose." (Shao)

On peut discerner dans cette présentation une double tendance à la singularisation, par la diversification des modes de vie, d'une part, et à la hiérarchisation, au travers des niveaux d'éducation, d'autre part. Dans cette vision d'une société différentiée et hiérarchisée, les citoyens sont automatiquement considérés comme corporatistes, égoïstes, en tous cas 
partiaux. Chaque catégorie est considérée comme ayant ses propres intérêts, qui sont nécessairement contradictoires avec ceux des autres catégories, sans que ces catégories soient toujours solides ni pérennes. Chacun, au fond, ne représente que lui-même avec ses particularités, et nul ne peut prétendre représenter les intérêts d'autres personnes.

"Dans les commentaires, chacun a son avis, on ne peut pas prendre la place des autres, ni attendre des autres qu'ils parlent pour nous." (Luo)

Cette vision d'une société morcelée n'est pas contradictoire avec la prétention à l'unanimité, mais bien au contraire elle la justifie. A l'incommensurabilité des différences entre les individus correspond pied à pied le discours sur la cohésion nationale et l'unanimisme de la nation chinoise. L'individu est coincé entre des particularismes au nom desquels il peut s'exprimer et l'identité nationale qui ne doit pas souffrir la discussion, puisque nul n'est habilité à représenter les autres ni la nation tout entière. L'insistance sur l'unanimité et l'harmonie n'est donc qu'une réponse à la segmentation de la société, un ersatz de liant pour cette société qui ne peut se tenir ensemble autrement. Globalement, la présentation de la société, morcelée, conflictuelle, où chacun ne peut parler que pour ses intérêts propres, et qui doit taire ses conflits, a des accents très hobbesiens ${ }^{2}$.

En effet, les internautes interrogés semblent résoudre la question de la multiplicité des intérêts particuliers en affirmant la nécessité d'une représentation publique unique et non contredite de la nation, et qui soit indépendante des positions individuelles de chacun. L'ensemble des justifications que les internautes emploient pour valoriser la notion d'unanimité pourrait donc ressortir d'une forme de "solidarité négative" ${ }^{3}$, une forme de coopération qui permet de neutraliser les nécessaires antagonismes de la société en les passant

2 "Une multitude d'hommes devient une seule personne quand ces hommes sont représentés par un seul homme ou une seule personne, de telle sorte que cela se fasse avec le consentement de chaque individu singulier de cette multitude. Car c'est l'unité de celui qui représente, non l'unité du représenté, qui rend une la personne. Et c'est celui qui représente qui assume la personnalité, et il n'en assure qu'une seule. On ne saurait concevoir l'unité dans une multitude sous une autre forme." HOBBES Thomas. Léviathan : traité de la matière, de la forme et du pouvoir de la république ecclésiastique et civile, Paris : Sirey, 1971, p. 166.

3 "Là où Locke, 'père spirituel des Lumières', voit une solidarité positive fondée sur la citoyenneté (idéale) d'individus moraux oeuvrant en fonction de l'intérêt général, je décris une solidarité négative fondée sur l'atomisme foncier des relations sociales: contrairement à la vision holiste, héritée de maints travaux d'orientalistes, les comportements référés à l'Islam ne sont en rien, ici, des actualisations de la communauté des croyants (umma) et de la solidarité communautaire. Ils sont, tout au contraire, des comportements d'évitement et de moindre coût." FERRIE Jean-Noël. Le régime de la civilité en Egypte, Paris: CNRS Editions, 2004, p.124. 
sous silence. Comme le montre Ferrié dans le cas de l'Egypte, une telle coopération ne s'accommode pas d'une expression publique du pluralisme, et elle suppose de renvoyer l'expression des différences dans la sphère du privé.

\section{2 - Parole publique et représentation}

\section{La parole publique comme représentation de la nation}

L'unanimité et la cohésion qui ne peuvent exister au plan des intérêts particuliers est défendue au plan de la représentation, de l'image. La transgression suprême consiste à mettre au jour les dissensions de la société face à des observateurs extérieurs. C'est vrai pour Internet tout autant que pour d'autres médias qui peuvent tenir lieu de vitrine de la société, comme par exemple le cinéma ou la littérature.

Ecrire sur l'identité ethnique en s'écartant du dogme officiel de l'unité nationale constitue certainement le Rubicon le plus manifeste de tous. [...] les prémisses dont découlent les jugements du Comité ou du Bureau révèlent aussi un intéressant mélange idéologique dans lequel on retrouve des éléments de la pensée marxiste traditionnelle (les personnages ou les scénarios doivent représenter une classe ou une nation, ou mettre en scène une forme de lutte entre classes ou nations) et d'autres, appartenant à une nouvelle forme de pensée 'harmonieuse'. Les conflits entre les riches et les pauvres, entre les parents et les enfants, ne doivent pas être mis en avant; les travailleurs migrants doivent être dépeints avec compassion, et non en tant que maîtres-chanteurs potentiels; la nouvelle bourgeoisie peut être montrée, même dans ses aspects les plus 'décadents', mais seulement si le film émet un jugement moral explicite à son égard. L'idée selon laquelle les films chinois 'représentent' la nation d'une manière ou d'une autre reste également répandue ${ }^{4}$.

Le rôle que les internautes chinois attribuent aux médias en général est singulièrement différent de celui qui leur est généralement attribué dans les différents modèles démocratiques occidentaux. En particulier, la presse n'est pas conçue comme un contre-pouvoir, un outil pluraliste indispensable à la construction d'une opinion publique démocratique, mais au contraire comme une forme de représentation, de communication ou de propagande (ces deux derniers mots se traduisent de la même manière: xuanchuan). En dépit des importantes

${ }^{4}$ VEG Sebastian. "Harmoniser les dissensions, exemples récents de censure dans la littérature et le cinéma chinois", Perspectives chinoises, ${ }^{\circ} 100,2007 / 3$, p.70-77, p.76. 
transformations qui ont affecté le secteur des médias depuis vingt ans, et des changements de rapports de forces qui en découlent ${ }^{5}$, les internautes interrogés continuent de concevoir les différents organes de presse comme la voix du Parti. Le travail de propagande effectué par les médias, au premier rang desquels figure le Quotidien du Peuple, est toujours d'actualité, même s'il peut sembler anachronique ${ }^{6}$. Cette conception sous-tend aussi la formation et l'accréditation des journalistes. Les journalistes doivent suivre des formations spécifiques, en particulier de politique ${ }^{7}$, et leur travail est bien sûr très strictement contrôlé. Seule cette accréditation officielle leur permet d'obtenir une carte de presse.

C'est à cause de cette différence de conception qu'une journaliste du International Herald Tribune fut prise à partie lors d'une conférence sur les médias à la London School of Economics par exemple. On lui reprochait de ne pas tenir compte des sentiments des Chinois lorsqu'elle écrivait sur des sujets qui pourraient les blesser dans leur fierté nationale, ou menacer la stabilité du pays, comme les événements tibétains de mars 2008. Elle témoigne dans son blog du malentendu qui avait causé ce reproche.

Quand j'ai dit que nous [médias occidentaux] ne tenions pas compte de la "stabilité sociale", je parlais de ne pas me plier à l'autocensure des mauvaises nouvelles que le Parti Communiste pourrait trouver "déstabilisantes". Cela n'induisait pas de ma part un manque de sympathie personnelle ou de sens de la responsabilité envers les gens dont nous parlons. ${ }^{8}$

Pour la plupart des internautes, ce rôle de représentation n'est pas spécifique à la presse chinoise, bien au contraire. C'est un phénomène universel et partout, la nécessité d'offrir une image unifiée du pays justifie des limitations à la liberté d'expression.

${ }^{5}$ LEE Chin-Chuan (dir.). Power, money, and media. Communication patterns and bureaucratic control in cultural China, Evanston: Northwestern University Press, 2000.

${ }^{6}$ BANDURSKI David. "Is Communist Party 'propaganda' a relic of China's past?" China Media Project, 15/05/2009, consulté le 24/10/2010 sur http://cmp.hku.hk/2009/05/15/1627/.

${ }^{7}$ Sur la manière dont les cours de politique contribuent à structurer le domaine officiel et les rôles qui s'y jouent, voir ARSENE Séverine. L'enseignement idéologique dans les universités chinoises, mémoire de Master Recherche, Paris: Institut d'études politiques, 2005. Les étudiants, eux aussi, sont encouragés à considérer leur responsabilité spécifique lorsqu'ils parlent en public.

8 HOR-CHUNG LAU Joyce. "God help India's terror victims. More random bits from the news room", Joyceland, 27/11/2008, consulté le 24/10/2010 sur http://joycelau1.spaces.live.com/blog/cns!DFE95C9AB5B43908!1601.entry. 
"C'est vrai qu'on ne peut pas dire tout ce qu'on veut, bien sûr. Mais [...] à l'étranger non plus on ne peut pas dire tout ce qu'on veut. En fait c'est comme ça dans n'importe quel pays, on protège partout la classe dirigeante. En Chine on se dit que les journalistes occidentaux répondent aux attentes de leurs gouvernements, ils leur rendent service, quand ils disent que l'expression n'est pas assez libre en Chine. Par exemple je me souviens qu'une fois la BBC avait couvert le suicide d'un ministre, très en détails, cette information a été récupérée. A l'époque Blair était au pouvoir, ils ont beaucoup manipulé l'information et à l'époque de Bush il y a aussi eu des cas de manipulation de l'information. Ce genre de situations montre clairement que tout le monde ne parle pas du tout de la liberté d'expression, mais ils remplissent tous des exigences politiques. En Chine aussi on répond certainement aux mêmes exigences. C'est pour ça que tous les journalistes étrangers critiquent la Chine en disant que l'information n'est pas libre. Comme la population chinoise est trop grande, et que les Chinois sont trop nombreux, ça donne l'impression que dès qu'on publie une information, le gouvernement sort une politique pour limiter beaucoup de gens. En fait c'est parce qu'on est très nombreux qu'ils limitent beaucoup de gens. S'il n'y avait qu'une seule loi sur l'information pour le monde entier, elle limiterait l'expression des gens du monde entier, ce serait la même chose. Donc libre ou pas libre c'est relatif, ça n'est pas absolu." (Zhao)

Les restrictions à la liberté d'expression sont considérées comme inhérentes à cette fonction de représentation de la presse. Le rôle de représentation que jouent les médias entraîne donc une "responsabilité" particulière de la part de tous les acteurs qui ont la possibilité d'y prendre la parole. Or Internet fournit des espaces d'expression aux formes très variées, dont une petite partie seulement est dédiée à traiter de problèmes publics. Beaucoup de blogs ou de forums ont un statut ambigu, traitant tour à tour de sujets très personnels ou plus collectifs. La dimension de représentation ne se déduit donc pas automatiquement à partir de la seule prise de parole. Elle oblige les internautes à se doter d'une définition plus fine de cette forme de publicité bien particulière.

\section{Les contours politiques de la parole publique}

Dans le discours des internautes, il semble y avoir plusieurs formes de prise de parole, qui ont des statuts différents, certaines étant plus publiques et d'autres plus privées. Les 
journalistes semblent dépositaires d'une forme de parole publique lourde de conséquences, ce qui distingue en quelque sorte leur parole de celle des autres internautes.

"Non, ça ce sont les sentiments des journalistes, c'est ce qu'ils expriment. Alors que les blogs, les forums ce sont nos sentiments à nous. Rapporter des informations c'est leur métier à l'origine, mais je trouve que ce qu'ils rapportent n'est pas forcément vrai. A Pékin le dernier truc c'est l'affaire des baozi, c'était une fausse information, il avait fait un faux reportage, il n'avait pas compris la situation en profondeur. Aujourd'hui les infos sont explosives, donc ces gens-là ne prennent pas leurs responsabilités." (Yang)

Ici, la parole des journalistes est séparée de la parole des internautes ordinaires ("nous"), car elle est porteuse d'un statut et d'une responsabilité spécifiques. Le fait de présenter la responsabilité des journalistes en mentionnant le caractère "explosif" des informations suppose que le contrôle s'étende bien au-delà de la seule vérification des faits. Compte tenu de ce rôle public, les journalistes sont désignés comme les premiers sur qui repose la stabilité et la cohésion de la société. Ils doivent donc éviter de publier toute information qui pourrait attiser des tensions. Ils sont, en ce sens, considérés comme les portes-parole du gouvernement, ce qui donne à leur parole un statut officiel, par opposition à ce qui est de l'ordre du "divertissement".

"Ce sont les informations qui ont un impact sur la société et qui sont publiées par des journalistes, je trouve que c'est ça les informations gouvernementales (zhengfuxing xinwen), ce qui concerne le pays, ce qui touche les problèmes de société, ce qui a un impact. En fait sur le divertissement, qu'il y ait des erreurs ça n'a pas d'importance, tout le monde sait que le divertissement de toute façon c'est un peu faux.

- Comment contrôler ces informations qui ont un impact?

- On sait bien que dans le système de surveillance des services du gouvernement il y a des fuites, et s'il n'y en avait pas, ce genre de choses n'arriverait pas. Donc maintenant que c'est arrivé, le mal est fait, c'est un peu tard, ça a déjà des conséquences sur ce que pensent les gens ordinaires. On ne peut pas revenir en arrière, donc les conséquences négatives sont plus importantes que les conséquences positives. A l'origine la société est très calme, elle donne à tous une certaine stabilité de vie, un sentiment de sécurité. Quand on fait un reportage comme ça tout à coup, ça détruit le sentiment de confiance chez les gens." (Yang) 
Le fait que les internautes distinguent de manière spécifique le travail des journalistes montre que toutes les formes de prise de parole n'ont pas le même statut sur Internet, ce qui en fait peut-être la spécificité. Lorsque les internautes évoquent des questions de "sécurité publique", ils distinguent des conséquences spécifiques liées à certains types de prise de parole. En particulier, la parole qui fait l'objet d'une mise en garde est celle qui peut avoir "un impact" sur la vie des autres, celle qui sort du cadre de l'expression purement individuelle, pour prendre une importance collective, c'est à dire une certaine forme de parole politique.

"Pour moi la sécurité publique, c'est difficile à dire, c'est quand on a une influence sur les autres, peut-être une influence sur une majorité de gens, quand on touche à la mise en place de la politique gouvernementale, peut-être que c'est ça toucher à la sécurité publique." (Zhang)

La manière dont les internautes décrivent cet "impact sur les autres" en termes d'ordre public rejoint très précisément la définition que John Dewey ${ }^{9}$ fait de ce qui est "public". Dewey ne s'intéresse pas vraiment à la question de la visibilité ou de l'intimité mais définit le public comme le domaine où les actions d'un individu peuvent avoir un impact sur les autres individus. Peu importe que ces actions aient lieu dans le secret de l'alcôve ou sur la place publique, car elles sont mesurées à l'aune de l'impact qu'elles ont sur les autres individus. En définissant ainsi la limite entre privé et public, les internautes donnent une limite très politique à la liberté d'expression.

Il se crée donc une distinction entre une certaine parole, officielle et politique, souvent animée par des journalistes, et d'autres types de parole, qui appartiennent plus souvent aux internautes ordinaires, comme le divertissement. La notion de représentation délimite ainsi les contours d'une parole politique que l'on peut qualifier de "publique", au sens où elle est porteuse de l'image publique de la nation, et qu'elle est soumise à des contraintes très particulières. Or la parole publique n'est plus réservée aux seuls journalistes depuis que tout internaute a la possibilité de prendre la parole sur diverses plates-formes. Dans ce champ très vaste offert à l'expression individuelle, les internautes sont tenus d'adapter leur discours au type de plates-formes sur lesquelles ils s'expriment. La principale exigence est certes d'afficher une certaine conformité, mais elle est surtout d'adopter le comportement adéquat

\footnotetext{
${ }^{9}$ DEWEY John. Le public et ses problèmes, Paris: L. Sheer, 2003.
} 
dans chaque type d'espace d'expression, en fonction de son degré d'importance politique ${ }^{10}$. Il s'agit en fait d'adopter des attitudes, des "façades" ${ }^{11}$ ou des modes de prise de parole ${ }^{12}$ appropriées en fonction du type de scène concerné.

\section{La publicité comme contrainte de rôle}

La presse officielle multiplie les articles qui rappellent aux internautes que leur parole sur Internet est publique, et qu'à ce titre ils doivent prendre leurs responsabilités et surveiller ce qu'ils disent.

Je crois que notre liberté de penser est absolue, qu'elle n'est sujette à aucune restriction, puisqu'elle relève purement de notre domaine personnel. D'un autre côté, la liberté d'expression et la liberté de mouvement, qui peuvent potentiellement avoir un impact sur la société, devraient être d'autant plus restreintes, sujettes à de plus grandes limitations venant de la loi ou de codes moraux. Nous devrions donc être responsables des discours et des actions tenus dans le domaine public. [...] Mais nous devons être clairs sur le fait que les mauvaises remarques de cette jeune fille ont été exprimées sur un espace QQ.com, ce qui veut dire sur un espace où les lignes entre le privé et le public sont l'objet d'une certaine controverse. ${ }^{13}$

Cet éditorial publié juste après l'arrestation d'une jeune fille, qui s'était publiquement réjouie du tremblement de terre au Sichuan en 2008, montre bien que les contraintes qui pèsent sur la prise de parole sur Internet reposent amplement sur un caractère public ambivalent. Cette notion est très bien relayée par les internautes eux-mêmes.

\footnotetext{
${ }^{10}$ Ce modèle ne semble pas spécifique à la Chine. Par exemple Ferrié pense possible de décrire la "genèse et la structuration de l'espace public au Maroc en tant que conséquences locales de la modernité". "La liberté individuelle n'est pas absorbée dans le public par les sociétés musulmanes mais au contraire reléguée dans le privé. En ce sens, 'public' et 'privé' doivent être précisément pensés comme des principes d'organisation et non comme des jugements sur les choses. On ne fait pas en privé des 'choses honteuses'; ce qui est honteux est de faire en public des choses censées être faites en privé." La société musulmane implique donc "une sélection plutôt drastique des degrés de publicité; cette sélection n'est autre que le choix d'un public selon les circonstances et ce que l'on montre". FERRIE Jean-Noël. "Lieux intérieurs et culture publique au Maroc", Politix, vol. 8, n 31, 1995, p. 187-202, p. 189.

${ }^{11}$ GOFFMAN Erving. La mise en scène de la vie quotidienne, Paris: Minuit, 1979, 2 vol.

${ }^{12}$ GOFFMAN Erving. Façons de parler, Paris: Minuit, 1987.

${ }^{13}$ YU Lisheng, "On what basis is the girl who cursed the disaster victims being detained?", RedNet, traduit dans BANDURSKI David. "Is it against Chinese law to be callous and pigheaded?", China Media Project, 10/06/2008, consulté le 22/07/2010 sur http://cmp.hku.hk/2008/06/10/1066/.
} 
"Je trouve que c'est assez bien en fait d'écrire sa propre opinion à propos de quelque chose en trois ou cinq phrases. Du moment qu'on exprime un avis objectif à propos de cette chose, je pense que c'est correct. Si en parlant de cette chose tu t'attaques à quelqu'un ou au pays par exemple, là je trouve que ce n'est pas correct. C'est une plate-forme publique (gonggong pingtai), ce n'est pas un endroit où on peut attaquer les gens comme ça. Peut-être que tu peux te faire plaisir comme ça sur le moment, mais la morale publique (shehui gongde), en fin de compte la morale (daode) c'est quand même important. Les Chinois en ont de moins en moins, et il faut l'améliorer." (Wu)

Là où cet internaute parle de "morale", la grande majorité des internautes utilisent le terme de "responsabilité". Celle-ci est toujours liée à l'idée que la parole publique est porteuse de nombreuses conséquences sur la société dans son ensemble, et qu'il faut adapter son comportement en fonction de l'importance de ces conséquences potentielles.

"Qu'est-ce que ça veut dire, le sens des responsabilités? Qu'est-ce que c'est qu'une chose fausse? Est-ce qu'il y a des règles formelles là-dessus?

- Il n'y a pas de règles précises, par exemple sur une question, il faut s'exprimer d'après sa pensée la plus réelle, il faut considérer cette question avec l'attitude la plus correcte. Il suffit que ce que tu dis ne soit pas tout à fait vrai, et on peut toujours t'attaquer violemment. Il faut considérer les choses avec un cœur pacifique, il faut réfléchir aux problèmes en gardant un comportement correct, il ne faut pas être violent. Parce que quand tu t'exprimes, c'est inévitable d'avoir un impact sur les autres, donc quand on parle de quelque chose il faut prendre ses responsabilités, surtout prendre ses responsabilités envers la société. Il ne faut pas penser qu'Internet n'est pas réel, que c'est faux. Internet a les deux aspects, il y a un côté faux, et il y a un côté réel aussi, donc quand on discute d'une question sur Internet c'est pareil, il faut traiter sérieusement les choses sérieuses." (Yang)

Ici, le caractère public d'Internet est synonyme de "réel" par opposition à "virtuel". L'internaute rappelle ainsi que l'expression publique n'est pas anodine, et que sous certaines conditions elle peut avoir des conséquences importantes sur la société, et a fortiori sur les internautes qui prennent la parole. Comme les médias, les internautes eux-mêmes signalent les dangers de considérer Internet comme un espace complètement virtuel. C'est surtout cette considération qui préside au respect des règles de conduite sur Internet. 
Il n'est donc possible de prendre position sur des sujets politiques qu'en adoptant la posture officielle patriotique. Dans ce cas, la responsabilité de chacun est de s'exprimer "en tant que Chinois" même si, à titre individuel, on ne partage pas la position officielle.

"J'y ai déjà pensé. En fait ça se passe comme ça. Je regarde beaucoup de choses, et je m'efforce de conserver une attitude objective. Par exemple quand il y a des choses [contrariantes], bien sûr personnellement je pense que le gouvernement a un problème, et qu'il ne faut pas restreindre la liberté de quelqu'un. Mais quand je regarde les choses en tant que Chinois, j'espère que ce genre de choses n'arrivent pas trop souvent, je veux dire des choses pas très bonnes pour le pays. Il ne faut pas trop en faire car si tout le monde fait attention à cet aspect-là, je trouve que globalement ça ne peut pas avoir une très bonne influence." (Cai)

Cependant, la plupart des internautes préfèrent s'écarter de ce domaine politique pour ne pas interférer avec la parole officielle. Que l'on choisisse de s'exprimer de manière conformiste "en tant que Chinois" ou de s'écarter du domaine politique, "être objectif" signifie tenir son rôle, adopter la "façade" adéquate en fonction de sa position particulière. Or le rôle des individus n'est pas de prendre parti au plan politique.

"Pour ce qui est des gens ordinaires, on s'intéresse un peu à notre environnement en général, et un petit peu aux affaires internationales, on s'intéresse un peu aux situations où il faut aider le pays et puis voilà, on fait un effort et puis voilà, et si on ne peut pas, on y fait un peu attention, ou on communique dessus (xuanchuan), en tous cas on ne fait pas obstacle. Mais je pense que c'est vrai pour la politique, et c'est aussi vrai individuellement, il faut toujours regarder les choses d'un point de vue très objectif. Ca ne vaut pas le coup de politiser des choses très ordinaires, ça crée trop de problèmes." (Zhao)

Le terme "objectif" est aussi fréquemment prononcé que l'est le terme "responsable". Ils sont généralement associés. Si l'idée de responsabilité indique que les internautes doivent tenir compte du caractère public d'Internet, celle d'objectivité précise la nature des précautions que cela implique. Au nom de la protection d'une représentation unique de la société, les internautes sont invités à ne pas adopter une attitude politique au sens large. 


\section{3 - La dévalorisation des conflits}

\section{Une difficile confrontation des opinions}

S'écarter des sujets politiques, qui supposent par définition un effort dans la direction de l'unanimisme, n'empêche pas l'existence de désaccords entre les différents internautes sur des sujets même mineurs. Il arrive que les internautes s'en amusent, et le prennent comme un jeu.

"Sur certains aspects nous sommes d'accord, mais sur beaucoup d'aspects, on dirait que j'aime bien me disputer (chao), parce que je trouve que les gens soutiennent certaines choses exagérément, et je trouve que si tu ne te disputes pas avec eux, c'est que tu les approuves, et alors ils ont l'avantage. Mais des fois quand on a un avis un peu différent, on peut en discuter très longtemps." (Gao)

Il y a ainsi des exemples d'enthousiasme devant la diversité et même la confrontation. Mais cet enthousiasme est relativement minoritaire et montre une certaine tension. Il n'est pas anodin que cette confrontation soit désignée avec le vocabulaire de la dispute. Même si elle est appréciée à titre personnel, comme une sorte de divertissement, elle n'en garde pas moins une connotation péjorative. Il existe une forme d'inconfort qui conduit à l'élaboration de recommandations sur la gestion des antagonismes.

"En fait on a tous des opinions différentes, et certaines m'énervent beaucoup quand je les lis. Par exemple, il y a le problème des pékinois et des provinciaux. Ils disent qu'on est comme ci ou comme ça. Moi j'ai un hukou de Pékin, et je ne veux pas me disputer là-dessus. Eux, ils sont très bizarres, on dirait que tous les provinciaux, quand ils arrivent à Pékin, disent que Pékin c'est très bien pour ceci et cela, et puis après ils disent que les Pékinois sont très mauvais pour ceci et cela. Mais qu'est-ce qu'ils viennent faire là, et nous on ne va pas leur faire de commentaires sur comment c'est chez eux. Par comparaison, la xénophobie est très grave à Shanghai, mais vraiment pas à Pékin, donc quand les gens ont répondu, ils se sont beaucoup disputés, beaucoup trop, et le modérateur a effacé leurs commentaires." (Luo)

Comme on le pressent, de telles confrontations entre des Chinois de différentes provinces sont vécues comme des menaces sur la stabilité de la société dans son ensemble.

"Je n'aime pas vraiment exprimer mon opinion par ce genre de moyens, ou bien c'est avec mes amis, en face à face, mais je ne dirais pas ce genre de choses sur Internet. 
- Mais tu disais qu'Internet était très libre?

- C'est à dire que, bien que la liberté d'expression soit une bonne chose, si on est trop libre, cela crée des problèmes dans la société, peut-être qu'à cause d'une toute petite affaire, la liberté d'expression attire des opinions extrêmes, ça peut créer, ça peut attirer de l'instabilité pour la Chine, et donc ce n'est pas très bien non plus." (Yuan)

Les discussions les plus futiles peuvent être prétexte à de tels débordements. Les débats autour de l'élection de Superboy deviennent ainsi un lieu de conflits potentiels, où il faut se comporter avec savoir-vivre.

"Par exemple Superboy sur Hunan TV, tout le monde s'y intéresse beaucoup, on peut en discuter sur le site. A ce moment-là j'ai regardé, il y en a un qui s'appelle Tang, que j'aime bien, sur un blog j'ai vu que quelqu'un disait qu'il n'était pas bien, il disait forcément ça pour attaquer, parce que chaque personne a ses préférés, et on a tous envie que tout le monde les aime, surtout dans les moments comme ça où il faut donner son avis. Il ne faut pas être trop méchant et c'est bon, sinon ça peut lui faire du mal, quand il y a des attaques, en fait ça peut rendre les gens malheureux, ça a trop de conséquences. Parce que sur ton blog, les autres le lisent aussi, donc ça les touche (yingxiang) directement, dans ces cas-là il faut que tout le monde exprime son avis amicalement." (Yang)

Le premier conseil donné par les internautes est évidemment de s'exprimer poliment, de ne pas attaquer les autres internautes et de toujours rester amical. D'une manière générale il ne faut pas, semble-t-il, "influencer" ou "toucher" (yingxiang) les autres internautes, ce qui pourrait les blesser. Bien sûr il s'agit d'abord de ne pas s'attaquer directement à eux.

"Le mieux c'est d'encourager les sentiments des autres, dire des paroles de soutien, il ne faut pas s'attaquer aux autres en leur faisant du mal, parce que je trouve que sur n'importe quel sujet, attaquer les gens... entre les gens il faut avoir un comportement pacifique, parce que ce qu'on dit peut influencer directement leur vie. Même si Internet est une très bonne plate-forme pour communiquer, il faut faire d'autant plus attention à ce qu'on dit." (Yang)

Il semble donc que l'idée de la liberté d'expression suppose de ne pas contredire les autres personnes, ce qui va plus loin que la simple politesse bienveillante.

"Oui, je trouve. En fait je respecte ça, que les gens aient besoin de se défouler (faxie) en ligne, je trouve qu'il faut qu'il y ait des endroits pour se défouler, là-dessus 
on ne peut pas blâmer chaque personne, ça c'est un problème lié à notre environnement. En plus notre environnement on ne peut rien y changer, on ne peut que s'en accommoder, donc même si cela conduit des gens à s'exprimer trop violemment en ligne, je ne suis pas vraiment contre ça, ça ne me fait rien. Puis je me dis qu'ils ont leur propre avis, ou bien ils ont encore plus de pression ou de problèmes que moi dans la vie, ce qui oriente leur point de vue, donc je ne peux pas contredire leur opinion. Tout le monde a le droit de dire ce qu'il veut." (Zeng)

Zeng, en répétant l'importance de pouvoir s'exprimer en ligne, excuse les excès de certains internautes pour lesquels il ressent de l'empathie. Cette empathie, si elle ne le conduit pas forcément à engager des conversations amicales avec les intéressés, le conduit au moins à éviter des discussions conflictuelles avec eux. La contradiction est conçue ici comme une entrave à la liberté d'expression.

"Il y a des choses qui ne me sont pas très familières, et ces choses-là sont très différentes selon les points de vue (jianrenjianzhi), chacun a un avis différent. Les niveaux d'intelligence et les degrés d'opinion de chaque personne sont différents, on n'a pas la même manière de comprendre les problèmes, on ne voit pas les choses sous le même angle, et moi peut-être que je vois encore une autre façade, donc je vais donner mon avis, et les autres donnent leur avis.

- Donc vous en discutez?

- D'abord ça peut créer des conflits, des fois ça crée des conflits.

- Alors que faites-vous dans ces cas-là?

- On comprend les choses de manière objective et puis c'est tout, je ne leur envoie pas mes commentaires, parce que dans la plupart des cas les commentaires sont subjectifs et pas objectifs. Donc je me contente d'observer les choses objectivement pour les comprendre et puis c'est tout, ça ne sert à rien d'exprimer mon avis, du moment que ça ne m'intéresse pas trop. " (Lin)

La confrontation des opinions serait donc source de conflits. Dans ce contexte, être exposé à des opinions divergentes peut conduire certaines personnes à éviter le débat. Insister pour débattre avec des personnes qui ne partagent pas les mêmes points de vue est perçu comme un comportement agressif, même dans des domaines qui sont tout à fait anodins. Lorsque je demande aux répondants quelle est leur manière de réagir lorsqu'ils rencontrent des opinions avec lesquelles ils ne sont pas d'accord, ils répondent très souvent en utilisant un 
vocabulaire péjoratif comme "polémiquer", "se disputer", contredire", autant de mots que je ne prononce pas dans mes questions.

"'Zhongyong' [juste milieu] ça veut dire quelque chose comme laisser faire, ne pas faire de polémique, ça ressemble un peu à ça, ça ressemble un peu à la pensée bouddhiste ou chrétienne, ça veut dire que je ne vais pas me disputer avec toi sur des problèmes, comme le pouvoir ou l'argent, même si j'ai forcément des désirs, mais mon comportement ou mon caractère ne sera pas comme ça. " (Zeng)

Pour Zeng, c'est la manière chinoise de tenir des discussions qui est plus subtile, et plus efficace que l'expression directe des antagonismes pour gérer des relations. Il s'en explique dans la suite de l'entretien.

"Il faut avoir un comportement accommodant (suihe), comme ça on facilite les relations, c'est plus facile de faire des choses ensembles (gongshi), en fait je trouve que sur tous les plans c'est plus facile de réussir. En Chine ça nous arrive souvent de tomber sur des situations, par exemple si tu n'es pas d'accord avec quelqu'un, avec ton patron, ou pas d'accord avec ton client, si vous vous êtes disputés violemment, même si tu as raison, il ne voudra peut-être pas s'aligner. A l'étranger c'est différent, si tu as un avis tu peux le dire directement, dire à tout le monde il faut qu'on fasse comme ça, et on fait comme ça, mais pas en Chine. Nous on parle de manière plus subtile (weiwan), on exprime notre point de vue de manière plus subtile, si c'est avec mon collègue, mon client, ou si c'est mon ami [...], on réfléchit plus à ces choses-là, pour régler les problèmes. Tu ne dois pas exiger des Chinois qu'ils règlent les problèmes à la manière des Occidentaux, ça embrouillerait les choses. Si je me mettais à débattre de questions violentes avec plein de gens, je ne crois pas du tout qu'après la dispute on puisse encore aller régler ensemble et en paix des questions communes. Donc je trouve qu'on peut comprendre que quand je lis sur Internet que les Chinois devraient s'inspirer de la pensée occidentale, je ne suis pas vraiment d'accord, on doit apprendre certaines choses, mais sur d'autres choses il faut qu'on se respecte soi-même, le contexte est différent."

Il semble qu'il soit impossible de résoudre un désaccord aux yeux de Zeng. Pour lui, les discussions n'ont pas le pouvoir de résoudre des questions, ni de les dépasser. Elles peuvent seulement souligner les désaccords et les rendre encore plus insurmontables. Dans ce cas les Chinois sont décrits comme fuyant systématiquement le conflit. Il arrive au contraire que le même facteur culturel soit invoqué pour dire que les Chinois veulent toujours convaincre les 
autres. Dans les deux cas cependant, la question du débat d'idées est considérée comme une entrave à la bonne entente entre les parties, en particulier dans le commerce.

"J'ai rencontré quelques Japonais, ils ne sont pas aussi mauvais que ce que j'imaginais, en fait ils ont un mode de vie plus réaliste que ce que j'imaginais, par exemple ils séparent très fortement la politique et le commerce. Ce n'est pas comme les Chinois, quand ils discutent avec toi, ils veulent absolument te convaincre et c'est seulement là qu'ils veulent bien faire du commerce avec toi." (Cai)

La diversité des opinions semble en fait ne pas constituer le problème central. S'il est possible d'exprimer une opinion, c'est le fait de confronter cette opinion à celle des autres et d'insister dans le but de convaincre qui crée le plus grand inconfort. C'est en ce sens que le débat est assimilé à un conflit. Il existe une sanction sociale forte, qui condamne la contradiction. Lorsque les internautes adoptent une position plus critique que la moyenne, et entrent ainsi dans le territoire du débat d'idées, ils sont souvent très mal reçus par les autres internautes. C'est ce qui est arrivé à deux au moins des personnes que j'ai rencontrées.

"Ca m'est déjà arrivé, je me suis déjà laissé aller plusieurs fois, après que je me suis éveillé (juexing). Je me suis rendu compte que ce processus [d'éveil à l'acquisition des connaissances] était trop douloureux, [...] parce que j'ai exprimé beaucoup d'idées sur des forums, des pensées personnelles, en espérant que tout le monde utiliserait Internet pour obtenir encore plus de connaissances. J'espérais éveiller (huanxing) encore plus de gens. Mais les gens m'ont pris pour un fou, pour un imbécile. Quand j'essayais de convaincre (quan) quelqu'un d'aller chercher plus d'informations, j'entendais les vulgarités les plus dégoûtantes, comme 'ignorant' (mengmei), 'imbécile'. On cache beaucoup trop de choses aux Chinois. En fait ce que je dis aujourd'hui est peut-être un peu triste." (Tian)

Cet internaute a donc voulu "convaincre", et c'est précisément cette tentative qui lui a valu les insultes des autres sur le Web. Il s'avère donc que certaines personnes ne souscrivent pas au modèle d'expression publique tel qu'il est décrit par la plupart des internautes que j'ai rencontrés. Ce modèle ne saurait donc être tenu pour un modèle absolu ni immuable. Cependant, il est dominant dans la mesure où il s'impose à la majorité et que celle-ci se charge de la faire observer par tous. D'ailleurs notre internaute déclare plus loin: "je trouve que ma pensée aujourd'hui est une pensée occidentale, je ne suis absolument pas un Chinois traditionnel." Il se désigne ainsi comme très peu représentatif des pratiques et des opinions qui 
circulent parmi les internautes chinois. En dépit de cela, il a fini par se plier lui aussi à ces pratiques, puisqu'il a cessé de tenter de convaincre les autres en ligne.

On assiste donc à un phénomène de marginalisation des internautes qui ne partagent pas la pensée dominante. Ils peuvent s'exprimer à côté, mais non de façon à débattre. Certains choisissent ainsi de changer de site, et passent dans des forums plus petits, plus confidentiels, qui ressemblent à de petites communautés.

"Oui, En général je m'exprime sur 55BBS, parce que je connais bien ce forum, je le regarde tous les jours, et je peux même reconnaitre les ID des gens, et je trouve qu'on peut y parler sans danger. Parce que des fois sur les sites publics (gongzhong), comme Sina ou Sohu, des fois quand on écrit un commentaire, si les gens ne sont pas d'accord avec toi, ils disent des choses vraiment très moches. Quand c'est comme ça je ne vois pas l'intérêt." (Guo)

Certaines personnes évoquent même la peur de représailles. Ils préfèrent alors se contenter de déposer leurs impressions sans les confronter à celles des autres.

"Toute chose a un bon et un mauvais côté. Les gens ont tous leur pensée, on ne peut pas toujours penser comme les autres. On ne peut pas dire il pense très mal à propos de cette chose, alors moi aussi je vais penser très mal. Il y a toujours une idée et son contraire. Par exemple, à propos de n'importe quelle chose, votre avis est celuici, le mien est celui-là. Votre avis est biaisé, et pas le mien. Mais des Chinois ne pourraient pas se contredire entre collègues, on ne peut que dire qu'on donne son avis. Bien qu'il y ait un avis correct et un avis incorrect, je ne peux pas insister, c'est comme ça, on est plutôt réservés, on a peur de mal faire. Ca a toujours été comme ça, les gens gardent leurs distances, des fois ils ont peur de mal faire. Il y a des gens qui sont très francs, qui disent 'vous avez tort', mais si je dis ça, et que ça ne vous plaît pas, le moment venu vous pouvez vous venger un peu, on a peur de ça." (Cai)

Les internautes manifestent donc une impression d'inconfort en situation de débat. Pour eux, la fonction de l'expression en ligne n'est visiblement pas l'échange d'arguments contradictoires pour se convaincre mutuellement. Il s'agit plus souvent d'un espace où déposer son témoignage personnel, enregistrer son journal, déposer des émotions intimes, de façon séparée des autres internautes. Les formes d'expression légitimes sont fortement "modalisées", c'est à dire que la position du locuteur y tient une place primordiale, dans un espace public conçu comme nécessairement polyphonique. On observe ainsi une disqualification du "régime 
de la critique" au profit des régimes "de l'opinion" et surtout "du partage", qui n'est pas sans rappeler la tendance observée en France, même si elle repose sur des ressorts différents ${ }^{14}$.

\section{Une "tyrannie de l'intimité"?}

Devant le risque de créer des conflits, les internautes s'abstiennent souvent de confronter leurs avis respectifs dans un débat contradictoire. Ils préfèrent généralement les juxtaposer, comme une collection d'opinions individuelles et particulières, et prôner une forme de tolérance qui décourage la montée en généralité.

"La vie de la société et les choses de ce genre n'ont pas un rapport très fort avec moi et mes amis, donc ce qu'on en pense n'a pas d'importance. Personne ne va penser à nous dans ces cas-là parce que si je ne touche pas aux intérêts des autres, il n'y a pas de raison qu'ils touchent aux miens. Tout ça n'a pas d'importance. Quand il se passe quelque chose il y a toujours beaucoup de monde pour donner plein d'avis, ça ne sert à rien d'essayer d'atteindre l'unanimité ou bien d'obliger les gens à écouter ton avis.

- Donc dans un cas comme ça, est-ce que tu préfères ne rien dire, ou bien écrire ton avis?

- J'écris mon opinion et puis c'est tout, par exemple si les gens ont des avis différents, je ne vais pas continuer à parler, parce qu'il n'y a pas de raison de se disputer, chacun a son opinion." (Pan)

Tous les internautes prônent une grande tolérance face aux paroles des autres internautes. Cette tolérance peut consister à encourager les autres internautes s'ils traversent une mauvaise passe, ou bien à les laisser s'exprimer de leur côté sans exprimer son opposition. Le plus souvent, les internautes déclarent rester simplement indifférents, et ils dévalorisent toujours l'idée de contredire les autres ouvertement. Chacun dispose de tout l'espace dont il a besoin pour écrire ce qu'il veut de son côté.

En fait, chacun est encouragé à exprimer son opinion personnelle à côté de celle des autres, mais il n'est pas encouragé à tenter de convaincre ses interlocuteurs. Plus que la contradiction en elle-même, c'est le fait de tenter de convaincre qui est dévalorisé.

\footnotetext{
${ }^{14}$ En France, cette disqualification repose sur le caractère plus sélectif du régime de la critique, car il demande des compétences argumentatives relativement élevées. CARDON Dominique, HEURTIN Jean-Philippe et LEMIEUX Cyril. "Parler en public", Politix, vol. 8, n³1, p. 5-19.
} 
"Si tu n'es pas d'accord avec l'avis d'un ami, est-ce que tu continues de lui écrire pour vous mettre d'accord?

- Non, parce que chacun pense différemment, je ne peux pas forcer les gens à penser comme moi! " (Dai)

La notion de débat étant systématiquement associée à celle de violence et de conflit, il en découle un rejet de cette pratique au profit d'une simple juxtaposition des opinions, sans échange poursuivi ni effort d'argumentation.

"On ne va pas avoir un débat (zhenglun) très violent, simplement quand il y a plusieurs points de vue, on donne le sien, mais personne n'essaie de convaincre les autres. Je veux seulement exposer mon point de vue et puis voilà. Par exemple sur des informations de divertissement, je trouve que ça ne sert à rien d'essayer de convaincre les autres, les avis et des points de vue de chaque personne sont forcément différents, non?" (Du)

Jusqu'à présent les personnes interrogées déclarent tout de même profiter de l'opportunité pour donner leur propre avis, qui est simplement déposé à côté de celui des autres. Cependant certains internautes vont jusqu'à s'abstenir de s'exprimer tout court. Il en résulte une certaine homogénéité des discours sur Internet, homogénéité qui est constatée par cet internaute, mais dont il est difficile d'évaluer l'étendue réelle.

"Je ne peux pas entraver la pensée des autres, et je ne peux pas contrôler ce qu'ils disent, et puis je m'intéresse seulement à ma propre pensée. En fait je pense en général la même chose que la plupart des gens, donc en général les pensées et les discussions sont assez unanimes (yizhi), donc je ne m'intéresse pas trop sérieusement au point de vue des autres, parce que pour la plupart ils sont comme moi.

- Pourquoi est-ce qu'il y a autant de points de vue identiques?

- Parce que nous sommes un groupe, une génération, donc notre pensée est à peu près identique. Par exemple quand Man Lian a obtenu la première place, il y a beaucoup de fans de Man Lian, et j'en suis un, donc j'ai écrit que je trouvais ça trop bien qu'il soit encore premier et tout ça, et $90 \%$ des gens ont écrit la même chose que moi, donc je n'ai pas vraiment besoin de lire leurs commentaires, parce qu'on est tous pareil.

- Qu'est-ce que ça t'apporte de publier ton propre avis? 
- Je n'ai pas de but particulier, je parle pour épancher (faxie chulai) mes émotions et c'est tout, c'est pour donner mon avis." (Dai)

Il faut donc relire les affirmations liées à la liberté d'expression et au défoulement à la lumière de la difficulté de gérer les conflits sur Internet. Chacun n'étant pas encouragé à s'engager dans un débat avec les autres et à proposer des arguments contradictoires, il en ressort que l'expression unilatérale des émotions personnelles prend une place prépondérante. Le récit de soi n'est pas seulement l'expression d'une diversité florissante. Il est aussi le reflet du cloisonnement et du difficile frottement des opinions individuelles entre elles ${ }^{15}$.

Pour la plupart des internautes, la parole publique sur Internet ne sert donc pas en priorité à débattre de questions communes, mais seulement à déposer les unes à côté des autres des impressions individuelles. La tolérance qui est mise en valeur dans ce modèle est celle de la diversité au sens strict, et elle s'oppose à la notion de pluralisme. Dans l'idéal-type du pluralisme, plusieurs points de vue peuvent émerger au sein d'un même groupe, et être partagés afin d'être discutés. Il existe un paradoxe naturel dans le concept de pluralisme ${ }^{16}$. Il doit exister un accord de base entre tous sur le fait que le désaccord est possible et même fructueux. Il n'y a rien de tel dans la conception de la diversité telle que décrite au cours de ces entretiens. Bien au contraire, les individus qui sont parfois tentés d'engager un débat sur Internet se voient conspués par les autres internautes, et marginalisés plus ou moins violemment.

Les notions de liberté d'expression, de tolérance, ou encore d'objectivité sont utilisées chez les internautes chinois à l'exact opposé de leur sens dans la tradition philosophique européenne, ce qui ne manque pas de conduire à une certaine confusion. Pour Sennett par exemple, "la civilité est une activité qui protège le moi des autres moi, et lui permet donc de jouir de la compagnie d'autrui. [...Elle] consiste à traiter les autres comme s'ils étaient des inconnus, à forger avec eux des liens sociaux respectant cette distance première. [...] L'incivilité pourrait se définir de manière inverse: c'est le fait de peser sur les autres de tout le

${ }^{15}$ Cela rejoint les observations de Cardon et Heurtin sur l'expression de l'indignation des auditeurs de France Inter, lorsqu'ils pensent ne rien pouvoir changer à leur situation. CARDON Dominique et HEURTIN Jean-Philippe. "La critique en régime d'impuissance, une lecture des indignations des auditeurs de France Inter", in FRANCOIS Bastien et NEVEU Erik (dir.). Espaces publics mosaïques, acteurs, arènes et rhétoriques des débats publics contemporains, Rennes: Presses universitaires de Rennes, 1999, p 85-119.

${ }^{16}$ LECA Jean. "Les paradoxes de la société civile", Sociétal, n9, juin 1997, p.59-62. 
poids de sa personnalité." Au contraire, le terme de civilité est seulement synonyme de politesse et de tolérance indifférente chez les internautes chinois interrogés. Ce sont les conditions supposées de l'évitement du conflit dans un régime de "solidarité négative"17.

Ici, les internautes sont donc sommés de faire valoir leur personnalité, de supprimer toute distance critique qui pourrait être une cause de conflit. L'expression publique doit être authentique, personnelle, intime. Dans cette situation, il est relativement difficile de monter en généralité, c'est à dire de traiter de manière contradictoire de problèmes communs avec des interlocuteurs, en mobilisant des arguments d'intérêt général. Pour reprendre l'analyse de Sennett, "le désir de sécurité, de tranquillité et de permanence, est le corollaire d'une peur de l'agressivité et de la vie publique" ${ }^{18}$.

Ce que Sennett appelle une "idéologie de l'intimité" empêche ainsi la création d'une véritable vie publique, car "la poursuite des intérêts communs est remplacée par la recherche d'une identité commune". En effet les règles qui s'appliquent à la prise de parole et à la gestion des désaccords au sein de la société sont intimement liées à la question du vivre ensemble. C'est donc bien la dimension politique de l'échange qui est niée ici, au sens de discussions autour de la chose publique. Ce n'est pas seulement la politique "politicienne" qui pose problème dans la parole publique, mais bien au-delà tout le domaine $d u$ politique, défini par Arendt comme "vivre-dans-un-monde-réel et discuter-de-lui-avec-d'autres"19.

Pour les internautes interrogés, la diversité et la stratification de la société chinoise rendent impossible et même peu souhaitable l'émergence de débats contradictoires. Au contraire, la nécessaire protection d'une image unifiée et "harmonieuse" de la nation impose à chacun de taire ces antagonismes. L'autocensure, lorsqu'elle consiste à éviter la mise en évidence des divergences, relève ainsi d'une forme de "responsabilité" qui incombe à tout individu patriote. Dans ce cadre, les individus ne peuvent prétendre donner que des témoignages personnels, des avis relativisés en permanence par leur position particulière. La variété des points de vue, qui n'est pas niée, ne peut être traitée que dans la juxtaposition, au nom de la diversité, et elle exclut la confrontation et la délibération. En d'autres termes, ce "régime de civilité" décourage les comportements politiques en ligne.

\footnotetext{
${ }^{17}$ FERRIE Jean-Noël. Le régime de la civilité en Egypte, Paris: CNRS Editions, 2004.

${ }^{18}$ SENNETT Richard. Les tyrannies de l'intimité, Paris: Seuil, 1979, p. 198 et p. 220.

${ }^{19}$ ARENDT Hannah. Qu'est-ce que la politique?, Paris: Seuil, 1995, p.92.
} 
Il ne faut pas oublier cependant que cette neutralisation du politique ne concerne que les dimensions les plus publiques de la prise de parole. Les nombreuses règles restrictives énoncées par les internautes délimitent très clairement des interdits politiques dans ce champ spécifique, mais il se dessine du même coup tout un monde dédié à la parole privée, individuelle et particulière, qui est le domaine du désordre et de la diversité, et où viennent se loger de nombreuses "conversations" informelles, y compris à propos de problèmes publics. 


\section{Chapitre VI - Les conversations en "clair-obscur"}

Les nombreuses règles de civilité énoncées par les internautes délimitent très clairement des interdits politiques dans le champ de la parole publique. Les internautes s'abstiennent le plus souvent de tenir ostensiblement des discours hétérodoxes, et ils jugent sévèrement les conflits qui pourtant éclatent relativement souvent sur Internet. Néanmoins, en décrivant ces interdits et en les attribuant au rôle de représentation de la parole publique, les internautes dessinent en creux tout un monde dédié à la parole privée, individuelle et particulière, qui a aussi sa place sur Internet. C'est le domaine du désordre et de la diversité, où viennent se loger leurs "conversations" informelles.

Ce domaine de la parole privée est d'abord un espace privilégié d'information et de formation des opinions, parce qu'il rassemble une multitude de points de vue individuels qui donnent un sentiment d'authenticité et de diversité. Les internautes sont capables de s'y repérer et d'y bricoler des opinions ${ }^{1}$ de manière plus éclairée que ne le suggère l'idée d'un blocage total de l'information. C'est aussi un univers en "clair-obscur", visible de tout un chacun et privatif à la fois, dont le statut est toujours ambivalent. Les internautes y investissent beaucoup plus volontiers de nombreuses niches d'expression. De la rumeur à la conversation entre amis sur une plate-forme publique, en passant par le double-langage et les allusions destinées à contourner la censure ${ }^{2}$, cette prise de parole informelle laisse en fait une place très importante à la critique, à la transgression et à la prise de distance.

${ }^{1}$ CERTEAU Michel. L'invention du quotidien 1. Arts de faire, Paris: Gallimard, 1990.

${ }^{2}$ SCOTT James. Domination and the arts of resistance: hidden transcripts, Yale University Press, 1990. 


\section{1 - La diversité des points de vue individuels}

\section{Un jeu de rôles}

D'après une enquête sur les usages d'Internet dans sept villes chinoises ${ }^{3}$, la confiance dans l'information sur Internet arrive loin derrière les sources plus traditionnelles que sont respectivement la télévision, la radio et les journaux.

Figure 5-10 The degrees of people's trust in various news sources

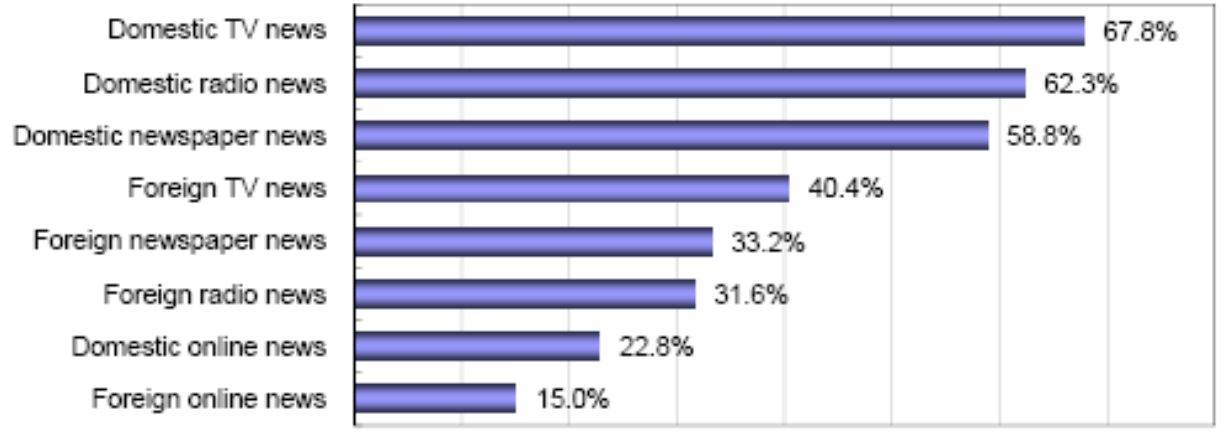

Ces déclarations semblent paradoxales car le même sondage indique qu'Internet est considéré comme une importante source d'informations, juste derrière les relations interpersonnelles, et devant les autres médias.

Figure 5-5 The importance of different media in providing information

- Very important $\mathbf{m}$ Important $\mathbf{m}$ Neutral/Undecided $\mathbf{m}$ Not important $\mathbf{a}$ Not important at all

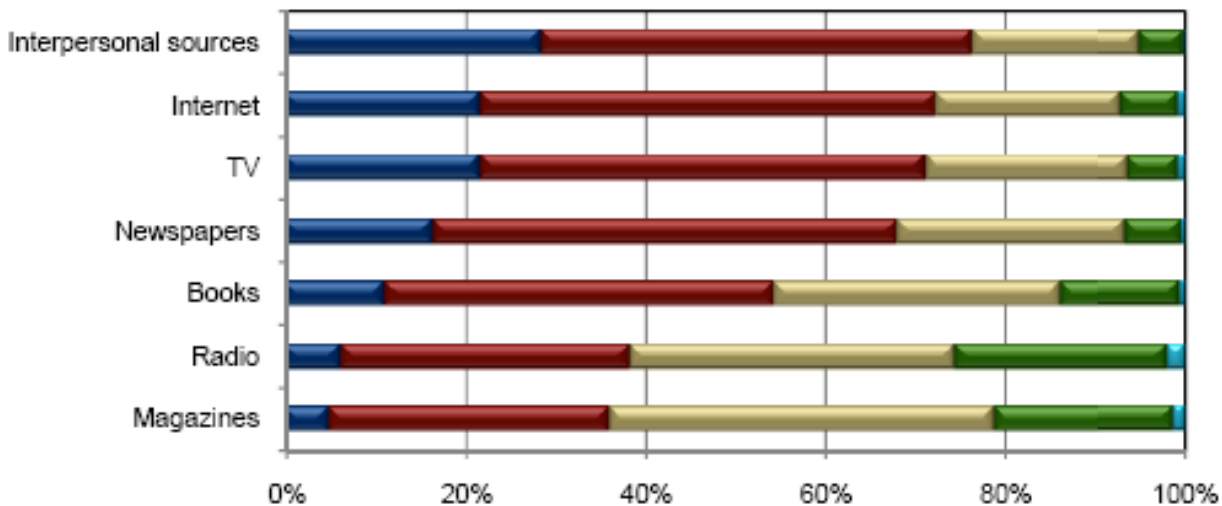

Il faut également remarquer que les informations nationales sont mieux classées que les informations internationales, ce qui doit être interrogé à la lumière du désintérêt pour la

${ }^{3}$ GUO Liang. Surveying Internet usage and its impact in seven Chinese cities, Markle Foundation, 2007. 
politique nationale affiché par les internautes et que j'ai documenté précédemment. Dans mes entretiens, les internautes déclarent bien plus souvent s'intéresser à la politique internationale ou à des enjeux très proches d'eux. Paradoxalement, il semblerait donc que les internautes soient plus attirés par les informations qu'ils considèrent comme les moins dignes de confiance, que ce soit en termes de sources ou de contenus! Que signifie la "confiance" accordée aux sources d'information officielles, si elle ne conduit pas les internautes à les adopter comme d'"importantes" sources d'information au quotidien?

Beaucoup d'internautes interrogés mentionnent la difficulté de s'assurer de la véracité des informations. Cette incertitude joue généralement en faveur des sources considérées comme les plus officielles, a fortiori les médias traditionnels, comme la télévision, et au détriment d'Internet.

"Des fois je lis les infos sur Internet, mais des fois, en fait j'ai l'impression que je ne sais pas si c'est vrai ou non. En fait avant, j'achetais souvent un magazine, j'achetais Le Confident (Zhiyin). Dans Le Confident il y a plein de choses très émouvantes, mais il y a des gens qui disent que ce qu'il y a dans Le Confident est entièrement faux, que ce n'est pas vrai et tout ça, donc maintenant je ne l'achète plus non plus, parce que c'est des choses que je lisais en passant, comme ça, je n'y faisais pas très attention. Maintenant en fait quand je veux voir les infos, les informations sur la société ou des choses comme ça, j'allume directement la télé. Tous les jours je regarde des choses comme les derniers procès, par exemple ils parlent de ça dans $L a$ Progression de la légalité (Fazhi jinxingzhi), ils attirent l'attention de tout le monde sur ceci ou cela, ils parlent très bien. Et puis il y a aussi Attention Spéciale (Tebie guanzhu), ils parlent aussi de ce qui s'est passé récemment, par exemple à tel ou tel endroit il y a eu telle chose. Quand j'ai regardé ça je sais ce qui s'est passé à Pékin, ça me donne une idée. Donc parfois je regarde aussi Internet, mais en général je ne clique que sur des choses qui m'intéressent, je vais voir les sujets que j'aime bien, voilà." (Shao)

La télévision et les médias écrits ont certes des défauts pratiques par rapport à Internet. D'abord, ils ne sont pas diffusés en temps réel, ce qui en fait des outils d'information bien moins performants qu'Internet. De plus ils n'offrent qu'un choix limité d'informations en termes de quantité et de diversité. Cependant ils bénéficient d'un statut officiel qui représente une certaine sécurité pour la fiabilité de l'information, et ce sont parfois eux que l'on croit en dernier ressort. 
"Il y a toutes sortes de gens, par exemple sur Internet il y a souvent de fausses informations.

- Comment sais-tu si une information est vraie ou fausse?

- Si je la vois pour la première fois, je pense généralement qu'elle est vraie, parce que tout peut arriver dans ce monde, mais au bout d'un moment, sur Internet ils finiraient pas dire si l'information était fausse. Pour les choses très graves, ils écriraient dans les journaux que sur Internet il y a telle ou telle information fausse qui circule, et donc je me dirais que c'est faux." (Yuan)

Les médias plus officiels comme la télévision ou les quotidiens, qui sont bien plus contrôlés que le Web, jouissent paradoxalement d'une confiance souvent accrue. Non pas que les auditeurs ou les lecteurs croient sur parole tout ce qui leur est rapporté, loin de là, mais ils pensent au moins pouvoir identifier clairement le statut des nouvelles, qui sont officielles. Ils ont, de toute manière, l'impression de pouvoir démêler ce qui tient de la propagande du reste, ou bien ils se résignent à l'idée que l'information est biaisée quelle que soit sa source. Dès lors, si les informations sont sélectionnées, et les commentaires orientés, les faits, eux, sont au moins supposés "vrais". Dans ces conditions, l'absence de commentaires est vécue comme un gage d'objectivité, et donc de fiabilité. Il n'y aurait que les faits, sans fards et donc sans manipulation.

"A la télé, il y a quand même moins d'informations, moins d'informations que sur Internet, parce que la télé a des programmes à des horaires précis, et qui durent une demi-heure en général, ou bien quarante minutes. Et puis à la télé ils rapportent les choses de manière plutôt objective, ils font très peu de commentaires." (Yuan)

Si les médias traditionnels reçoivent plus souvent la confiance des internautes, c'est aussi en grande partie parce qu'ils sont contrôlés, alors que les règles semblent beaucoup plus lâches pour ce qui est d'Internet.

"Je trouve que la loi chinoise n'impose en fait pas beaucoup de limites, ou de contraintes à l'information sur Internet, en fait très peu, et du coup ça a laissé la place à de fausses informations. Certains sites peuvent publier des choses assez scandaleuses ou violentes pour obtenir de forts taux de clics, pour attirer l'attention, mais ce ne sont pas forcément des choses vraies. Aujourd'hui ils disent noir, demain ils diront blanc, et du coup les gens n'ont pas confiance, ils préfèrent tout vérifier. Mais quand c'est des 
journaux, des journaux nationaux ou bien des chaînes de télévision nationales, d'une certaine façon c'est un peu plus digne de confiance." (Zhou)

Lorsqu' Internet est placé à égalité avec les autres, c'est que le niveau de contrôle y est perçu comme fort, ce qui n'est pas forcément présenté comme un inconvénient.

"En Chine les informations sur Internet sont assez fiables, c'est à peu près aussi fiable que les journaux. C'est un effet positif de ce, enfin, du contrôle de l'opinion publique, parce qu'ils censurent (kongzhi) des opinions qu'ils ne veulent pas que tu ailles répandre, et en même temps ils censurent certaines rumeurs, enfin des choses pas fiables (bu kexin de). Disons que les médias chinois, les médias de Chine continentale, je ne compte pas Hong Kong ou Taiwan, les médias de Chine continentale sont assez responsables." (Zhang)

A cause du contrôle plus strict dont ils sont l'objet, les médias de Chine continentale sont présentés comme "fiables" ou "responsables". Or comme on l'a vu précédemment, le terme de "responsable" ne renvoie pas nécessairement à la vérité de l'information, mais au respect d'une certaine position par rapport à la scène publique. Le fait que les médias soient qualifiés de responsables ne signifie pas qu'ils traitent l'information de manière impartiale, mais au contraire qu'ils présentent la position officielle, très clairement identifiable comme telle. Cette manière qu'ont les internautes d'identifier l'information officielle par opposition aux informations officieuses ne leur offre pas de garantie d'accéder à une information plus "vraie", plus vérifiée. En effet, la surveillance supposée de l'information n'empêche pas les journalistes de rapporter de fausses informations ni les rumeurs de circuler.

Une affaire en particulier peut illustrer ce phénomène, c'est l'affaire "des baozi en carton" au début de l'été 2007. Les baozi sont des petits pains fourrés à la viande de porc et cuits à la vapeur. Vendus dans la rue, ils sont généralement très bon marché et ils constituent donc un repas très populaire à prendre sur le pouce. Un journaliste aurait découvert que des fabricants de baozi remplaçaient la viande de porc, dont les prix avaient monté en flèche, par du carton auquel ils faisaient subir un traitement chimique. Le printemps 2007 a été émaillé de plusieurs scandales alimentaires très graves, qui ont conduit à la condamnation à mort du directeur de l'Administration d'Etat de l'alimentation et des médicaments en Chine ${ }^{4}$. C'est

${ }^{4}$ BOUGON François. "Chine: exécution d'un ministre corrompu, sur fond de scandales alimentaires", Chine Informations, 11/07/207, consulté le 24/10/2010 sur http://www.chineinformations.com/actualite/chine-execution-dun-ministre-corrompu-sur-fond-de-scandales_7019.html 
donc dans un contexte très tendu que ce nouveau scandale a été rendu public par un reportage sur la télévision d'Etat CCTV. Très peu de temps après la publication de cette information, elle a été officiellement démentie. Le journaliste qui avait publié le reportage a été arrêté pour diffusion de fausses informations et envoyé en prison pour un $\mathrm{an}^{5}$. Dès lors, il règne une certaine confusion. Plus personne ne sait bien quelle version il faut croire. Est-ce que l'arrestation du journaliste signifie que la nouvelle était vraiment fausse, ou bien que le gouvernement voulait donner un coup d'arrêt aux révélations de scandales alimentaires qui étaient déjà allées très loin? La rumeur dit même que le journaliste aurait été battu afin de lui extorquer des aveux ${ }^{6}$. On ne sait plus ce qui, de la révélation ou du démenti, constitue la fausse information ${ }^{7}$.

L'idée que le journaliste ait menti est tout à fait crédible pour les personnes que j'ai interrogées. En effet le développement très rapide du Net suscite des convoitises. Compte tenu des revenus publicitaires tirés par les sites les plus consultés, le nombre de clics est devenu un enjeu financier très important et il donne lieu à une fraude conséquente. Les internautes ne trouvent donc pas étonnant que des auteurs, blogueurs ou journalistes tentent d'attirer l'attention des lecteurs par tous les moyens. Citons un simple exemple à propos de la blogueuse $\mathrm{Mu} \mathrm{Zimei}^{8}$, qui est souvent représentée comme l'égérie de l'expression individuelle, parce qu'elle a publié des détails de sa vie sexuelle sur son blog, et à propos de Soeur Furong ${ }^{9}$, une fille ordinaire qui présente fièrement des photographies d'elle-même, mise en scène comme une pin-up.

"Par exemple Mu Zimei, je n'ai jamais vu son blog en fait, mais je sais de quoi il parle, ce qu'il y a dedans. Mais je ne la connais pas, je ne l'ai jamais vue. Je pense qu'elle écrit peut-être vraiment pour livrer ses états d'âme, et pas pour attirer

${ }^{5}$ ZHU Zhe. "TV report on cardboard buns 'fabricated'", China Daily, 18/07/2007, consulté en ligne le 24/10/2010 sur http://www.chinadaily.com.cn/china/2007-07/19/content_5438963.htm.

${ }^{6}$ GOLDKORN Jeremy. "Rumor: Cardboard baozi journalist beaten up to extract confession", Danwei.org, $\quad 07 / 08 / 2007, \quad$ consulté le $24 / 10 / 2010 \quad$ sur http://www.danwei.org/media_business/rumor_carboard_baozi_journalis.php.

${ }^{7}$ GOLDKORN Jeremy. "Is the fake news story fake news?", Danwei.org, 20/07/2007, consulté le 24/10/2010 sur http://www.danwei.org/media_regulation/fake_news_about_fake_news_abou.php.

${ }^{8}$ Pour quelques liens et un portrait succinct: http://en.wikipedia.org/wiki/Muzi_Mei (consulté le $24 / 10 / 2010)$.

${ }^{9}$ Pour quelques liens et un portrait succinct: http://en.wikipedia.org/wiki/Furong_Jiejie (consulté le 24/10/2010). 
l'attention. Après tout peut-être qu'elle se disait 'j'ai envie d'écrire, de mettre tout ça en ligne', sans vouloir être connue ou quoi, c'est possible. Mais d'un autre côté il y a beaucoup de gens sur Internet comme Soeur Furong, qui deviennent très célèbres. Il y a beaucoup de gens comme ça sur Internet, y compris des journalistes, ils fabriquent une fausse information avec une célébrité pour augmenter leur taux de clics. Ce genre de choses m'énerve beaucoup, mais je crois aussi que c'est inévitable, et comme on dit toujours, il faut de tout pour faire un monde." (Jiang)

Or les journalistes ne sont pas exempts de telles dérives, d'autant plus en tenant compte $\mathrm{du}$ risque de corruption auquel les uns et les autres sont potentiellement sujets ${ }^{10}$. La surveillance politique de l'information en conduit plus d'un à mentir dans le cadre de son travail, ce dont les internautes sont relativement conscients.

"En Chine on peut dire avec assurance, comment dire, ce n'est pas que tous les médias sont faux, mais tous les médias ne sont pas complètement vrais. Une célébrité du monde des médias avait l'habitude de dire: si on devait exécuter tous ceux qui ont écrit de fausses informations, et qu'on alignait tous les journalistes, même si on en épargnait un sur deux, on en fusillerait quand même la moitié.

- Qu'est-ce que ça veut dire?

- Ca veut dire que si on devait exécuter tous les journalistes qui écrivent de fausses informations, il faudrait tous les tuer, ça veut dire que tous les journalistes écrivent de fausses informations.

- Ils ont tous eu l'occasion d'écrire de fausses nouvelles?

- C'est une phrase que disait une célébrité du monde des médias, ça veut dire qu'il connaissait très bien le monde des médias de l'intérieur. Ca veut aussi dire qu'à la télé, dans les journaux, il y a des choses qu'on n'a pas le droit de dire, c'est interdit. Surtout à la télé, c'est le média roi, ils doivent toujours dire que les choses sont vraies, parfaites et belles, et ils ne doivent jamais dire qu'elles sont fausses, mauvaises et laides." (Tian)

L'obligation même d'assurer un rôle de représentation implique de travestir les informations afin de donner une bonne image à l'extérieur.

${ }^{10}$ LUPANO Emma. "Linfen 'gag fees' spark media ethics debate in China", China Media Project, 30/10/2008, consulté le 24/10/2010 sur http://cmp.hku.hk/2008/10/30/1322/. 
"Donc peu importe de quel pays il s'agit, quand les gouvernements parlent de choses qui les concernent, ils ne peuvent pas être objectifs?

- Oui, ils ne peuvent pas être objectifs, en fait peu importe de quoi ils discutent, ils ne sont jamais objectifs, à cause des nécessités politiques." (Zhao)

Toute information n'est que le reflet d'une position particulière, avec les omissions, voire les mensonges que cela implique. Il en résulte qu'aucune information n'est considérée comme vraie ou vérifiée. Les internautes sont donc quelque peu livrés à eux-mêmes pour évaluer le degré de crédibilité d'une information.

"Je ne peux pas dire en quoi j'aurais le plus confiance ou quoi, mais je me contente de regarder un peu, et j'essaie de comprendre. Parce qu'il y a des choses dans les informations sur Internet, c'est à dire dans notre société aujourd'hui, il n'y a pas les choses auxquelles on croit et celles auxquelles on ne croit pas, il faut regarder les choses et les comprendre en s'appuyant sur sa propre intuition ou sur ce qu'on aime, parce qu'ils ne peuvent pas dire les choses de manière trop originale (lipu). C'est peutêtre ça l'avantage avec la Chine, c'est qu'on n'ose pas trop écrire de fausses nouvelles, ce qu'on entend est plutôt vrai, donc on peut encore voir et comprendre l'actualité. C'est à dire qu'il y a trop de choses qui ne sont pas vraies, mais même dans les choses qui sont gravement fausses, on peut quand même comprendre quelques éléments qui sont vrais." (Deng)

Il y a là une certaine confusion. Sachant qu'aucune source n'est complètement impartiale, les internautes ne peuvent s'en remettre qu'à leur intuition pour comprendre l'actualité. Ils l'interprètent en fonction des positions particulières des différentes sources, comme s'il s'agissait d'un jeu de rôles.

"L'opinion individuelle (geren yanlun) n'est pas vraie ou fausse, c'est une impression sur le moment, et ce n'est pas comme l'information ou les activités commerciales, qui ont un caractère de vérité. Donc l'opinion individuelle, les avis différents sur une chose ne sont pas vrais ou faux, c'est juste sur le moment, peut-être qu'après quelques jours on peut changer d'avis, mais sur le moment on a ce point de vue là. C'est un jugement subjectif, on ne peut parler que d'objectif et de subjectif, mais pas de vrai ou faux. Internet est comme une scène.

- Une scène? 
- Oui, C'est comme une scène, les gens jouent des rôles différents, un peu comme dans une série télé, ou une pièce de théâtre. Que tu dises que c'est vrai ou faux, ce n'est que la véritable réaction psychologique et l'expression de quelqu'un à un moment et dans un contexte donné. Ce n'est ni vrai ni faux." (Lin)

Faute de moyens plus fiables pour évaluer la qualité de l'information et l'honnêteté des journalistes, le caractère officiel ne donne que quelques points de repères. Ces témoignages montrent surtout que les internautes savent interpréter l'information avec un recul non négligeable, qu'ils savent la décoder ${ }^{11}$. Dans le cadre de cette démarche, la prolifération des points de vue subjectifs qui s'expriment sur le Web constitue une ressource sans précédent.

\section{L'intérêt de la subjectivité}

Même si l'ensemble des médias sont perçus comme traitant l'information de manière biaisée, Internet a au moins l'avantage de permettre une mise en parallèle des différentes sources d'information, qu'elles soient chinoises ou internationales. Les presses hongkongaise et taiwanaise sont souvent lues avec l'intention de confronter les points de vue sur l'actualité de la Chine continentale.

"Je crois qu'on ne peut pas exprimer..., par exemple, ils doivent se placer du point de vue du pays, sinon, en Chine la parole n'est pas encore très libre, il y a de la liberté d'expression, mais elle n'est pas si libre que cela. Les journaux doivent se placer du point de vue de la Chine, mais sur Internet il n'y a pas cette obligation.

- Quel genre d'informations ne peut-on pas publier?

[...] - Par exemple les relations entre la Chine et Taiwan, ce que les Taïwanais en pensent au fond. Moi je trouve, je doute un peu, ce que j'ai vu à la télé, est-ce que c'était vrai à $100 \%$, je peux douter [...]. Ca s'est forcément vraiment passé, mais est-ce que ce n'était pas très limité, qu'ils auraient filmé un petit groupe de personnes? La plupart des gens, tous les citoyens taiwanais pensent-ils la même chose, j'ai un certain nombre de doutes, je me demande si c'est très objectif, est-ce que ça ne correspond pas à un but particulier? Dans un cas comme ça je ne fais pas trop confiance, $\mathrm{j}$ 'en crois une partie, donc là-dessus je peux facilement savoir comment c'est traité en Chine, et pour ce qui est de la manière dont c'est traité à Taiwan, je peux savoir comment les gens en parlent. Si je regarde des deux côtés je peux avoir une vision un peu plus objective.

${ }^{11}$ HALL Stuart. "Codage/décodage", Réseaux, vol. 12, n68, 1994, p. 27-39. 
- Donc Internet c'est mieux sur ce point?

- Oui, je trouve que comme ça on peut voir comment pensent les gens, quels commentaires ils font." (Sun)

Dans ce témoignage, l'internaute pense se faire une idée relativement claire des capacités et des limites de la censure. La grande diversité des contenus sur Internet permet de dépasser la principale limite des médias de masse traditionnels que sont la télévision, les journaux, la radio. Les informations imposées par les autorités prennent une part importante de l'espace et du temps disponibles sur ces médias, qui sont extrêmement limités. Au contraire, Internet ne pose pas cette limite et il est possible d'y trouver une grande diversité de points de vue. Si chaque point de vue n'est pas une garantie en soi, leur confrontation permet de s'approcher le plus possible d'une forme d'objectivité.

"J'ai l'impression que l'information en Chine est biaisée, bien sûr ça va de soi qu'elle penche en faveur de notre pays, elle se positionne du côté de notre pays. Mais si on allait voir des sites étrangers ce serait sûrement plus objectif, enfin ce ne serait pas la position de notre pays. Et puis on peut voir le monde plus objectivement à travers les yeux des autres, dans leurs commentaires. Des fois j'ai l'impression que chacun a sa propre position par rapport aux informations, donc quand quelqu'un dit que quelque chose est vrai, ce n'est pas forcément vrai." (Sun)

Il faut reconnaître qu'Internet n'a pas le monopole de cette approche de l'information, et que l'on trouve aussi dans la presse papier quelques initiatives pour proposer une plus grande diversité de points de vue, par exemple sous la forme d'une sorte de Courrier International, ce qui est très apprécié.

"En-dehors des journaux, d'Internet et des magazines, je lis aussi "La référence de l'information" (Cankao xiaoxi), c'est édité par le Quotidien du Peuple. Ils ne font pas de commentaires, c'est plutôt des résumés de beaucoup de médias importants à l'étranger, ou de commentaires sur la société du point de vue de ces journaux. Je le lis souvent. Et je l'aime bien parce que ça traite surtout des commentaires que font les journaux étrangers à propos de la Chine. On peut dire que c'est un regard extérieur." (Zhao)

Cependant les possibilités offertes par le Web en termes de diversification des sources d'informations sont décuplées. Il est possible d'y confronter des sources officielles différentes, mais aussi des sources plus officieuses, et également des blogs ou des forums. 
"En Chine, on n'a pas la même liberté d'expression qu'en Occident, même si en Occident non plus ce n'est pas parfait, mais en Chine c'est peut-être encore plus évident. Donc on étouffe souvent des informations, et puis, la même information ne sera pas traitée depuis la même position. On regarde les choses sous un certain angle, on les analyse sous un certain angle. C'est l'intérêt national qui compte le plus. Mais sur Internet, il y a beaucoup d'autres informations, qui viennent du portail de Sina, ou bien de n'importe quel blog ou revue, ça permet d'entendre un autre son de cloche." (Xu)

La confrontation de points de vue les plus diversifiés possibles fonctionne comme une meilleure garantie de la fiabilité de l'information. Or à la différence des médias traditionnels, Internet ne permet pas seulement d'accéder à des sources d'information plus diversifiées. Le réseau permet aussi d'accéder à de nombreux témoignages individuels, qui se présentent d'emblée comme une forme de parole particulière et subjective, et qui acquièrent ainsi la force de l'authenticité.

Les espaces d'expression offerts aux individus donnent à Internet un statut bien différent de celui des autres médias. Ils donnent à lire et à entendre la voix des individus, des "gens ordinaires". Sur eux, la règle qui impose de se placer "du point de vue du pays" ne s'applique pas. Bien au contraire il leur est demandé de ne parler que d'eux-mêmes et de leur quotidien. Ainsi la parole des gens ordinaires bénéficie-elle de l'authenticité, du crédit accordé au témoignage individuel, qui n'émane pas d'un organe officiel comme un journal, mais au contraire reflète les modes de vie des différentes couches la société. Au risque de croire des histoires montées de toutes pièces, les internautes apprécient ce reflet de la réalité la plus quotidienne, qui n'est disponible presque nulle part ailleurs avec autant d'authenticité.

C'est sans doute l'une des raisons pour lesquelles les forums ou BBS constituent une source d'information très importante pour de nombreux internautes. C'est ici en effet que renait la notion de vérité, au travers de l'authenticité de la parole des anonymes du Web.

"Par exemple pour une information, le plus important c'est sa véracité (zhenshixing), mais quand je lis les journaux chinois, il y a beaucoup de réductions. Donc c'est pour rechercher la vérité (zhen) que je vais sur Internet, pour voir les choses les plus vraies (zhenshi). Tout n'est pas forcément vrai sur Internet, mais tout est... Il y a une différence entre Internet et les autres médias, les journaux, les magazines, la télé, c'est que tout le monde dit ce qu'il a envie de dire. Ce n'est peut-être pas vrai, mais chacun a 
des pensées, ou un raisonnement. Ce n'est pas forcément le plus correct (zhengque), mais c'est le plus vrai (zhenshi)." (Tian)

Ici la vérité n'est plus mesurée à l'aune de la conformité politique ("correct") car elle sort de son champ. Il ne s'agit plus que de la parole, tout à fait relative, des autres internautes. Cette parole n'a pas vocation à fournir une information irréfutable. Elle est simplement authentique. La diversité des opinions particulières et subjectives est donc un avantage aux yeux des personnes interrogées. Elle permet de s'affranchir de l'aspect biaisé de toute information et de comprendre les faits sous des angles divers, et ainsi de manière plus complète. Internet tire donc une partie de son crédit de la place qu'il accorde à l'expression individuelle. A ce titre, Internet remplit un véritable rôle social en permettant aux uns et aux autres de partager des expériences et de trouver un portrait plus authentique de la société qui les entoure.

"On peut comprendre une chose sous de nombreux aspects au travers de personnes différentes. Je ne dis pas que sous prétexte que ces gens ont écrit un jugement subjectif sur quelque chose, il faudrait penser que c'est comme ils disent, mais il y a des choses qui ont beaucoup d'aspects." (Yuan)

L'authenticité est donc accompagnée de la diversité des points de vue. Chacun parlant de son expérience personnelle, tout le monde adopte des points de vue différents qu'il est enrichissant de consulter pour essayer de mieux comprendre les événements. La version la plus crédible d'un événement se trouve au croisement entre ces différentes versions.

"Je regarde un peu les points de vue des gens sur cet événement, parce que les informations c'est seulement quelque chose d'objectif, alors que dans les commentaires il y a tous les points de vue subjectifs des gens, et puis je me fais mon propre avis." (Li)

Finalement, nombreux sont ceux qui affirment tenir compte des avis des autres pour se faire une opinion sur les questions qui les intéressent.

"Il y a des gens qui s'expriment sur Internet, et tout le monde vient faire des commentaires. On lit un peu les avis des autres, leurs opinions.

- Est-ce que ça peut te faire changer d'opinion?

- Pas complètement, mais ça peut arriver, alors que si on n'en parle pas il n'y a aucune chance." (Wang) 
Alors même que les internautes refusent d'essayer de convaincre les autres, ils se forgent souvent une opinion en lisant les avis des autres, qui peuvent les influencer à des degrés divers. Le cloisonnement des opinions n'est donc peut-être pas aussi fort que ce que veulent bien affirmer les internautes au premier abord. De plus le caractère personnel des blogs permet d'évoquer certains sujets de manière plus critique, ce qui conduit Sun à les qualifier de plus "objectifs".

"On peut dire que c'est crédible, parce qu'Internet est assez objectif, et puis à la télé ils font des choix, ils diffusent de manière orientée, le côté gouvernemental est très fort. Par exemple les paroles qui ne vont pas dans le sens du gouvernement ne seront pas diffusées, mais sur Internet c'est différent, sur Internet on peut faire des commentaires assez objectifs, et pas seulement du point de vue $d u$ gouvernement." (Pan)

La "tyrannie de l'intimité" ${ }^{12}$ de Sennett trouve ici un avantage inattendu. Certes, c'est la parole la plus individuelle et intime qui trouve le plus de grâce aux yeux des internautes interrogés, même si elle les empêche de monter en généralité dans leurs discussions. En effet cette disqualification de la parole publique ou officielle, trop biaisée, ouvre un espace immense et riche pour l'expression croisée des subjectivités. C'est l'occasion pour beaucoup de former leurs opinions au contact d'une certaine diversité de points de vue, et bien souvent, au contact d'opinions relativement critiques.

Les internautes interrogés s'inscrivent en cela dans la même logique qu'un grand nombre d'outils dits participatifs, fondés sur une présentation la plus équitable possible des différentes opinions et non sur une recherche de vérité objective difficile et conflictuelle, comme Wikipédia par exemple ${ }^{13}$. Dans un tel modèle, le principe de la vérité est remplacé par celui de la pertinence ${ }^{14}$. Chacun se bricole ${ }^{15}$ une information qui l'intéresse, pertinente pour lui, à partir de sources diversifiées, et en interaction avec les autres internautes.

\footnotetext{
${ }^{12}$ SENNETT Richard. Les tyrannies de l'intimité, Paris: Seuil, 1979.

13 "La définition de l'objectivité utilisée par l'encyclopédie Wikipédia est antibachelardienne et consiste à rejeter la séparation entre les faits et les opinions sur les faits. Le principe de neutralité consiste à 'exposer ce que les gens croient, pas ce qui est'. La communauté opère une remontée critique et soupçonneuse des faits vers les opinions." AURAY Nicolas, HURAULT-PLANTET Martine, POUDAT Céline et JACQUEMIN Bernard. "La négociation des points de vue. une cartographie sociale des conflits et des querelles dans le Wikipédia francophone", Réseaux, n 154, 2009, p.15-50.

14 "Sur l'Internet, le régime de la pertinence remplace l'ancien royaume de la vérité". BOUGNOUX Daniel, cité dans GUILLAUD Hubert. "Réinventer la démocratie : Internet, nouvel espace
} 
Or les internautes ont accès à des formes d'expression beaucoup plus hétérodoxes qu'il ne peut sembler au premier abord. Si les opinions les plus critiques ne sont pas encouragées dans le discours public, elles ne sont pas non plus supprimées complètement, mais plus souvent renvoyées à des espaces marginalisés, moins visibles et surtout moins "publics", mais non moins nombreux. En effet, les ambiguïtés de la distinction entre privé et public offrent la possibilité de rendre visible, dans des espaces informels, une parole privée qui ne s'encombre pas beaucoup des contraintes de la représentation.

\section{2 - L'espace "clair-obscur" des conversations}

\section{Le royaume de la rumeur et de l'anonymat}

L'ambiguïté de la séparation entre privé et public est d'abord problématique pour les internautes eux-mêmes. Certains s'inquiètent de la surveillance plus faible qui règne sur une portion conséquente des plates-formes Web, et dénoncent l'anonymat qui permet toutes sortes d'abus. En même temps qu'ils le condamnent, ils décrivent très nettement un espace sans "conséquences légales", où nul n'est tenu de prendre ses responsabilités, autrement dit un espace où la contrainte de dire du bien de son pays est relâchée. Il n'est pas question de s'associer publiquement à l'existence de cet espace. Si plus d'un internaute affirme s'en inquiéter, la plupart disent cependant, mais toujours en parlant des autres, que "les Chinois aiment bien ça".

"On peut exprimer son propre avis avec encore moins de scrupules, c'est certain, parce qu'il n'y a pas de conséquences légales. Comment dire, les gens sont différents, il y a des gens qui ne veulent pas exprimer leur point de vue, même si c'est anonyme ils ne veulent pas. Les Chinois aiment bien ça, mais moi je n'aime pas, c'est comme les lettres anonymes avant, quand on s'attaquait à quelqu'un ou qu'on voulait se venger d'un officiel, on écrivait des lettres de dénonciation anonymes. Ce genre de dénonciations était très fréquent avant. Donc pourquoi est-ce qu'il y a autant de commentaires anonymes sur les forums? Premièrement parce qu'on n'est pas

démocratique ?", Internet Actu, 12/05/2009, consulté le 10/06/2009 sur http://www.internetactu.net/2009/05/12/reinventer-la-democratie-internet-nouvel-espacedemocratique/.

15 "Lire: un braconnage". CERTEAU Michel. L'invention du quotidien 1. Arts de faire, Paris: Gallimard, 1990, p.239s. 
responsable des conséquences légales, deuxièmement parce que les gens cherchent des moyens de se défouler." (Lin)

Il y a beaucoup d'ambivalence en effet dans la circulation de rumeurs diverses. Vraies ou fausses, elles ne sont cependant pas sans effet sur la population et sur le gouvernement, comme en témoigne la crise du SRAS en $2003^{16}$. Confronté à la circulation d'informations de plus en plus inquiétantes sur la pneumonie qui se répandait dans le pays, dont certaines étaient vraies et d'autres extravagantes, le gouvernement s'est vu contraint d'adopter une gestion plus transparente de la situation. Internet, mais aussi les SMS, avaient permis une circulation massive d'informations officieuses que nul ne pouvait ignorer. La censure des informations qui circulent ouvertement, "en haut", n'empêche pas la tenue, "en bas", d'autres formes de discours.

"Les questions intimes, c'est quel genre de choses?

- Par exemple des questions en rapport avec l'amour, comme les sentiments, ou des projets personnels, et toutes les choses qu'on ne peut pas dire ailleurs, c'est à dire, comme prenons l'exemple des chiens, comment faire pour la question des chiens. Les Chinois vivent tous selon le principe 'en haut il y a une mesure, en bas il y a une contre-mesure' (shang you zhengci xia you duici), c'est à dire que quand une règle sort, je dois avoir une façon d'y répondre, et en quoi consiste cette façon, ce sont des choses dont on ne peut pas discuter ouvertement." (Zhang)

De l'avis de tous, Internet, en fournissant des canaux plus petits pour faire circuler l'information, permet de parler de choses qui n'ont pas leur place dans d'autres médias. Beaucoup d'internautes semblent être à l'affût de ces "rumeurs", ces petites informations, qui en perdant leur caractère officiel peuvent circuler plus facilement.

"Par exemple il y a des informations de divertissement, comme les choses sur les stars, qu'on ne peut pas voir à la télé. Peut-être qu'ils brident ces médias-là, qu'on n'a pas le droit d'en parler dans les journaux, ni de voir ça à la télé, mais on peut trouver ces anecdotes (xiaodao xiaoxi) sur des sites Internet. Et puis il y a aussi des choses assez réactionnaires, des questions sur les dirigeants du pays, ou bien des questions sur Taiwan, y compris des rumeurs, qui ne passeraient forcément pas à la télé. Et il y a aussi des histoires de stars ou de fonctionnaires d'Etat qui touchent des

${ }^{16}$ SAUTEDE Eric. "Les leurres de la modernité, Internet, information et crise du "SRAS" en Chine", Perspectives chinoises, mars-avril 2003, $\mathrm{n}^{\circ} 76$, p. 21-27. 
pots de vin, ou ce genre de choses. C'est sûr que la télé ou les journaux ne peuvent pas en parler. Il y a des journalistes, sûrement des journalistes assez honnêtes (zhengyi), qui postent ça sur des forums, et tout le monde en parle." (Huang)

Les expressions utilisées pour désigner ces informations renvoient toujours à l'idée de prendre des voies détournées ou des petits chemins (xiaodao xiaoxi), ou à une forme de dissimulation dans les angles moins visibles des sites Internet (bianbian jiaojiao).

"Je m'intéresse à la politique depuis le collège. Au début, je n'aimais pas vraiment ça, à cette époque-là c'était vraiment pour l'école, parce qu'à l'école en Chine il y a un cours de politique, et que c'est en bonne partie lié aux informations. On peut dire que l'école m'a fait m'intéresser aux informations, et puis peu à peu, après la fin de ces cours, j'ai commencé à m'intéresser aux informations, surtout celles qui ne sont pas traitées dans les journaux, des informations périphériques (bianbian jiaojiao). Mais ces informations qui ne sont pas traitées dans les journaux, elles sont toutes sur Internet. Le Web chinois n'est pas encore très mûr, certaines informations sont publiées par des journalistes qui ne sont pas très responsables. Ils fouillent des rumeurs, ou bien ils brodent, pour leur intérêt personnel, sans prendre leurs responsabilités. Mais ce n'est pas complètement n'importe quoi, même si c'est la majorité, il y a quand même de l'information, et ça sort assez rapidement, et ça sort un peu plus tard dans les informations du gouvernement." (Zhao)

Les événements censurés ou les opinions critiques ne prennent pas les autoroutes de l'information que sont les grands portails et les sites des quotidiens en ligne. Ils n'en sont pas moins accessibles à qui veut les lire sur des espaces moins visibles, et ils peuvent circuler à grande échelle entre les internautes au travers de leurs conversations privées.

\section{Les conversations au sein de micro-publics}

Faut-il rappeler la fluidité avec laquelle une information peut circuler sur Internet, sous des formes plastiques et avec des degrés de visibilité très divers? Si les blogs ou les forums permettent de publier des billets, les messageries instantanées, les groupes QQ ou le courrier électronique permettent, eux, de faire circuler diverses informations de manière moins visible, plus privée, mais non moins massive. Cet internaute décrit d'une manière relativement claire et représentative le trajet que parcourt l'information dans son expérience personnelle. 
"On peut laisser des commentaires, faire des recherches, et transmettre les informations. Et avec QQ on peut l'envoyer à tout un groupe, et je peux aussi regarder ce que mes amis m'envoient. Ensuite on fait tous des commentaires, sur QQ. On va lire l'article, on lit les commentaires des gens, on renvoie les commentaires à des amis, et ils répondent." 17

D'une part cet internaute montre son rôle actif dans l'accès à l'information: il effectue une sélection en allant chercher des thèmes d'une manière proactive, selon le sujet, le temps dont il dispose, le degré de précision qu'il recherche. Il se rend acteur de l'information qu'il reçoit. D'autre part il décrit le passage de l'information dans des espaces dont les degrés de visibilité sont très divers. Si la source principale de l'information est un article posté sur le site Internet d'un journal, elle peut être transmise vers d'autres amis au travers de la messagerie instantanée, et assortie de commentaires, qui ne seront lisibles que pour une personne, ou un petit nombre de proches dans le cas d'un groupe. Ce groupe d'amis prête attention non seulement à l'article principal, mais également aux commentaires des internautes, qui bien souvent sont des inconnus. Dans l'hypothèse où l'un d'entre eux poste à son tour un commentaire sur cet espace visible, il aura bénéficié de la conversation privée qui aura eu lieu entre les proches, bouclant ainsi la boucle. L'espace d'expression n'est donc absolument pas unique, il est fluide, mouvant, plein de canaux alternatifs qui permettent la circulation d'information de manière plus ou moins visible.

Il n'y a pas en fait de cloisonnement formel des sujets de conversation dans les espaces de discussion. Au sein d'un forum a priori public, on peut finalement côtoyer autant d'inconnus que d'amis et discuter de tout et de rien, sans cesser de considérer le forum comme un espace privé. De nombreux internautes créent des fils de discussion qu'ils finissent par considérer comme des lieux privés où ils retrouvent leurs amis, par opposition aux fils principaux des grandes plates-formes qui peuvent réunir des milliers d'intervenants.

"En général les petits forums sont assez lâches. On s'en sert surtout pour discuter avec des amis. Il peut y avoir dix à vingt personnes, toujours les mêmes. Par contre sur les forums plus commerciaux, tout est très standardisé, les gens vont tous sur les plus grandes sections.

- Qu'est-ce que tu veux dire par standardisé?

\footnotetext{
${ }^{17}$ L.RF.
} 
- Standardisé, ça veut dire que c'est des forums plus grands. Ils sont plus contrôlés, plus spécialisés. C'est moins personnel." (Zhang)

La plupart des fils de discussion sont de fait appropriés par des internautes ou des groupes d'internautes, qui les considèrent comme des espaces personnels. Loin de recueillir une parole officielle, ces fils sont donc souvent considérés comme des espaces de conversation banals, entre des individus qui ne font que donner des impressions en leur nom propre. Les recommandations qui découragent la confrontation des opinions s'y estompent et de fait, chacun se trouve confronté aux impressions des autres, qui peuvent concerner des aspects très divers de la vie quotidienne et du monde social.

"On discute de tous les types de sujets [dans mes 40 groupes QQ], depuis la vie quotidienne jusqu'aux affaires du pays.

- Tu as un exemple?

- C'est difficile, par exemple sur le groupe de photo, ils parlent de modèles, de photo mais aussi des affaires du pays, tout le monde bavarde un peu, il y a de tout.

- Quand tu as envie de parler d'une question, tu vas sur quel groupe?

- En général je ne choisis pas, je parle sur le groupe où je me trouve, tout le monde est très bien, ils ne me disent pas 'ce groupe est fait pour parler de photo, on ne parle que de photo'. Tout le monde parle à bâtons rompus, des fois on parle d'autres choses, tout est possible.

- Est-ce qu'il y a un enregistrement de ces discussions?

- Oui, c'est dans les archives du groupe.

- C'est ouvert?

- Oui, c'est ouvert, tout est sur le groupe, tout est dans les fonctions du groupe, tout le monde peut le voir. Et puis tu peux dire ce que tu veux, personne ne se fâchera, c'est comme de plaisanter ensemble." (Deng)

Il s'agit ici de groupes QQ, qui sont la plupart du temps ouverts au public, et qui pourtant ne sont utilisés que par des groupes restreints d'amis. Ils sont utilisés comme des espaces privés, et les conversations qui y sont tenues sont considérées comme presque confidentielles. Elles le sont en tous cas par la petite taille des publics concernés. Quels que soient les dispositifs (forums, groupes, blogs), c'est généralement la taille de l'audience supposée qui détermine le statut accordé à la parole. La plupart s'adressent à des micro- 
publics, voire à personne en particulier, comme c'est le cas des blogs, lorsqu'ils sont conçus comme des journaux intimes.

"Donc tu me demandes quelle est la différence entre les trois? Sur QQ on s'adresse à une personne, sur un forum on s'adresse à moitié à quelqu'un, et sur les blogs on ne s'adresse à personne du tout. Je mets seulement mes trucs dessus, n'importe qui peut venir discuter, et la distance entre eux et moi va de très proche à très loin." (Tian)

Les blogs sont emblématiques de ce sentiment de disposer d'un espace privé, à soi. Les internautes se sentent d'autant plus libres de s'y exprimer comme ils le souhaitent.

"Pour ce qui représente vraiment l'avis personnel, comme par exemple sur la bourse, par exemple s'il s'est passé quelque chose à Pékin, on dirait que la liberté d'expression n'est pas limitée, ça n'a pas d'importance, du moment qu'il n'y a pas de calomnies, ça ne fait rien, tout le monde peut exprimer son opinion.

- Donc il n'y a pas de limites?

- C'est ça, il n'y a pas de limites.

- Entre les blogs, les forums, ou bien les groupes QQ par exemple, lequel est le plus adéquat pour discuter de phénomènes de société?

- Les phénomènes de société?

- Par exemple quand il y a un fait divers, tu préfères en parler sur les blogs ou sur les forums?

- Sur mon propre blog, les blogs sont plus appropriés.

- Pourquoi?

- Parce que sur les phénomènes de société, tu veux forcément exprimer un avis personnel, et quand tu l'as exprimé, alors c'est le tien. Tu peux aussi en discuter avec beaucoup de gens sur QQ, mais si personne ne veut en parler avec toi, si vous n'êtes pas sur la même longueur d'ondes (gongtong yuyan), tu peux le poster sur un forum et en même temps sur ton blog, [...] ça ne change rien. Je ne veux pas dire que tu es obligé de poster les articles sur ton blog en plus des forums. Mais je trouve que le blog c'est plus personnel, ça compte comme quelque chose qui t'appartient. Tu ne peux pas t'approprier un forum tout entier, mais ton blog est entièrement à toi. Ce que tu écris t'appartient, ce sont tes trucs, c'est ton point de vue. Surtout sur ces phénomènes de 
société, par exemple si une star dit quelque chose, et que tu as un avis là-dessus, ou bien qu'il se passe quelque chose dans le pays, tu peux aussi dire ton... par exemple ton point de vue. Si tu trouves que c'est comme ci ou comme ça, ça n'a pas d'importance, tu peux aussi le poster sur un forum, mais ça ne reste pas longtemps, au bout d'un moment tu finis par ne plus le retrouver. Il vaut mieux le mettre sur ton blog." (Luo)

Beaucoup d'internautes considèrent les blogs comme des espaces privilégiés à cause de leur caractère personnel. Ils sont parfois opposés aux grands forums qui peuvent brasser des publics nombreux, qui ne laissent pas toujours le temps de la réflexion à leurs participants, et qui peuvent sembler plus désordonnés.

"Je pense que pour les posts, comment dire, c'est comme une zone d'irrigation, il y a des gens qui disent de tout, c'est un peu n'importe quoi, et il est difficile de démêler le vrai du faux. Si j'écris un commentaire là-dessus, c'est sur des petites choses. Les blogs d'après moi, si on compare, c'est un espace plus pur, je trouve que les gens qui lisent vraiment les blogs, ils ont un état d'esprit différent de ceux qui irriguent les zones d'irrigation. Dans une zone d'irrigation, je peux dire des gros mots, je peux dire seulement un ou deux mots. Mais sur un blog c'est différent, on réfléchit à son blog, ses commentaires, à un niveau relativement plus élevé, et puis les productions sont relativement plus régulières, qu'elles soient positives ou négatives, tout mérite d'être lu. Dans la zone d'irrigation tout le monde fait des blagues, pour se défouler un peu. QQ c'est surtout pour discuter entre deux personnes, c'est encore plus privé. On s'adresse à un ami, c'est fait pour être entre soi, comme maintenant on est en tête à tête, et je ne dirais peut-être pas des choses aussi profondes que sur un blog. Je vais peut-être dire des choses simples, une discussion intime, mais si je suis d'accord pour révéler certaines de mes vraies pensées, je te présenterais mon blog, pour que tu regardes, ou je lui transférerais des choses que j'ai écrites." (Tian)

Les blogs sont généralement désignés comme le royaume de l'expression individuelle et intime, et ils sont à ce titre l'espace idéal d'exercice de la liberté d'expression. Le revers de la médaille est que la personnalisation est inversement proportionnelle à la taille de l'audience potentielle. Toutefois ils restent un outil d'autopublication important, en regard des outils d'échanges instantanés tels que QQ et MSN qui, eux, ne permettent pas l'archivage des échanges ni la publication ouverte. 
"Le plus libre, c'est les blogs, parce qu'ils ne sont pas contrôlés. Et sur QQ aussi il y a un espace de blog. Sur MSN aussi. Jusqu'à maintenant il ne faut pas donner beaucoup d'infos pour ouvrir son propre blog. Si le modérateur voit qu'il y a un problème, il peut te prévenir mais en général ils n'ont pas les moyens de contrôler. Encore plus libre que ça, c'est MSN ou QQ. Mais le défaut c'est qu'il n'y a pas une adresse fixe. Tout ce qu'on dit disparaît aussitôt. C'est comme le téléphone, on peut parler sans réfléchir.

[- Donc finalement tu trouves que ce sont les blogs qui sont les plus intéressants?]

- Oui, parce que tout d'abord pour parler de liberté d'expression, il faut que ce soit ouvert, parce que si ce n'est pas ouvert, on ne peut plus parler de liberté. Les blogs c'est d'abord un endroit ouvert, il peut y avoir des gens qui le lisent." (Zhang)

Cet internaute, qui est certainement l'un des plus politisés du panel interrogé, souligne l'importance du caractère public des blogs. Ils offrent une plus grande liberté d'expression à leurs utilisateurs car chaque auteur d'un blog maîtrise complètement le contenu de son espace. Si des sites ou des forums ne fournissent pas un environnement accueillant, les internautes ont la possibilité d'ouvrir leur propre page pour y exprimer leurs opinions. Mais que serait cette liberté sans la possibilité de s'adresser à un public, si petit soit-il? Les blogs sont un espace à la fois privé, au sens où ils sont personnels, et publics, au sens où ils sont visibles.

Si les internautes désignent le plus souvent les blogs comme le support privilégié de la liberté d'expression, on voit bien que chaque type de support peut également s'y prêter. Plus que le dispositif lui-même, c'est la démarche personnelle, ainsi que la taille restreinte du public qui placent les blogs, mais aussi les petits fils de discussion sur des forums, ou bien encore des groupes QQ, dans la catégorie des conversations privées. Alors que les internautes ont une tendance certaine à s'autocensurer sur des plates-formes plus médiatisées comme des forums très fréquentés (pensons à Qiangguo Luntan), il existe une multitude de plates-formes plus petites, qui s'adressent à des publics restreints, où les internautes ne s'expriment que pour eux-mêmes ou pour leurs $\operatorname{amis}^{18}$. Ces plates-formes, dans lesquelles une recherche

${ }^{18}$ A la date des entretiens, le service Twitter n'en était qu'à ses débuts et avait encore très peu d'utilisateurs. Je n'ai donc pas pu recueillir de témoignage sur son utilisation. Cependant il a été abondamment utilisé depuis comme un moyen de faire circuler de l'information en-dehors des canaux les plus surveillés, en Chine comme en Iran par exemple. Je le place donc dans la catégorie des espaces en "clair-obscur". 
systématique des différents propos est quasiment impossible, sont visibles pour les "passants", les "badauds". Ils se situent ainsi dans cet espace de "clair-obscur"19 qui rend visible les conversations sans forcément rendre publique l'identité de leurs auteurs, ou bien sans donner toutes les clés de compréhension de l'ensemble du discours. Les internautes s'y accordent une liberté de parole parfois surprenante.

\section{Les transcriptions cachées}

Cette présentation des formes ambivalentes de traitement de la parole publique ne serait pas complète sans mentionner les différentes manières dont les internautes peuvent subvertir le discours le plus public en ayant recours à des "transcriptions cachées" ${ }^{20}$. Le Web chinois est en effet truffé d'exemples de contournement des règles de la censure avec l'utilisation de double langage et d'allusions, qui respectent les règles formelles de la prise de parole publique, tout en faisant passer un discours hétérodoxe plus ou moins osé. Les internautes font ainsi preuve d'une relative habileté dans l'utilisation des différents supports d'Internet, que ce soit en tant que lecteurs ou lorsqu'ils s'expriment eux-mêmes.

En fonction de leur perception du degré de publicité des différents espaces, les internautes sont capables d'effectuer des arbitrages importants. Ils ne se positionnent pas de la même manière sur une page d'informations de Sina, sur leur blog, ou sur un forum de discussion très fréquenté. En fonction de leur évaluation personnelle des différentes situations, les internautes ne se donnent pas les mêmes contraintes.

"Quand on discute on n'est pas obligé de dire des choses vraies, ou bien si aujourd'hui je suis de bonne humeur, je vais avoir envie de plaisanter avec toi, et si je fais une blague, c'est sûr qu'il y aura du faux dedans, on peut le faire. Mais quand on s'exprime sur un forum, si on s'exprime sur des sujets qui impliquent un sens de la responsabilité, il faut absolument dire la vérité. C'est pour ça qu'au début [de l'entretien] on avait dit que pour des sujets différents, pour des choses différentes, on a un traitement différent. Sur Internet on peut s'exprimer comme on veut, mais sur certains sujets il faut quand même avoir un certain sens des responsabilités." (Yang)

${ }^{19}$ CARDON Dominique. "Le design de la visibilité. Un essai de cartographie du Web 2.0", Réseaux, $\mathrm{n}^{\circ} 152,2008$, p.93-137.

${ }^{20}$ SCOTT James. Domination and the arts of resistance: hidden transcripts, Yale University Press, 1990. 
Tous les individus, quelle que soit leur réaction, doivent naviguer dans les limites très mouvantes de la distinction entre espace intime et espace officiel, tout en espérant trouver une certaine audience. L'enjeu est donc ici d'atteindre une audience suffisamment large pour avoir la sensation de partager un message avec d'autres personnes, sans donner l'impression de quitter le domaine de la confidence intime, du témoignage, ou bien de l'expression conformiste, qui représentent des manières légitimes de s'exprimer publiquement sur Internet. Au moment de prendre la parole, les internautes les plus calculateurs se posent de nombreuses questions en fonction du contexte, du sujet, du moment, ainsi que du type d'espace choisi pour publier l'opinion.

"Tout dépend de comment on présente ses commentaires et tout dépend du sujet. Selon le niveau de liberté du sujet, on choisit la manière de le dire et aussi l'endroit." (Zhang)

Même dans le cas où un billet est effacé par les modérateurs, il peut être renvoyé sur d'autres plates-formes moins publiques, sous un autre pseudo, ou transformé légèrement pour passer les filtres. Certains internautes déploient ainsi de véritables stratégies pour faire passer leurs opinions sur Internet, même si cela implique parfois quelques modifications de leur propos.

"Je suis assez libre, je ne subis aucune limitation, parce que c'est mon caractère. Si tu veux m'imposer une limite, je ne t'adresserai aucune parole, ça a toujours été comme ça, si tu veux me contrôler, ceux qui veulent m'imposer des limites, je ne leur parlerai jamais. Si je trouve que tu ne m'apportes rien de bon, et que tu veux obtenir de moi quelque chose, tu ne pourras jamais m'imposer de limites. Si j'envoie quelque chose, et que tu dis on ne peut pas dire ça, alors soit, je ne t'adresse plus un mot, je considère que tu es quelqu'un qui ne veut pas avoir de vraies informations. A l'instant je disais que les forums sont un endroit relativement libre pour s'exprimer. En fait même si on efface mes trucs, on ne peut pas m'empêcher de changer d'endroit, ou de changer de pseudo, et je continue de m'exprimer. En résumé ce que je veux dire, je peux le dire, jusqu'à maintenant je ne peux pas dire que j'aie jamais eu de scrupules, à me dire 'je ne peux pas dire ça'. Bien sûr, je peux dire les choses d'une autre manière, ou sur un autre ton, mais ce que je veux dire personne ne peut m'empêcher de le dire. J'ai trop de techniques, mon niveau littéraire n'est pas mauvais, je peux faire des métaphores, j'ai beaucoup de méthodes, donc en termes de liberté, j'ai l'audace de dire que j'ai des techniques très subtiles pour dire ce que je veux." (Tian) 
De nombreuses solutions existent pour faire passer une idée un peu critique sur des plates-formes publiques. Ici cet internaute parle d'utiliser des "métaphores", de dire certaines choses à demi-mot. De manière classique, une solution souvent adoptée est la dérision, ou l'ironie, qui ramène la parole à un statut moins formel, non sans faire passer des idées importantes. La plupart des internautes sont tout à fait capables de lire entre les lignes, et de comprendre qu'une phrase nostalgique sur la Révolution culturelle peut être en réalité une critique acerbe de la situation contemporaine par exemple. Beaucoup d'internautes s'autorisent à faire des plaisanteries sur leurs dirigeants, même s'ils n'abordent pas directement le contenu de leur politique.

"Les gens en parlent beaucoup [de politique]?

- Oui, beaucoup. Mais ils en parlent indirectement [yinhui].

- Tu as un exemple?

- (rire). Euh... (se racle la gorge). C'est difficile. Par exemple dans chaque pays on fait des blagues sur ses propres dirigeants, donc en Chine, il y en a beaucoup, tout le monde, c'est à dire, comment dire... tout le monde peut discuter de quelques mouvements dans le monde ... politique, on peut discuter de choses comme des réformes du système par exemple... Mais on ne dirait pas cela directement, c'est à dire, bien sûr que si ce sujet est dit à voix haute, dans ce cas c'est pour s'opposer, si tout le monde est d'accord, mais en même temps on ne dirait pas directement que 'ceci n'est pas bien, cela n'est pas bien'. L'exemple, le plus simple, c'est la montée du prix des taxis, alors que le gouvernement leur donne une subvention pour l'essence. Alors beaucoup de gens ont dit en ligne qu'en fait cette méthode n'était pas très bien. Ils utilisent toutes sortes de méthodes pour s'opposer, mais ça ne sert à rien. En fait en Chine, enfin je ne sais pas si c'est pareil ailleurs, mais en Chine nous avons un point très spécial. Entre la vie de tous les jours et Internet, dans la vie on dit que le peuple vote blanc avec les pieds, mais sur Internet, on peut dire qu'ils votent (tout court) avec les pieds, ou on peut dire qu'ils votent leur approbation avec les yeux, c'est à dire que si ils restent c'est qu'ils approuvent, s'ils s'en vont ça veut dire qu'ils s'opposent. Donc on verra toujours des gens qui approuvent et qui s'opposent, mais il faut l'interpréter différemment par rapport à dans la vie." (Ren)

Cette remarque est importante. Pour cette personne, il n'est même pas nécessaire de rédiger des avis et de les transmettre à d'autres personnes pour faire savoir son opinion. Les opinions individuelles se lisent dans les taux de clics sur les différents sujets d'actualité. 
Lorsqu'un article sur un dirigeant corrompu est extrêmement lu, cela signifie que les gens sont préoccupés par la question de la corruption. Le taux de lecture des articles sur les scandales alimentaires révèle un déficit de confiance énorme dans les produits de consommation alimentaire. De plus en plus souvent, les internautes postent sur leurs pages personnelles des liens ou des photographies montrant des injustices sans commentaires, estimant qu'elles parlent d'elles-mêmes. Le message est souvent très fort alors même qu'il n'y a aucune critique explicite.

Les méthodes qui permettent de subvertir les règles formelles de l'expression en public sont de plus en plus nombreuses. Les "satires" (egao), ou parodies qui reprennent souvent des clichés de la propagande officielle, ont beaucoup de succès sur les sites de partage de vidéos en ligne. On poste également des plaisanteries tournant l'actualité en dérision, comme par exemple en décrivant une journée "ordinaire" qui consiste à éviter toutes sortes de dangers chimiques et alimentaires, qui circulait après des scandales alimentaires à répétition ${ }^{21}$. Pour contourner les moteurs de recherche, on peut intercaler des signes de ponctuation entre les différents caractères d'un mot interdit, sur le modèle: $\mathrm{Hu} / \mathrm{Jin} / \mathrm{Tao}$. On peut également utiliser des homophones pour remplacer des mots qui ne s'affichent pas sur les forums.

Ce qui n'est souvent qu'ironie ou humour finit parfois par devenir une véritable provocation, tournant la censure elle-même en dérision. Courant 2008, un jeu de mot a été formé à partir des mots "crabe de rivière", qui forment un homophone de "harmonie" (hexie), et de "porter trois montres", qui est à peu près l'homophone de "trois représentations" (sange daibiao), un autre slogan récent. Très rapidement des images de crabes portant trois montres se sont mises à circuler sur la Toile, provoquant l'hilarité générale ${ }^{22}$. Début 2009, alors que le gouvernement venait de lancer une nouvelle campagne de lutte contre la pornographie et la vulgarité, des internautes ont inventé une sorte de mascotte nommée "Cheval de l'herbe et de la boue", qui est aussi un homophone de "nique ta mère" (cao ni $m a)^{23}$. Cette mascotte a été mise en scène dans des clips musicaux, et on en a même fabriqué des peluches. Les

${ }^{21}$ XIAO Qiang. "Satire: a happy day in the life of an ordinary Chinese person (Sanlu edition)" [traduit par Christopher HUGHES], China Digital Times, 22/09/2008, consulté le 24/10/2010 sur http://chinadigitaltimes.net/2008/09/a-happy-day-in-the-life-of-an-ordinary-chinese-person-sanluedition/.

22 XIAO Qiang. "Under the Internet police's radar", China Digital Times, 28/08/2007, consulté le 24/10/2010 sur http://chinadigitaltimes.net/2007/08/under-the-internet-polices-radar/.

${ }^{23}$ NIVELLE Pascale. "'Caonima', le clip qui fait la nique à Pékin", Libération, 14/03/2009. 
internautes se sont également mis à inventer de nouveaux caractères chinois pour évoquer la censure de manière satirique, combinant par exemple les caractères $w u$ (cinq) et mao (dix centimes) en un seul qui se prononce wao, et qui désigne les internautes payés cinquante centimes par le gouvernement pour le défendre sur la toile ${ }^{24}$.

Par ailleurs, les internautes chinois ont exprimé un mécontentement général au sujet du projet de "Barrage vert" (Green Dam Youth Escort), un logiciel de contrôle "antipornographique" promu par le gouvernement, mais qui entrave aussi des utilisations non destinées à la publication. Certains internautes ont par exemple comparé ce logiciel à un "préservatif pour ordinateur", et d'autres ont parodié l'affiche de publicité, qui représente un lapin, mettant en scène de nombreux lapins en train de copuler ${ }^{25}$. Cela montre bien que les internautes ne sont pas prêts à accepter n'importe quelle intrusion dans leurs usages privatifs d'Internet, ce qui, pour eux, relève de pratiques archaïques ${ }^{26}$.

Comme le montrent Esarey et Xiao, il y a en fait beaucoup d'expression politique sur le Web chinois, mais elle est en quelque sorte invisible à qui n'y prête pas attention car elle est "en-dessous du radar", ou, pour le dire autrement, elle joue sur les nombreux registres en "clair-obscur" que permet Internet. Les Chinois "parlent vrai entre eux, et, en le faisant de manière si accessible, ils parlent vrai devant le pouvoir" ${ }^{27}$.

En même temps qu'une contrainte, l'obligation d'authenticité est saisie par les internautes comme une opportunité pour exprimer des opinions personnelles parfaitement subjectives. Lorsqu'ils s'en tiennent au cadre individuel, les internautes ont l'impression de bénéficier d'une grande liberté d'expression personnelle et de pouvoir consulter ainsi des sources d'information et des avis toujours plus diversifiés. Cette possibilité nouvelle n'est pas anodine du tout dans le contexte d'un pays qui n'a longtemps autorisé que la conformité

\footnotetext{
${ }^{24}$ LAM Oiwan. "China: newly invented Han characters", Global Voices Online, 01/09/2009, consulté le 24/10/2010 sur http://globalvoicesonline.org/2009/09/01/china-newly-invented-han-characters/

${ }^{25}$ FAUNA. "Chinese netizen reactions to 'Green Dam Youth Escort'", China Smack, 11/06/2009, consulté le 24/10/2010 sur http://www.chinasmack.com/stories/chinese-netizen-reactions-to-greendam-youth-escort/.

${ }^{26}$ ELEGANT Simon. "China netizens angry over Web porn crackdown", Time.com, 10/06/2009, consulté le 24/10/2010 sur http://www.time.com/time/world/article/0,8599,1903733,00.html.

${ }^{27}$ ESAREY Ashley et XIAO Qiang. "Political expression in the Chinese blogosphere. Below the radar", Asian Survey, vol. 48, n 5, 09/10 2008, p. 752-772.
} 
absolue jusque dans la vie privée. Elle est le corollaire de l'injonction à la prise en main autonome des destins individuels, et à la diversification des parcours.

Même s'il participe de la clôture du domaine public, cet enrichissement de l'expression individuelle à titre privé est porteur de conséquences considérables sur la circulation des informations et la formation des opinions en Chine. Tout d'un coup, les "transcriptions cachées", formes de discours critique tenues en privé ou encodées dans le discours public, qui ont toujours existé dans le quotidien des Chinois, deviennent infiniment plus visibles pour tous.

Cela suffit-il à considérer que l'ensemble de ces petites transgressions rendent la censure obsolète et ruinent à petit feu l'ordre établi? Comme le montre par exemple Regamey pour la cas de l'ex-URSS, la dérision, qui se fait toujours sous cape, rappelle la prééminence de la règle en même temps qu'elle en montre les défauts ${ }^{28}$. Pour Perry, en Chine, le fait que les protestations doivent suivre les lignes d'un langage officiel et l'existence de "transcriptions cachées" montrent une "conscience de la règle" plutôt qu'une "conscience des droits"29. Comme Eliasoph qui observe l'évaporation de la politique dans les situations publiques aux Etats-Unis ${ }^{30}$, on oscille entre le constat qu'il existe de nombreuses manières de tenir des discours hétérodoxes en privé, qui peuvent constituer une forme de résistance ${ }^{31}$, et l'impression que cette parole est malgré tout dérisoire. Tant que la parole publique reste le lieu d'une forme de représentation hégémonique qui exclut le débat public, les autres modalités d'expression ne peuvent constituer qu'une forme de défoulement, expression de l'impuissance politique des citoyens ${ }^{32}$.

${ }^{28}$ REGAMEY Amandine. Dérision et politique en URSS. Le rire contre la légitimité du pouvoir soviétique. Thèse de doctorat, IEP Paris, 2004.

${ }^{29}$ PERRY Elizabeth. "Studying Chinese politics: farewell to revolution?", The China Journal, ${ }^{\circ} 57$, janvier 2007, p. 1-22.

${ }^{30}$ ELIASOPH Nina. Avoiding politics. How Americans produce apathy in everyday life, Cambridge University Press, 1998.

${ }^{31}$ SCOTT James. Domination and the arts of resistance: hidden transcripts, Yale University Press, 1990.

${ }^{32}$ CARDON Dominique et HEURTIN Jean-Philippe. "La critique en régime d'impuissance, une lecture des indignations des auditeurs de France Inter", in FRANCOIS Bastien et NEVEU Erik (dir.). Espaces publics mosaïques, acteurs, arènes et rhétoriques des débats publics contemporains, Rennes: Presses universitaires de Rennes, 1999, p 85-119. 
En effet, c'est le passage à une forme de visibilité, qui suppose à la fois publicité et reconnaissance publique, qui détermine les effets politiques d'une prise de parole et ses conditions de félicité ${ }^{33}$. Tant qu'elle est dite sur le mode privé, une opinion, aussi contestataire qu'elle soit, n'a pas la force de la prise de parole publique qui est aussi une action politique ${ }^{34}$.

Par conséquent il faut rendre compte des mutations des formes de visibilité, ainsi que des stratégies de mise en visibilité dans l'espace public ${ }^{35}$ et sur Internet en particulier. Sous certaines conditions, Internet facilite la circulation des idées en privé, mais aussi leur incubation parmi des publics de plus en plus larges, ce qui leur permet d'être ensuite relayées de manière beaucoup plus publique. Internet permet ainsi des passages inédits du privé vers le public, des formes de "condensation" de cette parole politique "évaporée". Ces passages permettent la formation d'une certaine opinion publique et de ce que l'on pourrait appeler des formes d'action collective, dont il est important de détailler les spécificités.

${ }^{33}$ GOFFMAN Erving. Façons de parler, Paris: Minuit, 1987.

${ }^{34}$ ARENDT Hannah. Condition de l'homme moderne, Paris: Calmann-Lévy, 1961, p. $231 \mathrm{~s}$.

${ }^{35}$ VOIROL Olivier. "Les luttes pour la visibilité", Réseaux, n¹29-130, 2005, p. 89-122. 


\section{L'irruption du politique}


L"'évaporation de la politique" $^{1}$ qui se manifeste dans le discours des internautes chinois n'implique pas une absence de sensibilité pour la chose publique. Elle ressortit en fait d'une adhésion au projet de modernisation de la société, et à un modèle de civilité ${ }^{2}$ qui lui est propre. S'écarter de toute attitude politique est considéré comme une condition de préservation de l'ordre social, dans une société stratifiée et nécessairement conflictuelle. Cette "évaporation" n'est donc pas une disparition du politique, mais seulement son renvoi dans la sphère du privé, où les internautes disposent d'une grande liberté de conscience et d'expression. Or Internet a ceci de particulier qu'il donne la place tant à une information très surveillée, dépositaire d'une forme de représentation de l'unité nationale, qu'à d'innombrables formes de paroles individuelles à titre privé. Il propose ainsi de nombreux espaces en "clairobscur" où se tiennent des conversations de toutes natures. Depuis le témoignage d'une injustice vécue personnellement aux commentaires politiques qu'il faut lire entre les lignes, il y a de la place pour la circulation d'une expression relativement critique.

Celle-ci n'est cependant pas suffisante en elle-même pour porter des effets politiques. En effet, les "conditions de félicité" ${ }^{3}$ d'une parole dépendent en particulier de la reconnaissance ${ }^{4}$ de sa légitimité sur la place publique. C'est pourquoi partout dans le monde les groupes qui ne disposent pas de représentation officielle satisfaisante, les "sans" ${ }^{5}$, doivent trouver une forme de visibilité, et construire une opinion publique, pour faire entendre leurs causes $^{6}$. En ce sens, la visibilité est partout un enjeu de luttes politiques ${ }^{7}$. Comment passe-t-on alors d'une série de conversations privées, ou de l'expression d'indignations individuelles, à la formation de publics qui influencent les actions des autorités?

${ }^{1}$ ELIASOPH Nina. Avoiding politics. How Americans produce apathy in everyday life, Cambridge University Press, 1998.

${ }^{2}$ FERRIE Jean-Noël. Le régime de la civilité en Egypte, Paris: CNRS Editions, 2004.

${ }^{3}$ GOFFMAN Erving. Façons de parler, Paris: Minuit, 1987.

${ }^{4}$ HONNETH Axel. "Invisibilité: sur l'épistémologie de la "reconnaissance", Réseaux, vol.23, n¹29130, 2005, p. 39-58.

${ }^{5}$ SIMEANT Johanna. La cause des sans papiers, Paris: Presses de Sciences-po, 1998.

${ }^{6}$ BLEIL Susana. "Avoir un visage pour exister publiquement: l'action collective des sans terre au Brésil", Réseaux n¹29-130, 2005, p.123-154.

${ }^{7}$ VOIROL Olivier. "Les luttes pour la visibilité", Réseaux, n¹29-130, 2005, p. 89-122. 
Le modèle de modernité n'est pas seulement la source de limitations de la prise de parole publique. Il rend aussi légitimes d'autres registres de parole qui permettent la constitution de véritables mouvements collectifs autour de sujets consensuels. Les internautes se sentent ainsi autorisés à prendre la parole publiquement lorsqu'ils s'offusquent contre un cas de corruption avéré, ou qu'ils critiquent des atteintes à l'environnement par exemple. Ces registres ont leur place sur la scène publique parce qu'ils sont promus par les autorités ellesmêmes et qu'ils font partie du contrat social implicitement adopté par la population.

Le problème le plus délicat est donc le passage d'une cause de l'espace des conversations privées à celui de la discussion publique. Même si les grands traits de la rhétorique officielle peuvent fournir des registres de parole, leur interprétation peut rester problématique. Tous les acteurs ne sont alors pas dans la même position face à la perspective de rendre une cause publique. La plupart des internautes sont capables de s'informer de manière critique, mais aussi de réagir en transmettant une information, en postant un commentaire ou un avis. En revanche, il faut des compétences non négligeables pour pouvoir se servir des registres de prise de parole de manière tactique, en proposer une interprétation favorable à sa cause, et faire en sorte qu'elle soit relayée. Beaucoup de sujets tombent simplement dans l'oubli, ou ne franchissent pas les barrières de la censure et de l'autocensure. Seules quelques personnes trouvent ainsi les ressources pour donner une véritable publicité à des causes nouvelles. Ces initiatives peuvent ensuite être relayées par un public plus large.

Il se forme ainsi un jeu complexe où de nombreux acteurs interagissent ${ }^{8}$ autour de l'apparition et de la prise en compte de l'opinion publique. Même restreinte, la reconnaissance de l'opinion publique par les autorités est au fondement d'une légitimité qui se veut de plus en plus technocratique. Elle est donc surveillée étroitement, parfois écoutée, mais aussi "guidée" à tous les échelons du gouvernement chinois, ce qui passe par une prévention des crises et une propagande de plus en plus subtiles. La presse joue ainsi un rôle d'arbitre, en jouant le jeu de cette communication officielle tout en sélectionnant les angles les plus critiques possibles pour attirer le public. A ce jeu de cadrages réciproques, seuls les sujets les plus consensuels trouvent véritablement une audience sur la place publique. Les éléments les plus privilégiés

\footnotetext{
${ }^{8}$ NEVEU Erik. "Médias, mouvements sociaux, espaces publics", Réseaux, n98, 1999, p.17-85.
} 
de la société se trouvent ainsi en position favorable pour faire avancer des causes qui correspondent plus particulièrement à leurs préoccupations et à leurs valeurs. 


\section{Chapitre VII - La modernité: un registre polysémique}

Après avoir longuement décrit le processus qui conduit de nombreux internautes à ne pas prendre la parole en bien des circonstances, il faut immédiatement prendre acte d'un nombre croissant de cas où les internautes prennent effectivement la parole, individuellement et collectivement. L'utilisation du Web pour protester contre le bombardement de l'ambassade de Chine à Belgrade en $1999^{1}$, et surtout l'affaire Sun Zhigang en $2003^{2}$, ont marqué le point de départ de protestations de plus en plus nombreuses. De scandales de corruption en scandales alimentaires, en passant par des mouvements de défense des droits, il y a bien des situations où les internautes chinois s'autorisent à prendre la parole. A plus d'une reprise, ces mobilisations ont éclaboussé les autorités, les obligeant même parfois à changer la loi.

Plusieurs blogs d'observateurs, journalistes ou chercheurs chinois et étrangers, assurent une veille régulière des événements qui reçoivent un écho important sur le Web chinois ${ }^{3}$. Ces blogs spécialisés relèvent sur le Net des événements qui ont une notoriété très importante en ligne et hors ligne. Ils sélectionnent des contributions significatives et en traduisent des extraits. Parmi ces événements, ceux qui gagnent une portée plus générale ou politique sont particulièrement suivis et mis en lumière. Cette sélection des sujets, que l'on devine assez intuitive et qui est souvent très judicieuse, permet de suivre les différents thèmes qui se trouvent dans une sorte de "zone grise", ces sujets de société qui émergent à l'occasion de faits divers et qui prennent une ampleur peut-être insoupçonnée par les protagonistes eux-mêmes. Ces événements divers permettent, en somme, de repérer, dans un paysage informationnel très mouvant, la naissance des questions politiques dans l'espace public.

${ }^{1}$ WU Xu. Chinese Cyber Nationalism: Evolution, Characteristics and Implications, Plymouth: Lexington Books, 2007, p. 49s.

2 THIREAU Isabelle et HUA Linshan. "De l'épreuve publique à la reconnaissance d'un public: le scandale Sun Zhigang", Politix, vol.18, n71, 2005, p 137-164.

${ }^{3}$ Par exemple: China Digital Times: http://chinadigitaltimes.net/

Danwei: http://www.danwei.org/

East South West North: http://www.zonaeuropa.com/weblog.htm ou encore

China Media Project: http://cmp.hku.hk. 
Les causes qui font du bruit sont très diverses et elles sont suivies de manière très variable par les internautes. Pourtant elles semblent partager une sorte de rhétorique commune, fondée sur les grands principes de la modernité et de la civilisation "à la chinoise". Cette rhétorique, en conformité avec le discours officiel, mais dont la plasticité se prête à de nombreuses interprétations et qui est en perpétuelle redéfinition, permet de justifier la prise de parole sur la scène publique, y compris lorsqu'il s'agit de dénoncer des injustices mettant en cause des autorités locales ou une politique spécifique. Elle fonctionne en fait comme des registres discursifs dont certains internautes savent se servir pour faire passer des causes sur le devant de la scène.

\section{1 - L'idéal de modernité au cour des mobilisations}

\section{Une défense virulente de l'honneur national}

De nombreux scandales qui éclatent sur le Web chinois reflètent une pensée conformiste et très patriotique, au point que beaucoup y voient l'œuvre des petites mains payées 50 centimes pour poster des commentaires favorables au gouvernement. La prédominance de ces thématiques est certainement le fait d'une tendance à l'autocensure de la part de l'ensemble des internautes, mais elle n'est pas forcément complètement factice. Il faut prendre au sérieux l'ampleur et le nombre des réactions qui peuvent être exprimées dans ce type de cas.

Le cas d'un professeur anglais de Shanghai qui racontait dans son blog ses frasques avec ses étudiantes chinoises ${ }^{4}$ est emblématique des situations qui soulèvent une indignation généralisée à coloration patriotique, et même des réactions violentes. Un professeur chinois de psychologie a rendu ce cas très visible en lui adressant ses reproches dans son propre blog. Selon lui, le professeur d'anglais a eu un comportement immoral envers les jeunes femmes. Il leur a manqué de respect en leur faisant des promesses et des déclarations d'amour mensongères pour s'attirer leurs faveurs. Surtout, il a manqué de respect à ces jeunes femmes en tant que Chinoises lorsqu'il a parlé de leur naïveté sur son blog et lorsqu'il a insulté les hommes chinois. Pour lui, le fait que le professeur d'anglais a critiqué ouvertement la

${ }^{4}$ SOONG Roland. "The immoral foreign blogger", East South West North, 28/08/2008, consulté le 24/10/2010 sur http://www.zonaeuropa.com/20060828_1.htm 
politique du gouvernement chinois, le rapport des Chinois au Japon, et "s'engage ouvertement dans des activités de division de la Chine" en affirmant par exemple que Taiwan est un pays indépendant, est encore plus grave. Pour couronner le tout, il l'a fait dans le cadre de son enseignement, c'est à dire publiquement et officiellement. Le professeur de psychologie, sur son blog, s'indigne donc sur plusieurs plans à la fois, mais tous sont liés peu ou prou à une atteinte publique à l'image de la Chine.

A la suite de cette dénonciation, de nombreuses voix se sont élevées pour critiquer le professeur d'anglais. L'aspect le plus marquant de la mobilisation est certainement son caractère nationaliste. La critique est virulente contre les remarques condescendantes du professeur sur la Chine, qui sont désignées comme un relent d'impérialisme. Il faut donc "mettre dehors" celui qui s'obstine à humilier la Chine. La virulence du ton et l'extrémisme des mesures réclamées par nombre d'internautes répondent à cette atteinte qui est gravissime à leurs yeux. Cela s'exprime donc par une ruée sur la victime désignée, sans considération de proportionnalité de la sanction, et dans une apparente unanimité.

La plupart des scandales liés à la question nationaliste recueillent cet unanimisme, en particulier parmi ceux que l'on appelle les "jeunes en colère" ou fenqing ${ }^{5}$. Bien sûr ces sujets sont les plus sensibles politiquement, et donc soumis à une censure plus serrée. Dans le même temps, les internautes eux-mêmes font taire ceux qui "manquent de patriotisme". Les émeutes au Tibet en mars 2008 ont par exemple donné lieu à une condamnation unanime de la position française, exprimée avec un radicalisme parfois effrayant ${ }^{6}$. En effet beaucoup de mouvements s'accompagnent d'une vulgarité certaine, notamment dans les insultes adressées ici aux Français, là aux Japonais ou ailleurs aux Américains selon les situations. La virulence des réactions peut se lire comme le résultat d'un effort contrarié de construction et d'affirmation

\footnotetext{
${ }^{5}$ Ce terme, qui désigna d'abord les jeunes rendus amers par leur envoi à la campagne pendant la révolution culturelle, puis les étudiants des années 1980 réclamant une plus grande ouverture politique du pays, désigne désormais le patriotisme virulent de la jeune génération, soucieuse de réparer les humiliations des deux derniers siècles. ANDERLINI Jamil. "China's angry youth vent their feelings", The Financial Times, 02/05/2008; CHEN Shaoping. "Le blog de la colère", Courrier International, $\mathrm{n}^{\circ} 841,14-20 / 12 / 2006$.

${ }^{6}$ CHOW. "Chinese seethe on Web over rare riots in Tibet", 15/03/2008, traduit par Xiao Qiang, consulté le 24/10/2010 sur http://chinadigitaltimes.net/2008/03/chinese-seethe-on-web-over-rare-riotsin-tibet/. MACKINNON Rebecca. "Tibet... Is discussion possible?", Rconversation, 24/10/2010, consulté le 25/09/2009 sur http://rconversation.blogs.com/rconversation/2008/03/tibet-is-discus.html.
} 
de l'unité nationale. C'est pourquoi ces épisodes tolèrent moins que d'autres l'émergence de voix plus modérées appelant au débat. Il ne s'agit pas de discuter d'un problème sur le fond, mais de faire front pour défendre une cohésion, une image souffrant d'une certaine remise en cause.

Dans cette optique, les Chinois qui montrent une certaine modération sont eux-mêmes soupçonnés de trahir la cause nationale, et ils sont souvent traités avec beaucoup d'agressivité $^{7}$. Même la porteuse handicapée de la torche olympique, Jin Jing, héroïne de quelques jours pour avoir protégé la flamme contre des défenseurs de la cause tibétaine, a été vilipendée pour s'être prononcée contre le boycott des magasins Carrefour ${ }^{8}$. Les voix plus modérées qui s'expriment lors de ces occasions trouvent, il faut le reconnaître, un fort mauvais accueil.

Peut-être faudrait-il donc lire les grands mouvements nationalistes comme des mouvements d'imposition de l'ordre social au sein d'un "Peuple" pris dans son ensemble? Certaines remarques des personnes interrogées le suggèrent.

"C'est une forme de patriotisme. Le Japon a envahi la Chine pendant la seconde guerre mondiale. Surtout, ils ne le reconnaissent pas, et même ils disent le contraire, et ça nous met en colère. C'est une façon d'épancher (faxie) notre humeur patriotique. Il y a aussi la Corée. Beaucoup de Chinois admirent le développement de la Corée. Mais à l'origine, le développement de la Corée ne vaut pas celui de la Chine, et puis beaucoup d'aspects de leur culture envahissent la Chine, ça aussi nous met en colère. Donc on veut que tout le monde s'y oppose ensemble. Si on dit que la Fête de la Lune c'est chinois depuis des milliers d'années, ils prétendent que c'est à eux, et aussi les alcools, et ils disent aussi que c'est eux qui ont inventé la poudre, ça nous met très en colère. On fait très attention aux questions culturelles. Surtout quand les Américains et les Japonais tournent n'importe comment le film Voyage vers l'Occident (Xi you ji), ça nous met en colère, ça insulte notre culture.

- Pourquoi?

${ }^{7}$ WU Xu. Chinese Cyber Nationalism: Evolution, Characteristics and Implications, Plymouth: Lexington Books, 2007.

${ }^{8}$ LI Zhaohua. "Hero to traitor: the difference a day makes", China Digital Times, 19/04/2008, consulté le 24/10/2010 sur http://chinadigitaltimes.net/2008/04/nationalists-paris-torch-relay-hero-now-atraitor/. 
- C'est une sorte de sentiment national, quand il y a des choses injustes pour la Chine on réagit comme ça.

- Tu visites des forums spécialement dédiés à ces questions?

- Oui, quand j'en vois je rentre toujours dedans.

- Tu lis les posts des autres?

- Oui.

- Et ils sont tous du même avis?

- Oui, presque tout le monde est très patriote, mais il y en a qui disent que le mieux est encore de renforcer notre pays. Ils encouragent tout le monde à travailler à la régénération de la nation.

- Tu écris là-dessus sur ton blog?

- Oui.

- Et tu en discutes avec les autres?

- Oui, je veux que tout le monde s'unisse." (Gu)

Il est probable que pour certaines personnes, les questions impliquant un enjeu de souveraineté nationale soient particulièrement susceptibles de soulever le levier de l'indignation. Il est également probable que les autorités encouragent plus particulièrement ce type de mobilisations qui confirment l'unanimisme supposé de la nation chinoise, renvoient explicitement toute discorde dans la sphère du privé et entérinent donc le monopole du Parti sur la sphère publique et politique. La bienveillance des autorités rend ces causes beaucoup plus visibles que d'autres et elles peuvent parfois devenir envahissantes.

Cependant il serait réducteur de considérer les internautes comme de simples marionnettes de la politique gouvernementale. Dans bien des cas, d'ailleurs, les mobilisations à caractère nationaliste, en ligne ou hors ligne, échappent au contrôle des autorités chinoises pour prendre des dimensions imprévues ${ }^{9}$. En fait, les internautes s'insurgent surtout contre tout ce qui peut nier le développement de la nation et son retour sur la scène internationale en

${ }^{9}$ Pour des exemples, voir WU Xu. Chinese Cyber Nationalism: Evolution, Characteristics and Implications, Plymouth: Lexington Books, 2007. 
tant que grande puissance "moderne". Or l'idée de la modernité qui est ainsi défendue comporte de nombreux volets qui s'écartent de la seule question nationaliste.

\section{Promotion d'un projet de civilisation}

En effet, le modèle de "modernité à la chinoise" est un modèle culturel global, qui englobe tous les aspects de la vie individuelle et collective. Les internautes se sentent bien souvent légitimes à le défendre, dans la mesure où il est devenu un mot d'ordre officiel pour tous. Toutes les questions de la vie quotidienne peuvent ainsi donner lieu à des prises de parole indignées. Les internautes que j'ai rencontrés en témoignent: ils s'expriment surtout lorsqu'ils sont confrontés à une situation ou un comportement qu'ils considèrent comme contraire au projet de civilisation dans lequel la Chine est engagée.

De manière très significative, les questions de propreté et de présentation de soi font l'objet de nombreuses interventions sur Internet, surtout dans le contexte de l'ouverture croissante du pays à l'étranger. Dans la perspective des Jeux Olympiques de 2008, ainsi que de l'Exposition Universelle de 2010 à Shanghai, l'Etat a mené une intense campagne de sensibilisation auprès de la population pour l'inciter à adopter un comportement plus "civilisé". Cela passe par des campagnes d'affichage dans les rues et dans le métro, mais aussi par des reportages relayés dans la presse et a fortiori sur Internet. Il faut apprendre à faire la queue aux guichets, parler moins fort au téléphone etc... Autant de consignes destinées à améliorer l'image des Chinois auprès des visiteurs occidentaux. Entre autres consignes, figure par exemple celle de ne plus cracher par terre en public. Cette consigne est relayée sur le Web par certains internautes.

"Sur Internet, je dis ce que j'ai sur le cœur, que tout le monde doit faire attention à l'hygiène, tout le monde doit faire des efforts là-dessus, mais aujourd'hui beaucoup de gens ne le font pas et ça me met très en colère quand je vois ça. C'est comme les crachats par terre, les gens qui marchent sur les tables et les chaises dans les lieux publics. Je trouve que tout ça c'est très mal, surtout les crachats, c'est très sale. Je dis ce que j'ai sur le cœur, 'pensez aux étrangers, regardez votre pays, il y a vraiment un crachat par personne, que va devenir votre pays?' Du point de vue de l'hygiène ça me met très mal à l'aise. Des fois quand je prends le bus, et qu'il y a quelqu'un avec un enfant, si l'enfant marche sur le siège de devant, ou qu'il se permet de faire n'importe 
quoi, je trouve que le minimum, c'est que l'adulte dise quelque chose, parce que l'enfant ne comprend pas. Mais des fois on voit des gens, on a envie de leur dire, mais on ne peut pas, ce n'est pas notre enfant, on a peur de leur dire, donc on ne sait pas quoi faire. Je pense qu'on va bientôt être en 2008, et même si on ne faisait pas les Jeux Olympiques, il faudrait faire quelque chose pour ça dans ce pays. Mais dans des situations comme ça, dans un pays aussi grand, personne n'y changera rien, chaque chose en son temps. [...] Même si on peut dire que la capacité de propagande (xuanchuan li) est faible, ça fait quand même du bruit. Ces derniers jours on en parlait aussi à la télé, ils ont distribué des petits sacs pour cracher, il y en dans les arrêts de bus. Mais cette habitude devient déjà naturelle, il faut respecter ces bonnes habitudes, les respecter spontanément, se retenir, être naturellement propre. Quand on fait ce qu'on veut, il n'y a pas cette spontanéité. Va savoir pourquoi, c'est peut-être parce que les Chinois sont trop nombreux." (Cai)

Autre exemple, il y a eu en 2006 un grand débat autour du port du pyjama dans les rues de la ville de Shanghai ${ }^{10}$. Le port du pyjama en pleine rue, qui est très fréquent dans les vieux quartiers des villes chinoises, nuirait désormais à l'établissement d'une image plus "moderne" du pays, aux dires mêmes d'un sociologue convoqué par la presse pour l'occasion. Il se trouve que cette habitude est essentiellement celle des générations plus âgées. Cette critique du port du pyjama est le fait des plus jeunes générations, qui se sentent investies d'une autre culture, plus moderne et plus soucieuse de l'image internationale de la Chine.

Ces cas peuvent faire sourire, mais la même logique peut conduire les internautes très loin. En 2006, une jeune femme a ainsi défrayé la chronique pour être apparue sur une vidéo en ligne, dans laquelle elle écrasait la tête d'un chaton avec son talon aiguille ${ }^{11}$. En quelques jours, la vidéo a fait scandale et les critiques se sont déchaînées sur l'ensemble du Web chinois. Une telle cruauté ne devait pas rester impunie. Les internautes ont donc mis en marche une véritable chasse à l'homme afin de retrouver la jeune femme et de la punir. Elle fut retrouvée à Harbin et harcelée jusqu à son domicile et sur son lieu de travail. Ce fut l'un

\footnotetext{
${ }^{10}$ MORGAN Benjamin. "Dans la Shanghai moderne, le pyjama en pleine rue fait débat", Aujourd'hui la Chine, 11/11/2006, consulté le 25/10/2010 sur http://chine.aujourdhuilemonde.com/dans-lashanghai-moderne-le-pyjama-en-pleine-rue-fait-debat.

${ }^{11}$ MARTINSEN Joel. "Animal cruelty in the mainland press", Danwei, 02/03/2006, consulté le 25/10/2010 sur http://www.danwei.org/media_and_advertising/animal_cruelty_in_the_mainland.php.
} 
des premiers cas en Chine de ce que l'on appelle depuis le "moteur de recherche de chair humaine" (renrou sousuo). ${ }^{12}$

Dans ces situations de plus en plus nombreuses, les internautes prennent l'initiative de traquer collectivement les fauteurs de troubles, les personnes qui transgressent une certaine morale, ou qui ont des comportements préjudiciables à l'image de la Chine. Ces réactions collectives, qui sont souvent assez violentes, sont condamnées officiellement par les autorités et elles ont déjà donné lieu à des poursuites judiciaires, comme ce fut le cas par exemple après une cabale lancée contre un mari adultère ${ }^{13}$. En effet il est illégal de faire justice soi-même en Chine comme ailleurs. Les internautes participent donc à un processus de "maintien de l'ordre" qui va bien au-delà de ce que l'Etat leur suggère. Ils exercent un contrôle sur le comportement de leurs concitoyens, qui peut prendre des formes si coercitives que cela inquiète même les autorités.

Pourtant il faut remarquer que toutes ces dénonciations et critiques ne sont pas tournées a priori contre les autorités. Bien au contraire, elles s'appuient sur les valeurs promues par les autorités: modernité, civilisation, comportement responsable etc... Ces valeurs servent de justification pour lancer des critiques sur la scène publique ${ }^{14}$. Les internautes participent d'autant plus facilement à ces mouvements qu'ils ne sont pas subversifs ni contestataires politiquement. Bien au contraire, participer à l'un de ces mouvements, ne serait-ce qu'en acquiesçant sur un forum, est souvent perçu comme une manière d'affirmer son adhésion au modèle de société avancée qui semble être défendu par de nombreux internautes.

\section{L'apprentissage du débat: la modernité, c'est quoi?}

Or ce modèle est loin d'être clairement défini. Chacun des termes "moderne", "civilisé", "avancé" peut donner lieu à des interprétations variables. Les critères partagés sont imprécis, voire ambivalents ou contradictoires. Pour cette raison, de nombreux scandales mettent en

${ }^{12}$ FRENCH Howard W. " Mob rule on China's Internet: The keyboard as weapon", The New York Times, 31/05/2006, consulté le 25/10/2010 sur http://www.nytimes.com/2006/05/31/technology/31ihtchinet.1861775.html?_r=1\&pagewanted=2.

${ }^{13}$ PHILIP Bruno. "Un tribunal chinois condamne les "justiciers" de l'Internet", Le Monde, 23/12/2008.

${ }^{14}$ BOLTANSKI Luc et THEVENOT Laurent. De la justification. Les économies de la grandeur, Paris: PUF, 1987. 
lumière les limites ou les excès du processus de modernisation. Les discussions auxquelles ils donnent lieu contribuent à remodeler en permanence et à tâtons les valeurs qui doivent fonder la modernité chinoise.

Les questions débattues sur Internet montrent combien les transformations du modèle de société bousculent les repères des individus dans leur expérience personnelle et quotidienne, et jusque dans les aspects les plus intimes de leur vie. Elles les concernent dans les moments de leurs vies où ils doivent faire des choix importants et concrets comme l'orientation de leur carrière, la scolarisation de leurs enfants, ou leur mariage. Ainsi un cas d'adultère révélé sur Internet donnera-t-il lieu à une réflexion sur la fidélité. L'adultère est-il acceptable ou non dans la société d'aujourd'hui, qui offre une plus grande liberté de choix de son partenaire? Faut-il se marier, quand, avec qui et dans quelles conditions? Que penser des mariages arrangés, qui peuvent assurer un meilleur statut social, mais qui ne correspondent pas à l'idéal de liberté de choix des intéressés?

Par exemple, une controverse importante a eu lieu au sujet d'un article de blog intitulé "il n'y a pas d'hommes qui me conviennent en Chine", par une étudiante shanghaienne ${ }^{15}$. Celle-ci se décrit elle-même comme une jeune femme éduquée et jolie (donc de qualité élevée), et elle attend beaucoup de ses prétendants. Il lui faut naturellement un confort matériel important, avec les traditionnels appartement et voiture, mais elle recherche aussi un certain niveau de distinction chez les hommes, ce qu'elle ne trouve pas chez les Chinois. Au passage, elle égrène avec un mépris rare tous les stéréotypes sur la pauvreté et l'arriération. Elle déclare par exemple avoir besoin d'une voiture parce qu'une fille de son rang ne doit pas se retrouver collée aux vêtements sales des migrants dans les transports en commun. Bien sûr, l'affront fait à l'honneur des hommes chinois n'est pas pour rien dans le fait que son article ait été abondamment relayé. Cependant les thématiques abordées par les internautes dans les commentaires sont plus larges et elles interrogent les contradictions du modèle de société incarné par cette jeune femme. L'aspiration à un meilleur niveau de vie doit-elle justifier le mépris et l'insulte pour les catégories sociales moins élevées? La modernité, si elle prend des formes inspirée des séries TV occidentales, peut-elle réellement servir l'honneur de la nation

${ }^{15}$ CANON. "China does not have any men suitable for me", China Smack, 18/10/2008, consulté le 25/10/2010 sur http://www.chinasmack.com/2008/stories/china-does-not-have-any-men-suitable-forme.html /. 
chinoise, ou au contraire n'aboutit-elle qu'à souligner ses manques? Les aspects les plus triviaux de la vie quotidienne peuvent ainsi donner lieu à des débats normatifs importants.

La plupart de ces questionnements sont liés, d'une manière ou d'une autre, aux conséquences de l'individualisation et aux arbitrages nouveaux que celle-ci implique. En témoigne ce scandale, où une dame ayant trébuché à la sortie du bus a été rattrapée dans sa chute par un passant. Tout de même blessée, la dame a attaqué le passant en justice, en l'accusant d'être la cause de sa blessure, pour obtenir de lui une somme d'argent. La plupart des internautes en ont cyniquement conclu qu'il valait mieux ne pas aider les inconnus. L'article du blog qui relate l'épisode en anglais est très justement sous-titré "le samaritain solitaire"16. C'est bien l'attitude du Samaritain, telle qu'elle est théorisée dans le livre de Boltanski ${ }^{17}$, qui pose désormais problème. Un acte de générosité ou de solidarité purement gratuit semble presque louche dans un univers où le chacun-pour-soi semble devenir le mode de fonctionnement par défaut. Un autre cas de confrontation entre l'individualisme nouveau et des valeurs dites "traditionnelles" est la création du groupe "never give up your seat" sur le site Douban ${ }^{18}$. Désormais, certains jeunes ont décidé de ne plus céder leur place dans le bus, considérant que les personnes âgées ne méritaient pas forcément plus qu'eux le confort.

Témoins de faits divers encore plus graves, les internautes en viennent à s'interroger sur la valeur de la vie humaine, question inévitablement posée par la rhétorique liée à la "qualité". Ils sont amenés à ce débat au travers de cas comme celui dit "de la BMW"19. Dans un marché de Harbin, un tracteur rempli d'oignons a rayé la carrosserie d'une BMW. La conductrice de l'automobile, furieuse, a alors successivement insulté le paysan, repris le volant, et démarré en fendant la foule attroupée, faisant au passage un mort et plusieurs blessés. Devant le tribunal, la conductrice, disposant d'appuis influents, n'a été condamnée qu'à de la prison avec sursis. La disproportion entre la gravité des faits et la sanction a causé un très grand émoi sur le Net,

${ }^{16}$ CHEN Bob. "China: fragile morality, the lonely Samaritan", Global Voices Online, 13/10/2007, consulté le 25/10/2010 sur http://globalvoicesonline.org/2007/10/13/china-fragile-morality/.

${ }^{17}$ BOLTANSKI Luc. La souffrance à distance, Paris: Métailié, 1993, p.21.

${ }^{18}$ MU Eric. "Screw the elderly, I'm keeping my bus seat", Danwei.org, 08/10/2008, consulté le 25/10/2010 sur http://www.danwei.org/front_page_of_the_day/chinese_good_traditions.php.

19 "China's 'BMW killer' case upheld", BBC News, 29/03/2004, consulté le 25/10/2010 sur http://news.bbc.co.uk/2/hi/3577829.stm 
sur le thème: comment peut-on en arriver à considérer que la carrosserie d'une voiture a plus de valeur que la vie d'un paysan?

Les véhicules de luxe sont très fréquemment l'objet de controverses sur ce thème, comme en témoigne cette autre histoire, concernant cette fois une Mercedes.

"Il y a deux ans en province, une voiture a blessé une petite fille en la heurtant, et la compensation standard quand on blesse quelqu'un ou qu'on le tue en voiture c'est différent, quand on blesse la personne il faut lui donner de l'argent toute sa vie, quand on la tue on donne une fois une certaine somme d'argent. Du coup le chauffeur a fait demi-tour avec la voiture, et a écrasé la petite fille comme ça, il l'a tuée, et ensuite il a averti la police. Il n'avait pas intérêt à la blesser seulement car s'il la blessait il fallait l'indemniser toute sa vie, alors qu'en la tuant il lui suffisait de payer 30000,40000 RMB et voilà. Tu crois qu'en conduisant une Mercedes il n'avait pas l'argent? Du coup tout le monde en a parlé, comment se fait-il qu'il y ait ce genre de règles dans la loi, et il y a des questions sur le système, donc il a commencé à y avoir des forums sur la question, où ils disaient que le chauffeur a pensé comme ça, qu'il ferait mieux de revenir la tuer, sans même la sauver, mais il ne fallait pas la tuer. Sur le plan de la morale et dans les faits il y avait beaucoup d'avis, certaines personnes soutenaient le chauffeur, d'autres le traitaient de tous les noms, et d'autres insultaient le système." (Chu)

C'est d'abord l'émotion qui attire l'intérêt des lecteurs dans ce type de cas. Comment ne pas céder à l'effroi devant le comportement du chauffeur de la Mercedes? Beaucoup trouvent légitime de venir déposer sur Internet l'émotion ressentie. A l'indignation originelle succède cependant une forme de débat qui permet de démêler plusieurs positions contradictoires. D'une part les tenants d'une morale altruiste s'insurgent du fait que l'on puisse donner un prix à la vie d'un homme. D'autre part les tenants d'un certain cynisme prennent le parti du chauffeur en vertu de la primauté de son intérêt individuel. Il s'agit là d'une interprétation particulièrement extrême de l'individualisme, et qui n'est pas partagée par tous.

Il y a bien ici l'émergence d'une certaine incertitude sur la valeur qui doit primer. Cette incertitude donne lieu à une discussion, et finalement à un arbitrage, et qui permet de stabiliser la nouvelle norme sociale admise. Les grands portails Internet rassemblent ces grands débats, dont le point de départ est souvent un fait divers sordide, et qui donnent lieu à 
des questions aussi vastes que "quel est le prix de la vie humaine?", dans des dossiers spéciaux très bien documentés et dont la liberté de ton est souvent impressionnante ${ }^{20}$.

Dans le même ordre d'idées se trouve le cas d'un professeur qui a fui la salle de classe sans se préoccuper de ses élèves lors du tremblement de terre au Sichuan au printemps 2008. Le tollé général que ce cas, qui n'est pas isolé, a créé, a donné lieu à un véritable débat sur la place nouvelle qui est donnée à l'individu dans la société, à la valeur respective des différents individus. En effet, contre toute attente, le professeur a été largement excusé par de nombreuses personnes. Celles-ci, non contentes de prendre acte de sa panique, entérinent l'argument du professeur selon lequel "[s]a vie ne vaut pas moins que celle de [s]es élèves". S'opposant à ce point de vue, d'autres personnes font appel à une morale dite confucéenne pour s'indigner de l'inversion de valeurs que cet argument représente à leurs yeux ${ }^{21}$. Cette confrontation entre deux positions contradictoires est louée par Li Datong, célèbre éditorialiste de l'ancien magazine "Bingdian".

Cependant dans les discussions sur les actions et la moralité de Fan, les visions du public et des médias sur les événements sont devenus progressivement plus rationnelles; une tolérance envers l'opinion de la minorité et pour ceux qui vont à contre-courant a émergé. C'est l'avantage du débat libre et juste - un débat qui démontre que la société chinoise est en train de changer. ${ }^{22}$

Il s'agit probablement plus de relever les contradictions inhérentes au modèle d'individualisation et de modernisation, et d'en proposer des interprétations différentes, que d'aller véritablement à "contre-courant". Il serait par exemple inimaginable pour un internaute de s'opposer à l'idée même de modernisation du pays. Pourtant, il est vrai que ces cas montrent à quel point la définition du concept de modernisation se construit par tâtonnements successifs, et donne lieu à des débats contradictoires sur la définition précise à donner à la modernité chinoise. Les "scandales" cèdent ainsi de plus en plus souvent la place aux

\footnotetext{
${ }^{20}$ Sina, dossier "zhuangshang buru zhuangsi" (il vaut mieux tuer au volant que blesser au volant) http://auto.sina.com.cn/z/ZRTY/index.shtml, consulté le 25/10/2010.

${ }^{21}$ LIU Shinan. "Warped view of right and wrong", China Daily, 28/05/2008.

${ }^{22}$ LI Datong. "China: after the quake, the debate", Open Democracy, 20/06/2008, consulté le 25/10/2010 sur http://www.opendemocracy.net/article/china-after-the-quake-the-debate.
} 
"affaires" selon la distinction de Claverie ${ }^{23}$. Le scandale, lorsqu'il dévoile une norme partagée, fait unanimité, alors que l'affaire donne lieu à une opposition entre plusieurs camps. Dans l'affaire, une accusation portée contre quelqu'un peut se trouver elle-même critiquée au nom d'une autre conception des valeurs à défendre. Cette situation de controverse oblige les protagonistes à se justifier, à développer des arguments et donc à monter en généralité. C'est la naissance du débat.

La virulence et l'unanimisme apparent qui se déploient dans les mobilisations à caractère nationaliste ne doivent donc pas donner l'illusion d'une unité parfaite des internautes dans leurs mobilisations, ni d'un alignement exclusif sur les positions des autorités. Le plus souvent, les scandales sont le reflet d'une certaine perception de ce que doit être la modernité en Chine. Cela comporte bien un caractère normalisateur et même coercitif pour les victimes du "moteur de recherche de chair humaine". Cependant il faut souligner que ce modèle n'est pas complet ni clair, et qu'il peut être interprété de diverses manières. C'est ce que Ferrié identifie en Egypte comme une forme de "solidarité sans consensus": tous se rassemblent autour d'un mot d'ordre, mais sans en accepter la même définition ${ }^{24}$. C'est pourquoi il est en permanence redéfini, remis en question et critiqué par les internautes, à mesure qu'ils rencontrent des cas problématiques. La diversité même des définitions dont la modernité fait l'objet, et les débats qu'elle suscite montrent l'intensité des efforts que les internautes font pour se l'approprier et la mettre en actes. Au terme de ce processus, les normes liées au modèle de la modernité débordent largement du champ d'influence des autorités. Or la définition de ces normes est un véritable enjeu de pouvoir ${ }^{25}$.

${ }^{23}$ CLAVERIE Elisabeth., "La naissance d'une forme politique : l'Affaire du Chevalier de La Barre", in ROUSSIN Philippe (dir.). Critique et affaires de blasphème à l'époque des Lumières, 1998, Paris: Honoré Champion, p.185-260.

${ }^{24}$ FERRIE Jean-Noël. Le régime de la civilité en Egypte, Paris: CNRS Editions, 2004, d'après KERTZER David. "Rituel et symbolismes politiques des sociétés occidentales", L'Homme, vol. 32, n 121, 1992, p. 79-89.

${ }^{25}$ DUPRET Baudouin et FERRIE Jean-Noel. "Participer au pouvoir, c'est édicter la norme: sur l'affaire Abu Zayd (Egypte, 1992-1996)", RFSP, vol. 47, nº, 1997, p. 762-775. 


\section{2 - Un registre discursif pour la défense des droits des citoyens}

\section{L'affaire Sun Zhigang comme épreuve publique}

Ce fut mis en évidence lors de l'affaire Sun Zhigang, finement analysée par Isabelle Thireau et Hua Linshan ${ }^{26}$ et qui représente peut-être le premier cas très visible de l'influence politique des débats qui ont lieu sur Internet. Le jeune Sun Zhigang, styliste, originaire de la province du Hubei, s'est installé à Canton début 2003 à sa sortie de l'école des Beaux Arts. Quelques jours plus tard, le 17 mars 2003, il a subi un contrôle d'identité au cours duquel il n'a pas été en mesure de montrer un certificat de résidence provisoire dans la ville de Canton. Les policiers l'ont donc considéré comme un migrant en situation illégale et conduit dans un "centre de détention et de rapatriement" ${ }^{27}$. Il est mort de coups et blessures dans un hôpital pénitentiaire trois jours plus tard, le 20 mars. La mobilisation de son entourage pour faire la lumière sur cette affaire, et l'utilisation d'Internet pour la rendre publique ont transformé ce cas malheureusement banal en un événement fondateur. Cela a donné lieu à de nombreuses prises de position dans divers médias en ligne et hors ligne de la part de leaders d'opinion, d'avocats et d'intellectuels, et à l'indignation unanime des internautes dans les forums de discussion. Forcées de réagir, les autorités locales ont mené une enquête et des coupables ont été désignés parmi les malades et le personnel de l'hôpital pénitentiaire. Des peines très sévères ont été prononcées à leur encontre, allant jusqu'à la condamnation à mort ${ }^{28}$. De plus, ce cas est rapidement devenu un prétexte pour dénoncer la situation des migrants en général, et l'arbitraire policier dont ils sont l'objet dans les centres de détention et de rapatriement. Le débat prenait dès lors une ampleur nationale. Finalement, la loi régissant ces centres a été modifiée, ce qui constitue un événement d'une importance cruciale.

Il faut interpréter ces résultats avec circonspection. En effet la sanction judiciaire très sévère qui a été prononcée n'est pas totalement satisfaisante car elle manque de transparence,

${ }^{26}$ THIREAU Isabelle et HUA Linshan. "De l'épreuve publique à la reconnaissance d'un public: le scandale Sun Zhigang", Politix, vol.18, ${ }^{\circ} 71,2005, \mathrm{p}$ 137-164. Les termes entre guillemets sont empruntés à Thireau.

${ }^{27}$ Ces centres étaient des camps de transit où les migrants étaient enfermés quelque temps avant un rapatriement vers leurs provinces d'origine. Ils avaient parfois à y travailler.

28 "Sun Zhigang's brutal killers sentenced", China Daily, 10/06/2003, consulté le 25/10/2010 sur http://www.chinadaily.com.cn/en/doc/2003-06/10/content_168514.htm. 
ce qui peut laisser penser que tous les responsables n'ont pas forcément été poursuivis. En même temps qu'elles changeaient la loi régissant les migrants, les autorités rendaient plus sévère le contrôle des médias qui avaient aidé à rendre ce cas public. Pourtant, cet événement a montré l'importance nouvelle du public dans le jeu politique chinois.

Ce cas a mis en évidence pour la première fois le fait que les individus avaient la possibilité de rendre publics sur Internet des cas d'injustice manifeste et de contourner les pouvoirs locaux pour obtenir justice auprès du pouvoir central et même, fait extraordinaire, pour faire changer la loi. L'efficacité de la mobilisation était d'autant plus forte que toutes les voix qui se sont élevées étaient unanimes. En effet un public, constitué d'abord des personnes qui se reconnaissaient comme des victimes potentielles de ce type d'arbitraire, s'est reconnu dans la situation vécue par le jeune Sun, et ce public a réagi unanimement par une interpellation des autorités. Cette action du public n'aurait peut être pas suffi à obtenir gain de cause sans l'intervention d'experts en faveur de la notion d'Etat de Droit ou le relais par des médias de masse. En revanche la modification de la loi qui a suivi ce scandale a donné une légitimité a posteriori à la pression de ce public qui s'était constitué spontanément. Ce scandale a donc été une véritable épreuve ${ }^{29}$ qui, en exprimant un "dépassement des limites" (guofen) de la part des policiers, a révélé une "communauté de jugement". Cet épisode a conduit à délégitimer officiellement des pratiques arbitraires qui avaient cours auparavant, pour installer à leur place une conception de l'ordre public qui ne tolère plus les bavures policières.

Thireau souligne que le scandale Sun Zhigang "a rétabli la validité du jugement et la légitimité d'une pluralité normative". Une affaire telle que celle-ci conduit à "l'affirmation d'une conviction partagée, irréversible, concernant la validité des principes et conduisant à la formulation d'attentes particulières à l'égard de l'Etat". En d'autres termes, on ne juge plus les événements à la seule aune de leur conformité politique, mais surtout selon une certaine

${ }^{29}$ DE BLIC Damien et LEMIEUX Cyril. "Le scandale comme épreuve", dossier de Politix, vol.18 $\mathrm{n}^{\circ} 71$, 2005. GLUCKMAN Max. "Papers in honor of Melville J. Herskovits: Gossip and scandal", Current Anthropology, vol.4, n³, 1963, p. 307-316. 
appréciation de ce qui est "juste" ${ }^{30}$ dans une société moderne. Désormais, la conformité ellemême autorise et même requiert ce processus de recherche de justice.

Grâce aux discussions qu'il permet sur tous ces principes, Internet fait donc désormais partie de ces lieux de plus en plus nombreux où s'élaborent de nouvelles normes sociales, "multiples mais communes" ${ }^{31}$. Ces nouvelles normes s'imposent même à l'Etat parce qu'elles sont communément partagées et que leur autorité semble donc légitime. Le Parti se trouve donc d'une certaine façon contraint d'agir en cohérence avec son propre discours.

\section{Le Parti sous la contrainte de son discours}

Le discours officiel sur la modernisation comprend entre autres des promesses importantes en termes d'amélioration de la "gouvernance". Le Parti fait notamment une importante publicité autour de la formation et de la sélection de ses cadres, et surtout de la lutte contre la corruption. Dans le discours officiel, la réussite individuelle doit être fondée sur le mérite et la vertu, ce qui suppose de moraliser le comportement des fonctionnaires et des cadres du Parti. Pour conserver une certaine cohérence avec ces principes, et ainsi préserver sa propre légitimité au pouvoir, le Parti est obligé de sanctionner très sévèrement les cas de corruption ou d'abus graves qui sont rendus publics. Or les révélations publiques sur ces sujets sont de plus en plus nombreuses, et Internet leur donne un écho particulier.

Par exemple, le public chinois a été soulevé d'horreur en 2007 après la diffusion de photographies d'enfants et de handicapés réduits en esclavage dans des briqueteries de la province du Shanxi ${ }^{32}$. Des pères d'enfants disparus ont achevé de bouleverser l'opinion en publiant sur Internet un appel pour qu'on les aide à retrouver leurs enfants. Or les propriétaires de ces briqueteries étaient pour la plupart des membres influents de la cellule locale du Parti. Devant l'ampleur du scandale et la gravité des faits, les autorités ont envoyé bruyamment des centaines de policiers dans la province, qui ont délivré plus de mille personnes et condamné un certain nombre de responsables.

${ }^{30}$ BOLTANSKI Luc et THEVENOT Laurent. De la justification. Les économies de la grandeur, Paris: PUF, 1987.

${ }^{31}$ THIREAU Isabelle et WANG Hansheng. Disputes au village chinois, Paris: Maison des Sciences de l'Homme, 2001, p. 13.

${ }^{32}$ ELEGANT Simon. "Slave labor in china sparks outrage", Time, 20/06/2007. 
Les années 2007 et 2008 ont vu une accélération impressionnante des révélations de ce type. En particulier, plusieurs scandales liés à la mauvaise qualité de certains produits commercialisés en Chine ou exportés se sont succédés en quelques mois. Les jouets retournés à leur usine par Mattel $^{33}$ par exemple ont porté un coup très sévère à l'image de la Chine dans le commerce mondial, entraînant une vague de contrôles de qualité aux douanes de nombreux pays. Dans la foulée, on a découvert des dentifrices mélangés à des produits toxiques ${ }^{34}$, de la nourriture pour animaux empoisonnée qui a tué des chiens aux Etats-Unis, mais aussi des poissons aux antibiotiques etc... L'ensemble de ces découvertes a provoqué une grave crise de confiance de la part des consommateurs chinois ${ }^{35}$.

Ces scandales ont mis à nu de nombreux dysfonctionnements en hauts lieux, et en particulier dans la délivrance des autorisations de mise sur le marché pour un certain nombre de produits réglementés. A nouveau, l'intégrité des autorités risquant d'être mise en cause, des mesures particulièrement sévères ont été prises. Cible de cette prise en main très symbolique, le directeur de l'Administration d'Etat de l'alimentation et des médicaments, Zheng Xiaoyu, a été jugé en quelques semaines, convaincu de corruption, et condamné à mort en juillet $2007^{36}$. Dans le même temps, un coup d'arrêt était donné aux scandales alimentaires avec l'arrestation d'un journaliste pour "diffusion de fausse nouvelle", à cause d'un reportage "truqué" sur des pains à la vapeur, des "baozi" dans lesquels la viande aurait été remplacée par du carton ${ }^{37}$. Après la reprise en main du secteur par les autorités centrales, il est soudain devenu hors de question de rendre publiques de nouvelles affaires.

Cette reprise en main n'aura pas suffi à couvrir, un an plus tard, l'affaire du lait de marque Sanlu, empoisonné à la mélamine, une substance toxique utilisée pour falsifier les

${ }^{33}$ MACCARTNEY Jane. "How toy recall affects Brand China", Times Online, 14/08/2007, consulté le 25/10/2010 sur http://www.timesonline.co.uk/tol/news/world/article2259492.ece.

${ }^{34}$ BOGDANICH Walt. "Toxic toothpaste made in China is found in U.S.", The New York Times, 02/06/2007.

${ }^{35}$ WATTS Jonathan. "Made in China: tainted food, fake drugs and dodgy paint", The Guardian, 05/07/2007.

${ }^{36}$ BOUGON François. "Chine: exécution d'un ministre corrompu, sur fond de scandales alimentaires", Chine Informations, 11/07/2007, consulté le 25/10/2010 sur http://www.chineinformations.com/actualite/chine-execution-un-ministre-corrompu-sur-fond-de-scandales_7019.html.

37 ZHU Zhe. "TV report on cardboard buns 'fabricated'", China Daily, 18/07/2007, consulté 25/10/2010 sur http://www.chinadaily.com.cn/china/2007-07/19/content_5438963.htm. 
taux de protéines dans les tests de qualité, et qui a apparemment causé la mort de plusieurs bébés en Chine, ainsi que l'hospitalisation de milliers d'autres enfants. On aurait retrouvé des traces de mélamine dans un grand nombre de produits alimentaires commercialisés en Chine et hors de Chine. Là encore, tous les ingrédients d'un scandale de grande ampleur sont présents, au cœur même du mode de vie et des préoccupations de la "classe moyenne". Il s'agit en effet de menaces sérieuses sur la santé des enfants, sur les modes de consommation et sur la confiance des citoyens dans le modèle de développement économique chinois. Surtout, ce cas pose à nouveau la question des limites qui doivent être posées à l'enrichissement personnel et de l'interdiction formelle de la corruption. Pour toutes ces raisons, les autorités ont réagi très sévèrement, et cette affaire a également donné lieu à des condamnations à mort ${ }^{38}$.

Désormais, la publication des scandales à grande échelle oblige les autorités à intervenir et surtout à faire savoir qu'elles réagissent efficacement. A la suite de ces précédents d'envergure, les internautes n'hésitent plus à dénoncer un fonctionnaire local lorsqu'il transgresse manifestement une règle légitimée par le discours officiel. Ils sont aidés en cela par les grandes possibilités de diffusion de l'information et en particulier de l'image, qui se passe bien souvent de commentaires. C'est par exemple ce qui est arrivé à Lin Jiaxiang ${ }^{39}$, un membre de l'administration maritime de Shenzhen, qui fut filmé en novembre 2008 par la caméra de sécurité d'un restaurant en train de suivre une petite fille de onze ans en direction des toilettes du restaurant. La vidéo montre la jeune fille sortir aussitôt en courant et aller chercher son père. Elle déclare que Lin a voulu la pousser avec lui dans les toilettes des hommes. Ensuite, l'officiel menace la famille devant de nombreux témoins et devant la caméra, se présentant comme un membre du ministère des transports à Pékin. Il propose même de leur donner de l'argent sans même tenter de nier sa tentative d'agression. La vidéo a

\footnotetext{
${ }^{38}$ BARBOZA David. "Death sentences in Chinese milk case", The New York Times, 22/01/2009.

${ }^{39}$ XIAO Qiang. "Government Official Attacks 11-Year-Old Girl", China Digital Times, 01/11/2008, consulté le 25/10/2010 sur http://chinadigitaltimes.net/2008/11/government-official-attacks-11-yearold-girl/.
} 
très vite circulé et les internautes ont lancé contre lui le "moteur de recherche de chair humaine", conduisant en dernier lieu à son limogeage ${ }^{40}$.

Alors que les membres du Parti doivent officiellement se mettre "au service du peuple", il est de moins en moins tolérable aux yeux du grand public qu'ils se considèrent au-dessus de la loi, ou qu'ils tirent de leurs fonctions des privilèges indus. Dans le contexte de la lutte contre la corruption et de la promotion de la bonne gestion de l'argent public, certaines incongruités sont systématiquement relevées par les internautes. Ainsi, des photographies de locaux officiels particulièrement luxueux circulent-elles comme une forme de dénonciation. Les bâtiments officiels, qui abritent les bureaux des cadres locaux, construits à des prix faramineux, pour ressembler à la Maison Blanche ou à des immeubles de Dubai ${ }^{41}$ ne passent plus inaperçus. Ils font désormais l'objet d'un recensement officiel ${ }^{42}$, et les dépenses publiques sont désormais beaucoup plus contrôlées. Fortes de la rhétorique du gouvernement lui-même, les demandes du public en termes de transparence se font toujours plus hardies. Une caricature circulant de blog en blog demande même la publication des comptes publics ${ }^{43}$.

En somme, la rhétorique officielle, appropriée et réinterprétée par les Chinois en général et les internautes en particulier, fait partie d'un registre qui permet de justifier un grand nombre de prises de paroles, révélations, indignations publiques, de natures très variées. Certains internautes mobilisent les foules autour du credo patriotique officiel, tandis que d'autres utilisent l'idée de modernisation comme levier dans la défense d'intérêts collectifs ou

${ }^{40}$ BRANIGAN Tania. "Chinese official sacked over attack on girl, 11", The Guardian, 05/11/2008, p. 27.

${ }^{41}$ LIU Zhen et BLANCHAR Ben. "'Dubai-style' office stokes anger in China", Reuters, 24/12/2008, consulté le 25/10/2010 sur http://www.reuters.com/article/lifestyleMolt/idUSTRE4BN0HZ20081224?feedType=RSS\&feedName $=$ lifestyleMolt.

${ }^{42}$ CHAI Jing. "Ji guanjian haohua bangonglou diaocha: difang lingdao quanli quefa zhiyue", CCTV, 23/09/2008, transcrit le 26/06/2009 sur ca315.com.cn, consulté le 25/10/2010 sur http://news.ca315.com.cn/ts/2009/0626/157457.html.

${ }^{43}$ XIAO Qiang. "Cartoon: 'Take it off. Now it's your turn!'", China Digital Times, 31/03/2009, consulté le 25/10/2010 sur http://chinadigitaltimes.net/2009/03/cartoon-take-it-off-now-its-your-turn/. 
individuels, y compris contre les autorités lorsque cela s'avère nécessaire. Le Parti se trouve ainsi piégé (entrapped) par sa propre rhétorique ${ }^{44}$.

Les internautes qui prennent la parole ainsi ont des postures et des opinions politiques très diversifiées. C'est ce que montre par exemple Zhou en tirant le portrait de trois blogueurs dont les opinions sont radicalement différentes ${ }^{45}$. Il a choisi d'étudier des "outsiders mais non activistes", c'est à dire des personnes qui tiennent un discours à tonalité politique, sans se positionner contre l'Etat. Il définit l'espace occupé par ces internautes comme "minjian", c'est à dire "non officiel" ou "indépendant", "marginal" ou "hors du système" (traductions proposées par lui), par opposition au monde "guanfang", "officiel". Il souligne bien le fait que tous présentent leurs intentions comme favorables à la nation et à l'Etat, ce qui les distingue des dissidents. De la sorte, Zhou prend en compte l'effet de cadrage imposé par les lignes rouges du discours officiel, tout en montrant la diversité de points de vue que ce cadrage permet. Le point commun de tous ces porteurs de causes, ou de ces "écrivains politiques minjian" est d'affirmer travailler pour le bien de la nation. Cependant, leur positionnement hors de la sphère officielle leur permet de s'arroger le droit d'interpréter l'intérêt national à leur manière ${ }^{46}$, repoussant sans cesse les limites floues de cette marge d'interprétation.

Les règles de la censure et de l'autocensure n'interdisent donc pas toutes les mobilisations. Il semble que la parole publique en Chine soit ouverte à de nombreuses prises de positions, à condition d'utiliser une certaine grammaire de présentation des problèmes. Ce registre, ou cette grammaire, peut dans certains cas être utilisée comme une ressource pour faire émerger des problèmes sur la scène publique. La mise en récit des sujets abordés sur Internet devient donc un véritable enjeu stratégique, ce dont les internautes sont parfaitement conscients. Certains savent véritablement "mettre en scène" leurs causes pour qu'elles trouvent un écho auprès d'un public très large.

\footnotetext{
${ }^{44}$ LYALL Jason. "Pocket protests. Rhetorical coertion and the micropolitics of collective action in semiauthoritarian regimes", World politics, vol. 58, n³, 2006, p. 378-412.

${ }^{45}$ ZHOU Yongming. "Living on the cyber border. Minjian political writers in Chinese cyberspace", Current anthropology, vol.46 n5, Dec.2005, p 779-803.

${ }^{46}$ Comme le rappellent les commentateurs de l'article de Zhou, cela n'est pas un phénomène nouveau en Chine. Au moins depuis la dynastie Qing, des personnes se sont présentées comme des "voix autoproclamées du peuple". Randolph Kluver cite par exemple Liang Qichao lors du mouvement du 4 mai 1919, ou le phénomène des Dazibao.
} 


\section{3 - Stratégies de mise en récit}

Internet a donc rendu possibles un certain nombre de dénonciations qui n'épargnent pas toujours les autorités. L'utilisation d'Internet peut ainsi s'inscrire dans une stratégie de défense des droits qui consiste à prendre appui sur les textes de loi et sur le discours officiel pour le retourner contre les autorités lorsqu'elles les transgressent. C'est une stratégie particulièrement utilisée par les catégories défavorisées ou discriminées, comme les migrants ${ }^{47}$, mais il est vrai que des mobilisations de plus en plus nombreuses prennent appui sur les intérêts spécifiques des catégories plus favorisées. Les questions qui les concernent, comme le droit de propriété, ou la protection de l'environnement, trouvent un écho assez large sur Internet.

\section{La défense d'intérêts particuliers}

Des citoyens de plus en plus nombreux ont compris l'efficacité de l'image et du symbole dans la défense de leurs droits face aux autorités ou à des entités puissantes, comme par exemple des promoteurs immobiliers (souvent associés aux autorités municipales). Le cas de la "Maison-clou" (Dingzi $h u)^{48}$ est très illustratif de cette utilisation stratégique de la rhétorique de l'intérêt national. Il s'agit d'une maison qui se trouvait sur le site d'un projet immobilier à Chongqing et dont la propriétaire, Wu Ping, a longtemps refusé l'expropriation, arguant de compensations trop faibles. Comme dans le cas de Sun Zhigang, l'injustice des compensations financières trop faibles rencontre un écho auprès d'une partie importante de la population. Nombreux sont les Chinois concernés par un projet immobilier dans leur entourage. "C'est précisément l'universalité dans le pays de cette expulsion et de cette

\footnotetext{
${ }^{47}$ Sur le rapport entre légalité et défense des droits, voir par exemple HAND Keith J. "Using law for the righteous purpose: the Sunzhigang incident and evolving forms of citizen action in the people's republic of China", Columbia Journal of transnational law, $\mathrm{n}^{\circ} 114,2006$, YU Jianrong. "Conflits dans les campagnes, la naissance d'une conscience politique paysanne", Perspectives chinoises, $\mathrm{n}^{\circ} 100$, 2007/3, p.30-36, FROISSART Chloé. Quelle citoyenneté pour les travailleurs migrants en République Populaire de Chine? L'expérience de Chengdu, Thèse de doctorat, Paris: Institut d'études politiques, 2007.

48 FRENCH Howard. "In China, fight over development creates a star", The New York Times, $26 / 03 / 2007$.
} 
démolition, de la compensation insuffisante et retardée, qui a généré une telle sympathie et un tel soutien pour la 'Maison-clou'"'49.

Cependant la méthode employée par la principale protagoniste souligne bien à quel point elle a su se servir de tous les symboles qui placeraient son action dans la droite ligne du discours officiel. Wu Ping défend ses indemnités au nom de la reconnaissance de la propriété privée et de l'application du Droit, qui sont des principes fondateurs de l'idée de modernisation du pays. Au moment le plus fort de la médiatisation de sa situation, Wu Ping a brandi un drapeau chinois par la fenêtre de sa maison, qui se trouvait surélevée de manière impressionnante du fait de l'excavation de toutes les parcelles qui l'entouraient. C'est donc une image spectaculaire de la défense de la propriété privée, sous une bannière patriotique, qui a circulé sur Internet, au travers des photographies relayées sur de nombreux blogs. Depuis, les cas de "maisons-clous" se sont multipliés et les photographies circulent sur Internet, presque comme un concours.

Cette tendance à mobiliser les divers aspects de la rhétorique de l'intérêt national dans la défense d'intérêts particuliers se révèle également dans des cas de plus en plus nombreux de mobilisations sur le modèle "not in my backyard" (NIMBY). Par exemple, des citoyens de Xiamen $^{50}$ ont réussi à faire geler le projet d'implantation d'une énorme usine chimique en faisant valoir les risques que ce projet faisait peser sur l'environnement dans cette ville déjà hautement polluée. Ils se sont ainsi opposés aux arguments des promoteurs et de la municipalité selon lequel l'ensemble de la population bénéficierait de la richesse produite par l'usine, en lui opposant une rhétorique plus générale sur la santé et la défense de l'environnement, laquelle était plus favorable à leurs intérêts particuliers.

Dans le même ordre d'idées se trouve le report de l'extension de la ligne du train Maglev à Shanghai ${ }^{51}$ à la suite des protestations des riverains. Les arguments défendus par ceux-ci étaient très typiques des préoccupations des catégories favorisées de la population. Ils ont ainsi souligné les risques que le projet comportait pour leur santé (ils sont particulièrement

\footnotetext{
${ }^{49}$ YANG Zhizhu. "Dingzihushijian he jie yangai zhidu quexian" Sina.com.cn, 03/04/2007, consulté le 14/10/2010 à l'adresse http://news.sina.com.cn/c/2007-04-03/090612682608.shtml.

50 "Chinese city freezes plans for chemical plant after barrage of complaints", International Herald Tribune (Reuters), 30/05/2007.

${ }^{51}$ DYER Geoff. "Protests suspend work on Shanghai maglev", The Financial Times, 06/03/2008.
} 
inquiets des radiations potentielles), ainsi que la perte de valeur de leurs biens immobiliers aux alentours du passage du $\operatorname{train}^{52}$.

La manière dont ces groupes mobilisent la rhétorique officielle montre bien la marge d'interprétation dont ils disposent et sur laquelle ils ne se privent pas de jouer. Ils peuvent faire valoir tour à tour des arguments divergents, voire contradictoires, pourvu qu'ils réussissent à les relier d'une manière ou d'une autre à certaines valeurs défendues par l'Etat, dans une perspective de modernisation et de civilisation très vaguement définies.

\section{L'exemple de la cause des chiens}

Un exemple en particulier, au cours de mon enquête, s'est révélé très illustratif de la faculté des internautes à identifier les thématiques qui constituent ce registre, et à s'en servir pour prendre la parole de manière critique, sans prendre de risques. Il s'agit d'une forme de "protestation de poche" (pocket protest ${ }^{53}$ ), dont l'objet est circonscrit à une cause très spécifique et qui semble presque dérisoire, mais qui révèle bien la manière dont des individus sont capables de "piéger" les autorités avec leur propre rhétorique, pour défendre quelque chose qui leur tient à cœur.

$\mathrm{Au}$ moment où j'ai effectué les entretiens, en 2007, avait lieu une controverse importante à Pékin autour de nouvelles règles restreignant la possibilité d'élever des chiens, dans la perspective des Jeux Olympiques de 2008. Prenant pour prétexte une épidémie de rage, les autorités présentaient les chiens comme un danger sanitaire, qu'il fallait contrôler et maîtriser. Ils ont donc appliqué strictement un système de "hukou", sur le modèle des permis de résidence des citoyens chinois. Les propriétaires devaient ainsi payer des sommes différentes selon qu'ils souhaitaient enregistrer leur chien en centre-ville ou à la campagne. De plus, les chiens de grande taille (au-dessus de $35 \mathrm{~cm}$ ) ont été strictement interdits en zone urbaine, à l'intérieur du quatrième périphérique de Pékin, sous peine d'amendes. L'application de ce règlement s'est soldée par de véritables battues, et par l'euthanasie de dizaines de milliers de chiens "errants" ou considérés arbitrairement comme tels.

${ }^{52}$ DYER Geoff. "Train dispute tests activism in China", The Financial Times, 11/01/2008.

${ }^{53}$ LYALL Jason. "Pocket protests. Rhetorical coertion and the micropolitics of collective action in semiauthoritarian regimes", World politics, vol. 58, n³, 2006, p. 378-412. 
Ces mesures, qui étaient officiellement motivées par des préoccupations d'ordre public, touchaient en fait à un élément emblématique du mode de vie des "couches moyennes". Avec la voiture et l'appartement, les chiens sont un symbole de ce style de vie privilégié ${ }^{54}$, car ils sont à la fois un objet d'affection, un signe extérieur de richesse relative, et l'affirmation d'un statut de responsabilité en tant que "maître" de l'animal ${ }^{55}$. Le nombre de chiens augmente ainsi sensiblement depuis plusieurs années. Les restrictions imposées sans préavis ont non seulement mis de nombreux propriétaires de chiens en situation d'illégalité, mais elles ont été vécues comme une contradiction avec la direction générale que la société est invitée à prendre. Touchés dans leur vie privée, de nombreux internautes ont protesté contre ces mesures, en ligne et hors ligne, mettant en évidence ces contradictions.

Constatant que ses protestations sur Internet avaient été censurées, un blogueur est même allé jusqu'à porter plainte.

"Je n'ai jamais violé la loi", dit Liu, dont le cas est en appel. "Tous les cas dont j'ai parlé avaient déjà été publiés. Je voulais seulement exprimer mon opinion làdessus. Un blog doit être une plate-forme pour que les gens expriment leurs idées. Ce n'est pas correct de juger le blog de quelqu'un s'il ne viole pas la loi." [...] Pour soutenir son argument, il cite le Président Hu Jintao qui a demandé au 17ème Congrès du PCC en octobre que la Chine suive la voie du "développement scientifique" et rende le gouvernement plus transparent. ${ }^{56}$

On voit bien dans cette référence au discours du Président lui-même une stratégie qui consiste à montrer sa propre démarche sous un jour favorable et conformiste. La même stratégie est utilisée dans la presse par d'autres personnes mobilisées dans la défense des chiens. Beaucoup soulèvent l'argument du manque de "scientificité" de cette mesure. En effet,

\footnotetext{
${ }^{54}$ TOMBA Luigi. "Creating an urban middle class: social engineering in Beijing", The China Journal, $\mathrm{n}^{\circ} 51,2004$, p. 1-26.

${ }^{55}$ Les chiens, symbole d'un niveau de vie élevé, ont été chassés pour cette même raison dans l'empire soviétique. "Parce que la chasse à l'homme est en notre siècle la chasse aux privilégiés: à ceux qui lisent des livres ou qui ont un chien." KUNDERA Milan. La valse aux adieux, Paris: Gallimard, 1999, p. 137.

${ }^{56}$ CODY Edward. "Dog owner takes on china's web censors", Washington Post, 26/12/2007.
} 
selon eux les grands chiens sont souvent plus calmes que les petits ${ }^{57}$, et il n'est donc pas rationnel de limiter la taille des chiens en ville dans un objectif de sécurité publique.

Parmi les internautes que j'ai interrogés, beaucoup étaient sensibles de près ou de loin à cette question des animaux domestiques. Tous montrent qu'ils ont compris les objectifs affichés par les autorités, et ce que cela implique en termes de "cadrage" des discours sur Internet. La simple prolifération d'un sujet sur différentes sources d'information indique déjà que le sujet a été mis à l'agenda officiel, et sous un angle particulier.

"Par exemple hier j'ai ouvert QQ et il y avait quelque chose, ça disait qu'a partir d'aujourd'hui on n'a le droit d'avoir qu'un seul chien, qu'un seul animal de compagnie, et puis j'ai regardé un peu le journal aussi, ils disaient la même chose sur le journal, et aussi a la télé c'était pareil. C'est forcement très important, il faut que tout le monde y fasse attention." (Shao)

L'un des internautes interrogés était lui-même concerné par la nouvelle réglementation, possédant un labrador, trop gros pour être gardé en centre-ville légalement. Pour cette raison, il s'est montré particulièrement sensible à la campagne d'information menée par les autorités, sur laquelle il avait une opinion très tranchée. Cet internaute a décidé d'utiliser Internet pour faire connaître sa situation et plaider la cause des chiens auprès de l'opinion publique. En particulier, il voulait rétablir certaines vérités sur la dangerosité des chiens, et l'importance du dressage. Il veut surtout convaincre autour de lui qu'il n'y a rien à craindre de la présence de chiens en ville et qu'a fortiori la nouvelle règle est inutile. Pour cela, il est s'attaché à présenter son message de la manière la plus consensuelle possible.

"Par exemple sur le forum qui parle d'animaux domestiques, sur la question d'élever des chiens, c'est à dire la question du contrôle des chiens, j'ai dit 'nous le peuple, qu'est-ce qu'on peut faire?' En fait d'après moi nous pouvons faire très peu de choses. D'abord il ne faut pas faire de concurrence à la politique du gouvernement, non. Ce qu'il faut faire, c'est dire aux gens ordinaires autour de nous, avec une attitude pondérée, à quelle place les chiens devraient se trouver par rapport aux gens, pourquoi je pense que ce sont des amis, pourquoi ils n'ont pas leur place dans nos assiettes, et dire pourquoi nous pensons qu'élever un chien ne peut pas déranger (yingxiang) la vie

57 WEI Michael. "Dog owners maddened by Beijing canine restrictions", Reuters, 17/07/2008, consulté le 25/10/2010 sur http://www.reuters.com/article/inDepthNews/idUSPEK26979420080717. 
des gens, et pourquoi nous pensons que même pour des choses comme la rage, la question du contrôle ne repose pas sur les chiens, cela devrait reposer sur les gens, et pourquoi nous pensons que ce qu'on dit sur les chiens par rapport à la pollution, en fait c'est un problème de gestion de la ville, pas un problème de chiens. Quand j'ai dit ça, j'ai dit en gros, que nous pouvons en discuter avec nos amis, nos voisins, et que nous pouvons leur dire que les gens qui élèvent des chiens ont des règles, et qu'on ne toucherait pas à l'intérêt public (dajia de gongzhong liyi)." (Zhang)

Prenant la parole publiquement, cet internaute a une manière intéressante de poser le problème. Il ne nie pas le problème de l'hygiène publique tel qu'il est défini par le gouvernement, et même au contraire, il exprime très directement sa volonté de ne pas contredire les autorités. Il se contente d'essayer de montrer que les chiens ne sont pas à la source de ce problème, et que les pourchasser n'est pas la bonne solution. Pour justifier encore mieux sa position, il parle de ne pas déranger les gens, ni de toucher à l'intérêt général. Il procède en fait à une opération de cadrage très méticuleuse pour donner des chances à son discours d'être à la fois entendu par les autres internautes et autorisé par les censeurs.

Cette démarche n'est pas inconsciente ou spontanée. Bien au contraire, elle est réfléchie et elle repose sur une analyse très froide de l'agenda des autorités et des techniques de propagande utilisées pour l'imposer.

"Il y a encore des choses dont on peut discuter, et dans le domaine de ce qu'on peut discuter il y a des choses intéressantes, parce qu'en fait ce contrôle dépend des moments. Il y a une certaine coordination avec le gouvernement. Quand on ne se coordonne pas avec le gouvernement, prenons un exemple, aujourd'hui avoir un chien à Pékin est devenu très compliqué. En ce moment le gouvernement ne laisse pas passer dans la presse tous les reportages qui parlent des avantages des animaux domestiques, comme l'idée que les chiens sont nos amis, et tous les articles qui traitent de ce type de questions. Pourquoi? Parce qu'en ce moment ils veulent que tout le monde comprenne le problème de la rage, qu'il y en a en Chine, et que d'après les dernières statistiques, la rage est la maladie contagieuse qui tue le plus. Ils concentrent la propagande là-dessus, pourquoi? Ce n'est pas pour que tout le monde comprenne à quel point la rage est grave, en fait ils ont un but, et le but c'est quoi? C'est de préparer la prochaine étape du travail qu'ils veulent faire, et qui est d'attraper tous les chiens de la ville. Ils veulent éliminer complètement tous les chiens errants et qui n'ont pas de 
maître. Ils veulent remplir leurs objectifs, et faire en sorte que tout le monde accepte leur discours, et après c'est comme du lavage de cerveau, ils le disent tous les jours, tous les ans, tous les mois, tous les jours, ils le disent toutes les heures, par tous les canaux qui atteignent tes oreilles. Au début tu penses peut-être que ce n'est pas ce que tu aurais dit, et progressivement tu te dis qu'ils doivent avoir raison, et avec le temps tu finiras par penser que c'est peut-être vraiment comme ça, et si le temps passe encore, même si tu n'es pas d'accord tu finis par être assimilé (bei tonghua), et tu te dis que puisque tout le monde pense comme ça, c'est forcément comme ça." (Zhang)

L'internaute tire des conséquences très concrètes de cette analyse sur sa propre manière de communiquer sur Internet. Pour faire passer un message, il doit "se coordonner", trouver dans le discours officiel lui-même les sujets sur lesquels il est possible de s'exprimer, et le ton sur lequel on peut les aborder.

Un autre internaute théorise ce cadrage de la parole publique comme une forme de "canalisation", en se servant d'une parabole célèbre.

"'Le grand Yu canalise les eaux', c'est à une époque où il y avait des inondations très graves. Ce roi, le grand $\mathrm{Yu}$, son père s'appelait Gun, il avait reçu de l'empereur Shun la responsabilité de canaliser les eaux. La technique qu'il avait choisie était de les boucher, et de résister envers et contre tout. Mais il y a eu une inondation trop forte, et tout ce qu'il avait bouché a été balayé. Donc il a été exécuté par l'empereur Shun. Son fils a suivi ses dernières volontés, il a continué à contenir les eaux, mais sa technique était de draguer les cours d'eaux, de creuser des canaux, de transformer les grands en petits et d'éliminer les petits, c'est comme ça qu'il a pu canaliser les eaux. [...] Je ne parle pas de choses négatives, je ne parle pas des évaluations du gouvernement chinois qui sont faites par des médias étrangers, je trouve aussi que critiquer le gouvernement c'est troubler l'ordre public aveuglément. Si tu vois un abus du gouvernement, il faut réfléchir à un moyen de le résoudre. Si je vois quelque chose qui est contraire à mon intérêt, je réfléchis à une solution pour résoudre le problème, je cherche une méthode pour que cela m'arrange, et si je m'y intéresse, ce n'est pas pour faire de l'agitation inutile. Si, quand tu vois ça, tu te mets à lancer des plaintes, ça ne sert à rien. Quand tu veux faire quelque chose, et que tu te rends compte que le gouvernement est en ta défaveur, alors tu ne le fais pas. Tu vas faire des choses dont le gouvernement ne se préoccupe pas. Tu penses surtout à trouver des occasions. En 
Chine on a une phrase comme ça: les gens qui ne sont pas très intelligents, les gens stupides, font ce que la loi les autorise à faire; mais les gens intelligents font les choses qui ne sont pas interdites par la loi. Il n'y a que comme ça qu'on peut trouver des occasions. La loi chinoise est très incomplète. Pour trouver ces occasions, tu peux aller sur des forums, dans les commentaires, dans les médias et tu découvres ces trous, tu peux saisir ces opportunités. En Chine, on ne peut pas réussir de grandes choses sans spéculer." (Tian)

Cette technique de "canalisation", consiste donc à éviter les attaques frontales, et à aborder les problèmes par les angles les moins contestataires, en utilisant comme levier des valeurs très consensuelles pour les retourner contre des mesures ponctuelles prises par les autorités. Le cas des animaux de compagnie se prête bien à un tel retournement du discours officiel, car il se présente comme une question domestique avant d'être une question d'ordre public.

Le passage ou non d'un problème sur la scène publique et son écho plus ou moins important dépend donc de la manière dont il est mis en récit. Les variantes du discours officiel dans le temps fournissent un "cadre" ${ }^{58}$, ou si l'on veut un registre, qu'il faut suivre attentivement pour connaître en un moment donné les thématiques qu'il est possible d'aborder, ou au contraire déconseillé de critiquer.

Ce registre peut, dans certains cas, favoriser une prise de parole massive de la part des internautes, reproduisant ainsi, en quelque sorte, le principe des grands mouvements politiques dont la Chine est familière. Comme le montre Zhou Xueguang en effet, ces mouvements créent à la fois des contraintes et des occasions pour qui sait s'en saisir. "Les réponses individuelles aux changements de la politique d'Etat ne suggèrent pas l'adhésion à la ligne politique établie par l'Etat. Participer à des campagnes politiques initiées par l'Etat fournit aux individus et aux groupes l'occasion de poursuivre leurs propres agendas en exploitant de nouvelles opportunités" ${ }^{59}$.

${ }^{58}$ GOFFMAN Erving. Les cadres de l'expérience, Paris: Minuit, 1991.

${ }^{59}$ ZHOU Xueguang. "Unorganized interests and collective action in communist China", American sociology review, vol.58, $\mathrm{n}^{\circ} 1,1993$, p. 54-73, p. 61. 
En effet, les définitions imprécises et plastiques des valeurs portées sur la place publique laissent de l'espace pour des négociations, et, dans certains cas, pour de véritables retournements des causes défendues. De plus en plus d'internautes en sont conscients et certains savent jouer de ces imprécisions pour faire passer des idées ou donner de la visibilité à des causes. Ils savent profiter des ouvertures momentanées de ce que Gamson et Meyer appellent des "fenêtres d'opportunités discursives" ${ }^{60}$. Ainsi, les individus défendant des intérêts de manière "non-organisée" se retrouvent-ils le long des mêmes lignes politiques, utilisant les mêmes formulations, et formant ainsi de manière presque spontanée ce que l'on peut appeler, avec Zhou, une forme d'action collective "non-organisée".

Pour l'instant j'ai mis sur le même plan des actions de "maintien de l'ordre" de type patriotique avec d'autres qui consistent à défendre des intérêts personnels, qui se servent de la même rhétorique parce qu'elle est pratiquement la seule possible. Bien sûr, ces différents modèles de mobilisations, s'ils convergent par le type de justifications mobilisées, n'ont pas pour autant la même valeur, les mêmes sources ou objectifs. Il semble donc utile de détailler la complexité du processus de formation de l'opinion et les jeux complexes auxquels s'adonnent une multiplicité d'acteurs aux positions, intérêts et agendas très différents.

${ }^{60}$ GAMSON William et MEYER David. "Framing political opportunity", in MCADAM Doug, MCCARTHY John et ZALD Mayer (dir.). Comparative perspectives on social movements, Cambridge University Press, 1996, p. 275-290. 


\section{Chapitre VIII - La prise de parole: des postures diverses}

Les mobilisations qui prennent de l'ampleur sur le Web chinois sont de plus en plus nombreuses et elles sont particulièrement variées, du fait de la marge d'interprétation importante que comporte la rhétorique officielle. Cependant il existe des causes qui échouent, soit qu'elles soient censurées, soit qu'elles ne trouvent pas un écho suffisant chez les internautes, auquel cas elles tombent dans l'oubli. Il ne suffit donc pas d'identifier la voie étroite que constituent les fenêtres d'opportunités offertes par le projet officiel de modernisation du pays. Il faut aussi comprendre comment les causes se forment et viennent peu à peu s'imposer publiquement, comment se jouent les tâtonnements successifs qui conduisent à des définitions sans cesse renouvelées des valeurs communément acceptées.

Les entretiens ont permis d'envisager cette question du point de vue des internautes euxmêmes, et d'observer à la loupe les circonstances spécifiques qui les conduisent à s'exprimer ou non. Il existe tout un continuum de postures $^{1}$ possibles, qui dépendent de nombreux facteurs, comme le degré de proximité avec la cause défendue, ou le statut de l'internaute en ligne et hors ligne par exemple. Ils peuvent prendre l'initiative de porter eux-mêmes des sujets sur la place publique, pour s'étonner, s'amuser, s'indigner ou parfois interpeller les autorités sur un problème. Ils peuvent aussi tout simplement manifester en un mot leur soutien ou leur désaccord, venant grossir les rangs d'un public de plus en plus influent. Au contraire ils peuvent choisir de rester silencieux, ce qui n'implique pas nécessairement une complète indifférence. C'est dans ce silence attentif, souvent accompagné de conversations hors ligne, que se forgent de nombreux points de vue.

$\mathrm{Au}$ travers des différentes attitudes, on peut voir que la prise de parole sur Internet, même si elle est ouverte à tous, reste tributaire de compétences ${ }^{2}$ et de ressources qui sont inégalement distribuées ${ }^{3}$, et qui sont d'autant plus importantes que le cadrage politique impose

${ }^{1}$ GOFFMAN Erving. Façons de parler, Paris: Minuit, 1987.

${ }^{2}$ CARDON Dominique, HEURTIN Jean-Philippe et LEMIEUX Cyril. "Parler en public", Politix, vol. $8, n^{\circ} 31$, p. 5-19.

${ }^{3}$ BOURDIEU Pierre. Ce que parler veut dire : l'économie des échanges linguistiques, Paris: Fayard, 1982. 
de la subtilité. De là découle une grande variabilité dans la croyance dans l'efficacité de la prise de parole publique. Pour certains, la publicité est devenue une véritable ressource dans la défense de leurs droits, alors que d'autres considèrent qu'elle est parfaitement inutile et vaine. Tous jouent pourtant un rôle important dans le filtrage et la diffusion des informations, et dans le développement des différentes causes.

\section{1 - La circulation silencieuse des causes politiques}

\section{Une "majorité silencieuse"?}

Les internautes les plus prompts à affirmer qu'ils ne prennent pas la parole sur Internet sont aussi ceux qui affichent un soutien sans faille aux autorités, et qui déclarent qu'il vaut mieux ne pas se mêler des affaires de l'Etat. Ils justifient ainsi leur silence en ligne dans les mêmes termes que la censure, en soulignant la nécessité de l'harmonie et l'envie de ne pas critiquer publiquement la société chinoise. Dans deux cas, les internautes se présentent comme des personnes "traditionnelles"(chez Zeng par exemple), qui n'aiment pas exposer leur personnalité sur Internet, que ce soit dans le cadre de forums de discussion ou pour rédiger un blog, même s'ils peuvent comprendre, avec une sorte d'indulgence, que d'autres personnes puissent ressentir ce besoin de défoulement.

Dans ces deux cas, l'entretien lui-même a été l'occasion de recueillir une parole aux accents très officiels et patriotiques, avec la reprise presque mot pour mot de tous les slogans très souvent entendus dans le discours officiel sur la difficulté de gérer un si grand pays, le besoin d'harmonie et d'adhésion populaire aux politiques du gouvernement central etc... De toute évidence, ces deux entretiens au moins, ainsi que certains passages des autres entretiens doivent s'analyser comme des performances, des transcriptions publiques exemplaires. Confronté à une question quelque peu politique, l'un d'eux a demandé à changer de sujet, et plus tard il a tenu un discours particulièrement conformiste.

"Je ne veux pas parler de politique avec toi [...]

Tout le monde peut avoir son propre avis là-dessus, je le reconnais. Il y a toujours des avantages et des inconvénients, mais il faut toujours faire ce qui convient au pays où on est. Je trouve que la Chine est un très bon pays, j'adore ce pays. Je 
trouve que le système de parti unique du Parti Communiste Chinois est plutôt bien adapté à la Chine. La Chine est trop grande, la population est trop nombreuse, s'il y avait je ne sais combien de partis, elle serait ingérable. En plus, dans la tradition chinoise, on a un gouvernement familial, on ne peut pas être quatre ou cinq chefs de famille, il n'y en a qu'un, n'est-ce pas? Il faut que la famille soit grande pour que l'activité soit grande aussi. Ce n'est pas un problème d'avoir une ou deux provinces autonomes, il y a cinquante-six minorités nationales, la population est trop nombreuse, si on avait plusieurs partis pour finir ils feraient sécession, non? Donc pour la Chine on ne peut avoir qu'un seul parti, c'est la seule façon d'être unis tous ensemble, même si le développement de la Chine n'est pas équilibré du tout pour le moment, n'est-ce pas? Il y a beaucoup de problèmes sociaux, mais je crois que la Chine se réforme petit à petit, à partir des changements à Pékin, à Shanghai. Quand j'étais petit toute cette partie de Pékin [le district de Haidian] c'était des champs, la campagne, et dix ans et quelque après c'est devenu comme ça, c'est très rapide. Sans le Parti Communiste ce serait difficile de faire changer les choses. " (Wu)

En d'autres termes, l'absence de critique est pour lui la contrepartie du développement rapide du pays. Ce comportement semble assez typique de ce que Hirschman appelle la "loyauté" ${ }^{4}$. Pourtant, l'internaute évoque un peu plus tard certaines mobilisations qui ont permis de faire réagir le gouvernement, notamment dans le cas de manifestations contre le Japon. Il semble se féliciter à ce moment-là que l'Etat se montre à l'écoute des citoyens, sur un certain nombre de questions bien ciblées. Il se montre ainsi attentif à l'actualité et à certaines mobilisations, auxquelles il ne prendrait certainement pas part, mais dont il se fait un spectateur alerte.

Un tel alignement sur la parole officielle est en fait plutôt rare dans les entretiens que j'ai menés. La plupart des internautes préfèrent montrer une grande indifférence à l'ensemble des questions politiques, dès lors qu'elles ne les concernent pas personnellement. Ceux-là choisissent, en quelque sorte la "sortie" (exit) au sens de Hirschman.

"On ne peut rien y changer. Récemment, un enfant a été mordu par un chien, donc maintenant ils attrapent à nouveau les chiens assez sévèrement. Maintenant on ne

${ }^{4}$ HIRSCHMAN Albert. Exit, voice, and loyalty : responses to decline in firms, organizations, and states, Cambridge: Harvard University Press, 1970, traduction française: Défection et prise de parole, Paris: Fayard, 1995 
peut plus avoir qu'un chien, mais beaucoup de gens en ont encore deux ou trois, tu crois qu'on peut tous les attraper? C'est impossible. Beaucoup d'entre eux sont de grosses bêtes, [...] mais finalement je pense que c'est beaucoup de bruit pour rien. Des fois je crois qu'ils le disent comme ça, mais en réalité ce n'est pas si sévère, donc je pense qu'il me suffit de le savoir. Maintenant ils les attrapent, mais je pense que ça n'a pas grand chose à voir avec moi, donc je n'y fais pas attention." (Shao)

Cette personne n'ignore pas la controverse autour du traitement des chiens par les autorités, mais elle s'abstient d'émettre un jugement sur la question. Elle s'y autorise en minimisant l'ampleur du phénomène. Cependant elle montre, elle aussi, que son indifférence est choisie délibérément, après un calcul qui la conduit à se décourager et à se détourner de la question, alors même qu'elle y avait initialement prêté une attention particulière.

\section{Le faible poids de la parole individuelle}

Un grand nombre d'individus se montrent ainsi potentiellement intéressés par des problèmes de société, et finalement découragés par le faible poids de la parole individuelle sur la scène publique.

"En général on parle très peu de politique, on ne parle pas des questions nationales, parce qu'on n'y comprend pas grand chose. En plus pour moi, personnellement je ne m'y intéresse pas beaucoup. Les relations entre les pays n'ont pas beaucoup de rapport avec les gens ordinaires. Et puis je comprends de travers le vocabulaire politique, donc je ne peux pas trop en discuter. Et puis les individus n'ont pas trop leur place dans ces sujets-là, ça concerne surtout des organisations ou le gouvernement, très rarement des individus. La parole des individus est très faible, ce n'est pas nécessaire de gaspiller trop de temps là-dessus." (Lin)

Les individus se sentent exclus des questions politiques. Affirmant "comprendre de travers", cet internaute se dévalorise, montrant que pour lui, prendre la parole est affaire de compétence et d'éducation. Etant une affaire de spécialistes et d'experts, la politique serait trop complexe pour les simples individus comme lui. Ce phénomène d"'auto-déshabilitation" 5 ,

\footnotetext{
${ }^{5}$ GAXIE Daniel. "Cognition, auto-habilitation et pouvoirs des 'citoyens'", RFSP, vol.57, nº, 2007, p737-758.
} 
causé par un sentiment d'infériorité, ressemble beaucoup au "cens caché"6 observé en France par exemple. Beaucoup pensent par exemple que les questions politiques sont réservées aux experts.

"Tant que ça ne me concerne pas, je ne m'y intéresse pas beaucoup, et puis quand tu exprimes des opinions sur des choses que tu n'as pas vécues toi-même il y a un biais subjectif, si tu n'es pas un spécialiste qui a fait des recherches là-dessus, ça ne sert à rien de t'exprimer dessus.

- Donc tu penses que les experts seuls devraient publier des opinions?

- Au moins ça devrait être des experts ou des gens qui ont vécu les choses euxmêmes, qui les ont vraiment traversées personnellement." (Zhou)

Pourtant, cela n'empêche pas forcément les internautes de lire et de partager l'information.

"Alors pourquoi est-ce que tu ne combats pas ça sur les forums?

- Peut-être parce que mes sources d'informations sont limitées. Si je vais sur les forums, j'obtiens ces informations, mais sinon, par moi-même je ne lis pas ces sujets de société, je n'y fais pas spécialement attention. Mais une fois que je suis tombée dessus, je veux que mes amis, et tout le monde soit au courant, donc je les attire dans cette rubrique." $(\mathrm{Gu})$

Cette tendance est renforcée par le fait que beaucoup sentent que les autorités ne tiennent aucun compte de leurs préoccupations, et que les décisions semblent tomber d'en haut, irrémédiablement, comme mécaniquement. Certains l'expriment avec un accent fataliste qui n'est pas sans rappeler les paysans italiens décrits par Lévi, qui subissaient la politique comme une série d'événements extérieurs auxquels ils ne pouvaient rien ${ }^{7}$.

"Je n'ai jamais écrit de commentaires.

- Pourquoi?

- Parce que s'il s'agit de politiques nationales, ça ne sert à rien de commenter.

- Comment ça?

${ }^{6}$ GAXIE Daniel. Le cens caché: inégalités culturelles et ségrégation politique, Paris: Seuil, 1978.

${ }^{7}$ LEVI Carlo. Le Christ s'est arrêté à Eboli, Paris: Gallimard, 1948. 
- Parce que pour les politiques nationales, ils ne font pas d'enquête, ils ne nous demandent pas notre avis, donc je trouve qu'on perd son temps à faire des commentaires.

- Tu penses que les gens ne liraient pas tes commentaires?

- C'est parce qu'ils ne changeraient pas la politique à cause d'un commentaire.

- Mais beaucoup de gens écrivent quand même des commentaires...

- C'est seulement pour épancher leurs propres plaintes, parce que quand le gouvernement a une politique, il ne peut pas la modifier sous prétexte que quelques personnes, peu importe combien, ont écrit des commentaires, parce que ces choses-là se décident après avoir réfléchi très longtemps, et pesé le pour et le contre. Prends le prix des abonnements, en fait ça ne me concerne pas, mais si ça doit augmenter, ça augmente, et si ça doit baisser, ça baisse. Qu'on en discute ou pas, je trouve que ça revient au même. Ils feront la même politique de $\mathrm{A}$ à $\mathrm{Z}$, peu importe qu'on la soutienne ou qu'on s'y oppose, ils la feront, même si le peuple chinois en entier s'y oppose. S'ils l'ont décidé, ils le réaliseront, et donc, ça ne sert à rien." (Han)

La même expression fataliste revient sans cesse: "mei banfa", "on n'y peut rien".

"On en a parlé un moment et puis on a arrêté, parce que ça ne sert à rien. Maintenant c'est déjà réglé, on ne peut rien y changer.

- Que disent les gens?

- Ils disent qu'il n'y a que la parole du gouvernement qui compte, quoi qu'on dise, ça ne sert à rien. On ne peut qu'aller dans le sens du gouvernement et puis c'est tout." (Tang)

Cette impression est très partagée. Pourtant, plusieurs personnes interrogées réaffirment en même temps leur intérêt pour des sujets liés à la politique officielle, comme l'éducation des enfants, qui a des conséquences très lourdes sur les choix de vie qu'ils doivent faire. Dans ces conditions, l'expression ne peut servir qu'à se soulager de la pression ressentie individuellement. Elle ne peut pas servir à changer la situation, mais bien au contraire à "prendre sur soi" les difficultés rencontrées.

"Je n'écris pas de commentaires, j'en reste à l'étape de la lecture.

- Pourquoi? 
- Parce qu'il y a des choses qu'il ne faut pas trop commenter.

- C'est à dire?

- Par exemple dans l'actualité nationale, que l'on fasse des commentaires ou pas, franchement, ça ne sert à rien.

- Pourquoi ça?

- Parce que dans ce pays, la parole individuelle n'a pas de poids. Des fois, quand tu vois des choses, tu as envie de t'exprimer, mais à la base personne ne t'écoute. Donc je me contente de comprendre le développement actuel, et puis l'éducation des enfants, et puis tous les sujets qui intéressent les gens ordinaires. Les gens ordinaires s'intéressent tous aux produits de première nécessité, aux assurances, pour les voitures ou ce genre de choses, voilà, c'est ça que les gens lisent sur Internet. Et surtout il y a l'éducation des enfants. Et puis c'est parce qu'on a l'intention de faire un enfant, c'est notre projet, donc on regarde un peu. [...]

- Alors quand tu veux donner ton avis, comment fais-tu?

- En général, j'attends que mon mari rentre pour qu'on discute, ça arrive. Mais en général on ne discute pas des grandes affaires nationales, parce que franchement, ce ne sont pas des choses dont on parle, ça n'a pas d'intérêt d'en discuter pendant des heures. Parler de l'augmentation du prix de la viande ou bien de l'éducation, ça ne sert à rien du tout." (Deng)

La prise de parole à laquelle les internautes renoncent explicitement est celle qui interpelle les autorités en vue d'obtenir un changement de politique. Cette prise de parole, envisagée comme tentative de prendre part au processus de décision, ou au moins de l'influencer, n'a pas de crédit auprès de la majorité des personnes que j'ai rencontrées. Pour eux, elle est vouée à l'échec. Elle ne peut apporter individuellement aucun bénéfice. Mieux vaut encore agir soi-même à son échelle, ou bien se replier sur des dimensions plus ludiques du Web.

"En ce qui me concerne je pense que je pourrais encore moins faire ce genre de choses, ça n'a aucun intérêt d'aller écrire mon avis dans un endroit virtuel comme Internet. Si vraiment j'ai un avis sur quelque chose, je n'ai qu'à agir directement dessus, ça sert à quoi d'aller en parler, ça n'a aucun intérêt d'en parler tout haut. Il n'y a aucune chance d'en tirer une récompense. Donc pour donner mon avis sur Internet, je 
suis peut-être différent des autres gens, je pense que ça n'est qu'une plate-forme, pour communiquer, ou bien une plate-forme pour se faire des amis. Mais pour d'autres choses je n'y ai jamais vraiment pensé.

- Donc finalement tu donnes ton avis, ou bien est-ce que tu cherches ta propre plate-forme, par exemple entre amis?

- Des fois j'en parle, des fois ça nous arrive de parler de certaines affaires du pays avec des amis, des choses sensibles, et à ce moment-là chacun donne son point de vue. Ca ne veut pas dire qu'en général on n'ose pas en parler, mais c'est simplement que le mode de pensée des Chinois, c'est que dans les moments détendus on ne dit pas de choses très profondes. Dans ces moments-là on va donner un peu son avis personnel sur des choses, les choses qu'on trouve bien, et on peut aussi parler de certaines choses qu'on ne trouve pas bien. On en parle avec de bons amis, des amis très proches." (Cai)

Cette frustration vis-à-vis du politique, qui est ressentie par anticipation, conduit donc les individus à se replier sur la quête du "bonheur privé", faute de croire dans le potentiel de l'action politique ${ }^{8}$.

\section{Le public comme pépinière des causes politiques}

En revanche, rien n'empêche ces internautes de partager les mêmes préoccupations sous d'autres formes, avec des amis et dans un cadre privé, ce qu'ils font très souvent pour la plupart.

"Quand une nouvelle loi est promulguée, si elle ne te plaît pas, est-ce que tu peux en parler?

- En privé oui, ou bien entre amis on peut en parler, mais pas avec des inconnus. Mais maintenant il n'y a pas de quoi être insatisfait, ça s'est déjà un peu ouvert. Quand on était petits il y avait encore des crimes politiques, si on n'était pas satisfait de la société on pouvait se faire enfermer." (Lin)

\footnotetext{
${ }^{8}$ HIRSCHMAN Albert. Bonheur privé, action publique, Paris: Fayard, 1983.
} 
Selon plusieurs répondants, il vaut mieux, dans ce cas, choisir ses interlocuteurs, en fonction de leurs centres d'intérêts, de leur capacité d'écoute, et, on le devine, en fonction du degré de confiance réciproque.

"Je ne fais pas de commentaires [sur la politique], je crois qu'il n'y a pas d'endroit pour ça.

- Pourquoi?

- J'en discute seulement avec mes amis. Sur Internet, ça ne m'intéresse pas de faire des commentaires sur ce genre de choses, non, je n'en fais pas. En plus on ne peut pas être complet sur ces questions, parce qu'on ne sait pas tout, et surtout ce n'est pas demandé.

- Donc tu préfères en discuter avec tes amis?

- Oui, pas tous mes amis, seulement quelques uns, ceux qui aiment ce genre de choses, on en discute." (Hong)

Ces discussions entre amis sont importantes. Elles font partie des sources d'informations qui tiennent certaines personnes informées de nombreux sujets, surtout s'ils sortent des canons autorisés par la presse officielle. L'information se diffuse ainsi sur Internet, mais aussi hors ligne, dans les familles et les groupes d'amis, c'est à dire souvent bien au-delà du simple lectorat d'un article ou d'un commentaire posté à sa suite.

"Apparemment en dessous de chaque information, il y a un lien sur lequel on peut cliquer, pour aller sur un forum, mais moi je ne clique jamais là-dessus.

- Pourquoi?

- Parce que ça prend beaucoup de temps, et je ne passe pas tant de temps que ça sur Internet. Je lis des choses dans un temps assez limité finalement. Peut-être que je trouve que les forums ne servent pas à grand chose, ça gaspille du temps, donc je n'y vais pas.

- Mais ces questions-là, est-ce que tu en discutes avec des amis?

- Oui, dans notre dortoir tout le monde discute, et il y a des sujets dont je ne suis au courant que parce qu'on en discute dans le dortoir." (Li) 
Finalement, ce large public silencieux apprécie dans sa grande majorité le fait que les autres internautes s'expriment en ligne. Cela contribue à nourrir leurs propres sources d'informations, même s'ils effectuent un tri personnel entre les différentes contributions. Les internautes semblent plébisciter une certaine forme de pluralisme, ou tout au moins de diversité.

"C'est une question de caractère, il y a des gens qui veulent à tout prix exprimer leur opinion, qui veulent que tout le monde connaisse leur point de vue. Ils ont vraiment le désir de publier leur opinion, pour que les autres la connaissent. [...]

- Tu trouves ça important qu'il y ait des gens comme ça?

- Oui, assez, il faut des gens comme ça. Si tout le monde était comme moi ce ne serait pas bien, personne ne réagirait à rien. Que ce soit correct ou erroné, ou bien n'importe comment, les gens expriment leurs opinions, et tout le monde exprime des opinions, et je trouve ça bien comme ça. Les gens n'arrêtent pas d'exprimer des opinions, et ils n'arrêtent pas de discuter, de débattre, de communiquer, d'échanger. $\mathrm{Ca}$ permet de s'approcher de la vérité (zhenli yue bian yue ming).

- Qu'est-ce que ça veut dire?

- La vérité, ça veut dire que ce n'est que quand tout le monde discute et débat ensemble, qu'on peut trouver le point de vue correct. C'est un dicton chinois, zhenli yue bian yue ming.

- OK. Et tu crois que toutes ces discussions ont une influence sur toi?

- Oui, sûrement. Parce qu'il y a des gens qui ont un point de vue différent du mien, donc ça permet de comprendre pourquoi les gens ont des points de vue différents. Ca nous encourage à aller voir, par exemple si j'étais à sa place, peut-être que je verrais aussi les choses comme lui, alors que du point de vue où je suis aujourd'hui je ne le peux pas." (Chen)

S'ils renoncent à prendre la parole sur de nombreux sujets, ces internautes ne sont pas pour autant complètement apathiques. Ils se montrent en effet relativement attentifs à de nombreux sujets de société, et ils sont bien plus enclins à en discuter dans des conversations privées que sur des plates-formes publiques. On peut reconnaître ici la notion de braconnage 
chez Certeau'. Les internautes, même lorsqu'ils ne s'expriment pas en ligne, sont actifs dans leur manière de recevoir les informations. Ils sélectionnent les sources auxquelles ils s'intéressent, les partagent, en discutent, les critiquent pour eux-mêmes. Ils jouent aussi un rôle de relais et procèdent à une sélection de l'information pour d'autres lecteurs lorsqu'ils informent leurs amis de l'existence de certains sujets en particulier.

Aussi les informations circulent-elles beaucoup plus que l'on ne pourrait le supposer au premier abord, mais de manière discontinue, informelle, et forcément intraçable. Ces conversations, au travers desquelles les différents cas deviennent célèbres, et qui permettent aux internautes de développer des argumentaires, fonctionnent ainsi comme une pépinière dans laquelle se développent les différentes causes politiques.

\section{2 - L'acquiescement comme chambre de résonance}

Ne croyant pas à l'influence de la parole individuelle sur la prise de décision politique, beaucoup choisissent donc le silence pur et simple sur les espaces qu'ils considèrent comme publics. Il arrive cependant que certains internautes ressentent le besoin de déposer en ligne des "indignations sans forces"10, qui ne sont pas destinées à faire pression sur quiconque, mais qui prennent simplement la forme d'une réaction émotionnelle à un événement, ou d'un questionnement sur un choix de vie personnel. Cette forme de prise de parole donne aux internautes l'impression de rester politiquement neutres, car ils ne font généralement qu'acquiescer, ou renchérir sur des positions déjà entérinées par d'autres et par les médias. En hochant la tête de la sorte, les internautes forment une chambre d'écho puissante pour de nombreuses questions proches de la vie quotidienne des "citoyens ordinaires". C'est ainsi que l'on voit croître en ligne certains sujets plutôt que d'autres.

\footnotetext{
${ }^{9}$ CERTEAU Michel. L'invention du quotidien 1. Arts de faire, Paris: Gallimard, 1990, p. 239s.: "Lire: un braconnage".

${ }^{10}$ CARDON Dominique et HEURTIN Jean-Philippe. "La critique en régime d'impuissance, une lecture des indignations des auditeurs de France Inter", in FRANCOIS Bastien et NEVEU Erik (dir.). Espaces publics mosaïques, acteurs, arènes et rhétoriques des débats publics contemporains, Rennes: Presses universitaires de Rennes, 1999, p.95: "La particularité des indignations sans force de France Inter est que, ne sachant ou ne voulant plus activer des conventions politiques ou militantes préétablies, refusant de déléguer à quiconque le droit de représenter leur colère, bref ne croyant aucunement à leur pouvoir d'influence, elles contribuent à défaire l'architecture de croyances sur laquelle l'efficace fiction du Tribunal du public s'est érigée".
} 


\section{L'indignation}

L'envie de s'exprimer, ou de partager son indignation, naît souvent dans l'émotion créée par une situation que les internautes jugent touchante, surtout s'ils se sentent proches ou concernés par cette situation. Au plus proche du quotidien des Pékinois, la question des animaux familiers a été l'occasion de voir des internautes plutôt indifférents à la politique sortir de l'apathie pure et simple pour partager en ligne leurs sentiments attristés.

"Tout ce qui est chiens ou chats abandonnés, je déteste ça, donc je ne les lis absolument pas, parce que je n'y peux rien, après avoir lu ce genre de choses je me sens très triste.

- Tu as déjà eu un chien?

- Je pourrais, et puis sur Internet un de mes collègues tient une page sur les animaux familiers. Il y a des gens qui y parlent de leurs petits chiens et chats. D'abord c'est très marrant, et puis les petits chats portent leurs propres petits vêtements, bien sûr ce ne sont pas des vrais vêtements, et puis ils leurs racontent beaucoup d'histoires, c'est très marrant. Mais ça parle aussi de la rage, et à Pékin ils ont tué 50000 chiens, c'est trop triste, alors je ne veux pas cliquer sur ces choses-là, quand je les lis ça me rend trop triste. Le gouvernement fait ça parce qu'il y a des gens qui sont morts après avoir été mordus par des chiens, mais ils en ont tué beaucoup trop. Je trouve que tous ces chiens étaient innocents. En tuer 50 000, qui s'empilent comme une montagne, quand j'y pense c'est très triste. Dans les forums habituels, on dirait que les gens n'en parlent pas beaucoup. Une fois je m'en suis plainte à mon mari, mais ça ne sert à rien du tout.

- Pourquoi est-ce que les gens écrivent des commentaires là-dessus?

- Pour en appeler à l'affection de tout le monde, pour que les gens refusent cette situation.

- Et ça marche?

- Oui sûrement, par exemple ils vont souvent chez une vieille dame qui a 200 chats, tous des chats errants. Elle ne gagne que 100 kuais [10 euros] environ, mais les chats lui coûtent chaque jour 200 kuais. Elle nous fait pitié en se privant elle-même de nourriture, et elle est très bonne avec les chats, et puis elle s'en occupe spécialement. Et puis il y a aussi des étudiantes qui viennent leur donner du lait, il y en a beaucoup, 
et puis des fois elles organisent des activités, tout le monde se donne rendez-vous pour y aller ensemble. Donc si tout le monde aime les animaux et y va ensemble, je trouve ça très bien." (Luo)

A la pitié ou à la tristesse, succède la colère, sans que les internautes ne croient toujours à l'efficacité de la prise de parole en elle-même. Pourtant ces sentiments ne s'évacuent pas immédiatement dans l'indifférence, car les chiens ont un capital de sympathie important. En dépit de l'inutilité supposée des actions, beaucoup d'internautes partagent leurs impressions et s'indignent publiquement, même s'ils pensent que cela ne sert à rien.

"Des fois je regarde d'autres choses, par exemple ces derniers jours la municipalité de Pékin a été contre les chiens, et beaucoup de chiens sont morts. Je suis très en colère à cause de ça, des fois je lis des articles sur des forums, tout le monde en parle, mais il n'y a pas de solution. La seule chose que les internautes peuvent faire est de s'envoyer leurs messages indignés et leurs opinions les uns aux autres, mais en réalité ça ne changera rien. Des fois je trouve qu'Internet est assez inutile pour ça." (Guo)

L'émotion est un ressort assez efficace de la prise de parole indignée sur de nombreux sujets. Par exemple, la situation des travailleurs migrants peut soulever des mouvements de compassion, en particulier lorsqu'elle est envisagée sous des angles auxquels les citoyens urbains sont très sensibles, comme l'éducation.

"Je lis aussi des choses sur les ouvriers migrants, leurs petits frères, leurs enfants. On les appelle 'population flottante', ils ont des problèmes pour mettre leurs enfants à l'école. Je compatis pour eux, et je sais que ce n'est pas facile pour le gouvernement, mais il y a beaucoup de choses, on est tous des hommes. Il y a deux jours j'ai vu un reportage tourné dans le Sud de la Chine. Avant, le gouvernement de Pékin leur avait construit une école, et puis ils l'ont détruite. Il y a eu plein de photos sur Internet, avec des petits enfants qui voulaient absolument aller à l'école, c'était très émouvant, beaucoup de gens avaient écrit des commentaires, et moi aussi." (Tian)

Or, quoi de plus lié à l'action de l'Etat que l'organisation de l'école sur tout le territoire? Les internautes ne peuvent que mentionner l'importance politique de la question, même lorsque leur attention est initialement attirée par l'image pathétique d'un enfant qui ploie sous le poids de son cartable. 
"Oui, on fait des commentaires [sur les forums], et puis on en discute aussi dans des groupes QQ. Pourquoi est-ce que la Chine ne fait toujours pas de réforme, il y a beaucoup trop de cours dans une journée. Est-ce que tu as déjà vu ce dessin animé chinois, c'est un petit écolier qui porte un cartable extrêmement lourd. Dans son cartable il y a tous ses livres de classe. C'est beaucoup de pression, parce que l'éducation n'est pas personnalisée comme en Occident, c'est une éducation imposée." (Ye)

\section{L'implication personnelle}

Outre les cas où les internautes sont touchés émotionnellement, ils s'expriment aussi sur des questions qui les concernent personnellement, et dont, bien souvent, ils ne perçoivent pas au premier abord la dimension politique.

Par exemple, une jeune mère se pose des questions au sujet d'une pratique très répandue en Chine: faire des cadeaux aux enseignants. C'est à la fois une habitude ancrée dans les usages pour marquer la considération de la famille envers le professeur, et, dans un contexte de concurrence accrue, une manière de soudoyer le professeur pour assurer la réussite de ses enfants. La modernisation des institutions tend à délégitimer ces pratiques, mais l'injonction de réussir par le moyen de l'éducation et l'étendue de la corruption représentent un dilemme pour cette jeune maman.

"Ce forum est fait pour les mamans, on y dit ce qu'on a sur le cœur. Et puis on pose aussi des questions sur notre situation, par exemple au sujet de l'éducation des tout petits, faut-il donner des cadeaux aux instituteurs du jardin d'enfants, et est-ce qu'ils ont le droit de les recevoir. Il y a des gens qui trouvent que c'est inconvenant, d'autres qui pensent que c'est interdit, que les instituteurs n'ont le droit de rien recevoir. Ca me permet de lire les avis l'un après l'autre. Parce que sur des questions comme ça, chacun a forcément un avis différent, et puis ça n'implique l'intérêt de personne en particulier. Ce genre de choses générales n'a certainement pas d'importance." (Shao)

Les difficultés de la vie quotidienne sont donc l'occasion de prendre la parole, essentiellement pour poser des questions, mais cela consiste au passage à souligner certains 
travers de la société, qu'il faut bien décrire lorsqu'on y est confronté. De ce fait, la plupart des internautes s'autorisent à aborder de nombreux sujets.

"Ce ne sont pas des grandes questions, on peut dire que ce sont des questions de société, des informations sur des questions de société. Il y en a beaucoup en ce moment sur Internet. Ca comprend des faits divers, bien sûr on ne parle pas des sujets trop politiques, comme le sommet Chine-Afrique. Par contre on donne notre opinion sur le prix des logements, ces derniers temps ils ont beaucoup augmenté. Ce sont des sujets en rapport avec notre vie, et puis on en discute aussi avec nos collègues, mais ce sont toujours des sujets très liés à notre vie." (Lei)

Les questions de logement dont parle cette internaute sont en réalité très politiques, mais elles sont cadrées comme un élément du mode de vie des individus, rentrant ainsi dans le domaine de ce qui peut se discuter. Tous les internautes prennent la peine de souligner qu'ils ne prennent la parole que sur des sujets qui les concernent de près ou de loin, comme une garantie contre le fait d'empiéter sur des questions politiques. Tous les sujets ne se prêtent donc pas à ce type d'interventions, aussi anodines soient-elles, et les internautes font souvent attention à choisir des sujets consensuels. Il y a déjà, dans cette attitude, une dimension stratégique, qui consiste à évaluer assez précisément la nature de la question et ce qu'elle comporte de risques.

"Oui, mais par exemple si un officiel est corrompu, s'il n'a pas détourné mon argent, ce n'est pas mon affaire. Quand ça ne touche pas mon intérêt, je ne vais pas trop faire de commentaires, parce que j'ai peur que ça me porte tort. Mais par contre si l'argent détourné est à moi, ou bien si on utilise un procédé pour s'approprier mon argent, alors bien sûr je serai très énervé. Je ne peux pas aller faire un sit-in ou une manifestation devant la porte du gouvernement municipal. Si je fais ça, personne ne le verra, mais sur Internet c'est différent, tout le monde est sur Internet, tout le monde pourra voir ce qui se passe, alors que si tu vas devant la porte du gouvernement, bien sûr les gens qui s'y trouvent le verront, ce sont les gens importants qui le verront, alors qu'Internet crée une sorte de force de l'opinion. C'est plus persuasif que quand il n'y a qu'une seule personne. Mais il faut que le problème soit lié à tes intérêts, pour que tu prennes la peine de te battre. Si ça n'a pas de rapport avec tes intérêts, ça ne vaut pas le coup de dire quoi que ce soit. Si quelque chose n'a pas beaucoup de rapport avec moi, je ne peux que faire deux lignes de commentaire, mais je ne peux pas dire beaucoup 
de choses en m'énervant. Non, mais par contre si ça touchait mon intérêt, c'est certainement ce que je ferais, parce que je voudrais d'abord faire connaitre aux autres ma façon de penser." (Huang)

Dans cet extrait, l'internaute exprime clairement le sentiment d'être une petite voix parmi d'autres, et qu'ensemble ces voix forment un tribunal public dont le pouvoir est effectif. Elle semble ainsi croire un peu plus que d'autres en la valeur de la parole publique, qui est, certes, très faible à l'échelle individuelle, mais qui devient puissante avec le nombre. Par contre, pour elle, l'initiative de porter un nouveau sujet sur la place publique ne peut venir que d'une personne véritablement lésée personnellement, et de manière grave, car elle comporte encore des risques.

L'émotion ou la compassion d'une part, ou bien le fait d'être soi-même concerné par un problème, constituent deux ressorts possibles d'une implication plus active dans les discussions qui ont lieu en ligne. Ces formes variables d'implication personnelle, ou ce que Boltanski appelle le "concernement"11, permettent aux internautes de se sentir en droit de participer à des causes, voire de les proposer au regard des autres, amis ou inconnus. La plupart du temps, cette participation se limite à une réaction de quelques mots ou de quelques lignes à la suite d'une information, ou d'un message d'encouragement sur le blog d'un internaute en difficulté. Ces internautes donnent ainsi un écho important aux causes dans lesquelles ils se reconnaissent, celles qui se rapprochent de leur mode de vie ou de leur idée de ce mode de vie. Ils sont de plus en plus conscients du poids que représentent ces petites contributions, insignifiantes individuellement, mais très importantes lorsqu'elles sont agrégées. Des affaires comme le cas Sun Zhigang ne prendraient pas une telle importance sans le poids de ces internautes faiblement engagés, qui papillonnent d'une cause à l'autre, mais qui sont capables de s'indigner occasionnellement lorsque les valeurs auxquelles ils souscrivent sont bafouées.

Le faible engagement de ces internautes dans les différentes causes qui les font réagir, ainsi que la manière dont ils minimisent les enjeux pour les rapprocher de leur vie quotidienne, en occultant les dimensions politiques qu'elles peuvent contenir, montre les effets des opérations de cadrage sur la sélection des sujets sur la place publique. En effet, sans

\footnotetext{
${ }^{11}$ BOLTANSKI Luc. La souffrance à distance, Paris: Métailié, 1993.
} 
les multiples petits relais que représentent ces internautes, une cause, une histoire, un fait divers ont peu de chance de trouver une audience importante. Il y a fort à parier que même disponible en ligne, une information fortement contestataire tombera rapidement dans l'oubli, même si elle recueille l'assentiment de certains lecteurs. Faute de percevoir cette information comme consensuelle, et ne voulant pas polémiquer, de nombreuses personnes peuvent renoncer à donner leur avis.

Enfin ces diverses réactions individuelles ne constituent pas en elles-mêmes des transgressions $^{12}$, au sens où elles ne sortent pas des registres autorisés pour prendre la parole. Il ne s'agit, après tout, que de réagir à des sujets qui ont été mis sur la place publique par d'autres, tout en tâchant de ne pas trop s'éloigner de ses propres préoccupations. Or, c'est justement quand les intérêts personnels sont touchés que les internautes commencent à envisager Internet comme une ressource pour rendre publiques de nouvelles causes.

\section{3 - Le rôle charnière des porteurs de causes}

Les divers types de réactions décrits ici ne sont jamais purs ni définitifs. Il est tout à fait possible pour un internaute de passer d'une position à une autre en fonction des sujets, et même au cours d'un même entretien, en fonction des exemples que les internautes ont en tête quand ils parlent de leur comportement habituel. Beaucoup décrivent des expériences qui les ont conduits à modifier leurs habitudes. En particulier, deux internautes ont mentionné des épisodes au cours desquels ils étaient passés d'une position de membre du public, un peu en retrait par rapport à l'actualité, à une position d'investissement plus actif, postant des articles plus approfondis pour défendre leurs idées.

Dans un cas, cette démarche a été un échec total et a conduit à un mutisme durable de l'internaute. Dans l'autre cas, le sujet a rencontré une bonne réception de la part d'autres internautes. Deux types de problèmes différents, deux manières différentes de signaler ces problèmes, de les analyser et de les rendre publics conduisent à deux résultats opposés. Ces deux exemples montrent à quel point le cadrage d'un sujet est important, et ils soulignent ainsi

${ }^{12}$ CEFAI Daniel. Pourquoi se mobilise-t-on?, Paris: La Découverte, 2007, p.490. "Sur chacune de ces scènes publiques, certains genres dramatiques, rhétoriques et narratifs sont pertinents et d'autres pas l'innovation doit être mesurée dans sa transgression des genres autorisés sous peine d'être disqualifiée." 
le rôle central des personnes qui les présentent au public en tout premier lieu, ainsi que l'importance de leur positionnement, de leur stratégie de mise en récit et, a fortiori, de leurs ressources discursives.

\section{Les échecs de la critique frontale}

Vivant seul avec sa mère enseignante, Tian a abandonné ses études supérieures au bout de deux ans, sans obtenir de diplôme. Il est employé dans une entreprise étrangère et gagne un salaire moyen pour Pékin (environ 3500 RMB par mois). Il ne se considère pas comme faisant partie d'une classe privilégiée, mais bien au contraire comme quelqu'un d'ordinaire, ayant fait une école "moyenne", sans bénéficier de passe-droit, faisant les choses simplement, vivant une expérience de "la vraie vie". En même temps, il se voit comme un intellectuel, quelqu'un qui sait voir la réalité des choses de manière critique. Il déclare avoir une "pensée occidentale" et ne pas être un Chinois très traditionnel. Pourtant ses nombreuses références à des personnages historiques et ses citations de dictons, qui ponctuent tout l'entretien, tendent à le contredire au moins sur la question de la connaissance de la culture chinoise.

Il aurait été intéressant d'en savoir plus sur la manière dont Tian a forgé ses convictions sur la Chine et le monde. Nul doute que son histoire personnelle et familiale a contribué à le rendre plus critique que la moyenne, et qu'elle influence la manière dont il choisit de s'exprimer en ligne ${ }^{13}$. Malheureusement la durée de l'entretien n'a pas permis d'en savoir beaucoup plus. En revanche il a livré un témoignage plus qu'intéressant sur sa manière d'envisager la prise de parole sur Internet.

Cet internaute a été témoin de nombreuses injustices qui l'ont marqué et qui nourrissent une certaine révolte face au fonctionnement de la société chinoise. Il a notamment beaucoup à dire sur le système éducatif chinois qui, selon lui, rend les enfants passifs jusqu'à un âge avancé ("23 ans"), alors que les enfants d'autres pays sont amenés à faire des choix de vie autonomes dès quinze ans ${ }^{14}$. De plus les conditions d'éducation des élèves sont très inégales en fonction des établissements. Cette discrimination est d'autant plus grave à ses yeux que

${ }^{13}$ JASPER James. The art of moral protest. Culture, biography and creativity in social movements, Chicago: University of Chicago Press, 1997.

${ }^{14} \mathrm{Il}$ idéalise certainement les systèmes éducatifs occidentaux de manière exagérée. 
l'éducation détermine le rang social, la "qualité" d'une personne. De plus l'école, au lieu d'inculquer aux enfants des valeurs critiques, ne fait que les habituer à un système clientéliste dès le plus jeune âge. Il en veut pour preuve son instituteur qui, quand il avait dix ans, a réclamé à tous les parents d'élèves de lui apporter un chou à l'approche de l'hiver. Ce fut l'occasion d'une surenchère pour les parents qui voyaient là une possibilité d'assurer de meilleures notes à leurs enfants. Il raconte également des cas de chantage par la police auprès de restaurants, ou le fait que "l'argent achète tout", comme par exemple des papiers administratifs difficiles à obtenir.

Son discours, qui se veut critique, reflète en réalité une certaine adoption du discours dominant sur la qualité des individus, qui se développe par l'éducation, et par le mérite personnel. Il prend au pied de la lettre la question de la lutte contre la corruption, et il observe autour de lui le fait que cette lutte ne porte pas de fruits. Au regard de cette expérience très amère, Internet fonctionne comme une sorte de revanche ou de seconde chance. C'est un moyen de s'éduquer autrement que par l'école, de manière plus autonome et plus critique. Surtout, il envisage Internet comme une occasion d'entraîner les autres dans sa démarche. Il compte pour cela, dans un premier temps, écrire de nombreux articles sur les maux de la société chinoise et les poster en ligne.

Pour lui, la censure en tant que telle n'est pas un problème. J'ai cité plus tôt un paragraphe où il explique comment la contourner très facilement. Le frein vient véritablement des autres internautes. En effet, il ne reçoit pas l'accueil escompté, et, après avoir été refoulé par d'autres internautes, il montre des signes de repli sur soi, sur le même modèle que les autres internautes désabusés.

"Je n'envoie plus ce genre de choses, parce que ça pourrait s'opposer à des gens, ça pourrait s'opposer à des internautes, de leur donner des conseils, donc tout ce qui est à propos du gouvernement, maintenant je n'en parle plus. Ca ne sert à rien, tu ne peux rien y changer. C'est comme un bateau qui prendrait l'eau de toutes parts, si tu comptes sur une seule personne munie d'une bassine pour vider l'eau, c'est impossible. Je fais cette comparaison pour dire que s'ils veulent réparer immédiatement le bateau, il y a encore de l'espoir. Mais s'ils ne veulent pas réparer, le bateau finira par couler tôt ou tard. En Chine il y a une histoire très ancienne qui parle d'un empereur célèbre, Tang Taizong, qui a dit la phrase suivante: 'l'empereur est un bateau, le peuple est 
l'eau'. L'eau peut renverser le bateau, elle peut le retourner et le faire couler. Le gouvernement chinois aujourd'hui ressemble à un bateau, un bateau qui prend l'eau de toutes parts, et si on peut le réparer, si on répare les trous, et qu'on ne fait pas sortir l'eau, il y a encore de l'espoir, mais ça ne repose pas sur une seule personne. En fait beaucoup de gens ne voient pas cette crise, y compris au gouvernement, beaucoup de gens ne le voient pas, ils ne veulent pas se transformer, et ils ne veulent pas voir plus de choses vraies. Je connaissais des amis qui travaillaient dans un ministère, je leur en parlais, je leur disais il y a des choses qui se passent, voulez-vous regarder, voulezvous écouter un peu? Et qu'est-ce qu'ils me disaient? 'Ca ne sert à rien, même si je savais ces choses-là, mon salaire ne serait pas plus élevé, je n'aurais pas un meilleur poste. Au contraire, si je savais ces choses-là, je me ferais peut-être virer'. Tout le monde pense comme ça. [Il explique longuement que les postes à responsabilité sont surtout des moyens d'enrichissement personnel et d'abus divers.] En somme l'argent est prioritaire en Chine. En Chine on dit toujours qu'en Occident, les pays comme les Etats-Unis et l'Angleterre ont le culte de l'argent, mais en fait maintenant c'est la Chine qui rend le plus grand culte à l'argent, et moi aussi. [... Quand j'en parlais dans mon blog] les gens disaient que ce n'était pas un scoop, que tout le monde le savait déjà, et qu'on ne peut pas empêcher ces choses-là."

Cet internaute ressent une colère évidente devant les injustices que représentent la corruption et l'égoïsme généralisés. Il exprime une certaine volonté de se mobiliser, mais de manière assez classique, il se heurte à une résistance très forte de ses propres concitoyens, ce qui le réduit au silence. Découragé, il finit par adopter une attitude et un vocabulaire fatalistes, de manière presque mimétique. Son cas rend très évidente la nécessité du sentiment d'être à l'unisson avec les autres internautes pour prendre la parole et pour qu'un mouvement "prenne". A l'inverse, quand ce sentiment est absent, c'est le découragement qui prime. A quoi bon défendre une cause qui n'est pas partagée par ses concitoyens? La conséquence presque naturelle de ce processus est le passage à l'indifférence, comme une sorte de durcissement face à l'adversité.

[A propos d'un passe-droit flagrant accordé à un personnage important et dont il a été témoin sur la voie publique.] "J'en ai parlé sur un forum. Les commentaires en dessous ce n'était que des oh! et des ah! Ces choses-là, personne ne trouvait ça anormal, ou bizarre, ou très inhabituel. Ils trouvent que c'est nécessaire, en Chine c'est 
comme ça, c'est le prestige des officiels. Ils sont déjà devenus indifférents. Je ne sais pas si tu as déjà lu un article de Lu Xun, sur l'invasion de la Chine par le Japon. Pendant qu'un groupe de japonais massacrait un Chinois, les autres Chinois tout autour riaient. Tout le monde est déjà devenu indifférent à ces choses-là, ils n'émettent plus de jugements. Avant je n'étais pas indifférent, mais maintenant je le suis aussi, je ne fais plus de commentaires. Je m'en tiens à essayer de m'améliorer moi-même, de me changer moi-même, et je pense que je ne peux pas changer les autres, je ne peux que me changer moi-même. J'espère qu'en vivant dans cet environnement, je trouverai des endroits où manifester mes talents. Et puis en comprenant ces choses-là, je sais aussi comment je dois me comporter à l'avenir, comment faire pour me coordonner avec le gouvernement."

Tian ne peut que renoncer et se replier sur la sphère de l'intime qui, elle, ne lui est pas refusée. Là, il a une prise pour se lancer dans un projet positif (très confucéen) de "développement personnel" et, surtout, de telle manière que ce développement soit "coordonné" avec l'action du gouvernement. La stratégie d'évitement devient presque une forme de camouflage, d'accompagnement de la tonalité principale.

"Dans le passé, le présent et l'avenir de la Chine, c'est une règle de développement, je dois faire les choses selon cette règle de développement si je veux m'améliorer, améliorer mes connaissances. Bien sûr je sais que ces choses-là ne sont pas normales, et il y a des choses que je trouve vraiment honteuses, mais je suis en Chine, je dois apprendre, je dois faire comme ça. Les gens disent que ce n'est pas correct de faire comme ça, mais on n'y peut rien."

Pour lui, un homme seul ne peut rien devant le poids du nombre et surtout face à une tradition millénaire. C'est finalement devant cette "culture" chinoise millénaire que Tian s'incline, comme pour se faire une raison devant une fatalité implacable et qui ne relève plus de la décision individuelle. Dès lors, cet internaute ne recherche plus le savoir que pour luimême et renonce à toute ambition prosélyte, sauf pour qui souhaiterait explicitement connaître son avis. Tout l'entretien respire ainsi le dépit, la colère parfois, lorsqu'il critique les organes de presse officiels, mais également un certain espoir dans les vertus de l'éducation pour les générations futures. 
Comment interpréter alors les dernières phrases de l'entretien où il juge nécessaire de rappeler son patriotisme très fort ? Est-ce une volonté d'affirmer sa participation au jeu de la cohésion sociale? Il prend même la peine de louer les qualités des deux personnages principaux du PCC, Mao et Deng. Cependant les termes qu'il emploie sont surtout élogieux pour Deng, et en particulier pour la politique d'ouverture que ce dernier a engagée. De même, le patriotisme, vécu comme une volonté d'améliorer son pays, motive sa volonté de changement, là où le même patriotisme invite ses contemporains à opter pour le conservatisme politique.

" La Chine a commencé les réformes dans les années 1980, c'est là qu'elle a commencé à accepter une pensée correcte, donc parmi les gens du Parti, il n'y en a que deux que j'admire, l'un est Mao Zedong, l'autre est Deng Xiaoping. Mao Zedong était un grand homme, c'était un paysan, et Deng Xiaoping était un réformateur. S'il n'y avait pas eu Deng Xiaoping il n'y aurait pas la Chine d'aujourd'hui. Donc ce que Deng Xiaoping a apporté d'après moi, c'est de faire entrer les gens qui sont nés dans les années 1980 dans un endroit correct.[...] Bien que j'aie dit beaucoup de choses négatives sur la Chine, je suis chinois, j'aime beaucoup mon pays, j'aime l'histoire tellement longue de mon pays, sa culture brillante, et j'espère que ce pays va continuer sur la même voie, pour devenir une grande puissance mondiale. Bien sûr il y a des choses que je ne peux pas faire, que je n'ai pas la capacité de faire, et j'espère que mes descendants pourront obtenir un véritable savoir par n'importe quel canal, par n'importe quelle voie, qu'ils pourront augmenter leurs capacités, qu'ils pourront chercher une situation stable dans cette société, comment dire, disons pour se construire (zuo hao ziji)."

Dans ce mot qui fut la conclusion de l'entretien, se dessine toute la complexité du rapport de cet individu à la nation chinoise. Il lui est profondément attaché et il partage un enthousiasme très fort pour l'idée de la grandeur retrouvée du pays sur la scène internationale. Il nourrit l'espoir que l'accès à une connaissance plus libre soit possible pour les générations futures, mais cela ne peut passer que par un travail sur soi-même et non par la prise de parole publique. Paradoxalement, sa critique du régime s'accompagne d'une attitude très confucéenne qui consiste à prendre sur lui. 
Cet entretien illustre donc la manière dont un discours formulé de manière relativement contestataire peut être découragé, même sans être formellement interdit, simplement à cause de l'absence d'écho positif de la part des autres internautes. Le fait que la critique porte sur le régime lui-même est ici rédhibitoire, et Tian ne peut pas trouver l'assentiment de ses concitoyens qui pensent qu'un changement de régime est impossible. En revanche, d'autres personnes réussissent à faire passer des messages, y compris relativement politiques, lorsqu'ils savent les formuler dans des termes plus acceptables par le grand public et surtout lorsqu'ils utilisent les diverses plates-formes du Web avec perspicacité. Bien sûr, cela ne peut concerner que des questions plus ponctuelles ou anecdotiques.

\section{Des tactiques subtiles d'expression publique}

C'est le cas d'un internaute qui défend la cause des chiens, en ligne et hors ligne. Zhang, propriétaire d'un labrador, est personnellement concerné par la législation sur les chiens dans le centre ville de Pékin. J'ai déjà montré comment la cause des chiens avait été "cadrée", par lui comme par d'autres, de manière à utiliser de nombreux éléments du discours officiel dans un sens plus favorable, mais il faut aller plus loin et relire son explication plus en détails.

"D'abord il ne faut pas faire de concurrence à la politique du gouvernement, non. Ce qu'il faut faire, c'est dire aux gens ordinaires autour de nous, avec une attitude pondérée [, que] la question du contrôle ne repose pas sur les chiens, cela devrait reposer sur les gens, et pourquoi nous pensons que ce qu'on dit sur les chiens par rapport à la pollution, en fait c'est un problème de gestion de la ville, pas un problème de chiens. Quand j'ai dit ça, j'ai dit en gros, que nous pouvons en discuter avec nos amis, nos voisins, et que nous pouvons leur dire que les gens qui élèvent des chiens ont des règles, et qu'on toucherait pas à l'intérêt public."

Le premier réflexe de Zhang est de ramener la question des chiens dans la sphère domestique: élever des chiens est une question individuelle qui concerne les propriétaires de chiens et ne doit pas avoir d'influence sur les autres. D'ailleurs, cette question "politique" ne l'intéresse lui-même que dans la mesure où elle le touche personnellement.

"Je ne m'intéresse vraiment pas à la politique, mais les infos j'y fais attention, parce que ce qui se passe autour de moi, ça a un rapport avec moi, donc j'y fais quand même attention." 
Ce positionnement lui permet de présenter sa cause comme apolitique, ce qui l'autorise à développer ses arguments en public. Cependant il ne peut pas échapper à la question de réclamer une législation plus souple puisque le problème se situe bel et bien dans la gestion de l'espace urbain. On voit donc combien cette situation engendre un dilemme ${ }^{15}$, c'est à dire une tension entre deux principes contradictoires qui s'entrechoquent à cause de l'ambivalence de la situation: il s'agit à la fois d'aborder publiquement un problème individuel, et en même temps de circonscrire les questions politiques à la sphère privée. Les deux positions ne sont pas tenables en même temps, dès lors que le problème individuel prend ses racines dans une décision politique. Zhang trouve finalement une issue en cadrant son discours d'une manière audible sur la scène publique. Cela résulte d'une compréhension très claire de la stratégie de communication du gouvernement.

"Sinon il y a aussi des sujets que les médias ont tendance à éviter, comme la question des chiens en ce moment. Maintenant le gouvernement, comment dire, vu que la Chine est un pays avec une grande population, c'est une situation très spéciale, dans ces circonstances on est obligés de contrôler l'opinion. Donc en Chine l'opinion est contrôlée, on n'a pas entièrement la liberté d'opinion. Ça, c'est la situation actuelle, donc les médias ont tendance à éviter certaines questions. Par exemple en ce moment le gouvernement dit qu'il faut attraper les chiens, et pendant ce mouvement les médias ne vont pas dire que les chiens c'est bien, que ça a beaucoup d'avantages, et montrer comment on peut très bien coexister en harmonie avec les chiens. Ce ne sera peut-être pas ce genre d'informations. A ce moment-là il n'y aura pas de reportages complets pour dire 'aujourd'hui on a encore attrapé tant de chiens'. Comme il faut contrôler l'hygiène pour les masses, on ne peut pas avoir ce genre de reportages. Dans ces moments-là il n'y a que dans certains forums un peu discrets ou sur certains espaces de discussion comme les groupes $\mathrm{QQ}$, c'est à dire sur des petits forums internes à nos petits groupes QQ, que l'information se répand."

Zhang a donc recours à des espaces ouverts au public, mais dont l'audience est plus limitée pour faire passer son message, ce qui lui permet tout de même de le faire circuler largement. Une remarque d'une autre internaute vient faire écho à ce mode de circulation de l'information.

${ }^{15}$ La notion de dilemme est développée notamment à partir d'exemples de la vie quotidienne, dans FERRIE Jean-Noël. "Lieux intérieurs et culture publique au Maroc". 
"En fait je trouve que des choses comme Sohu, Sina et les quotidiens comme Beijing Evening News, Beijing Youth Daily, ils reflètent tous l'opinion du gouvernement.

- Pourquoi as-tu cette impression?

- Avant cette impression n'était pas si forte, mais avec les événements sur les chiens pourchassés, dans les journaux et sur Sina ils font de la propagande qui dit que les chiens errants sont mauvais pour les gens. Mais j'ai lu sur de petits forums, et aussi sur les blogs de certaines personnes, qu'on s'attaque directement aux chiens, et il y a beaucoup de petits chiens errants, mais ils sont battus à mort parce qu'ils n'ont pas de hukou. Et ils disaient que les chiens élevés chez des gens ne pouvaient pas attraper la rage. Mais il y avait message sur Sina, et il a été effacé, les gens ne peuvent plus le voir.

- Il a été effacé après un long moment?

- En à peu près cinq minutes. Il a été effacé, et puis la personne a renvoyé l'article sur un petit forum, et tout le monde lui a répondu, et il a été transmis sur d'autres BBS, et sur des boîtes mail." (Guo)

Dans son cas, la censure rend presque plus visible l'information, car la disparition subite d'un article attire l'attention. Ce n'est pas un cas isolé. En effet, suite à ses prises de position sur Internet, Zhang a reçu des réponses de la part d'autres internautes. Ceux-ci ont adopté un mode de communication plus prudent, en privé, ce qui leur permet de faire des propositions beaucoup plus fortes politiquement.

"Alors à ce moment-là j'ai reçu des messages de beaucoup de gens, ils demandaient en privé, ils discutaient en privé, pas très ouvertement, pour dire 'est-ce qu'on ne devrait pas entreprendre quelque chose comme ça, par exemple à petite échelle on pourrait imprimer nos propres matériaux de propagande, ce ne serait opposé à personne bien sûr, nous imprimons des choses pour encourager les gens à élever leurs chiens de manière civilisée, nous proposons une législation pour protéger les animaux, est-ce qu'on devrait faire ça...' C'était notre discussion, parce qu'ils pensaient peut-être que ce que je disais était relativement sensé, assez précis, que cela correspondait à leur opinion. Et puis il y a des gens qui m'ont demandé si je ne voulais pas qu'on organise un, c'est à dire un mouvement, par exemple on organiserait une pétition, et on la donnerait au département concerné. Est-ce qu'on ne devrait pas 
rassembler des textes et documents d'autres pays, d'organisations internationales ou étrangères de protection des animaux. On pourrait leur demander des conseils ou bien de l'aide. Et puis on a eu ces discussions en privé. Ils pensaient que, ils pensaient que peut être ce n'est pas convenable de parler de ce genre de choses dans des situations publiques. Ils avaient peut être ce genre de scrupules, donc ils m'ont contacté en privé, et aussi certains d'entre eux sont devenus mes amis."

Les modes de cadrage sont les mêmes: réaffirmer que nul ne s'oppose au gouvernement en tant que tel, rassembler une argumentation sérieuse, et plaider pour un traitement "civilisé" des chiens. Les espaces choisis pour entrer en contact peuvent cependant varier en fonction de la perception que les internautes ont de la limite à ne pas franchir publiquement. En effet il revient à chacun de choisir de manière stratégique sa façon d'utiliser les différentes platesformes d'expression, en fonction de leur degré de publicité et du type de propos tenus.

"Notre génération, on est moins touchés par les sujets sensibles. On est moins influencés par la Révolution culturelle. Prends l'exemple de l'ambassade de Chine qui a été attaquée par les Etats-Unis, il y a eu beaucoup de réactions de toutes sortes en ligne. Ce n'est pas qu'on n'a aucune liberté, mais cette liberté n'est pas complète. Par exemple si tu parles des dirigeants, là c'est sensible. Mais il y a des sujets qu'on peut aborder.

- Comment sait-on quels sont ces sujets?

- D'abord on voit si l'info est traitée clairement dans les médias. On doit adapter la manière de parler de la question. Je réfléchis bien avant d'envoyer un post en général. Je me demande par exemple: faut-il absolument poster cet avis, et comment est-ce que je dois argumenter. Je peux choisir aussi de le faire sur un forum ou sur ICQ. Il y a plusieurs manières de contrôler Internet. Tous les forums marchent à peu près pareil donc on peut choisir autre chose comme ICQ.

- Quelles sont les meilleures plates-formes pour ça?

- Le plus libre, c'est les blogs, parce qu'ils ne sont pas contrôlés."

Il y a beaucoup de choses importantes dans cette stratégie. L'internaute lui-même prend soin de s'informer pour connaître la position officielle sur une question, ce qui lui permet d'adapter son propos. Cela lui donne également des indications sur le degré de publicité qu'il peut s'autoriser. Avec les blogs cependant, il peut jouer sur une ambiguïté profonde: ils 
peuvent être considérés comme des espaces privés, puisque leurs auteurs ne s'expriment qu'en leur nom propre. En même temps, ils sont visibles de tout un chacun.

Dans sa démarche, la presse joue un rôle important, car c'est elle qui signale les sujets sensibles et les sujets plus ouverts en fonction du traitement qu'elle leur accorde. J'y reviendrai plus en détails. Zhang sait utiliser ces données pour adapter son discours. En même temps, il sait aussi repousser les limites et négocier ses marges de manœuvre avec ses premiers censeurs, les modérateurs des forums.

En effet, les personnes comme lui sont des moteurs pour les différentes discussions qui ont lieu sur Internet. En postant des sujets sensibles, ils attirent du trafic sur les plates-formes qui les accueillent. C'est donc sur eux que repose une grande partie du succès des forums et des blogs (et a fortiori de leurs revenus publicitaires). Zhang affirme qu'il en fait un moyen de pression pour repousser sans cesse les limites de l'expression en ligne.

"En plus, comme je passe beaucoup de temps sur Internet, et que je vais beaucoup sur des forums, je connais beaucoup d'amis en ligne. Donc très souvent les modérateurs sont obligés de réfléchir aux points de vue personnels et collectifs, pour eux c'est une sorte de pression, parce que nous poussons souvent, comment dire, par exemple à ce que les forums soient de bonne qualité. Les gens comme nous sont souvent, sont sûrement la cheville ouvrière des activités d'un forum, donc le plus souvent on prête attention à nos avis, et puis si ça n'implique pas des politiques, si ça n'implique pas de départements du gouvernement, si c'est seulement des activités du forum, on peut dire tout d'abord 'je n'ai rien fait de violent, je n'ai rien dit de violent'. Dans ces conditions notre parole n'est en principe pas limitée, personne ne nous limite de manière inflexible, en principe non."

De toute évidence, Zhang sait utiliser efficacement tous les relais qui sont à sa disposition pour faire passer ses messages, et cela inclut l'entretien auquel il a bien voulu participer avec moi. La conversation a en effet duré plus de trois heures trente, et Zhang s'est montré très heureux d'avoir l'occasion de partager son témoignage avec une chercheuse française. Il a conclu en disant qu'il n'aurait jamais pu parler de la même façon à un sociologue chinois.

Ces deux exemples montrent à quel point il est important pour un internaute qui souhaite porter un sujet sur la place publique de bien maîtriser les codes d'utilisation 
d'Internet. Il est indispensable d'avoir une bonne connaissance du discours officiel pour connaître les lignes rouges, et lorsque l'occasion s'y prête, de profiter de certaines imprécisions pour repousser les limites. Les internautes efficaces dans leur démarche savent également jouer sur les différents registres de discours ainsi que sur les différents degrés de publicité disponibles sur Internet, tout en sachant qu'eux aussi peuvent prêter à confusion, ce qui laisse, là encore, une marge de manœuvre.

\section{Les porteurs de causes}

Il est parfois difficile de connaître la généalogie précise de telle ou telle histoire qui émerge sur Internet, mais la plupart des affaires médiatisées ont eu au moins un promoteur identifié, qu'on peut appeler un "tireur d'alarme" ${ }^{16}$ ou encore un porteur de cause. Ce sont des personnes qui ont cru à un moment donné à la force de la publicité et qui ont décidé de s'en servir pour arriver à leurs fins, le plus souvent pour faire pression sur des autorités. Ils ont su formuler leurs causes dans des termes adéquats, à la fois pour franchir les barrières de la censure, mais aussi pour trouver une meilleure crédibilité et ainsi trouver du soutien auprès d'un public plus large.

Il arrive que ces personnes soient concernées elles-mêmes par un problème, comme c'était le cas de Lian Yue, leader de la contestation contre le projet d'implantation d'une usine polluante près de Xiamen ${ }^{17}$. Ce peuvent être des proches de victimes, comme les pères d'enfants disparus qui, grâce à une lettre ouverte postée sur Tianya, ont conduit à l'affaire des briqueteries du Shanxi ${ }^{18}$. Ce peuvent aussi être des témoins directs d'un scandale, ou des

${ }^{16}$ BERNSTEIN Mary et JASPER James. "Les tireurs d'alarme dans les conflits sur les risques technologiques. Entre intérêts particuliers et crédibilité", Politix, vol. 11, n 44, 1998, p. 109-134.

${ }^{17}$ MO Ming. "The biggest chemical project puts Xiamen in danger", China Digital Times, 06/04/2007, consulté le 25/10/2010 sur http://chinadigitaltimes.net/2007/04/the-biggest-chemical-project-putsxiamen-in-danger/.

${ }^{18}$ ZHAO Kate. "Who can save our children? - Fathers of 400 enslaved children", China Digital Times, 14/06/2007, consulté le 25/10/2010 sur http://chinadigitaltimes.net/2007/06/who-can-save-ourchildren-fathers-of-400-enslaved-children/. 
personnes impliquées dans une organisation, comme c'était le cas des médecins qui ont donné l'alerte sur l'épidémie de SRAS en 2003, désobéissant à leur hiérarchie ${ }^{19}$.

Ces porteurs de causes sont donc souvent concernés, de près ou de loin, par les revendications qu'ils défendent. Cependant il n'est pas rare qu'ils changent de posture avec le temps, soit pour se donner plus de crédibilité, soit que leur but est atteint mais qu'ils décident de poursuivre leur action au travers de nouvelles causes. C'est par exemple le cas du blogueur "Zola" (ou Zuola), de son vrai nom Zhou Shuguang. Celui-ci s'est d'abord rendu sur le site déjà célèbre de la "Maison-Clou" de Chongqing. Ayant remarqué que le cas était très peu traité dans la presse, il a décidé de s'y rendre et de faire un reportage sur son blog, pour se faire connaître, dans l'espoir de quitter sa condition de petit commerçant. Il est ainsi devenu aux yeux de tous le "premier journaliste-citoyen" chinois ${ }^{20}$.

Pour Zhou, ce fut un moment de révélation. Il a décidé que, en tant que citoyen chinois célibataire et sans emploi, qui 'croit fermement à la liberté individuelle', il était parfaitement indiqué pour se consacrer à 'aider les personnes exclues et déplacées'. Il a aussi déclaré joyeusement que son but était de faire du bruit pour devenir célèbre afin de 'ne jamais rentrer vendre des légumes. ${ }^{21}$

Fort de cette expérience, Zola, désormais célèbre, a décidé de poursuivre dans cette direction. Se faisant financer par des dons individuels, il a décidé d'enquêter sur d'autres cas. Zola est ainsi devenu un "chercheur de causes"22 pendant quelque temps, jusqu'à ce qu'il soit frappé par un policier alors qu'il enquêtait sur une affaire d'arnaque dans le Liaoning. Renvoyé chez lui après plus de vingt-quatre heures d'interrogatoire, il a écrit sur son blog que sa mission était terminée, et que le travail de défense de la vérité qu'il avait entrepris était désormais entre les mains de chaque citoyen. Il a pourtant continué son activité de blogueur. Empêché d'aller à Pékin pendant les Jeux Olympiques de 2008, il s'y rendra plus tard pour

${ }^{19}$ SAUTEDE Eric. "Les leurres de la modernité, Internet, information et crise du "SRAS" en Chine", Perspectives chinoises, mars-avril 2003, $\mathrm{n}^{\circ} 76$, p. 21-27.

${ }^{20}$ KENNEDY John. "China: Nation's first citizen reporter?", Global Voices Online, 30/03/2007, consulté le 25/10/2010 sur http://globalvoicesonline.org/2007/03/30/china-nations-first-citizenreporter/.

${ }^{21}$ ELEGANT Simon. "Spinning a Web", Time, 24/01/2008.

${ }^{22}$ SIMEANT Johanna. La cause des sans papiers, Paris: Presses de Sciences-po, 1998. 
mener, avec d'autres blogueurs, une enquête très sensible sur les "prisons secrètes" qui auraient remplacé les centres de détention et de rapatriement supprimés après l'affaire Sun Zhigang $^{23}$.

Zola n'est qu'un exemple de ces blogueurs qui décident de poursuivre des causes dépassant leur cas personnel. Il aurait été intéressant de connaître les motivations particulières, ainsi que les parcours biographiques d'un nombre conséquent de ces internautes pour mieux connaître ce qui détermine leur passage à un engagement plus soutenu. Cependant mon objet ici est de montrer comment le positionnement de ces individus, ainsi que leurs stratégies d'expression influencent le déroulement des causes qu'ils poursuivent.

On voit ici que certains blogueurs finissent par prendre des postures très proches de celles de journalistes, même s'ils n'en ont pas l'accréditation officielle, et c'est là qu'ils encourent les risques les plus sévères. D'abord, ils peuvent subir des représailles de la part des malfaiteurs qu'ils dénoncent, comme, suppose-t-on, ce fut le cas de Xu Lai, dit "la Prostate enflammée", poignardé par des inconnus lors d'une conférence ${ }^{24}$. Pour la première fois, l'un de ces citoyens-journalistes, Wei Wenhua, est mort au cours de son enquête sur des émeutes dans le Hubei début $2008^{25}$. Sa mort a été perçue comme un scandale et largement dénoncée en ligne.

Cependant le risque principal encouru par ces "chercheurs de causes" reste l'arrestation politique. En témoigne l'arrestation de $\mathrm{Hu} \mathrm{Jia}^{26}$ et sa condamnation à trois ans et demi de

${ }^{23}$ ZHANG Meng. "China: co-operation 2.0 on Beijing's black jails", Global Voices Online, 17/10/2008, consulté le 25/10/2010 sur http://globalvoicesonline.org/2008/10/17/china-co-operation20-on-beijing\%E2\%80\%99s-black-jails/.

${ }^{24}$ PEDROLETTI Brice. "La Prostate enflammée victime d'une attaque au couteau", Quand la Chine déblogue, 16/02/2009, consulté le 25/10/2010 sur http://chinedesblogs.blog.lemonde.fr/2009/02/16/laprostate-enflammee-victime-d\%E2\%80\%99une-attaque-au-couteau/.

${ }^{25}$ OLIVER Laura. "Death of Chinese 'citizen journalist' sparks online outrage", Journalism.co.uk, 15/01/2008, consulté le 25/10/2010 sur http://blogs.journalism.co.uk/editors/2008/01/15/death-ofchinese-citizen-journalist-sparks-online-outrage/.

${ }^{26}$ HASKI Pierre. "L'arrestation de Hu Jia, dissident chinois privé de webcam", Rue89, 29/12/2007, consulté le 25/10/2010 sur http://www.rue89.com/chinatown/larrestation-de-hu-jia-dissident-chinoisprive-de-webcam. 
prison $^{27}$. Hu Jia avait enquêté entre autres sur le scandale de la contamination de paysans par le Sida dans la province du Henan, et, transgression s'il en est, il avait fait la publicité de ces événements à l'étranger, accordant une audition sur la question des Droits de l'Homme en Chine au Parlement européen. Hu Jia, comme Zola, maintiennent pourtant une argumentation cadrée de manière stratégique, reprenant à tout instant les textes des lois chinoises qui garantissent la liberté d'expression et en proposant l'interprétation la plus littérale possible pour justifier du fait qu'ils ne font qu'agir dans l'intérêt du pays.

Le positionnement de ces internautes prend donc une importance capitale. Ils ne sont pas traités de la même manière en fonction de leur identité, de leur notoriété, et encore de la manière dont ils présentent leur discours. Ainsi Tan Zuoren, militant écologiste, a-t-il été arrêté alors qu'il tentait d'enquêter sur les défauts de construction des écoles qui se sont effondrées lors du tremblement de terre du Sichuan. Il a finalement été condamné à cinq ans d'emprisonnement le 9 février 2010 pour "incitation à a subversion du pouvoir de l'Etat" ${ }^{28}$. Le célèbre artiste Ai Weiwei ${ }^{29}$ a repris la même démarche. Il est le fils du célèbre poète Ai Qing, il a vécu à l'étranger, et son propre travail d'artiste est très reconnu à une échelle internationale. Cela lui donne un statut très particulier. Il s'est lancé dans un décompte des enfants morts dans ces écoles, car les chiffres n'étaient pas rendus publics par les autorités. Il a pu recenser 4383 noms d'écoliers et de lycéens morts au cours du séisme ${ }^{30}$. Il a pu aller beaucoup plus loin que Zola avant que son blog Sina ne soit fermé (il en a rouvert un autre depuis), et il se sert activement de sa célébrité pour résister aux "invitations à prendre le thé" que lui fait régulièrement la police ${ }^{31}$. Même si des limites certaines se manifestent dans les

${ }^{27}$ HASKI Pierre. "Malgré les JO, la Chine condamne le dissident Hu Jia à la prison", Rue89, consulté le 21/04/2009 sur http://www.rue89.com/chinatown/malgre-les-jo-la-chine-condamne-le-dissident-hujia-a-la-prison.

${ }^{28}$ Cette condamnation s'appuie également sur des écrits critiques à propos des événements de Tiananmen le 4 juin 1989. "Profile: Tan Zuoren", BBC News, 09/02/2010, consulté le 25/10/2010 sur http://news.bbc.co.uk/2/hi/8506763.stm.

${ }^{29}$ PEDROLETTI Brice. "Le journal d'enquête citoyen d'Ai Weiwei sur les écoles du Sichuan", Quand la Chine déblogue, 07/04/2009, consulté le 25/10/2010 sur http://chinedesblogs.blog.lemonde.fr/2009/04/07/le-journal-d\%E2\%80\%99enquete-citoyen$\mathrm{d} \% \mathrm{E} 2 \% 80 \% 99$ ai-weiwei-sur-les-ecoles-du-sichuan

${ }^{30}$ Voir son blog: http://www.bullogger.com/blogs/aiww/ (consulté le 25/10/2010).

${ }^{31}$ HARTONO Paulina. "Ai Weiwei's blog closed", China Digital Times, 28/05/2009, consulté le 25/10/2010 sur http://chinadigitaltimes.net/2009/05/ai-weiweis-blog-closed. 
deux cas, la différence de position entre Tan Zuoren et Ai Weiwei ne leur permet pas d'agir de la même façon, ni de s'exprimer dans les mêmes termes, alors qu'ils poursuivent le même objectif.

Les porteurs de causes jouent un rôle crucial dans le passage des causes à la postérité. Ce sont eux qui effectuent une sélection entre les sujets, les mettent en récit, et construisent une argumentation suffisamment bien cadrée pour qu'elle puisse être rendue publique. Compte tenu des contraintes très serrées qui s'imposent dans ce processus, des internautes relativement éduqués, ou bien disposant de soutiens conséquents, ont plus de ressources que les autres pour faire passer des causes qui les concernent ou qui les touchent sur la place publique. C'est d'autant plus vrai que les contraintes varient en fonction du statut de ces porteurs de causes, et qu'une prise de risque plus importante est accordée à des personnages déjà plus privilégiés ou plus publics. Il y a bien entendu des causes portées initialement par des individus ou des groupes moins dotés en ressources, mais elles se distinguent souvent par leur gravité (pour les cas d'esclavage par exemple) et leur succès est souvent dû à des relais efficaces qui disposent de meilleures ressources, comme des avocats, des intellectuels, des artistes ou, désormais, des "journalistes citoyens". Ce sont eux qui fournissent aux autres citoyens l'occasion d'acquiescer ou de s'indigner, de réagir d'une façon ou d'une autre.

En effet toutes ces causes, pour avoir un véritable effet, doivent trouver un écho auprès de portions plus larges de la population. Seules celles qui peuvent toucher, d'une manière ou d'une autre, une portion importante des internautes ont une chance d'aboutir à un mouvement de masse et, peut-être, à un règlement du problème favorable aux personnes concernées. Dans le bouillonnement des lectures individuelles d'informations et des conversations privées s'effectue en fait une sélection très sévère des causes qui deviendront publiques, à la faveur de celles portées par les populations les plus représentées parmi les internautes. Pour l'heure, les personnes urbaines, éduquées, en cours d'ascension sociale restent très sur-représentées. A l'effet déjà très réducteur de la démographie des internautes s'ajoute un effet d'autodisqualification chez les internautes les moins éduqués, ou ceux qui se sentent le moins à l'aise avec la prise de parole et l'argumentation. S'ils s'autorisent à montrer brièvement leur accord ou leur désaccord avec un point de vue, plus rares sont ceux qui oseront apporter de nouveaux sujets sur la place publique ou proposer un point de vue hétérodoxe. 
On voit donc que les mouvements de masse qui se forment sur Internet sont loin de représenter une opinion publique structurée par le débat, et encore plus loin de représenter l'opinion d'une majeure partie de la population chinoise. Ces mouvements se forment ad hoc, à l'occasion de scandales ou d'affaires, et ils sont suivis ou non en fonction des stratégies de mise en récit de certains acteurs, et de leur capacité à toucher les internautes les plus enclins à s'exprimer en ligne. L'opinion, ou si l'on veut les opinions, qui se forment en ligne, sont certes publiques, mais elles sont plus particulièrement représentatives des préoccupations des portions les plus prospères de la population chinoise, celles qui, de fait, sont souvent plus conformes au modèle de développement "moderne" du pays ${ }^{32}$.

C'est cette opinion publique, que j'appellerai donc "restreinte", qui s'impose de plus en plus en plus souvent dans les décisions politiques. Elle est centrale dans le modèle de gouvernement prôné par l'Etat, qui lui offre une reconnaissance officielle. Dans ce nouveau modèle, l'opinion publique, plus que jamais dirigée, est considérée comme utile à l'instauration d'un mode de gouvernance technocratique sur l'ensemble du territoire chinois, ce que la plupart des internautes décrivent avec beaucoup de lucidité.

\footnotetext{
${ }^{32}$ En cela, Internet n'est donc pas différent des autres médias comme la télévision, qui couvre plus souvent des affaires urbaines que des affaires rurales. ZHAO Yuezhi et SUN Wusan. "Public opinion supervision: possibilities and limits of the media in constraining local officials", in GOLDMAN Merle et PERRY Elizabeth (dir.). Grassroots political reform in contemporary China, p. 300-324, p. 314; ZHAO Yuezhi. Media, market, and democracy in China. Between the party line and the bottom line. Urbana et Chicago: University of Illinois Press, 1998.
} 


\section{Chapitre IX - La gouvernance chinoise de l'opinion publique}

L'existence de plates-formes d'expression en ligne permet la formation de mouvements de masse qui ont des conséquences importantes sur la vie politique chinoise. Le gouvernement ne peut plus l'ignorer, il n'est plus entièrement maître de l'agenda et il doit montrer qu'il réagit. Beaucoup y voient l'avènement d'une opinion publique chinoise capable de s'opposer au gouvernement, à condition qu'elle trouve les formes appropriées pour former un jeu "gagnant-gagnant" avec l'Etat, une sorte de coopération, ou d"'interaction stratégique" qui conditionne une certaine tolérance de la part des autorités ${ }^{1}$. Or il faut aussi penser la relation entre les leaders d'opinion et les publics d'une part, ainsi que les enjeux de pouvoir au sein même de l'Etat, et enfin, le rôle de la presse dans cet imbroglio d'influences réciproques.

Il faut d'abord prendre le temps de caractériser l'opinion exprimée en ligne. Il s'agit d'une opinion restreinte, portée par une certaine catégorie de la population comme je l'ai montré précédemment. Elle ne se définit pas par opposition au gouvernement, mais par sa capacité à apparaître ad hoc et à disparaître aussitôt, en se faufilant dans les creux du discours officiel. Les revendications qu'elle porte sont loin d'être uniformes ou construites, et elles s'adressent à l'Etat plutôt qu'elles ne le dénoncent.

Compte tenu de ce cadrage, les autorités sont placées en situation d'arbitrer de l'extérieur des conflits considérés comme locaux. Cela a pour effet de renforcer et de crédibiliser l'image très officielle d'un gouvernement central technocratique luttant contre les féodalismes de l'échelon local. L'Etat central y a bien saisi son intérêt en évoquant de manière croissante la notion de "supervision par l'opinion publique" ${ }^{2}$. Ainsi, le regard des internautes

\footnotetext{
${ }^{1}$ ZHENG Yongnian. Technological empowerment. The Internet, State, and society in China, Stanford University Press, 2008, p.135s. Zheng argumente longuement pour une "déconstruction" des deux entités que sont la société et l'Etat, sans parvenir cependant à les présenter autrement qu'opposés l'un à l'autre.

${ }^{2}$ ZHAO Yuezhi et SUN Wusan. "Public opinion supervision: possibilities and limits of the media in constraining local officials", in GOLDMAN Merle et PERRY Elizabeth (dir.). Grassroots political reform in contemporary China, p. 300-324.
} 
devient-il un outil du maintien de l'autorité de l'Etat central sur son propre appareil et dans l'ensemble du territoire chinois.

C'est dans cette configuration complexe que les différents échelons de l'Etat participent tant bien que mal à la compétition collective qui se joue autour de la mise en récit des problèmes. La propagande devient ainsi plus subtile, au travers d'une "direction" de l'opinion publique renouvelée, qui est tributaire d'un relais de plus en plus incertain par la presse, et, en dernier ressort, d'une audience de plus en plus exigeante.

\section{1 - L'avènement d'une opinion publique restreinte}

\section{Une opinion publique restreinte mais reconnue}

La parole publique en Chine est l'objet d'effets de cadrage très importants, très au-delà de la seule censure, comme je l'ai montré dans les chapitres précédents. Seuls certains thèmes sont susceptibles de franchir les barrières de la surveillance politique, et surtout de retenir l'attention des internautes. Le profil sociologique de ces derniers est donc lui-même un facteur de cadrage important, qui tend à privilégier pour l'instant des problématiques propres aux catégories urbaines et plutôt aisées, même si cela pourra évoluer avec le temps. De plus, il faut probablement disposer d'un capital culturel et social important pour mettre en oeuvre des tactiques de mise en récit efficaces. Il en résulte que les causes portées en ligne ne sont représentatives que des préoccupations d'une petite partie de la population. Elles forment en somme une version très restreinte et très biaisée de l'opinion publique.

Quelques uns des internautes interrogés semblent sentir ces limites. Interrogés par exemple sur les sondages d'opinions qui sont de plus en plus nombreux en ligne, ils émettent des doutes sur leur représentativité.

"Est-ce qu'ils pourraient se servir d'Internet pour connaître l'opinion des gens?

- Il y a un site qui fait ça, "Fenêtre sur la capitale" (Shoudu zhi hu), mais je n'y vais jamais. A l'origine, leur intention affichée était de recueillir l'opinion publique, mais d'après ce que je ressens, en fait d'opinion publique, ce sont des gens sélectionnés qui répondent aux enquêtes d'opinion, et on ne peut pas voter ou s'exprimer quand on fait partie des masses." (Han) 
Ces internautes repèrent un caractère artificiel, voire manipulatoire dans ces sondages. Ils ne servent, pour beaucoup, qu'à maintenir une façade de consultation populaire. Certains pensent que c'est le cas pour l'expression sur Internet en général.

"Je pense que les chefs des gouvernements de tous les pays doivent faire attention [à l'opinion publique], parce que maintenant tous les pays essaient d'établir la démocratie. La Chine établit le centralisme démocratique, et le problème clé est ce terme de 'centralisme'. Qu'est-ce que ça centralise, est-ce que ça centralise la démocratie ou bien l'opinion de quelques personnes?" (Lin)

Pourtant, le terme d'opinion publique est utilisé de manière extensive dans la presse comme dans le discours officiel, et il l'est également dans le discours de la majorité des personnes interrogées. La plupart ont l'impression qu'il se forme en ligne une opinion publique qui a de plus en plus d'influence sur le gouvernement. Ils parlent de ce phénomène de manière plutôt positive.

"L'utilité principale d'Internet, c'est qu'on peut contacter beaucoup de gens en très peu de temps, et créer un consensus. En fait, Internet me donne l'impression, comment dire, que c'est moderne, c'est rapide, et puis c'est assez efficace.

-Pourquoi est-ce que c'est aussi important?

- Parce que tout le monde peut comprendre Internet, et on peut exprimer son opinion sur les forums, on peut faire des commentaires, laisser des messages, et créer une sorte d'opinion sociale, ça sert à ça." (Ye)

Cette opinion "sociale" n'est pas celle de tous les internautes. Certains ne s'expriment pas du tout en ligne, d'autres expriment leur désaccord. Cependant, même en l'absence d'un accord parfait, certains internautes pensent qu'il est possible de trouver un socle sur lequel tout le monde se rejoint.

"Quand il y a de trop grandes différences, il faut chercher le plus petit dénominateur commun (qiutongcunyi). Au travers de ces discussions certains points de vue peuvent peut-être faire consensus, même si bien sûr il restera toujours quelques divergences." (Chen)

Pour pouvoir être exprimée ouvertement et massivement, c'est à dire pour devenir publique, l'opinion doit donc être débarrassée des dissensions. Elle doit rassembler, montrer 
une union, un accord commun. Cette opinion publique peut parfois être effrayante, comme dans le cas des chasses à l'homme. Elle montre ainsi son efficacité de manière très excessive.

"A Harbin, c'était aussi dans le Nord-Est, c'était une infirmière de Harbin qui avait écrasé la tête d'un petit chat avec ses talons aiguille, on appelle ça l'affaire du chat. Quand c'est sorti, simplement quand la photo est sortie, après ça, c'était déjà à la télé, on a trouvé dans quel hôpital la femme travaillait, ils ont appelé l'hôpital, sa maison, ils l'ont empêchée de vivre normalement, c'est comme ça que ça se passe. Après l'hôpital l'a renvoyée, c'était obligé, sinon il leur était impossible de travailler normalement, c'est ça la pression de l'opinion publique." (Ye)

Cette opinion-là n'est ni pondérée, ni réfléchie en termes politiques. Dans d'autres cas elle permet la défense de causes soutenues par de nombreuses personnes. Alors elle a véritablement le pouvoir d'influencer les dirigeants, que ce soit pour les critiquer ou au contraire pour les conforter dans leur action.

"Mais alors les dirigeants ne font pas du tout attention à l'opinion des gens?

- Je viens de te le dire, une seule personne ils n'y font pas attention, mais si tout le monde se met à regarder une question, et si les gens sont très nombreux, alors ils vont forcément s'y intéresser, ils ne peuvent pas dire non plus qu'ils sont complètement indifférents. Parce que les dirigeants sont un groupe, les masses sont aussi un groupe, la force de l'union, la force de la collectivité, il suffit que la majorité des gens soient d'accord avec lui pour qu'on ne puisse pas changer une décision d'un dirigeant." $(\mathrm{Chu})^{3}$

En plus de l'unanimité, le nombre est important, que ce soit pour soutenir ou pour critiquer une politique. Les causes qui réussissent reposent, pour plusieurs internautes, sur le fait que des personnes extrêmement nombreuses s'expriment sur Internet, ressuscitant les mouvements de "masses" sous une autre forme. Le principe ne change donc pas fondamentalement, mais cette fois, le Parti n'a plus le monopole de l'initiative de ces mouvements. Il ne s'agit donc plus pour le gouvernement de lancer des mouvements politiques à grands renforts de propagande, mais bien de se montrer à l'écoute d'une opinion

${ }^{3}$ On voit bien combien l'Etat lui aussi peut tirer profit de cette opinion, qui contribue parfois à légitimer son action, quitte à ce qu'elle soit manipulée. 
publique restreinte et de prouver qu'il sait répondre efficacement aux attentes exprimées de cette manière.

\section{Une nouvelle source de légitimité}

L'importance de la formation de cette opinion publique est devenue si grande aux yeux de certains internautes qu'ils ont lancé une véritable campagne d'accusation contre Chen Hua, directeur adjoint de la gestion des informations sur Internet au Ministère de la Propagande de Pékin. Celui-ci aurait déclaré en privé: "C'est moi qui octroie la liberté d'expression aux internautes. Je leur en donne autant que cela me plait"t". Avant même que cette phrase ait été vérifiée, elle a immédiatement été considérée comme inadmissible par de nombreux internautes. Même les annonces récurrentes de lutte contre les contenus illicites sont sévèrement critiquées en ligne ${ }^{5}$. Désormais, les autorités doivent manifester publiquement du respect et de l'écoute envers ce que tous appellent l'opinion publique.

Autre exemple, le traitement du tremblement de terre du Sichuan a montré qu'il est devenu impossible de cacher des catastrophes importantes au grand public ${ }^{6}$. C'est pourquoi un internaute, qui se présente comme un universitaire, s'est félicité de ce phénomène et a déclaré sur son blog l'année 2008 "année zéro de la transparence politique" (wenzheng) ${ }^{7}$. Il oppose le temps révolu de la politique dirigée par les élites à celui enfin arrivé de la politique sous le contrôle de l'opinion. Pour lui, les internautes sont ainsi élevés au rang de troisième force, qui s'adresse directement aux autorités et les oblige à agir de manière transparente. A son tour, le sociologue Sun Liping défend l'idée que les conflits, la contestation ne sont pas néfastes, mais bien au contraire qu'ils sont un phénomène social naturel et indispensable à l'équilibre d'une

${ }^{4}$ XIAO Qiang. "Internet censorship official targeted by Chinese netizens", China Digital Times, 22/01/2009, consulté le 25/10/2010 sur http://chinadigitaltimes.net/2009/01/internet-censor-officialtargeted-by-chinese-netizens/.

5 XIAO Qiang. "Chinese bloggers' respond to the Internet crackdown", China Media Project, 29/01/2009, consulté le 25/10/2010 sur http://chinadigitaltimes.net/2009/01/chinese-bloggers-respondto-the-internet-crackdown/.

6 "From Tangshan to Wenchuan: A fault line through China", China Daily, 16/06/2008.

${ }^{7}$ LIANG Jiang Shu Sheng. "2008 wangshi: zhongguo wangluo wenzheng yuannian" ["Les affaires sur Internet en 2008: l'année de la transparence politique sur Internet en Chine"], Tianya, 20/12/2008, consulté le 25/10/2010 sur http://www.tianya.cn/publicforum/content/news/1/112759.shtml 
société. Par conséquent, le gouvernement doit leur ouvrir des canaux d'expression, afin de mieux identifier les problèmes et d'être capable de les résoudre ${ }^{8}$.

Même le Président Hu Jintao déclare vouloir tenir compte de la "sagesse du peuple", dont acte. Il a répondu à des questions d'internautes sur un chat en juin $2008^{9}$. Il n'y avait évidemment que trois questions, soigneusement sélectionnées, mais qu'importe, le ton était donné: désormais le Parti ne peut plus se permettre d'afficher une indifférence totale envers l'opinion publique. Se prêtant au même exercice de questions-réponses, le premier ministre Wen Jiabao a confirmé la tendance en février 2009 en déclarant:

Les gens ont le droit de critiquer la politique du gouvernement et le gouvernement doit aussi se montrer ouvert et démocratique dans ses prises de décision. ${ }^{10}$

La reconnaissance officielle de l'importance de l'opinion publique s'accompagne d'efforts importants pour recueillir ostensiblement l'avis des citoyens, par exemple au travers de sites de e-gouvernement. Chaque municipalité, région, ministère a son site ${ }^{11}$ et procède régulièrement à des consultations du public par des sondages en ligne, tout en donnant un maximum de signes de transparence. Les dirigeants chinois ont rarement fait autant d'efforts pour mettre la responsabilité et la transparence au cœur de leur communication politique. Il leur importe apparemment de se montrer réactifs et responsables (accountable) vis-à-vis de cette opinion publique, aussi restreinte et cadrée soit-elle.

\footnotetext{
${ }^{8}$ SUN Liping. "Maodun, chongtu benlai jiu shi shehui changtai" ["Les contradictions et les conflits sont des situations sociales naturelles"], Zhongguo Jiangxi Wang, 27/03/2009, consulté le 25/10/2010 sur http://news.163.com/09/0327/09/55DDGSC400012Q9L.html.

9 "Hu Jintao talks to netizens via People's Daily Online", 20/06/2008, People's Daily Online, consulté le 25/10/2010 sur http://english.people.com.cn/90001/90776/90785/6433952.html.

10 "People have right to criticize gov't, says Chinese Premier Wen", China View, 28/02/2009, consulté le 25/10/2010 sur http://news.xinhuanet.com/english/2009-02/28/content_10917646.htm.

${ }^{11}$ Voir par exemple un annuaire des sites de e-gouvernement chinois sur le site du Congrès américain. http://www.cecc.gov/pages/prcEgovDir/dirEgovPRC.php (consulté le 25/10/2010).
} 


\section{2 - L'opinion publique au service de la gouvernance}

\section{L'opinion publique comme outil de gestion technocratique}

Les internautes déclarent tous apprécier la place nouvelle que prend l'opinion publique sur Internet. Paradoxalement, ils ne s'attendent pas à ce que leurs attentes soient satisfaites pour autant. Les entretiens montrent en effet qu'une majorité des internautes restent silencieux parce qu'ils ne croient pas vraiment à l'influence politique de la parole individuelle. Un sondage de l'Académie des Sciences Sociales de Chine montre également que les internautes attribuent bien un certain pouvoir politique à Internet, mais que la croyance dans son efficacité tend à décliner.

Comment expliquer tout à la fois le discours enthousiaste sur l'impact de l'opinion publique et le sentiment d'impuissance qui se dégage à l'échelle individuelle? En fait, la plupart des internautes interrogés ne voient pas véritablement l'opinion publique comme une forme de participation individuelle ou collective à la prise de décision politique, et encore moins comme une représentation fidèle des opinions de la population. Bien au contraire, la plupart d'entre eux voient l'opinion publique comme un outil de gestion politique qui reste entre les mains de l'Etat. L'attention portée aux idées exprimées sur Internet n'est rien de plus, en fait, qu'une forme de consultation.

"En principe on comprend très peu de choses à la politique, je pense que c'est un moyen, mais ce moyen n'est pas forcément efficace. C'est juste une consultation, seulement une façon de participer, c'est à dire que pour nous, Internet est seulement un moyen de participer, ça ne peut pas devenir un principe. Nous, les individus, je crois que les individus et le gouvernement doivent avoir le même point de vue. Ce n'est qu'un produit, si c'est utile ils s'en servent, sinon ils l'écartent, mais ils ne s'en servent pas pour décider." (Lin)

L'opinion des individus ne serait donc requise qu'occasionnellement, pour faciliter la prise de décision, et plus encore, pour asseoir la légitimité des mesures adoptées. En aucun cas cependant, les individus ne sont invités à prendre part à la décision politique. L'arbitre ultime des problèmes de la société chinoise reste l'Etat, dont la volonté s'impose à tous. 
Dans cette vision, Internet fonctionne comme un système d'information sophistiqué dans une entreprise. Les internautes décrivent le pays comme une entreprise où l'information aurait du mal à circuler efficacement à cause des pesanteurs hiérarchiques. Personne n'osant révéler les dysfonctionnements, il est difficile de gouverner efficacement, phénomène bien connu en Chine ${ }^{12}$. Internet a donc l'avantage de pallier cette difficulté en aidant l'information à mieux circuler du bas vers le haut.

"En fait Internet c'est comme une entreprise. C'est pareil qu'une entreprise. Les internautes sont comme les employés, et les dirigeants ont intérêt à entendre l'avis des employés, il n'y a que comme ça que l'entreprise peut se développer, n'est-ce pas? Si les employés d'en bas disent tout ce qu'ils pensent, mais qu'ils ne le disent qu'entre eux, si les dirigeants ne l'entendent pas, et qu'ils s'appuient là-dessus pour travailler, c'est très difficile, très difficile de se développer. Ils ne peuvent pas se défendre si en dessous il y a des gens qui leur mettent des bâtons dans les roues, qui les trompent. Donc on dirait que sur Internet quand il y a des insultes, c'est comme les employés malicieux, non? " (Wu)

Les questions politiques sont dès lors réduites à leur aspect technique, administratif. Il s'agit d'atteindre un meilleur développement économique et de déployer les meilleures stratégies pour y arriver. C'est pourquoi il peut être intéressant de faire remonter certaines informations, mais pas de donner un pouvoir de décision aux citoyens qui ne sont pas des experts. C'est ce qu'explique le même internaute dans la suite de la conversation.

"Je ne fais pas de commentaires sur des sujets de société, parce que je crois que la société, comment dire, les problèmes de société, si on veut les commenter c'est quelque chose de très compliqué, tu sais? Prenons la Chine d'aujourd'hui, parlons de la situation de la circulation, aujourd'hui pour conduire c'était encore assez fluide, je suis vite arrivé, mais en général il y a beaucoup d'embouteillages, non? Ca bouchonne énormément, en fait à Pékin on construit des routes sans arrêt, ça se développe sans arrêt, pourquoi est-ce qu'il y a ce problème, c'est un problème de planification et un problème administratif. Pour une rue toute simple, comme la rue en bas, il est écrit que c'est une piste cyclable, au milieu ils ont en plus délimité un passage pour les voitures, et à côté du passage il y a un stop. Qui est responsable de cela? C'est quelqu'un de

${ }^{12}$ LEYS Simon. "Les habits neufs du président Mao", in Essais sur la Chine, Paris: Robert Laffont, 1998, p. 1-227. 
l'administration qui a fait ça, ce n'est pas un problème de société, c'est un acte administratif, donc si on veut faire des commentaires sur ces problèmes-là, ça ne sert pas à grand-chose, a moins que, en fait aujourd'hui, si par exemple on ouvrait quelque chose sur le web comme une séance d'audition ça permettrait peut-être de donner son avis personnel, mais si c'est après avoir vu la nouvelle qu'on envoie son commentaire, ça ne m'intéresse pas, et je crois que ça m'ennuie en fait."

Il y a là une relation proche du clientélisme entre les individus et l'Etat. Les individus sont encouragés à se concevoir comme consommateurs des services de l'Etat, ou même créditeurs des politiques publiques dans le cas des expropriations.

"Sur ce point, je trouve que la société se développe dans une très bonne direction. Bien sûr il faudra faire des sacrifices, mais je crois qu'on ne peut pas obtenir de meilleures choses si on ne fait pas de sacrifices. Cela ira à l'encontre des intérêts de certaines personnes, mais tant pis, parce que même si le gouvernement détruit ta maison, il te donnera forcément une compensation, non? Donc ce n'est pas grave. Il y a même des Pékinois qui veulent que le gouvernement détruise leur maison, pour gagner de l'argent." (Jiang)

De même, on fréquente notamment les sites Internet des différentes administrations dans l'optique clairement exprimée de profiter d'un certain nombre de bénéfices matériels.

"Quel type d'informations recherches-tu sur une plate-forme du gouvernement comme ça?

- Je cherche des informations fiables, sur la situation de la vie des gens ordinaires. Par exemple au tout début de la carte IC, la carte de transports publics, quand elle venait juste de sortir, ils donnaient des conseils aux gens, on pouvait faire des commentaires, on disait si c'était bien de faire cette carte ou non, et ils prenaient en compte notre avis. Donc si quelque chose a peut-être un rapport avec la vie des gens ordinaires, tu peux leur donner vraiment ton avis, ils le prennent sûrement en compte. Chacun ne peut que donner son avis personnel, mais si on exprime son avis là-dessus, à la fin ça représente forcément l'avis de tout le monde. Il y a aussi des plates-formes de marché, tu peux directement t'exprimer dessus, tu peux exprimer ton opinion sur la société, si tu trouves qu'une assurance maladie, ou que le minimum de prise en charge maladie est trop faible, tu peux tout dire là-dessus. Peu importe s'ils le regardent ou 
non, c'est seulement en exprimant nos véritables sentiments et réactions qu'ils peuvent connaître l'opinion des gens ordinaires." (Yang)

Dans cette perspective, l'expression de l'opinion personnelle n'est plus seulement un droit, mais c'est surtout un outil de gestion, car elle permet une meilleure maîtrise des besoins et des attentes des citoyens. Cette expression rend en fait possible une gestion technicienne et centralisée des préoccupations particulières des individus.

"En fait les dirigeants du pays, ou bien les dirigeants au niveau de la ville de Pékin, regardent souvent des sites Internet, ils lisent les réactions directes des gens. Par exemple, depuis un an ou deux, quand les dirigeants du pays ont diffusé un discours à la télévision, s'il ne se passe rien, ils vont voir les commentaires des gens sur Internet." (Zhou)

En permettant aux individus de déposer leurs plaintes, Internet rend même un service aux autorités centrales. Il fournit en effet une possibilité de "scientificiser" la connaissance de l'opinion publique, de la quantifier de la manière la plus précise qui soit. Les sondages d'opinion qui foisonnent sur le Web permettent de recueillir de manière très précise les souhaits des individus. Ceux-ci en viennent à considérer que les politiques du gouvernement sont élaborées en tenant compte de l'opinion publique mesurée par ces sondages.

"Je publie très rarement des commentaires. Parce que je pense que quand le gouvernement sort une nouvelle politique, elle a sûrement été élaborée par des spécialistes, ça ne peut pas venir d'un coup de tête, on fait comme ça ce qui nous passe par la tête. Y compris certains articles qui sont publiés par le gouvernement, il y a forcément eu des sondages d'opinion avant (minyi ceyan), ce qu'ils décident va dans le sens de certains intérêts des gens ordinaires, ça va dans le sens des masses, ça n'est pas fait pour les gens des couches supérieures. Aujourd'hui on divise les gens en différentes couches hein, je trouve que maintenant la politique du gouvernement, les lois, vont dans le sens des gens ordinaires." (Xie)

Ce portrait de l'Etat comme une entreprise, qui vise essentiellement l'efficacité économique (mais aussi de plus en plus sociale), et qui l'atteint notamment au moyen de mesures de l'opinion publique, est révélateur d'une évolution profonde dans la manière de gouverner du PCC. 
La vision d'un Etat chargé de gérer le plus efficacement possible les affaires du pays rejoint une conception technocratique de la légitimité du régime qui s'est développée depuis une trentaine d'années ${ }^{13}$ au moins ${ }^{14}$ en Chine. Balme note le fait que la technocratie est un phénomène propre aux sociétés modernes, et qu'il repose sur la conviction que le gouvernement des hommes peut répondre à des règles scientifiques, des techniques de gestion. Cette conception est commode car elle rejoint une tendance mondiale à promouvoir la notion de "gouvernance"15, en particulier dans les organisations internationales comme la Banque mondiale. Le critère ultime pour juger les gouvernants d'un pays n'est pas tant le respect des Droits de l'Homme ou la légitimité démocratique de l'équipe dirigeante, que la compétence technique de l'administration et sa capacité à mettre en place les meilleurs outils de développement économique ${ }^{16}$. Les nouvelles technologies de la communication en général, et Internet en particulier, se voient dès lors attribuer un rôle central ${ }^{17}$. Cette conception de la gouvernance technocratique ressort assez nettement dans le discours des internautes sur l'utilisation de l'opinion publique par le gouvernement chinois.

L'opinion publique restreinte qui s'exprime sur Internet n'est donc souvent conçue, par les internautes eux-mêmes, que comme un outil dans la gouvernance technocratique exercée par le Parti. Pourtant son rôle n'est pas neutre et elle transforme les relations de pouvoir au sein même de l'Etat chinois. En facilitant la remontée de certaines informations, l'opinion

${ }^{13}$ BALME Stéphanie. Entre soi, l'élite du pouvoir dans la Chine contemporaine, Paris: Fayard, 2004, p. 361s. ZHANG Junhua. "China's "government online" and attempts to gain technical legitimacy", ASIEN, $\mathrm{n}^{\circ} 80,2001$.

${ }^{14}$ Il est intéressant de noter que Rigby parlait déjà dans les mêmes termes du mode de légitimation de l'Etat communiste avant le début de la réforme: "Ce qui est spécifique des systèmes communistes est l'intégration organisationnelle et le management centralisé de toute la société dans une seule 'entreprise' dans laquelle l'autorité est légitimée dans des termes rationnels-objectifs (goal-rational)." RIGBY Thomas H. et FEHER Ferenc (dir.). Political legitimacy in communist states, New York: St. Martin's Press, 1982, p. 12.

\section{${ }^{15}$ HOWELL Jude (dir.). Governance in China, Oxford: Rowman \& Littlefield, 2004.}

${ }^{16}$ HERMET Guy. "La gouvernance serait-elle le nom de l'après-démocratie? L'inlassable quête du pluralisme limité", in HERMET Guy, KAZANCIGIL Ali et PRUD'HOMME Jean-François (dir.). La gouvernance: un concept et ses applications, Paris: Karthala, 2005.

${ }^{17}$ Sur cette question, voir par exemple FLICHY Patrice. L'imaginaire d'Internet, Paris: La Découverte, 2001; MATTELART Armand. Histoire de la société de l'information, Paris: La Découverte, 2001; MIEGE Bernard. La société conquise par la communication, Grenoble: Presses universitaires de Grenoble, 2007. 
publique permet d'asseoir l'autorité de l'Etat central sur l'ensemble du territoire, et en particulier sur les différents niveaux hiérarchiques du Parti. Le cadrage de l'expression de l'opinion publique devient donc un enjeu de pouvoir entre les différents échelons de l'Etat. Ce balancement entre la reconnaissance de l'opinion et son cadrage, souvent maladroit, donne parfois l'impression d'un tâtonnement ${ }^{18}$, mais ce ne sont que les deux pentes de la voie étroite sur laquelle s'est engagé le pays. Cette tension est nettement présente dans deux doctrines importantes du Parti, l'une née lors des réformes dans les années 1980, et l'autre introduite au moment de la reprise en main des médias après 1989: la "supervision" par l'opinion publique d'une part, et la "direction" de l'opinion publique d'autre part ${ }^{19}$.

\section{La "supervision", ou l'opinion publique au service de l'Etat central}

La plupart des internautes ont très nettement la sensation que l'information est essentielle dans la prise de décision politique. Les dirigeants doivent donc trouver les moyens de s'informer le mieux possible sur ce qui se passe dans le pays. Tout leur est utile: rapports de synthèse, audit, comptes-rendus etc... La plus grande difficulté est pour eux de faire remonter l'information de la base sans que les problèmes ne soient cachés par les niveaux hiérarchiques intermédiaires.

"D'après moi, les canaux par lesquels les dirigeants obtiennent des informations ne sont en fait pas très nombreux. Ils doivent surfer un peu sur Internet, mais ce n'est pas leur moyen principal pour obtenir de l'information. Les dirigeants ont plusieurs façons d'obtenir de l'information, ils reçoivent des rapports de synthèse, faits exprès pour eux, et comme ils doivent survoler de grandes quantités d'informations très rapidement, une façon c'est les rapports de synthèse, c'est à dire condenser en un document toutes sortes d'informations nationales et internationales, qui viennent d'Internet, des journaux, de la télévision, et ça devient un petit cahier, qu'on leur donne tous les jours, ça c'est un canal assez important. Une autre voie assez importante, on l'appelle l'audit, (neibucankao), qu'est-ce que c'est, ce sont des choses que seuls

\footnotetext{
${ }^{18}$ PING Ke. "Two steps forward, one step back? a review of the Chinese Internet in 2008", Southern Weekend via East South West North, 21/01/2009, traduit par Roland Soong, consulté le 25/10/2010 sur http://www.zonaeuropa.com/20090124_1.htm.

${ }^{19}$ QIAN Gang. "Guidance • Supervision • Reform • Freedom: Plotting the direction of Chinese media through an analysis of the all-important buzzword" [traduit par David Bandurski], China media project, 13/07/2005, consulté le 25/10/2010 sur http://cmp.hku.hk/2005/07/13/33/.
} 
quelques médias ont le droit de traiter, par exemple des médias très spécialisés, et ils ont le droit de remettre leurs rapports à des consultants internes. Alors peut-être qu'ils prennent en compte de ce que ces médias leur rapportent, et qu'ils peuvent savoir comme cela l'opinion des gens. Ils peuvent même rassembler tout ça en un dossier, et le donner aux dirigeants. Et sinon les gens ont aussi d'autres moyens de faire connaître leurs points de vue, il faut faire remonter l'information étage par étage dans la hiérarchie, ça a aussi une influence sur la politique chinoise. Aujourd'hui il y a beaucoup de gens au pouvoir qui ne savent pas du tout ce que pensent les gens (on s'écarte un peu de la discussion). Des fois, en fait, dans beaucoup de régimes politiques il peut y avoir de la corruption, comment est-ce que ça arrive, si on regarde bien, si sa politique n'est pas raisonnable du tout, comment est-ce possible que des politiques pas raisonnables soient appliquées? C'est parce qu'ils n'entendent pas la voix des gens, et quand ils se retrouvent au pouvoir, tout en haut, ceux qui ont les idées au plus haut niveau, ils n'ont aucun moyen de voir ce qui se passe vraiment, ils ne comprennent pas, en soi c'est corrompu. La corruption à la base n'a rien à voir avec les gens de ce niveau, mais qui est-ce qui nous donne une influence corrompue au moment de mettre en place les politiques, ce sont les responsables qui les appliquent concrètement, ce sont eux qui nous créent cette influence. Et avec eux au milieu, comment est-ce que notre opinion peut atteindre les oreilles des gens non corrompus, elle n'y arrive pas. Ils sélectionnent pour nous les opinions, et leur font dire ce qui les arrange, c'est la situation la plus courante." (Zhang)

Cet internaute reproduit une doctrine plus ou moins officielle: l'Etat central est animé de bonnes intentions, mais il rencontre les pires difficultés à faire appliquer ses mesures par les autorités locales et les différents échelons administratifs, qui sont touchés par un fort taux de corruption. L'enjeu est donc d'établir un contact direct entre la population et l'Etat central, pour pouvoir signaler ces injustices. Si Internet peut désormais jouer ce rôle de manière plus ouverte, le type de relations que cela implique entre la population et l'Etat central n'est pas nouveau. Il remonte à la Chine impériale.

"Ca a aussi de l'influence sur le gouvernement, il doit plus tenir compte de l'opinion des masses et de ce que pensent les gens ordinaires. Avant ils avaient très peu de contacts avec les gens ordinaires, ils ne pouvaient les contacter que dans le cas des "lettres et visites". Avec Internet ils peuvent entendre la voix des gens de toutes les couches, ils peuvent atteindre directement des choses vraies, ils peuvent directement 
les voir. Par exemple quand des enfants tombent malades, il y en a qui attrapent la leucémie, ils peuvent toucher ça directement. Ca a raccourci le temps de résolution des problèmes entre les gens ordinaires et le gouvernement." ( $\mathrm{Lu})$

Internet ranime ici le principe ancien du bureau des "lettres et visites" ${ }^{20}$. Il s'agit d'une institution qui permet aux citoyens lésés par un officiel local, ou victimes d'une injustice, de venir à Pékin pour obtenir réparation auprès du pouvoir central. Cette institution existe toujours mais il est extrêmement difficile, pour ne pas dire impossible, d'atteindre effectivement les bureaux des visites. Celui-ci en effet est surveillé de très près par des émissaires de chaque province qui ont pour mission d'intercepter ceux qu'on appelle les "pétitionnaires" avant qu'ils n'aient l'occasion de déposer leur revendication. Le sort des pétitionnaires est généralement très mauvais. Ils peuvent être simplement renvoyés chez eux, battus ou même enfermés dans des asiles psychiatriques ${ }^{21}$. Internet est perçu ici comme une manière nouvelle et plus sûre de déposer des revendications, une sorte de résurgence du droit de pétition sous une autre forme. Chacun peut raconter son histoire, et rencontrer (ou pas) l'assentiment d'autres individus, et ainsi contourner l'omnipotence du pouvoir local en faisant connaître sa situation directement au pouvoir central.

De fait, le cadrage des problèmes publiables sur Internet fait qu'il est impossible de critiquer le régime ou le Parti, mais qu'il est possible de raconter une injustice particulière, qui s'est déroulée dans une circonscription particulière. De cette manière, la faute n'est donc pas imputable au système en général, mais à des individus ou des groupes d'individus faillibles. Ceux-ci sont souvent difficiles à surveiller par l'Etat central, et lorsque les injustices sont trop flagrantes, ils nuisent à l'image du Parti, menaçant la stabilité sociale et a fortiori politique. Les internautes le sentent, et ils ont l'impression de rendre service à l'Etat en fustigeant les écarts des dirigeants locaux.

"Je fais très peu de commentaires, ça m'arrive, mais très peu. Dans quels cas? Contre des gouvernements locaux, quand ils vont trop loin, dans ces cas-là, je fais des commentaires.

\footnotetext{
${ }^{20}$ THIREAU Isabelle et HUA Linshan. Les ruses de la démocratie, Paris: Seuil, 2010.

${ }^{21}$ MU Eric. "Local authorities in Shandong put petitioners in mental hospital", Danwei.org, 08/12/2008, consulté le $\quad 25 / 10 / 2010$ sur http://www.danwei.org/front_page_of_the_day/the_beijing_newsdecember_8_200.php.
} 
- Pourquoi?

- A mon avis la gestion du gouvernement central, son point de départ est souvent bon, mais le problème, c'est quand le gouvernement local l'exécute. D'après moi, les Chinois sont trop nombreux, et le système d'exécution en dessous est certainement... Ce système s'écarte facilement des intentions du gouvernement chinois, donc cela crée beaucoup de choses qui mettent les gens en colère. J'écris assez énergiquement là-dessus, ou à chaque fois qu'il y a une absurdité." (Zhao)

Là encore, ce n'est pas aux internautes de trouver des solutions, ni même d'analyser la situation en détails. Une fois que le problème est signalé, l'Etat central est chargé de le régler.

"Je trouve que ce n'est pas vraiment une question de content ou pas content, parce que poster des commentaires c'est très excitant. Surtout, ça permet à tout le monde de créer un consensus, on peut dénoncer des choses, c'est surtout ça. Mais concrètement, on ne peut pas résoudre ces problèmes, pas sur Internet. Tout ce qu'on peut faire c'est de s'y intéresser, de les dénoncer, mais concrètement c'est le gouvernement local qui peut résoudre ces problèmes, c'est dommage. Mais je me rends compte que quand une information reçoit beaucoup d'attention et qu'elle est dénoncée sur Internet, le gouvernement intervient tout de suite pour régler le problème. Par exemple à Tangshan, le président d'un grand groupe avait commandé des véhicules militaires, des voitures blindées, il en avait commandé sept. Ça s'est répandu assez vite sur Internet. Peut-être une heure après que l'information soit sortie, les médias gouvernementaux ont commencé à parler de ce problème, et ils parlaient de la manière dont ce serait résolu." (Zhao)

Dénoncer un abus localement reviendrait donc à montrer sa loyauté envers le gouvernement central, comme l'explique un internaute parmi les plus conformistes. Internet permet une alliance entre les "couches les plus basses" (qui en réalité sont bien plus souvent des couches favorisées) et l'Etat central, contre les dirigeants corrompus, conformément à la doctrine officielle de prendre "l'individu comme base".

"Quand les gens ordinaires ne sont pas très satisfaits, et qu'ils veulent écrire ce qu'ils pensent sur Internet, tu penses que c'est utile?

- Apparemment il y a déjà eu des exemples comme ça, c'est à dire des avis différents sur quelque chose, et tout le monde qui en discute sur Internet, et que ça 
attire l'attention du gouvernement, par exemple au Henan le gouvernement local d'un endroit a fait construire un bâtiment officiel qui avait 27 colonnes, comme la Maison Blanche aux Etats-Unis, très somptueux. C'est une ville très pauvre, pourquoi est-ce qu'ils ont dépensé autant d'argent pour construire leur propre bâtiment? Plus tard la photo a été mise sur Internet, elle a circulé, et leurs supérieurs hiérarchiques ont vérifié, je trouve que ça a quand même une influence, et cette influence est relativement grande, parce qu'elle vient de la couche la plus basse, ou parce que c'est le point de vue des gens qui sont le plus à la base, qui réagissent sur ce qui est tout en haut, c'est un canal très efficace. [...] C'est une attitude très compliquée, c'est à dire que je sais qu'il y a beaucoup de plaintes dans le pays, mais on a formé une sorte de concept de clan familial, une sorte de conception de la nation qui me dit que je ne dois pas faire beaucoup d'opposition, ou m'écarter de notre affaire d'objectif commun (gongtong mubiao de shiqing), je dois le soutenir, la pensée de tous les Chinois est comme ça." (Zeng)

Il ne s'agit donc pas de manifester une quelconque opposition au régime, mais de montrer son attachement aux valeurs qu'il promeut, en dénonçant les entorses les plus graves. Les internautes exercent ainsi une sorte de surveillance au service des autorités centrales, et souvent à l'encontre des autorités locales, effectuant ainsi un travail de remontée de l'information qui était auparavant effectué par les journalistes dans le secret des rédactions des journaux officiels ${ }^{22}$. Ce phénomène est tout aussi utile au gouvernement qu'aux individus, et l'Etat est même plus facilement en mesure d'en contrôler l'issue. Cette surveillance exercée d'en bas, ou "sousveillance" ${ }^{23}$ si l'on veut, n'est donc pas une forme de résistance contre les autorités, mais elle se révèle être l'un des éléments d'une distribution très complexe de la coercition au sein de la société et au sein même de l'Etat.

\footnotetext{
${ }^{22}$ ZHAO Yuezhi et SUN Wusan. "Public opinion supervision: possibilities and limits of the media in constraining local officials", in GOLDMAN Merle et PERRY Elizabeth (dir.). Grassroots political reform in contemporary China, p. 300-324.

${ }^{23}$ MANN Steve, NOLAN Jason and WELLMAN Barry. "Sousveillance: Inventing and using wearable computing devices for data collection in surveillance environments", Surveillance and Society, vol. 1, $\mathrm{n}^{\circ} 2$ 2, 2003, p. 331-335, consulté le 28/04/2009 sur http://www.surveillance-andsociety.org/articles1(3)/sousveillance.pdf.
} 
Il faut prendre en compte cette complexité des rapports entre autorités locales et centrales pour comprendre la doctrine officielle de la "supervision par l'opinion publique", telle qu'elle est présentée par exemple dans un article de Xinhuanet.

Les gens ont le droit de savoir la vérité, donc le fait de dire que les masses sont 'coupées de la réalité relève de la fuite devant les responsabilités par ceux qui sont au pouvoir dans certaines localités. Par conséquent, si certains lieux sont attaqués et bombardés par des 'masses coupées de la réalité', ceux qui sont au pouvoir devraient commencer par se retourner et revoir leurs propres déficiences. Les 'masses coupées de la réalité' mettent en évidence l'aveuglement de certains officiels envers l'opinion publique, tout comme leur aversion pour des idées de l'Etat de droit comme 'le pouvoir doit accepter une supervision'. Dans l'esprit d'un petit nombre d'officiels, ils méprisent fondamentalement les droits fondamentaux des citoyens, sans parler du 'droit de savoir des citoyens'. ${ }^{24}$

Seul un petit nombre d'officiels sont visés dans cette présentation. La transparence et la prise en compte de l'opinion publique sont désignées comme les attributs principaux d'une gouvernance moderne et rationnelle, laquelle serait l'apanage de l'Etat central. Internet seraitil l'outil idéal pour parvenir à une modernisation de l'appareil administratif dans les provinces les plus récalcitrantes? C'est ce que suggère Lagerkvist en étudiant les "techno-cadres" ${ }^{25}$ qui concoctent la réforme numérique en Chine. De fait, de plus en plus de scandales sont l'occasion pour les autorités centrales de remettre les pendules à l'heure dans certaines provinces, tout en insistant sur la légitimité nouvelle de la supervision par l'opinion publique.

24 HUANG Huo. "Meiti ping quntixing shijian qunzhong 'bumingzhenxiang' shi guanyuan shizhi"["Les commentaires sur les incidents de masse: 'l'idée que les masses soient détachées de la réalité' est un manquement au devoir de la part des officiels"], Xinhuanet, 01/12/2008. Traduction intégrale par KELLY David dans XIAO Qiang. "Media commentary on mass incidents: masses 'out of touch with the facts' is official dereliction of duty", China Digital Times, 01/12/2008, consulté le 25/10/2010 sur http://chinadigitaltimes.net/2008/12/media-commentary-on-mass-incidents-massesout-of-touch-with-the-facts-is-official-dereliction-of-duty

${ }^{25}$ LAGERKVIST Johan. "The techno-cadre's dream: administrative reform by electronic governance in China today?", China Information, $n^{\circ} 19,2005$, p. 189-216. 


\section{3 - L'opinion publique comme enjeu de pouvoir}

\section{La "direction" de l'opinion publique}

La propagande gouvernementale, loin d'avoir été oblitérée par la reconnaissance officielle de l'opinion publique, prend tout au contraire un souffle nouveau. Ainsi Hu Jintao appelle-t-il à une nouvelle forme de "guidage" ou de "direction" de l'opinion (yulun daoxiang), par exemple dans un important discours sur la presse à l'occasion du soixantième anniversaire du Quotidien du Peuple en juin $2008^{26}$. Sans revenir sur le principe du contrôle de l'opinion publique, qui est, plus que jamais, une priorité, il semble se prononcer pour de nouvelles méthodes. Il mentionne expressément le fait que l'information circule désormais très rapidement, et qu'elle a une influence très forte sur les mentalités. Prenant acte de cette réalité, il annonce une nouvelle stratégie avec l'adoption d'une sorte de "gouvernance de l'opinion publique" ${ }^{27}$, qui repose bien plus sur des méthodes comme le contrôle de l'agenda, le cadrage des décisions, l'utilisation d'événements marquants pour imposer une thématique sur la scène publique par exemple, que sur la censure pure et simple, qui est inefficace et peut nuire à l'image "moderne" du Parti. Lagerkvist nomme "ideotainment" ${ }^{12}$ la transformation des techniques de propagande dans une direction plus informative et ludique. Il faut désormais influencer sans cesser de plaire, car le public peut fuir plus facilement.

Localement, la consigne est diversement comprise et appliquée sur le territoire, ce qui conduit parfois à des reprises en main sévères. Prenons l'exemple des émeutes causées par la mort d'une jeune fille à Wengan dans le Guizhou en juin 2008. Alors que la famille soupçonne le fils d'un officiel du canton de l'avoir violée et assassinée, la police a conclu à un suicide, sans fournir les résultats de l'autopsie demandée. Le fait que le meurtre de la jeune fille ait été caché, ainsi que les rumeurs qui se sont très vite répandues, ont rapidement conduit des milliers de personnes à se rassembler devant les bâtiments de la police, qu'ils ont

${ }^{26}$ BANDURSKI David. "Propaganda leaders scurry off to carry out the 'spirit' of Hu Jintao's 'important' media speech", China Media Project, 25/06/2008, consulté le 14/10/2010 sur http://cmp.hku.hk/2008/06/25/1079/.

${ }^{27}$ GERSTLE Jacques. "Gouverner l'opinion publique", in BRECHON Pierre (dir.), La gouvernance de l'opinion publique, Paris: L'Harmattan, 2003, p. 19-34.

28 LAGERKVIST Johan. "Internet Ideotainment in the PRC: national responses to cultural globalization", Journal of Contemporary China, vol. 17, n54, 2008, p. 121-140. 
incendiés. De nombreuses images ont été prises et rapidement postées en ligne, interdisant d'étouffer l'affaire. C'est à ce stade que les événements ont pris une tournure relativement nouvelle. Le secrétaire du Parti de la province du Guizhou s'est rendu officiellement sur place pour désavouer les autorités locales, affirmant que le fait d'avoir négligé les intérêts de la population pendant des années a conduit à cette situation ${ }^{29}$. Il a pris des mesures disciplinaires contre trois officiels locaux avant même de s'attaquer aux responsables de l'émeute. Il a ainsi montré que désormais les autorités locales sont tenues pour responsables de ces débordements et que l'usage de la force est découragé. Par la suite, l'enquête a été rendue publique pour apaiser les rumeurs. Ce traitement de la situation est emblématique du changement des règles $\mathrm{du} \mathrm{jeu}^{30}$. Il n'est plus question d'étouffer des scandales s'ils ont déjà été rendus publics. La consigne officielle est donc de publier tout de suite les mauvaises nouvelles plutôt que de les voir publiées par d'autres ${ }^{31}$, tout en assurant un cadrage très serré de leur mise en récit. Il faut les prendre en main au plus tôt, pour retourner l'opinion publique dans un sens plus favorable.

Peut-être est-ce l'exemple désastreux de Wengan qui a convaincu le département de la propagande du Yunnan de recourir à une stratégie toute différente début 2009. Un scandale a éclaté suite à la mort de Li Qiaoming dans une prison du Yunnan, que la police a expliquée par une chute accidentelle lors d'un jeu de colin-maillard entre détenus (duo maomao). Bien sûr les commentaires incrédules se sont rapidement multipliés sur Internet. Répondant de manière tout à fait inattendue, les autorités locales ont annoncé la création d'un comité d'internautes qui pourraient mener eux-mêmes l'enquête ${ }^{32}$. Un comité a bel et bien été constitué, dans une transparence à peu près nulle, et il a pu effectuer une visite très guidée de la prison en question, sans pouvoir accéder à quelque information significative que ce soit ${ }^{33}$.

${ }^{29}$ WANG Hongjiang. "Commentary: Public grievances should be channeled not suppressed", China View, 08/07/2008, consulté le 25/10/2010 sur http://news.xinhuanet.com/english/200807/08/content_8512767.htm.

30 LI Datong. "The Weng'an model: China's fix-it governance", Open Democracy, 30/07/2008, consulté le 25/10/2010 sur http://www.opendemocracy.net/article/the-wengan-model-china-s-fix-itgovernance.

31 "The Internet drives China to loosen its grip on the media", The New York Times, 20/10/2008.

32 PEDROLETTI Brice. "Pékin invite les internautes à enquêter sur la mort d'un gardé à vue", Le Monde.fr, 20/02/2009.

33 MACKINNON Rebecca. "'Eluding the Cat' - Bloggers investigate Yunnan prison death", RConversation, 24/02/2009, consulté le $25 / 10 / 2010$ sur 
Cependant l'essentiel n'est pas là, comme l'a indiqué l'initiateur même de ce comité. Son objectif n'est autre que de canaliser l'opinion publique en donnant les signes extérieurs de la transparence. Dans cette perspective, Internet devient un instrument de propagande d'un genre nouveau ${ }^{34}$.

Diverses stratégies sont possibles au cas par cas et montrent toute la difficulté de trouver le bon ton, la bonne méthode de communication. De plus en plus, les autorités sont amenées à anticiper sur la publication des faits divers et à élaborer des stratégies pour contrer très en amont l'émergence des scandales. Observons par exemple les évolutions de l'affaire Lin Songlin. A la sortie d'un bar, le jeune homme, probablement alcoolisé, a eu une altercation avec six policiers, probablement non moins alcoolisés, qui l'ont finalement battu à mort. Les circonstances de l'altercation et l'état des sept protagonistes n'étant pas déterminés précisément, un débat est né. Comme souvent dans de tels cas, les internautes se sont violemment attaqués aux policiers, auteurs d'une grave bavure. Cependant de nouvelles informations ont été postées sur Internet au sujet de la victime, provoquant un retournement de situation des plus inattendus. Le jeune homme appartiendrait à la classe privilégiée (il aurait acheté son diplôme et obtenu son emploi en faisant jouer des relations familiales par exemple), et dans une vidéo, on le voit provoquer l'altercation avec un comportement arrogant et violent. Très rapidement, de nombreux commentateurs se sont attaqués à lui, et les six policiers ont été déchargés d'une part de leur responsabilité. Cette nouvelle version a "transformé l'histoire de 'six policiers battent à mort un étudiant d'université dans la rue' en 'six gardiens ont frappé le privilégié du gouvernement Lin'"'35. Un tel retournement de situation n'a pas manqué d'attirer les soupçons: ne serait-ce pas le fait d'armées de petites mains rémunérées au service de la police ${ }^{36}$

http://rconversation.blogs.com/rconversation/2009/02/eluding-the-cat---bloggers-investigate-yunnanprison-death.html.

${ }^{34}$ BANDURSKI David. "How Control 2.0 found its poster boy in Yunnan", China Media Project, 24/02/2009, consulté le 25/10/2010 sur http://cmp.hku.hk/2009/02/24/1483/.

${ }^{35}$ SOONG Roland. "The habitual way of thinking about "'the police killed someone'", East South West North, 18/10/2008, traduction d'après Wei Yingjie sur My1510.cn, consulté le 14/10/2010 sur http://www.zonaeuropa.com/200810b.brief.htm.

${ }^{36}$ SOONG Roland. "How big is the army of fifty-cent gang?", East South West North, 17/10/2008, consulté le 14/10/2010 sur http://www.zonaeuropa.com/200810b.brief.htm. 
Le traitement médiatique de cette affaire a été, en effet, pris en main très rapidement par les autorités locales, qui ont manifesté le plus de transparence possible, affichant les exigences de la famille ainsi que le compromis trouvé avec la police. En effet, le vice-maire de Harbin et le directeur du bureau de la sécurité publique de Harbin ont négocié avec la famille sur les cinq points suivants:

1. La police aurait dû faire preuve de plus de maîtrise d'elle-même;

2. La famille ne devrait pas croire les rumeurs sur Internet et elles ne devraient pas être utilisés par d'autres personnes qui ont d'autres motivations.

3. La police ne protégera personne dans ses rangs

4. La police n'utilisera pas de mesures de rétorsions contre les proches de Lin Songlin et ne les tiendra pas pour responsables.

5. La communication sera maintenue entre la famille Lin et la police et [le directeur du bureau de la sécurité publique] donnera son numéro de téléphone mobile personnel. ${ }^{37}$

L'insistance particulière sur Internet et la qualification des "rumeurs" n'est pas étrangère à cette démarche. Il s'agit pour les autorités de renvoyer le débat dans la sphère peu respectable des "on-dits", afin de mettre un terme au déferlement des opinions indignées des internautes. Cette reprise en main de la communication officielle sur le sujet par les autorités ne se fait qu'au prix d'une intense négociation avec la famille, et sous une exigence de transparence rarement encore égalée jusque là. Visiblement, l'ensemble des acteurs concernés sont conscients des enjeux de la publication de l'information, et ils maîtrisent de mieux en mieux l'opinion publique comme outil stratégique.

Même si les stratégies adoptées par les autorités locales sont d'une inégale efficacité, on observe des réactions de plus en plus rapides et de mieux en mieux coordonnées, un peu partout dans le pays. Il semble que le mot d'ordre national soit de mieux en mieux appliqué, comme par une forme de normalisation des comportements.

${ }^{37}$ SOONG Roland. "The six policemen kicked Lin Songling for five to six minutes", East South West North, 20/10/2008, traduit d'après Beijing News, consulté le 14/10/2010 sur http://www.zonaeuropa.com/200810b.brief.htm. 
Par exemple, le maire de la ville de Changzhou a opté pour une stratégie de communication très directe. Alors que des rumeurs circulaient au sujet de son exercice du pouvoir, il a décidé non pas de les faire taire, mais d'y répondre point par point pour les démentir ${ }^{38}$ (l'histoire ne dit pas ce qu'il en est en réalité). Cette réaction n'est peut-être pas tout à fait inédite, mais elle est pour le moins rare en Chine. En effet, par cette action, le maire se montre transparent devant l'opinion publique. De ce fait, il échange une autorité traditionnelle, moquée sous cape mais, qui était, au sens propre, indiscutable, contre une crédibilité nouvelle due à son attitude de transparence, et qui lui demandera certainement beaucoup de responsabilité dans l'avenir.

L'enjeu pour tout dirigeant est désormais d'être informé au plus tôt de tout incident qui pourrait causer un scandale public. De ce point de vue la démarche du maire de Changzhi de faire distribuer sa carte de visite en 960000 exemplaires pour permettre à tout travailleur de lui signaler des atteintes à sa sécurité est tout à fait exemplaire ${ }^{39}$. Là encore, la possibilité effective que des travailleurs s'adressent au maire pour signaler des problèmes est très faible, mais l'essentiel réside dans l'affichage d'un gouvernement local à l'écoute des citoyens. Peutêtre cela permettra-t-il également d'intercepter les conflits les plus graves avant qu'ils ne soient rendus publics.

Là est peut-être aussi le véritable enjeu des outils de filtrage toujours plus fins des données en ligne ${ }^{40}$. Il faut pouvoir détecter les sujets populaires dès leur naissance, pour préparer au plus vite les réponses adéquates ${ }^{41}$. On peut lire de la même façon le recours à de nombreuses personnes chargées de poster des commentaires favorables au gouvernement. Ils sont un relais indispensable pour mettre en place une stratégie de mise en récit de

${ }^{38}$ SOONG Roland. "Changzhou city mayor responds to Internet rumor", East South West North, 20/10/2008, traductions d'après DWNews et Southern Metropolis Daily, consulté le 25/10/2010 sur http://www.zonaeuropa.com/200810b.brief.htm\#026.

${ }^{39}$ BANDURSKI David. "Even if the mayor transforms into an octopus...", China Media Project, 23/10/2008, consulté le 25/10/2010 sur http://cmp.hku.hk/2008/10/23/1298/.

${ }^{40}$ HILLE Kathrin. "China bolsters Internet censors' scrutiny", The Financial Times, 05/01/2009.

${ }^{41}$ BANDURSKI David. "Taxi strikes in China highlight changing press controls", China Media Project, 12/11/2008, consulté le 25/10/2010 sur http://cmp.hku.hk/2008/11/12/1344/. 
l'information $^{42}$. Dans cette véritable course de vitesse entre les différents acteurs de l'information, le Parti dispose en effet d'avantages indéniables, mais insuffisants. Il lui est facile de faire circuler sa version des faits au travers des dépêches de l'agence officielle Xinhua qui sont largement reprises, mais le statut officiel de ces informations conduit beaucoup de lecteurs à les lire avec distance. Il est donc plus stratégique de recourir à la parole d'individus anonymes pour s'assurer de toucher les internautes d'une autre manière.

En effet, le Parti est loin d'avoir conservé une mainmise totale sur l'agenda politique. Pour relayer ses messages, il doit désormais compter avec une presse diversifiée, soumise aux contraintes du marché et à ce titre, beaucoup plus sensible aux centres d'intérêt des lecteurs.

\section{La consécration des causes par les médias de masse}

Les médias dits traditionnels, c'est à dire la télévision et la radio, les journaux et les magazines, mais également les pages d'informations les plus contrôlées des grands portails Internet, sont censés représenter la voix officielle du Parti. A ce titre, ils permettent à tout un chacun de connaître les lignes rouges à ne pas franchir en fonction de l'actualité. Cependant, ces médias sont toujours à la recherche de sujets proches des préoccupations de leurs publics, ce qui les oblige à se faire l'écho de sujets de plus en plus sensibles.

J'ai déjà montré que les médias traditionnels servent d'indicateurs à tous les acteurs lorsqu'ils décident de la manière dont ils vont s'exprimer en ligne, c'est pourquoi je n'y reviendrai que très brièvement. Tous les internautes cités précédemment mentionnent le fait qu'ils sont attentifs au traitement de l'information par les médias officiels. Très surveillés, ces médias sont considérés comme la voix du Parti, et ils permettent de connaître, dans l'actualité, la position officielle que chacun peut adopter ouvertement. Ce sont donc eux qui signalent les sujets qui offrent des opportunités pour dénoncer telle ou telle injustice. En particulier, la thématique de la lutte contre la corruption offre beaucoup de place pour tenir un discours critique dans divers espaces.

42 XIAO Qiang. "Authorities' attempts to bring online public opinion under control", China Digital Times, 28/10/2008, consulté le 25/10/2010 sur http://chinadigitaltimes.net/2008/10/cyber-police-tryto-avoid-mass-incident-with-a-preemptive-post. 
Par exemple, Wang Shaoguang rappelle que quelques articles avaient paru dans la presse en 2000 et 2001 sur la question des centres de détention et de rapatriement qui ont donné lieu à l'affaire Sun Zhigang en 2003. Ces articles n'avaient pas suscité beaucoup de réactions à l'époque, mais la publication de l'affaire aurait peut-être été plus difficile sans ces signaux $^{43}$. Alors que la famille avait épuisé toutes les voies de recours dont elle disposait, le bureau des lettres et des visites lui-même leur a conseillé d'alerter la presse ${ }^{44}$. C'est en effet à la suite de la publication de leur histoire dans le Southern Metropolis Daily (Nanfang Dushibao), que les premières réactions se sont manifestées. L'article a été rapidement repris par de nombreux journaux très officiels dont le Quotidien du Peuple, et posté sur Internet où il a été abondamment commenté. De plus, des intellectuels et en particulier des juristes ont saisi l'occasion pour écrire des pétitions adressées aux autorités. Ce cas montre bien la conjonction très particulière qui s'est faite entre les intérêts et les calendriers de divers types d'acteurs, médias, autorités, leaders d'opinion ou publics.

Surtout, il montre que le balisage des sujets n'est pas effectué à sens unique par les médias ou les autorités. Les différents publics se sont invités dans le processus de définition de l'agenda politique, tel qu'il est reflété dans la presse. Les internautes ont clairement le sentiment que les médias officiels sont très attentifs aux histoires qu'ils publient, car elles représentent une source d'informations sans équivalent, et surtout elles les informent sur les centres d'intérêts de leurs publics.

"Par exemple, quand il se passe quelque chose, peut-être que chacun a un point de vue différent dessus, et beaucoup de gens peuvent en discuter. Et il peut y avoir des gens de toutes sortes, y compris des étudiants, des fonctionnaires du gouvernement, ou des représentants à l'Assemblée, qui s'intéressent à cet événement. Alors tout le monde discute de la meilleure manière de résoudre le problème, et au travers de la discussion, peut-être qu'on peut trouver une très bonne solution.

- Les gens qui participent à la discussion ont ce pouvoir-là?

\footnotetext{
${ }^{43}$ WANG Shaoguang. "Changing models of China's policy agenda setting", Modern China, vol.34, $\mathrm{n}^{\circ} 1,2008$, p. 56-87.

${ }^{44}$ THIREAU Isabelle et HUA Linshan. "De l'épreuve publique à la reconnaissance d'un public: le scandale Sun Zhigang", Politix, vol.18, n71, 2005, p 137-164.
} 
- Bien sûr, il y a de plus en plus de monde, et parmi eux il y a certainement des gens qui ont du pouvoir, peut-être qu'ils participent, et ensuite ils agissent.

- Grâce à la discussion?

- Dès qu'une question est discutée sur Internet, et si c'est une question importante, elle passe dans les médias, par exemple dans les journaux, ou bien au journal télévisé, ou bien ils en parlent dans la presse écrite. Une fois que c'est sorti, le gouvernement y fait peut-être plus attention, surtout le ministère de la surveillance. De cette manière, peut-être que les discussions permettent de résoudre le problème.

- Donc tu veux dire que le gouvernement s'intéresse à ce que les gens veulent rendre public?

- En fait voilà, grâce au fait que les informations sur Internet sont instantanées, et vraies, ça a de la force. Par exemple s'il se passe quelque chose n'importe où, peutêtre que ça va d'abord apparaître sur Internet, et peut-être que d'autres médias n'y auront pas fait attention. Alors tout le monde en discute, tout le monde s'y intéresse beaucoup, et alors les journalistes des médias s'exprimeront aussi, et ils vont faire des enquêtes en profondeur, ils vont vérifier la véracité des faits, si ça s'est bien passé comme ça, et s'il n'y a pas de problème, ils le publieront dans les journaux, aux informations. Ensuite, le ministère de la surveillance du gouvernement va s'y intéresser, et ils vont résoudre le problème." (Chen)

En reprenant des informations qui circulent sur Internet, en les vérifiant et en les étayant par de nouvelles enquêtes, les médias entérinent une nouvelle légitimité de l'opinion publique.

"Depuis toujours en Chine, l'opinion publique, les sentiments du peuple, c'est comme l'eau qui peut porter un bateau ou le renverser. Ca veut dire qu'aujourd'hui les médias se mettent plus souvent dans le rôle de l'opinion publique, pour gagner la sympathie du peuple, ils expriment les sentiments du peuple. Avant les sentiments du peuple n'étaient exprimés que dans les discussions privées, ou bien dans des commentaires sur telle ou telle chose, mais maintenant c'est publié sur la couverture ou bien dans le texte." (Zhou)

Cette situation transforme le traitement médiatique qui est fait sur de nombreuses causes. Au sujet d'une pétition antijaponaise qui avait circulé en ligne, un journaliste de la télévision d'Etat CCTV raconte que "c'est l'opinion publique qui a joué un rôle décisif sur la 
manière dont les médias d'Etat ont traité cette affaire, pas l'inverse. Après la vague de réactions sur Internet, le gouvernement a changé, et nous avons dû changer aussi" ${ }^{45}$. De plus en plus d'éditorialistes de presse osent plaider publiquement pour une libéralisation de l'information qui ne serait qu'une reconnaissance de sa circulation horizontale de fait. ${ }^{46}$

La sortie d'un nouveau magazine entièrement constitué d'extraits de blogs est peut-être encore plus significative. Dans une publicité, ses fondateurs déclarent explicitement que désormais l'opinion publique a sa place comme source d'information.

La croissance d'Internet a ouvert un nouvel espace pour l'opinion publique dans la société chinoise et elle a eu une profonde influence sur la formation et les méthodes de transmission de l'opinion publique dans la société, ce qui change la vie à tous les niveaux.

En tant que transcription directe d'Internet, Blog Weekly se félicite des opportunités historiques d'une société connectée avec un esprit de responsabilité, de partage et d'engagement personnel, et il utilisera le pouvoir d'Internet pour entraîner la Chine vers l'avant.

A l'ère des blogs, nous sommes tous journalistes. ${ }^{47}$

Bien sûr, ce magazine se doit d'effectuer une sélection très serrée des blogs qu'il publie, mais il rend désormais très visible le fait qu'Internet est devenu une source d'information pour les médias plus traditionnels. Cela leur permet de connaître au mieux les attentes et les préoccupations de leur lectorat, dont ils ne peuvent pas trop s'éloigner pour des raisons commerciales.

\footnotetext{
${ }^{45}$ HASKI Pierre. Internet et la Chine, Paris: Seuil, 2008.

${ }^{46}$ YAN Lieshan. "The liberalization of news and the flattening of the society", People's Daily Net, 03/02/2008, traduit sur China Digital Times le 08/02/2009, consulté le 25/10/2010 sur http://chinadigitaltimes.net/2009/02/yan-lieshan-the-liberalization-of-news-and-the-flattening-of-thesociety/

${ }^{47}$ MARTINSEN Joel. "Grass-roots journalism meets the modern news weekly", Danwei, 11/08/2008, consulté le 25/10/2010 sur http://www.danwei.org/magazines/a_crowdsourced_news_weekly.php.
} 


\section{La presse, le Parti et le marché}

L'une des spécificités des médias chinois est en effet d'être à la fois très contrôlés dans leurs contenus, et d'être tenus à la rentabilité économique ${ }^{48}$. Or le public représente un enjeu commercial majeur. En effet, lorsque l'engouement des internautes s'est déjà déclaré pour une affaire, il n'est plus si simple de mettre une sourdine à tous les commentaires. Une thématique excitante pour le public, ou un scandale, représentent des millions de clics, et autant de crédits publicitaires pour le portail en question. Ces portails comme Sina, Sohu ou encore QQ, sont des entreprises commerciales énormes, en concurrence les unes avec les autres, et ce sont des fleurons du développement économique du pays. Ils ne peuvent pas aborder l'actualité à la manière des journaux officiels, dont le lectorat est réduit à peau de chagrin depuis des décennies.

Cette situation est très bien analysée par Lagerkvist au travers d'une série d'entretiens avec des éditeurs de portails d'informations ${ }^{49}$. La bataille du marché force les portails à trouver des angles qui attirent toujours plus de lecteurs, et les incite donc à traiter de l'information politique d'une manière différente des grands organes de presse. En même temps, la propagande elle-même devient plus subtile, car elle repose sur l'audience que peuvent attirer les portails en traitant l'actualité de manière plus ouverte.

Cela signifie donc que l'Etat ne peut pas leur imposer la totalité des thématiques traitées, sous peine d'entraver le développement commercial qu'il encourage par ailleurs. Il est même obligé de payer les portails pour donner toute l'importance qu'il souhaite à certains sujets... Ou au contraire pour censurer des sujets épineux! C'est ce qu'a révélé le scandale du lait contaminé à la mélamine ${ }^{50}$. L'Etat aurait acheté des espaces publicitaires en échange de la coopération de certains sites à la surveillance de ce sujet. De même, l'Etat a recours à des procédés bien connus du marketing, et il paie des moteurs de recherches comme Baidu pour

\footnotetext{
${ }^{48}$ Pour des études de cas sur la position ambivalente des différents organes de presse vis-à-vis de l'Etat et du marché, voir LEE Chin-Chuan (dir.). Power, money, and media. Communication patterns and bureaucratic control in cultural China, Evanston: Northwestern University Press, 2000.

${ }^{49}$ LAGERKVIST Johan. "In the crossfire of demands. Chinese news portals between propaganda and the public", in DAMM Jens et THOMAS Simona, Chinese cyberspaces. Technological changes and political effects, Londres et New York: Routledge, 2006, p. 42-63.

${ }^{50}$ BANDURSKI David. "Conscience sold to the highest bidder in China's 'melamedia'", China Media Project, 25/10/2010, http://cmp.hku.hk/2008/10/09/1275.
} 
faire référencer ses sites ${ }^{51}$. De toute évidence, la répression pure et simple ne suffit plus et la censure devient littéralement payante pour l'Etat ${ }^{52}$ ! Evidemment, cette pratique n'est pas indépendante des questions d'intégrité des journalistes, qui reçoivent souvent de l'argent pour présenter leurs articles de manière plus ou moins flatteuse, voire pour passer sous silence certains scandales $^{53}$. Les sites Internet importants se trouvent désormais au cœur de jeux d'influences impliquant de multiples acteurs individuels, collectifs ou institutionnels. Ce rôle est devenu si évident qu'il a même circulé une blague à ce propos.

- Le journaliste: plus je suis prêt à payer, plus haut je serai dans le référencement?

- Baidu: Oui!

- Je suis de CCTV!

- Pas de problème, du moment que vous payez plus cher que Hunan TV. ${ }^{54}$

De ce rapport de forces, certainement très informel, il est au moins possible de tirer une conclusion simple: le Parti n'a plus le monopole de l'établissement de l'agenda. Désormais, il existe une dynamique qui lui échappe, dont sont responsables un certain nombre d'acteurs, particuliers et institutionnels, selon une chaîne compliquée d'amplifications ou de restrictions successives.

En somme, pour les autorités chinoises, la reconnaissance officielle d'une certaine opinion publique ne se confond pas avec l'idée d'une représentation populaire ni d'une participation politique de la population. Elle est au contraire conçue comme une forme de consultation, mise au service d'une conception technocratique de la légitimité du Parti communiste chinois. Loin d'être anecdotique pour autant, cette irruption de l'opinion sur la

${ }^{51}$ SIJIA. "Baidu's search methodology controversy gets heated up as CCTV steps in", China Digital Times, 16/11/2008, consulté le 25/10/2010 sur http://chinadigitaltimes.net/2008/11/baidus-searchmethodology-being-questioned/.

${ }^{52}$ LUPANO Emma. "In China, speech and silence both come with price tags", China Media Project, 28/11/2008, consulté le 25/10/2010 sur http://cmp.hku.hk/2008/11/28/1390/.

${ }^{53}$ LUPANO Emma. "Linfen 'gag fees' spark media ethics debate in China", China Media Project, 30/10/2008, consulté 25/10/2010 sur http://cmp.hku.hk/2008/10/30/1322/.

${ }^{54}$ SIJIA. "Baidu's search methodology controversy gets heated up as CCTV steps in", China Digital Times, 16/11/2008, consulté le 25/10/2010 sur http://chinadigitaltimes.net/2008/11/baidus-searchmethodology-being-questioned/. 
scène publique contribue à transformer les relations de pouvoir entre les différents échelons du gouvernement.

Les internautes exercent une surveillance de fait des dirigeants locaux sur les problèmes qui les concernent, les contraignant, au moins en apparence, à mieux se conformer aux directives du gouvernement central. Ainsi, ce qui pouvait passer pour un mouvement de "retrait" de l'Etat n'est-il en fait qu'un "recentrage" ${ }^{55}$ autour de l'Etat central, qui se fait probablement au prix d'une plus grande dépendance des échelons de gouvernement locaux. Ce processus peut être considéré comme le pendant indispensable de l'autonomisation et de la responsabilisation des individus dans le processus de modernisation ${ }^{56}$.

Réciproquement, cette "supervision" par l'opinion publique s'accompagne d'efforts très importants pour mieux anticiper les problèmes, et cadrer leur présentation dans des termes compatibles avec les grandes orientations du régime et aussi favorables que possible aux intéressés. L'enjeu majeur pour l'ensemble des acteurs de ce jeu complexe devient donc la définition des normes autour desquelles se fixent les nouveaux rapports de pouvoir. Chaque événement nouveau est l'occasion d'une course de vitesse pour proposer un récit, une interprétation, une définition de ce que doit être une société "moderne".

Plusieurs conditions doivent donc être remplies pour qu'un type de récit soit porté avec succès sur la place publique. Il doit être formulé par des porteurs de causes capables de cadrer efficacement leur propos, c'est à dire de raconter l'événement dans des termes compatibles avec l'idéologie officielle. Il doit toucher une part importante des internautes de manière suffisamment directe pour que certains d'entre eux aient envie, et se sentent en droit, de poster quelques mots de soutien. Cela montre l'importance de la composition sociologique de la population internaute. A mesure que le taux de personnes connectées augmente, en particulier dans les zones les moins favorisées du pays, les sujets ainsi promus pourront évoluer, mais compte tenu de la place majoritaire des citadins dans la population actuelle d'internautes, leurs préoccupations ont bien plus de chances d'être portées publiquement. Enfin, ces questions doivent être relayées à un moment donné par la presse, qui entérine le caractère consensuel

55 SIGLEY Gary. "Chinese governmentalities: government, governance and the socialist market economy", Economy and society, vol.35, n 4, 2006, p. 487-508.

${ }^{56}$ YANG Dali. Remaking the Chinese Leviathan, Stanford University Press, 2004. 
des valeurs défendues, alors même que l'absence de couverture contribuera à renvoyer l'information dans le domaine de la rumeur ou tout simplement dans une "spirale du silence". Seuls certains sujets, parmi les plus consensuels au sein d'une population bien spécifique, peuvent franchir tous ces obstacles. Face à ce mode de fonctionnement, tout le monde n'est cependant pas équipé de la même manière, que ce soit en termes de statuts, de ressources ou de compétences. L'Etat central d'un côté, et certains individus porteurs de l'idéologie de la classe moyenne supérieure de l'autre, semblent les plus à même de tirer leur épingle du jeu, en imposant un système de normes commun. 


\section{Conclusion}

$\mathrm{Au}$ terme de cette démonstration, j'espère avoir montré comment les mobilisations qui ont lieu sur le Web chinois sont cadrées $^{1}$ au travers d'une certaine idéologie dominante. Celleci est le fruit d'un compromis par lequel le PCC a accordé plus d'autonomie aux individus en échange du maintien de son rôle de leader incontestable du pays. Ce compromis est mis en scène dans le discours officiel sous les traits d'une "classe moyenne" jeune, active, entrepreneuse et riche, et qui délaisse les questions politiques au nom de l'unité de la nation et de la stabilité du pays. L'ensemble de la population est invitée à tendre vers ce style de vie, qui est présenté comme un idéal dans de nombreux domaines de la vie quotidienne, notamment au travers de la publicité, ou dans les magazines. Cette norme sociale est comprise de diverses manières, parfois critiquée ou rejetée, parfois embrassée sans réserve, ou seulement endossée en public par les individus. Elle n'est cependant jamais ignorée, comme en témoignent les cinquante entretiens réunis ici.

L'usage d'Internet, et plus particulièrement l'expression personnelle sur Internet, tiennent une place privilégiée au sein de cette idéologie. En effet, la connexion au réseau est vue comme un facteur de développement du pays, et par extension l'usage d'Internet est considéré par les internautes comme une forme de participation à la modernisation de la société. Les répondants associent généralement le fait de tenir un blog ou de donner leur opinion sur un forum comme une manière de prendre en charge leur développement personnel et d'affirmer leur identité de manière "moderne".

Les internautes interrogés revendiquent ainsi une forme d'individualisme, qui selon eux est l'apanage de la jeune génération, et qui les conduit à rejeter toute idée de participation politique. Pourtant, la plupart des répondants manifestent un certain attrait pour des questions d'intérêt public, dès lors qu'elles sont formulées dans des termes compatibles avec leurs centres d'intérêt personnels. Cette "évaporation" ${ }^{2}$ de la politique suggère le fait que l'intérêt

${ }^{1}$ GOFFMAN Erving. Les cadres de l'expérience, Paris: Minuit, 1991.

${ }^{2}$ ELIASOPH Nina. Avoiding politics. How Americans produce apathy in everyday life, Cambridge University Press, 1998. 
déclaré pour la politique est lié au type de scène où s'expriment les individus ainsi qu'à la manière dont les différents problèmes sont formulés.

Cela peut s'expliquer par le fait que l'effort partagé vers la modernisation du pays fonctionne comme un "principe de civilité" ${ }^{3}$, qui codifie les comportements publics des individus. Il n'est pas de bon ton d'adopter en public un comportement qui rompe avec la norme dominante, à partir du moment où celle-ci est considérée comme un fondement de l'ordre social. Cela implique en particulier que très peu d'internautes s'autorisent à critiquer l'idée d'une nation unie et les conditions politiques du développement économique, sous peine d'être l'objet de remontrances par les autres internautes, voire, dans les cas les plus extrêmes, d'être exclus des plates-formes de discussion. Dans un pays où la plupart des individus conçoivent les relations sociales comme très conflictuelles, toute attitude politique hétérodoxe est perçue comme un danger pour la cohésion de la société.

Il est cependant important d'analyser ce principe comme une simple "convention", c'est à dire une codification des comportements en public qui permet de réguler la coexistence des individus, mais qui n'implique pas nécessairement une adhésion profonde de chacun. Cette approche permet d'éclairer toutes les formes de distanciation dont font preuve les internautes, les critiques formulées en privé, mais aussi les nombreuses transgressions et les discours à double sens ${ }^{4}$. En démultipliant les degrés de visibilité et les formes de prise de parole, Internet rend poreuse la séparation entre les scènes publiques et celles qui sont plus privatives. Toute une pluralité d'opinions individuelles circulent ainsi, sous forme de rumeurs et de conversations informelles, dans les interstices de nombreux espaces en clair-obscur. Les individus sont probablement mieux informés des différents problèmes, et d'une manière plus critique, qu'il n'y paraît au premier abord.

Cette civilité laisse également la place à des malentendus, des conflits d'interprétation, et des ajustements au jour le jour sur ce qui se dit et ce qui ne se dit pas publiquement. Du fait de ces tâtonnements, les divergences de vues des internautes sur ce que doit être un style de

\footnotetext{
${ }^{3}$ FERRIE Jean-Noël. Le régime de la civilité en Egypte, Paris: CNRS Editions, 2004.

${ }^{4}$ SCOTT James. Domination and the arts of resistance: hidden transcripts, Yale University Press, 1990.
} 
vie moderne et civilisé peuvent donner lieu à des scandales ${ }^{5}$ inattendus. Loin d'être immuable, la norme évolue chaque jour au gré des événements, et ses dimensions échappent bien souvent au champ d'intervention des autorités, ce qui la rend tour à tour plus restrictive et plus permissive que la seule censure. Il n'est pas rare que le gouvernement soit ainsi pris au piège de sa propre rhétorique ${ }^{6}$, réinterprétée au quotidien et mise à l'épreuve des faits, y compris lorsqu'il s'agit de défendre les droits des citoyens.

A la lumière de ce contexte normatif, les mobilisations en ligne ne sont plus seulement un jeu du chat et de la souris entre des individus revendicatifs et la censure des autorités. Ce sont des jeux complexes d'influences, de filtrages, de cadrages entre les nombreux acteurs impliqués, "porteurs de causes", médias, autorités locales et centrales, et publics divers. Sur cet échiquier, les positions de ces différents acteurs sont très variables. Les "porteurs de causes" effectuent un véritable travail de mise en récit des problèmes, qui résonnent plus ou moins auprès d'autres individus. Ceux-ci, en fonction de leurs préoccupations personnelles, de leurs compétences, de leur personnalité ou d'autres facteurs encore, peuvent ignorer, se contenter de lire ou transférer un article, mais ils peuvent aussi y répondre par un bref commentaire en ligne. Ils jouent ainsi un rôle central de chambre d'écho pour de nombreuses causes. Les médias traditionnels, enfin, entérinent ou non l'intérêt de ces causes et les valeurs qui les sous-tendent, en leur offrant une couverture plus ou moins importante, ou en ouvrant leurs colonnes à des experts, qui cadrent à leur tour le débat dans des termes spécifiques.

Les conséquences de ces mobilisations sur les autorités sont ambivalentes. Pour préserver une image de transparence et de "bonne gouvernance", les autorités sont obligées d'intervenir pour mettre fin aux injustices dénoncées, au moins en apparence. La maîtrise de ce processus engendre une stratégie de propagande plus subtile, et qui intervient très en amont des problèmes. Cependant l'Etat central peut tirer parti de la surveillance des dirigeants exercée de fait par les internautes au niveau local, car elle est plus efficace que la bureaucratie pour déceler les dysfonctionnements au sein du Parti. Entre les doctrines de "direction" de l'opinion publique d'une part, et de "supervision" de l'appareil du Parti par l'opinion publique

${ }^{5}$ DE BLIC Damien et LEMIEUX Cyril. "Le scandale comme épreuve", dossier de Politix, vol.18 $\mathrm{n}^{\circ} 71,2005$.

${ }^{6}$ LYALL Jason. "Pocket protests. Rhetorical coertion and the micropolitics of collective action in semiauthoritarian regimes", World politics, vol. 58, n³, 2006, p. 378-412. 
d'autre part, le gouvernement dispose d'une voie très étroite, car il n'a plus le monopole de l'établissement de l'agenda politique.

Il convient donc de tirer un bilan nuancé du rôle d'Internet dans le fonctionnement du régime politique chinois. Au regard de la manière dont les citoyens conçoivent l'impact de leur parole sur les décisions qui les concernent, il est impossible de parler de démocratisation $\mathrm{du}$ pays. Internet ne change pas l'apparent désintérêt des citoyens pour la politique "politicienne". Il ne les conduit pas à remettre en question la place qui leur est réservée dans l'organisation du régime. En interpellant parfois les autorités sur des questions qui les préoccupent, ils semblent parfois se comporter en usagers ou en clients plutôt qu'en citoyens. De ce point de vue, Internet ressemblerait presque à un instrument de sondage de l'opinion publique, un outil d'aide à la décision au service d'un gouvernement technocratique ${ }^{7}$.

Cela souligne cependant à quel point l'opinion publique est centrale dans la légitimation du régime politique chinois. Invoquée de toutes parts, sa définition et sa représentation font l'objet d'une lutte d'influence permanente, qui se joue de plus en plus sur Internet, mais aussi au travers des autres médias. C'est en ralliant des publics à leur cause que les citoyens ordinaires peuvent dénoncer des injustices, obtenir des changements de comportement de leurs dirigeants, ou encore faire évoluer la loi. Ils disposent pour cela d'argumentaires qui s'appuient sur des valeurs partagées, des représentations idéales de la société chinoise. Cellesci sont parfois remises en question, réinterprétées, mises à l'épreuve de nouveaux événements. Internet est ainsi le théâtre de nombreux conflits, qui naissent dans les problèmes du quotidien et qui mobilisent de nombreux individus ordinaires. Le partage d'informations, l'acquiescement, l'indignation, le témoignage individuel, et mille autres petites actions quotidiennes que les internautes font presque incidemment en ligne, constituent ainsi un mode de participation politique si fluide qu'il en est presque inconscient. Il n'en est pas moins puissant.

\footnotetext{
${ }^{7}$ C'est ce que suggère BEJA Jean-Philippe. "The changing aspects of civil society in China".
} 


\section{Liste des entretiens}

La liste ci-dessous réunit les profils des cinquante répondants par ordre alphabétique. Les trois groupes principaux sont donc mélangés: jeunes actifs de 20-30 ans (30 personnes), actifs de 30-40 ans (10 personnes) et étudiants (10 répondants). Dans un souci de confidentialité, seules les initiales des répondants ont été conservées.

Les niveaux de revenus sont présentés sous des formes différentes selon les groupes parce qu'ils ont été recrutés selon un protocole légèrement différent (indication de fourchette ou question ouverte sur le revenu). Cela est dû au délai important qui a séparé le recrutement des différents groupes. De plus certains répondants ont préféré se situer dans une fourchette de revenus plutôt que de donner une information très précise. Dans ce cas de figure, j'ai préféré restituer l'information telle quelle plutôt que d'inférer un niveau de revenu qui aurait été de toute manière approximatif.

Les termes Zhuanke, Benke et Master indiquent les diplômes obtenus par les répondants (ou en cours de préparation dans le cas des étudiants). Le Zhuanke est une formation de premier cycle, généralement professionnalisante, en deux ans. Le Benke est un diplôme universitaire obtenu en quatre ans, reconnu en France par une équivalence de niveau licence. Le Master s'obtient généralement en deux ans après le Benke. Quant au Gaokao, qui est à peu près l'équivalent d'un baccalauréat, il valide la fin des études secondaires et constitue surtout un concours d'entrée à l'université. La mention "Gaokao" ici n'indique donc pas nécessairement une réussite à ce concours, mais elle signifie seulement que le répondant a suivi des études secondaires jusqu'au lycée (ce qui est le cas d'une grande majorité de la population urbaine).

On notera dans cet échantillon une forte sur-représentation du niveau d'études "Benke" à cause du groupe d'étudiants qui préparent tous ce diplôme. D'autre part les personnes n'ayant pas poursuivi leurs études jusqu'au Gaokao sont un peu moins nombreuses (9 personnes) car elles se sont révélées très difficiles à recruter au sein de la population internaute. On peut également imputer cette difficulté à l'introduction d'une question sur l'intérêt pour l'actualité, qui tend également à biaiser l'échantillon en faveur des personnes les plus éduquées. 


\begin{tabular}{|c|c|c|c|c|c|c|c|}
\hline PSEUDONYME & GENRE & AGE & $\begin{array}{c}\text { NIVEAU } \\
\text { D'ETUDES }\end{array}$ & $\begin{array}{c}\text { ACTIVITE } \\
\text { PROFESSIONNELLE }\end{array}$ & $\begin{array}{l}\text { REVENU EN } \\
\text { RMB }\end{array}$ & $\begin{array}{c}\text { DISTRICT DE } \\
\text { RESIDENCE OU } \\
\text { UNIVERSITE }\end{array}$ & $\begin{array}{c}\text { DATE DE } \\
\text { L'ENTRETIEN }\end{array}$ \\
\hline CAI & $\mathrm{F}$ & 30 & Gaokao & Secrétaire dans une usine & 4500 & Chao Yang & $25 / 07 / 2007$ \\
\hline CAO & $\mathrm{H}$ & 35 & Zhuanke & $\begin{array}{l}\text { Commercial dans la gestion } \\
\text { immobilière }\end{array}$ & 3200 & Xuan Wu & $01 / 08 / 2007$ \\
\hline CHEN & $\mathrm{H}$ & 37 & Master & $\begin{array}{l}\text { Dirigeant, entreprise de } \\
\text { finance }\end{array}$ & $>10000$ & Hai Dian & $16 / 07 / 2007$ \\
\hline CHU & $\mathrm{H}$ & 26 & Zhuanke & Cuisinier & 2500 & Feng Tai & $14 / 06 / 2007$ \\
\hline DAI & $\mathrm{H}$ & 26 & Benke & Cadre dans le bâtiment & 6000 & Chao Yang & $16 / 05 / 2007$ \\
\hline DENG & $\mathrm{F}$ & 28 & $\begin{array}{c}2 \text { années } \\
\text { d'université }\end{array}$ & Femme au foyer & 0 & Feng Tai & $18 / 06 / 2007$ \\
\hline DONG & $\mathrm{H}$ & 26 & Benke & Coach sportif & 20000 & Xuan Wu & $01 / 06 / 2007$ \\
\hline DU & $\mathrm{F}$ & 24 & Benke & $\begin{array}{l}\text { Employé de bureau dans } \\
\text { l'immobilier }\end{array}$ & $3000-4999$ & Xi Cheng & $12 / 11 / 2006$ \\
\hline FANG & $\mathrm{H}$ & 22 & $\begin{array}{c}\text { Benke, 3ème } \\
\text { année }\end{array}$ & Etudiant en comptabilité & NA & $\begin{array}{c}\text { Ren Min University of } \\
\text { China }\end{array}$ & $19 / 11 / 2006$ \\
\hline GAO & $\mathrm{F}$ & 25 & Gaokao & Employée & 2000 & Xi Cheng & $19 / 05 / 2007$ \\
\hline GONG & $\mathrm{H}$ & 25 & Gaokao & Chef d'entreprise & 12000 & Chong Wen & $25 / 06 / 2007$ \\
\hline GU & $\mathrm{F}$ & 21 & $\begin{array}{c}\text { Benke, 4ème } \\
\text { année }\end{array}$ & Etudiant en commerce & NA & $\begin{array}{c}\text { Beijing Science } \\
\text { Technology and } \\
\text { Management College }\end{array}$ & $15 / 11 / 2006$ \\
\hline GUO & $\mathrm{F}$ & 23 & Benke & $\begin{array}{l}\text { Cadre dans une entreprise } \\
\text { d'assurances }\end{array}$ & 5200 & Chong Wen & $15 / 06 / 2007$ \\
\hline HAN & $\mathrm{H}$ & 28 & Gaokao & Chef d'entreprise & 15000 & Chong Wen & $27 / 06 / 2007$ \\
\hline HE & $\mathrm{H}$ & 25 & $\begin{array}{c}\text { Master, 3ème } \\
\text { année }\end{array}$ & $\begin{array}{c}\text { Etudiant en biologie } \\
\text { moléculaire }\end{array}$ & NA & $\begin{array}{l}\text { Beijing University of } \\
\text { Chinese Medicine }\end{array}$ & $20 / 11 / 2006$ \\
\hline HONG & $\mathrm{H}$ & 23 & Benke & Etudiant en gestion & NA & $\begin{array}{c}\text { University of Science } \\
\text { and Technology } \\
\text { BeiJing }\end{array}$ & $18 / 11 / 2006$ \\
\hline HuAng & $\mathrm{F}$ & 21 & Zhuanke & $\begin{array}{l}\text { Employée dans une usine } \\
\text { chimique }\end{array}$ & 1500 & Feng Tai & $11 / 06 / 2007$ \\
\hline JIANG & $\mathrm{H}$ & 26 & Benke & Vendeur de chaussures & $3000-4999$ & Hai Dian & $10 / 11 / 2006$ \\
\hline LEI & $\mathrm{F}$ & 27 & Benke & Enseignante & $3000-4999$ & Dong Cheng & 03/11/2006 \\
\hline LI & $\mathrm{F}$ & 21 & $\begin{array}{c}\text { Benke, 3ème } \\
\text { année }\end{array}$ & Etudiante en cinéma & NA & $\begin{array}{l}\text { Beijing Normal } \\
\text { University }\end{array}$ & $14 / 11 / 2006$ \\
\hline
\end{tabular}




\begin{tabular}{|c|c|c|c|c|c|c|c|}
\hline PSEudonyme & GENRE & AGE & $\begin{array}{l}\text { NIVEAU } \\
\text { D'ETUDES }\end{array}$ & $\begin{array}{c}\text { ACTIVITE } \\
\text { PROFESSIONNELLE }\end{array}$ & $\begin{array}{l}\text { REVENU EN } \\
\text { RMB }\end{array}$ & $\begin{array}{l}\text { DISTRICT DE } \\
\text { RESIDENCE OU } \\
\text { UNIVERSITE }\end{array}$ & $\begin{array}{c}\text { DATE DE } \\
\text { L'ENTRETIEN }\end{array}$ \\
\hline LiANG & $\mathrm{F}$ & 25 & Benke & $\begin{array}{l}\text { Employée dans une } \\
\text { entreprise de design }\end{array}$ & $5000-9999$ & Xuan Wu & $11 / 11 / 2006$ \\
\hline Lin & $\mathrm{H}$ & 28 & Zhuanke & Coach sportif & $5000-9999$ & Chong Wen & $24 / 10 / 2006$ \\
\hline LiU & $\mathrm{F}$ & 21 & $\begin{array}{c}\text { Benke, 4ème } \\
\text { année }\end{array}$ & Etudiante en finances & NA & \begin{tabular}{|c|} 
Central University of \\
Finance and Economics
\end{tabular} & $16 / 11 / 2006$ \\
\hline Lu & $\mathrm{F}$ & 39 & Zhuanke & Employée de maison & 2000 & Chao Yang & $30 / 07 / 2007$ \\
\hline LuO & $\mathrm{F}$ & 25 & Gaokao & Femme au foyer & 0 & Shi Jingshan & 19/06/2007 \\
\hline MA & $\mathrm{F}$ & 21 & Zhuanke & Secrétaire dans l'immobilier & 2200 & Chong Wen & 05/06/2007 \\
\hline PAN & $\mathrm{F}$ & 24 & Zhuanke & $\begin{array}{c}\text { Employé de bureau dans une } \\
\text { agence de voyages }\end{array}$ & 5000 & Xuan Wu & $15 / 05 / 2007$ \\
\hline QIAN & $\mathrm{H}$ & 22 & $\begin{array}{c}\text { Benke, 4ème } \\
\text { année }\end{array}$ & $\begin{array}{c}\text { Etudiant en sport (tennis de } \\
\text { table) }\end{array}$ & NA & $\begin{array}{l}\text { Beijing Sport } \\
\text { University }\end{array}$ & $23 / 11 / 2006$ \\
\hline REN & $\mathrm{H}$ & 25 & Benke & Marketing & $>10000$ & Haidian & $00 / 08 / 2006$ \\
\hline SHAO & $\mathrm{F}$ & 27 & Benke & $\begin{array}{l}\text { Professeur de piano et } \\
\text { assistante maternelle }\end{array}$ & & Chong Wen & $08 / 11 / 2006$ \\
\hline SHEN & $\mathrm{H}$ & 22 & Zhuanke & Aide soignant & Below 300 & Shi Jingshan & $24 / 10 / 2006$ \\
\hline SHI & $\mathrm{F}$ & 21 & $\begin{array}{c}\text { Benke, 3ème } \\
\text { année }\end{array}$ & Etudiant en sciences de la vie & NA & Beijing University & $21 / 11 / 2006$ \\
\hline SONG & $\mathrm{H}$ & 27 & Benke & Consultant sportif & $3000-4999$ & Hai Dian & $10 / 11 / 2006$ \\
\hline Sun & $\mathrm{F}$ & 23 & $\begin{array}{l}\text { Benke, 3ème } \\
\text { année }\end{array}$ & Etudiante en anglais & NA & $\begin{array}{l}\text { China Women's } \\
\text { University }\end{array}$ & $22 / 11 / 2006$ \\
\hline TANG & $\mathrm{H}$ & 21 & Gaokao & $\begin{array}{l}\text { Secrétaire dans la gestion } \\
\text { immobiliaire }\end{array}$ & 1200 & Hai Dian & $11 / 06 / 2007$ \\
\hline TIAN & $\mathrm{H}$ & 27 & $\begin{array}{c}2 \text { ans } \\
\text { d'université }\end{array}$ & $\begin{array}{c}\text { Employé dans une entreprise } \\
\text { étrangère }\end{array}$ & 3500 & Feng Tai & $20 / 06 / 2007$ \\
\hline WANG & $\mathrm{H}$ & 23 & Benke & Ouvrier & 2800 & Shi Jingshan & $18 / 05 / 2007$ \\
\hline Wu & $\mathrm{H}$ & 28 & Benke & Commercial dans un hôtel & $>10000$ & Dong Cheng & $17 / 10 / 2006$ \\
\hline XIE & $\mathrm{H}$ & 35 & Benke & Cadre dans l'immobilier & 4500 & Chao Yang & $31 / 07 / 2007$ \\
\hline $\mathbf{X u}$ & $\mathrm{H}$ & 33 & Zhuanke & Ouvrier dans le bâtiment & 2000 & Xi Cheng & $24 / 07 / 2007$ \\
\hline YANG & $\mathrm{F}$ & 32 & Zhuanke & Employée dans l'automobile & 3200 & Hai Dian & $26 / 07 / 2007$ \\
\hline YAO & $\mathrm{H}$ & 20 & Gaokao & Serveur & 1500 & Chao Yang & $07 / 06 / 2007$ \\
\hline
\end{tabular}




\begin{tabular}{|c|c|c|c|c|c|c|c|}
\hline PSEUdonyme & GENRE & AGE & $\begin{array}{l}\text { NIVEAU } \\
\text { D'ETUDES }\end{array}$ & $\begin{array}{c}\text { ACTIVITE } \\
\text { PROFESSIONNELLE }\end{array}$ & $\begin{array}{l}\text { REVENU EN } \\
\text { RMB }\end{array}$ & $\begin{array}{l}\text { DISTRICT DE } \\
\text { RESIDENCE OU } \\
\text { UNIVERSITE }\end{array}$ & $\begin{array}{c}\text { DATE DE } \\
\text { L'ENTRETIEN }\end{array}$ \\
\hline YE & $\mathrm{F}$ & 23 & Benke & $\begin{array}{c}\text { Cadre dans une agence de } \\
\text { voyages }\end{array}$ & 5000 & Chao Yang & $13 / 06 / 2007$ \\
\hline Yuan & $\mathrm{F}$ & 26 & Benke & Ingénieur & $3000-4999$ & Xi Cheng & $04 / 11 / 2006$ \\
\hline ZENG & $\mathrm{H}$ & 25 & Benke & $\begin{array}{l}\text { Cadre dans le domaine } \\
\text { médical }\end{array}$ & 8000 & Chao Yang & 06/06/2007 \\
\hline ZHANG & $\mathrm{H}$ & 26 & Benke & Décorateur & $3000-4999$ & Chao Yang & $05 / 11 / 2006$ \\
\hline ZHAO & $\mathrm{H}$ & 30 & Benke & $\begin{array}{l}\text { Cadre dans une entreprise de } \\
\text { communication }\end{array}$ & 4000 & Chao Yang & $17 / 07 / 2007$ \\
\hline ZHONG & $\mathrm{F}$ & 38 & Zhuanke & $\begin{array}{l}\text { Secrétaire dans une } \\
\text { entreprise d'assurances }\end{array}$ & 2500 & Chao Yang & 19/07/2007 \\
\hline ZHOU & $\mathrm{H}$ & 24 & Benke & $\begin{array}{l}\text { Dirigeant d'un magasin } \\
\text { d'électroménager }\end{array}$ & 10000 & Feng Tai & 04/06/2007 \\
\hline ZHU & $\mathrm{H}$ & 23 & $\begin{array}{c}\text { Master, 2ème } \\
\text { année }\end{array}$ & Etudiant ingénieur & NA & Beihang University & $17 / 11 / 2006$ \\
\hline
\end{tabular}




\section{Bibliographie}

\section{Ouvrages généraux}

ALDRIN Philippe. Sociologie politique des rumeurs, Paris: PUF, 2005.

ARENDT Hannah. Condition de l'homme moderne, Paris: Calmann-Lévy, 1961.

ARENDT Hannah. Qu'est-ce que la politique?, Paris: Seuil, 1995.

BAUMAN Zygmunt. Liquid Modernity, Cambridge: Polity, 2000.

BAYART Jean-François, MBEMBE Joseph-Achille et TOULABOR Comi. Le politique par le bas en Afrique noire, Paris, Karthala, 1992.

BECKER Howard. Outsiders, Paris: Métailié, 1985.

BENTHAM Jeremy et FOUCAULT Michel. Le Panoptique, Paris: Belfond, 1977.

BIRNBAUM Pierre et LECA Jean (dir.). Sur l'individualisme, Paris: Presses de la FNSP, 1986.

BLONDIAUX Loïc. La fabrique de l'opinion : une histoire sociale des sondages, Paris: Seuil, 1998.

BOLTANSKI Luc et THEVENOT Laurent. De la justification. Les économies de la grandeur, Paris: PUF, 1987.

BOLTANSKI Luc, CLAVERIE Elisabeth, OFFENSTADT Nicolas, Van DAMME Stéphane (dir.). Affaires, scandales et grandes causes, de Socrate à Pinochet, Paris: Stock, 2007.

BOLTANSKI Luc. Les cadres: la formation d'un groupe social, Paris: Minuit, 1982

BOURDIEU Pierre. Ce que parler veut dire : l'économie des échanges linguistiques, Paris: Fayard, 1982.

BOURDIEU Pierre. La distinction : critique sociale du jugement, Paris: Minuit, 1979.

CALHOUN Craig (dir). Habermas and the public sphere, Cambridge, Massachusetts.: MIT Press, 1992.

CEFAI Daniel. Pourquoi se mobilise-t-on?, Paris: La Découverte, 2007.

CERTEAU Michel. La prise de parole, Paris: Seuil, 1994.

CERTEAU Michel. L'invention du quotidien 1. Arts de faire, Paris: Gallimard, 1990.

DABENE Olivier, GEISSER Vincent et MASSARDIER Gilles (dir.). Autoritarismes

démocratiques et démocraties autoritaires au XXIè siècle. Convergences Nord/Sud, Paris: La Découverte, 2008.

DEWEY John. Le public et ses problèmes, Paris: L. Sheer, 2003. 
Education counts benchmarking progress in 19 WEI Countries, World Education Indicators 2007, Montreal: UNESCO Institute for Statistics, 2007, consulté le 23/12/2008 sur http://www.uis.unesco.org/template/pdf/wei/2007/WEI2007report.pdf.

ELIASOPH Nina. Avoiding politics. How Americans produce apathy in everyday life, Cambridge University Press, 1998.

FERRIE Jean-Noël. Le régime de la civilité en Egypte, Paris: CNRS Editions, 2004.

FISHKIN James. The voice of the people. Public opinion and democracy, New Haven et Londres: Yale University Press, 1995.

FOUCAULT Michel. Histoire de la sexualité. 1 La volonté de savoir, Paris: Gallimard, 1976. FOUCAULT Michel. Surveiller et punir, Paris: Gallimard, 1975.

FRANCOIS, Bastien, et NEVEU, Erik (dir.), Espaces publics mosaïques, acteurs, arènes et rhétoriques des débats publics contemporains, Presses universitaires de Rennes, 1999.

GAMSON William. Talking Politics, Cambridge: Cambridge University Press, 1992.

GAXIE Daniel. Le cens caché: inégalités culturelles et ségrégation politique, Paris: Seuil, 1978.

GIDDENS Anthony. Modernity and self-identity: self and society in the late modern age, Stanford University Press, 1991.

GOFFMAN Erving. Façons de parler, Paris: Minuit, 1987.

GOFFMAN Erving. La mise en scène de la vie quotidienne, Paris: Minuit, 1979, 2 vol.

GOFFMAN Erving. Les cadres de l'expérience, Paris: Minuit, 1991.

GOFFMAN Erving. Les moments et leurs hommes, Paris: Seuil, 1988.

GRAMSCI Antonio. Cahiers de prison. 1, Cahiers 1, 2, 3, 4, 5, Paris : Gallimard, 1996.

GUSFIELD Joseph. La culture des problèmes publics: l'alcool au volant: la production d'un ordre symbolique, Paris: Economica, 2009.

HABERMAS Jürgen. L'espace public, Paris, Payot, 1992.

HIRSCHMAN Albert. Bonheur privé, action publique, Paris: Fayard, 1983.

HIRSCHMAN Albert. Exit, voice, and loyalty: responses to decline in firms, organizations, and states, Cambridge: Harvard University Press, 1970, traduction française: Défection et prise de parole, Paris: Fayard, 1995.

HOBBES Thomas. Léviathan : traité de la matière, de la forme et du pouvoir de la république ecclésiastique et civile, Paris : Sirey, 1971.

ION Jacques (dir.). L'engagement politique, Saint-Etienne: Publications de l'Université, 2001.

ION Jacques. La fin des militants?, Paris: L'Atelier, 1997.

JASPER James. The art of moral protest. Culture, biography and creativity in social movements, Chicago: University of Chicago Press, 1997.

LAHIRE Bernard. L'homme pluriel : les ressorts de l'action, Paris: Nathan,1998. 
LE BART Christian. L'individualisation, Paris: Presses de Sciences-po, 2008.

LIPOVETSKY Gilles. L'ère du vide : essais sur l'individualisme contemporain, Paris :

Gallimard, 1983.

MAIGRET Eric. Sociologie de la communication et des médias, Paris: Armand Colin, 2003.

MAYER Nonna et PERRINEAU Pascal. Les comportements politiques, Paris: Armand Colin, 1992.

MILLS Charles Wright. Les cols blancs : essai sur les classes moyennes américaines, Paris: Seuil, 1970.

MOORE Barrington Jr. Injustice. The social bases of obedience and revolt, New York: M.E. Sharpe, 1978.

MUXEL Anne. Les jeunes et la politique, Paris: Hachette, 1996.

QUERE Louis. Des miroirs équivoques, Paris: Aubier Montaigne, 1982.

REGAMEY Amandine. Dérision et politique en URSS. Le rire contre la légitimité du pouvoir soviétique. Thèse de doctorat, IEP Paris, 2004.

REYNIE Dominique. Le triomphe de l'opinion publique, Paris: Odile Jacob, 1998.

SCOTT James. Domination and the arts of resistance: hidden transcripts, Yale University Press, 1990.

SENNETT Richard. Les tyrannies de l'intimité, Paris: Seuil, 1979.

SIMEANT Johanna. La cause des sans papiers, Paris: Presses de Sciences-po, 1998.

SUNSTEIN Cass. Republic.com, Princetown: Princetown University Press, 2001.

THOMPSON Edward Palmer. La Formation de la classe ouvrière anglaise, Paris: Seuil, 1988.

WEBER Max. Economie et société, Paris: Pocket, 1995.

\section{Articles}

"La compétence politique", dossier, Revue Française de Science Politique, vol. 57, n6, 2007.

BAYART Jean-François. "L'énonciation du politique", Revue française de science politique, vol. $35, \mathrm{n}^{\circ} 3,1985$, p. 343-373.

BERNSTEIN Mary et JASPER James. "Les tireurs d'alarme dans les conflits sur les risques technologiques. Entre intérêts particuliers et crédibilité", Politix, vol. 11, n 44, 1998, p. 109-134.

BLEIL Susana. "Avoir un visage pour exister publiquement: l'action collective des sans terre au Brésil", Réseaux n¹29-130, 2005, p.123-154.

BLONDIAUX Loïc et SINTOMER Yves. "L'impératif délibératif", Politix, vol.15, n57, 2002, p 17-35. 
CEFAI Daniel. "La construction des problèmes publics. Définitions de situations dans des arènes publiques", Réseaux, n75, 1996, p. 43-65.

CLAVERIE Elisabeth. "La naissance d'une forme politique : l'Affaire du Chevalier de La Barre", in ROUSSIN Philippe (dir.). Critique et affaires de blasphème à l'époque des Lumières, 1998, Paris: Honoré Champion, p.185-260.

CONVERSE Philip. "The nature of belief systems in mass publics", in APTER David (dir.), Ideology and discontent, New York: Free Press, 1964.

DE BLIC Damien et LEMIEUX Cyril. "Le scandale comme épreuve", dossier de Politix, vol. $18 \mathrm{n}^{\circ} 71,2005$.

DUPRET Baudouin et FERRIE Jean-Noel. "Participer au pouvoir, c'est édicter la norme: sur l'affaire Abu Zayd (Egypte, 1992-1996)", RFSP, vol. 47, n6, 1997, p. 762-775.

FERRIE Jean-Noël. "La gifle, sur la mise en place d'un espace public 'municipal' au Maroc", Politique africaine, $\mathrm{n}^{\circ} 74$, juin 1999.

FERRIE Jean-Noël. "Lieux intérieurs et culture publique au Maroc", Politix, vol. 8, n 31 , 1995, p. 187-202.

FOUCAULT Michel. "L'écriture de soi", in Correspondance Dits et Ecrits : tome $I V$, texte ${ }^{\circ}$ 329.

FRASER Nancy. "Repenser la sphère publique: une contribution à la critique de la démocratie telle qu'elle existe réellement", Hermès, n³1, 2001, p. 125-158.

GAXIE Daniel. "Cognition, auto-habilitation et pouvoirs des 'citoyens'", RFSP, vol.57, n6, 2007, p737-758.

GERSTLE Jacques. "Gouverner l'opinion publique", in BRECHON Pierre (dir.), La gouvernance de l'opinion publique, Paris: L'Harmattan, 2003, p. 19-34.

GRANOVETTER Mark. "The strength of weak ties", The American Journal of Sociology, vol. $78, \mathrm{n}^{\circ} 6$, mai 1973 , p. 1360-1380.

HALL Stuart. "Codage/décodage", Réseaux, vol. 12, n68, 1994, p. 27-39.

HERMET Guy. "La gouvernance serait-elle le nom de l'après-démocratie? L'inlassable quête du pluralisme limité", in HERMET Guy, KAZANCIGIL Ali et PRUD'HOMME JeanFrançois (dir.). La gouvernance: un concept et ses applications, Paris: Karthala, 2005.

HONNETH Axel. "Invisibilité: sur l'épistémologie de la "reconnaissance", Réseaux, vol.23, n¹29-130, 2005, p. 39-58.

JOIGNANT Alfredo. "Compétence politique et bricolage. Les formes profanes du rapport au politique", Revue française de science politique, vol. 57, nº 6, p. 799-817.

LECA Jean. "Les paradoxes de la société civile", Sociétal, n9, juin 1997, p.59-62.

LIVINGSTONE Sonia et LUNT Peter. "Se faire entendre dans l'espace public. Les femmes, la télévision et le citoyen-spectateur", Réseaux, vol.12, n63, 1994, p. 59-74.

LYALL Jason. "Pocket protests. Rhetorical coertion and the micropolitics of collective action in semiauthoritarian regimes", World politics, vol. 58, n³, 2006, p. 378-412. 
NEVEU Erik. "Médias, mouvements sociaux, espaces publics", Réseaux, n98, 1999, p.1785.

NOELLE-NEUMANN Elisabeth. "The theory of public opinion : the concept of the spiral of silence", Communication Yearbook, n¹4, 1991, p. 256-287.

PUTNAM Robert. "Bowling alone : America's declining social capital", Journal of Democracy, vol. 6, $\mathrm{n}^{\circ} 1,1995$, p. 65-78.

QUERE Louis. "Opinion: l'économie du vraisemblable. Introduction à une approche praxéologique de l'opinion publique", Réseaux, vol. 8 n43, 1990, p. 33-58.

SANDERS Lynn. "Against deliberation", Political theory, vol. 25, n³, p. 347-376.

SCHNEIDER Steven. "Creating a democratic sphere through political discussion", Social science computer review, vol.14, n% , p. 373-393.

SCHUDSON Michael. "Why conversation is not the soul of democracy", Critical studies in mass communication, $\mathrm{n}^{\circ} 14,1997$, p. 297-309.

SOMERS Margaret. "Citizenship and the place of public sphere: law, community, and political culture in the transition to democracy", American sociological review, vol 58, n5, 1993.

THOMPSON John B., "La nouvelle visibilité", in VOIROL Olivier (dir.), Visibilité/invisibilité", Réseaux, vol.23, n¹29-130, 2005

TOURAINE Alain. "Classes moyennes", Encyclopaedia Universalis [CD ROM], 2008.

VOIROL Olivier. "Les luttes pour la visibilité", Réseaux, n¹29-130, 2005, p. 89-122.

\section{Internet et politique (Europe et monde anglo-saxon)}

BLANCHARD Gersende. La communication politique partisane sur Internet: des pratiques et des stratégies nouvelles?, Thèse pour le doctorat en Sciences de l'information et de la communication, Université Stendhal Grenoble 3, 3 vol, 2007.

COLEMAN Stephen et GOTZE John. Bowling together: Online Public Engagement in Policy Deliberation, London: Hansard society, consulté en ligne le 04/11/2008 sur http://bowlingtogether.net.

FLICHY Patrice. L'imaginaire d'Internet, Paris: La Découverte, 2001.

GRANJON Fabien. L'Internet militant, Rennes: Apogée, 2001.

MCCAUGHEY Martha et AYERS Michael D. Cyberactivism, New York et Londres:

Routledge, 2003.

RHEINGOLD Howard. The virtual community : homesteading on the electronic frontier, Cambridge: MIT Press, 2000.

WOJCIK Stéphanie. Délibération électronique et démocratie locale: le cas des forums municipaux des régions Aquitaine, Languedoc-Roussillon et Midi-Pyrénées, Thèse pour l'obtention du doctorat de science politique, Université Toulouse 1, 2005. 


\section{Articles}

AGUITON Christophe et CARDON Dominique. "The strength of weak cooperation : an attempt to understand the meaning of Web2.0 ", Communications \& Strategies, n ${ }^{\circ} 65,2007, \mathrm{p}$. 51-65.

AURAY Nicolas, HURAULT-PLANTET Martine, POUDAT Céline et JACQUEMIN Bernard. "La négociation des points de vue. une cartographie sociale des conflits et des querelles dans le Wikipédia francophone", Réseaux, $\mathrm{n}^{\circ}$ 154, 2009, p.15-50.

CARDON Dominique et DELAUNAY-TETEREL Hélène. "La production de soi comme technique relationnelle. Un essai de typologie des blogs par leurs publics", Réseaux, ${ }^{\circ} 138$, 2006, p. 15-72.

CARDON Dominique et HEURTIN Jean-Philippe. "La critique en régime d'impuissance, une lecture des indignations des auditeurs de France Inter", in FRANCOIS Bastien et NEVEU Erik (dir.). Espaces publics mosaïques, acteurs, arènes et rhétoriques des débats publics contemporains, Rennes: Presses universitaires de Rennes, 1999, p 85-119.

CARDON Dominique, HEURTIN Jean-Philippe et LEMIEUX Cyril. "Parler en public", Politix, vol. 8, n³1, p. 5-19.

CARDON Dominique. "Comment se faire entendre? La prise de parole publique des auditeurs de RTL", Politix, vol.8 n³1, 1995, p145-186.

CARDON Dominique. "Le design de la visibilité. Un essai de cartographie du Web 2.0", Réseaux, $\mathrm{n}^{\circ} 152$, 2008, p.93-137.

FLICHY Patrice. "Internet, un outil de la démocratie?", laviedesidees.fr, 2008.

FLUCKIGER Cédric. "La sociabilité juvénile instrumentée. L'appropriation des blogs dans un groupe de collégiens", Réseaux, vol.24, n¹38, 2006, p. 109-138.

GRANJON Fabien. "Les répertoires d'action télématiques du néo-militantisme", Le Mouvement social, $\mathrm{N}^{\circ} 200,2002$, p. 11-32.

GREFFET Fabienne et WOJCIK Stéphanie. "Parler politique en ligne. Une revue des travaux français et anlo-saxons", Réseaux, vol. 26, n¹50, p. 19-50.

LEV-ON Azi et MANIN Bernard. "Internet: la main invisible de la délibération", Esprit, n5, 2006, p.195-212.

PAPACHARISSI Zizi. "Democracy online: civility, politeness, and the democratic potential online political discussion groups", New media and society, vol.6, n² 2, 2004, p. 259-283.

SAVIGNY Heather. "Public opinion, political communication and the Internet", Politics, vol. 22, $\mathrm{n}^{\circ} 1,2002$, p. 1-8.

TRAUTMANN Flore. "Internet au service de la démocratie? Le cas d'ATTAC", Les Cahiers $d u$ CEVIPOF, $\mathrm{n}^{\circ} 30$. 
WITSCHGE Tamara. "Online deliberation: possibilities of the Internet for deliberative democracy", in SHANE Peter (dir.) Democracy online. Prospects for political renewal through the Internet, London: Routledge, 2004, p. 109-122.

WOJCIK Stéphanie. "Les modérateurs des forums de discussion municipaux, des intermédiaires démocratiques?", Questions de communication, n¹2, p. 335-354.

WOJCIK Stéphanie. "The Three key roles of moderator in municipal online forums", communication à Politics: Web 2.0: An international conference, Royal Holloway, University of London, 2008.

WRIGHT Scott et STREET John. "Democracy, deliberation and design: the case of online discussion forums", New media \& society, vol.9, n5, p. 849-869.

\section{Internet et politique (contextes autoritaires)}

ALAVI Nasrin. We are Iran, Londres: Portobello Books, 2006.

BOAS Taylor et KALATHIL Shanthi. Open networks, closed regimes: the impact of the Internet on authoritarian rule, Washington DC: Carnegie Endowment for International Peace, 2003.

DEIBERT Ronald et al., Access controlled, Cambridge (Mass.): MIT Press, 2010.

DEIBERT Ronald, PALFREY John, ROHOZINSKI Rafal et ZITRAIN Jonathan (dir.). Access denied, Cambridge (Mass.): MIT Press, 2008.

ELTING Bruce et al. Mapping the Arabic blogosphere: politics, culture, and dissent, Internet and democracy case studies, Harvard University: Berkman Center for Internet and Society, 2009.

LYSENKO Volodymyr et DESOUZA Kevin. Cyberprotest in contemporary Russia: The cases of Ingushetiya.ru and Bakhmina.ru, Technological Forecasting \& Social Change, 2010.

RONFELDT David. The zapatista "social netwar" in Mexico, New York: Rand Corporation, 1998.

WEIMANN Gabriel. Terror on the Internet: the new arena, the new challenges, US Institute of Peace Press, 2006.

\section{Articles}

ABBOTT Jason. "Democracy@internet.asia? The challenges to the emancipatory potential of the net: lessons from China and Malaysia", Third World Quarterly, Vol. 22, №1, 2001, p. 99114.

ADHAMI Wael. "The strategic importance of the Internet for armed insurgent groups in modern warfare", International Review of the Red Cross, vol. 89, n868, 2007, p. 857-878. 
AMIR-EBRAHIMI Masserat. "La jeunesse iranienne dans le miroir du blog", Les Cahiers de l'Orient, $\mathrm{n}^{\circ} 79,2005$, p. 43-56.

BRAY John. "Tibet, democracy and the Internet bazaar", Democratization, vol.7, $\mathrm{n}^{\circ} 1,2000$, p.157-173.

CHOWDHURY Mridul. The role of the Internet in Burma's Saffron Revolution, Berkman Center for Internet and Society, 2008, consulté le 17/07/2010 sur http://cyber.law.harvard.edu/publications/2008/Role_of_the_Internet_in_Burmas_Saffron_Re volution.

CHROUST Peter. "Neo-Nazis and Taliban on-line: anti-modern political movements and modern media", Democratization, vol. 7, n²1, 2000, p. 102-118.

DAVIS Creighton Powell. "The Internet as a Source of Political Change in Egypt and Saudi Arabia", Al Noor, 1(1), 2008, p. 32-41.

FROEHLING Oliver. "The cyberspace "war of ink and Internet" in Chiapas, Mexico", Geographical Review, vol. 87, $\mathrm{N}^{\circ}$ 2, 1997, p. 291-307.

HILL Kevin et HUGHES John. "Is the internet an instrument of global democratization?", Democratization, Vol. 6, n², 1999, p. 99 - 127.

LONKILA Markku. "The Internet and anti-military activism in Russia", Europe-Asia Studies, vol. $60, \mathrm{n}^{\circ} 7,2008$, p.1125-1149.

MANN Steve, NOLAN Jason and WELLMAN Barry. "Sousveillance: Inventing and using wearable computing devices for data collection in surveillance environments", Surveillance and Society, vol. 1, n 2, 2003, p. 331-335, consulté le 28/04/2009 sur http://www.surveillance-and-society.org/articles1(3)/sousveillance.pdf.

O'LEAR Shannon. "Networks of engagement: electronic communication and grassroots environmental activism in Kaliningrad networks of engagement: electronic communication and grassroots environmental activism in Kaliningrad", Geografiska Annaler. Series B, Human Geography, vol. 81, n 3, 1999, p. 165-178.

OTT Dana et ROSSER Melissa. "The electronic republic? The role of the Internet in promoting democracy in Africa", Democratization, vol.7, $\mathrm{n}^{\circ} 1,2000$, p.137-156.

RAHIMI Babak. "Cyberdissent: the Internet in revolutionary Iran", Middle East review of, international affairs, vol.7, $\mathrm{n}^{\circ} 3,2003$.

RAHIMI Babak. "The politics of the Internet in Iran", dans SEMATI Mehdi (dir.). Media, culture and society in Iran: living with globalization and the Islamic state, Londres:

Routledge, 2008, p. 37-55.

RUTTEN Ellen. "More than a poet? Why Russian writers didn't blog on the 2008 elections", Russian Cyberspace, vol. 1, $\mathrm{n}^{\circ}$ 1, 2009, p. 25-30.

SCHLICHTING Florian. "The freedom to speak freely: casual blogging as a way to a better life", International Symposium on "Information and Communication Technologies (ICT) and Transformation in China”, 14-15/06/2007, The University of Nottingham, résumé disponible sur 
http://www.nottingham.ac.uk/cpi/events/event_reports/ict_symposium_14_15_06_2007/highl ights_and_abstract/schlichting_florian.php.

\section{La Chine}

ARSENE Séverine. L'enseignement idéologique dans les universités chinoises, mémoire de Master Recherche, Paris: Institut d'études politiques, 2005.

BALME Stéphanie. Entre soi, l'élite du pouvoir dans la Chine contemporaine, Paris: Fayard, 2004.

BEJA Jean-Philippe. A la recherche d'une ombre chinoise, Paris: Seuil, 2004.

BERGERE Marie-Claire. Capitalismes et capitalistes en Chine: XIXe-XXIe siècle, Paris: Perrin, 2007.

CHANG Leslie. Factory girls. From village to city in a changing China, Spiegel \& Grau, 2008.

CHEN Jie. Popular political support in urban China, Washington: Woodrow Wilson Center Press et Stanford (California): Stanford University Press, 2004.

DAVIS Deborah (dir.). The consumer revolution in urban China, Berkeley: California University Press, 2000.

DAVIS Deborah, KRAUS Richard, NAUGHTON Barry, et PERRY Elizabeth. Urban spaces in contemporary China, Woodrow Wilson Center press et Cambridge University press, 1995.

FROISSART Chloé. Quelle citoyenneté pour les travailleurs migrants en République Populaire de Chine? L'expérience de Chengdu, Thèse de doctorat, Paris: Institut d'études politiques, 2007.

GOLDMAN Merle et PERRY Elizabeth (dir.). Grassroots political reform in contemporary China, Cambridge: Harvard University Press, 2007.

GOODMAN David (dir.). The new rich in China. Future rulers, present lives, London: Routledge, 2008.

HEWITT Duncan. China: getting rich first : a modern social history, New York: Pegasus Books, 2008.

HOOK Brian (dir). The individual and the state in China, Oxford: Clarendon Press, 1996.

HOWELL Jude (dir.). Governance in China, Oxford: Rowman \& Littlefield, 2004.

HSU Carolyn. Creating market socialism. How ordinary people are shaping class and status in China, Durham et Londres: Duke University Press, 2007.

KLUVER Randy et POWERS John H. (dir.). Civic discourse, civil society, and Chinese communities, Stamford: Ablex Publishing Corporation, 1999.

LALIBERTE André et LANTEIGNE Marc (dir.). The Chinese Party-state in the 21st century. Adaptation and the reinvention of legitimacy, londres et New York: Routledge, 2008. 
LU Sheldon. Chinese modernity and global biopolitics, University of Hawaii Press, 2007.

LU Xueyi. Dangdai Zhongguo shehui jieceng yanjiu baogao [Rapport d'étude sur les couches sociales de la Chine actuelle], Beijing: Shehui kexue wenxian chubanshe, 2001.

MEYER Eric. Sois riche et tais-toi! Portrait de la Chine d'aujourd'hui, Paris: Robert Laffont, 2002.

PARISH William L. et TANG Wenfang. Chinese urban life under reform, Cambridge University Press, 2000.

RIGBY Thomas H. et FEHER Ferenc (dir.). Political legitimacy in communist states, New York: St. Martin's Press, 1982.

ROCCA Jean-Louis. La condition chinoise, Paris: Karthala, 2006.

ROFEL Lisa. Desiring China. Experiments in neoliberalism, sexuality, and public culture, Durham et Londres: Duke University Press, 2007.

SAICH Tony. Governance and politics of China, New York: Palgrave, 2001.

TANG Wenfang. Public opinion and political change in China, Stanford: Stanford University Press, 2005.

THIREAU Isabelle et HUA Linshan. Les ruses de la démocratie, Paris: Seuil, 2010

THIREAU Isabelle et WANG Hansheng. Disputes au village chinois, Paris: Maison des Sciences de l'Homme, 2001.

YAN Yunxiang. Private life under socialism. Love, intimacy and family change in a Chinese village 1949-1999, Stanford University Press, 2003.

YANG Dali. Remaking the Chinese Leviathan, Stanford University Press, 2004.

\section{Articles}

"China in transition", dossier, Social Research, vol. 73, $n^{\circ} 1,2006$.

"China's changing of the guard", dossier, Journal of Democracy, vol. 14, n¹, 2003.

"L'énigme chinoise", dossier, Le Débat, n¹17, 2001, p. 73-135.

BAUM Richard. "The limits of 'authoritarian resilience' in China", conférence au CERI, Paris, 2007, consulté le 15/10/2010 sur http://www.ceri-sciences-po.org/archive/jan07/art_rb.pdf.

BEJA Jean-Philippe. "Après les émeutes au Xinjiang: répression et communication", CERI/Alternatives Internationales, 2009, consulté le 24/08/2009 sur http://www.cerisciencespo.com/archive/2009/juillet/chr_jb.pdf.

BEJA Jean-Philippe. "Chine: la vie difficile des censeurs", Esprit, n³36, 2007, p. 67-74.

BEJA Jean-Philippe. "The changing aspects of civil society in China", Social research, vol. $73, \mathrm{n}^{\circ} 1,2006$, p. 53-74. 
BILLIOUD Sébastien et THORAVAL Joël. "Jiaohua: le renouveau confucéen en Chine comme projet éducatif", Perspectives Chinoises, n¹01, 2007/4, p. 4-21.

CABESTAN Jean-Pierre. "La Chine évoluerait-elle vers un autoritarisme 'éclairé' mais ploutocratique?", Perspectives chinoises, n84, 2004, p.21-29.

CAI Yongshun. "China's moderate middle class", Asian Survey, vol.45, n5, 2005, p.777-799.

CHANG Chen-pang. "The resurgence of the bourgeoisie", Issues and Studies, vol. 30, n5, mai 1994, p. 31-44.

CHEN Jie et LU Chunlong. "Does China's middle class think and act democratically? Attitudinal and behavioral orientations towards self-government", Journal of Chinese Political Science, vol.11, n², automne 2006, p.1-20.

CHEVRIER Yves. "De la révolution à l'Etat par le communisme", Le Débat, n¹17, 2001, p. 92-113.

DAVIS Deborah. "Urban Chinese homeowners as citizen-consumers" in GARON Sheldon et MACLACHLAN Patricia (ed.). The ambivalent consumer : questioning consumption in East Asia and the West, New York: Ithaca, Cornell University Press, 2006.

FAURE Guy Olivier. "Chinese society and its new emerging culture", Journal of contemporary China, vol. 17, $\mathrm{n}^{\circ}$ 56, août 2008, p. 469-491.

FROISSART Chloé. "Le système du 'hukou': pilier de la croissance chinoise et du maintien du PCC au pouvoir", Etudes du CERI, n²149, septembre 2008, p.1-47.

GENTELLE Pierre. "Une société en mouvement", Questions internationales, ${ }^{\circ} 6$, mars-avril 2004, p.26-38.

GIROIR Guillaume. "Les fractures socioterritoriales en Chine, ou l'impossible 'société harmonieuse'?", Perspectives chinoises, n²007/3, p. 88-97.

GODEMENT François. "Chine: la transition sans fin", Politique étrangère, Vol. 64, $\mathrm{n}^{\circ} 3$, 1999, p. 557-571.

HAND Keith J. "Using law for the righteous purpose: the Sunzhigang incident and evolving forms of citizen action in the people's republic of China", Columbia Journal of transnational law, n¹14, 2006.

HOFFMAN Lisa. "Autonomous choices and patriotic professionalism: on governmentality in late-socialist China", Economy and society, vol. 35, n² 4, novembre 2006, p. 550-570.

KIPNIS Andrew. "Neoliberalism reified: suzhi discourse and tropes of neoliberalism in the People's Republic of China", Journal of the Royal Anthropological Institute, n²13, 2007, p. 383-400.

KIPNIS Andrew. "Suzhi: a keyword approach", The China Quarterly, n¹86, juin 2006, p. 294-313.

KWONG Julia. "Ideological crisis among China's youths: values and official ideology", The British journal of sociology, vol. 45, n², juin 1994, p. 247-264.

LEIB Ethan. "The Chinese communist party and deliberative democracy", Journal of public deliberation, vol.1, n $1,2005$. 
LEYS Simon. "Les habits neufs du président Mao", in Essais sur la Chine, Paris: Robert Laffont, 1998, p. 1-227.

LI He. "Emergence of the Chinese middle class and its implications", Asian Affairs, vol.33, n², été 2006, p. 67-83.

LI Jian et NIU Xiaohan. "Accès à la propriété et formation d'une classe moyenne à Pékin", Perspectives chinoises , $\mathrm{n}^{\circ} 74,11-12 / 2008$, p. 4-20.

LI Peilin. "The building of a harmonious society and the 'China experience'", Social sciences in China, vol.28, automne 2007, p. 98-107.

MURPHY Rachel. "Turning peasants into modern Chinese citizens: 'population quality' discourse, demographic transition and primary education", The China Quarterly, ${ }^{\circ} 177$, mars 2004, p. 1-20.

NATHAN Andrew. "Authoritarian resilience", Journal of democracy, vol.14, n¹, p. 6-17, 2003.

NEE Victor. "The emergence of a market society", American sociological review, ${ }^{\circ} 56$, vol.3, p. 267-282.

PERRY Elizabeth. "Studying Chinese politics: farewell to revolution?", The China Journal, n'57, janvier 2007, p. 1-22.

ROCCA Jean-Louis. "Political crossroad, social representations and academic intervention: the formation of a middle class in China", communication au International Forum on Comparative Studies on MiddleClass, Changsha (Hunan), Juillet 2007.

ROFEL Lisa. "Liberation nostalgia and yearning for modernity", in GILMARTIN Christina, HERSHATTER Gail, ROFEL Lisa et WHITE Tyrene. Engendering China, Cambridge (Mass.): Harvard University Press, 1994, p. 226-249.

ROSEN Stanley. "The victory of materialism: aspirations to join China's urban moneyed

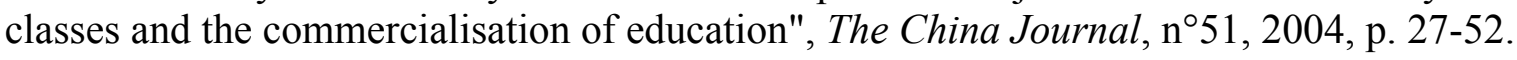

SIGLEY Gary. "Chinese governmentalities: government, governance and the socialist market economy", Economy and society, vol.35, n4, 2006, p. 487-508.

SIGLEY Gary. "Liberal despotism: population planning, subjectivity, and government in contemporary China", Alternatives, n²9, 2004, p. 557-575.

THIREAU Isabelle et HUA Linshan. "De l'épreuve publique à la reconnaissance d'un public: le scandale Sun Zhigang", Politix, vol.18, n71, 2005, p 137-164.

TOMBA Luigi. "Creating an urban middle class: social engineering in Beijing", The China Journal, n51, 2004, p. 1-26.

TOMBA Luigi. "Of quality, harmony and community: civilization and the middle class in urban China", Positions: East Asia cultures critique, 2009.

WANG Shaoguang. "Changing models of China's policy agenda setting", Modern China, vol. $34, \mathrm{n}^{\circ} 1,2008$, p. 56-87. 
WANG Xin. "Divergent identities, convergent interests: the rising middle-income stratum in China and its civic awareness", Journal of contemporary China, vol. 17, $\mathrm{n}^{\circ}$ 54, 2008, p. 53 69.

ZANG Xiaowei. "The consolidation of political technocracy in china: The fourteenth and fifteenth central committees of the CCP", Journal of Communist Studies and Transition Politics, vol. 15, n³, p.101, 1999.

ZHANG Houyi. "The position of the private entrepreneur stratum in China's social structure", Social Sciences in China, vol.16, n4, 1995, p.29-36.

ZHOU Xiaohong. "La classe moyenne chinoise: réalité ou illusion?", traduit par Mylène Hardy et Jean-Louis Rocca, Actes du colloque Quels outils pour analyser les sociétés contemporaines?, Pékin: Université Tsinghua, 19 et 20 mai 2007.

ZHOU Xueguang. "Unorganized interests and collective action in communist China", American sociology review, vol.58, ${ }^{\circ} 1,1993$, p. 54-73.

\section{Médias et Internet en Chine}

CHASE Michael et MULVENON James. You've got dissent! Chinese dissident use of the Internet and Beijing's counter-strategies, Santa Monica: RAND, 2002.

CHINE. Voyage au cour de la censure d'Internet, Reporters sans frontières, octobre 2007, consulté le 20/01/2009 sur

http://www.rsf.org/IMG/pdf/Voyage_au_coeur_de_la_censure_FR.pdf.

COLOMB Dominique. Médias et communication en Chine. Au-delà des paradoxes, Paris:

L'Harmattan, 2008.

GUO Liang. Surveying Internet usage and its impact in seven Chinese cities, Markle

Foundation, 2007.

HASKI Pierre. Internet et la Chine, Paris: Seuil, 2008.

Internet Filtering in China, Opennet Initiative, consulté le 25/06/2009 sur

http://opennet.net/sites/opennet.net/files/ONI_China_2009.pdf.

LEE Chin-Chuan (dir.). Power, money, and media. Communication patterns and bureaucratic control in cultural China, Evanston: Northwestern University Press, 2000.

LYNCH Daniel. After the propaganda State. Media, politics, and "thought work" in reformed China, Stanford: Stanford University Press, 1999.

MENGIN Françoise (dir). Cyber China, Reshaping national identities in the age of information, New York: Palgrave Macmillan, 2004.

QIU Jack. Working-class network society. Communication technology and the information have-less in urban China, Cambridge (Mass.): MIT Press, 2009.

Statistical survey report on the Internet development in China, Pékin: CNNIC, 2009, consulté sur www.cnnic.net.cn.

TAI Zixue. The Internet in China. Cyberspace and civil society, New York et Londres: Routledge, 2006. 
TSUI Lokman. Internet in china: big mama is watching you, Mémoire de Master, Chinese Languages \& Cultures, University of Leiden, July 2001, consulté le 18/11/2008 sur http://lokman.nu/thesis/.

WU Xu. Chinese Cyber Nationalism: Evolution, Characteristics and Implications, Plymouth: Lexington Books, 2007.

ZHAO Yuezhi. Communication in China : political economy, power, and conflict, Lanham: Rowman \& Littlefield, 2008.

ZHAO Yuezhi. Media, market, and democracy in China. Between the party line and the bottom line. Urbana et Chicago: University of Illinois Press, 1998.

ZHENG Yongnian. Technological empowerment. The Internet, State, and society in China, Stanford University Press, 2008.

ZHOU Yongming. Telegraphy, the Internet, and Political Participation in China, Stanford University Press, 2006.

\section{Articles}

BERG Daria. "Reality shows and urban utopia: cyberspace, consumerism and the new cultural discourse in twenty-first century China", International Symposium on "Information and Communication Technologies (ICT) and Transformation in China”, 14-15/06/2007, The University of Nottingham, résumé disponible sur http://www.nottingham.ac.uk/cpi/events/event_reports/ict_symposium_14_15_06_2007/highl ights_and_abstract/berg_daria.php.

CHAN Alex. "Guiding public opinion through social agenda-setting: China's media policy since the 1990s", Journal of contemporary China, vol.16 n53, 2007, p. 547-559.

DAMM Jens. "The Internet and the fragmentation of Chinese society", Critical Asian Studies, vol. 39, n 2, 2007, p. 273-294.

DEIBERT Ronald. "Dark Guests and Great Firewalls: the Internet and Chinese security policy", Journal of social issues, vol.58 $\mathrm{n}^{\circ} 1,2002$, p. 143-159.

DOUZET Frédérick. "Les frontières chinoises de l'Internet", Hérodote, n¹25, 2007, p. 127 143.

ESAREY Ashley et XIAO Qiang. "Political expression in the Chinese blogosphere. Below the radar", Asian Survey, vol. 48, n 5, 09/10 2008, p. 752-772.

FALLOWS Deborah. "Most Chinese say they approve of government Internet control", PEW Internet and American Life Project, 27/03/2008, consulté le 26/06/2009 sur http://www.pewinternet.org/Reports/2008/Most-Chinese-Say-They-Approve-of-GovernmentInternet-Control.aspx.

GIESE Karsten. "Speakers corner or virtual panopticon: discursive construction of Chinese identities online", in MENGIN Françoise (dir). Cyber China, Reshaping national identities in the age of information, New York: Palgrave Macmillan, 2004, p. 19-36. 
HOM Sharon, TAI Amy et NICHOLS Gabriel. "The rise of the Internet and advancing human rights", Human Rights in China, China Rights Forum n ${ }^{\circ} 3$, 2004, consulté en ligne le 06/08/2008 sur http://www.hrichina.org/fs/view/downloadables/pdf/downloadableresources/The_Rise_of_the_Internet_and_Advancing_Human_Rights.pdf

HU Yong. "Why remain independent and how to be alternative?", in HU Yong, FENG Chien San et MCKINNON Rebecca. Info-Rhizome: Report on independent media in the Chinesespeaking world, Hong Kong: Hong Kong In-Media, 2009, p. 13-16.

HUGHES Christopher. "Controlling the Internet architecture within Greater China", in MENGIN Françoise (dir). Cyber China, Reshaping national identities in the age of information, New York: Palgrave Macmillan, 2004, p. 71-90.

HUGHES Christopher. "Pourquoi Internet ne démocratisera pas la Chine", Critique internationale, $\mathrm{n}^{\circ} 15$, avril 2002, p.85-104.

HUNG Ching-Fu. "Public discourse and 'virtual' political participation in the PRC: the impact of the Internet", Issues \& Studies, vol. 39, n²4, 2003, p1-38.

LAGERKVIST Johan. "In the crossfire of demands. Chinese news portals between propaganda and the public", in DAMM Jens et THOMAS Simona, Chinese cyberspaces. Technological changes and political effects, Londres et New York: Routledge, 2006, p. 4263.

LAGERKVIST Johan. "Internet Ideotainment in the PRC: national responses to cultural globalization", Journal of Contemporary China, vol. 17, n54, 2008, p. 121-140.

LAGERKVIST Johan. "The rise of online public opinion in the People's Republic of China", China: An international journal, vol.3, ${ }^{\circ} 1$, mars 2005, p. 119-130.

LAGERKVIST Johan. "The techno-cadre's dream: administrative reform by electronic governance in China today?", China Information, n¹9, 2005, p. 189-216.

LATHAM Kevin. "Nothing but the truth: news media, power and hegemony in South China", The China Quarterly, ${ }^{\circ} 163,2000$, p. 633-654.

LEE Hsiao-Wen. "The popular press and its public in contemporary China", article présenté au Congrès annuel de l'International Communication Association - ICA, Montréal, mai 2008.

LI Yanling, DAI Guanzhong, ZHU Yehang et QIN Sen, "A high-performance extraction method for public opinion on internet ", Wuhan: Wuhan University Journal of Natural Sciences, vol.12 n5, septembre 2007, p. 902-906.

MCKINNON Rebecca. "China's censorship 2.0: how companies censor bloggers", First Monday, Vol.14, n², février 2009, consulté le 13/02/2009 sur http://www.uic.edu/htbin/cgiwrap/bin/ojs/index.php/fm/article/view/2378/2089.

MCKINNON Rebecca. "Flatter world and thicker walls ? Blogs, censorship and civic discourse in China", Public Choice, ${ }^{\circ}$ 134, 2008, p. 31-46.

MIN Jiang. "Authoritarian deliberation: public deliberation in China", article présenté au Congrès annuel de l'International Communication Association - ICA, Montréal, mai 2008.

SAUTEDE Eric. "Les leurres de la modernité, Internet, information et crise du "SRAS" en Chine", Perspectives chinoises, mars-avril 2003, nº76, p. 21-27. 
SHIE Tamara Renee. "The tangled web: does the internet offer promise or peril for the Chinese Communist Party?", Journal of contemporary China, vol.13 n40, 2004, p. 523-540.

SIMA Yangzi et PUGSLEY Peter. "The rise of a 'me culture' in postsocialist China", The International Communication Gazette, vol. 72, n³, 2010, p. 287-306.

SONG Gang. "Transcending e-government: a case of mobile government in Beijing", communication, Brighton, 2005, consulté le 05/11/2008 sur http://mobility.grchina.com.

SUN Sun Lim. "From cultural to information revolution. ICT domestication by middle-class Chinese families", in BERKER Thomas, HARTMANN Maren, PUNIE Yves et WARD Katie. Domestication of media and technology, Maidenhead: Open University Press, 2007.

TAUBMAN Geoffry. "A not-so World Wide Web: The Internet, China and the challenges to nondemocratic rule", Political Communication, vol. 15, n² 2, 1998, p.255-273.

TSUI Lokman. "An inadequate metaphor: the Great Firewall and Chinese Internet censorship”, Global Dialogue, vol.9, $n^{\circ} 1-2,2007$, p.60-68.

WEBER Ian et LU Jia, "Internet and self-regulation in China: the cultural logic of controlled commodification", Media, Culture \& Society, Vol. 29, n 5, 2007, p. 772-789.

WU Mei. "E-publics: political participation in Chinese discussion forums", communication à la International Conference on Media and Democracy in the Knowledge Society, Séoul, 2006.

XIAO Qiang. "The rise of online public opinion and its political impact", communication à la Chinese Internet Research Conference, Université de Hong Kong, 2008, consulté le 09/07/2009 sur http://jmsc.hku.hk/blogs/circ/files/2008/06/xiao_qiang.pdf.

YANG. Guobin. "The co-evolution of the Internet and civil society in China", Asian Survey, vol. XLIII, n³, mai-juin 2003, p.405-422.

ZHANG Junhua. "China's "government online" and attempts to gain technical legitimacy", ASIEN, $\mathrm{n}^{\circ} 80,2001$.

ZHANG Junhua. "Will the government 'serve the people'? The development of Chinese egovernment", New media and society, vol. 4, n² 2, p. 163-184.

ZHAO Yuezhi et SUN Wusan. "Public opinion supervision: possibilities and limits of the media in constraining local officials", in GOLDMAN Merle et PERRY Elizabeth (dir.). Grassroots political reform in contemporary China, p. 300-324.

ZHOU Xiang. "Is an online public sphere emerging in China? A comparative study of deliberativeness of Chinese online discussion forums", Article présenté à la Convention de l'Association internationale de communication, Montréal, Mai 2008.

ZHOU Yongming. "Living on the cyber border. Minjian political writers in Chinese cyberspace", Current anthropology, vol.46 n5, Dec.2005, p 779-803.

ZYW Anna. "Les avocats chinois, promoteurs d'un réseau juridique virtuel", Hermès, $\mathrm{n}^{\circ}$ 55, 2009, p. 65-70. 


\section{Table des matières}

$\begin{array}{ll}\text { Remerciements } & 2\end{array}$

$\begin{array}{ll}\text { Avant-propos } & 4\end{array}$

Introduction Internet et prise de parole politique en Chine 12

1 - La formation d'un espace public complexe et conflictuel 13

2 - Le cadrage de la prise de parole 38

3 - Méthodologie 63

INCARNER L'IMAGE D'UNE CHINE "MODERNE" 76

Chapitre I - La quête de "modernité" des internautes chinois 79

1 - Etre un internaute en Chine $\quad 79$

2 - Internet, l'attribut d'une génération "moderne" 88

$\begin{array}{ll}\text { Chapitre II - Vers une forme singulière d'individualisme } & 104\end{array}$

1 - L'expression individuelle au cœur des contradictions de la modernité $\quad 107$

2 - Le triomphe des liens faibles $\quad 121$

Chapitre III - L'évaporation de la politique $\quad 135$

$\begin{array}{ll}1 \text { - La politique confisquée } & 137\end{array}$

2 - La politique dévoyée 149

3 - Les voies détournées de la curiosité politique 157

LES CONTRAINTES D'UNE CIVILITE "MODERNE" 165

$\begin{array}{lc}\text { Chapitre IV - La responsabilisation de l'individu } & 168\end{array}$

$\begin{array}{ll}1 \text { - Les gardiens de l'ordre public: de la police aux individus } & 168\end{array}$

2 - Les règles de la civilité 183

$\begin{array}{lr}\text { Chapitre V - La clôture du domaine public } & 198\end{array}$ 
1 - Une fragile unité nationale 198

2 - Parole publique et représentation 203

3 - La dévalorisation des conflits 211

Chapitre VI - Les conversations en "clair-obscur" 222

1 - La diversité des points de vue individuels 223

2 - L'espace "clair-obscur" des conversations 235

$\begin{array}{ll}\text { L'IRRUPTION DU POLITIQUE } & 250\end{array}$

Chapitre VII - La modernité: un registre polysémique $\quad 254$

1 - L'idéal de modernité au cœur des mobilisations 255

2 - Un registre discursif pour la défense des droits des citoyens 267

3 - Stratégies de mise en récit 274

Chapitre VIII - La prise de parole: des postures diverses $\quad 283$

1 - La circulation silencieuse des causes politiques 284

2 - L'acquiescement comme chambre de résonance 293

3 - Le rôle charnière des porteurs de causes 299

Chapitre IX - La gouvernance chinoise de l'opinion publique 316

1 - L'avènement d'une opinion publique restreinte 317

2 - L'opinion publique au service de la gouvernance 322

3 - L'opinion publique comme enjeu de pouvoir 333

$\begin{array}{ll}\text { Conclusion } & 346\end{array}$

$\begin{array}{ll}\text { Liste des entretiens } & \mathbf{3 5 0}\end{array}$

$\begin{array}{ll}\text { Bibliographie } & 354\end{array}$

Ouvrages généraux 354

Internet et politique (Europe et monde anglo-saxon) 358

Internet et politique (contextes autoritaires) 360

$\begin{array}{ll}\text { La Chine } & 362\end{array}$ 
Médias et Internet en Chine

Table des matières 